\title{
Second-generation drug-eluting stents and beyond
}
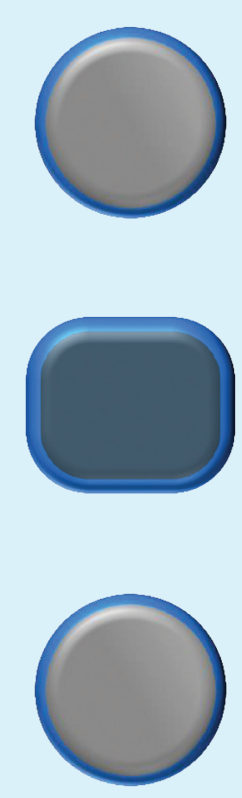

Liefke van der Heijden
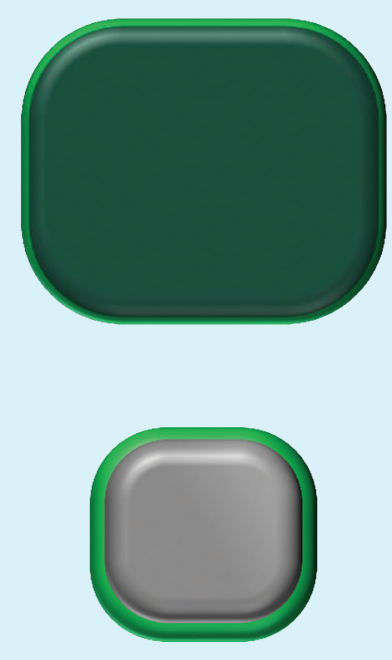


\section{Second-generation drug-eluting stents and beyond}

Liefke Clementine van der Heijden 


\section{COLOFON}

Lay-out: Legatron Electronic Publishing, Rotterdam

Printed by: Ipskamp Printing, Enschede

ISBN: 978-90-365-4419-1

This thesis is a part of the Health Sciences Series of the Health Technology and Services Research Department, University of Twente, the Netherlands: HSS 17-19. ISSN: 1878-4968.

C2017 Liefke Clementine van der Heijden.

All rights reserved. No parts of this thesis may be reproduced, stored in a retrieval system or transmitted in any form or by any means without prior permission of the author.

Financial support for printing this thesis was funded by Stichting Kwaliteitsverbetering Cardiologie, Lide Jannink Stichting namens het KNMG district Twente, Stichting Thoraxcentrum Twente, CICON B.V., Medical School Twente, and HSS 14-012, department of Health Technology and Services Research, University of Twente, Enschede. ISSN 1878-4968.

Financial support by the Dutch Heart Foundation for the publication of this thesis is gratefully acknowledged. 


\title{
SECOND-GENERATION DRUG-ELUTING STENTS AND BEYOND
}

\author{
DISSERTATION \\ to obtain \\ the degree of doctor at the University of Twente \\ on the authority of the rector magnificus, \\ Prof. dr. T.T.M. Palstra, \\ on account of the decision of the graduation committee, \\ to be publicly defended \\ on Wednesday 6 December 2017 at 16.45
}

by

Liefke Clementine van der Heijden

Born on 30 July 1989

In Apeldoorn, the Netherlands 
This dissertation has been approved by the promotors:

Prof. dr. Clemens von Birgelen

Dr. Carine J.M. Doggen 


\section{Members of the committee}

\section{Chairman/Secretary}

Prof. dr. Th. A.J. Toonen

\section{Promotor}

Prof. dr. C. von Birgelen

\section{Co-promotor}

Dr. C.J.M. Doggen

\section{Other members}

Prof. dr. J.G. Grandjean

Prof. dr. J. van der Palen

Prof dr. R.H. Geelkerken

Prof dr. J.C.A. Hoorntje

Prof. dr. R.J. de Winter

Prof. dr. J.J. Kolkman
University of Twente, Enschede

University of Twente, Enschede

University of Twente, Enschede

University of Twente, Enschede

University of Twente, Enschede

University of Twente, Enschede

University of Maastricht

University of Amsterdam

University Medical Center Groningen 



\section{Table of contents}

Part I: General introduction

CHAPTER 1 General Introduction

Outline of the Thesis

Part II: Assessment of broad patient populations

CHAPTER 2 Five-Year Outcome After Implantation of Zotarolimus- and Everolimus-Eluting Stents in Randomized Trial Participants and Non-Enrolled Eligible Patients:

A Secondary Analysis of a Randomized Clinical Trial

JAMA Cardiol. 2017;2:268-76.

CHAPTER 3 Three-Year Safety and Efficacy of Treating All-Comers With Newer-Generation Resolute Integrity or Promus Element Stents in the Randomized DUTCH PEERS (TWENTE II) Trial Eurointervention. 2017;12:2128-31.

CHAPTER 4 Three-Year Clinical Outcome of Patients With Coronary Disease and Increased Event Risk Treated With Newer-Generation Drug-Eluting Stents: From the Randomized DUTCH PEERS Trial

Cardiology. 2017;137:207-17.

CHAPTER 5 Clinical Outcomes After Percutaneous Coronary Intervention With the COMBO Stent Versus Resolute Integrity and Promus Element Stents: A PropensityMatched Analysis Eurointervention 2017. Epub ahead of print.

CHAPTER 6 Very Thin Strut Biodegradable Polymer Everolimus-Eluting and SirolimusEluting Stents Versus Durable Polymer Zotarolimus-Eluting Stents in Allcomers With Coronary Artery Disease (BIO-RESORT): A Three-Arm, Randomised, Non-Inferiority Trial Lancet. 2016;388:2607-17

CHAPTER 7 Safety and Efficacy of Everolimus-Eluting Bioresorbable Vascular Scaffolds Versus 111 Durable Polymer Everolimus-Eluting Metallic Stents Assessed at 1-Year Follow-Up: A Systematic Review and Meta-Analysis of Studies Int J Cardiol. 2016;221:1087-94. 
CHAPTER 7.1 Reply to Letter to the Editor entitled: "Bioresorbable stent thrombosis, lactic acid release and Kounis syndrome"

Int J Cardiol. 2017; 247:16.

CHAPTER 8 Bioresorbable Polymer-Coated Orsiro Versus Durable Polymer-Coated Resolute Onyx Stents (BIONYX): Rationale and Design of the Randomized TWENTE IV Multicenter Trial

Submitted

\section{Part III: Contemporary drug-eluting stents for complex lesions}

CHAPTER 9 Long-Term Outcome of Consecutive Patients With Previous Coronary Bypass

Surgery, Treated With Newer-Generation Drug-Eluting Stents

Submitted

CHAPTER 10 Bifurcation Treatment With Novel, Highly Flexible Drug-Eluting Coronary Stents in All-Comers: 2-Year Outcome in Patients of the DUTCH PEERS Trial Clin Res Cardiol. 2016;105:206-15.

CHAPTER 11 Long-Term Outcome and Chest Pain in Patients With True Versus Non-True Bifurcation Lesions Treated With Second-Generation Drug-Eluting Stents in the TWENTE Trial Heart and Vessels. 2016;31:1731-9

CHAPTER 12 Clinical Outcome of All-Comer Patients With Severely Calcified Bifurcation 199 Lesions Treated With New-Generation Coronary Drug-Eluting Stents: A Patient-Level Pooled Analysis From TWENTE and DUTCH PEERS (TWENTE II) Submitted

CHAPTER 13 Small Vessel Treatment With Contemporary Newer-Generation Drug-Eluting Coronary Stents in All-Comers: Insights From 2-Year DUTCH PEERS (TWENTE II) Randomized Trial

Am Heart J. 2016;176:28-35

CHAPTER 14 Impact of Severe Lesion Calcification on Clinical Outcome of Patients with Stable Angina, Treated with Newer Generation Permanent Polymer-Coated Drug-Eluting Stents: A Patient-Level Pooled Analysis From TWENTE and DUTCH PEERS (TWENTE II)

Am Heart J. 2016;175:121-9. 
CHAPTER 15 Two-Year Outcome After Treatment of Severely Calcified Lesions With Newer-Generation Drug-Eluting Stents in Acute Coronary Syndromes: A Patient-Level Pooled Analysis From TWENTE and DUTCH PEERS J Cardiol. 2017;69:660-5.

CHAPTER 16 Three-Year Outcome of Patients With Severely Calcified Coronary Lesions Treated With Different Everolimus-Eluting Stents: A Propensity Score-Adjusted Analysis of TWENTE and DUTCH PEERS Trial Participants, Treated by the Same Operators

Submitted

Part IV: General discussion and future perspectives

Portfolio 



\section{Part I}

General introduction 


\section{CHAPTER 1}

General Introduction

Outline of the Thesis

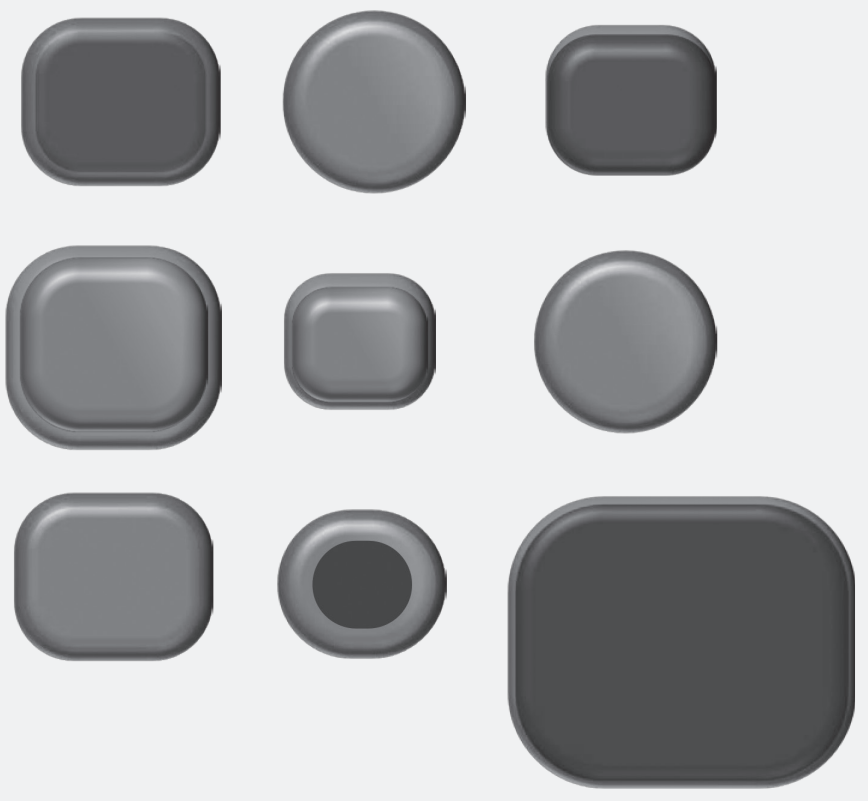




\section{General Introduction}

\section{From plain old balloon angioplasty to second-generation drug-eluting stents}

In patients with severe coronary artery lesions, interventional procedures are performed to improve myocardial perfusion. Historically, coronary artery bypass grafting (CABG) was the standard revascularization therapy for symptomatic patients. ${ }^{1}$ In 1977, Andrea Grüntzig introduced a new technique, the 'plain old balloon angioplasty' (POBA), which is used to dilate a narrowed coronary vessel by advancing and inflating a small balloon in the obstructive atherosclerotic lesion. ${ }^{2}$ Nevertheless, the use of POBA was limited by the risk of abrupt vessel closure due to coronary dissections and the relatively high incidence of restenosis during the first 6 months after the interventional procedure. ${ }^{3}$ Then, coronary stents were (initially) developed to counteract the risk of acute vessel closure following POBA. ${ }^{4}$

Bare metal stents (BMS), the first coronary stents, were first applied in humans in $1986 .{ }^{5}$ The BMS is able to provide a platform that seals dissection flaps and prevents acute lesion recoil ${ }^{5}$, which resulted in a significant reduction of lesion recurrence during follow-up. ${ }^{6}$ Nevertheless, despite the use of BMS, $20-30 \%$ of patients still required a repeat revascularization procedure. ${ }^{7}$

In order to resolve this problem of restenosis, drug-eluting stents (DES) were developed. DES consist of three components: a metallic stent platform, a polymer coating, and an anti-proliferative drug. ${ }^{8}$ After the implantation of a DES, the coating releases the anti-proliferative drug over a period of time in order to prevent an excess of the local proliferative response of the traumatized vessel wall. As a result of the anti-proliferative effects of the drug, endothelialization and healing processes are delayed in coronary lesions treated with DES. Several randomized trials demonstrated the effectivity of these "first-generation" DES in reducing neointimal proliferation and the need for repeat revascularization. ${ }^{9-11}$ Despite an improvement in morbidity with first-generation DES, mortality was not reduced; this was mainly related to the increased incidence of late and very late stent thrombosis following stenting with first-generation DES. ${ }^{12,13}$ In addition, while DES reduced overall the need for repeat revascularization, the rate of this adverse event was still quite high in patients with advanced coronary disease. ${ }^{14}$ The lack of biocompatibility of the coating of first-generation DES was shown to sometimes be associated with hypersensitivity and vessel wall inflammation which both can promote stent thrombosis. ${ }^{15,16}$ Secondgeneration DES were developed to overcome the limitations of the first-generation DES, in particular the increased risk of stent thrombosis.

\section{Second-generation drug-eluting stents and beyond}

Second-generation DES had, as compared to first-generation DES, novel stent coatings with greater biocompatibility, which reduced their potential of inducing an inflammatory response of the vessel wall and ultimately reduced the risk of late and very late stent thrombosis. An important secondgeneration DES is the everolimus-eluting stents (EES), which was non-inferior to the first generation sirolimus-eluting stent after 18 months of follow-up. ${ }^{17}$ The first-generation paclitaxel-eluting stent had a significantly higher rate of adverse outcome than the second-generation EES. ${ }^{18}$ Later on, randomized comparison of second-generation zotarolimus-eluting Resolute ZES (R-ZES) and EES showed noninferiority of the R-ZES versus the EES..$^{16,19}$ 
Further refinement of DES involved an increase in flexibility of the metallic stent platform to increase deliverability and stent apposition to the vessel wall to tackle the most challenging lesions and anatomies. ${ }^{20}$ Disadvantage of such increase in flexibility may be a reduction in longitudinal stability of the device, which may be related to procedure-related complication such as longitudinal stent deformation. ${ }^{21}$ In the randomized DUTCH PEERS trial, two of the newer-generation DES, the cobalt chromium-based Resolute Integrity ZES and the platinum chromium-based Promus Element EES, were assessed for the first time in all-comer patients. While short-term follow-up showed similar effectivity and safety of both stents, no long-term data are available. ${ }^{21,22}$ Long-term follow-up of randomized clinical trials is a prerequisite for trustworthy judgment of the long-term effects of $\mathrm{PCl}$, as certain between-stent differences may only be discovered after years. So far only limited long-term data are available from large-scale randomized trials in all-comers. ${ }^{23,24}$

In parallel with the refinement of durable-polymer coated DES, stents with biodegradable coatings were developed. This advancement in coronary device technology aims at reducing (and ultimately perhaps eliminating) very late stent thrombosis and excessive growth of neointimal tissue with formation of neo-atherosclerosis - issues that are still relevant to the latest generation of durable polymer DES. ${ }^{25}$ Growing awareness of these risks associated with durable polymer DES motivated the development of stents with biodegradable coatings that leave only a bare metal stent after polymer resorption. Early biodegradable polymer stents had thick stainless steel struts and showed in large all-comer trials similar efficacy and better long-term safety as compared to early-generation durable polymer stents that also had thick struts. ${ }^{26,27}$ However, in another all-comers trial, similar thick-strut biodegradable polymer stents did not show non-inferiority as compared to early-generation durable polymer stents. ${ }^{28}$ Equivocal results were reported when comparing early biodegradable polymer stents with new-generation durable polymer stents with thin cobalt-chromium struts. ${ }^{29-31}$

The latest technical development are bioresorbable vascular scaffolds (BVS) that aim at providing a finite period of vascular support after device implantation. ${ }^{32,33}$ The theoretical advantages of BVS include the preservation of vessel geometry, adaptive vascular remodeling, and restoration of physiological vasomotion, which offer the prospect of late luminal expansion. ${ }^{32,34-36}$ Safety and efficacy of BVS have not yet been fully established, and in some types of these devices there are concerns for a greater risk of scaffold thrombosis before the human body has resorbed the device. ${ }^{37}$

\section{Complex lesions}

While short-term follow-up of second-generation and newer-generation stents are well examined in randomized clinical trials in all-comer patients, improvements in DES technology may be of benefit in patients with challenging target lesions such as lesions in degenerated saphenous vein grafts, small coronary vessels, and bifurcated or severely calcified coronary segments.

Patients with a history of CABG are often older, have more comorbidities, and require treatment of more complex lesions. ${ }^{38-40} \mathrm{Gradual}$ failure of a bypass graft, lesion recurrence, and disease progression in native coronary vessels are the main causes of repeat revascularization in patients with a history of CABG. ${ }^{41-44}$ While $\mathrm{PCl}$ in patients with previous CABG are often complex and more often associated with adverse clinical outcome, $\mathrm{PCl}$ is first choice for treating late graft failure because of an increased mortality risk associated with redo-CABG. ${ }^{45-49}$ In patients with previous $C A B G$ undergoing $\mathrm{PCl}$, the use 
of first-generation DES was associated with improved long-term clinical outcomes as compared to bare metal stents, but there is little known about the results of using second-generation DES in this setting. ${ }^{50-52}$

Stenting of bifurcated target lesions is among the most challenging procedures in the field of $\mathrm{PCl}$ and associated with an increased risk of adverse events, in particular of target vessel myocardial infarction (MI). ${ }^{53,54}$ True bifurcation lesions are characterized by an advanced atherosclerotic disease burden that obstructs at bifurcation level both the main vessel and the side-branch. PCl of true bifurcation lesions are often technically more challenging, require more often two-stent techniques, and have previously been associated with somewhat lower technical success rate and a higher restenosis risk than $\mathrm{PCl}$ of non-true bifurcation lesions. ${ }^{55-59}$ Virtual bench tests to assess the impact of technical characteristics of DES platforms on stenting in bifurcated lesions have shown that technical features of DES platforms lead to differences in response to similar procedural steps of provisional stenting, such as final kissingballoon inflation. ${ }^{60}$ Therefore, it is of interest to assess in the complex patient and lesion populations of randomized all-comer trials the potential impact of the type of DES implanted on clinical outcome. Percutaneous treatment of lesions in small coronary vessels has always been associated with a higher incidence of restenosis as compared to PCl in larger sized vessels. ${ }^{61-65}$ Several studies have shown an impact of the stent type used on clinical outcome, especially on the incidence of lesion recurrence. ${ }^{62,63,66-68}$ Strut thickness, lesion length, and the minimum stent lumen diameter were previously identified as independent predictors of restenosis in DES implanted in small vessel lesions. .4,65,69-71 $^{\text {The development }}$ of new-generation DES with thinner struts and high flexibility may be of benefit for the treatment of small vessel lesions.

$\mathrm{PCl}$ in patients with severely calcified target lesions is associated with an increased risk of suboptimal procedural results and adverse clinical events..$^{72}$ In patients treated with BMS and first-generation DES, severe target lesion calcification was a strong predictor of adverse outcome, in particular an increase need for repeat target lesion revascularization. ${ }^{73,74}$ The increased event risk following stenting of calcified coronary lesions has been related to an impaired expansion and incomplete apposition of stents. ${ }^{75,76}$ In addition, coronary calcium is radiopaque and interferes with the X-ray-based visualization of stents. ${ }^{77}$ Therefore, refinements in DES, which aim at improving the radial force and radiographic visibility of stents as well as the conformability of these implants to vascular geometry, may be particularly relevant for treating severely calcified coronary lesions. ${ }^{77}$ 


\section{Outline of the Thesis}

The first part of this thesis focuses on the safety and efficacy of treating all-comer patients with newergeneration DES and BVS.

- In Chapter 1, a general introduction is provided about the development of newer-generation DES and the importance of stent design and characteristics in patients with complex lesions.

- In Chapter 2, we assess the long-term outcome following treatment with second-generation drugeluting stents in a consecutive series of "most-comer" patients - a broad study population with minimum exclusion criteria.

- In Chapter 3, we evaluate the three-year outcome data of all-comer patients treated in a randomized fashion with the newer-generation zotarolimus-eluting Resolute Integrity versus everolimus-eluting Promus Element stents.

- In Chapter 4, we assess the three-year safety and efficacy of treating high-risk patients with newergeneration DES.

- In Chapter 5, we performed a propensity matched analysis of clinical outcomes after PCI with the COMBO stent versus Resolute Integrity or Promus Element stents.

- In Chapter 6, we describe the one-year clinical outcome of a large, prospective, randomized, threearm, multicenter trial, comparing three contemporary DES with either durable or bioresorbable polymer coatings.

- In Chapter 7, we perform a meta-analysis to compare the safety and efficacy of everolimus-eluting BVS versus durable polymer-coated everolimus-eluting metallic stents.

- In Chapter 8, we discuss the rationale and design of the BIONYX trial, a randomized comparison of a biodegradable polymer and a novel newer-generation DES in all-comer patients.

In the second part of this thesis we discuss the impact of complex lesions on clinical outcome of $\mathrm{PCl}$ with newer-generation DES.

- In Chapter 9, we evaluate the impact of previous CABG on long-term clinical outcome after PCI with newer-generation DES.

- In Chapter 10, we assess the impact of treatment of bifurcation lesions with newer-generation DES on clinical outcome at two-year follow-up in a randomized all-comer trial that compares two newergeneration durable polymer DES.

- In Chapter 11, we describe the three-year clinical outcome of patients who underwent PCI with second-generation DES implantation for true- and non-true bifurcation lesions.

- In Chapter 12, we evaluated in patients with bifurcation lesions the additional risk of severe lesion calcification on the outcome of $\mathrm{PCl}$ with durable polymer DES.

- In Chapter 13, we evaluate the impact of small vessel treatment on the clinical outcome of patients treated with newer-generation durable polymer DES.

- In Chapter 14, we investigate the impact of severe lesion calcification on clinical outcome in patients with stable angina, treated with newer-generation durable polymer DES.

- In Chapter 15, we assess in patients who presented with an acute coronary syndrome the safety and efficacy of treating severely calcified lesions with newer-generation durable polymer DES. 
- In Chapter 16, we assessed the clinical outcome of patients, who were treated by PCl for target lesions with or without severe calcifications, to compare two durable polymer everolimus-eluting stents that differ only in alloy and design of their metallic stent backbone.

- In Chapter 17, we present a general discussion of the findings of this thesis.

- In Chapter 18, we provide the summary and conclusions of the thesis. 


\section{References}

1. Favaloro RG. Saphenous vein autograft replacement of severe segmental coronary artery occlusion: operative technique. Ann Thorac Surg 1968;5:334-9.

2. Gruntzig AR, Senning A, Siegenthaler WE. Nonoperative dilatation of coronary-artery stenosis: percutaneous transluminal coronary angioplasty. N Engl J Med 1979;301:61-8.

3. Sigwart U, Puel J, Mirkovitch V, Joffre F, Kappenberger L. Intravascular stents to prevent occlusion and restenosis after transluminal angioplasty. N Engl J Med 1987;316:701-6.

4. de Feyter PJ, de Jaegere PP, Serruys PW. Incidence, predictors, and management of acute coronary occlusion after coronary angioplasty. Am Heart J 1994;127:643-51.

5. Iqbal J, Gunn J, Serruys PW. Coronary stents: historical development, current status and future directions. Br Med Bull 2013;106:193-211.

6. Serruys PW, Kutryk MJ, Ong AT. Coronary-artery stents. N Engl J Med 2006;354:483-95.

7. Arjomand H, Turi ZG, McCormick D, Goldberg S. Percutaneous coronary intervention: historical perspectives, current status, and future directions. Am Heart J 2003;146:787-96.

8. Stefanini GG, Holmes DR,Jr. Drug-eluting coronary-artery stents. N Engl J Med 2013;368:254-65.

9. Morice MC, Serruys PW, Sousa JE, et al. Randomized Study with the Sirolimus-Coated Bx Velocity BalloonExpandable Stent in the Treatment of Patients with de Novo Native Coronary Artery Lesions. A randomized comparison of a sirolimus-eluting stent with a standard stent for coronary revascularization. N Engl J Med 2002;346:1773-80.

10. Moses JW, Leon MB, Popma JJ, et al. Sirolimus-eluting stents versus standard stents in patients with stenosis in a native coronary artery. N Engl J Med 2003;349:1315-23.

11. Stone GW, Ellis SG, Cox DA, et al. A polymer-based, paclitaxel-eluting stent in patients with coronary artery disease. N Engl J Med 2004;350:221-31.

12. Kastrati A, Mehilli J, Pache J, et al. Analysis of 14 trials comparing sirolimus-eluting stents with bare-metal stents. N Engl J Med. 2007;356:1030-9.

13. Daemen J, Wenaweser P, Tsuchida K, et al. Early and late coronary stent thrombosis of sirolimus-eluting and paclitaxel-eluting stents in routine clinical practice: data from a large two-institutional cohort study. Lancet 2007;369:667-78.

14. Serruys PW, Morice MC, Kappetein AP, et al. Percutaneous coronary intervention versus coronary-artery bypass grafting for severe coronary artery disease. N Engl J Med 2009;360:961-72.

15. Serruys PW, Silber S, Garg S, et al. Comparison of zotarolimus-eluting and everolimus-eluting coronary stents. N Engl J Med 2010;363:136-46.

16. von Birgelen C, Basalus MW, Tandjung K, et al. A randomized controlled trial in second-generation zotarolimuseluting Resolute stents versus everolimus-eluting Xience V stents in real-world patients: the TWENTE trial. J Am Coll Cardiol 2012;59:1350-61.

17. Jensen LO, Thayssen P, Hansen HS, et al. Randomized comparison of everolimus-eluting and sirolimus-eluting stents in patients treated with percutaneous coronary intervention: the Scandinavian Organization for Randomized Trials with Clinical Outcome IV (SORT OUT IV). Circulation 2012;125:1246-55.

18. Kedhi E, Joesoef KS, McFadden E, et al. Second-generation everolimus-eluting and paclitaxel-eluting stents in real-life practice (COMPARE): a randomised trial. Lancet 2010; 375: 201-9.

19. Serruys PW, Silber S, Garg S, et al. Comparison of zotarolimus-eluting and everolimus-eluting coronary stents. N Engl J Med 2010;363:136-46.

20. von Birgelen C, Sen H, Lam MK, et al. Third-generation zotarolimus-eluting and everolimus-eluting stents in all-comer patients requiring a percutaneous coronary intervention DUTCH PEERS): a randomized, single-blind, multicenter, non-inferiority trial. Lancet 2014;383:413-23.

21. Williams PD, Mamas MA, Morgan KP, et al. Longitudinal stent deformation: a retrospective analysis of frequency and mechanisms. Eurointervention 2012;8:267-74.

22. Sen H, Lam MK, Löwik MM, et al. Clinical events and patient-reported chest pain in all-comers treated with Resolute Integrity and Promus Element stents: 2-year follow-up of the DUTCH PEERS (Durable polymer-based stent challenge of Promus Element versus Resolute Integrity) randomized trial (TWENTE II). J Am Coll Cardiol Intv 2015;8:889-99. 
23. Sarno G, Lagerqvist B, Carlsson J, et al. Initial clinical experience with an everolimus eluting platinum chromium stent (Promus Element) in unselected patietns from the Swedish Coronary Angiography and Angioplasty Registry (SCAAR). Int J Cardiol 2013;167:146-50.

24. Nakamura M, Muramatsu T, Yokoi $\mathrm{H}$, et al. Three-year follow-up outcomes of SES and PES in a randomized controlled study stratified by the presence of diabetes mellitus: J-DEsSERT trial. Int J Cardiol 2016;208:4-12.

25. Wiebe J, Nef HM, Hamm CW. Current status of bioresorbable scaffolds in the treatment of coronary artery disease. J Am Coll Cardiol 2014;64:2541-51.

26. Windecker S, Serruys PW, Wandel S, et al. Biolimus-eluting stent with biodegradable polymer versus sirolimuseluting stent with durable polymer for coronary revascularisation (LEADERS): a randomised non-inferiority trial. Lancet 2008;372:1163-73.

27. Serruys PW, Farooq V, Kalesan B, et al. Improved safety and reduction in stent thrombosis associated with biodegradable polymer-based biolimus-eluting stents versus durable polymer-based sirolimus-eluting stents in patients with coronary artery disease: final 5-year report of the LEADERS (limus eluted from a durable versus erodable stent coating) randomized, noninferiority trial. JACC Cardiovasc Interv 2013;6:777-89.

28. Christiansen EH, Jensen LO, Thayssen $\mathrm{P}$, et al. Biolimus-eluting biodegradable polymer-coated stent versus durable polymer-coated sirolimus-eluting stent in unselected patients receiving percutaneous coronary intervention SORT OUT V): a randomised non-inferiority trial. Lancet 2013;381:661-69.

29. Smits PC, Hofma S, Togni M, et al. Abluminal biodegradable polymer biolimus-eluting stent versus durable polymer everolimus-eluting stent (COMPARE II): a randomised, controlled, non-inferiority trial. Lancet 2013;381:651-60.

30. Raungaard B, Jensen LO, Tilsted HH, et al. Zotarolimus-eluting durable-polymer-coated stent versus a biolimuseluting biodegradable-polymer-coated stent in unselected patients undergoing percutaneous coronary intervention SORT OUT VI): a randomised non-inferiority trial. Lancet 2015;385:1527-35.

31. Palmerini T, Biondi-Zoccai G, Della Riva D, et al. Clinical outcomes with bioabsorbable polymer- versus durable polymer-based drug-eluting and bare-metal stents: evidence from a comprehensive network meta-analysis. J Am Coll Cardiol 2014;63:299-307.

32. Waksman R. Biodegradable stents: they do their job and disappear. J Invasive Cardiol 2006;18:70-4.

33. Iqbal J, Onuma Y, Ormiston J, Abizaid A, Waksman R, Serruys P. Bioresorbable scaffolds: rationale, current status, challenges, and future. Eur Heart J 2014;35:765-76.

34. Gomez-Lara J, Garcia-Garcia HM, Onuma Y, et al. A comparison of the conformability of everolimus-eluting bioresorbable vascular scaffolds to metal platform coronary stents. J Am Coll Cardiol Intv 2010;3:1190-8.

35. Serruys PW, Garcia-Garcia HM, Onuma Y. From metallic cages to transient bioresorbable scaffolds: change in paradigm of coronary revascularization in the upcoming decade? Eur Heart J 2012;33:16-25b.

36. Haude M, Ince H, Abizaid A, et al. Safety and performance of the second-generation drug-eluting absorbable metal scaffold in patients with de-novo coronary artery lesions (BIOSOLVE-II): 6 month results of a prospective, multicenter, non-randomised, first-in-man trial. Lancet 2016;387:31-9.

37. Raber L, Brugaletta S, Yamaji K, et al. Very Late Scaffold Thrombosis: Intracoronary Imaging and Histopathological and Spectroscopic Findings. J Am Coll Cardiol 2015;66:1901-14.

38. Tejada JG, Velazquez M, Hernandez F, et al. Percutaneous revascularization in patients with previous coronary artery bypass graft surgery. Immediate and 1-year clinical outcomes. Int J Cardiol 2009;134:201-6.

39. Scarsini R, Zivelonghi C, Pesarini G, Vassasnelli C, Ribichini FL. Repeat revascularization: percutaneous coronary intervention after coronary bypass graft surgery. Cardiovasc Revasc Med 2016;17:272-8.

40. Ribichini F, Pugno F, Ferrero V, et al. Long-term histological and immunohistochemical findings in human venous aorto-coronary bypass grafts. Clin Sci (Lond) 2008;114:211-20.

41. Sabik JF, Blackstone EH, Gillinov AM, Smedira NG, Lytle BW. Occurrence and risk factors for reintervention after coronary artery bypass grafting. Circulation 2006;114[Suppl. I]:I-454-60.

42. Tatoulis J, Buxton BF, Fuller JA. Patency of 2127 arterial to coronary conduits over 15 years. Ann Thorac Surg 2004;77:93-101.

43. Escaned J. Secondary revascularization after CABG surgery. J Nat Rev Cardiol 2012;9:540-9.

44. Garg P, Kamaruddin H, Iqbal J, Wheeldon N. Outcomes of primary percutaneous coronary intervention for patients with previous coronary artery bypass grafting presenting with ST-segment elevation myocardial infarction. Open Cardiovasc Med J 2015;9:99-104. 
45. Morrison DA, Sethi G, Sacks J, et al. Percutaneous coronary intervention versus repeat bypass surgery for patients with medically refractory myocardial ischemia: AWESOME randomized trial and registry experience with post-CABG patients. J Am Coll Cardiol 2002;40:1951-4.

46. Windecker S, Kohl P, Alfonso F, et al. 2014 ESC/EACTS Guidelines on myocardial revascularization: the Task Force on myocardial revascularization of the European Society of Cardiology (ESC) and the European Association for Cardio-Thoracic Surgery (EACTS) developed with the special contribution of the European Association of Percutaneous Cardiovascular Interventions (EAPCI). Eur Heart J 2014;35:2541-19.

47. Nikolsky E, McLaurin BT, Cox DA, et al. Outcomes of patients with prior coronary artery bypass grafting and acute coronary syndromes: analysis from the ACUITY (acute catheterization and urgent intervention triage strategy) trial. J Am Coll Cardiol Intv 2012;5:919-26.

48. Berry C, Pieper KS, White HD, et al. Patients with prior coronary artery bypass grafting have a poor outcome after myocardial infarction: an analysis of the valsartan in acute myocardial infarction trial (VALIANT). Eur Heart J 2009;30:1450-6.

49. Teixeira R, Vieira MJ, Ribeiro MA, Goncalves L, Gersh BJ. Prognosis following acute coronary syndromes according to prior coronary artery bypass grafting: meta-analysis. Eur Heart J Acute Cardiovasc Care 2015;4:518-27.

50. Sen H, Lam MK, Tandjung K, et al. Impact of previous coronary artery bypass surgery on clinical outcome after percutaneous interventions with second generation drug-eluting stents in TWENTE trial and Non-Enrolled TWENTE registry. Int J Cardiol 2014;176:885-90.

51. Brilakis ES, Lichtenwalter C, Abdel-Karim AR, et al. Continued benefit from paclitaxel-eluting compared with bare-metal stent implantation in saphenous vein graft lesions during long-term follow-up of the SOS (stenting of saphenous vein grafts) trial. J Am Coll Cardiol Intv 2011;4:176-82.

52. Agostoni $\mathrm{P}$, Vermeersch $\mathrm{P}$, Semeraro $\mathrm{O}$, et al. Intravascular ultrasound comparison of sirolimus-eluting stent versus bare metal stent implantation in diseased saphenous vein grafts (from the RRISC [reduction of restenosis in saphenous vein grafts with Cypher sirolimus-eluting stent] trial). Am J Cardiol 2007;100:52-8.

53. Lassen JF, Holm NR, Stankovic G, et al. Percutaneous coronary intervention for coronary bifurcation disease: consensus from the first 10 years of the European Bifurcation Club meetings. Eurolntervention 2014;10:545-60.

54. von Birgelen C, van Houwelingen KG, Lam MK. Coronary bifurcations: still the touchstone of drug-eluting stents and bioresorbable vascular scaffolds? Rev Esp Cardiol 2014;67:797-803.

55. Jim MH, Wu EB, Fung RC, et al. Angiographic result of T-stenting with small protrusion using drug-eluting stents in the management of ischemic side branch: the ARTEMIS study. Heart Vessels 2015;30:427-431.

56. Fan L, Chen L, Luo Y, et al. DK mini-culotte stenting in the treatment of true coronary bifurcation lesions: a propensity score matching comparison with T-provisional stenting. Heart Vessels 2016;31:308-21.

57. Al Suwaidi J, Berger PB, Rihal CS, et al. Immediate and longterm outcome of intracoronary stent implantation for true bifurcation lesions. J Am Coll Cardiol 2000;35:929-936.

58. Niemelä M, Kervinen K, Erglis A, et al. Randomized comparison of final kissing balloon dilatation versus no final kissing balloon dilatation in patients with coronary bifurcation lesions treated with main vessel stenting: the Nordic-Baltic Bifurcation Study III. Circulation 2011;123:79-86.

59. Louvard Y, Lefevre T, Morice MC. Bifurcation lesions. In: Eekhout E, Serruys PW, Wijns W, Vahanian A, van Sambeek M, and de Palma R, editors. Percutaneous interventional cardiovascular medicine: the PCR-EAPCI textbook. 2012; Toulouse, France: Europa Edition, pp 283-320.

60. Burzotta F, Mortier P, Trani C. Characteristics of drug-eluting stent platforms potentially influencing bifurcated lesion provisional stenting procedure. Eurolntervention 2014;10:124-32.

61. Schunkert H, Harrell L, Palacios IF. Implications of small reference vessel diameter in patients undergoing percutaneous coronary revascularization. J Am Coll Cardiol 1999;34:40-8.

62. Elezi S, Kastratie A, Neumann FJ, Hadamitzky M, Dirschinger J, Schömig A. Vessel size and long-term outcome after coronary stent placement. Circulation 1998;98:1875-80.

63. Biondi-Zoccai G, Moretti C, Abbate A, Sheiban I. Percutaneous coronary intervention for small vessel coronary artery disease. Cardiovasc Revasc Med 2010;11:189-98.

64. Bartorelli AL, Serruys PW, Miquel-Hébert K, Yu S, Pierson W, Stone GW. An everolimus-eluting stent versus a paclitaxel-eluting stent in small vessel coronary artery disease: a pooled analysis from the SPIRIT II and SPIRIT III trials. Catheter Cardiovasc Interv 2010;76:60-6.

65. Akiyama T, Moussa I, Reimers B, et al. Angiographic and clinical outcome following coronary stenting of small vessels: a comparison with coronary stenting of large vessels. J Am Coll Cardiol 1998;32:1610-8. 
66. Tanimoto S, Daemen J, Tsuchida K, et al. Two-year clinical outcome after coronary stenting of small vessels using 2.25-mm sirolimus- and paclitaxel-eluting stents: insight into the RESEARCH end T-SEARCH registries. Catheter Cardiovasc Interv 2007;69:94-103.

67. Claessen BE, Smits PC, Kereiakes DJ, et al. Impact of lesion length and vessel size on clinical outcomes after percutaneous coronary intervention with everolimus- versus paclitaxel-eluting stents: pooled analysis from the SPIRIT (clinical evaluation of the XIENCE V everolimus eluting coronary stent system) and COMPARE (secondgeneration everolimus-eluting and paclitaxel-eluting stents in real-life practice) randomized trials. J Am Coll Cardiol Intv 2011:11;1209-15.

68. Teirstein PS, Meredith IT, Feldman RL, et al. Two-year safety and effectiveness of the platinum chromium everolimus-eluting stent for the treatment of small vessels and longer lesions. Catheter Cardiovasc Interv 2015;85:207-15.

69. Kasaoka S, Tobis JM, Akiyama T, et al. Angiographic and intravascular ultrasound predictors of in-stent restenosis. J Am Coll Cardiol 1998;32:1630-5.

70. Kastrati A, Mehilli J, Dirschinger J, et al. Intracoronary stenting and angiographic results: strut thickness effect on restenosis outcome (ISAR-STEREO) trial. Circulation 2001;103:2816-21.

71. Briguori C, Sarais C, Pagnotta P, et al. In-stent restenosis in small coronary arteries: impact of strut thickness. J Am Coll Cardiol 2002;40:403-9.

72. Madhavan MV, Tarigopula M, Mintz GS, et al. Coronary artery calcification: pathogenesis and prognostic implications. J Am Coll Cardiol 2014;63:1703-14.

73. Bangalore S, Vlachos HA, Selzer F, Wilensky RL, Kip KE, Williams DO, Faxon DP. Percutaneous coronary intervention of moderate to severe calcified coronary lesions: insights from the National Heart, Lung, and Blood Institute Dynamic Registry. Catheter Cardiovasc Interv 2011;77:22-8.

74. Généreux P, Madhavan MV, Mintz GS, et al. Ischemic outcomes after coronary intervention of calcified vessels in acute coronary syndromes. Pooled analysis from the HORIZONS-AMI (Harmonizing Outcomes With Revascularization and Stents in Acute Myocardial Infarction) and ACUITY (Acute Catheterization and Urgent Intervention Triage Strategy) trials. J Am Coll Cardiol 2014;63:1845-54.

75. Wiemer M, Butz T, Schmidt W, et al. Scanning electron microscopic analysis of different drug eluting stents after failed implantation: from nearly undamaged to major damaged polymers. Catheter Cardiovasc Interv 2010;75:905-11.

76. Gudmundsdottir I, Adamson P, Gray C, et al. Optical coherence tomography versus intravascular ultrasound to evaluate stent implantation in patients with calcific coronary artery disease. Open Heart 2015;2:e000225.

77. Menown IBA, Noad R, Garcia EJ, Meredith I. The platinum chromium element stent platform: from alloy, to design, to clinical practice. Adv Ther 2010;27:129-41. 


\section{Part II}

Assessment of broad patient populations 


\section{Conflicts of interest statement}

The research department of Thoraxcentrum Twente has received educational and/or research grants funded by AstraZeneca, Biotronik, Boston Scientific, Medtronic. Dr. von Birgelen has been an unpaid consultant to Boston Scientific and Medtronic, and he received a speaker's honorarium from AstraZeneca and Biotronik. All other authors declare that they have no conflicts.

\section{Acknowledgments}

We thank Mrs Renate Wiggers-van der Leest at CardioResearch Enschede for collecting the TWENTE trial follow-up data with such great thoroughness and dedication. We gratefully acknowledge the invaluable support of the study by the staff of the research department, catheterization laboratory, wards, and administrative department of Thoraxcentrum Twente. In addition, we thank our colleagues in the cooperating hospitals, and the general physicians and pharmacists in the Twente region for their support and for treating the patients so well.

\section{Copyright}

Reprinted with permission from

(c) American Medical Association

JAMA Cardiology 2017 Mar 1;2(3):268-276 


\section{CHAPTER 2}

Five-Year Outcome After Implantation of Zotarolimus- and Everolimus-Eluting Stents in Randomized Trial Participants and Non-Enrolled Eligible Patients

A Secondary Analysis of a Randomized Clinical Trial

Clemens von Birgelen*, Liefke C. van der Heijden*, Mounir W.Z. Basalus, Marlies M. Kok, Hanim Sen, J.(Hans) W. Louwerenburg, K. Gert van Houwelingen, Martin G. Stoel, Frits H.A.F. de Man, Gerard C.M. Linssen, Kenneth Tandjung, Carine J.M. Doggen, Job van der Palen, Marije M. Löwik

*Both authors contributed equally to this manuscript.
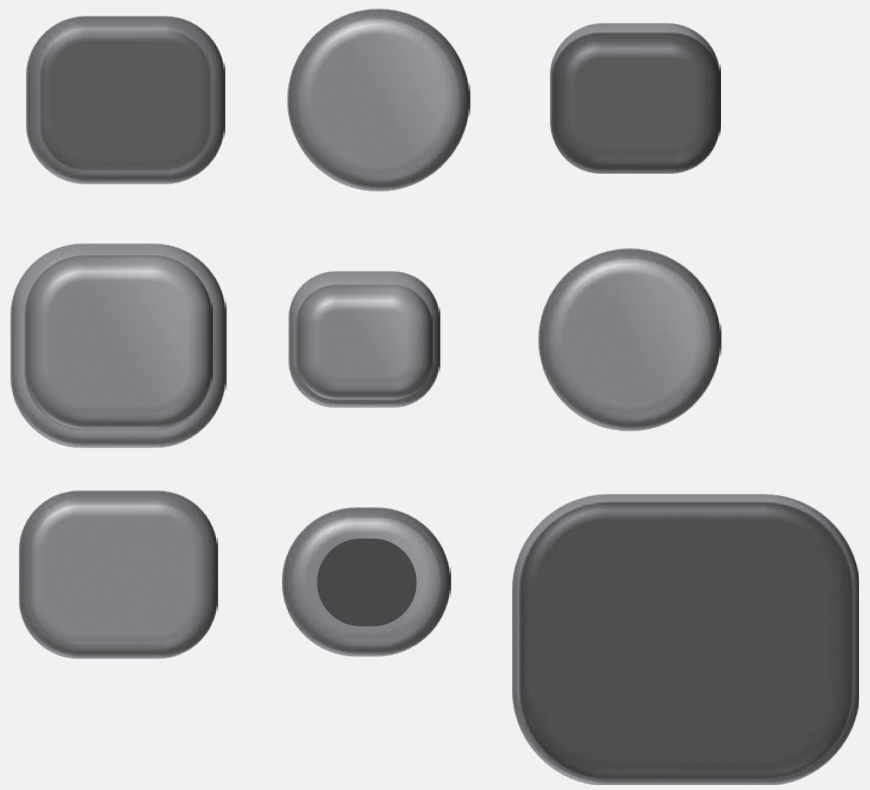


\begin{abstract}
Importance: Long-term follow-up after a randomized clinical trial of 2 often-used, newer-generation, drug-eluting stents (DESs) in a broad patient population is of interest. Comprehensive long-term outcome of eligible non-enrolled patients has never been reported.

Objective: To assess 5-year safety and efficacy of 2 newer-generation DESs in randomized trial participants with non-ST-elevation acute coronary syndromes or stable angina, and to evaluate longterm outcomes of non-enrolled eligible patients, treated with the same DESs.

Design, setting, and participants: The TWENTE (Real-World Endeavor Resolute vs Xience V Drug-Eluting Stent Study in Twente) trial is an investigator-initiated, patient-blinded, randomized, comparative DES trial that enrolled patients from June 18, 2008 and August 26, 2010. In a broad study population most of the patinets had non-ST-elevation acute coronary sndromes and complex lesions. Of all 1709 eligible patients, 1391 (81.4\%) were treated in the TWENTE trial with zotarolimus-eluting $(n=697)$ or everolimus-eluting $(n=694)$ cobalt-chromium stents (ZES and EES, respectively). The remaining 318 eligible patients (18.6\%) were not enrolled but underwent non-randomized treatment with the same DESs. Analyses were based on intention to treat. Event rates (\%) were derived from log-rank analysis and may differ from straightforward calculation (nominator/denominator). The 5-year follow-up of the TWENTE participants was prespecified in the trial protocol; that of the nonenrolled participants was ad hoc.
\end{abstract}

Main Outcome: Target vessel failure (TVF), a composite of cardiac death, target vessel-related myocardial infarction, or target vessel revascularization.

Results: A total of 1709 eligible participants (1233 men [72.1\%]; 476 women [27.9\%]; mean [SD] age, 64.6 [10.6] years) were included in the analysis. Among the 1370 of 1391 TWENTE trial participants (98.5\% follow-up), TVF was similar between those in the ZES (110 of 697) [16.1\%] and EES (123 of 694 [18.1\%]) groups ( $p=0.36)$. Definite stent thrombosis rates (7 of 697 [1.0\%] vs 4 of 694 [0.6\%]; $p=0.37$ ) were low and occurred after more than 1 year in $3(0.4 \%)$ vs $4(0.6 \%)(p=0.69)$. The 318 nonenrolled eligible patients (96.9\% follow-up) were older and had more advanced disease than trial participants. Their TVF rate was higher than that of trial participants (71 of 318 [23.3\%] vs 233 of 1391 [17.1\%]; $p=0.02$ ), which partly reflects a difference in cardiac mortality (23 of 318 [7.7\%] vs 60 of 1391 [4.5\%]; $p=0.03$ ). Similar 5-ear rates were found for myocardial infarction (91 of 1391 [6.7\%] vs 22 of 318 [7.2\%]; $p=0.80$ ) and target vessel revascularization (129 of 1391 [9.7\%] vs 34 of 318 [11.4\%]; $p=0.36$ ) between trial participants and nonenrolled eligible patients. In all eligible patients (ie, trial participants plus nonenrolled eligible patients), the TVF rate was only slighl higher than in trial participants only (18.3\% vs $17.1 \%)$.

Conclusions and Relevance: Long-term outcome data from non-enrolled eligible patients support the validity of the randomized trial and present, together with the trial, a strong case of long-term safety and efficacy for the newer-generation DESs used. 


\section{Introduction}

Randomized clinical trials that compare novel drug-eluting stents (DESs) in large, greatly unrestricted patient populations are indispensable because they permit a reliable evaluation of the efficacy and safety of these devices. ${ }^{1-8}$ Availability of long-term follow-up from such trials in all comers is a prerequisite for trustworthy judgment of the long-term effects of percutaneous coronary interventions (PCIs) using these devices. ${ }^{5}$ However, almost all randomized clinical trials (RCTs) in broad patient and allcomer populations involve some selection. ${ }^{9-11}$ Information on the clinical characteristics and short-term mortality of patients who did not participate in an RCT is infrequently reported. ${ }^{4,12,13}$

The zotarolimus-eluting stent (ZES) (Resolute; Medtronic Inc) and the everolimus-eluting stent (EES) (Xience V; Abbott Vascular) are newer-generation DESs that were developed to increase device biocompatibility and improve long-term outcomes. ${ }^{2,3,14}$ The randomized TWENTE (Real-World Endeavor Resolute vs Xience V Drug-Eluting Stent Stud in Twente) trial demonstrated noninferiority of the ZES vs EES in 1391 patients, representing 81.4\% of all 1709 eligible patients in a broad population. ${ }^{7}$ The Nonenrolled TWENTE registry compared the clinical characteristics and outcomes of the 1391 trial participants vs 318 nonenrolled eligible patients who were treated with the same DESs. ${ }^{13}$ After 12 months, the rates of adverse events were fairly similar for all trial participants (treatment arms pooled) vs the nonenrolled eligible patients. ${ }^{13}$

Five-year outcome data from randomized comparisons of the ZES and EES are of clinical interest but are so far only available from the RESOLUTE AC (A Randomized Comparison of a Zotarolimus-Eluting Stent With an Everolimus-Eluting Stent for Percutaneous Coronary Intervention All-Comers) trial. ${ }^{15}$ In addition, long-term outcome data from eligible nonenrolled patients treated with the same stents as the randomized patients have not yet been reported. In the present study, we report the 5-year clinical outcomes of patients enrolled in the randomized TWENTE trial and eligible nonenrolled patients.

\section{Methods}

\section{Study Design and Patient Populations}

The TWENTE trial is an investigator-initiated, patient-blinded, randomized, comparative DES trial with limited exclusion criteria. ${ }^{7}$ During the RCT, nonenrolled eligible patients were treated at the operator's discretion with one of the European Conformity-certified DESs that were also examined in the randomized trial (i.e., ZES or EES), using the same routine clinical and procedural strategies. The RCT and registry complied with the Declaration of Helsinki ${ }^{16}$ for investigation in human beings and were approved by the medical ethical committee of Twente and the institutional review board. All participants in the RCT provided written informed consent. For the nonenrolled TWENTE registry, patients were not required to change their behavior or taking action other than following their regular treatment; therefore, according to Dutch law and as approved by the medical ethical committee of Twente, written informed consent from patients in this registry was not required.

Patients were enrolled in the TWENTE tiral from June 18, 2008, to August 26, 2010, at Thoraxcentrum Twente, Enschede, the Netherlands. Patients were randomized in a 1:1 ratio for treatment with cobalt- 
chromium ZES or EES. No limit for lesion length, reference vessel size, number of target lesions, or number of vessels to be treated was applied. The main exclusion criterion was an ST-segment elevation myocardial infarction (MI) prior to $\mathrm{PCl}\left(<48\right.$ hours). ${ }^{7}$ Outcomes of the TWENTE trial participants were reported until the 3 -year follow-up. ${ }^{17,18}$ Operators generally avoided the use of different stents in a single patient. Procedural details and the 12-month clinical course of the nonenrolled eligible patients have been published elsewhere. ${ }^{13}$ The outcome of all eligible patients were followed up as participants of the TWENTE trial or within the nonenrolled TWENTE registry. Stent-level analyses were only performed in participants of the TWENTE trial.

\section{Definition of Clinical End Points}

The same end point definitions were applied in the nonenrolled TWENTE registry as used for the randomized TWENTE trial. ${ }^{7,13}$ Clinical end points, including stent thrombosis, were defined according to the Academic Research Consortium (ARC), including the addendum on definition of MI. ${ }^{19,20}$ The pre-specified main end point of the RCT-target vessel failure (TVF) - was composed (in hierarchical order) of cardiac death, target vessel-related $\mathrm{Ml}$, or clinically indicated target vessel revascularization. ${ }^{9}$ Death was considered cardiac unless an unequivocal noncardiac cause could be established. Myocardial infarction was defined by any creatine kinase concentration of more than double the upper reference limit with elevated confirmatory cardiac biomarkers. ${ }^{20} \mathrm{~A}$ target vessel-related $\mathrm{MI}$ was related to the target vessel or could not be related to another vessel; further MI classification was based on laboratory, electrocardiographic, angiographic, and/or clinical data. ${ }^{7,20}$ Revascularization procedures were considered clinically indicated if the visually assessed angiographic percent diameter stenosis was at least $50 \%$ in the presence of ischemic signs or symptoms or if the diameter stenosis was at least $70 \%$ irrespective of ischemic signs or symptoms. ${ }^{20}$

Prespecified secondary end points included the individual components of TVF, all-cause mortality, and stent thrombosis. Further composite end points included target lesion failure (cardiac death, target vessel-related $\mathrm{MI}$, or clinically indicated target lesion revascularization), major adverse cardiac events (all-cause death, any MI, emergent coronary bypass surgery, or clinically indicated target lesion revascularization), and a more global patient-oriented composite endpoint (all-cause death, any MI, or any coronary revascularization).

\section{Acquisition and Analysis of Clinical Follow-up Data}

We obtained clinical follow-up data at visits to outpatient clinics, by medical questionnaire, and/or by telephone follow-up (with staff blinded to assigned DES). The contract research organization CardioResearch Enschede BV, Enschede, the Netherlands, coordinated the trial and data management. The independent contract research organizations Cardialysis, Rotterdam, the Netherlands, and Diagram, Zwolle, the Netherlands, which were blinded to the assigned DES, performed the adjudication of adverse clinical events for the RCT participants and nonenrolled eligible patients. Angiographic analysts performed a visual assessment of target lesion characteristics offline. 


\section{Assessment of Reasons for Nonenrollment and Potential Selection}

To identify the explicitly stated and implicit reasons for nonenrollment of eligible patients, 5 members of the research group reviewed all medical files available. If the reason for nonenrollment was not explicitly stated, the presumed reason for nonenrollment was retrospectively established by consensus of the committee members. Reasons for nonenrollment were classified into 1 of the following categories: (1) explicit refusal of the informed patient; (2) inability of the operator to obtain informed consent (e.g. owing to severe anxiety or partial deafness); (3) logistic aspects eg, no randomization envelopes available, forgotten to randomize, or time pressure); (4) omission of informing the patient before treatment on the ward; and (5) unknown. In the absence of (conceivable) reasons, the committee also searched for indications of potential selection, which was suspected if vessels with particularly high risk were treated (eg, left main stem, degenerated vein grafts, or all 3 coronary arteries) or if operators indicated an increased procedural risk, high technical complexity of the procedure, or serious co-morbidities.

\section{Statistical analysis}

Data were analyzed from August 26, 2015, to October 11, 2016. The 5-ear follow-up of the TWENTE participants was prespecified in the trial protocol; that of the nonenrolled participants was ad hoc. Analyses were based on intention-to-treat using SPSS software (version 22.0; SPSS Inc). Categorical variables were assessed with the use of $\chi^{2}$ or Fisher's exact tests, whereas continuous variables were assessed with the Wilcoxon rank-sum test or 1-sample $t$ test. The time to the main end point and its components was assessed according to the Kaplan-Meier method, and the log-rank test was applied to compare groups. Therefore, percentages of outcome parameters may slightly differ from the results of a straightforward calculation of nominator devided by denominator. We calculated hazard ratios using Cox-regression analysis. Logistic regression was used to test for interaction between subgroups and stent type with respect to the main endpoint. Unless otherwise specified, P values and Cis were 2 sided. $\mathrm{P}<0.05$ was considered significant.

\section{Results}

\section{Clinical Outcome of the TWENTE Trial}

A total of 1709 eligible participants (1233 men [72.1\%] and 476 women [27.9\%]; mean [SD] age, 64.6 [10.6] years) were included in the analaysis. Of 1391 TWENTE trial participants (1009 men [72.5\%]; 382 women [27.5\%]), 5-year follow-up was obtained from 1370 (98.5\%), including 683 of 697 patients (98.0\%) randomized to ZES and 687 of 694 (99.0\%) randomized to EES (eFigure 1 in the Supplement). Between stent groups, no difference was found in baseline patient, lesion, and procedural characteristics (eTable 1 in the Supplement). At the 5-year follow-up 61 patients (5.0\%) were receiving dual antiplatelet therapy without any between-stent difference.

The main end point of TVF was met by 110 of 697 patients (16.1\%) in the ZES group vs 123 of 694 (18.1\%) in the EES group (hazard ratio, $0.89,95 \%-\mathrm{Cl}$ : 0.69-1.15, $\mathrm{p}=0.36$ ) (Table 1 and Figure 1 ). No significant difference was seen in the individual components of TVF, including cardiac death 25 of 697 
[3.7\%] vs 35 of 694 [5.2\%], p=0.18), target vessel-related MI (46 of 697 [6.8\%] vs 45 of 694 [6.6\%], $\mathrm{p}=0.94$ ), and clinically driven target vessel revascularization (60 of 697 [8.9\%] vs 69 of 694 [10.5\%], $p=0.41$ ). In addition, the rates of other composite endpoints were similar for both DESs (Table 1). An exploratory subgroup analysis of the main end point of TVF showed consistent results across subgroups, with the only exception being single vessel treatment, favoring ZES (67 of 523 [12.8\%] vs 92 of 532 [17.3\%], $p=0.05, p=0.03$ for interaction) (eFigure 2 in the Supplement).

Table 1: Five-year clinical outcome of TWENTE randomized trial participants according to assigned DES.

\begin{tabular}{|c|c|c|c|c|}
\hline & & TWEN & $\begin{array}{l}\text { ial participants* } \\
=1,391\end{array}$ & \\
\hline & $\begin{array}{c}\text { ZES } \\
n=697\end{array}$ & $\begin{array}{c}\text { EES } \\
n=694\end{array}$ & $\begin{array}{c}\text { HR } \\
(95 \%-\mathrm{Cl})\end{array}$ & P logrank \\
\hline Death (any) & $62(9.0)$ & $80(11.6)$ & $0.77(0.55-1.07)$ & 0.12 \\
\hline Cardiac death & $25(3.7)$ & $35(5.2)$ & $0.71(0.42-1.18)$ & 0.18 \\
\hline Any myocardial infarction & $49(7.2)$ & $52(7.7)$ & $0.94(0.63-1.38)$ & 0.73 \\
\hline Target vessel myocardial infarction & $46(6.8)$ & $45(6.6)$ & $1.02(0.67-1.53)$ & 0.94 \\
\hline Any revascularization & $95(14.1)$ & 105 (15.9) & $0.90(0.68-1.18)$ & 0.43 \\
\hline $\begin{array}{l}\text { Clinically indicated target vessel } \\
\text { revascularization }\end{array}$ & $60(8.9)$ & $69(10.5)$ & $0.86(0.61-1.22)$ & 0.41 \\
\hline $\begin{array}{l}\text { Clinically indicated target lesion } \\
\text { revascularization }\end{array}$ & $47(7.0)$ & $50(7.7)$ & $0.94(0.63-1.40)$ & 0.77 \\
\hline Target vessel failure & $110(16.1)$ & $123(18.1)$ & $0.89(0.69-1.15)$ & 0.36 \\
\hline Target lesion failure & $102(15.0)$ & $110(16.2)$ & $0.93(0.71-1.21)$ & 0.58 \\
\hline Major adverse cardiac events & $138(19.9)$ & $157(22.7)$ & $0.88(0.70-1.10)$ & 0.26 \\
\hline Patient-oriented composite endpoint & $176(25.4)$ & $196(28.4)$ & $0.89(0.73-1.10)$ & 0.27 \\
\hline Definite or probable stent thrombosis & 13 (1.9) & $14(2.1)$ & $0.92(0.43-1.96)$ & 0.83 \\
\hline Definite stent thrombosis & $7(1.0)$ & $4(0.6)$ & $1.74(0.51-5.94)$ & 0.37 \\
\hline
\end{tabular}

Abbreviations: DES, drug-eluting stent; EES, everolimus-eluting stent; HR, hazard ratio; MI, myocardial infarction; TWENTE, RealWorld Endeavor Resolute vs Xience V Drug-Eluting Stent Study in Twente; ZES, zotarolimus-eluting stent.

* Five-year follow-up information was obtained from 1370 of 1391 participants (98.5\%) in the TWENTE trial, including 683 of 697 (98.0\%) in the ZES group (Resolute; Medtronic Inc) and 687 of 694 (99.0\%) in the EES group (Xience V; Abbott Vascular). Data were analyzed using the Kaplan-Meier method, which implies that patients who could not be followed up for the entire 5 years because of death, consent withdrawal, or loss to follow-up were censored at the exact moment of dropout. Please note that the percentages provided in the Table may therefore differ slightly from the results of straightforward calculations of nominator divided by denominator.

For ZES and EES, the rates of definite (7 of 697 [1.0\%] vs 4 of 694 [0.6\%], $p=0.37$ ) and definite or probable (13 of 697 [1.9\%] vs 14 of 694 [2.1\%], p=0.83) stent thrombosis were low and similar (Figure 2). Very late definite stent thrombosis ( $>1$ year) occurred in 3 patients in the ZES group (0.4\%) vs 4 patients in the EES group (0.6\%) ( $p=0.69)$. In eTable 2 in the Supplement, annual cumulative event rates for both stent groups are presented. 


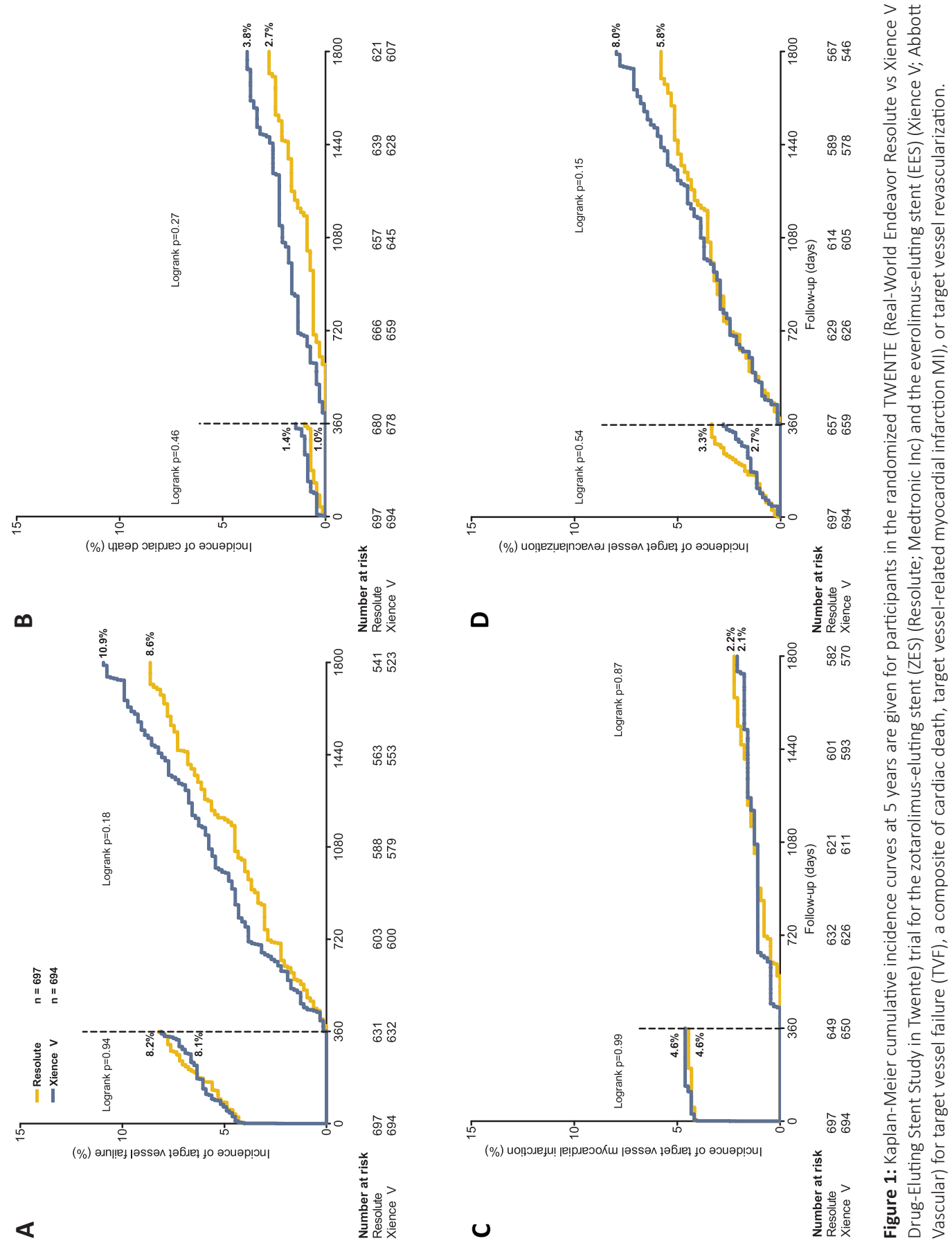




\section{Nonenrolled Eligible Patient Population}

Five-year follow-up data were available for 308 of 318 eligible patients (96.9\%) (224 men [70.4\%], 94 women [29.6\%]) who had not been enrolled in the randomized trial (eFigure 1 in the Supplement). Reasons for nonenrollment included (1) explicit refusal to participate (33 of 318 [10.4\%]); (2) inability to obtain informed consent (57 of 318 [17.9\%]); (3) logistic aspects (40 of 318 [12.6\%]); (4) omission of informing the patient before treatment (113 of 318 [35.5\%]); and (5) unknown reasons (75 of 318 [23.6\%]). Indications of potential selection were found in 61 of 318 [19.2\%] nonenrolled eligible patients, representing $3.6 \%$ of all 1709 eligible patients. Nonenrolled eligible patients were significantly older than randomized trial participants (66.0 [10.9] vs 64.2 [10.8] years; $p=0.01$ ), significantly more often had a history of $\mathrm{MI}(137$ [43.1\%] vs 450 [32.4\%]; $\mathrm{p}<0.001), \mathrm{PCl}$ (92 [28.9\%] vs 288 [20.7\%], p=0.001), and bypass surgery (54 [17.0\%] vs 148 [10.6\%]; $p=0.002)$, and significantly more often had chronic renal failure (21 [6.6\%] vs 38 [2.7\%]; $p=0.001$ ) and a left ventricular ejection fraction of less than $30 \%$ (13 of 199 [6.5\%] vs 32 of 1051 [3.0\%], p=0.02). In addition, they were treated more often for complex target lesions (355 of 466 [76.2\%] vs 1484 of 2116 [70.1\%]; p=0.009), bypass lesions (101 of 466 [21.7\%] vs 518 of 2116 [24.5\%]; $p=0.20$ ), and in-stent restenosis (37 of 466 [7.9\%] vs 75 of 2116 [3.5\%]; $p<0.001$ ) (eTable 3 in the Supplement). At 5-year follow-up, 19 (7.2\%) of these patients were receiving dual antiplatelet therapy ( $p=0.15 \mathrm{vs}$. RCT participants).

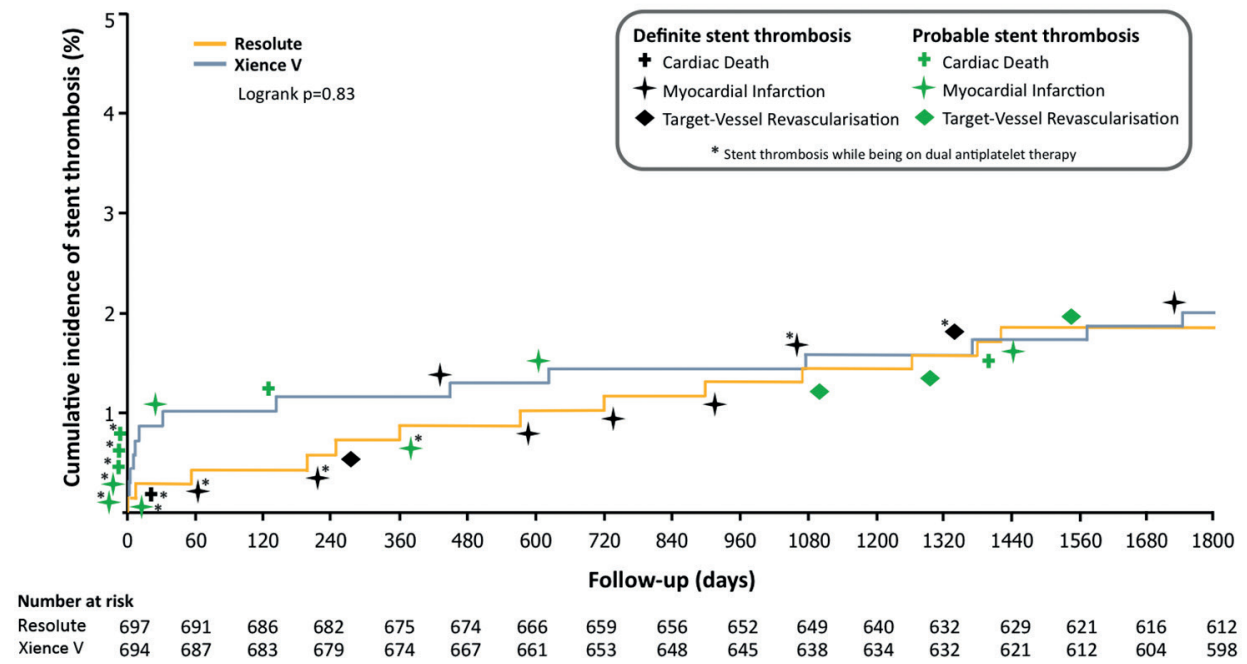

Figure 2: Data include participants in the randomized TWENTE (Real-World Endeavor Resolute vs Xience V DrugEluting Stent Study in Twente) trial for the zotarolimus-eluting stent (ZES) (Resolute; Medtronic Inc) and the everolimus- eluting stent (EES) (Xience V; Abbott Vascular). Stent thrombosis was adjudicated by the Academic Research Consortium definition. 


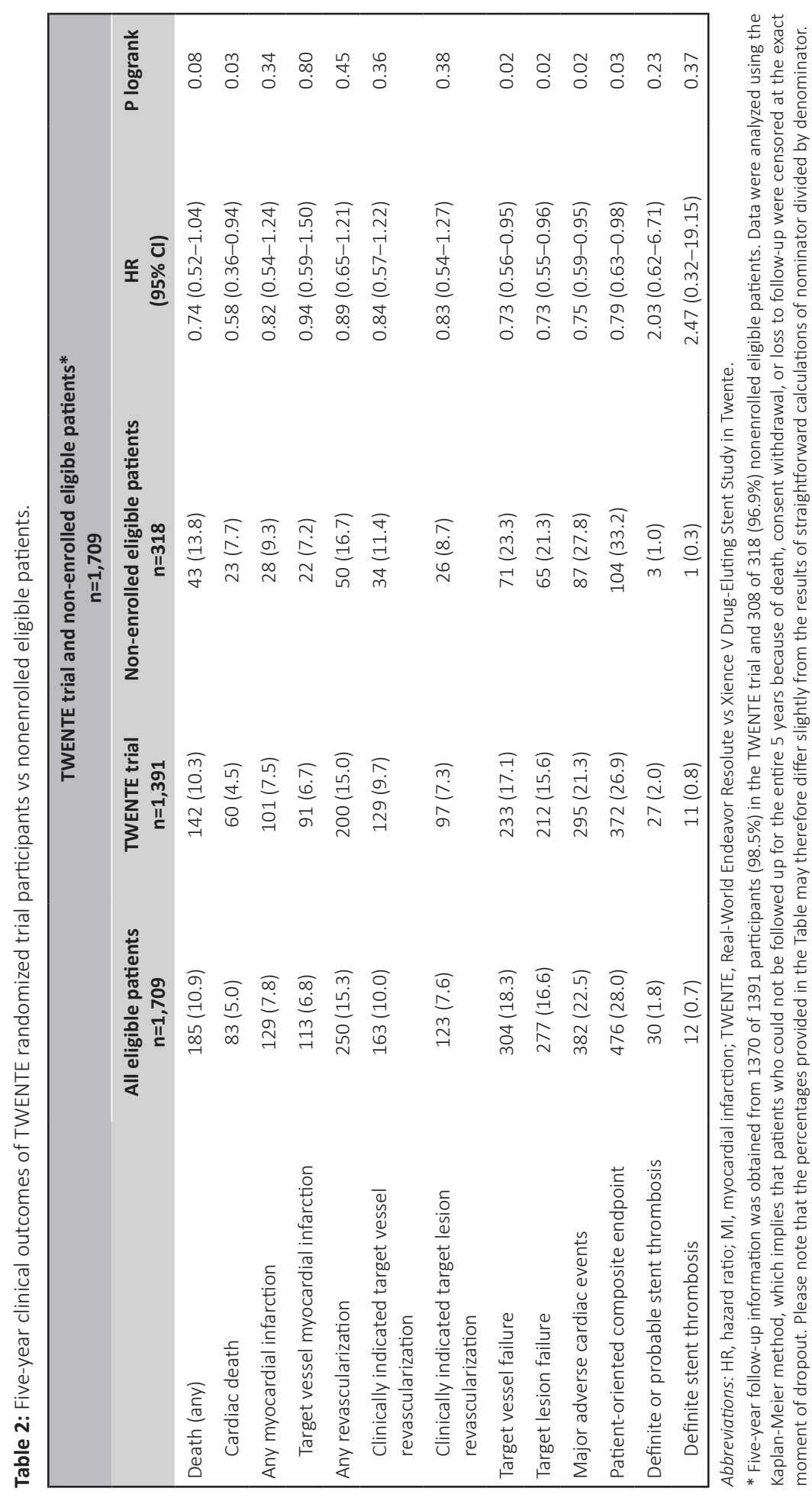




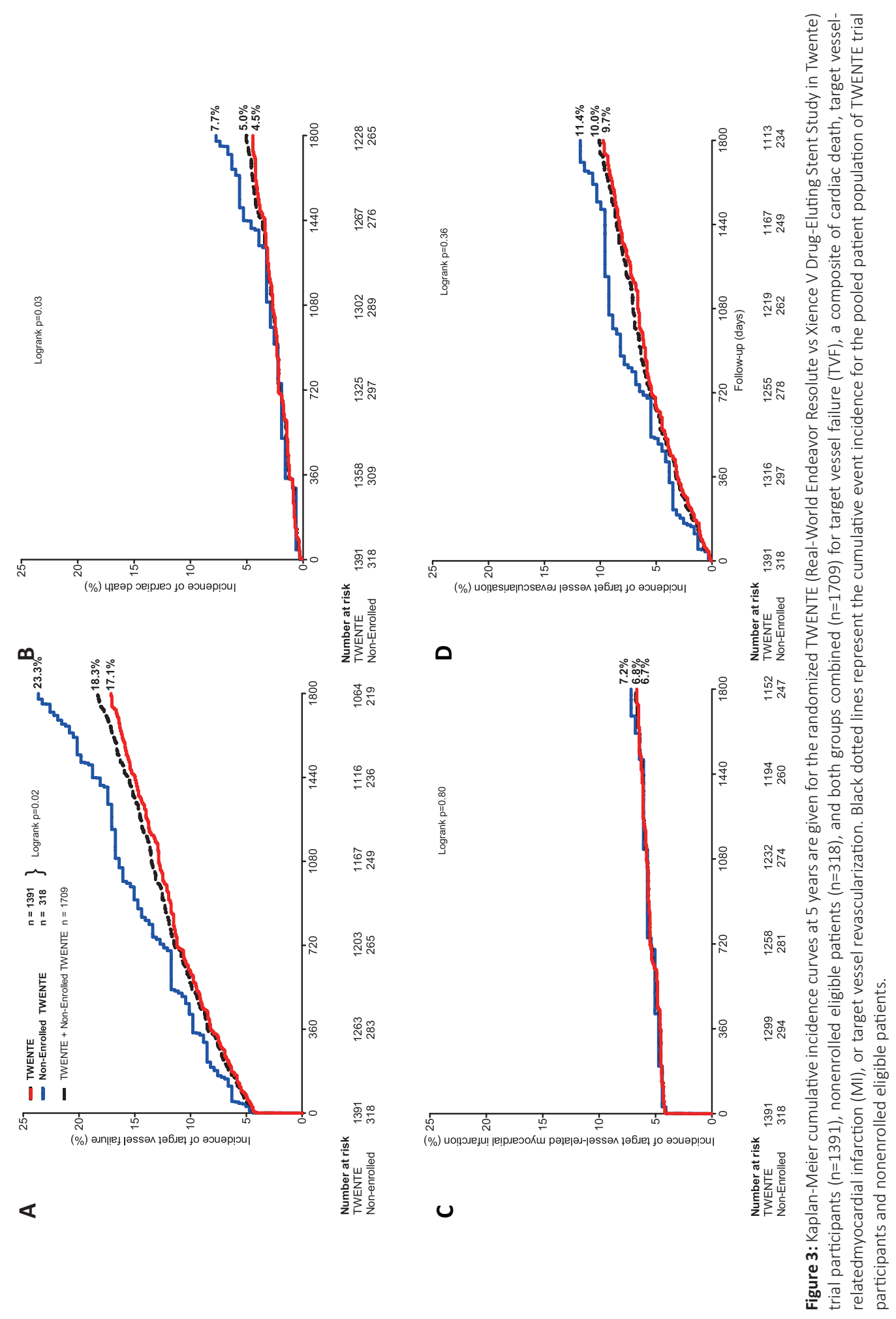




\section{TWENTE Trial Participants versus Nonenrolled Eligible Patients}

The TWENTE trial participants and nonenrolled eligible patients differed in TVF (233 of 1391 [17.1\%] vs 71 of 318 [23.3\%], $p=0.02$ ), which was partly attributable to a difference in cardiac death (60 of 1391 [4.5\%] vs 23 of 318 [7.7\%], $p=0.03)$. The Kaplan-Meier curves showed that, until the 4-year followup, the cardiac death rates were similar, after which the slope of the curve increased for nonenrolled patients (Figure 3). Both patient groups had quite similar 5-year rates of target vessel-related $\mathrm{MI}$ (91 of 1391 [6.7\%] vs 22 of 318 [7.2\%], $p=0.80$ ) and TVR (129 of 1391 [9.7\%] vs 34 of 318 [11.4\%], $p=0.36$ ). The 5 -year definite or probable stent thrombosis rate was low in trial participants and nonenrolled eligible patients (27 of 1391 [2.0\%] vs 3 of 318 [1.0\%], p=0.23). Further outcome data are presented in Table 2 , and landmark analyses for TVF and its components are presented in eFigure 3 in the Supplement.

Table 2 also shows the event rates of all eligible patients (ie, a pooled population of trial participants and nonenrolled eligible patients). The 5-year TVF rate was 304 of 1709 (18.3\%) in all eligible patients and 233 of 1391 (17.1\%) in randomized trial participants only (stent arms pooled); this finding is also visualized in eFigure 4 in the Supplement.

\section{Discussion}

Randomized studies generally do not randomize every eligible patient and are therefore susceptible to selection. ${ }^{9-11}$ The TWENTE trial enrolled a large proportion of all eligible patients (81.4\%), whereas excellent multicenter DES trials ${ }^{9-11}$ previously enrolled $40 \%$ of eligible patients or did not report such details. Minor selection cannot be excluded and may - from a clinician's perspective - sometimes appear reasonable in patients with end-stage coronary heart disease or excessive co-morbidities. The present analysis is special in that it reports long-term outcome data from the randomized trial and a registry of nonenrolled eligible patients, which together provide unique, complementary insights.

In the present study, TWENTE trial participants treated with ZES vS EES showed 5 -year TVF rates that were relatively low (16.1\% vs $18.1 \%$ ) in both treatment arms, as were the rates of the individual components of TVF. Our findings are in line with those of other RCTs that compared similar ZESs and EESs, ${ }^{3,8,21,22}$ network meta-analyses that - among other DESs - constituted the 2 DESs assessed in the TWENTE trial, ${ }^{23,24}$ and a dedicated meta-analysis. ${ }^{25}$

Comprehensive information was available also on the long-term outcome of nonenrolled eligible patients, who were treated in a nonrandomized fashion with the same DESs. The adverse events of these 2 patient populations were adjudicated by the same independent clinical event committee, which further increases the usefulness of these data. Moreover, 5-year follow-up rates of trial participants and nonenrolled eligible patients were high (98.5\% and $96.9 \%$, respectively). In nonenrolled eligible patients, who were older and more often had a history of coronary revascularizations and $\mathrm{Ml}$ than RCT participants, the TVF rate was higher (23.3\% vs $17.1 \%$ ). Until 4-year follow-up, the rate of cardiac death remained similar in both patient groups. During the fifth year of follow-up, cardiac mortality increased more noticeably in the nonenrolled patients. The 5 -year cardiac death rate $(7.7 \%$ vs $4.5 \%)$ reflected the more advanced age and disease stage of the nonenrolled patients and contributed to the aforementioned difference in TVF. Our data suggest that if all eligible patients had been randomized, 
clinical event rates might still have been favorable and, in general, only slightly higher than those actually obtained for the randomized population. Hence, the long-term outcome data from the nonenrolled eligible patients support the external validity and findings of the TWENTE trial.

\section{Previous Studies Addressing Nonenrolled Patients}

Patients enrolled in coronary intervention trials are often not fully representative of patients in clinical practice. ${ }^{9-11}$ Nevertheless, data on the clinical outcome of eligible nonenrolled patients or of all nonparticipants (ie, nonenrolled eligible plus per-protocol excluded patients) are scarce. Highquality clinical trials occasionally report the clinical characteristics of the nonparticipating patients to provide insight into the degree (or the absence) of selection. ${ }^{4} \mathrm{~A}$ single high-volume $\mathrm{PCl}$ center reported baseline characteristics and 12-month all-cause mortality of 579 patients who participated in 2 randomized all-comer trials ${ }^{12}$ and compared these data with the mortality of 663 nonparticipants. Baseline characteristics differed significantly between trial participants and nonparticipants, with the latter being older and having more heart failure and unstable clinical syndromes and also a significantly higher 1-year all-cause mortality (6.9\% vs 3.1\%). ${ }^{12}$ Because the nonparticipants included patients with exclusion criteria (eg, cardiogenic shock) and the outcome was focused on 12-month all-cause mortality, ${ }^{12}$ a meaningful comparison with the 5-year outcome of the nonenrolled TWENTE registry cannot be made. Nevertheless, in our study, 5-year all-cause mortality also tended to be higher in nonenrolled eligible patients than in trial participants (43 [13.8\%] vs 142 [10.3\%]).

\section{Previous Randomized Studies With 5-Year Follow-up Comparing Newer-Generation DESs}

Newer-generation durable polymer DESs have previously been assessed in randomized trials with longterm follow-up. In patients with low to moderate procedural risk in the SPIRIT III (Clinical Evaluation of the Xience V Everolimus Eluting Coronary Stent System) trial, the 5-year rate of TVF was lower in patients using the EES than in those using early-generation paclitaxel-eluting stents (19.3\% vs $24.5 \%) .{ }^{26}$ At the 5-year follow-up of the COMPARE (A Trial of Everolimus-Eluting Stents and Paclitaxel-Eluting Stents for Coronary Revascularization in Daily Practice) trial, all comers treated with the EES (Xience V) showed significantly lower rates of various composite end points than patients treated with paclitaxel-eluting stents, including TVF (12.6\% vs $17.8 \%$ ) and definite or probable stent thrombosis (3.1\% vs $5.9 \%) .{ }^{27}$ In the 5-year results of the SORT OUT IV (Danish Organization of Randomized Trials with Clinical Outcome) trial, the composite end point of major adverse cardiac events and definite stent thrombosis was significantly lower in all-comer patients treated with the EES (Xience V) than in patients treated with early-generation sirolimus-eluting stents ( $14.0 \%$ vs $17.4 \%$ and $0.4 \%$ vs $2.0 \%$, respectively). ${ }^{28}$ Moreover, in the EXAMINATION (Clinical Evaluation of the Xience V Stent in Acute Myocardial Infarction) trial, the use of EES in patients with acute ST-segment elevation MI resulted in a lower 5-year mortality than treatment with bare-metal stents ( $8.7 \%$ vs $11.8 \%) .{ }^{29}$ The randomized RESOLUTE AC trial has reported 5 -year outcome data, showing similar efficacy and safety of ZES (Resolute) and EES in an all-comer population (rate of TVF, $20.0 \%$ vs $19.1 \%$ ). ${ }^{15}$ The outcome of the randomized TWENTE trial supports these findings in general. The exploratory subgroup analysis for the main endpoint of TVF also showed consistent results, with singlevessel treatment being the only exception (favoring ZES), which may most likely reflect a play of chance. 


\section{Limitations of the study}

Scientific evidence from multicenter trials is generally considered higher ranking than that from singlecenter trials. Our conclusions do not apply to patients with acute ST-segment elevation MI undergoing primary PCl because such patients were not studied. Patients with acute ST-segment elevation MI are of considerable interest for the assessment of novel stents, ${ }^{30}$ whereas their enrollment may be challenging. The subgroup analysis was not prespecified but assessed the same subgroups as the RESOLUTE AC trial. ${ }^{3}$ Results of comparisons between nonenrolled eligible patients and the trial population are hypothesisgenerating.

\section{Conclusions}

At 5-year follow-up, the second-generation DESs used in the RCT showed favorable and similar longterm results in a broad patient population, most of whom were treated for non-ST-segment elevation acute coronary syndromes and complex coronary lesions. Outcome data from nonenrolled eligible patients support the validity of the randomized TWENTE trial and present, with the results of the randomized trial, a strong case for the long-term safety and efficacy of both devices. Moreover, these data underline the importance of aiming at high study enrollment.

\section{Key Points}

Questions: Are newer-generation drug-eluting stents safe and efficacious at the 5-year follow-up, and do outcomes of eligible nonenrolled patients treated with the same stents support the randomized clinical trial findings?

Findings: In this secondary analysis of the TWENTE (Real-World Endeavor Resolute vs Xience V DrugEluting Stent Study in Twente) randomized clinical trial, which examined $81 \%$ of all the eligible patients, the rate of the main composite clinical endpoint of target vessel failure was similar for zotarolimus- and everolimus-eluting stents. In all eligible patients, the event rate was only slightly higher than in trial participants only.

Meaning: At long-term follow-up of this randomized clinical trail, newer-generation stents were safe and efficacious, and the outcomes of nonenrolled eligible patients support the trials validity. 


\section{References}

1. Windecker S, Serruys PW, Wandel S, et al. Biolimus-eluting stent with biodegradable polymer versus sirolimuseluting stent with durable polymer for coronary revascularisation (LEADERS): a randomised non-inferiority trial. Lancet 2008;372:1163-7

2. Kedhi $E$, Joesoef KS, McFadden E, et al. Second-generation everolimus-eluting and paclitaxel-eluting stents in real-life practice (COMPARE): a randomised trial. Lancet 2010;375:201-9.

3. Serruys PW, Silber S, Garg S, et al. Comparison of zotarolimus-eluting and everolimus-eluting coronary stents. N Engl J Med 2010;363:136-46.

4. Christiansen EH, Jensen LO, Thayssen P, et al; SORT OUT $\vee$ investigators. Biolimus-eluting biodegradable polymer-coated stent versus durable polymer-coated sirolimus-eluting stent in unselected patients receiving percutaneous coronary intervention (SORT OUT V): a randomised non-inferiority trial. Lancet 2013;381:661-9.

5. Maeng M, Tilsted HH, Jensen LO, et al. Differential clinical outcomes after 1 year versus 5 years in a randomised comparison of zotarolimus-eluting and sirolimus-eluting coronary stents (the SORT OUT III study): a multicentre, open-label, randomised superiority trial. Lancet 2014;383:2047-56.

6. Bligaard N, Thuesen L, Saunamäki K, et al.; SORT OUT II investigators. Similar five-year outcome with paclitaxeland sirolimus-eluting coronary stents. Scand Cardiovasc J 2014;48:148-55.

7. von Birgelen C, Basalus MW, Tandjung K, et al. A randomized controlled trial in second-generation zotarolimuseluting Resolute stents versus everolimus-eluting Xience $V$ stents in real-world patients: The TWENTE trial. J Am Coll Cardiol 2012;59:1350-61.

8. von Birgelen C, Sen H, Lam MK, et al. Third-generation zotarolimus-eluting and everolimus-eluting stents in all-comer patients requiring a percutaneous coronary intervention (DUTCH PEERS): a randomised, single-blind, multicentre, non-inferiority trial. Lancet 2014;383:413-23.

9. Hordijk-Trion M, Lenzen M, Wijns W, et al. Patients enrolled in coronary intervention trials are not representative of patients in clinical practice: results from the Euro Heart Survey on Coronary Revascularization. Eur Heart J 2006;27:671-8.

10. Hutchinson-Jaffe AB, Goodman SG, Yan RT, et al. Comparison of baseline characteristics, management and outcome of patients with non-ST-segment elevation acute coronary syndrome in versus not in clinical trials. Am J Cardiol 2010;106:1389-96.

11. Rothwell PM. External validity of randomised controlled trials: "To whom do the results of this trial apply?" Lancet 2005;365:82-93.

12. de Boer SPM, Lenzen MJ, Oemrawsingh RM, et al. Evaluating the 'all-comers' design: a comparison of participants in two 'all-comers' PCl trials with non-participants. Eur Heart J 2011;32:2161-7.

13. Sen $\mathrm{H}$, Tandjung $\mathrm{K}$, Basalus MW, et al. Comparison of eligible non-enrolled patients and the randomised TWENTE trial population treated with Resolute and Xience V drug-eluting stents. Eurolntervention 2012;8:664-71.

14. Meredith IT, Worthley S, Whitbourn R, et al. Clinical and angiographic results with the next-generation Resolute stent system: A prospective, multicenter, first-in-human trial. J Am Coll Cardiol Intv 2009;2:977-85.

15. Iqbal J, Serruys PW, Silber S, et al. Comparison of zotarolimus- and everolimus-eluting coronary stents: final 5year report of the RESOLUTE all-comers trial. Circ Cardiovasc Interv 2015;8:e002230.

16. World Medical Association. World Medical Association Declaration of Helsinkis: ethical principles for medical research involving human subjects. JAMA 2013;310:2191-4.

17. Tandjung K, Sen H, Lam MK, et al. Clinical outcome following stringent discontinuation of dual antiplatelet therapy after 12 months in real-world patients treated with second-generation zotarolimus-eluting resolute and everolimus-eluting Xience $V$ stents: 2-year follow-up of the randomized TWENTE trial. J Am Coll Cardiol 2013;61:2406-16.

18. Löwik MM, Lam MK, Sen $\mathrm{H}$, et al. Safety of second-generation drug-eluting stents three years after randomised use in the TWENTE trial. Eurolntervention 2015;10:1276-9.

19. Cutlip DE, Windecker S, Mehran R, et al. Clinical end points in coronary stent trials: A case for standardized definitions. Circulation 2007;115:2344-51.

20. Vranckx P, Cutlip DE, Mehran R, et al. Myocardial infarction adjudication in contemporary all-comer stent trials: Balancing sensitivity and specificity. addendum to the historical $\mathrm{Ml}$ definitions used in stent studies. Eurolntervention 2010;5:871-4. 
21. Mehilli J, Richardt G, Valgimigli M, et al.; ISAR-LEFT-MAIN 2 Study Investigators. Zotarolimus- versus everolimuseluting stents for unprotected left main coronary artery disease. J Am Coll Cardiol 2013;62:2075-82.

22. Park KW, Kang SH, Kang HJ, et al.; HOST-ASSURE Investigators. A randomized comparison of platinum chromiumbased everolimus-eluting stents versus cobalt chromium-based Zotarolimus-Eluting stents in all-comers receiving percutaneous coronary intervention: HOST-ASSURE, a randomized, controlled, noninferiority trial. J Am Coll Cardiol 2014;63:2805-16.

23. Navarese EP, Tandjung K, Claessen B, et al. Safety and efficacy outcomes of first and second generation durable polymer drug eluting stents and biodegradable polymer biolimus eluting stents in clinical practice: comprehensive network meta-analysis. BMJ 2013;347:f6530.

24. Palmerini $T$, Benedetto $U$, Biondi-Zoccai $G$, et al. Long-term safety of drug-eluting and bare-metal stents: evidence from a comprehensive network meta-analysis. J Am Coll Cardiol 2015;65:2496-507.

25. Piccolo R, Stefanini GG, Franzone A, et al. Safety and efficacy of resolute zotarolimus-eluting stents compared with everolimus-eluting stents: a meta-analysis. Circ Cardiovasc Interv 2015;8:e0022

26. Gada H, Kirtane AJ, Newman W, et al. 5-year results of a randomized comparison of XIENCE V everolimus-eluting and TAXUS paclitaxel-eluting stents: final results from the SPIRIT III trial (clinical evaluation of the XIENCE V everolimus eluting coronary stent system in the treatment of patients with de novo native coronary artery lesions). J Am Coll Cardiol Intv 2013;6:1263-6.

27. Smits PC, Vlachojannis GJ, McFadden EP, et al. Final 5-year follow-up of a randomized controlled trial of everolimus- and paclitaxel eluting stents for coronary revascularization in daily practice: The COMPARE trial (A Trial of Everolimus-Eluting Stents and Paclitaxel-Eluting Stents for Coronary Revascularization in Daily Practice). J Am Coll Cardiol Intv 2015;8:1157-65.

28. Jensen LO, Thayssen P, Christiansen EH, et al. Safety and efficacy outcomes of everolimus- versus sirolimuseluting stents: the SORT OUT IV 5-year results. J Am Coll Cardiol 2016;67:751-62.

29. Sabaté M, Brugaletta S, Cequier A, et al. Clinical outcomes in patients with ST-segment elevation myocardial infarction treated with everolimus-eluting stents versus bare-metal stants (EXAMINATION): 5-year results of a randomised trial. Lancet 2016;387:357-66.

30. von Birgelen C, Kok MM, van der Heijden LC, et al. Very thin-strut biodegradable polymer everolimus-eluting and sirolimus-eluting stents versus durable polymer zotarolimus-eluting stents in all-comers with coronary artery disease (BIO-RESORT): a three-arm, randomised, non-inferiority trial. Lancet 2016;388:2607-17. 


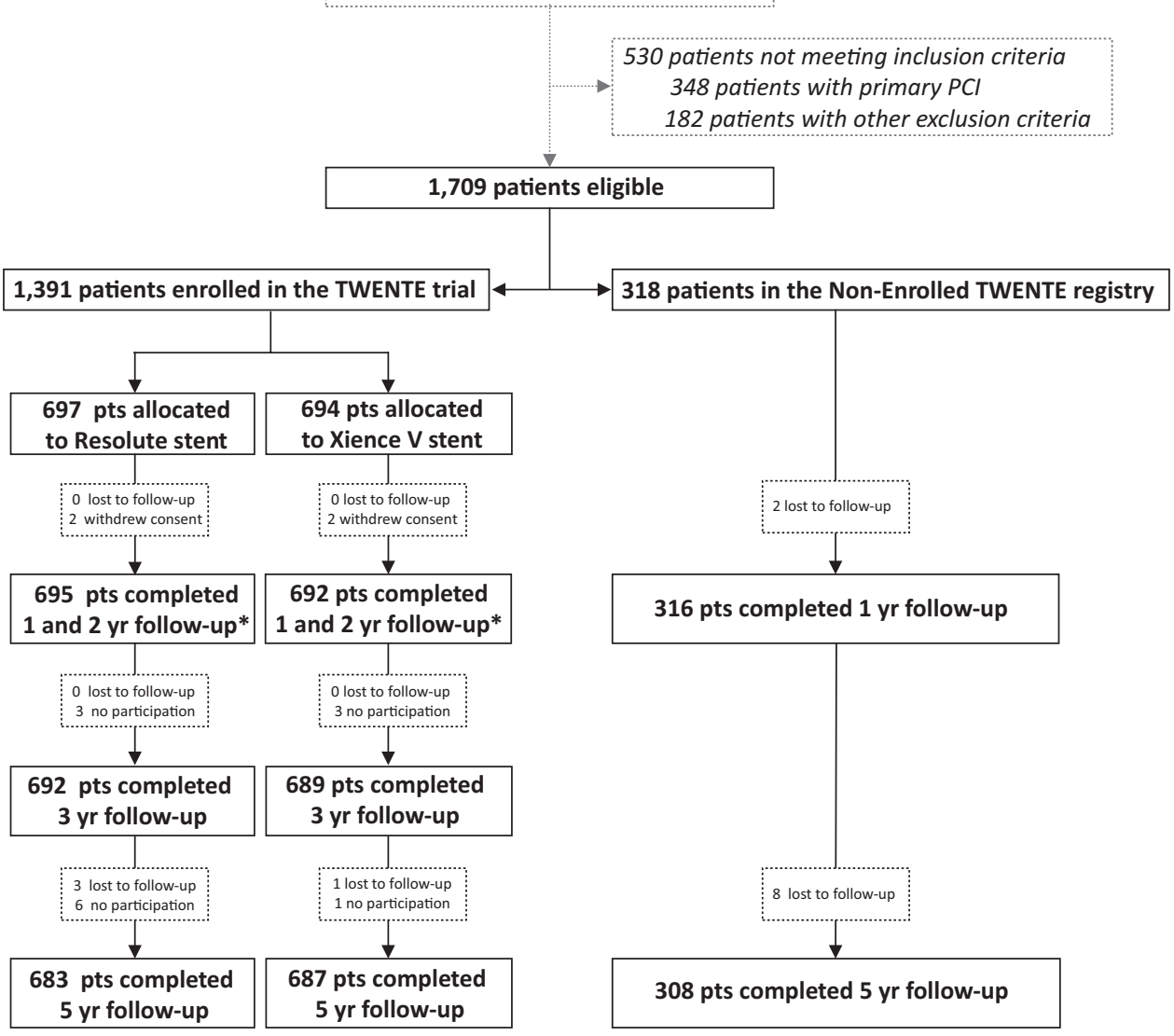

eFigure 1: Study flow diagram of randomized TWENTE trial and Non-Enrolled TWENTE registry. Abbreviations: $\mathrm{DES}=$ drug-eluting stent(s); $\mathrm{PCl}=$ percutaneous coronary intervention; $\mathrm{Pts}=$ patients; $y r=y e a r .{ }^{*}$ No patients were lost or withdrew consent in the second year of follow-up 


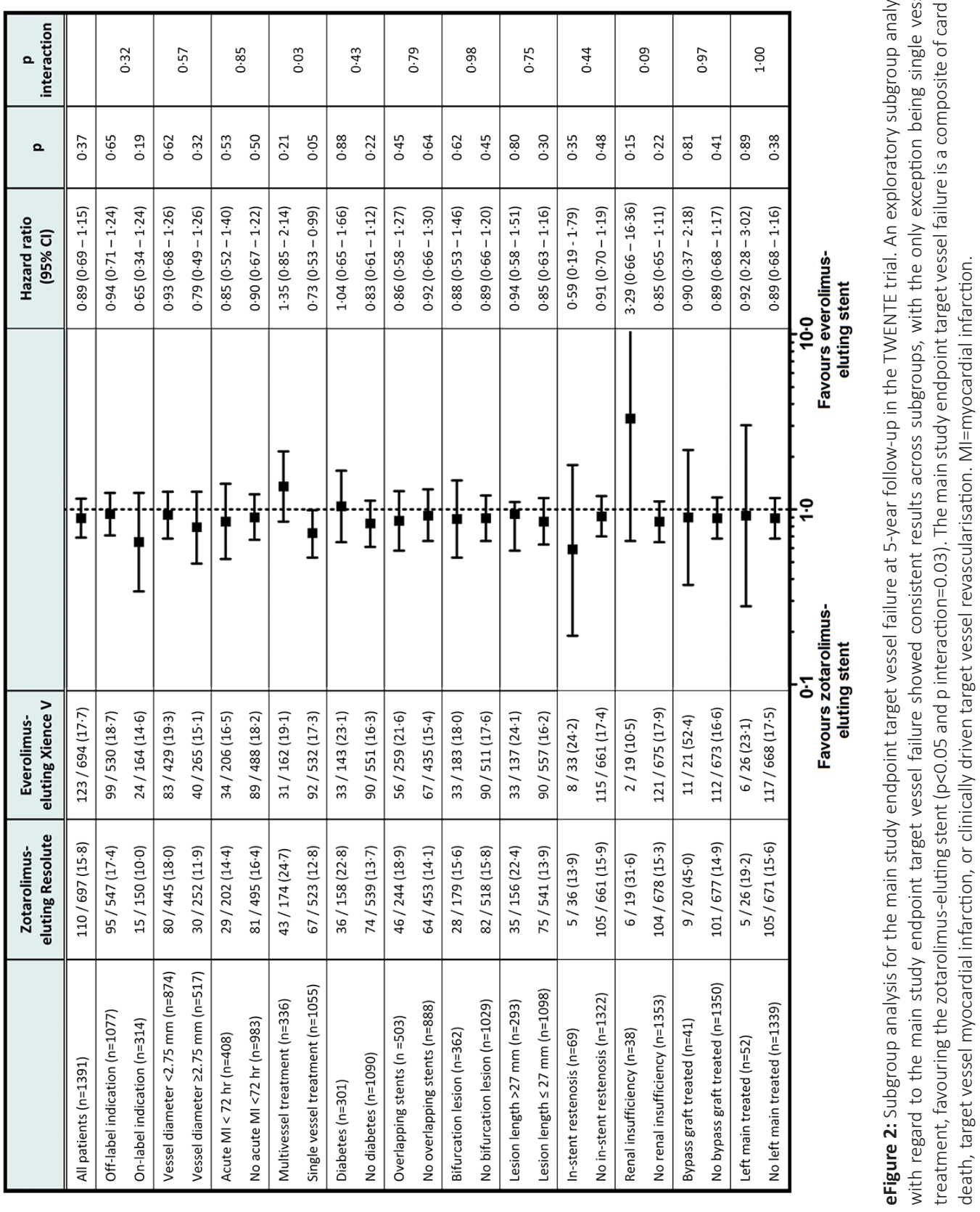



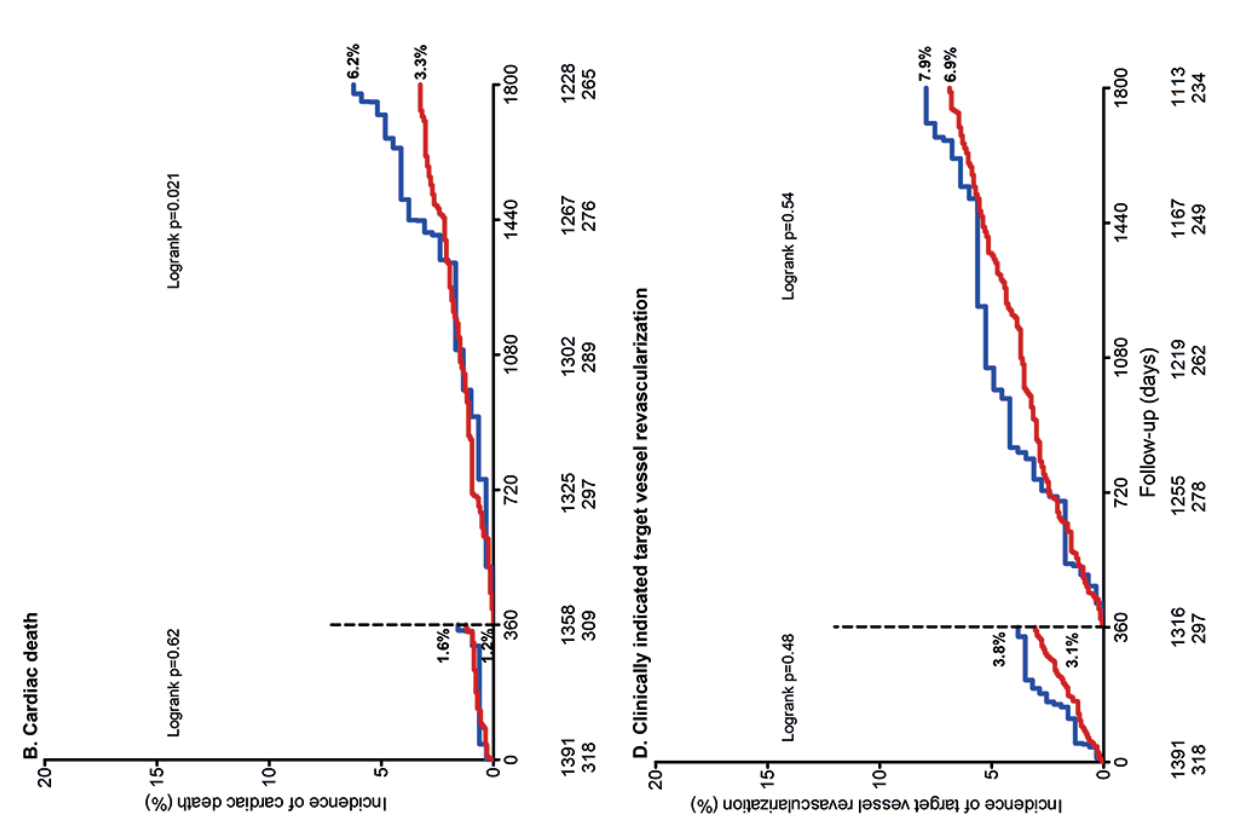

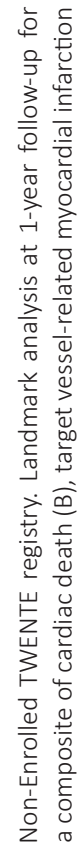
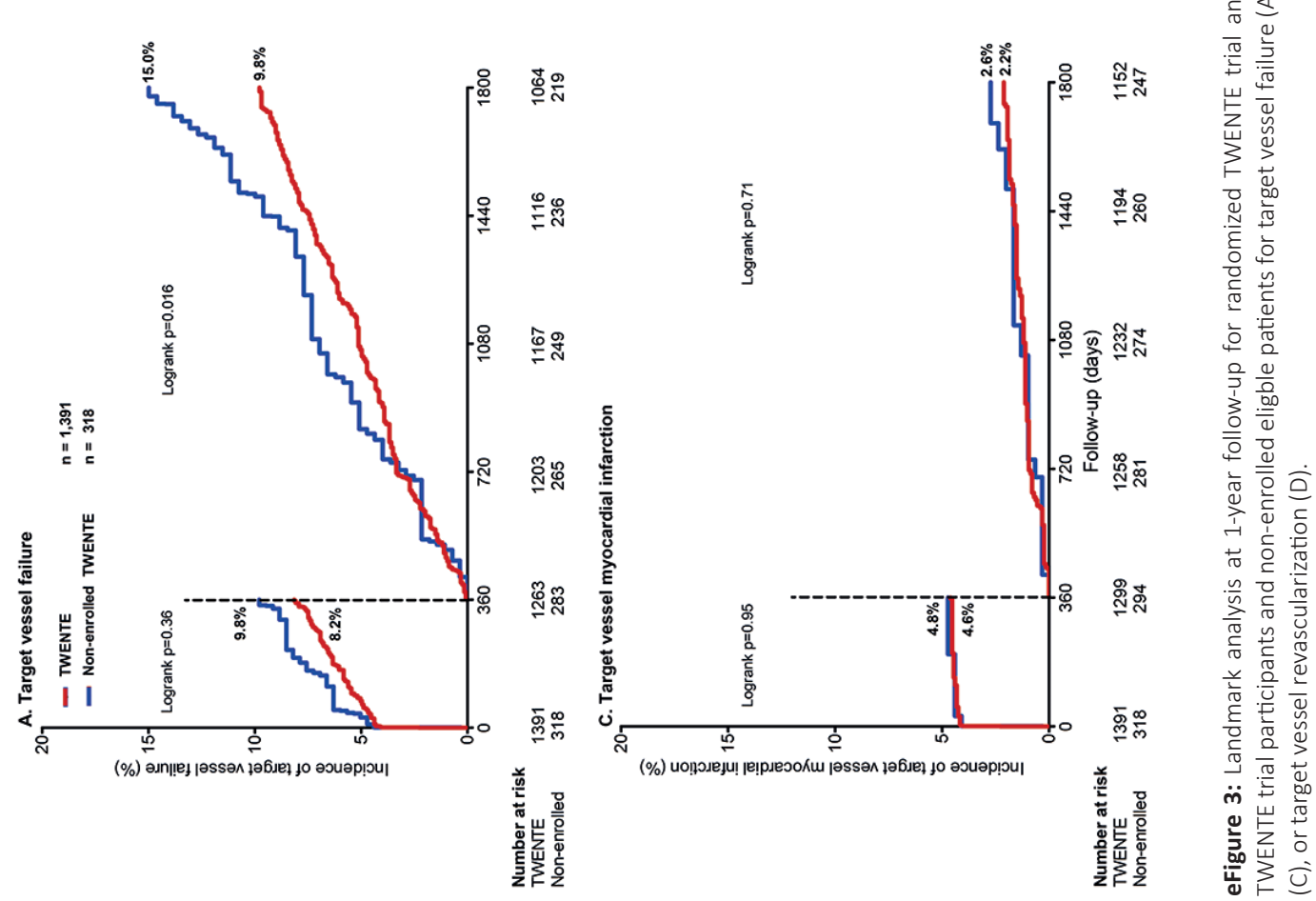


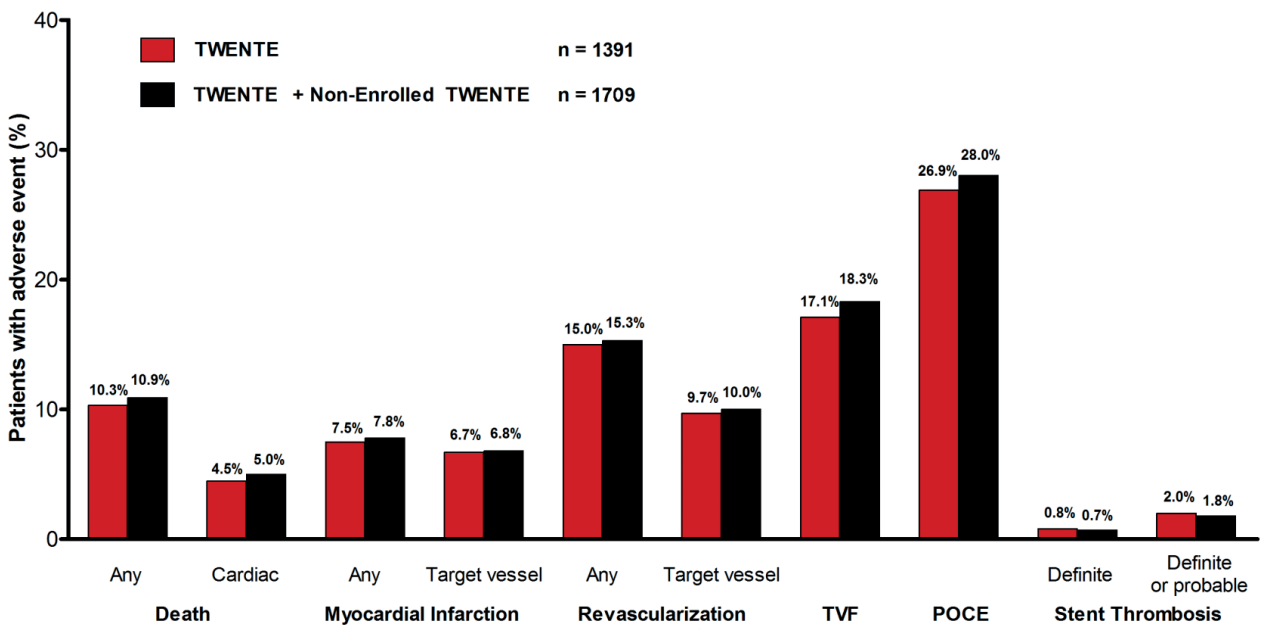

eFigure 4: Mean overall event rates for randomized TWENTE trial participants versus all eligible patients. If all eligible patients had participated in the randomized TWENTE trial (i.e. 100\% enrollment), event rates (black bars) would still have been similar to those of the randomized trial participants (red bars). POCE=patient-oriented composite endpoint; TVF=target vessel failure. 
eTable 1: Baseline characteristics of TWENTE trial participants according to the assigned stent

\begin{tabular}{|c|c|c|c|}
\hline & Resolute stent & Xience V stent & $\mathbf{p}$ \\
\hline Patients & $n=697$ & $n=694$ & \\
\hline Age (yrs) & $63.9(10.9)$ & $64.5(10.7)$ & 0.32 \\
\hline Men & $505(72.5)$ & $504(72.6)$ & 0.94 \\
\hline Diabetes mellitus (any) & $158(22.7)$ & $143(20.6)$ & 0.35 \\
\hline Arterial hypertension & $386(55.4)$ & $387(55.8)$ & 0.89 \\
\hline Hypercholesterolaemia & $392 / 688(57.0)$ & $411 / 669(61.4)$ & 0.10 \\
\hline Current smoker & $176(25.3)$ & $164(23.6)$ & 0.48 \\
\hline Family history of coronary artery disease & $370 / 660(53.1)$ & $370 / 649(53.3)$ & 0.73 \\
\hline Previous myocardial infarction (any) & $213(30.6)$ & $237(34.1)$ & 0.15 \\
\hline Previous $\mathrm{PCl}$ & 139 (19.9) & $149(21.5)$ & 0.48 \\
\hline Previous CABG & $68(9.8)$ & $80(11.5)$ & 0.28 \\
\hline Clinical characteristics & & & 0.77 \\
\hline - Stable angina pectoris & $335(48.1)$ & $339(48.8)$ & \\
\hline - NSTE acute coronary syndrome & $362(51.9)$ & $355(51.2)$ & \\
\hline Multivessel treatment & $174(25.0)$ & $162(23.3)$ & 0.48 \\
\hline No lesions treated per patient & $1.4 \pm 0.5$ & $1.4 \pm 0.5$ & 0.41 \\
\hline At least one small-vessel (RVD $<2.75 \mathrm{~mm}$ ) & $445(63.8)$ & $429(61.8)$ & 0.43 \\
\hline At least one lesion length $>27 \mathrm{~mm}$ & $156(22.4)$ & $137(19.7)$ & 0.23 \\
\hline Lesions & $n=1,080$ & $n=1,036$ & \\
\hline ACC/AHA lesion class type B2/C & $762(70.6)$ & $722(69.7)$ & 0.67 \\
\hline Lesion length (mm) & $14.51(9.85-22.54)$ & $14.30(9.66-21.83)$ & 0.35 \\
\hline Bifurcated lesion & $258(23.9)$ & $260(25.1)$ & 0.59 \\
\hline Chronic total occlusion & $53(4.9)$ & $47(4.5)$ & 0.69 \\
\hline Stents implanted per patient & $2.03 \pm 1.19$ & $2.02 \pm 1.18$ & 0.91 \\
\hline Total stent length per patient (mm) & $41.84 \pm 27.66$ & $40.09 \pm 26.02$ & 0.22 \\
\hline Direct stenting & 416 (38.5) & 408 (39.4) & 0.68 \\
\hline Post-dilation & $876(81.1)$ & $848(82.1)$ & 0.54 \\
\hline
\end{tabular}

Data are number (\%), mean (SD) or median (interquartile range).

Abbreviations: $\mathrm{PCl}=$ percutaneous coronary intervention. $\mathrm{CABG}=$ coronary artery bypass grafting. NSTE=Non-ST-elevation. $\mathrm{RVD}=$ reference vessel diameter. $\mathrm{ACC}=$ American College of Cardiology. $\mathrm{AHA}=$ American Heart Association. 


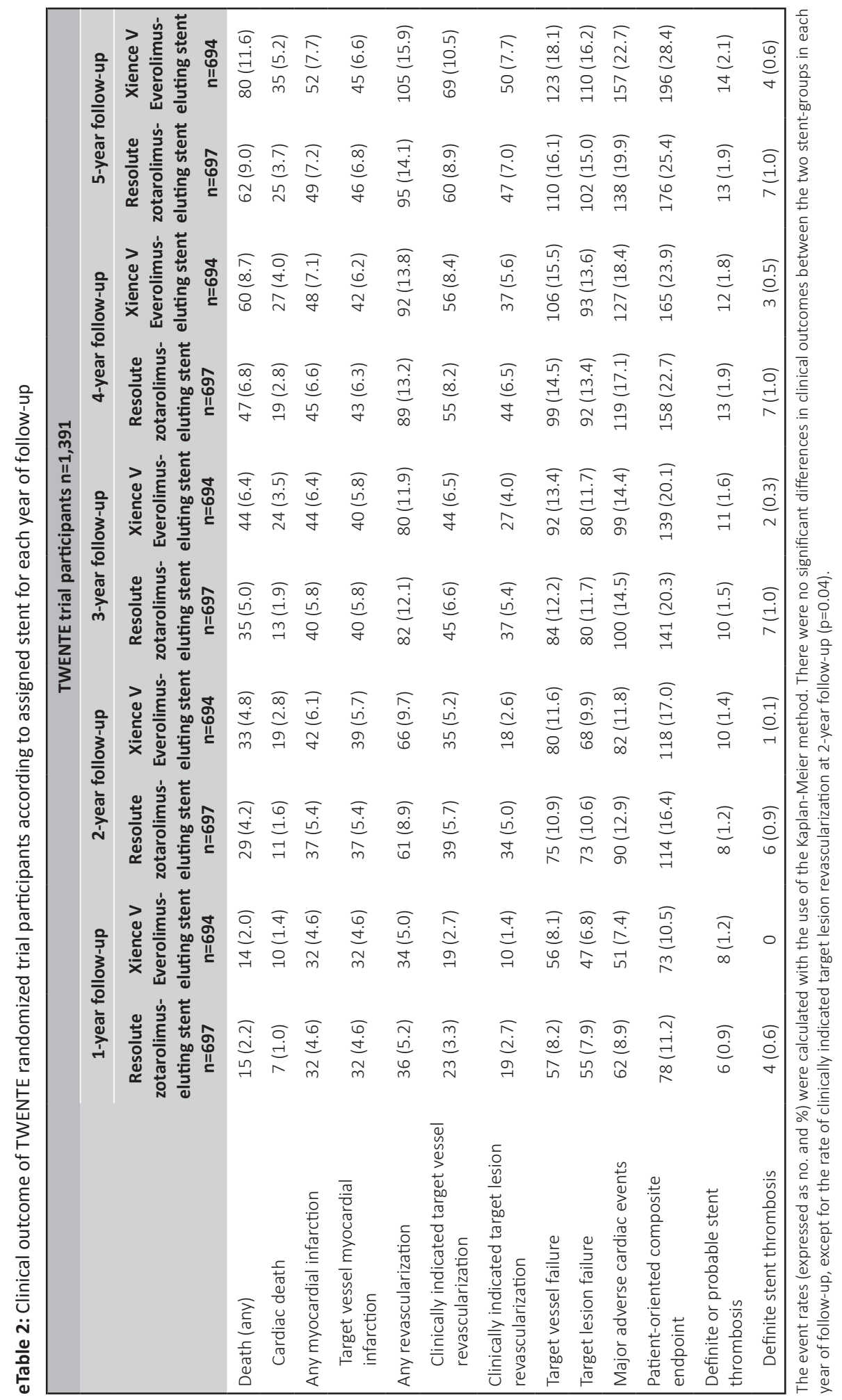


eTable 3: Baseline characteristics of TWENTE randomized trial participants versus non-enrolled eligible patients

\begin{tabular}{|c|c|c|c|}
\hline \multirow[b]{2}{*}{ Patients } & \multicolumn{3}{|c|}{$\begin{array}{l}\text { TWENTE trial and non-enrolled eligible patients } \\
\qquad n=1,709\end{array}$} \\
\hline & $\begin{array}{c}\text { TWENTE } \\
\text { trial population } \\
n=1,391\end{array}$ & $\begin{array}{c}\text { Non-enrolled } \\
\text { eligible patients } \\
\qquad n=318\end{array}$ & $\mathrm{p}$ \\
\hline Age(yrs) & $64.2(10.8)$ & $66.0(10.9)$ & 0.01 \\
\hline Men & 1009 (72.5) & $224(70.4)$ & 0.45 \\
\hline Diabetes mellitus (any) & $301(21.6)$ & $72(22.6)$ & 0.66 \\
\hline Arterial hypertension & $773(55.6)$ & $185(58.2)$ & 0.40 \\
\hline Hypercholesterolemia & $803 / 1357(59.2)$ & $193(60.7)$ & 0.06 \\
\hline Current smoker & $340(24.4)$ & $70(22.0)$ & 0.36 \\
\hline Family history of coronary artery disease & $740 / 1309(53.2)$ & $102 / 193(52.8)$ & 0.93 \\
\hline Previous myocardial infarction (any) & $450(32.4)$ & $137(43.1)$ & $<0.001$ \\
\hline Previous $\mathrm{PCl}$ & $288(20.7)$ & $92(28.9)$ & 0.001 \\
\hline Previous CABG & $148(10.6)$ & $54(17.0)$ & 0.002 \\
\hline Clinical characteristics & & & 0.76 \\
\hline - Stable angina pectoris & $674(48.5)$ & $151(47.5)$ & \\
\hline - NSTE acute coronary syndrome & $717(51.5)$ & $167(52.5)$ & \\
\hline Left ventricular ejection fraction $<30 \%$ & $13 / 199(6.5)$ & $32 / 1051(3.0)$ & 0.015 \\
\hline Multivessel treatment & $336(24.2)$ & $61(19.2)$ & 0.06 \\
\hline No lesions treated per patient & $1.4 \pm 0.5$ & $1.4 \pm 0.6$ & 0.12 \\
\hline At least one in-stent restenosis & $43(13.5)$ & $69(5.0)$ & $<0.001$ \\
\hline Lesions & $n=2,116$ & $n=466$ & \\
\hline Complex lesion* & $1484(70.1)$ & $355(76.2)$ & 0.009 \\
\hline De novo lesion ${ }^{+}$ & $409(87.8)$ & 1999 (94.5) & $<0.001$ \\
\hline Bifurcated lesion & $518(24.5)$ & $101(21.7)$ & 0.20 \\
\hline In stent restenosis & $37(7.9)$ & $75(3.5)$ & $<0.001$ \\
\hline Chronic total occlusion & $100(4.7)$ & $30(6.4)$ & 0.13 \\
\hline
\end{tabular}

Data are number (\%) or mean (SD).

Abbreviations: $\mathrm{PCl}=$ percutaneous coronary intervention. $\mathrm{CABG}=$ coronary artery bypass grafting. NSTE=Non-ST-elevation. *Complex lesion was defined as American College of Cardiology/American Heart Association lesion types B2/C. ${ }^{+}$De novo lesions include chronic total occlusion, but not grafts and in-stent restenosis. 



\section{Conflicts of interest statement}

CvB was consultant to Boston Scientific and Medtronic; he received lecture fees from AstraZeneca and Biotronik. The institution has received research grants, provided by AstraZeneca, Biotronik, Boston Scientific, and Medtronic. All other authors declare that they have no conflict of interest.

\section{Copyright}

Reprinted with permission from

Eurointervention 2017 Apr 20;12(17):2128-2131

(c) Europa Digital \& Publishing 


\section{CHAPTER 3}

\section{Three-Year Safety and Efficacy of Treating All-Comers With Newer-Generation Resolute Integrity or Promus Element Stents in the Randomized DUTCH PEERS (TWENTE II) Trial}

Liefke C. van der Heijden, Marlies M. Kok, Marije M. Löwik, Peter W. Danse, Gillian A.J. Jessurun, Raymond W.M. Hautvast, K. Gert van Houwelingen, Martin G. Stoel, Marc Hartmann, Gerard C.M. Linssen, Carine J.M. Doggen, Clemens von Birgelen
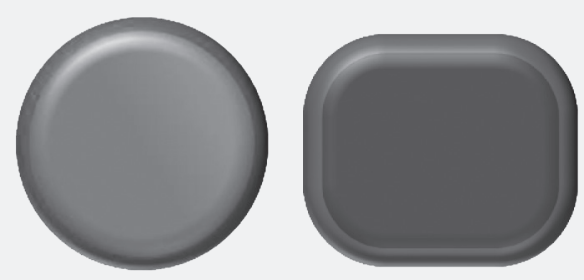


\begin{abstract}
Aim: The aim of this report was to assess the three-year safety and efficacy of implanting newergeneration Resolute Integrity zotarolimus-eluting stents (ZES) versus Promus Element everolimuseluting stents (EES) in all-comers.

Methods and results: In the randomised, multicentre, investigator-initiated DUTCH PEERS trial, a total of 1,811 all-comers were 1:1 randomly assigned to treatment with ZES versus EES. A total of 1,293 patients (72\%) were treated for complex lesions and 455 patients (25\%) were treated for multiple lesions. The primary endpoint target vessel failure (TVF) is a composite of cardiac death, target vessel-related myocardial infarction or target vessel revascularisation. Adverse clinical events were independently adjudicated.

Three-year follow-up data were obtained in 1,807 patients (99.8\%, four withdrawals). Both the ZES and EES groups, showed favourable outcomes with a similar incidence of TVF (10.7\% vs $10.3 \%$; p-Logrank $=0.77$ ) and the individual components thereof: cardiac death (3.2\% vs 3.1\%; $p$-Log-rank=0.87), target vessel-related myocardial infarction ( $2.8 \%$ vs $2.2 \%$; P-Log-rank $=0.44$ ) and target vessel revascularisation (6.0\% vs 6.2\%; $p=$ Log-rank=0.87). In addition, the incidence of definite-or-probable stent thrombosis was similar for patients treated with ZES versus EES (1.4\% vs $1.1 \%$; $p$-Log-rank=0.66).
\end{abstract}

Conclusion: The safety and efficacy of treating all-comers with newer-generation Resolute Integrity and Promus Element stents was found to be extended up to three years. 


\section{Introduction}

Newer-generation metallic drug-eluting stents (DES), such as the cobalt-chromium-based Resolute Integrity zotarolimus-eluting stent (ZES) (Medtronic, Minneapolis, MN, USA) and the platinum chromiumbased Promus Element everolimus-eluting stent (EES) Boston Scientific, Marlborough, MA, USA), have stent designs that were developed to facilitate deliverability and further improve DES apposition. ${ }^{1,2}$ Both DES were compared for the first time in the randomised DUTCH PEERS trial, which was also the first trial ever to investigate Resolute Integrity. In 1,811 all-comers, this study demonstrated non-inferiority of the ZES versus the EES at 12-month follow-up. ${ }^{1}$ Long-term data from the DUTCH PEERS trial are of interest, as certain between-stent differences may only be discovered after years. We therefore assessed the three-year clinical outcome of percutaneous coronary interventions in the two DES arms.

\section{Methods}

The design of the study, definitions of clinical endpoints and characteristics of patients, lesions and procedures of the multicentre, patient-blinded, investigator-initiated, 1:1 randomised DUTCH PEERS (TWENTE II) trial (ClinicalTrials.gov NCT01331707) have been reported previously. ${ }^{1}$ DUTCH PEERS enrolled 1,811 patients with any type of clinical syndrome, including $20.4 \%$ of patients with ST-elevation myocardial infarction (MI), 24.7\% with non-ST-elevation $\mathrm{MI}$ and $71.7 \%$ with complex coronary lesions. ${ }^{1}$ The external CRO Diagram (Zwolle, the Netherlands) monitored clinical outcome in $10 \%$ of randomly selected patients and organised the adjudication of adverse events by an independent clinical events committee for both two- and three-year follow-up. The DUTCH PEERS trial complied with the Declaration of Helsinki and was approved by the Medical Ethics Committee Twente and the institutional review boards of all participating centers. All patients provided written informed consent. Clinical endpoints were defined according to the Academic Research Consortium (ARC), including the addendum on MI.,4 Primary endpoint was target vessel failure (TVF) at one year, a composite of cardiac death, target vesselrelated $\mathrm{MI}$ and clinically-indicated target vessel revascularisation. P-values and confidence intervals were two-sided and $p$-values $<0.05$ were considered significant. Statistics were performed as previously reported, ${ }^{4}$ using SPSS, Version 22.0 (IBM Corp. Armonk, NY, USA).

\section{Results}

Three-year follow-up was obtained from 1,807 patients (99.8\%; four consent withdrawals). Patients treated with Resolute Integrity ZES ( $n=906)$ and Promus Element EES ( $n=905)$ showed favourable outcomes with similar TVF rates ( $10.7 \%$ vs. $10.3 \%$; p-Log-rank=0.77) (Table 1, Figure 1). The incidence of the individual components of TVF was similar for both DES: cardiac death (3.2\% vs $3.1 \%$; p-Logrank=0.87); target vessel-related $\mathrm{MI}(2.8 \%$ vs $2.2 \%$; $\mathrm{p}$-Log-rank=0.44); and target vessel-related $\mathrm{MI}$ ( $2.8 \%$ vs $2.2 \%$; $p$-Log-rank=0.44); and target vessel revascularisation ( $6.0 \%$ vs $6.2 \%$; $p$-Log-rank=0.87) (Table 1, Figure 1). At three-year follow-up, $6.1 \%$ of patients treated with Resolute Integrity ZES and $7.1 \%$ of patients treated with Promus Element ZES were on dual antiplatelet therapy. 


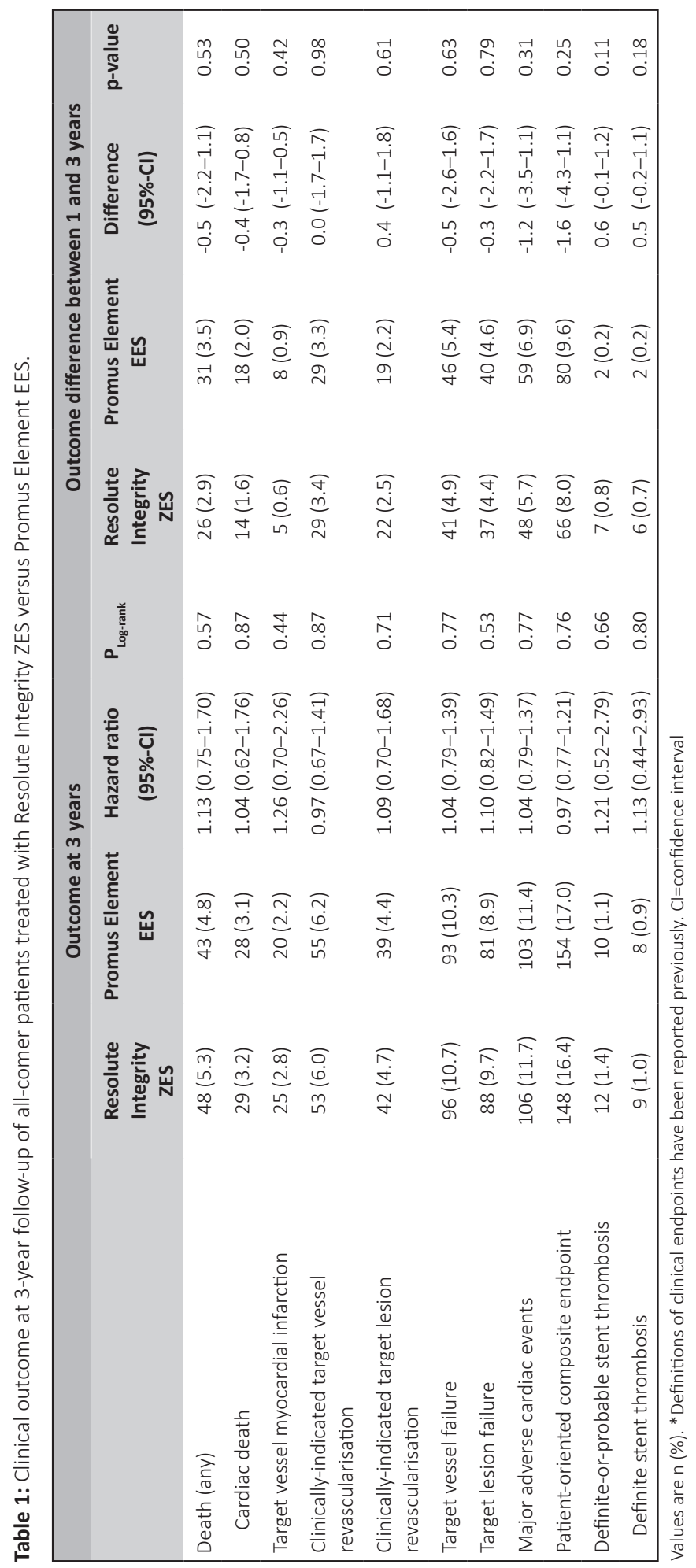



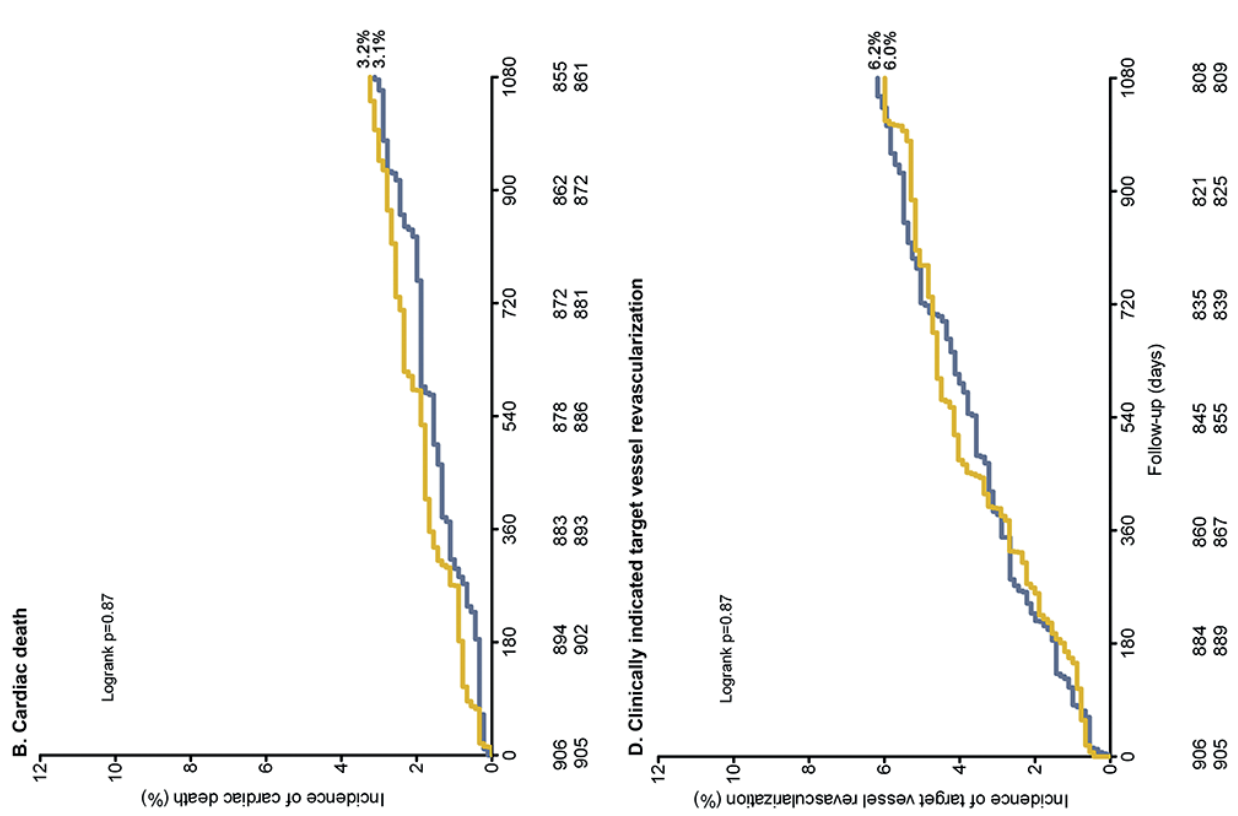

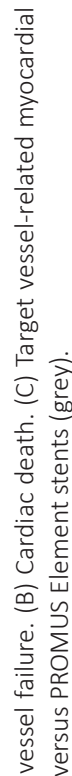
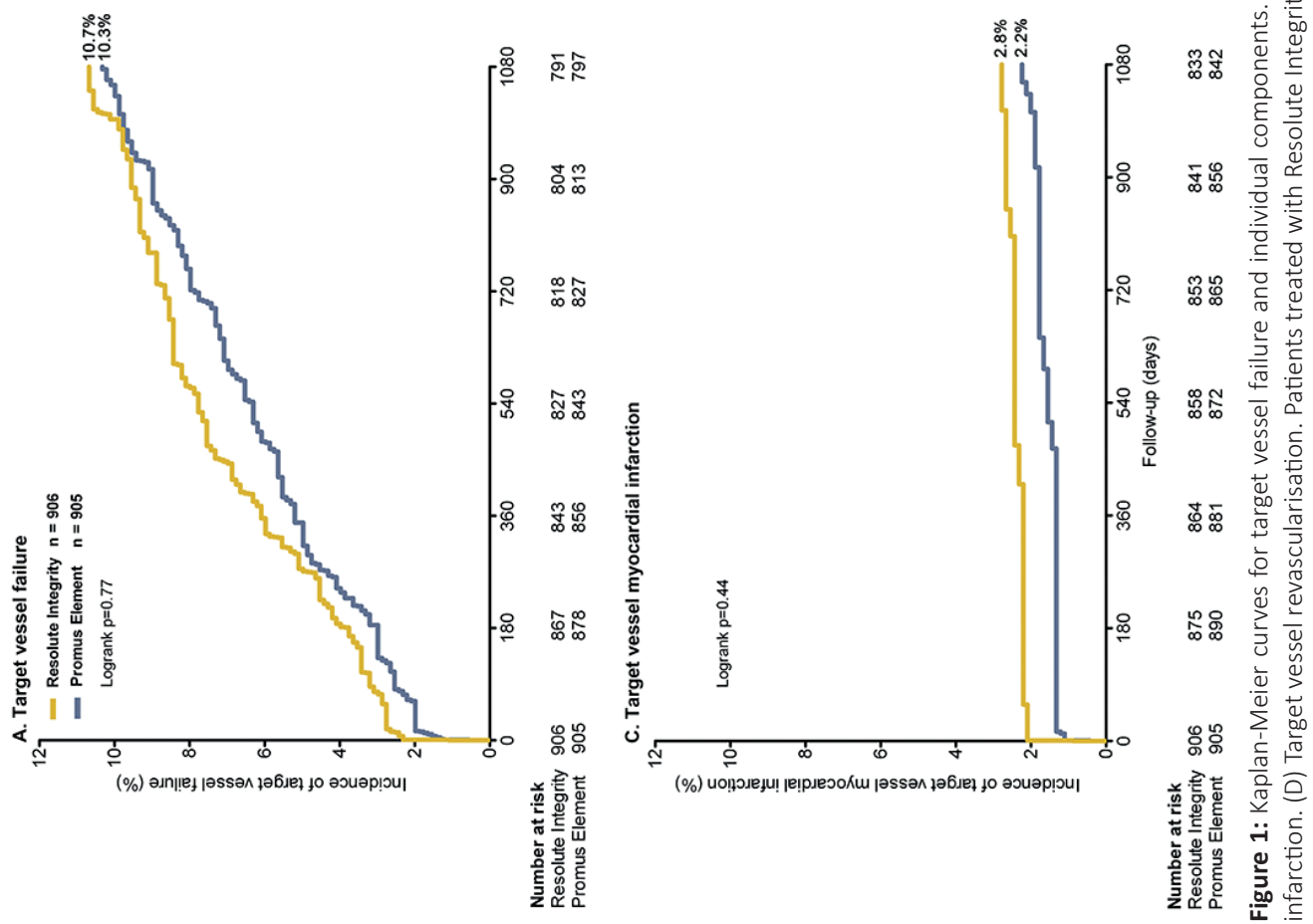


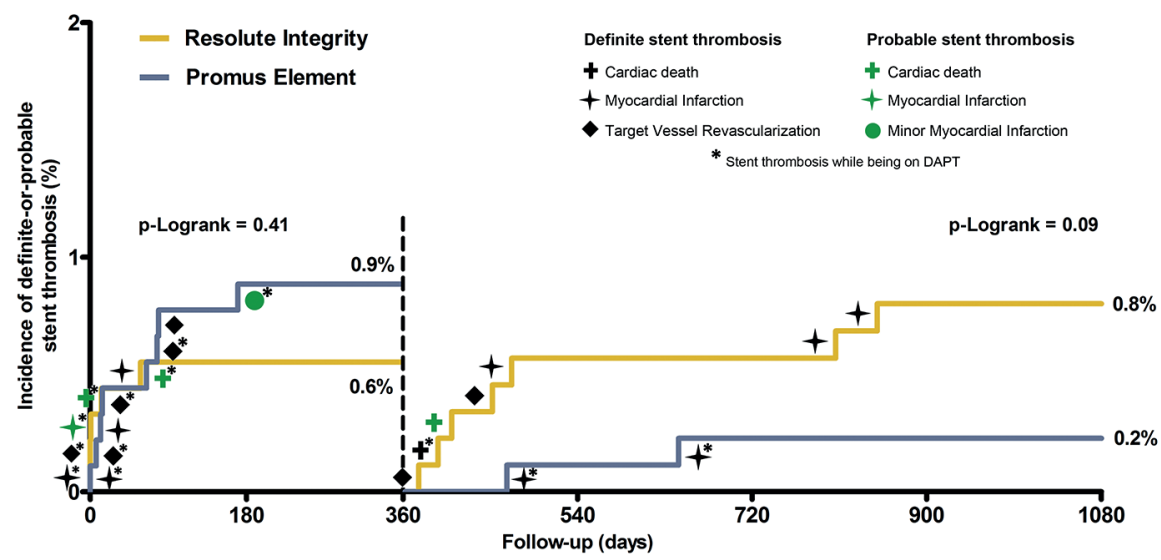

Figure 2: Kaplan-Meier curve for stent thrombosis. Patients treated with Resolute Integrity (yellow) versus PROMUS Element stents (grey). Dual antiplatelet therapy means acetylsalicylic acid plus P2Y12 receptor antagonist.

As shown in Table 1 and Figure 2, the rates of definite and definite-or-probable stent thrombosis were low for patients treated with Resolute Integrity ZES and Promus Element EES (1.0\% vs $0.9 \%$; p-Logrank $=0.80$ and $1.4 \%$ vs $1.1 \% ; p=$ Log-rank $=0.66$, respectively). Due to an apparent dissimilarity between the Resolute Integrity ZES and Promus Element EES groups in the course of their time-to-event curves for definite or probable stent thrombosis (catch-up after $>1$ year vs. main increase within first 12 months) (Figure 2), we performed post hoc a landmark analysis at 12-month follow-up. Definite or probable stent thrombosis occurred during the first year in $0.6 \%$ vs $0.9 \%$ ( $p$-Log-rank $=0.41$ ) of patients and during the second plus third years in $0.8 \%$ vs. $0.2 \%$ (p-Log-rank $=0.09)$ of patients.

\section{Discussion}

The current study reports the three-year clinical outcome of the DUTCH PEERS trial, which is the first randomised study to compare Resolute Integrity ZES versus Promus Element EES in all-comers. ${ }^{1}$ Patients of both stent arms had similar and relatively low rates of the main clinical endpoint TVF $(10.7 \%$ vs $10.3 \%$ ), and the incidence of definite or probable stent thrombosis was low and comparable (1.4\% vs $1.1 \%)$.

Our findings corroborate with the results of previous randomised studies, that compared the predecessors of these newer-generation DES in broad patient populations. ${ }^{5,6}$ Long-term outcome data about Resolute Integrity ZES and Promus Element EES in all-comers are scarce. The only randomised trial other than DUTCH PEERS that studied Resolute Integrity in all-comers is the SORT OUT VI study², but definite long-term results of that study have not yet been published. In addition, the PLATINUM trial is the only randomised trial that has published three-year follow-up data on the use of Promus Element in low-to-moderate risk patients (e.g. $\leq 2$ de novo lesions in vessels $\geq 2.5 \mathrm{~mm}$ ), showing similar 
safety and efficacy for both Promus Element and Xience V (Abbott Vascular, Santa Clara, CA, USA). ${ }^{7}$ Our current three-year follow-up data support these favourable findings in a broader, greatly unrestricted patient population.

As the present study was not powered to assess between-group differences in secondary clinical endpoints, these findings should be considered hypothesis-generating.

\section{Conclusion}

The safety and efficacy of treating all-comers with newer-generation Resolute Integrity and Promus Element stents in the randomised DUTCH PEERS trial was extended up to three years.

\section{Impact on daily practice}

The three-year results of the DUTCH PEERS trial are the first long-term data in all-comers from a randomised comparison of the newer-generation Resolute Integrity ZES and the Promus Element EES, two stents that are often used in routine clinical practice. The consistently low rates of adverse clinical events, such as target vessel myocardial infarction, target vessel revascularisation and definite or probable stent thrombosis, provide a strong signal of sustained safety and efficacy of both metallic drugeluting stents in this broad patient population. These long-term outcome data fill a gap in the literature and might in the future be useful for interpreting long-term data following broader applications of bioresorbable scaffolds.

\section{Funding}

This investigator-initiated study was supported equally by Boston Scientific and Medtronic. 


\section{References}

1. von Birgelen C, Sen H, Lam MK, et al. Third-generation zotarolimus-eluting and everolimus-eluting stents in all-comer patients requiring a percutaneous coronary intervention (DUTCH PEERS): a randomised, single-blind, multicentre, non-inferiority trial. Lancet 2014;383:413-23.

2. Raungaard B, Okkels Jensen L, Tilsted $\mathrm{HH}$, et al. Zotarolimus-eluting durable-polymer-coated stent versus a biolimus-eluting biodegradable-polymer-coated stent in unselected patients undergoing percutaneous coronary intervention (SORT OUT VI): a randomised non-inferiority trial. Lancet 2015;385:1527-35.

3. Cutlip DE, Windecker S, Mehran R, et al. Clinical end points in coronary stent trials: a case for standardized definitions. Circulation 2007;115:2344-51.

4. Vranckx P, Cutlip DE, Mehran R, Kint PP, Silber S, Windecker S, Serruys PW. Myocardial infarction adjudication in contemporary all-comer stent trials: balancing sensitivity and specificity: addendum to the historical $\mathrm{MI}$ definititons used in stent studies. Eurolntervention 2010;5:871-4.

5. Iqbal J, Serruys PW, Silber S, et al. Comparison of zotarolimus- and everolimus-eluting coronary stents: final 5-year report of the RESOLUTE all-comers trial. Circ Cardiovasc Interv 2015;8:e002230.

6. Lowik MM, Lam MK, Sen H, et al. Safety of second-generation drug-eluting stents three years after randomised use in the TWENTE trial. Eurolntervention 2015;10:1276-9.

7. Meredith IT, Teirstein PS, Bouchard A, et al. Three-year results comparing platinum-chromium PROMUS element and cobalt-chromium XIENCE $V$ everolimus-eluting stents in de novo coronary artery narrowing (from the PLATINUM trial). Am J Cardiol 2014;113:117-23. 



\section{Conflicts of interest statement}

CVB was consultant to Boston Scientific and Medtronic; he received lecture fees from AstraZeneca and a travel grant from Biotronik. The institution has received research grants, provided by AstraZeneca, Biotronik, Boston Scientific, and Medtronic. All other authors declare that they have no conflict of interest.

\section{Copyright}

Reprinted with permission from

Cardiology 2017;137(4):207-217

(c) S. Karger AG, Basel 


\title{
CHAPTER 4
}

\section{Three-Year Clinical Outcome of Patients With Coronary Disease and Increased Event Risk Treated With Newer-Generation Drug-Eluting Stents}

\author{
From the Randomized DUTCH PEERS Trial
}

Liefke C. van der Heijden, Marlies M. Kok, Marije M. Löwik, Peter W. Danse,

Gillian A.J. Jessurun, Marc Hartmann, Martin G. Stoel, K. Gert van Houwelingen,

Raymond W.M. Hautvast, Gerard C.M. Linssen, Carine J.M. Doggen, Clemens von Birgelen
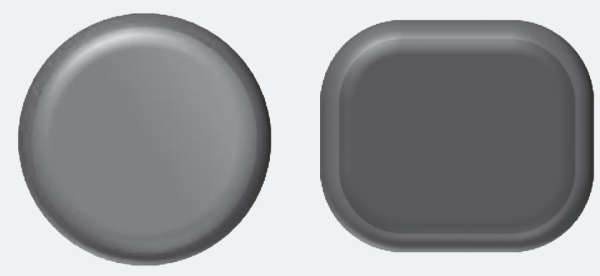


\begin{abstract}
Objective: Limited data is available on the long-term outcome of patients with increased cardiovascular event risk, treated with newer-generation durable polymer drug-eluting stents (DES).

Methods: We therefore assessed 3-year follow-up data of high-risk versus low- to intermediate- risk patients of the randomized DUTCH PEERS trial (NCT01331707). In both risk groups we also compared patients treated with Resolute Integrity versus Promus Element DES. Patients were categorized as 'highrisk' if they met $\geq 1$ of the following criteria: (1) diabetes (17.9\%); (2) previous myocardial infarction (21.9\%); (3) previous coronary revascularization (25.8\%); (4) chronic renal failure (3.5\%); (5) left ventricular ejection fraction $\leq 30 \%$ (1.5\%); and (6) age $\geq 75$ years $(17.3 \%)$.

Results: At the 3-year follow-up, the incidence of the composite endpoint target vessel failure (TVF) $(13.2 \%$ vs $7.5 \%$; p-Logrank<0.001) and two of its components-cardiac death $(4.7 \%$ vs $1.5 \%$; p-Logrank $<0.001)$ and target vessel revascularization (7.3\% vs $4.7 \%$; $p$-Logrank $=0.03)$ - was higher in high-risk ( $n=957)$ versus low-to-intermediate risk patients $(n=854)$. Among high-risk patients, treatment with Resolute Integrity $(n=481)$ and Promus Element stents $(n=476)$ was similarly safe and efficacious (TVF: $13.3 \%$ vs 13.1\%; p-Logrank=0.95; definite-or-probable stent thrombosis: $1.7 \%$ vs $1.7 \%$; p-Logrank=1.00).
\end{abstract}

Conclusions: The newer-generation Resolute Integrity and Promus Element stents showed similar results in terms of safety and efficacy for treating high-risk patients, who had significantly higher event rates than low-to-intermediate risk patients. 


\section{Introduction}

The development of drug-eluting stents (DES), used in percutaneous coronary interventions (PCI), has revolutionized the treatment of patients who suffer from obstructive coronary artery disease. Firstgeneration DES were associated with an increased risk of late and very late stent thrombosis, and these devices had stent designs with limited flexibility. ${ }^{1}$ Second-generation durable polymer DES, showed an improved biocompatibility that resulted in improved safety profiles in various clinical settings. ${ }^{2,3}$ Newer-generation durable polymer-based drug-eluting stents (DES) with modified stent designs were then developed to facilitate device deliverability in challenging anatomical situations and to further improve the apposition to the vessel wall. ${ }^{4-8}$ Examples of such devices are the cobalt chromium-based Resolute Integrity zotarolimus-eluting stents (ZES) and the platinum chromium-based Promus Element everolimus-eluting stents (EES), which were compared for the first time in the randomized all-comer DUTCH PEERS trial. ${ }^{4}$

Certain 'high-risk' patients, treated with various types of DES, had an increased risk of encountering adverse clinical events. Among these patients are diabetics, elderly patients, and patients with previous myocardial infarction or prior coronary revascularization., ${ }^{9,10}$ Previous studies have shown that, even among high-risk patients, between-DES differences may not be seen during the first year of follow-up, when most patients are on dual anti-platelet therapy. ${ }^{10}$ So, information on the long-term outcome of such high-risk patients treated with the newer-generation DES, assessed in the DUTCH PEERS trial, is clinically relevant but rare. ${ }^{7,8,11}$ Therefore, we evaluated the 3 -year clinical outcome of high-risk patients of the DUTCH PEERS trial.

\section{Methods}

\section{Study design and patient population}

Details of the DUTCH PEERS (TWENTE II) trial (ClinicalTrials.gov NCT01331707) have previously been reported. ${ }^{4}$ In brief, DUTCH PEERS is a multicenter, prospective, randomized, single-blinded, investigatorinitiated trial in an all-comers patient population. Patients were enrolled between November 25, 2010 and May 24, 2012. All-comer patients who were aged 18 years and older, were capable of providing informed consent and required a percutaneous coronary intervention with implantation of a drugeluting stent were randomly assigned for treatment with one of the two study stents. All coronary syndromes, de novo and restenotic lesions, and coronary artery or bypass stenosis were permitted (with no limit for lesion length, reference size, or number of lesions or diseased vessels). The trial complied with the Declaration of Helsinki and was approved by the Medical Ethics Committee Twente and the institutional review boards of all participating centers. All patients provided written informed consent. Patients were categorized as high-risk patients (versus low-to-intermediate risk patients), if they met at least one of the following criteria: (1) diabetes mellitus; (2) previous Ml; (3) previous coronary revascularization; (4) chronic renal failure (serum creatinine level $\geq 130 \mu \mathrm{mol} / \mathrm{L}$ ); (5) left ventricular ejection fraction $\leq 30 \%$ : (6) age $\geq 75$ years. ${ }^{9,10,12-17}$ 


\section{Stents and interventional procedure}

The newer-generation Resolute Integrity stent releases zotarolimus from a permanent polymer coating, which has been highly effective on Resolute stents. ${ }^{2,3,18}$ It uses a novel stent platform with an opencell design that is made from a single, sinusoid-shaped cobalt-chromium wire. ${ }^{19}$ The newer-generation Promus Element stent releases everolimus from a permanent fluoropolymer coating that recently demonstrated its efficacy in other patient populations. ${ }^{2,3,12,20-22}$ Its novel stent platform has an open-cell design that is laser-cut from radiopaque platinum-chromium alloy. $4,7,23$

Interventional procedure and administration of concomitant medication were performed in accordance to medical guidelines, clinical standards, and the physician's judgment. Dual anti-platelet therapy was generally prescribed for 1 year after stenting. Systematic laboratory and electrocardiographic testing were performed as previously described ${ }^{4}$ to identify periprocedural myocardial infarction (MI).

\section{Clinical follow-up, angiographic analysis, monitoring, and adjudication}

The follow-up procedures of this study have previously been reported. ${ }^{4,11}$ Clinical follow-up data were obtained at visits to outpatient clinics or, if not feasible, by a medical questionnaire, telephone followup, or both (with staff blinded to assigned study stents). Angiographic analysts from Thoraxcentrum Twente, formally blinded to the assigned type of stent and clinical outcome, performed offline quantitative coronary angiographic analyses with Qangio XA 7.2 (Medis, Leiden, the Netherlands) for all patients. The external contract research organization Diagram (Zwolle, the Netherlands) monitored clinical outcome and organized the adjudication of adverse events by an independent clinical event committee.

\section{Clinical endpoints}

The definitions of the clinical endpoints have previously been described in detail. ${ }^{4}$ Clinical endpoints, including stent thrombosis, were defined according to the Academic Research Consortium, including the addendum on MI. ${ }^{24,25}$ In brief, target vessel failure (TVF), the prespecified composite primary endpoint of the DUTCH PEERS trial, was composed of (in hierarchical order): cardiac death, target vessel related $\mathrm{MI}$, or clinically indicated target vessel revascularization. Prespecified secondary endpoints included: the individual components of TVF, all-cause mortality, any myocardial infarction, clinically indicated target lesion revascularization, and stent thrombosis. Other composite clinical endpoints were: target lesion failure, a composite of cardiac death, target vessel related $\mathrm{MI}$, or clinically indicated target lesion revascularization; major adverse cardiac events, a composite of all-cause death, any MI, emergent coronary bypass surgery, or clinically indicated target lesion revascularization; and patient-oriented composite endpoint (POCE), a composite of all-cause death, any MI, or any revascularization.

\section{Statistical analysis}

Data were reported as frequencies and percentages for dichotomous and categorical variables, as mean \pm standard deviation for continuous normally distributed variables, and as median and interquartile range for not normally distributed variables. Chi-square test and Fisher's exact test were used as appropriate. Differences between groups in continuous variables were assessed with the Student's $t$ test or the Wilcoxon rank-sum test. The Kaplan-Meier method was used to calculate the time to clinical 
endpoints and the Logrank test was applied to compare groups. In a multivariate analysis, parameters were considered as potential confounders if in univariate analyses associations were found with a $p$-value $<0.15$. A multivariate Cox regression model was then used to adjust for potential confounders. All $p$-values and confidence intervals were two-sided and $p$-values $<0.05$ were considered significant. No adjustment was performed for multiple testing. Data analysis was performed with SPSS (version 22.0, SPSS Inc., Chicago, IL, USA).

\section{Results}

\section{Study population and procedural details}

Of all trial participants, 957 (52.8\%) were categorized as high-risk patients while 854 (47.2\%) patients had a low-to-intermediate risk. The baseline clinical, procedural, and angiographic characteristics of both groups are shown in Table 1. High-risk patients were predominantly men, presented more often with stable angina, and underwent more often treatment in lesions in left main stems or vein grafts ( $p<0.001$, for all parameters).

Among the high-risk patients, 481 (50.2\%) were treated with Resolute Integrity and 476 (49.8\%) with Promus Element. The characteristics of patients, lesions, and procedures did not differ significantly between DES groups, except for a higher rate of male patients treated with Resolute Integrity $\mathbf{7 4 . 6 \%}$ vs. 67.9\%; $p=0.02$ ) (Web-based Table 1).

\section{Clinical outcome of high-risk versus low-to-intermediate risk patients}

The 3-year follow-up was obtained from all but 4 patients, who withdrew consent (Consort diagram shown in Web-based Figure 1). High risk patients, as compared to low-to-intermediate risk patients, showed a significant difference in the primary endpoint TVF (13.2\% vs 7.5\%; p-Logrank<0.001) (Table 2 , Figure 1). The incidences of cardiac death ( $4.7 \%$ vs $1.5 \%$; p-Logrank $<0.001)$ and target vesselrelated revascularization (7.3\% vs $4.7 \%$; $p$-Logrank=0.03), two of the individual components of TVF, were significantly higher among high-risk patients. In addition, the rate of definite-or-probable stent thrombosis tended to be higher in high-risk patients ( $1.7 \%$ vs $0.7 \%$; $p$-Logrank=0.06) (Table 2, Figure 2). At 3-year follow-up, more patients in the high-risk group used dual antiplatelet therapy (acetylsalicylic acid plus P2Y12 receptor antagonist) ( $8.6 \%$ vs $5.2 \% ; \mathrm{p}=0.006$ ). In addition, patients in the high-risk group more often used oral anticoagulant therapy $(18.8 \%$ vs $6.1 \% ; p<0.001)$ and less often aspirin (77.3\% vs $89.1 \% ; p<0.001$ ). 
Table 1: Patient, lesion, and procedural characteristics of high-risk patients versus low-to-intermediate risk patients.

\begin{tabular}{|c|c|c|c|}
\hline \multirow[b]{2}{*}{ Patient characteristics } & \multicolumn{2}{|c|}{$\begin{array}{c}\text { All patients } \\
n=1,811\end{array}$} & \multirow[b]{2}{*}{ p } \\
\hline & High-risk patients & $\begin{array}{c}\text { Low-to-intermediate risk } \\
\text { patients }\end{array}$ & \\
\hline Number of patients treated & 957 & 854 & \\
\hline Age (yrs) & $68.0 \pm 10.5$ & $59.3 \pm 9.2$ & $<0.001$ \\
\hline Women & $275(28.7)$ & $214(25.1)$ & 0.08 \\
\hline $\mathrm{BMI}\left(\mathrm{kg} / \mathrm{m}^{2}\right)$ & $28.2 \pm 4.6^{a}$ & $27.6 \pm 4.8^{b}$ & 0.01 \\
\hline Diabetes mellitus & $324(33.9)$ & 0 & \\
\hline Chronic renal failure & $63(6.6)$ & 0 & \\
\hline LVEF $<30 \%$ & $28(2.9)$ & 0 & \\
\hline Previous myocardial infarction & $397(41.5)$ & 0 & \\
\hline Previous $\mathrm{PCl}$ & $349(36.5)$ & 0 & \\
\hline Previous CABG & $173(18.1)$ & 0 & \\
\hline Clinical syndrome at presentation & & & $<0.001$ \\
\hline Stable angina pectoris & $439(45.9)$ & $310(36.3)$ & \\
\hline Unstable angina pectoris & $140(14.6)$ & $105(12.3)$ & \\
\hline Non-ST-elevation myocardial infarction & $270(28.2)$ & $177(20.7)$ & \\
\hline ST-elevation myocardial infarction & $108(11.3)$ & $262(30.7)$ & \\
\hline \multicolumn{4}{|l|}{ Lesion and procedural characteristics } \\
\hline Multivessel treatment & $179(18.7)$ & $117(13.7)$ & 0.004 \\
\hline \multicolumn{4}{|l|}{ Treated vessel } \\
\hline Left main & $33(3.4)$ & $7(0.8)$ & $<0.001$ \\
\hline Right coronary artery & $347(36.3)$ & $322(37.7)$ & 0.53 \\
\hline Left anterior descending artery & $437(45.7)$ & $417(48.8)$ & 0.18 \\
\hline Circumflex artery & $286(29.9)$ & $237(27.8)$ & 0.32 \\
\hline Graft & $54(5.6)$ & $1(0.1)$ & $<0.001$ \\
\hline De novo lesion ${ }^{c}$ & $812(84.8)$ & $815(95.4)$ & $<0.001$ \\
\hline Reference vessel diameter pre $\mathrm{PCl}(\mathrm{mm})$ & $2.69 \pm 0.60$ & $2.75 \pm 0.55$ & 0.02 \\
\hline Diameter stenosis pre $\mathrm{PCl}(\%)$ & $65.5(54.7-76.1)$ & $69.4(58.2-86.0)$ & $<0.001$ \\
\hline Number of stents per patient & $2.0(1.0-2.0)$ & $1.0(1.0-2.0)$ & $<0.001$ \\
\hline Total stent length per lesion (mm) & $32.0(20.0-52.0)$ & $28.0(18.0-44.0)$ & $<0.001$ \\
\hline Postdilation & $767(80.1)$ & $636(74.5)$ & 0.004 \\
\hline Diameter stenosis post $\mathrm{PCl}(\%)$ & $15.1(10.6-20.9)$ & $15.7(11.0-21.9)$ & 0.19 \\
\hline
\end{tabular}

Values are $n(\%)$, mean $\pm S D$, or median (IQR), as appropriate. CABG, coronary artery bypass grafting; LVEF=left ventricular ejection fraction; $\mathrm{Ml}=$ myocardial infarction; $\mathrm{PCl}=$ percutaneous coronary intervention. ${ }^{\mathrm{a}} \mathrm{n}=784 .{ }^{\mathrm{b}} \mathrm{n}=640 .{ }^{\mathrm{c}}$ Including chronic total occlusion, but not grafts or in-stent restenosis. 
Table 2: Clinical outcome at the 3-year follow-up of high-risk patients versus low-to-intermediate-risk patients.

\begin{tabular}{|c|c|c|c|c|}
\hline & \multicolumn{4}{|c|}{$\begin{array}{c}\text { All patients } \\
n=1,811\end{array}$} \\
\hline & $\begin{array}{l}\text { High risk } \\
\text { patients } \\
n=957\end{array}$ & $\begin{array}{l}\text { Low-to- } \\
\text { intermediate risk } \\
\text { patients } \\
n=854\end{array}$ & $\begin{array}{l}\text { Hazard ratio } \\
\qquad(95 \% \mathrm{Cl})\end{array}$ & p-Log-rank \\
\hline Death (any) & $70(7.3)$ & $21(2.5)$ & $3.01(1.88-4.98)$ & $<0.001$ \\
\hline Cardiac death & $44(4.7)$ & $13(1.5)$ & $3.01(1.67-5.75)$ & $<0.001$ \\
\hline Target vessel myocardial infarction & $29(3.1)$ & $16(1.9)$ & $1.64(0.89-3.02)$ & 0.11 \\
\hline $\begin{array}{l}\text { Clinically indicated target vessel } \\
\text { revascularization }\end{array}$ & $68(7.3)$ & $40(4.7)$ & $1.56(1.06-2.30)$ & 0.03 \\
\hline $\begin{array}{l}\text { Clinically indicated target lesion } \\
\text { revascularization }\end{array}$ & $49(5.3)$ & $32(3.8)$ & $1.40(0.90-2.19)$ & 0.13 \\
\hline Target vessel failure* & $125(13.2)$ & $64(7.5)$ & $1.79(1.33-2.42)$ & $<0.001$ \\
\hline Target lesion failure & $111(11.8)$ & $58(6.9)$ & $1.76(1.28-2.41)$ & $<0.001$ \\
\hline Major adverse cardiac events & $139(14.5)$ & $70(8.2)$ & $1.83(1.37-2.43)$ & $<0.001$ \\
\hline Patient-oriented composite endpoint & $197(20.6)$ & $105(12.3)$ & $1.73(1.37-2.20)$ & $<0.001$ \\
\hline Definite stent thrombosis & $12(1.3)$ & $5(0.6)$ & $2.18(0.77-6.19)$ & 0.13 \\
\hline Definite-or-probable stent thrombosis & $16(1.7)$ & $6(0.7)$ & $2.42(0.95-6.18)$ & 0.06 \\
\hline
\end{tabular}

Values are $\mathrm{n}(\%)$. MI, myocardial infarction. ${ }^{a}$ The main clinical endpoint target vessel failure is a composite of cardiac death, target vessel-related myocardial infarction, and clinically indicated target vessel revascularization.

Among the high-risk patients, patients with $\geq 3$ risk factors had a significantly higher incidence of TVF than patients who had 1 and who had 2 risk factors ( $23.5 \%$ vs $10.9 \%$ vs. $13.1 \%$, respectively; $p=0.003$ ), which was mainly due to a significantly higher incidence of cardiac death $(15.3 \%$ vs $2.5 \%$ vs $4.6 \%$, respectively; $p<0.001$ ) (Table 3 ).

\section{DES groups among high-risk and low-to-intermediate risk patients}

Table 4 presents the 3-year clinical event rates for the two DES groups in both, high-risk patients and low-to-intermediate risk patients. Low-to-intermediate risk patients showed no significant betweenDES difference in various clinical endpoints. Likewise, among the high-risk patients, time-to-event analysis showed similar rates of TVF and its individual components for patients treated with Resolute Integrity versus Promus Element stents (Figure 3, Table 4) ( $p$-interaction=0.80 for high-risk vs low-tointermediate risk patients). Multivariate analysis for TVF in high-risk patients demonstrated that, after adjustment for potential confounders, the rate of TVF was still similar for both DES groups (HR 1.02; 95\% Cl: 0.71-1.44, p-Log-rank=0.94). 

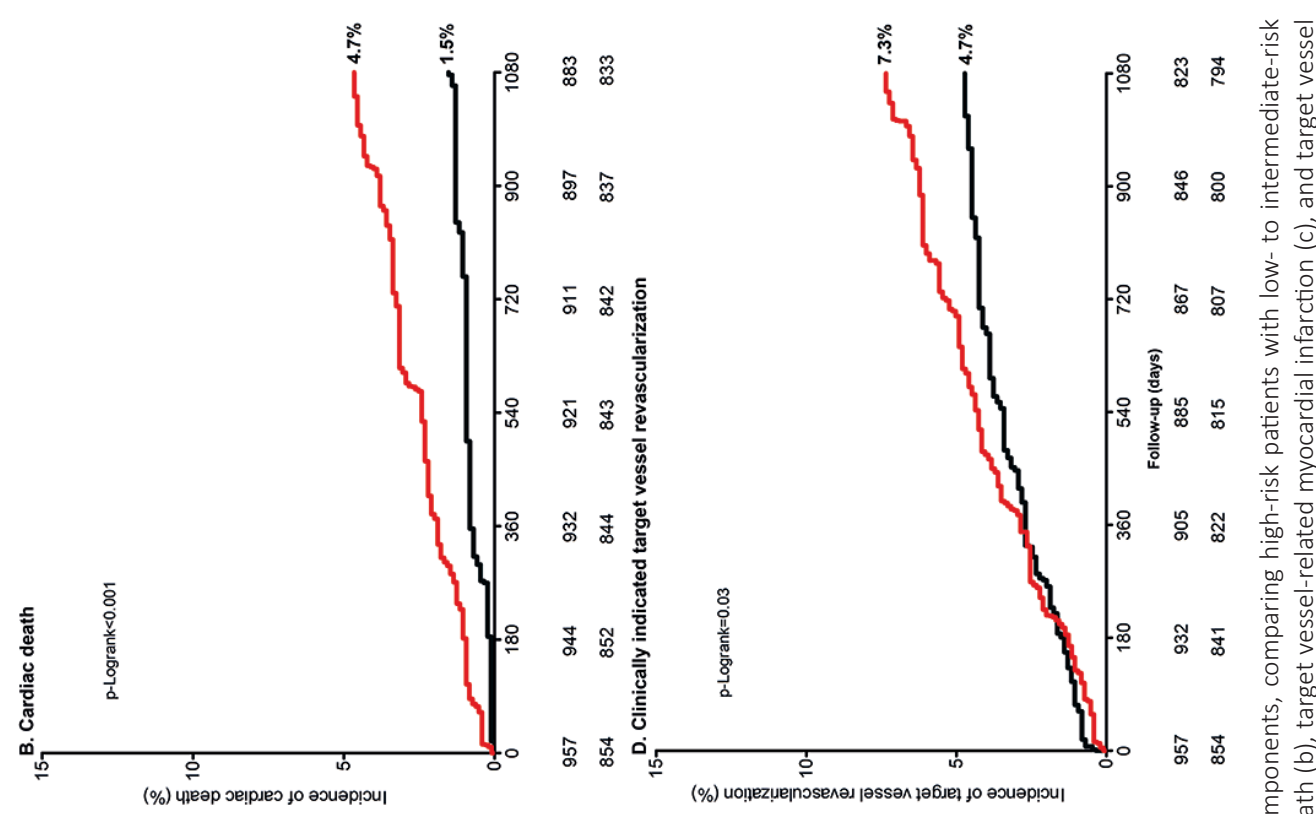

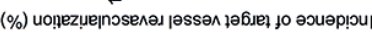
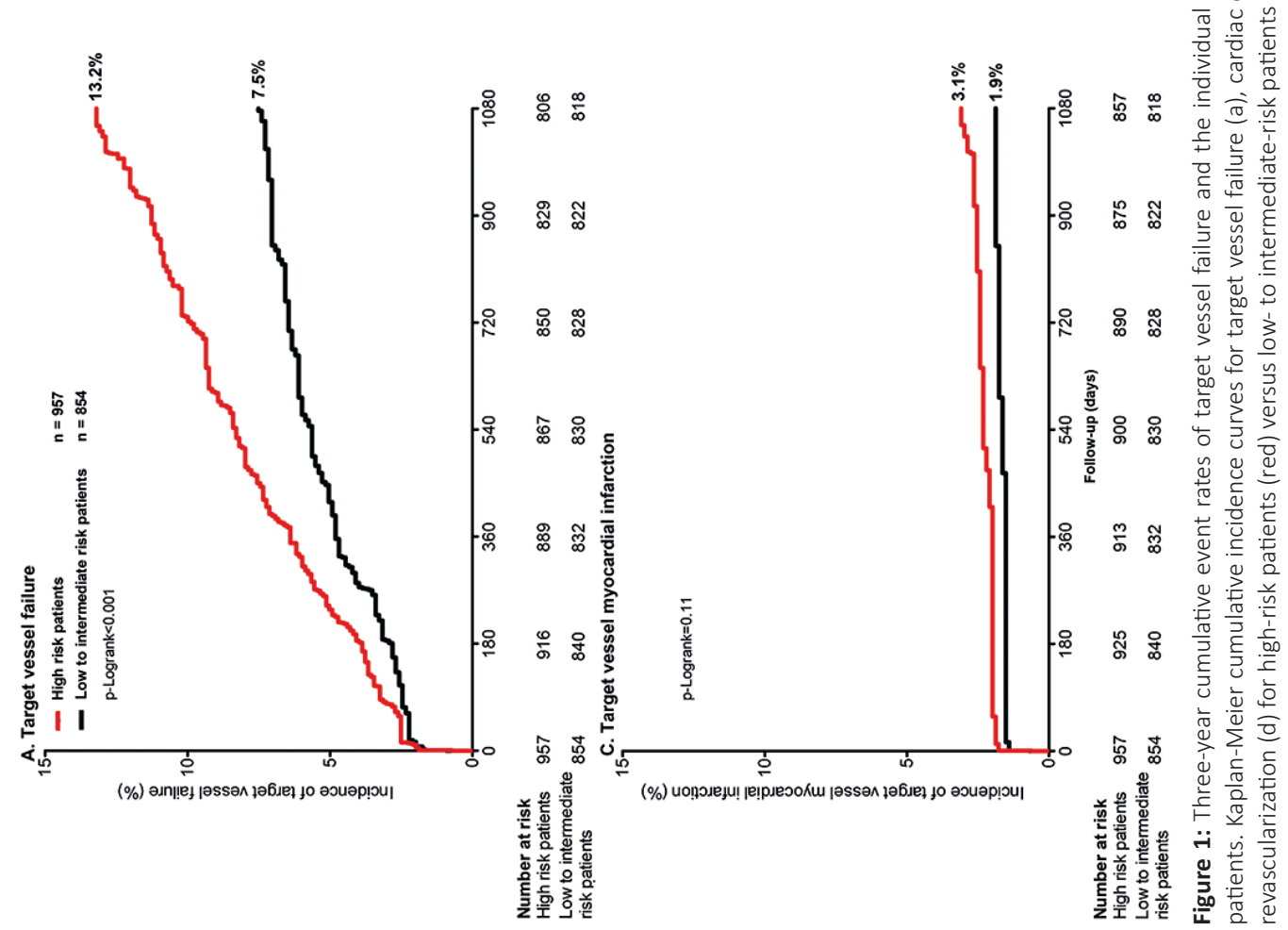


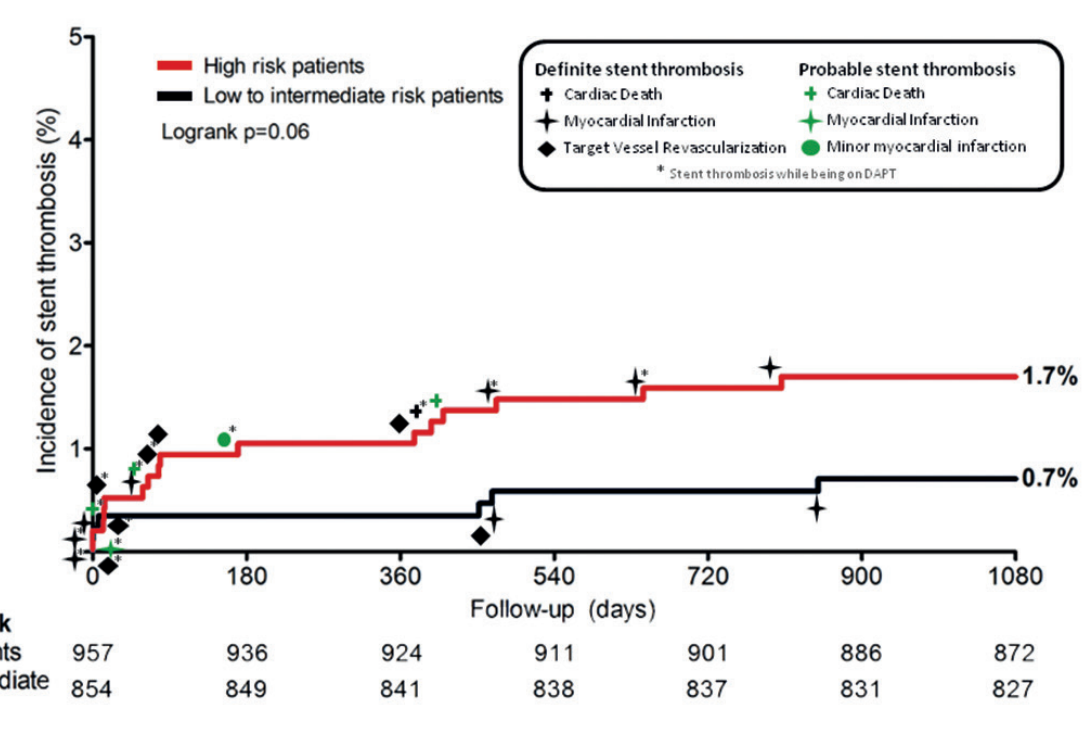

Number at risk High risk patients Low to intermediate risk patients

Figure 2: Kaplan-Meier curve for stent thrombosis at the 3-year follow-up. Cumulative incidence curve for stent thrombosis in high-risk patients (red) versus low- to intermediate- risk patients (black). Dual antiplatelet therapy means acetylsalicylic acid plus P2Y12 receptor antagonist.

Table 3: Clinical outcome at the 3-year follow-up of high-risk patients with 1, 2, or $\geq 3$ high-risk factors.

\begin{tabular}{|lcccc|}
\hline & \multicolumn{4}{c|}{ High risk patients } \\
$\mathbf{n}=\mathbf{9 5 7}$ \\
Number of high-risk criteria & $\mathbf{1}$ factor & $\mathbf{2}$ factors & $\mathbf{2 3}$ factors & $\mathbf{p}$ \\
$\mathbf{n = \mathbf { n } = \mathbf { 3 7 3 }}$ & $49(13.1)$ & $23(23.5)$ & 0.003 \\
Target vessel failure & $53(10.9)$ & $17(4.6)$ & $15(15.3)$ & $<0.001$ \\
Cardiac death & $12(2.5)$ & $9(2.4)$ & $2(2.0)$ & 0.46 \\
Target vessel myocardial infarction & $18(3.7)$ & $28(7.5)$ & $10(10.2)$ & 0.34 \\
Target vessel revascularization & $30(6.2)$ & & $\mathbf{n}$ & \\
\hline
\end{tabular}

Values are $\mathrm{n}(\%)$. Ml, myocardial infarction. ${ }^{a}$ The main clinical endpoint target vessel failure is a composite of cardiac death, target vessel-related myocardial infarction, and clinically indicated target vessel revascularization.

Furthermore, the 3-year rate of definite-or-probable stent thrombosis was similar for high-risk patients treated with both stents (1.7\% vs 1.7\%; $p$-Log-rank=0.99) (Figure 4). Due to an apparent dissimilarity between the Resolute Integrity and Promus Element groups in the course of their time-to-event curves for definite-or-probable stent thrombosis (Figure 4, catch-up after $>1$ year vs main increase within first 12 months), we performed a landmark analysis at 12-month follow-up: definite-or-probable stent thrombosis occurred in the Resolute Integrity versus Promus Element group during the first year in $0.6 \%$ vs $1.5 \%$ ( $p$-Log-rank=0.20) of patients and during the second-plus-third year in $1.1 \%$ vs. $0.2 \%$ ( $p$-Logrank=0.10). 

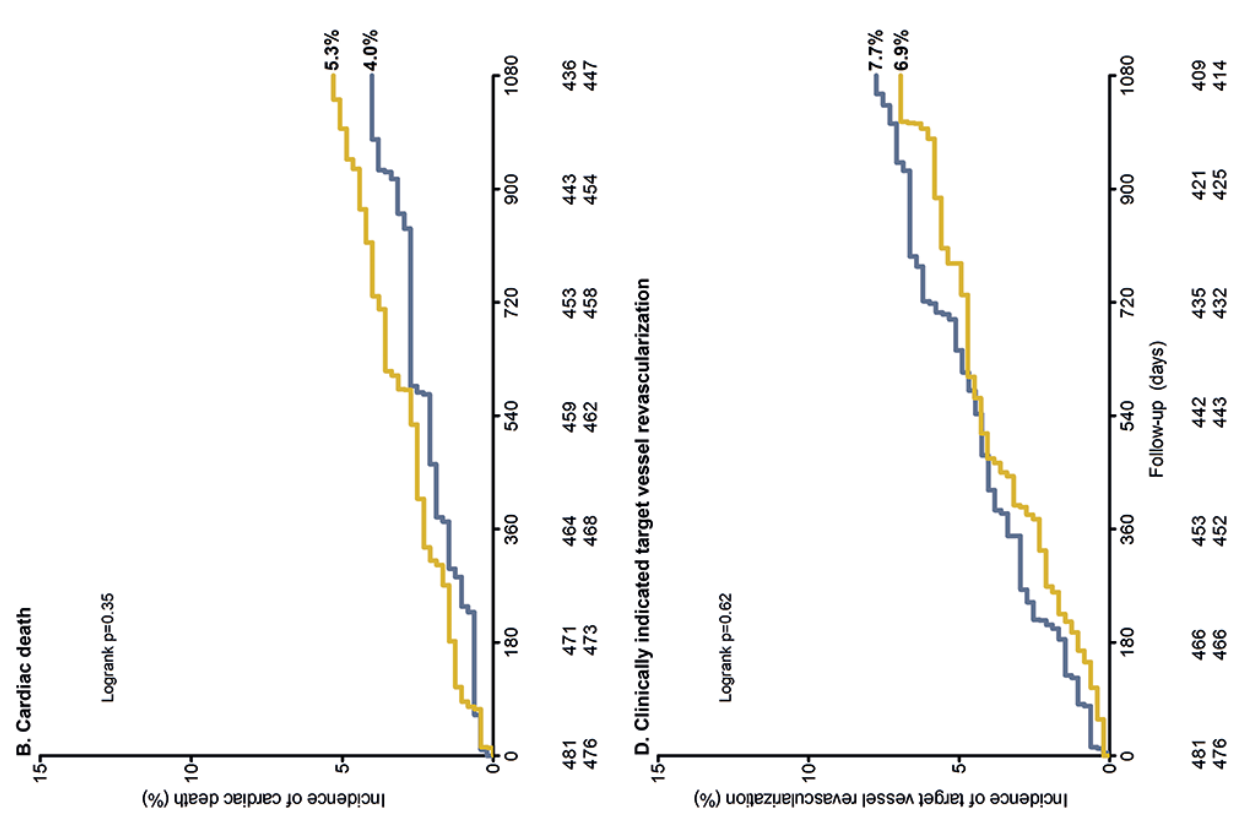

तิ

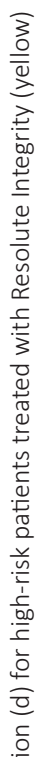
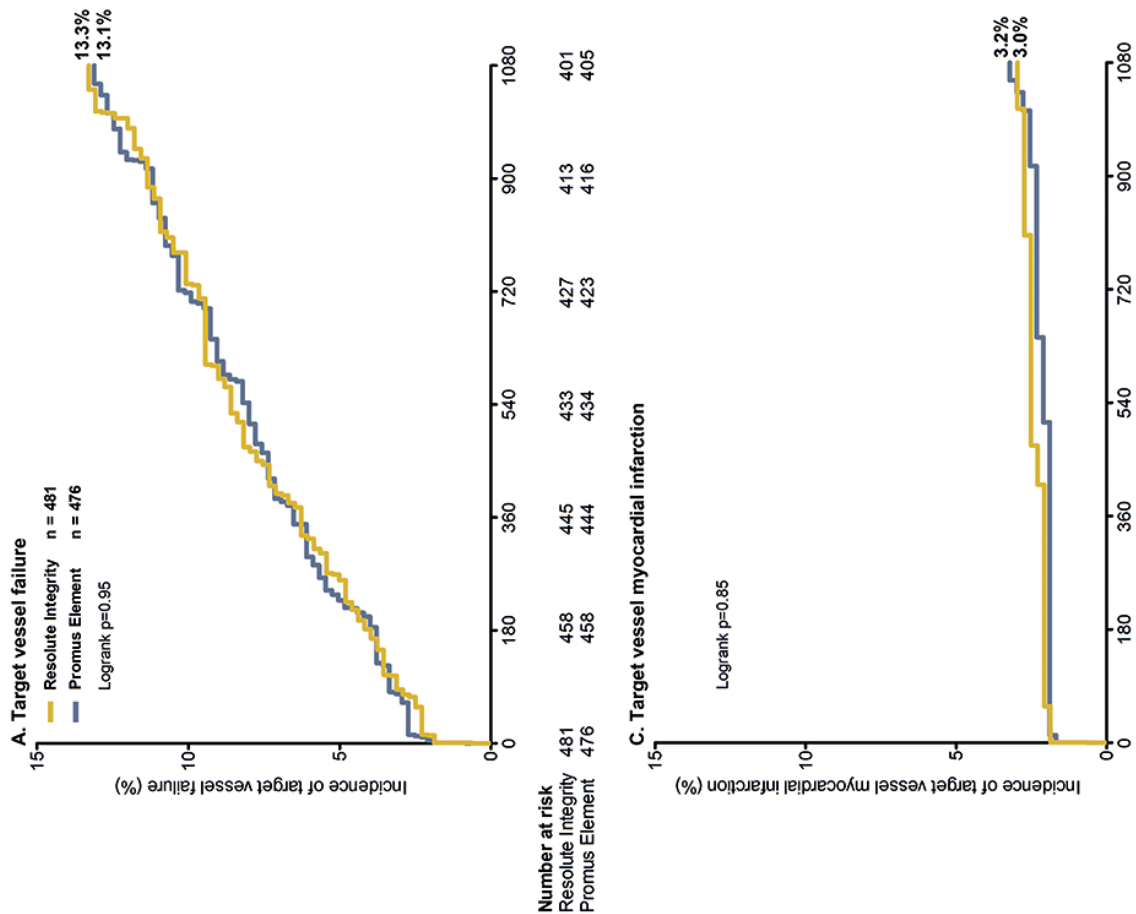

范区

辛学

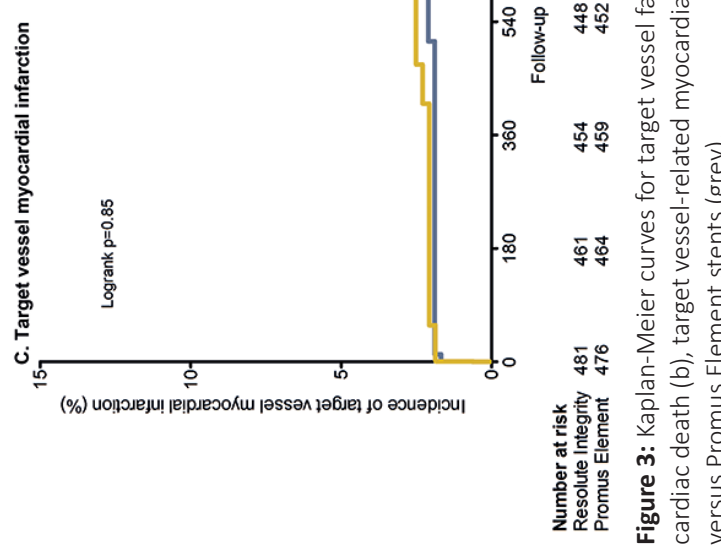




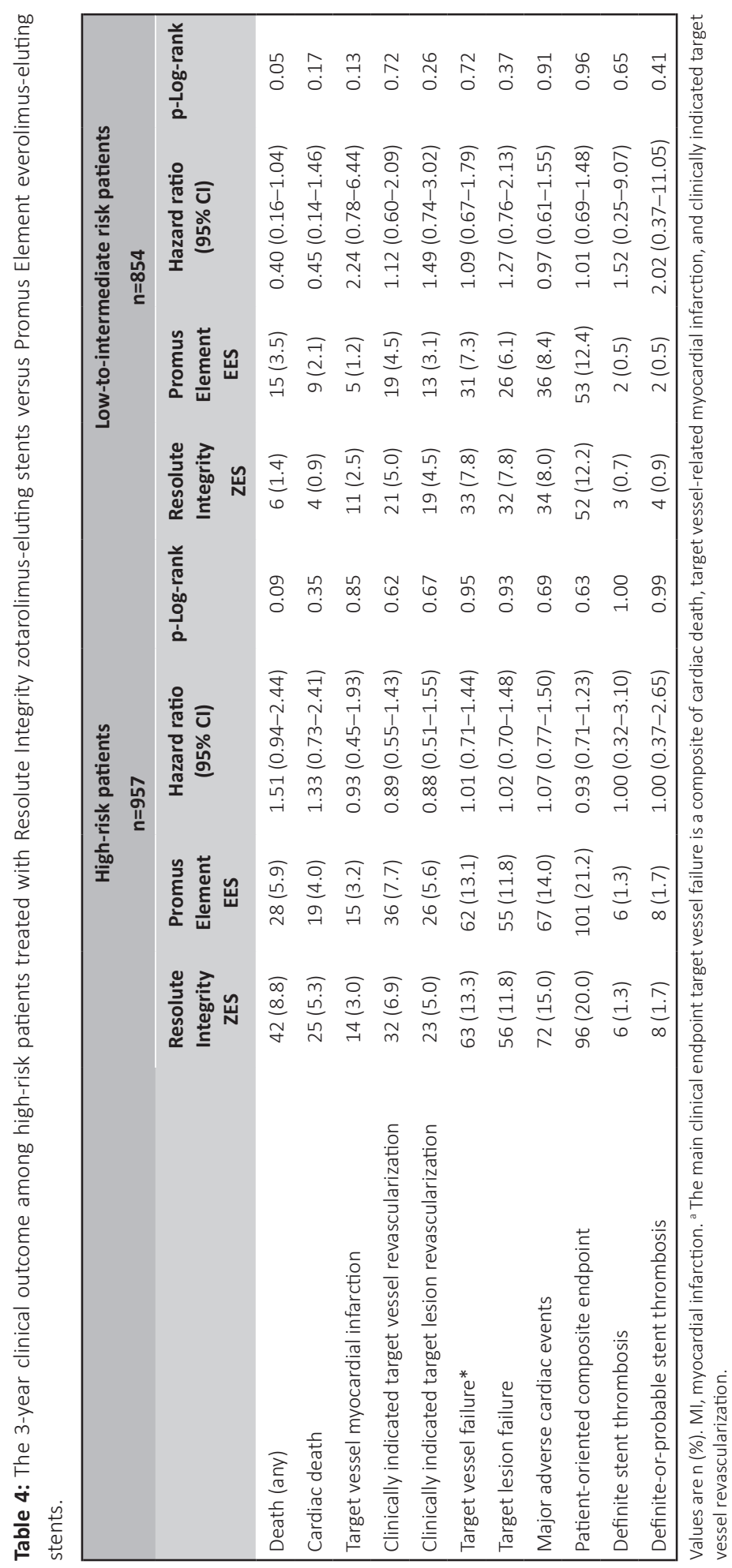




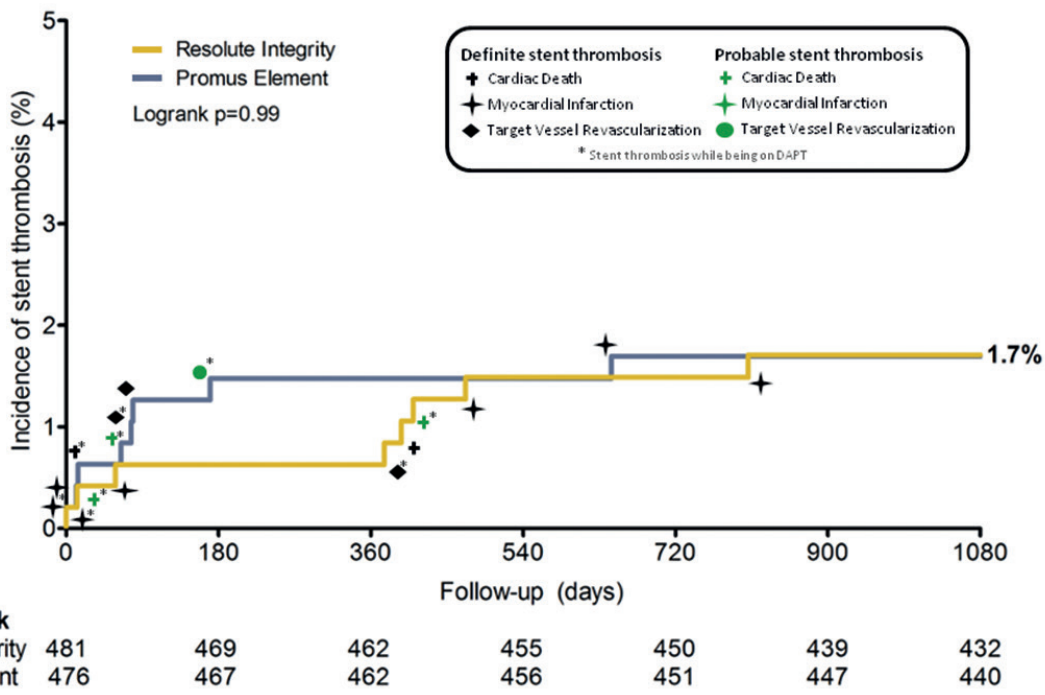

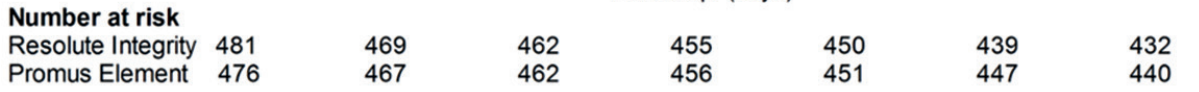

Figure 4: Kaplan-Meier curve for stent thrombosis at the 3-year follow-up in high-risk patients. Cumulative incidence curve of stent thrombosis for high-risk patients treated with Resolute Integrity (yellow) versus Promus Element stent (grey). Dual antiplatelet therapy means acetylsalicylic acid plus P2Y12 receptor antagonist.

\section{Discussion}

\section{Major findings of the study}

High-risk patients of the DUTCH PEERS trial, as compared to low-to-intermediate risk patients, had significantly higher rates of various clinical endpoints including the primary endpoint TVF (13.2\% vs 7.5\%). Both Resolute Integrity and Promus Element stents were shown to be similarly safe and efficacious for treating these patients with a particularly high risk of adverse clinical events. In addition, in patients with a low-to-intermediate risk there was no significant between-stent difference in clinical outcome.

\section{Clinical outcome of DES in high-risk patients}

Why is the assessment of high-risk patient populations of all-comer trials of significant interest? In fact, there are several reasons to perform such analyses. One (most recent) reason is that clinical use of completely bioresorbable coronary scaffolds is expected to increase soon. ${ }^{26,27}$ However, calcified lesions and diffuse disease may be less suitable targets for these scaffolds, which currently offer only limited radial force. ${ }^{26,28,29}$ Therefore, 'high-risk' patient populations of current all-comer DES trials may represent in the near future a 'standard patient population' for treatment with metallic DES. In addition, as current event rates following treatment with modern DES are relatively low, differences between devices may only be seen in patients known to have the highest adverse event risk.

The definition of 'high-risk' patients may always be a matter of discussion and a moving target. However, certain patient characteristics are known to increase the probability of adverse clinical events. 
A patient-level pooled analysis of seven stent trials investigated whether adding patient characteristics to the SYNTAX score was of additional value for the prediction of mortality during the first 3 years after stenting. ${ }^{14}$ That study demonstrated that advanced age, renal dysfunction, left ventricular systolic dysfunction, and diabetes mellitus were predictors of 3-year all-cause mortality. ${ }^{14}$ In addition, diabetics were shown to have an increased 2-year target lesion revascularization rate that was independent of lesion complexity. ${ }^{15}$ In the multicenter prospective EXCELLENT and RESOLUTE-Korea registries, chronic renal failure was shown to be a highly important predictor for target lesion failure at 3-year follow-up. ${ }^{16}$ And at the 4-year follow-up of the Resolute All-comers trial, advanced age, diabetes mellitus, and a history of PCl predicted an increased risk of repeat revascularization. ${ }^{13}$ Therefore, we used the patient characteristics diabetes mellitus, previous $\mathrm{MI}$, previous coronary revascularization, chronic kidney disease, reduced left ventricular ejection fraction( $\leq 30 \%)$, and advanced age ( $\geq 75$ years) to define the high-risk patient cohort in this analysis.

In the present study, patients who met at least one high-risk criterion showed approximately $70 \%$ higher rates of the composite endpoints TVF and POCE than the low-to-intermediate risk patients. Nevertheless, event rates were still reasonable for both DES groups. The more frequent use of dual anti-platelet therapy in high-risk patients is not unexpected and can be greatly explained by the higher incidence of repeat revascularization. In high-risk patients oral anticoagulants were more often used, which may be most likely related to a higher incidence of comorbidities such as atrial fibrillation. Considering the clustering of risk factors in the high-risk patients, the clinical outcome data obtained should be regarded as reassurance about the long-term safety and efficacy of both DES assessed.

High-risk patients with 3 or more risk factors had a significantly higher TVF risk than patients with 1 or 2 risk factors - a difference that was mainly related to a difference in cardiac death. The treatment of patients with multiple high-risk criteria is part of our clinical work, and, indeed, these patients had considerably higher adverse event rates. Our findings underline the importance of enrolling high-risk patients with a particularly high event risk in studies that assess broad patient populations, such as all-comers. In addition, knowledge about a patient's pattern of risk criteria prior to $\mathrm{PCl}$ does allow to better estimate the adverse event risk following PCl. Such information can be very useful for improving discussions of the Heart Team and individualizing the informed consent.

Even among high-risk patients, between-DES differences in the risk of stent thrombosis may not be seen during the first year of follow-up, when most patients are on dual anti-platelet therapy. This was recently shown by a patient-level pooled analysis of 3-year follow-up data from female high-risk patients from 26 randomized DES trials. During the second and third year of follow-up, newer-generation DES showed a significant advantage in terms of a lower stent thrombosis rate versus early-generation DES, while during the first year of follow-up stent thrombosis rates were similar. ${ }^{10}$ While the Resolute Integrity and Promus Element stent have previously shown to be safe and efficacious in low-to-intermediate risk patients and all-comers ${ }^{5,7,30}$, the known between-stent differences ${ }^{31}$ in design, maximum expansion capacity, and radial force theoretically could have been related to difference in long-term safety and efficacy in high-risk patients. As in daily clinical practice decisions have to be made for an individual patient, it is relevant to know whether both stents are similarly safe and efficacious in patients, who fulfill criteria of increased clinical risk. We observed, in the present analysis, a higher 3-year incidence of definite-orprobable stent thrombosis in high-risk patients versus low-to-intermediate risk patients $(1.7 \%$ vs $0.7 \%$, 
$p=0.06)$, as might be expected. Among the high-risk patients, definite and definite-or-probable stent thrombosis rates of the Resolute Integrity and Promus Element stent groups were identical. A landmark analysis revealed that a visually recognizable dissimilarity between the stent groups in the course of the time-to-event curves of definite-or-probable stent thrombosis (i.e. more events during the first year of follow-up in Promus Element stents, and late catch-up in Resolute Integrity stents) was statistically nonsignificant and most likely a play of chance.

Long-term outcome data about Resolute Integrity and Promus Element stents, the DES examined in the DUTCH PEERS trial, are scarce. ${ }^{30}$ The only other randomized trial studying Resolute Integrity in allcomers is the SORT OUT VI study ${ }^{5}$, but definite long-term results have not yet been published. The PLATINUM trial is the only other randomized trial that has published 3-year follow-up data of Promus Element; however, this study was performed in patients with no more than low-to-moderate event risk. $^{\text {? }}$

In parallel with the refinement of the durable polymer DES, biodegradable polymer DES were developed. As these stents may facilitate arterial healing, their long-term event risk could theoretically be lower than the risk associated with the permanent presence of a durable polymer-coated DES in the coronary vessel. Various all-comer trials have shown non-inferiority of (very) thin strut biodegradable polymer DES to newer-generation durable polymer DES. ${ }^{32-35}$ Additional data about the clinical long-term followup after treatment with newer biodegradable polymer-coated DES is of interest, including data about long-term safety and efficacy of these devices in the high-risk patient subset.

\section{Limitations}

As this study is not powered to assess between-group differences in subgroup analyses, our findings should be considered hypothesis generating. Angiographic features of increased risk were not considered in the present study, which focused on established clinical risk factors.

\section{Conclusions}

The newer-generation Resolute Integrity and Promus Element stents showed similar results in terms of safety and efficacy for treating high-risk patients, who had significantly higher event rates than patients with low-to-intermediate risk. 


\section{References}

1. Stefanini GG, Holmes DR Jr. Drug-eluting coronary-artery stents. N Engl J Med 2013; 368:254-65.

2. Serruys PW, Silber S, Garg S, et al. Comparison of zotarolimus-eluting and everolimus-eluting coronary stents. N Engl J Med 2010; 363:136-46.

3. von Birgelen C, Basalus MW, Tandjung K, et al. A randomized controlled trial in second-generation zotarolimuseluting resolute stents versus everolimus-eluting Xience $V$ stents in real-world patients: the TWENTE trial. J Am Coll Cardiol 2012; 59:1350-61.

4. von Birgelen $\mathrm{C}$, Sen $\mathrm{H}$, Lam MK, et al. Third-generation zotarolimus-eluting and everolimus-eluting stents in all-comer patients requiring a percutaneous coronary intervention (DUTCH PEERS): a randomised, single-blind, multicentre, non-inferiority trial. Lancet 2014; 383:413-23.

5. Raungaard B, Okkels Jensen L, Tilsted HH, et al. Zotarolimus-eluting durable-polymer-coated stent versus a biolimus-eluting biodegradable-polymer-coated stent in unselected patients undergoing percutaneous coronary intervention (SORT OUT VI): a randomised non-inferiority trial. Lancet 2015; 385:1527-35.

6. Park KW, Kang SH, Kang HJ, et al. A randomized comparison of platinum chromium-based everolimus-eluting stents versus cobalt chromium-based zotarolimus-eluting stents in all-comers receiving percutaneous coronary intervention: HOST-ASSURE (Harmonizing optimal strategy for treatment of coronar artery stenosis-safety \& effectiveness of drug-eluting stents \& anti-platelet regimen), a randomized, controlled, noninferiority trial. J Am Coll Cardiol 2014; 63:2805-16.

7. Meredith IT, Teirstein PS, Bouchard A, et al. Three-year results comparing platinum-chromium PROMUS element and cobalt-chromium XIENCE $\vee$ everolimus-eluting stents in de novo coronary artery narrowing (from the PLATINUM trial). Am J Cardiol 2014; 113:117-23.

8. Sarno G, Lagerqvist B, Carlsson J, et al. Initial clinical experience with an everolimus eluting platinum chromium stent (Promus Element) in unselected patients from the Swedish Coronary Angiography and Angioplasty Registry (SCAAR). Int J Cardiol 2013; 167:146-50.

9. Nakamura M, Muramatsu T, Yokoi H, et al. Three-year follow-up outcomes of SES and PES in a randomized controlled study stratified by the presence of diabetes mellitus: J-DEsSERT trial. Int I Cardiol 2016; 208:4-12.

10. Giustino G, Baber U, Salianski O, et al. Safety and efficacy of new-generation drug-eluting stents in women at high risk of atherothrombosis: from the women in innovation and drug-eluting stents collaborative patientlevel pooled analysis. Circ Cardiovasc Interv. 2016; 9:e002995. DOI:10.1161/CIRCINTERVENTIONS.115.002995, accessed July 8, 2016.

11. Sen H, Lam MK, Löwik MM, et al. Clinical events and patient-reported chest pain in all-comers treated with Resolute Integrity and Promus Element stents: 2-year follow-up of the DUTCH PEERS (Durable Polymer-Based Stent Challenge of Promus ElemEnt Versus ReSolute Integrity) Randomized Trial (TWENTE II). J Am Coll Cardiol Intv 2015; 8:889-99.

12. Chen PF, Wang DN, Chen $\mathrm{K}$, et al. Outcomes of percutaneous coronary intervention in patients $\geq 75$ years: onecenter study in a Chinese patient group. J Geriatr Cardiol 2015; 12:626-33.

13. Taniwaki M, Stefanini GG, Silber S, et al. 4-year clinical outcomes and predictors of repeat revascularization in patients treated with new-generation drug-eluting stents: a report from the RESOLUTE All-comeres trial (a randomized comparison of a zotarolimus-eluting stent with an everolimus-eluting stent for percutaneous coronary intervention). J Am Coll Cardiol 2014; 63:1617-25.

14. Iqbal J, Vergouwe Y, Bourantas CV, et al. Predicting 3-year mortality after percutaneous coronary intervention: updated logistic clinical SYNTAX score based on patient-level data from 7 contemporary stent trials. J Am Coll Cardiol Intv 2014; 7:464-70.

15. Koskinas KC, Siontis GC, Piccolo R, et al. Impact of diabetic status on outcomes after revascularization with drugeluting stents in relateion to coronary artery disease complexity: patient-level pooled analysis of 6081 patients. Circ Cardiovasc Interv. 2016; 9:e003255.DOI:10.1161/CIRCINTERVENTIONS.115.003255, accessed July 8, 2016.

16. Lee JM, Park KW, Han JK, et al. Three-year patient-related and stent-related outcomes of second-generation everolimus-eluting Xience $V$ stents versus zotarolimus-eluting resolute stents in real-world practice (from the Multicenter Prospective EXCELLENT and RESOLUTE-Korea Registries). Am J Cardiol 2014; 114:1329-38.

17. Stefanini GG, Taniwaki M, Kalesan B, et al. The impact of renal impairment on long-term safety and effectiveness of drug-eluting stents. PloS One 2014;9:e106450.DOI:10.1371/journal.pone.0106450, accessed July 8, 2016. 
18. Yeung $A C$, Leon $M B$, Jain $A$, et al. Clinical evaluation of the resolute zotarolimus-eluting coronary stent system in the treatment of de novo lesions in native coronary arteries: the RESOLUTE US Clinical Trial. J Am Coll Cardiol 2011; 57:1778-83.

19. Turco MA. The Integrity bare-metal stent made by continuous sinusoid technology. Expert Rev Med Devices 2011; 8:303-06.

20. Kedhi E, Joesoef KS, McFadden E, et al. Second-generation everolimus-eluting and paclitaxel-eluting stents in real-life practice (COMPARE): a randomised trial. Lancet 2010; 375:201-9.

21. Jensen LO, Thayssen P, Hansen HS, et al. Randomized comparison of everolimus-eluting and sirolimus-eluting stents in patients treated with percutaneous coronary intervention: the Scandinavian Organization for Randomized Trials With Clinical Outcome IV (SORT OUT IV). Circulation 2012; 125:1246-55.

22. Stone GW, Rizvi A, Newman W, et al. Everolimus-eluting versus paclitaxel-eluting stents in coronary artery disease. N Engl J Med 2010; 362:1663-74.

23. Stone GW, Teirstein PS, Meredith IT, et al. A prospective, randomized evaluation of a novel everolimus-eluting coronary stent: the PLATINUM (a prospective, randomized, multicenter trial to assess an everolimus-eluting coronary stent system [PROMUS ELEMENT] for the treatment of up to two de novo coronary artery lesions) trial. J Am Coll Cardiol 2011; 57:1700-8.

24. Cutlip DE, Windecker $S$, Mehran R, et al. Clinical end points in coronary stent trials: a case for standardized definitions. Circulation 2007; 115:2344-51.

25. Vranckx P, Cutlip DE, Mehran R, et al. Myocardial infarction adjudication in contemporary all-comer stent trials: balancing sensitivity and specificity: addendum to the historical MI definititons used in stent studies. Eurolntervention 2010; 5:871-4.

26. Iqbal J, Onuma Y, Ormiston J, Abizaid A, Waksman R, Serruys P. Bioresorbable scaffolds: rationale, current status, challenges, and future. Eur Heart J 2014; 35:765-76.

27. Haude $M$, Ince $H$, Abizaid $A$, et al. Safety and performance of the second-generation drug-eluting absorbable metal scaffold in patients with de-novo coronary artery lesions (BIOSOLVE-II): 6 month results of a prospective, multicentre, non-randomised, first-in-man trial. Lancet 2016; 387:31-9.

28. von Birgelen C, Basalus MWZ. Oversized post-dilatation of current bioresorbable vascular scaffolds: kill or cure? Eurolntervention 2016; 11:1330-3.

29. Capodanno D. Bioresorbable scaffolds for calcified lesions: not a free lunch! Eurolntervention 2016; 11:1334-6.

30. van der Heijden LC, Kok MM, Löwik MM, et al. Three-year safety and efficacy of treating all-comers with newergeneration Resolute Integrity or Promus Element stents in the randomised DUTCH PEERS (TWENTE II) trial. Eurointervention 2017;12:2128-31.

31. Foin $N$, Alegria $E$, Sen $S$, et al. Importance of knowing stent design threshold diameters and post-dilatation capacities to optimise stent selection and prevent stent overexpansion/incomplete apposition during PCI. Int J Cardiol 2013;166:755-8.

32. von Birgelen C, Kok MM, van der Heijden LC, et al. Very thin strut biodegradable polymer everolimus-eluting and sirolimus-eluting stents versus durable polymer zotarolimus-eluting stents in allcomers with coronary artery disease (BIO-RESORT): a three-arm, randomised, non-inferiority trial. Lancet 2016; 388:2607-17.

33. Pilgrim $T$, Heg $D$, Roffi $M$, et al. Ultrathin strut biodegradable polymer sirolimus-eluting stent versus durable polymer everolimus-eluting stent for percutaneous coronary revascularisation (BIOSCIENCE): a randomised, single-blind, non-inferiority trial. Lancet 2014; 384:2111-22.

34. Kereiakes DJ, Meredith IT, Windecker S, et al. Efficacy and safety of a novel bioabsorbable polymer-coated, everolimus-eluting coronary stent: the EVOLVE II randomized trial. Circ Cardiovasc Interv. 2015;8:e002372. Doi: 10.1161/circinterventions.114.002372.

35. Saito S, Valdes-Chavarri M, Richardt G, et al. A randomized, prospective, intercontinental evaluation of a bioresorbable polymer sirolimus-eluting coronary stent system: the CENTURY II (clinical evaluation of new terumo drug-eluting coronary stent system in the treatment of patients with coronary artery disease) trial. European Heart Journal 2014; 35:2021-31. 



\section{Conflicts of interest statement}

CvB was a consultant to device-manufacturing companies, among them Biotronik, Boston Scientific, and Medtronic; institutional grant support from AstraZeneca, Biotronik, Boston Scientific, Medtronic. RJdW received grant support and consultant fee from OrbusNeich, grant support from Abbott, grant support from AstraZeneca, grat support from Stentys, and grant support from Tryton. Other authors declare that they have no conflicts of interest.

\section{Acknowledgments}

We would like to acknowledge all patients for their participation in both studies and all interventional cardiologists and study nurses for conducting these studies. We especially would like to thank all principle investigators of the DUTCH PEERS and REDEMEDEE Registry: PW Danse, GAJ Jessurun, R W M Hautvast, GK van Houwelingen, AR Schramm, RM Tjon Joe Gin, JW Louwerenburg, FHAF de Man, MG Stoel, G C M Linssen, SAM Saïd, MB Nienhuis, PMJ Verhorst, MWZ Basalus, CJM Doggen, K Tandjung, P den Heijer, IBA Menown, A Erglis, H Suryapranata, KE Arkenbout, A Iñiguez, AWJ van 't Hof and P Muller. 


\title{
CHAPTER 5
}

\section{Clinical Outcomes After Percutaneous Coronary Intervention With the COMBO Stent Versus Resolute Integrity and Promus Element Stents}

\author{
A Propensity-Matched Analysis
}

Deborah N. Kalkman*, Marlies M. Kok*, Liefke C. van der Heijden*, Pier Woudstra, Marcel A.M. Beijk, Jan G.P. Tijssen, Clemens von Birgelen, Robbert J. de Winter

*First, second, and third author contributed equally to this manuscript.
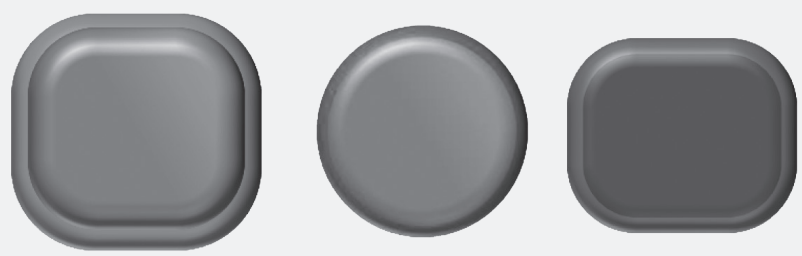


\begin{abstract}
Aims: The COMBO stent combines a sirolimus-elution with an endothelial progenitor cell-capturing layer to promote early endothelialization. There has not been a head to head comparison of this novel device with any other currently used drug-eluting stent (DES). We compare clinical outcome at 2-years after COMBO stent placement with the Resolute Integrity or Promus Element stent in an all-comers cohort.
\end{abstract}

Methods and results: Patients from the REMEDEE Registry (COMBO, $n=1000$ ) were matched with patients from the DUTCH PEERS trial (Promus Element/Resolute Integrity, $n=1811$ ). Propensity scorematching on 13 baseline characteristics was applied to create two balanced cohorts of patients treated with COMBO versus (Promus Element/Resolute Integrity). Propensity-score matching yielded 771 patient pairs, representing all-comers patients, with a median age of 65 years, 27\% females, and more than $50 \%$ of patients presenting with acute coronary syndrome. Target lesion failure (TLF, a composite of cardiac death, target vessel $\mathrm{MI}$ and any target lesion revascularization) at 2-year follow-up was 7.9\% in COMBO and 6.4\% in Promus Element/Resolute Integrity, HR 1.24 (95\% Cl: 0.85-1.81), p=0.26. Definite stent thrombosis (ST) was not significantly different between groups (0.8\% vs $0.9 \%, p=0.79)$.

Conclusions: In a propensity-matched analysis; the COMBO stent shows similar rates of TLF and ST rates at 2-year follow-up, compared to Resolute Integrity and Promus Element.

\title{
Condensed abstract
}

The COMBO stent is a stent with dual-therapy technology. The sirolimus-elution on the abluminal side of the stent is combined with an endothelial progenitor cell-capturing layer allow rapid endothelialization. This device has not been studied in relation to contemporary drug-eluting stents. A propensity score matched analysis is performed with data from the REMEDEE registry (patients treated with COMBO stent) and DUTCH PEERS trial (Promus Element and Resolute Integrity stents). The analysis found similar safety and efficacy outcomes among 771 all-comers patient-pairs treated with either COMBO or Resolute/Promus stents. 


\section{Introduction}

Clinical outcomes after percutaneous coronary intervention ( $\mathrm{PCl}$ ) with stent placement are strongly related to the device type that is implanted. Drug eluting stents (DES) improved outcomes compared to the bare metal stent in terms of in-stent restenosis. ${ }^{1-3}$ Further developments in coronary DES led to different metal platforms, drug types, mechanisms of drug-release, and polymer coatings. ${ }^{4} \mathrm{~A}$ novel stent that has been developed to further reduce adverse outcomes after PCl is the dual-therapy stent, the COMBO stent (OrbusNeich Medical, The Netherlands). 5,6

The СOMBO stent combines a biodegredable abluminal sirolimus-eluting polymer with a unique luminal endothelial progenitor cell-capturing layer. This stent technique aims to promote true vessel healing and thereby improve clinical outcome after PCI. Two-year clinical outcomes after COMBO stent treatment have been evaluated in the all-comers REMEDEE Registry. ${ }^{7}$ Thus far, no randomized data is available comparing the dual therapy stent technology with new generation DES such as the Resolute Integrity zotarolimus-eluting stent (Medtronic, USA) and the Promus Element everolimus-eluting stent (Boston Scientific, USA). ${ }^{8-10}$ These newer-generation durable polymer drug-eluting stents have been shown to be similarly safe and efficacious. ${ }^{10}$

The objective of the current analysis is to compare 2-year clinical outcomes of the COMBO stent with the Resolute Integrity and Promus Element stent therapy in a balanced cohort. This analysis in the first to compare results after COMBO stent placement with other DES and will provide additional insight in the clinical performance of the COMBO stent.

\section{Methods}

\section{Devices}

The three investigational devices are the Promus Element, the Resolute Integrity and the COMBO stent. All stents are CE-marked (available on the European market) and used in daily clinical practice. Promus Element consists of a thin fluoropolymer-everolimus coating on a stent platform made from a platinumchromium alloy which has a novel, laser-cut, open-cell stent design, consisting of serpentine rings connected by helically distributed links. ${ }^{8}$ Resolute Integrity consists of a BioLinx-zotarolimus coating on a cobalt-chromium alloy stent platform that has a sinusoidal design. The BioLinx polymer system of this stent consists of a blend of three different polymers: the hydrophobic C10 polymer, aiding drug release; a hydrophilic C19 polymer, supporting biocompatibility; and a polyvinyl pyrro-lidone, which increases the initial drug burst and enhances elution rate. ${ }^{9}$ COMBO consists of a $316 \mathrm{~L}$ stainless steel alloy in a helical sinusoidal design and an abluminal biodegradable sirolimus-eluting polymer layer, with a bioengineered anti-CD34+ antibody layer to attract endothelial progenitor cells.

\section{Trials}

The DUTCH PEERS trial is a prospective, investigator-initiated, all-comers, randomized (1:1), noninferiority trial comparing clinical outcomes after Promus Element and Resolute Integrity stent placement. The study design and primary results have been published in more detail elsewhere. ${ }^{10}$ In 
short, a total of 1811 patients were enrolled between November 2010 and May 2012 at four PCl centers in the Netherlands (Promus Element, $\mathrm{N}=905$, Resolute Integrity, $\mathrm{N}=906$ ). Based on the primary endpoint target vessel failure, non-inferiority of the Resolute Integrity stent was proven, compared with the Promus Element stent, and both stents were similarly efficacious and safe in an all-comer population. ${ }^{10}$ At 2-year follow-up, similar clinical outcomes were reported for both DES examined.

The REMEDEE registry is a prospective, investigator-initiated, all-comers, single-arm registry, of 1000 patients treated with the COMBO stent. The study was conducted in nine European sites across The Netherlands, Latvia, Luxembourg, Northern-Ireland and Spain. Enrollment started in June 2013 and was completed in March 2014. Registry design and primary results have been published previously. All events were adjudicated by an independent clinical event committee. ${ }^{7}$

In the REMEDEE Registry DAPT regimen was advised to follow current guidelines: 6-12 months after elective $\mathrm{PCl}, 12$ months after acute coronary syndrome (ACS). DUTCH PEERS recommended prescription of Aspirin and Clopidogrel for 12 months after PCl. Both studies complied with the Declaration of Helsinki, and were approved by independent Medical Ethics Committees and institutional review boards of all participating centers. The DUTCH PEERS complied to the CONSORT 2010 statement. Patients in the studies all provided written informed consent.

\section{Patient population}

Inclusion criteria were identical between the two studies. The following inclusion criteria were used: Patients undergoing $\mathrm{PCl}$ with stent treatment, $>18$ years old and willing and able to cooperate with study requirements. Exclusion criteria in both trials were: High probability of non-adherence to the follow-up requirements (due to social, psychological or medical reasons), currently participating in another investigational drug or device study in which a routine angiographic follow-up is planned, a life expectancy of $<1$ year. Additional exclusion criteria for the DUTCH PEERS trial were: known pregnancy or intolerance to a P2Y12 receptor antagonist, aspirin, heparin or any of the components of the DES.

\section{Clinical endpoints and definitions}

The primary outcome of interest was target lesion failure (TLF): a composite endpoint of cardiac death, target vessel-related myocardial infarction (MI), or any target lesion revascularization at 2 years. Stent thrombosis: (definite, definite and probable) at 2 years. Two-year follow-up is defined as 720 days post index procedure. Endpoints were defined according to the Academic Research Consortium including the addendum on myocardial infarction. ${ }^{11,12}$ Furthermore, we looked at the individual endpoint of TLF separately, cardiac death, target vessel-MI and target lesion revascularization.

\section{Statistical analysis entire cohort}

Categorical variables are presented as numbers and percentages and are compared for the entire cohort with Fisher's exact test. Based on their distributions, continuous variables are presented as mean \pm standard deviation or median with interquartile ranges and compared with student's $t$ or Wilcoxon rank sum test, or Kruskal-Wallis test in case of non-normally distributed variables. 


\section{Propensity score matching}

A pre-specified analysis plan was made prior to the conduct of the analysis to eliminate (potential) model bias, including a consensus on the matching variables. Propensity score matching was performed using the following selected thirteen baseline variables: age, gender, insulin treated Diabetes Mellitus, hypertension, previous $\mathrm{MI}$, previous $\mathrm{PCl}$, previous bypass, ACS, number of treated lesions, target vessel location, stent length and diameter and American College of Cardiology/American Heart Association (ACC/AHA) classification (see appendix I). A logistic multivariable regression was used with device type (Promus Element or Resolute Integrity versus COMBO) as dependent variable and the 13 above listed baseline variables as independent predictors to calculate the propensity score. Patients were 1-to-1 greedy matched using the nearest-neighbor method, COMBO versus either Promus Element or Resolute Integrity. The caliper for the propensity match was set at 0.2 .

All statistical analyses were conducted in R Studio and R version 3.2.2 and the package Matchlt for propensity matching 17 and 18 . All reported $p$ values were 2-tailed, and $p<0.05$ was considered statistically significant.

\section{Sensitivity analyses}

Sensitivity analyses is performed per stent device (COMBO versus Resolute Integrity, COMBO versus Promus) using the same method of propensity score matching.

\section{Analysis of the matched cohort}

Baseline variables of the matched cohort were compared with the same methods of the unmatched cohorts. Kaplan-Meier estimates were used for the cumulative incidence of outcomes at two-year follow-up. P-values and hazard ratios are calculated using Cox proportional hazards ( $\mathrm{CPH}$ ) models. $\mathrm{CPH}$ assumptions are visually inspected by plotting Schoenfeld residuals. P-values $<0.05$ are considered statistically significant.

\section{Results}

\section{Patient and angiographic characteristics from the DUTCH PEERS and REMEDEE Registry}

Baseline characteristics of both trials have been published previously,8, and are summarized in Table 1. REMEDEE Registry patients are older, have more hypertension, more previous $\mathrm{PCl}$ and previous MI. Acute coronary syndrome was more present in the DUTCH PEERS, and the RCA was treated more frequently in DUTCH PEERS. Significant differences were also found in the number of treated lesions, AHA/ACC lesion type and lesion length. 
Table 1: Baseline patient, lesion, and stent characteristics in the REMEDEE Registry and DUTCH PEERS trial before matching.

\begin{tabular}{|c|c|c|c|c|}
\hline & & $\begin{array}{l}\text { All patients } \\
n=2,811\end{array}$ & & \\
\hline & $\begin{array}{l}\text { Promus Element/ } \\
\text { Resolute Integrity } \\
\quad n=1,811\end{array}$ & $\begin{array}{l}\text { СОМво } \\
n=1,000\end{array}$ & p-value & SMD \\
\hline Female & $489(27.0)$ & $261(26.1)$ & 0.636 & 0.020 \\
\hline Age at randomization & $63.94(10.83)$ & $65.25(11.08)$ & 0.002 & 0.120 \\
\hline Diabetes Mellitus & $324(17.9)$ & $184(18.6)$ & 0.694 & 0.018 \\
\hline Insulin treated DM & $113(6.2)$ & $64(6.5)$ & 0.884 & 0.009 \\
\hline Hypertension & $984(54.3)$ & $580(59.2)$ & 0.015 & 0.098 \\
\hline Previous CABG & $173(9.6)$ & $68(6.9)$ & 0.018 & 0.098 \\
\hline Previous $\mathrm{PCl}$ & $349(19.3)$ & $301(30.4)$ & $<0.001$ & 0.260 \\
\hline Previous MI & 397 (21.9) & $253(25.9)$ & 0.020 & 0.093 \\
\hline Acute coronary syndrome & $1062(58.6)$ & 498 (49.9) & $<0.001$ & 0.176 \\
\hline LAD treated & $854(47.2)$ & $484(48.4)$ & 0.553 & 0.025 \\
\hline LCX treated & $523(28.9)$ & $264(26.4)$ & 0.175 & 0.055 \\
\hline Left main treated & $40(2.2)$ & $20(2.0)$ & 0.818 & 0.015 \\
\hline RCA treated & 669 (36.9) & $312(31.2)$ & 0.003 & 0.121 \\
\hline Graft treated & $55(3.0)$ & $23(2.3)$ & 0.308 & 0.046 \\
\hline Number of treated lesions & & & 0.014 & 0.127 \\
\hline 0 & $0(0.0)$ & $1(0.1)$ & & \\
\hline 1 & $1356(74.9)$ & $797(79.7)$ & & \\
\hline 2 & $373(20.6)$ & $163(16.3)$ & & \\
\hline 3 & $82(4.5)$ & 39 (3.9) & & \\
\hline AHA/ACC lesion type (worst) & & & $<0.001$ & 0.429 \\
\hline A & $77(4.3)$ & $146(15.1)$ & & \\
\hline B1 & $436(24.1)$ & $231(23.8)$ & & \\
\hline B2 & $647(35.7)$ & $366(37.8)$ & & \\
\hline C & 651 (35.9) & $226(23.3)$ & & \\
\hline Max stent diameter & $3.00(3.0-3.5)$ & $3.00(3.0-3.5)$ & 0.021 & 0.110 \\
\hline Total stent length & $30.0(18.0-50.0)$ & $18.0(15.0-23.0)$ & $<0.001$ & 0.824 \\
\hline
\end{tabular}

Values are mean \pm SD, median (IQR) or $n(\%)$. SMD: standard mean difference. In DUTCH PEERS; Promus Element $n=905$, Resolute Integrity $n=906$.

\section{Patient and angiographic characteristics of patients in the matched cohorts}

The propensity score calculation was performed, taking into account the thirteen baseline and angiographic variables. The propensity score match resulted 771 balanced patient pairs, as illustrated in Table 2. AHA/ACC lesion type could not be perfectly matched (taking into account the four lesion types). The combined type B2/C was the same, both $59.8 \%$. 
Table 2: Baseline patient, lesion, and stent characteristics in the matched cohort.

\begin{tabular}{|c|c|c|c|c|}
\hline & & $\begin{array}{c}\text { All patients } \\
n=1,542\end{array}$ & & \\
\hline & $\begin{array}{l}\text { Promus Element/ } \\
\text { Resolute Integrity } \\
\qquad n=771\end{array}$ & $\begin{array}{l}\text { COMBO } \\
n=771\end{array}$ & p-value & SMD \\
\hline Female & $215(27.9)$ & $206(26.7)$ & 0.647 & 0.026 \\
\hline Age at randomization & $64.06(10.93)$ & $64.78(11.21)$ & 0.204 & 0.065 \\
\hline Diabetes Mellitus & $138(17.9)$ & $141(18.3)$ & 0.895 & 0.010 \\
\hline Insulin treated DM & $44(5.7)$ & $47(6.1)$ & 0.829 & 0.017 \\
\hline Hypertension & $436(56.5)$ & $440(57.1)$ & 0.877 & 0.010 \\
\hline Previous CABG & $54(7.0)$ & $52(6.7)$ & 0.920 & 0.010 \\
\hline Previous $\mathrm{PCl}$ & $187(24.3)$ & $200(25.9)$ & 0.481 & 0.039 \\
\hline Previous MI & $164(21.3)$ & $177(23.0)$ & 0.462 & 0.041 \\
\hline Acute coronary syndrome & $420(54.5)$ & $406(52.7)$ & 0.507 & 0.036 \\
\hline LAD treated & $369(47.9)$ & $373(48.4)$ & 0.878 & 0.010 \\
\hline LCX treated & $188(24.4)$ & $199(25.8)$ & 0.557 & 0.033 \\
\hline Left main treated & $12(1.6)$ & $12(1.6)$ & 1.000 & $<0.001$ \\
\hline RCA treated & $235(30.5)$ & $234(30.4)$ & 1.000 & 0.003 \\
\hline Graft treated & $11(1.4)$ & $14(1.8)$ & 0.687 & 0.031 \\
\hline Number of treated lesions & & & 0.294 & 0.098 \\
\hline 0 & $0(0.0)$ & $1(0.1)$ & & \\
\hline 1 & $669(86.8)$ & $651(84.4)$ & & \\
\hline 2 & $93(12.1)$ & $103(13.4)$ & & \\
\hline 3 & $9(1.2)$ & $16(2.1)$ & & \\
\hline AHA/ACC lesion type (worst) & & & $<0.001$ & 0.360 \\
\hline A & $53(6.9)$ & $127(16.5)$ & & \\
\hline B1 & $257(33.3)$ & $183(23.7)$ & & \\
\hline B2 & $321(41.6)$ & $287(37.2)$ & & \\
\hline C & $140(18.2)$ & $174(22.6)$ & & \\
\hline Max stent diameter & $3.00[3.00,3.50]$ & $3.00[3.00,3.50]$ & 0.883 & 0.008 \\
\hline Total stent length & $20.00[15.00,28.00]$ & $18.00[15.00,28.00]$ & 0.751 & 0.060 \\
\hline
\end{tabular}

Values are mean $\pm S D$, median (IQR) or $n(\%)$. SMD: standard mean difference. In DUTCH PEERS; Promus Element $n=375$, Resolute Integrity $n=396$.

\section{Clinical outcomes at two years in the matched cohorts}

TLF at 2-year follow-up in the matched cohort by Kaplan-Meier Estimate is shown in Figure 1. TLF occurred in $7.9 \%$ in patients treated with $\mathrm{COMBO}$, and $6.4 \%$ in patients treated with Promus Element/ Resolute Integrity $(p=0.26, \mathrm{HR} 1.24(95 \% \mathrm{Cl} 0.85-1.81))$. Cardiac death occurred in $2.9 \%$ vs $2.1 \%$ ( $p=0.31$, HR 1.39 (95\% Cl 0.73-2.65)); target vessel MI in 1.2\% vs 1.3\% ( $p=0.90, \mathrm{HR}(95 \% \mathrm{Cl} 0.37-2.22)$ ); and TLR in $5.6 \%$ vs $3.8 \%(p=0.1, H R 1.48$ (95\% Cl 0.92-2.37)), respectively. Definite stent thrombosis 
occurred in $0.8 \%$ of the COMBO patients (all early ST) and in $0.9 \%$ of the Promus Element/Resolute Integrity patients, $p=0.79$. Definite or probable stent thrombosis was observed in $0.9 \%$ vs $1.0 \%$ patients ( $p=0.8$, HR $0.88(95 \% \mathrm{Cl} 0.32-2.43))$, respectively.

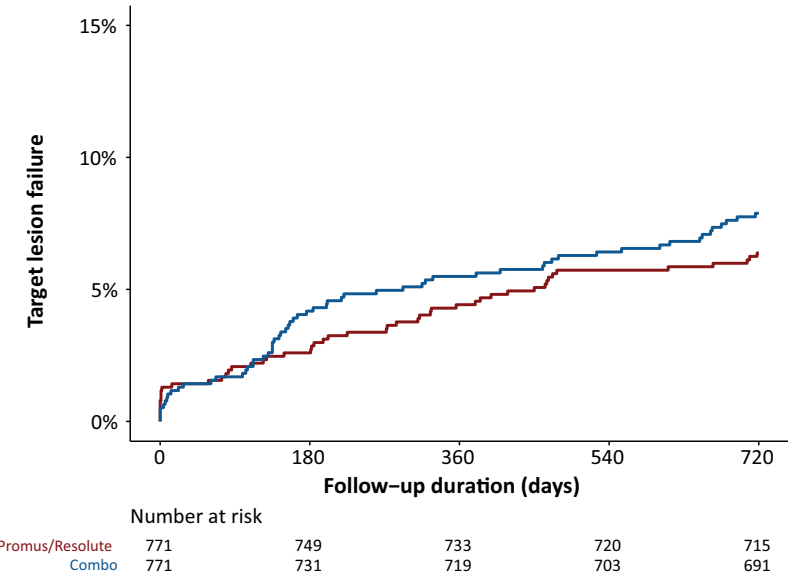

Figure 1: Target Lesion Failure. Cumulative event rate of target lesion failure by Kaplan meier method.
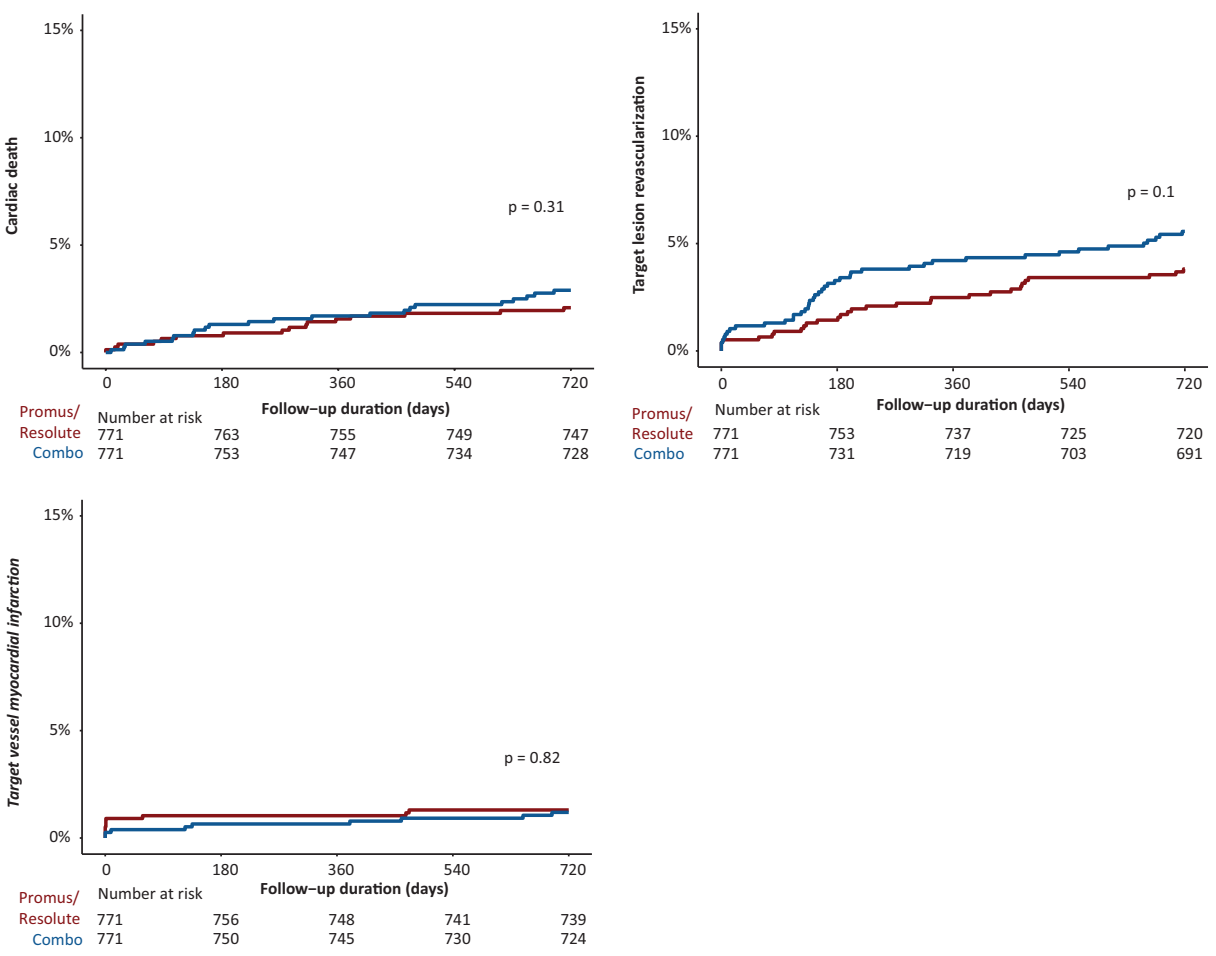

Figure 2: Cardiac death, target vessel MI, and TLR. Cumulative event rate of cardiac death, target vessel MI, and TLR by Kaplan Meier method. 
Table 3: Kaplan-Meier estimates and treatment effects of COMBO and Resolute Integrity/Promus Element at 2-year follow-up.

\begin{tabular}{|lccc|}
\hline & & $\begin{array}{c}\text { All patients } \\
\mathbf{n = 1 , 5 4 2}\end{array}$ & p-value \\
& $\begin{array}{c}\text { Promus Element/ } \\
\text { Resolute Integrity } \\
\mathbf{n = 7 7 1}\end{array}$ & $\mathbf{C O M B O}$ & $\mathbf{n = 7 7 1}$ \\
TLF & $60(7.9)$ & $49(6.4)$ & 0.26 \\
Cardiac death & $22(2.9)$ & $16(2.1)$ & 0.32 \\
Target vessel MI & $9(1.2)$ & $10(1.3)$ & 0.82 \\
TLR & $42(5.6)$ & $29(3.8)$ & 0.11 \\
Stent thrombosis (definite) & $6(0.8)$ & $7(0.9)$ & 0.79 \\
Stent thrombosis (definite or probable) & $7(0.9)$ & $8(1.0)$ & 0.80 \\
\hline
\end{tabular}

Values are $\mathrm{n}(\%)$.

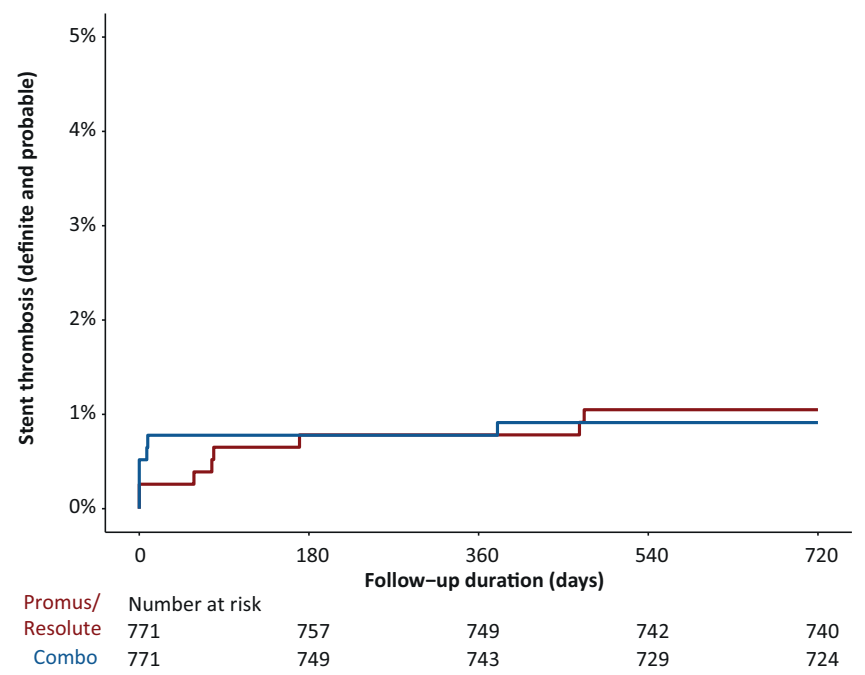

Figure 3: Definite and probable stent thrombosis. Cumulative event rate of the secondary endpoint stent thrombosis by Kaplan Meier method.

\section{Sensitivity analyses}

Sensitivity analyses have been conducted per device type. Two patient pairs were made for COMBO and Resolute Integrity (both $\mathrm{n}=588$ ). Baseline and angiographic characteristics were nicely balanced. The evaluation of TLF at two-year follow-up shows 8.4\% for COMBO and 7.2\% Resolute Integrity $(p=0.44)$. Sensitivity analysis for the comparison of COMBO with Promus Element resulted also in two balanced cohorts (both $n=617$ ), and evaluation of TLF shows $7.4 \%$ for COMBO and $5.5 \%$ for Promus Element $(p=0.19)$. 


\section{Discussion}

This is the first analysis comparing the bio-engineered COMBO stent with newer-generation DES, showing no significant differences in clinical outcomes between the COMBO and Promus/Resolute Integrity in a balanced cohort at two-year follow-up. Furthermore, the current analysis shows that baseline clinical and angiographic characteristics are different among all-comers trials, and low rates of target lesion failure are observed at two-year follow-up after DES implantation.

\section{COMBO versus current mono-therapy DES}

This analysis is the first to compare the COMBO stent with current DES. No significant differences were observed in terms of TLF, any individual endpoints and definite/and probable stent thrombosis. Target lesion revascularization is non-significantly higher in patients treated with COMBO, with an increase of revascularization between 6-9 months post index procedure. Although there was no scheduled repeat angiography the REMEDEE registry, there might be a slight recall bias due to the fact that the COMBO stent is a newer device, and patients with persistent angina were more easily scheduled for repeat angiography.

\section{All-comers PCl cohorts}

In the present analysis we found that the baseline characteristics of two large all-comers $\mathrm{PCl}$ patient cohorts are different, despite similar inclusion and exclusion criteria. When comparing clinical and angiographic characteristics, there are notable differences in age, hypertension, previous $\mathrm{PCl}$ or $\mathrm{MI}$, location of lesion, number of lesions, lesion type and lesion length. This could be explained by the fact that the DUTCH PEERS is a Dutch trial, where patients were enrolled in the Netherlands only, while the REMEDEE Registry enrolled patients in five European countries. Also, there might be differences in patients who want to participate in a randomized trial or registry. De Boer et al. previously compared participants and non-participants of a single high-volume center in two 'all-comer' randomized PCl trials and found that these two groups differed significantly in baseline characteristics and clinical outcome; the authors explained this by the fact that only half of the target population was enrolled. ${ }^{13}$ While the all-comers design of clinical studies may not always fully represent 'real world' clinical practice, von Birgelen et al. recently found that 5-year clinical outcome was similar for participants in a randomized 'most-comer' DES study with a very high enrolment rate versus the complete cohort of patients, who had been eligible for trial enrolment.

If we compare the baseline characteristics with other trials; LEADERS $^{14}$, COMPARE ${ }^{15}$, SPIRIT IV ${ }^{16}$, baseline characteristics are similar but do alter frequently; e.g. age (lowest mean: 63 years, highest: 65 years), hypertension (lowest: 44\%, highest: 77\%), previous PCl (lowest: 13\%, highest: 37\%), previous MI (lowest: 15\%, highest: $33 \%$ ). Attention to baseline characteristics should be given, when interpreting all-comers PCI trial results. In this study we accounted for multiple confounders by propensity score matching, there were no differences between $\mathrm{COMBO}$ and Promus Element/Resolute Integrity patients. 


\section{Clinical outcomes}

2 years after stent placement In the Prodigy study, subgroup analyses were performed comparing the clinical combined endpoint of death, $\mathrm{MI}$ and target vessel revascularization after BMS or DES placement at two years. The endpoint occurred in $32.1 \%$ of patients treated with BMS. ${ }^{17}$ In this same study the endpoint occurred $27.8 \%$ in patients treated with zotarolimus-eluting Endeavor Sprint stents (ZES-S), $26.2 \%$ in paclitaxel-eluting stents (PES) and $19.2 \%$ in everolimus-eluting stents (EES). A major decrease in adverse events was noted with the use of newer DES.

The adverse event rates after $\mathrm{PCl}$ continue to decrease. In the LEADERS trial two year results a further decline in event rates was noticed, comparing the first generation DES, sirolimus-eluting stent, with second generation DES, a biolimus-eluting stent. Combined cardiac death, $\mathrm{MI}$ and clinically indicated TLR (rate was $11.9 \%$ in BES and $13.6 \%$ in SES. ${ }^{18}$ The COMPARE trial two year results also showed lower event rates with second generation DES (rate of all death, MI, and TVR: 9.0\% in EES and $13.7 \%$ in PES)..$^{19}$ SPIRIT IV two year results were also in line with these findings, showing lower events in second generation DES. ${ }^{20}$ To evaluate the efficacy of the bio-engineered anti-CD34 antibody layer comparison might be done with a DES eluting sirolimus. If we compare the 2-year results of this balanced cohort with mono- SES from LEADERS, we notice a lower event rate in the COMBO stent group.

The theoretical benefit of the novel dual-therapy stent technology is to reduce the healing time and potentially reduce the DAPT duration. This could specifically benefit patients who have a high risk of bleeding, are scheduled for operation or patients who are unlikely to adhere to medication. The BioFreedom stent (BioFreedom, Biosensors Interventional Technologies, Singapore) with 1 month DAPT has been demonstrated to be safer than BMS in patient with a high risk of bleeding. ${ }^{20}$ Myocardial infarction and/or stent thrombosis was observed in $8.2 \%$ of high risk bleeding patients treated with BioFreedom at two year follow-up. These data cannot be directly compared to our data, due to patient selection. Future trials would be needed to address the differences in performance between BioFreedom and Combo stent.

\section{Strengths and limitations}

First, the main limitation of these analyses is that the data is not randomized. The propensity score was calculated based on 13 pre-specified baseline and angiographic characteristics, but there could be other factors playing a role in clinical outcome that were not taken into account. We could not correct the fact that the patients treated in DUTCH PEERS were all patients in the Netherlands, and the REMEDEE Registry consisted of European patients. Also, the REMEDEE Registry did not have core laboratoryadjudicated data on angiographic characteristics. Angiographic data was entered in the database by the sub-investigator of the site. All angiographic data in the DUTCH PEERS was core laboratory-adjudicated and obtained by means of Quantitative Coronary Angiography (QCA, Medis, Leiden). However all events were adjudicated by an independent clinical event committee in DUTCH PEERS but also in the REMEDEE Registry. COMBO is compared with two different DES together (consisting of either Resolute Integrity or Promus Element). The results of the randomized DUTCH PEERS showed non-inferiority of Resolute Integrity to Promus Element, allowing these DES results to be pooled. For the main analysis we used the pooled set because of the increased power of a larger set of matched pairs. The separate analyses shown in the electronic supplement show comparable outcomes. ${ }^{21}$ 
This analysis is the first to compare the COMBO stent with other newer-generation DES in a balanced cohort at 2-year follow-up. Results of the study evaluating short (3 months) versus standard (12 months) duration of DAPT in ACS patients, the REDUCE study, are being awaited. Randomized data comparing the $\mathrm{COMBO}$ with the everolimus-eluting Xience stent (Abbott Vascular) are expected from the forthcoming HARMONEE trial (NCT02073565).

\section{Conclusion}

In a propensity matched cohort of patients treated with the novel COMBO stent, and patients treated with either a Promus Element or Resolute Integrity DES, no differences were found in the clinical endpoint target lesion failure, with overall low adverse event rates. However, randomized trials are needed to demonstrate the equivalence of the COMBO stent to other current generation DES.

\section{Impact on daily practice}

The dual-therapy stent technology has been evaluated in all-comers patients and has shown good clinical results. There is currently no data comparing the clinical results with other commonly used newer-generation DES, such as the Promus Element and Resolute Integrity. Two-year clinical follow-up of the COMBO stent shows low event rates, not significantly different from the Promus Element and Resolute Integrity stents in a balanced cohort. Randomized controlled trials evaluating the clinical results after COMBO stent treatment are currently being conducted and their results are highly anticipated. 


\section{References}

1. Moses JW, Leon MB, Popma JP, et al. Sirolimus-Eluting Stents versus Standard Stents in Patients with Stenosis in a Native Coronary Artery. N Engl J Med. 2003;349:1315-23.

2. Stettler C, Wandel S, Allemann S, et al. Outcomes associated with drug-eluting and bare-metal stents: a collaborative network meta-analysis. Lancet. 2007;370:937-48.

3. Stone GW, Ellis SG, Cox DA, Hermiller J, et al. A Polymer-Based, Paclitaxel-Eluting Stent in Patients with Coronary Artery Disease. N Engl J Med. 2004;350:221-31.

4. Stefanini GG, Holmes DR. Drug-Eluting Coronary-Artery Stents. N Engl J Med. 2013;368:254-65.

5. Granada JF, Inami S, Aboodi MS, et al. Development of a novel prohealing stent designed to deliver sirolimus from a biodegradable abluminal matrix. Circ Cardiovasc Interv. 2010;3:257-66.

6. Larsen K, Cheng C, Tempel D,et al. Capture of circulatory endothelial progenitor cells and accelerated reendothelialization of a bio-engineered stent in human ex vivo shunt and rabbit denudation model. Eur Heart J. 2012;33:120-8.

7. Woudstra P, Kalkman DN, den Heijer P, et al. 1-Year Results of the REMEDEE Registry: Clinical Outcomes After Deployment of the Abluminal Sirolimus-Coated Bioengineered (Combo) Stent in a Multicenter, Prospective AllComers Registry. JACC Cardiovasc Interv. 2016;9:1127-34.

8. Stone GW, Teirstein PS, Meredith IT, et al. A Prospective, Randomized Evaluation of a Novel Everolimus-Eluting Coronary Stent: The PLATINUM (A Prospective, Randomized, Multicenter Trial to Assess an Everolimus-Eluting Coronary Stent System [PROMUS Element] for the Treatment of up to Two De Novo Co. J Am Coll Cardiol. 2011;57:1700-8.

9. Yeung AC, Leon MB, Jain A, et al. Clinical Evaluation of the Resolute Zotarolimus-Eluting Coronary Stent System in the Treatment of De Novo Lesions in Native Coronary Arteries. J Am Coll Cardiol. 2011;57:1778-83.

10. von Birgelen C, Sen H, Lam MK, et al. Third-generation zotarolimus-eluting and everolimus-eluting stents in allcomer patients requiring a percutaneous coronary intervention (DUTCH PEERS): a randomised, single-blind, multicentre, non-inferiority trial. Lancet. 2014;383:413-23.

11. Cutlip DE, Windecker S, Mehran R, et al. Clinical end points in coronary stent trials: A case for standardized definitions. Circulation. 2007;115:2344-51.

12. Vranckx P, Cutlip DE, Mehran R, Kint PP, Silber S, Windecker S, Serruys PW. Myocardial infarction adjudication in contemporary all-comer stent trials: Balancing sensitivity and specificity: Addendum to the historical MI definitions used in stent studies. Eurolntervention. 2010;5:871-4.

13. De Boer SPM, Lenzen MJ, Oemrawsingh RM, et al. Evaluating the "all-comers" design: A comparison of participants in two "all-comers" PCl trials with non-participants. Eur Heart J. 2011;32:2161-7.

14. Garg S, Sarno G, Serruys PW, et al. The twelve-month outcomes of a biolimus eluting stent with a biodegradable polymer compared with a sirolimus eluting stent with a durable polymer. Eurolntervention. 2010;6:233-9.

15. Kedhi E, Joesoef KS, McFadden E, Wassing J, van Mieghem C, Goedhart D, Smits PC. Second-generation everolimus-eluting and paclitaxel-eluting stents in real-life practice (COMPARE): a randomised trial. Lancet. 2010;375:201-9.

16. Stone GW, Rizvi A, Newman W, et al. Everolimus-Eluting versus Paclitaxel-Eluting Stents in Coronary Artery Disease. N Engl J Med. 2010;362:1663-74.

17. Valgimigli $M$, Tebaldi $M$, Borghesi $M$, et al. Two-year outcomes after first- or second-generation drug-eluting or bare-metal stent implantation in all-comer patients undergoing percutaneous coronary intervention: A prespecified analysis from the prodigy study (prolonging dual antiplatelet treatment after grading stent-induced intimal hyperplasia study). J Am Coll Cardiol Intv. 2014;7:20-8.

18. Klauss V, Serruys PW, Pilgrim T, et al. 2-Year clinical follow-up from the randomized comparison of biolimuseluting stents with biodegradable polymer and sirolimus-eluting stents with durable polymer in routine clinical practice. J Am Coll Cardiol Intv. 2011;4:887-95.

19. Smits PC, Kedhi E, Royaards KJ, Joesoef KS, Wassing J, Rademaker-Havinga TAM, McFadden E. 2-year follow-up of a randomized controlled trial of everolimus- and paclitaxel-eluting stents for coronary revascularization in daily practice: COMPARE (Comparison of the everolimus eluting XIENCE-V stent with the paclitaxel eluting TAXUS LIBERT stent in all-comers: A randomized open label trial). J Am Coll Cardiol. 2011;58:11-8. 
20. Stone GW, Rizvi A, Sudhir K, et al. Randomized comparison of everolimus- and paclitaxel-eluting stents: 2-year follow-up from the SPIRIT (clinical evaluation of the XIENCE v everolimus eluting coronary stent system) IV trial. J Am Coll Cardiol. 2011;58:19-25.

21. Elze MC, Gregson J, Baber U, et al. Comparison of Propensity Score Methods and Covariate Adjustment: Evaluation in 4 Cardiovascular Studies. J Am Coll Cardiol. 2017;69:345-57. 



\section{Conflicts of interest statement}

The research department of Thoraxcentrum Twente has received research grants from AstraZeneca, Biotronik, Boston Scientific, and Medtronic. CvB was a consultant to device-manufacturing companies, among them Abbott, Boston Scientific, and Medtronic; he has received travel expenses from Biotronik and speakers honoraria from AstraZeneca and MSD. All other authors declare no competing interests.

\section{Acknowledgments}

We thank our patients for their confidence and willingness to participate in this randomised trial. We gratefully acknowledge the invaluable support of this trial by the cardiologists and the staff of the research departments, catheterisation laboratories, wards, and administrative departments of the study centres and referring hospitals, and by the general physicians and pharmacists of our patients. Biotronik, Boston Scientific, and Medtronic equally supported this investigator-initiated study and the present 1 year follow-up.

\section{Copyright}

Reprinted with permission from

Lancet 2017 Nov 26;388(10060):2607-2617.

(C) Elsevier 


\title{
CHAPTER 6
}

\section{Very Thin Strut Biodegradable Polymer Everolimus-Eluting and Sirolimus-Eluting Stents Versus Durable Polymer Zotarolimus-Eluting Stents in Allcomers With Coronary Artery Disease (BIO-RESORT)}

A Three-Arm, Randomised, Non-Inferiority Trial

\begin{abstract}
Clemens von Birgelen*, Marlies M. Kok*, Liefke C. van der Heijden*, Peter W. Danse, Carl E. Schotborgh, Martijn Scholte, R. Melvyn Tjon Joe Gin, Samer Somi, K. Gert van Houwelingen, Martin G. Stoel, Frits H.A.F. de Man, J.(Hans) W. Louwerenburg, Marc Hartmann, Paolo Zocca, Gerard C.M. Linssen, Job van der Palen, Carine J.M. Doggen, Marije M. Löwik.
\end{abstract}

* First, second, and third author contributed equally to this manuscript.
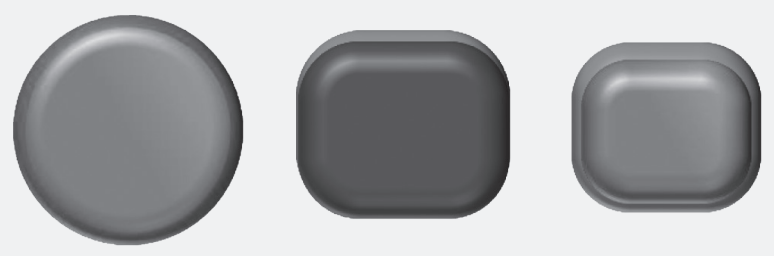


\section{Abstract}

Background: In patients with coronary artery disease, treated with durable polymer-coated drugeluting stents, the life-long presence of the polymer might delay arterial healing. Novel very thin strut biodegradable polymer stents, which leave only a bare metal stent after polymer resorption, might improve long-term outcome. We investigated in allcomers the safety and efficacy of three stents eluting either everolimus, sirolimus, or zotarolimus, often clinically used but never compared, of which the biodegradable polymer everolimus-eluting stent was never before assessed in allcomers.

Methods: The large-scale, investigator-initiated, multicentre, assessor and patient blinded, threearm, randomised, BIO-RESORT non-inferiority trial was done at four clinical sites in the Netherlands. All-comer patients were aged 18 years or older, capable of providing informed consent, and required a percutaneous coronary intervention with drug-eluting stent implantation according to clinical guidelines or the operators' judgment. Exclusion criteria were: participation in another randomised drug or device study before reaching the primary endpoint of that study; planned surgery necessitating interruption of dual antiplatelet therapy within the first 6 months; known intolerance to components of the investigational product or medication required; uncertainty about the adherence to follow-up procedures or an assumed life expectancy of less than 1 year; or known pregnancy. Web-based computergenerated allocation sequences randomly assigned patients (1:1:1) to treatment with very thin strut biodegradable polymer everolimus-eluting or sirolimus-eluting stents (which differ substantially in type, amount, distribution, and resorption speed of their respective coating), or thin strut durable polymer zotarolimus-eluting stents. The primary endpoint was a composite of safety (cardiac death or target vessel-related myocardial infarction) and efficacy (target vessel revascularisation) at 12 months of follow up with a very thin strut biodegradable polymer of either everolimus-eluting or sirolimus-eluting stents, compared with durable polymer zotarolimus-eluting stents, analysed by intention to treat (noninferiority margin 3.5\%). This trial was registered with ClinicalTrials.gov, number NCT01674803.

Findings: From Dec 21, 2012, to Aug 24, 2015, 3514 patients were enrolled and analysed, of whom 2449 (70\%) had acute coronary syndromes, which included 1073 (31\%) ST-elevation myocardial infarctions. 12 month follow-up of 3490 (99\%) patients (three lost to follow-up; 21 withdrawals) was available. The primary endpoint was met by 55 (5\%) of 1172 patients assigned to everolimus-eluting stents, 55 (5\%) of 1169 assigned to sirolimus-eluting stents and 63 (5\%) of 1173 assigned to zotarolimuseluting stents. Non-inferiority of the everolimus-eluting stents and sirolimus-eluting stents compared with zotarolimus-eluting stents was confirmed (both-0.7\% absolute risk difference, $95 \% \mathrm{Cl}-+2 \cdot 4$ to $1 \cdot 1$; upper limit of one sided $95 \% \mathrm{Cl} 0 \cdot 8 \%$, pnon-inferiority<0.0001). Definite stent thrombosis (defined by the Academic Research Consortium) occurred in four (0.3\%) of 1172 patients who were allocated to everolimus-eluting stents, four (0.3\%) of 1169 patients who were allocated to sirolimus-eluting stents, and three $(0 \cdot 3 \%)$ of 1173 patients who were allocated to zotarolimus-eluting stents ( $p$-Log-rank=0.70 for both comparisons with zotarolimus-eluting stents). 
Interpretation: At 12 month follow-up, both very thin strut drug-eluting stents with dissimilar biodegradable polymer coatings (eluting either everolimus or sirolimus) were non-inferior to the durable polymer stent (eluting zotarolimus) in treating allcomers with a high proportion of patients with acute coronary syndromes. The absence of a loss of 1 year safety and efficacy with the use of these two biodegradable polymer-coated stents is a prerequisite before assessing their potential longer-term benefits.

Funding: Biotronik, Boston Scientific, and Medtronic. 


\section{Introduction}

The implantation of a drug-eluting stent is considered the standard approach for percutaneous coronary intervention. ${ }^{1}$ The elution of antiproliferative drugs from the stent's polymer coating reduces the risk of lesion recurrence. ${ }^{1,2}$ However, the lifelong presence of a durable polymer in a coronary artery might induce vessel wall inflammation, delay arterial healing, and occasionally cause serious complications such as stent thrombosis and myocardial infarction. ${ }^{3}$

Growing awareness of this risk motivated the development of stents with biodegradable coatings that leave only a bare metal stent after polymer resorption. ${ }^{4-14}$ Early biodegradable polymer stents had thick stainless steel struts $(120 \mu \mathrm{m})$ and in a large allcomers trial, ${ }^{6,7}$ showed similar efficacy and better long-term safety as compared with early-generation durable polymer stents that also had thick struts. However, in another allcomers trial, ${ }^{8}$ similar thick-strut biodegradable polymer stents did not show noninferiority as compared with early-generation durable polymer stents. Moreover, equivocal results were reported when comparing early biodegradable polymer stents with new-generation durable polymer stents with thin cobalt chromium struts, ${ }^{11-14}$ which are in line with previous research showing that thick struts increase the risk of stent thrombosis and lesion recurrence. ${ }^{15}$

Today, novel biodegradable polymer stents are available that have uncoated struts that are up to half as thick as the struts of the early biodegradable polymer stents. These very thin strut (60-81 $\mu \mathrm{m})$ biodegradable polymer stents have flexible designs and thin, refined coatings. ${ }^{4}$ The present trial assesses two stents that share these characteristics, but differ in the type, amount, distribution, and degradation speed of their respective coatings. ${ }^{16}$ One device, the everolimus-eluting platinum chromium stent (Synergy, Boston Scientific; Natick, MA, USA), ${ }^{4,17,18}$ is the first and currently only biodegradable polymer stent with US Food and Drug Administration approval. While the device has rapidly gained clinical acceptance, there are still no data from a randomised trial with allcomers. The other novel device is a sirolimus-eluting cobalt chromium stent (Orsiro, Biotronik; Bülach, Switzerland) that has shown its usefulness outside of the USA. ${ }^{19-22}$

So far, neither of these stents has been compared with the new-generation, thin-strut durable polymer zotarolimus-eluting stent (Resolute Integrity, Medtronic, Santa Rosa; CA, USA), an established device with excellent clinical outcomes. ${ }^{12-23}$ Therefore, the randomised, three-arm, BIO-RESORT non-inferiority trial assessed in allcomers the safety and efficacy of the two novel stents versus the zotarolimus-eluting stent.

\section{Methods}

\section{Study design and participants}

This randomised trial (BIO-RESORT) was done in an allcomers population, at four clinical centres in the Netherlands (Thoraxcentrum Twente, Medisch Spectrum Twente, Enschede; Rijnstate Hospital, Arnhem; Haga Hospital, The Hague; and Albert Schweitzer Hospital, Dordrecht). This investigator-initiated study is a three-group trial that assessed two independent non-inferiority hypotheses in allcomers that the 1 year safety and efficacy of the biodegradable polymer everolimus-eluting stent is non-inferior to the 
durable polymer zotarolimus-eluting stent, and that the 1 year safety and efficacy of the biodegradable polymer sirolimus-eluting stent is non-inferior to the durable polymer zotarolimus-eluting stent. The design of this study has been described previously. ${ }^{16}$

All-comer patients were eligible if they were aged 18 years or older, capable of providing informed consent, and required a percutaneous coronary intervention with drug-eluting stent implantation according to clinical guidelines or the operators' judgment. All coronary syndromes, de-novo and restenotic lesions, and coronary artery or bypass lesions were permitted. There was no limit for lesion length, reference size, number of lesions, or diseased vessels to be treated. The exclusion criteria were: participation in another randomised drug or device study before reaching the primary endpoint of that study; planned surgery necessitating interruption of dual antiplatelet therapy within the first 6 months; known intolerance to components of the investigational product or medication required (eg, intolerance to concomitant anticoagulation or antiplatelet therapy); uncertainty about the adherence to follow-up procedures or an assumed life expectancy of less than a year; or known pregnancy. The trial complied with the CONSORT 2010 Statement and Declaration of Helsinki and was approved by the Medical Ethics Committee Twente and the institutional review boards of all participating centres. All patients provided written informed consent.

\section{Randomisation and masking}

After guide wire passage or predilation, patients were randomly assigned in a 1:1:1 ratio to one of the three study stents. Web-based randomisation was done with the use of a custom-designed computer program in random block sizes of 6 and 3, stratified according to the presence of diabetes. Patients were blinded to the allocated stent but treating clinicians were not. Assessors such as the angiographic analysts or members of the independent clinical event committee were blinded to the assigned treatment. Blinding was maintained until the independent external event committee had judged all event triggers of the 1 year follow-up.

\section{Procedures}

The everolimus stent elutes its drug within 3 months from a $4 \mu \mathrm{m}$ biodegradable poly(lactic-co-glycolic acid) coating that is located only on the abluminal side of $74 \mu \mathrm{m}$ (for stent sizes $\leq 2.5 \mathrm{~mm}$ ), $79 \mu \mathrm{m}$ (for 3.0-3.5 mm stents), or $81 \mu \mathrm{m}$ (for $4.0 \mathrm{~mm}$ stents) platinum chromium struts and resorbed within 4 months. ${ }^{16,17}$ The sirolimus-eluting stent has $60 \mu \mathrm{m}$ (for stents $\leq 3.0 \mathrm{~mm}$ ) or $80 \mu \mathrm{m}$ (for stents $>3.0$ $\mathrm{mm}$ ) cobalt chromium struts that are circumferentially covered by an asymmetrical hybrid coating that is thicker on the abluminal side $(7.4 \mu \mathrm{m}$ vs $3.5 \mu \mathrm{m}) .{ }^{16}$ The zotarolimus-eluting stent ${ }^{16,23}$ has thin $91 \mu \mathrm{m}$ cobalt chromium struts, circumferentially covered by a $6 \mu \mathrm{m}$ zotarolimus-eluting blend of three durable polymers. During study enrolment, everolimus-eluting stents and zotarolimus-eluting stents with diameters of 2.25-4.0 $\mathrm{mm}$ and lengths of $8-38 \mathrm{~mm}$ were available; sirolimus-eluting stents had the same diameters $(2.25-4.0 \mathrm{~mm})$ and similar lengths $(9-40 \mathrm{~mm})$.

Coronary interventions were done according to standard techniques. Lesion predilation, direct stenting, and stent postdilation were left to the operator's discretion. Staged procedures with allocated stents were permitted within 6 weeks after the initial percutaneous intervention with coronary stenting (index procedure). Concomitant drugs did not differ from routine treatment; further treatment was given 
according to medical guidelines and the physician's judgment. ${ }^{16}$ Generally, dual antiplatelet therapy (DAPT) was prescribed for 6-12 months. Operators were encouraged to use the assigned stent if additional lesions required treatment during follow-up.

Electrocardiographs were systematically assessed and recommended at routine clinical followup. Laboratory tests included systematic assessment of cardiac markers after the intervention and subsequent serial measurements in case of suspected ischaemia. In patients with acute coronary syndromes, cardiac markers were generally also assessed before the intervention. Analysts, blinded for the stent type used, did angiographical analyses and offline quantitative coronary angiographical measurements according to present standards (QAngio XA, version 7.3).

\section{Outcomes}

Clinical endpoints were prespecified, with definitions according to the Academic Research Consortium (ARC). ${ }^{16,24,25}$ The prespecified primary composite endpoint of target vessel failure assessed by device efficacy and patient safety at 1 year follow-up comprised cardiac death, target vessel-related myocardial infarction, or clinically indicated target vessel revascularisation (components in hierarchical order). Death was considered as cardiac, unless an unequivocal non-cardiac cause could be established. Myocardial infarction was defined by any creatine kinase concentration of more than double the upper limit of normal with elevated confirmatory cardiac biomarkers. ${ }^{25}$ Target vessel-related myocardial infarction was related to the target vessel or could not be related to another vessel; further classification was on the basis of laboratory, electrocardiographical, angiographical, or clinical data. ${ }^{16}$ Revascularisation procedures were considered clinically indicated if the angiographical percent diameter stenosis of the then treated lesion was $50 \%$ or higher in the presence of ischaemic signs or symptoms, or if the diameter stenosis was $70 \%$ or higher irrespective of ischaemic signs or symptoms. ${ }^{16,25}$

Prespecified secondary endpoints included: all-cause mortality; any myocardial infarction; clinically indicated target lesion revascularisation; and stent thrombosis. ${ }^{16,24,25}$ Additional composite endpoints were (components in hierarchical order): a composite endpoint of target lesion failure, consisting of cardiac death, target vessel-related myocardial infarction, or clinically indicated target lesion revascularisation; a composite endpoint of major adverse cardiac events, consisting of all-cause death, any myocardial infarction, emergent coronary bypass surgery, or clinically indicated target lesion revascularisation; and the patient-oriented composite endpoint, consisting of all-cause death, any myocardial infarction, or any coronary revascularisation. A final residual diameter stenosis of less than $50 \%$ was defined as device success if achieved with assigned study stents only; lesion success if achieved with any approach; and procedure success if achieved without in-hospital major adverse cardiac events. A predefined subgroup analysis of the primary endpoint was done.

The 1 year clinical follow-up data were obtained at visits to outpatient clinics or, if not feasible, by telephone follow-up or a medical questionnaire (research staff blinded to assigned stent). There was no routine angiographic follow-up. The clinical research organisation Cardio Research Enschede (Enschede, Netherlands) coordinated trial and data management. A formal data safety monitoring committee reviewed the outcome data periodically. The clinical course of the study population will be assessed per protocol until 5 years from stenting. 
Data monitoring, processing of clinical outcome data, and independent clinical event adjudication were done by an independent clinical research organisation (Diagram; Zwolle, Netherlands). Monitoring comprised: informed consent and stent type (all patients); potential clinical events, reported by investigators or patients (all event triggers); and further in-depth monitoring of all demographical, procedural, and clinical outcome data (at random, 10\% of patients). The independent clinical event committee was at all times blinded to the assigned treatment.

\section{Statistical analysis}

For both main comparisons, we did non-inferiority analyses ${ }^{26}$ for the primary endpoint at 12 months. The time to primary endpoint and associated components were assessed according to Kaplan-Meier methods; the log-rank test was applied for between-group comparisons. Assuming a proportion of target vessel failure of 8.5\%, based on the early 2012 available outcome data of the RESOLUTE All comers 27 and TWENTE trials ${ }^{28}$ and the assumed enrolment of substantially more patients with STelevation myocardial infarction, we estimated that 3540 patients would provide a power of $85 \%$ or higher to show non-inferiority with a margin of $3.5 \%$, with a one-sided $\alpha$ level of $2.5 \%$ and $3.0 \%$ loss to follow-up. We calculated the sample size with PASS software (version 11.0.8). Analyses were based on the intention-to-treat principle. For the primary endpoint, we also did a per-protocol analysis. Pearson's $\chi^{2}$ test or Fisher's exact test were used to compare categorical variables and the $t$ test was done to compare continuous variables. Hazard ratios (HRs) were computed with Cox proportional hazards regressions analysis. To account for intrapatient correlation (due to interlesion dependence), additional lesion-based analyses were done with the generalised estimating equations method. Logistic regression was used to test for interaction between subgroups and treatment with regard to the primary endpoint. $p$ values of less than 0.05 were considered significant. $p$ values and confidence intervals were two sided, except those for non-inferiority testing of the primary endpoint. Data analysts remained blinded to the assigned treatment until the evaluation of 12 month follow-up was finished. No interim analysis was done. SPSS (version 22) was used for the statistical analysis. This trial is registered with ClinicalTrials.gov, number NCT01674803.

\section{Role of the funding source}

The funder of the study had no role in study design, data collection, data analysis, data interpretation, or writing of the report. CVB, MMK, LCVdH, and MML had full access to all the data in the study and had final responsibility for the decision to submit for publication.

\section{Results}

From Dec 21, 2012, to Aug 24, 2015, 3514 patients with 4663 target lesions were randomly assigned and assessed at four clinical sites, representing $44 \%$ of all 7928 patients who underwent percutaneous coronary interventions with drug-eluting stent implantation during the sites' participation in the trial (irrespective of inclusion and exclusion criteria; we have no reliable data for the total number of eligible patients; Figure 1). Of these allcomers aged 32-93 years, most (2449 [70\%] of 3514) were treated for acute coronary syndromes (Table 1). The proportion of patients with ST elevation myocardial 
infarction was very high (1073 [31\%] of 3514). Of all coronary lesions treated, 3357 (72\%) of 4663 were complex (American College of Cardiology and American Heart Association lesion class B2 or C; Table 2). In almost all patients (3480 [99\%] of 3514), at least one assigned stent was implanted. Of the 3514 patients, $30(<1 \%)$ received only non-assigned stents and four $(<1 \%)$ received no stent (Figure 1). Deviation from the assigned stent did not differ significantly between treatment groups $(p=0.33)$. Assignment to treatment was balanced among the participating research centres $(p=0.94)$. Overall, direct stent implantation (ie, without predilation of the lesion) was done in 589 (17\%) of 3514 patients, and stent postdilation was done in 2833 (81\%) of 3514 patients (Table 1); no significant difference between stent arms. Postprocedural cardiac biomarkers were available in 3412 (97\%) of 3514 patients (no significant difference between stent arms). A total of 3490 (99\%) of 3514 patients completed the 12 month follow-up or had died. Very few patients (three $[<0.1 \%]$ of 3514 patients) were lost to follow-up, and $21(<1.0 \%)$ of 3514 patients withdrew consent during the trial (censored; outcome data were used until time of withdrawal).

The clinical outcome data are presented in Table 3. The primary composite endpoint of target vessel failure at 1 year was met by 55 (5\%) of 1172 patients assigned to everolimus-eluting stents, 55 (5\%) of 1169 patients assigned to sirolimus-eluting stents and 63 (5\%) of 1173 assigned to zotarolimus-eluting stents. Non-inferiority of the everolimus-eluting stent versus zotarolimus-eluting stent was confirmed with an absolute risk difference of $-0.7 \%(95 \% \mathrm{Cl}-2.4$ to 1.1$)$ and an upper limit of the one sided $95 \% \mathrm{Cl}$ of $0.8 \%$ (pnon-inferiority<0.0001; Figure $2 \mathrm{~A}$ ). Moreover, non-inferiority of the sirolimus-eluting stent versus zotarolimus-eluting stent was confirmed with an absolute risk difference of -0.7\% $(95 \% \mathrm{Cl}-2.4$ to 1.1) and an upper limit of the one sided $95 \% \mathrm{Cl}$ of $0.8 \%$ (pnon-inferiority<0.0001; Figure 2A). For these two main comparisons, the results for the primary endpoint were consistent across subgroups (appendix).

Figure 2 also shows the event rates of the individual components of the primary endpoint, which were low for all treatment groups. Various other adverse events and composite clinical endpoints are reported in table 3. Further lesion-based results are provided in the appendix. To account for the possibility that deviation from the assigned stent might have affected the primary outcome, we also performed a per-protocol analysis of the primary endpoint, which gave results similar to the intentionto-treat analyses (appendix).

3419 (97\%) of 3514 patients at discharge and 2939 (86\%) of 3432 patients at 1 year follow-up were on DAPT, without any difference in DAPT rate or use of more potent P2Y12 inhibitors among the treatment groups (appendix). Among the three treatment groups, definite stent thrombosis was an infrequent event that occurred in four $(0.3 \%)$ of 1172 , four $(0.3 \%)$ of 1169 , and three $(0.3 \%)$ of 1173 patients ( $p$-Log-rank $=0.70$ for both comparisons with zotarolimus-eluting stents). Moreover, the 1 year rate of definite or probable stent thrombosis was similar among the treatment groups ( $p$-Log-rank=0.77; Table 3, Figure 3). In patients treated with everolimus-eluting stents, there were two non-fatal, late, definite stent thromboses in patients who were on DAPT. In patients treated with sirolimus-eluting stents, there was one, non-fatal, late, definite stent thrombosis in a patient who was not on DAPT. In patients treated with zotarolimus-eluting stents, there was one non-fatal, late, definite stent thrombosis in a patient on DAPT; there was also one fatal, late, probable stent thrombosis in a patient on DAPT (Figure 3). Further information on circumstances and clinical consequences of patients who developed a stent thrombosis is provided in the appendix. 


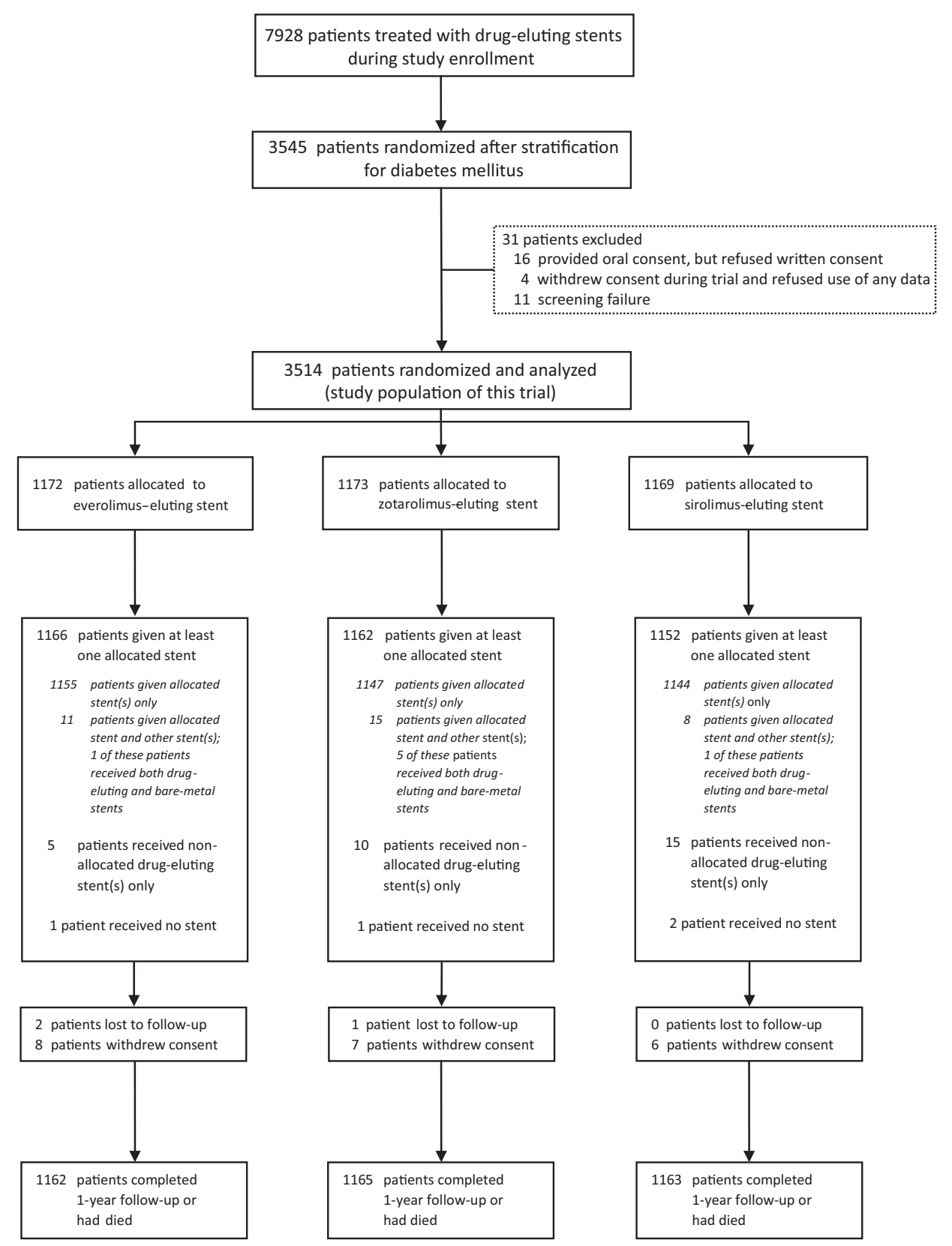

Figure 1: Trial profile. *Information on the number of patients treated with drug-eluting stents during the period of study enrolment is given irrespective of whether these patients fulfilled the inclusion and exclusion criteria, as we do not have reliable data on the total number of eligible patients. 
Table 1: Characteristics of patients and procedures.

\begin{tabular}{|c|c|c|c|c|}
\hline & $\begin{array}{c}\text { All } \\
\text { patients }\end{array}$ & $\begin{array}{l}\text { Everolimus- } \\
\text { eluting stent }\end{array}$ & $\begin{array}{l}\text { Zotarolimus- } \\
\text { eluting stent }\end{array}$ & $\begin{array}{l}\text { Sirolimus- } \\
\text { eluting stent }\end{array}$ \\
\hline Patients & $n=3514$ & $\mathrm{n}=\mathbf{1 1 7 2}$ & $n=1173$ & $n=1169$ \\
\hline Age - yrs & $63 \cdot 9 \pm 10 \cdot 8$ & $64 \cdot 0 \pm 10 \cdot 7$ & $63 \cdot 6 \pm 10 \cdot 9$ & $64 \cdot 2 \pm 10 \cdot 7$ \\
\hline Men & $2547(72 \cdot 5)$ & $845(72 \cdot 1)$ & $848(72 \cdot 3)$ & $854(73 \cdot 1)$ \\
\hline Body-mass index* & $27 \cdot 4 \pm 4 \cdot 2$ & $27 \cdot 6 \pm 4 \cdot 2$ & $27 \cdot 3 \pm 4 \cdot 0$ & $27 \cdot 4 \pm 4 \cdot 2$ \\
\hline Current smoker & $1031 / 3422(30 \cdot 1)$ & $336 / 1135(29 \cdot 6)$ & $354 / 1143(31 \cdot 0)$ & $341 / 1144(29 \cdot 8)$ \\
\hline Family history of coronary artery disease & $1557 / 3372(46 \cdot 2)$ & $512 / 1114(46 \cdot 0)$ & $529 / 1138(46 \cdot 5)$ & $516 / 1120(46 \cdot 1)$ \\
\hline \multicolumn{5}{|l|}{ Medical history } \\
\hline Diabetes mellitus, medically treated & $624(17 \cdot 8)$ & $203(17 \cdot 3)$ & $210(17 \cdot 9)$ & $211(18 \cdot 0)$ \\
\hline Hypertension & $1624(46 \cdot 2)$ & $520(44 \cdot 4)$ & $554(47 \cdot 2)$ & $550(47 \cdot 0)$ \\
\hline Hypercholesterolemia & $1335(38 \cdot 0)$ & $422(36 \cdot 0)$ & $450(38.4)$ & $463(39 \cdot 6)$ \\
\hline Previous myocardial infarction & $649(18 \cdot 5)$ & $192(16 \cdot 4)$ & $248(21 \cdot 1)$ & $209(17 \cdot 9)$ \\
\hline Previous stroke & $231(6 \cdot 6)$ & $74(6 \cdot 3)$ & $81(6 \cdot 9)$ & $76(6 \cdot 5)$ \\
\hline Renal insufficiency ${ }^{\dagger}$ & $108(3 \cdot 1)$ & $29(2 \cdot 5)$ & $33(2 \cdot 8)$ & $46(3.9)$ \\
\hline $\begin{array}{l}\text { Previous percutaneous coronary } \\
\text { intervention }\end{array}$ & $626(17 \cdot 8)$ & $214(18 \cdot 3)$ & $198(16 \cdot 9)$ & $214(18 \cdot 3)$ \\
\hline Previous coronary artery bypass grafting & $267(7 \cdot 6)$ & $91(7 \cdot 8)$ & $96(8 \cdot 2)$ & $80(6 \cdot 8)$ \\
\hline \multicolumn{5}{|l|}{ Clinical presentation } \\
\hline ST-elevation myocardial infarction & $1073(30 \cdot 5)$ & $377(32 \cdot 2)$ & $326(27 \cdot 8)$ & $370(31 \cdot 7)$ \\
\hline Non-ST-elevation myocardial infarction & $756(21 \cdot 5)$ & $247(21 \cdot 1)$ & $270(23 \cdot 0)$ & $239(20 \cdot 4)$ \\
\hline Unstable angina & $620(17 \cdot 6)$ & $192(16 \cdot 4)$ & $219(18 \cdot 7)$ & $209(17 \cdot 9)$ \\
\hline Stable angina & $1065(30 \cdot 3)$ & $356(30 \cdot 4)$ & $358(30 \cdot 5)$ & $351(30 \cdot 0)$ \\
\hline \multicolumn{5}{|l|}{ Lesion characteristics $^{\ddagger}$} \\
\hline At least one complex lesion & $2783(79 \cdot 2)$ & $903(77 \cdot 0)$ & $938(80 \cdot 0)$ & $942(80 \cdot 6)$ \\
\hline At least one bifurcation lesion & $1236(35 \cdot 2)$ & $415(35 \cdot 4)$ & $409(34 \cdot 9)$ & $412(35 \cdot 2)$ \\
\hline At least one chronic total occlusion & $139(4 \cdot 0)$ & $44(3 \cdot 8)$ & $48(4 \cdot 1)$ & $47(4 \cdot 0)$ \\
\hline At least one bypass graft lesion & $70(2 \cdot 0)$ & $18(1 \cdot 5)$ & $30(2 \cdot 6)$ & $22(1 \cdot 9)$ \\
\hline At least one ostial lesion & $252(7 \cdot 2)$ & $97(8 \cdot 3)$ & $81(6.9)$ & $74(6 \cdot 3)$ \\
\hline At least one severely calcified lesion & $783(22 \cdot 3)$ & $252(21 \cdot 5)$ & $265(22 \cdot 6)$ & $266(22 \cdot 8)$ \\
\hline \multicolumn{5}{|l|}{ Procedural characteristics } \\
\hline Implantation of assigned stents only & $3446(98 \cdot 1)$ & $1155(98 \cdot 5)$ & $1147(97 \cdot 8)$ & $1144(97 \cdot 9)$ \\
\hline Total stent length per patient (mm) & $31(20-50)$ & $32(20-48)$ & $30(22-52)$ & $30(18-49)$ \\
\hline Direct stenting & $589(16 \cdot 8)$ & $208(17 \cdot 7)$ & $174(14 \cdot 8)$ & $207(17 \cdot 7)$ \\
\hline Postdilation & $2833(80 \cdot 6)$ & $960(81 \cdot 9)$ & $927(79 \cdot 0)$ & $946(80 \cdot 9)$ \\
\hline Multivessel treatment & $640(18 \cdot 2)$ & $201(17 \cdot 2)$ & $220(18 \cdot 8)$ & $219(18 \cdot 7)$ \\
\hline Radial approach & $1597(45 \cdot 4)$ & $523(44 \cdot 6)$ & $544(46 \cdot 4)$ & $530(45 \cdot 3)$ \\
\hline Fractional Flow Reserve use & $440(12 \cdot 5)$ & $147(12 \cdot 5)$ & $155(13 \cdot 2)$ & $138(11 \cdot 8)$ \\
\hline
\end{tabular}

Data are $\mathrm{n}(\%)$, means (SD), or median (IQR). * Renal insufficiency was defined as an estimated glomerular filtration rate of less than $30 \mathrm{~mL}$ per min per $1.73 \mathrm{~m}^{2}$ of body-surface area or the need for dialysis. +Definitions of lesion characteristics are provided in the appendix. Lesion-based analysis corrected for intrapatient correlation with generalised estimating equations are available in the appendix. 


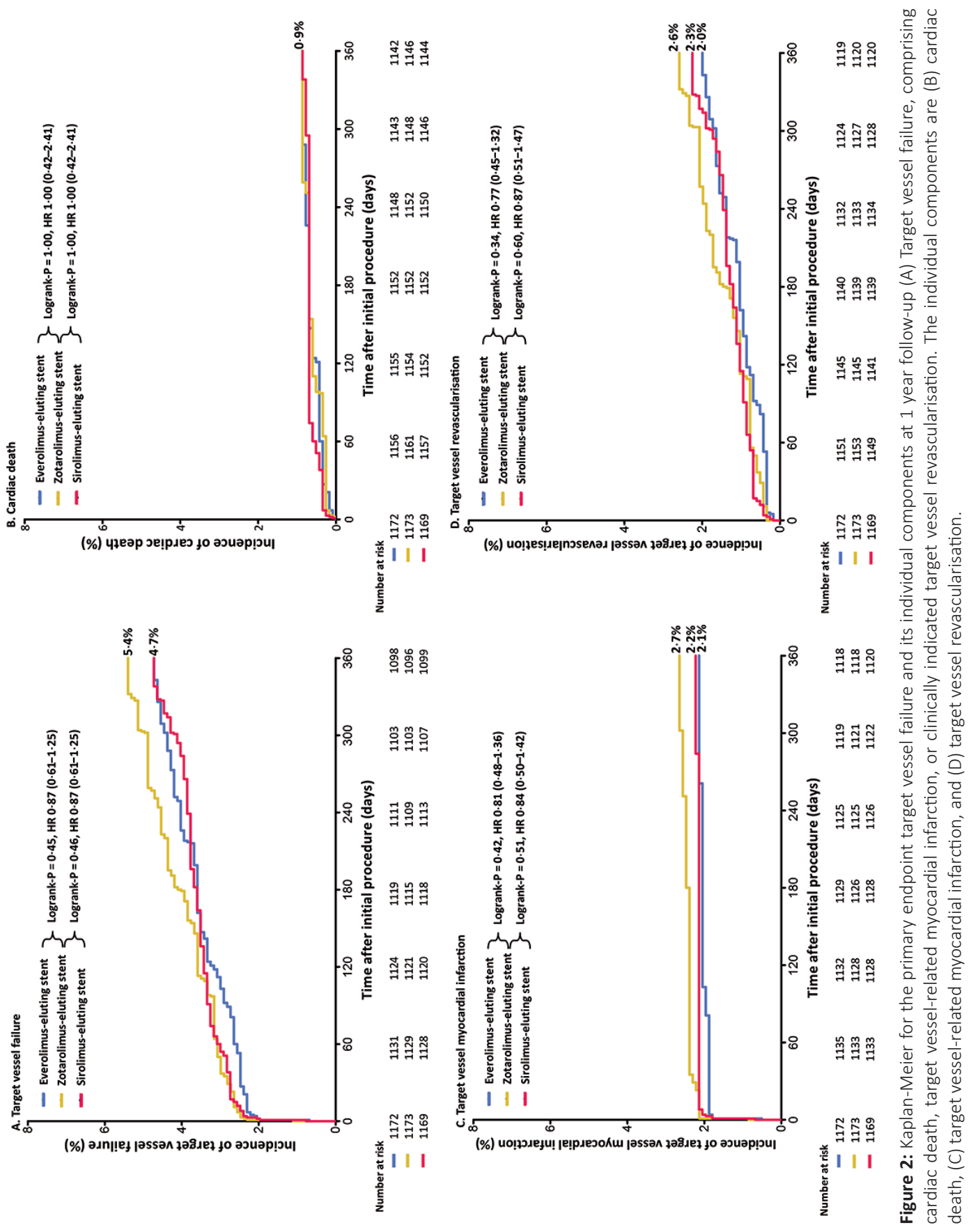


Table 2: Characteristics of target lesions.

\begin{tabular}{|c|c|c|c|c|}
\hline & All patients & $\begin{array}{c}\text { Everolimus-eluting } \\
\text { stent }\end{array}$ & $\begin{array}{c}\text { Zotarolimus-eluting } \\
\text { stent }\end{array}$ & $\begin{array}{c}\text { Sirolimus-eluting } \\
\text { stent }\end{array}$ \\
\hline Target lesions* & $n=4663$ & $n=1532$ & $n=1580$ & $n=1551$ \\
\hline Left main stem & $76(1.6)$ & $25(1 \cdot 6)$ & $28(1 \cdot 8)$ & $23(1.5)$ \\
\hline $\begin{array}{l}\text { Left anterior descending } \\
\text { artery }\end{array}$ & $1883(40 \cdot 4)$ & $616(40 \cdot 2)$ & $588(37 \cdot 2)$ & $679(43 \cdot 8)$ \\
\hline Left circumflex artery & $1091(23 \cdot 4)$ & $358(23 \cdot 4)$ & $395(25 \cdot 0)$ & $338(21 \cdot 8)$ \\
\hline Right coronary artery & $1530(32 \cdot 8)$ & $510(33 \cdot 3)$ & $535(33 \cdot 9)$ & $485(31 \cdot 3)$ \\
\hline Bypass graft & $86(1 \cdot 8)$ & $23(1.5)$ & $34(2 \cdot 2)$ & $29(1.9)$ \\
\hline ACC/AHA lesion class & $4645 / 4663$ & $1527 / 1532$ & $1573 / 1580$ & $1545 / 1551$ \\
\hline A & $225(4 \cdot 8)$ & $82(5 \cdot 4)$ & $68(4 \cdot 3)$ & $75(4 \cdot 9)$ \\
\hline B1 & $1063(22 \cdot 9)$ & $361(23 \cdot 6)$ & $370(23 \cdot 5)$ & $332(21 \cdot 5)$ \\
\hline B2 & $1826(39 \cdot 3)$ & $578(37.9)$ & $624(39 \cdot 7)$ & $624(40 \cdot 4)$ \\
\hline C & $1531(33 \cdot 0)$ & $506(33 \cdot 1)$ & $511(32 \cdot 5)$ & $514(33 \cdot 3)$ \\
\hline Chronic total occlusion & $151(3 \cdot 2)$ & $50(3 \cdot 3)$ & $49(3 \cdot 1)$ & $52(3.4)$ \\
\hline In-stent restenosis & $93(2 \cdot 0)$ & $30(2 \cdot 0)$ & $33(2 \cdot 1)$ & $30(1.9)$ \\
\hline Bifurcated lesion & $1327(28 \cdot 5)$ & $446(29 \cdot 1)$ & $438(27 \cdot 7)$ & $443(28.6)$ \\
\hline Severely calcified lesion & $940(20 \cdot 2)$ & $296(19 \cdot 3)$ & $327(20 \cdot 7)$ & $317(20 \cdot 4)$ \\
\hline \multicolumn{5}{|l|}{ Preprocedural † } \\
\hline $\begin{array}{l}\text { Lesion length }-\mathrm{mm} \\
\text { (range) }\end{array}$ & $14 \cdot 67(10 \cdot 53-22 \cdot 02)$ & $14.59(10 \cdot 34-21 \cdot 95)$ & $14.74(10.65-21.92)$ & $14 \cdot 63(10 \cdot 59-22 \cdot 30)$ \\
\hline $\begin{array}{l}\text { Minimum lumen } \\
\text { diameter (mm) }\end{array}$ & $0.71(0.38-1.02)$ & $0.71(0.36-1.02)$ & $0.70(0.42-1.02)$ & $0.71(0.37-1.01)$ \\
\hline $\begin{array}{l}\text { Reference vessel } \\
\text { diameter ( } \mathrm{mm})\end{array}$ & $2 \cdot 76 \pm 0.57$ & $2 \cdot 76 \pm 0 \cdot 56$ & $2 \cdot 76 \pm 0.59$ & $2 \cdot 75 \pm 0.56$ \\
\hline $\begin{array}{l}\text { Lumen diameter } \\
\text { stenosis (\%) }\end{array}$ & $73 \cdot 0(62 \cdot 4-85 \cdot 3)$ & $73 \cdot 8(62 \cdot 7-86 \cdot 3)$ & $72 \cdot 5(62 \cdot 5-84 \cdot 1)$ & $72 \cdot 8(62 \cdot 2-86 \cdot 3)$ \\
\hline \multicolumn{5}{|l|}{ Post-procedural * } \\
\hline $\begin{array}{l}\text { Minimum lumen } \\
\text { diameter (mm) }\end{array}$ & $2 \cdot 20(1 \cdot 83-2 \cdot 59)$ & $2 \cdot 23(1.84-2 \cdot 60)$ & $2 \cdot 20(1 \cdot 84-2 \cdot 59)$ & $2 \cdot 18(1.83-2 \cdot 57)$ \\
\hline $\begin{array}{l}\text { Lumen diameter } \\
\text { stenosis (\%) }\end{array}$ & $17 \cdot 3(13 \cdot 3-22 \cdot 9)$ & $17 \cdot 4(13 \cdot 4-22 \cdot 9)$ & $17 \cdot 3(13 \cdot 3-23 \cdot 0)$ & $17 \cdot 4(13 \cdot 3-22 \cdot 9)$ \\
\hline $\begin{array}{l}\text { Acute lumen gain in } \\
\text { segment }(\mathrm{mm})\end{array}$ & $1.47(1.03-1.96)$ & $1.50(1.06-1.99)$ & $1.45(1.04-1.95)$ & $1.46(1.01-1.95)$ \\
\hline
\end{tabular}

Data are $\mathrm{n}(\%)$, mean (SD), or median (IQR), unless otherwise stated. Lesion-based analysis corrected for intrapatient correlation with generalised estimating equations are available in the appendix. Abbreviations: ACC=American College of Cardiology. AHA=American Heart Association. * Data for 1527 lesions in the everolimus-eluting stent group, 1573 lesions in the zotarolimuseluting stent group, and 1545 lesions in the sirolimus-eluting stent group. †Data for 1526 lesions in the everolimus-eluting stent group, 1574 lesions in the zotarolimus-eluting stent group, and 1547 lesions in the sirolimus-eluting stent group. 


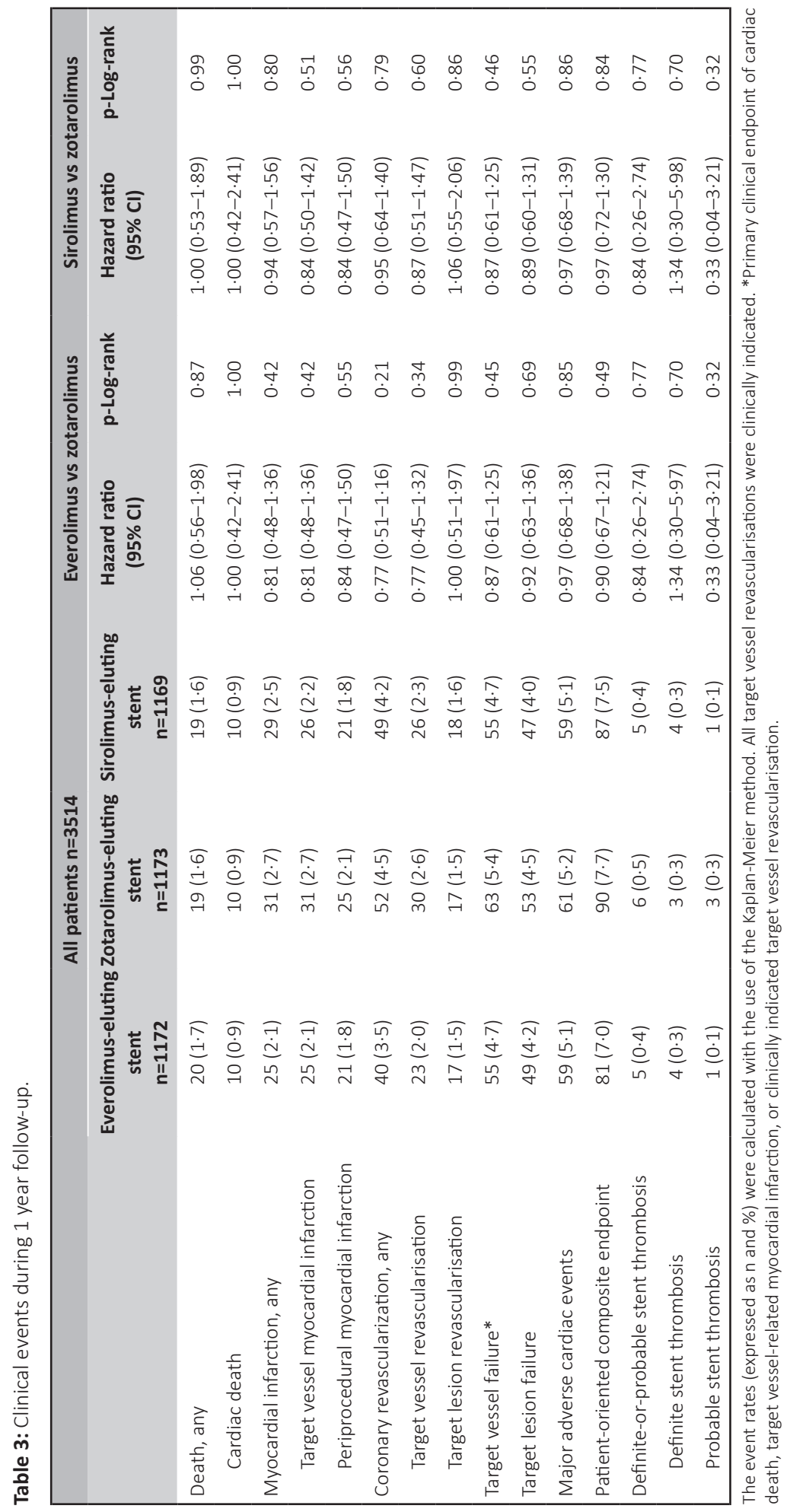




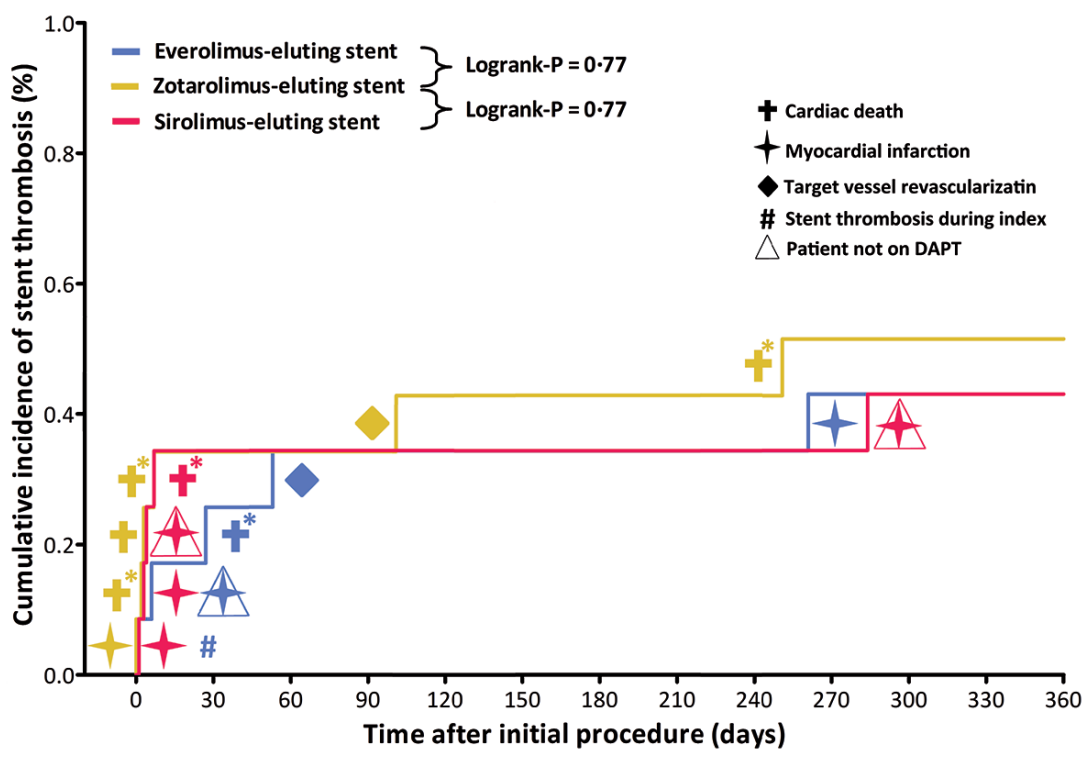

Figure 3: Incidence of definite or probable stent thrombosis up to 1 year. Symbols indicate the hierarchically highest adverse events associated with stent thromboses. Probable stent thromboses had five fatal events; all other events represent definite stent thromboses (one fatal event). DAPT consisted of aspirin $80 \mathrm{mg}$ or more daily and an adequate dose of a P2Y12 receptor antagonist. DAPT=dual antiplatelet therapy.

\section{Discussion}

To our knowledge, BIO-RESORT is the first randomised trial to compare the very thin strut biodegradable polymer everolimus-eluting or sirolimus-eluting stents with the durable polymer zotarolimus-eluting stent, to assess the everolimus-eluting stent in allcomers; and to assess more than one novel very thin strut biodegradable polymer stent. Between stent groups, there was no difference in the 12 month incidence of the composite primary endpoint. As a result, both the everolimus-eluting stent and the sirolimus-eluting stent met the criterion of non-inferiority compared with the zotarolimus-eluting stent. Moreover, in all three treatment groups the rates of individual components of the primary endpoint were relatively low.

The study population represents $44 \%$ of all potentially eligible patients (irrespective of inclusion and exclusion criteria). Additionally, 70\% of all 3514 allcomers were treated for acute coronary syndromes, and the enrolment rate of $31 \%$ patients with acute ST elevation myocardial infarction was much higher than in previous stent trials in allcomers. ${ }^{6,8,11,12,21-23,27,28}$ The fact that BIO-RESORT has such very high percentages of patients with these disorders suggests that the present study population might be a true representation of the full range of patients treated in routine clinical practice. Almost all patients (99\%) received the assigned stent. Moreover, postprocedural cardiac biomarkers were available in $97 \%$ of all patients, and the 1 year follow-up rate of $99 \%$ was very high. Considering the complex study population in BIO-RESORT, which included many patients with increased clinical, lesion-related, or procedural risk, 
the event rates were remarkably low and represent at 1 year an excellent safety signal for the stents assessed.

It has been suggested that the absence of a permanent polymer in the coronary artery might reduce vascular inflammation, which has been connected to delayed arterial healing, incomplete endothelial strut coverage, neoatherosclerosis, and potentially fatal complications such as (very) late stent thrombosis and myocardial infarction. ${ }^{3,29}$ So far, some clinical trials reported encouraging outcome data for biodegradable polymer stents. ${ }^{3,7,10}$ But comprehensive clinical proof is still pending to show that the theoretical advantages of biodegradable polymer stents translate into long-term clinical outcomes that are significantly better than with new-generation, durable polymer stents. ${ }^{13,30}$ Nevertheless, a substantial proportion of operators support the concept of biodegradable polymer stents and use them in routine clinical practice. However, evidence from clinical and experimental research has shown that specific durable polymer coatings might lower the risk of stent thrombosis as compared with biodegradable polymer coatings, ${ }^{13,31}$ but these durable polymer stents might still develop in-stent neoatherosclerosis and stent thrombosis. ${ }^{13,32}$

The potential benefits (or disadvantages) of biodegradable polymer stents might be noted later than after a year. ${ }^{7-9}$ But before consideration of potential long-term benefits of biodegradable polymer stents, the short-term usefulness of these devices should be determined - preferably in a complex allcomers population. BIO-RESORT shows that the 1 year efficacy and safety of the two novel biodegradable polymer stents are non-inferior to that of an established new-generation, durable polymer stent. The mid-term and long-term clinical outcome of these patients will also be of great interest.

The clinical results of the everolimus-eluting stent group in our present all-comer trial represent an important addition to scientific knowledge about this device. Previously, the EVOLVE ${ }^{18}$ randomised trial assessed 1684 patients with up to moderate complexity, treated with Synergy biodegradable polymer versus durable polymer everolimus-eluting platinum chromium stents, and reported noninferiority of the biodegradable polymer stent.

The outcome of the sirolimus-eluting Orsiro stent group of our trial corroborates the results of two previous multicentre trials that compared this device with an everolimus-eluting, durable polymer stent in 2119 allcomers of the BIOSCIENCE trial ${ }^{21}$ and versus a biolimus-eluting, early biodegradable polymer stent in 2525 allcomers from the SORT OUT VII trial. ${ }^{22}$ Both trials reported non-inferiority of the sirolimus-eluting stent compared with the respective comparator stent. ${ }^{21,22}$

The durable fluoropolymer coating of newer-generation everolimus-eluting stents has been shown to reduce the risk of thrombus formation as compared with bare metal stents, ${ }^{15}$ which corroborates clinical data that showed a particularly low risk of stent thrombosis in durable fluoropolymer stents. ${ }^{33}$ Resolute-type durable polymer (ie, blend of three durable polymers) zotarolimus-eluting stents, as used in the present trial, were previously shown to also have a highly favourable safety profile with relatively low stent thrombosis rates. ${ }^{30}$ Recent data suggested that biodegradable polymer stents, of which many have a polymer coating on the abluminal surface only, might be less thromboresistant than fluoropolymer-coated stents. ${ }^{31}$ Nevertheless, in the present study, both biodegradable polymer stents showed excellent clinical outcomes and low stent thrombosis rates. This finding might be related to their low strut thickness, because the overall surface that requires re-endothelialisation is much smaller in thin strut stents. Additionally, preclinical research has shown that thin struts reduced both thrombus 
formation and intimal proliferation. ${ }^{15}$ Consequently, the use of thinner struts might lower the risk of potentially fatal complications, such as stent thrombosis and myocardial infarction, as well as the risk of lesion recurrence. ${ }^{15}$

The struts of the novel biodegradable polymer stents are substantially thinner 60-81 $\mu \mathrm{m}$ ) than those of the early biodegradable polymer stents $(120 \mu \mathrm{m})$. We use the term very thin strut stents to characterise the novel devices and to emphasise this difference.

The minimum stent strut thickness required to ensure sufficient radial force to prevent elastic recoil of the dilated vessel depends on the stent material and design. The two novel biodegradable polymer stents use platinum chromium and cobalt chromium alloys, which permit the construction of stents with very thin struts and flexible designs. ${ }^{4}$ Such stent designs reduce the thrombogenicity by lowering the incidence of strut malapposition, a well-known cause of coronary flow disturbance and thrombus formation. ${ }^{15}$ However, very thin struts might also have disadvantages. Theoretically, the inferior radiographical visibility of thin stent struts, which is more marked in cobalt chromium stents than in platinum chromium stents, ${ }^{17,18}$ might increase the risk of geometrical miss and could have resulted in higher event rates. Nevertheless, our present study shows for both novel devices that there is no loss of 1 year efficacy and safety. Longer-term follow-up of the present and future studies will be required to assess potential long-term benefits of these devices. In the BIO-RESORT trial, the substantial differences in type, amount, distribution, and resorption speed of the polymer coating between the everolimus-eluting and sirolimus-eluting stent did not result in a significant between-stent difference in 1 year clinical outcome. Theoretically, the rapid polymer resorption in the everolimus-eluting stent might have justified a shorter DAPT in this treatment arm. Nevertheless, most patients in all three stent groups remained on DAPT for 12 months, and the use of the more potent P2Y12 inhibitors ticagrelor or prasugel did not differ between treatment groups. Shortening DAPT after drug-eluting stent implantation might be most advantageous in patients with increased bleeding risk. Yet, in the present trial, almost $70 \%$ of patients were treated for acute coronary syndromes, where shortening DAPT is generally not considered.

The present study has some limitations. When designing the BIO-RESORT trial in early 2012, the expected incidence of the primary endpoint was based on results of previous studies done in highvolume centres. ${ }^{27,28}$ At that time, no data were available about the clinical outcome after treatment of allcomers with the specific type of thin-strut durable polymer zotarolimus-eluting stent used in the present trial; these data were later reported by the DUTCH PEERS trial23 and the SORT OUT VI trial. ${ }^{12}$ We assumed that BIO-RESORT would enrol a complex allcomers population with a substantially higher proportion of patients with ST-elevation myocardial infarction than enrolled in the RESOLUTE All Comers tria ${ }^{27}$ and that this increase in patient complexity might result in a slightly higher event rate. As a result, we assumed a target vessel failure rate of $8.5 \%$ was reasonable. Moreover, the choice of a noninferiority margin of $3.5 \%$ was in line with previous stent trials in allcomers that used non-inferiority margins of $3.5-4.0 \%{ }^{6,11,23,27}$

While BIO-RESORT succeeded in enrolling $31 \%$ of patients with ST-elevation myocardial infarction, the actual event proportion of the primary endpoint target vessel failure was lower than expected. As in other randomised trials, under-reporting of adverse events cannot be completely excluded. Nevertheless, considering the systematic postprocedural assessment of biomarkers and 
electrocardiograph, high follow-up rates, and independent monitoring and event adjudication, underreporting might not have been substantial. Other randomised stent trials also have found lower than expected event rates; ${ }^{11,18,21-23,34}$ which suggests that the event rates of the present study are actually more representative of the outcome of present percutaneous coronary interventions, as opposed to when this trial was designed. Besides the improvement in stent systems, balloon catheters, and other equipment to facilitate interventional procedures, other aspects might also have contributed to the low event rates: wider use of the more potent P2Y12 antagonists (in $48 \%$ of patients receiving DAPT) as compared with our previous trials; ; $^{23,28}$ more frequent use of the radial access route (in $45 \%$ in this trial); and wider implementation of fractional flow reserve measurements ( $13 \%$ of patients in this trial) to identify clinically relevant target lesions and defer haemodynamically non-significant lesions. Moreover, in patients with acute coronary syndromes (70\% in BIO-RESORT) the probability of observing periprocedural myocardial infarction is reduced, which might have contributed to the low rates of target vessel myocardial infarction and various composite endpoints, including the primary endpoint.

Although the present trial is a large-scale study, it is not powered to reliably assess very rare adverse clinical events, for instance, stent thrombosis. However, stent thrombosis is an event that is too important to be ignored, in particular as one of the two novel stents was never before assessed in an allcomers trial. These data are no more than hypothesis generating. Future studies might assess the potential of novel biodegradable polymer stents to be treated with short-term DAPT.

\section{Conclusion}

In conclusion, the two very thin strut drug-eluting stents with highly dissimilar biodegradable polymer coatings were non-inferior to the durable polymer stent in treating a complex allcomers population with a high proportion of patients with acute coronary syndromes. The absence of a loss of 1 year safety and efficacy with the use of these biodegradable polymer-coated stents is a prerequisite before assessing their potential longer-term benefits.

\section{Research in context}

\section{Evidence before this study}

We searched PubMed and checked listings of the EuroPCR, European Society of Cardiology, Transcatheter Cardiovascular Therapeutics, and American College of Cardiology conferences for complete reports of randomised trials comparing the biodegradable polymer everolimus-eluting Synergy or sirolimuseluting Orsiro stents with the zotarolimus-eluting Resolute Integrity stent or with other stents (we used as search terms "coronary" and "stent" in combination with one or more of the following: "everolimus", "sirolimus", "zotarolimus", "Synergy", "Orsiro", "Resolute Integrity", "randomised", and "randomized", for reports published in English from Sept 15, 2011, to Sept 15, 2016.

Previously, the Synergy stent has been assessed in the EVOLVE first-in-man study and the EVOLVE II trial, which reported non-inferiority of Synergy as compared with a durable polymer everolimus- 
eluting stent in treating patients with up to moderate risk. The Orsiro stent showed non-inferiority compared with a durable polymer everolimus-eluting stent with respect to a primary angiographical endpoint in BIOFLOW-II, and versus durable polymer everolimus-eluting stents (BIOSCIENCE) and early biodegradable polymer biolimus-eluting stents (SORT OUT VII) in treating all-comers. Yet, no randomised clinical trial has ever compared the Synergy or Orsiro stents with the Resolute Integrity stent. Additionally, the Synergy stent has not yet been assessed in all-comers. Moreover, Synergy and Orsiro stents have never been assessed together in one randomised clinical trial.

\section{Added value of this study}

Our analysis shows that treatment with two very thin strut biodegradable polymer stents and the durable polymer zotarolimus-eluting stent was similarly efficacious and safe with excellent 1 year clinical outcomes in a complex population of all-comers. To our knowledge, BIO-RESORT is the first randomised trial to assess the everolimus-eluting platinum chromium stent in all-comers. Moreover, this trial is also the first randomised comparison of two biodegradable, very thin strut, sirolimus-eluting cobalt chromium and everolimus-eluting platinum chromium stents versus thin strut durable polymer zotarolimus-eluting stent.

\section{Implications of all the available evidence}

At 12 month follow-up, both very thin strut drug-eluting stents with dissimilar biodegradable polymer coatings were non-inferior to the durable polymer stent in treating all-comers with a high proportion of patients with acute coronary syndromes. 


\section{References}

1. Stefanini GG, Holmes DR Jr. Drug-eluting coronary-artery stents. N Engl J Med 2013; 368: 254-65.

2. Kastrati A, Mehilli J, Pache J, et al. Analysis of 14 trials comparing sirolimus-eluting stents with are-metal stents. N Engl J Med 2007; 356: 1030-39.

3. Joner M, Finn AV, Farb A, et al. Pathology of drug-eluting stents in humans: delayed healing and late thrombotic risk. J Am Coll Cardiol 2006; 48: 193-202.

4. Stefanini GG, Taniwaki M, Windecker S. Coronary stents: novel developments. Heart 2014; 100: 1051-61.

5. Räber L, Kelbæk H, Ostojic M, et al. Effect of biolimus-eluting stents with biodegradable polymer vs bare-metal stents on cardiovascular events among patients with acute myocardial infarction: the COMFORTABLE AMI randomized trial. JAMA 2012; 308: 777-87.

6. Windecker S, Serruys PW, Wandel S, et al. Biolimus-eluting stent with biodegradable polymer versus sirolimuseluting stent with durable polymer for coronary revascularisation (LEADERS): a randomised non-inferiority trial. Lancet 2008; 372: 1163-73.

7. Serruys PW, Farooq V, Kalesan B, et al. Improved safety and reduction in stent thrombosis associated with biodegradable polymer-based biolimus-eluting stents versus durable polymer-based sirolimus-eluting stents in patients with coronary artery disease: final 5-year report of the LEADERS (limus eluted from a durable versus erodable stent coating) randomized, noninferiority trial. JACC Cardiovasc Interv 2013; 6: 777-89.

8. Christiansen EH, Jensen LO, Thayssen P, et al. Biolimus-eluting biodegradable polymer-coated stent versus durable polymer-coated sirolimus-eluting stent in unselected patients receiving percutaneous coronary intervention (SORT OUT V): a randomised non-inferiority trial. Lancet 2013; 381: 661-69.

9. Byrne RA, Kastrati A, Massberg S, et al; ISAR-TEST 4 Investigators. Biodegradable polymer versus permanent polymer drug-eluting stents and everolimus- versus sirolimus-eluting stents in patients with coronary artery disease: 3-year outcomes from a randomized clinical trial. J Am Coll Cardiol 2011; 58: 1325-31.

10. Stefanini GG, Byrne RA, Serruys PW, et al. Biodegradable polymer drug-eluting stents reduce the risk of stent thrombosis at 4 years in patients undergoing percutaneous coronary intervention: a pooled analysis of individual patient data from the ISAR-TEST 3, ISAR-TEST 4, and LEADERS randomized trials. Eur Heart J 2012; 33: 1214-22.

11. Smits PC, Hofma S, Togni M, et al. Abluminal biodegradable polymer biolimus-eluting stent versus durable polymer everolimus-eluting stent (COMPARE II): a randomised, controlled, non-inferiority trial. Lancet 2013; 381: 651-60.

12. Raungaard B, Jensen LO, Tilsted $\mathrm{HH}$, et al. Zotarolimus-eluting durable-polymer-coated stent versus a biolimuseluting biodegradable-polymer-coated stent in unselected patients undergoing percutaneous coronary intervention (SORT OUT VI): a randomised non-inferiority trial. Lancet 2015; 385: 1527-35.

13. Palmerini T, Biondi-Zoccai G, Della Riva D, et al. Clinical outcomes with bioabsorbable polymer- versus durable polymer-based drug-eluting and bare-metal stents: evidence from a comprehensive network meta-analysis. J Am Coll Cardiol 2014; 63: 299-307.

14. Kaiser C, Galatius S, Jeger R, et al. Long-term efficacy and safety of biodegradable-polymer biolimus-eluting stents: main results of the Basel Stent Kosten-Effektivitäts Trial-PROspective Validation Examination II (BASKETPROVE II), a randomized, controlled noninferiority 2-year outcome trial. Circulation 2015; 131: 74-81.

15. Kolandaivelu K, Swaminathan R, Gibson WJ, et al. Stent thrombogenicity early in high-risk interventional settings is driven by stent design and deployment and protected by polymer-drug coatings. Circulation 2011; 123: 140009.

16. Lam MK, Sen H, Tandjung K, et al. Comparison of 3 biodegradable polymer and durable polymer-based drugeluting stents in all-comers (BIO-RESORT): rationale and study design of the randomized TWENTE III multicenter trial. Am Heart J 2014; 167: 445-51.

17. Meredith IT, Verheye S, Dubois CL, et al. Primary endpoint results of the EVOLVE trial: a randomized evaluation of a novel bioabsorbable polymer-coated, everolimus-eluting coronary stent. J Am Coll Cardiol 2012; 59: 1362-70.

18. Kereiakes DJ, Meredith IT, Windecker S, et al. Efficacy and safety of a novel bioabsorbable polymer-coated, everolimus-eluting coronary stent: the EVOLVE II randomized trial. Circ Cardiovasc Interv 2015; 8: e002372.

19. Hamon M, Niculescu R, Deleanu D, Dorobantu M, Weissman NJ, Waksman R. Clinical and angiographic experience with a third-generation drug-eluting Orsiro stent in the treatment of single de novo coronary artery lesions (BIOFLOW-I): a prospective, first-in-man study. Eurolntervention 2013; 8: 1006-11. 
20. Windecker S, Haude M, Neumann FJ, et al. Comparison of a novel biodegradable polymer sirolimus-eluting stent with a durable polymer everolimus-eluting stent: results of the randomized BIOFLOW-II trial. Circ Cardiovasc Interv 2015; 8: e001441.

21. Pilgrim $T$, Heg $D$, Roffi $M$, et al. Ultrathin strut biodegradable polymer sirolimus-eluting stent versus durable polymer everolimus-eluting stent for percutaneous coronary revascularisation (BIOSCIENCE): a randomised, single-blind, non-inferiority trial. Lancet 2014; 384: 2111-22.

22. Jensen LO, Thayssen $\mathrm{P}$, Maeng $\mathrm{M}$, et al. Randomized comparison of a biodegradable polymer ultrathin strut sirolimus-eluting stent with a biodegradable polymer biolimus-eluting stent in patients treated with percutaneous coronary intervention: the SORT OUT VII trial. Circ Cardiovasc Interv 2016; 9: e003610.

23. von Birgelen $\mathrm{C}$, Sen $\mathrm{H}$, Lam MK, et al. Third-generation zotarolimus-eluting and everolimus-eluting stents in all-comer patients requiring a percutaneous coronary intervention (DUTCH PEERS): a randomised, single-blind, multicentre, non-inferiority trial. Lancet 2014; 383: 413-23.

24. Cutlip DE, Windecker S, Mehran R, et al. Academic Research Consortium. Clinical end points in coronary stent trials: a case for standardized definitions. Circulation 2007; 115: 2344-51.

25. Vranckx P, Cutlip DE, Mehran R, et al. Myocardial infarction adjudication in contemporary all-comer stent trials: balancing sensitivity and specificity. Addendum to the historical $\mathrm{Ml}$ definitions used in stent studies. Eurolntervention 2010; 5: 871-74.

26. D’Agostino RB Sr, Massaro JM, Sullivan LM. Non-inferiority trials: design concepts and issues - the encounters of academic consultants in statistics. Stat Med 2003; 22: 169-86.

27. Serruys PW, Silber S, Garg S, et al. Comparison of zotarolimus-eluting and everolimus-eluting coronary stents. N Engl J Med 2010; 363: 136-46.

28. von Birgelen C, Basalus MW, Tandjung K, et al. A randomized controlled trial in second-generation zotarolimuseluting Resolute stents versus everolimus-eluting Xience $V$ stents in real-world patients: the TWENTE trial. J Am Coll Cardiol 2012; 59: 1350-61.

29. Basalus MW, Joner M, von Birgelen C, Byrne RA. Polymer coatings on drug-eluting stents: Samson's hair and Achilles' heel? Eurolntervention 2013; 9: 302-05.

30. Navarese EP, Tandjung K, Claessen B, et al. Safety and efficacy outcomes of first and second generation durable polymer drug eluting stents and biodegradable polymer biolimus eluting stents in clinical practice: comprehensive network meta-analysis. BMJ 2013; 347: f6530.

31. Otsuka F, Cheng Q, Yahagi K, et al. Acute thrombogenicity of a durable polymer everolimus-eluting stent relative to contemporary drug-eluting stents with biodegradable polymer coatings assessed ex vivo in a swine shunt model. JACC Cardiovasc Interv 2015; 8: 1248-60.

32. Otsuka F, Vorpahl M, Nakano M, et al. Pathology of second-generation everolimus-eluting stents versus firstgeneration sirolimus- and paclitaxel-eluting stents in humans. Circulation 2014; 129: 211-23.

33. Palmerini T, Biondi-Zoccai G, Della Riva D, et al. Stent thrombosis with drug-eluting and bare-metal stents: evidence from a comprehensive network meta-analysis. Lancet 2012; 379: 1393-402.

34. Saito S, Valdes-Chavarri M, Richardt G, et al. A randomized, prospective, intercontinental evaluation of a bioresorbable polymer sirolimus-eluting coronary stent system: the CENTURY II (Clinical Evaluation of New Terumo Drug-Eluting Coronary Stent System in the Treatment of Patients with Coronary Artery Disease) trial. Eur Heart J 2014; 35: 2021-31. 
Webbased Table 1: Extended baseline and procedure-related data.

\begin{tabular}{|c|c|c|c|c|}
\hline & All patients & $\begin{array}{l}\text { Everolimus- } \\
\text { eluting stent }\end{array}$ & $\begin{array}{l}\text { Zotarolimus- } \\
\text { eluting stent }\end{array}$ & $\begin{array}{l}\text { Sirolimus- } \\
\text { eluting stent }\end{array}$ \\
\hline Patients & $n=3514$ & $\mathrm{n}=1172$ & $\mathrm{n}=1173$ & $n=1169$ \\
\hline \multicolumn{5}{|l|}{ Medical history } \\
\hline Peripheral artery disease & $285(8 \cdot 1)$ & $98(8.4)$ & $95(8 \cdot 1)$ & $92(7 \cdot 9)$ \\
\hline Left ventricular ejection fraction $<30 \%$ & $50(1 \cdot 4)$ & $15(1 \cdot 3)$ & $18(1 \cdot 5)$ & $17(1 \cdot 5)$ \\
\hline Heart failure & $82(2 \cdot 3)$ & $26(2 \cdot 2)$ & $28(2 \cdot 4)$ & $28(2 \cdot 4)$ \\
\hline Previous gastro-intestinal bleeding & $54(1 \cdot 5)$ & $21(1 \cdot 8)$ & $17(1 \cdot 4)$ & $16(1 \cdot 4)$ \\
\hline \multicolumn{5}{|l|}{ Lesion characteristics } \\
\hline At least one instent restenosis & $89(2 \cdot 5)$ & $28(2 \cdot 4)$ & $31(2 \cdot 6)$ & $30(2 \cdot 6)$ \\
\hline At least one small vessel $(<2.75 \mathrm{~mm})$ & $2078(59 \cdot 1)$ & $680(58.0)$ & $667(56 \cdot 9)$ & $731(62 \cdot 5)$ \\
\hline At least one lesion length $>27 \mathrm{~mm}$ & $1073(30 \cdot 5)$ & $353(30 \cdot 1)$ & $369(31 \cdot 5)$ & $351(30 \cdot 0)$ \\
\hline \multicolumn{5}{|l|}{ Number of lesions treated per patient } \\
\hline One & $2580(73 \cdot 4)$ & $877(74 \cdot 8)$ & $850(72 \cdot 5)$ & $853(73 \cdot 0)$ \\
\hline Two & $746(21 \cdot 2)$ & $237(20 \cdot 2)$ & $254(21 \cdot 7)$ & $255(21 \cdot 8)$ \\
\hline Three or more & $188(5 \cdot 4)$ & $58(4 \cdot 9)$ & $69(5 \cdot 9)$ & $61(5 \cdot 2)$ \\
\hline Left main & $76(2 \cdot 2)$ & $25(2 \cdot 1)$ & $28(2 \cdot 4)$ & $23(2 \cdot 0)$ \\
\hline Left anterior descending artery & $1719(48.9)$ & $562(48 \cdot 0)$ & $543(46 \cdot 3)$ & $614(52 \cdot 5)$ \\
\hline Left circumflex artery & $1004(28 \cdot 6)$ & $331(28 \cdot 2)$ & $353(30 \cdot 1)$ & $320(27 \cdot 4)$ \\
\hline Right coronary artery & $1337(38.0)$ & $452(38.6)$ & $462(39 \cdot 4)$ & $423(36 \cdot 2)$ \\
\hline Bypass graft & $70(2 \cdot 0)$ & $18(1 \cdot 5)$ & $30(2 \cdot 6)$ & $22(1.9)$ \\
\hline Thrombus present & $817(23 \cdot 2)$ & $284(24 \cdot 2)$ & $249(21 \cdot 2)$ & $284(24 \cdot 3)$ \\
\hline \multicolumn{5}{|l|}{ Procedural characteristics } \\
\hline Thrombus aspiration & $656(18 \cdot 7)$ & $224(19 \cdot 1)$ & $202(17 \cdot 2)$ & $230(19 \cdot 7)$ \\
\hline Rotablation & $51(1 \cdot 5)$ & $15(1 \cdot 3)$ & $18(1 \cdot 5)$ & $18(1 \cdot 5)$ \\
\hline Guiding catheter extension & $267(7 \cdot 6)$ & $78(6 \cdot 7)$ & $106(9 \cdot 0)$ & $83(7 \cdot 1)$ \\
\hline Cutting balloon & $65(1 \cdot 8)$ & $21(1 \cdot 8)$ & $18(1 \cdot 5)$ & $26(2 \cdot 2)$ \\
\hline Target lesions* & $n=4663$ & $n=1532$ & $n=1580$ & $\mathrm{n}=1551$ \\
\hline Complex lesion & $3357(72 \cdot 0)$ & $1084(70 \cdot 8)$ & $1135(71 \cdot 8)$ & $1138(73 \cdot 4)$ \\
\hline Ostial lesion & $260(5 \cdot 6)$ & $100(6 \cdot 5)$ & $86(5 \cdot 4)$ & $74(4 \cdot 8)$ \\
\hline Implantation of assigned stents only & $4583(98 \cdot 3)$ & $1513(98 \cdot 8)$ & $1547(97 \cdot 9)$ & $1523(98 \cdot 2)$ \\
\hline Number of stents per lesion & $1.34 \pm 0.66$ & $1 \cdot 34 \pm 0.67$ & $1 \cdot 35 \pm 0 \cdot 66$ & $1 \cdot 34 \pm 0 \cdot 67$ \\
\hline Postdilation & $3495(75 \cdot 0)$ & $1190(77 \cdot 7)$ & $1158(73 \cdot 3)$ & $1147(74 \cdot 0)$ \\
\hline Device success ${ }^{\dagger}$ & $4553(98.0)$ & $1503(98 \cdot 5)$ & $1535(97 \cdot 5)$ & $1515(97 \cdot 9)$ \\
\hline Lesion success ${ }^{\dagger}$ & $4631(99 \cdot 7)$ & $1522(99 \cdot 7)$ & $1567(99 \cdot 6)$ & $1542(99 \cdot 7)$ \\
\hline
\end{tabular}

Plus-minus values are means \pm SD. There was no significant between-group difference in details of the interventional procedures or the success of treatment except for postdilation $(\mathrm{p}=0.0100) .{ }^{*}$ Lesion-based analysis corrected for intra-patient correlation with generalised estimating equations are available in Table W2. Definitions of lesions characteristics are provided on p5. +Data for 1526 lesions in the everolimus-eluting stent group, 1574 lesions in the zotarolimus-eluting stent group, and 1547 lesions in the sirolimus-eluting stent group. 


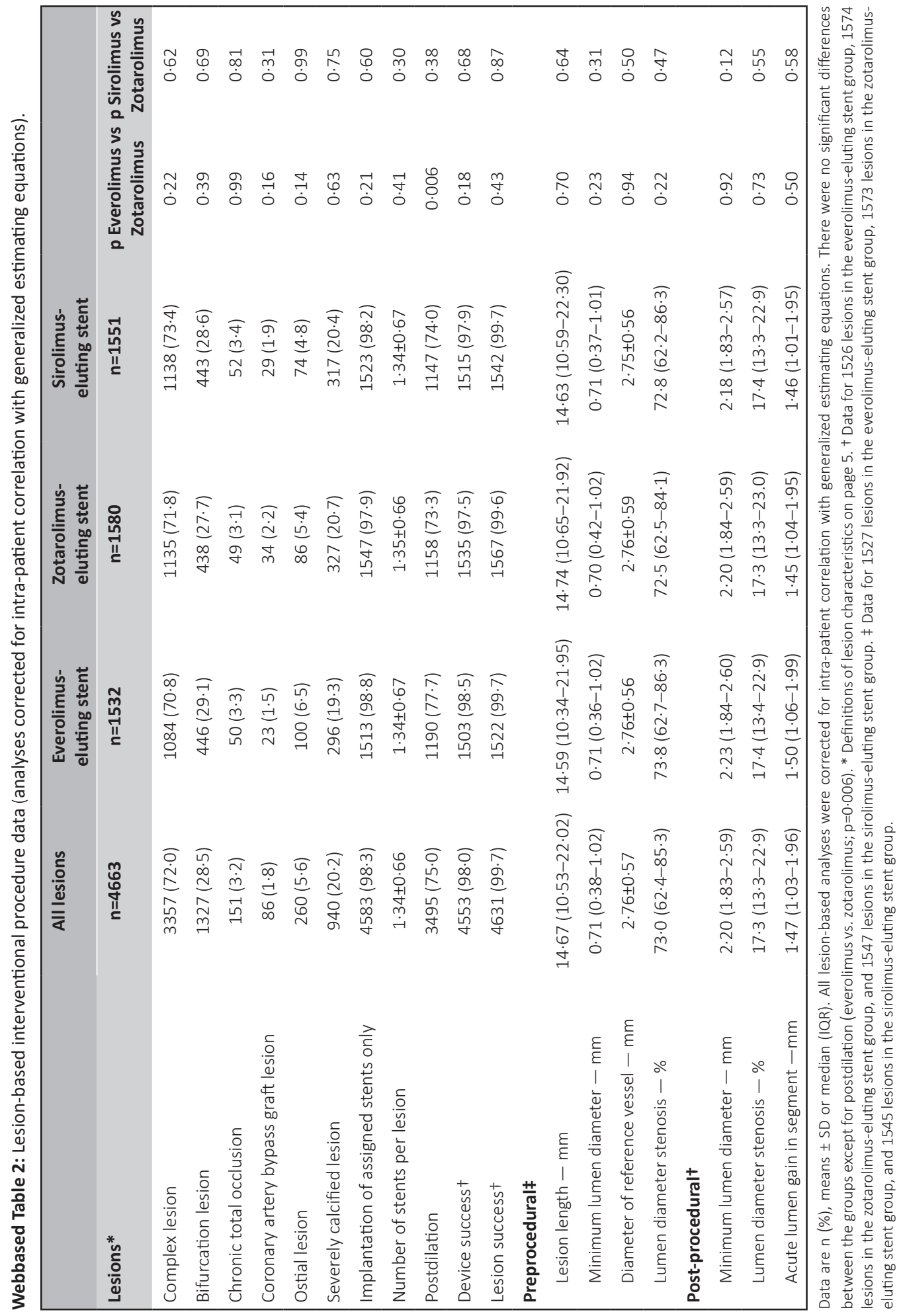




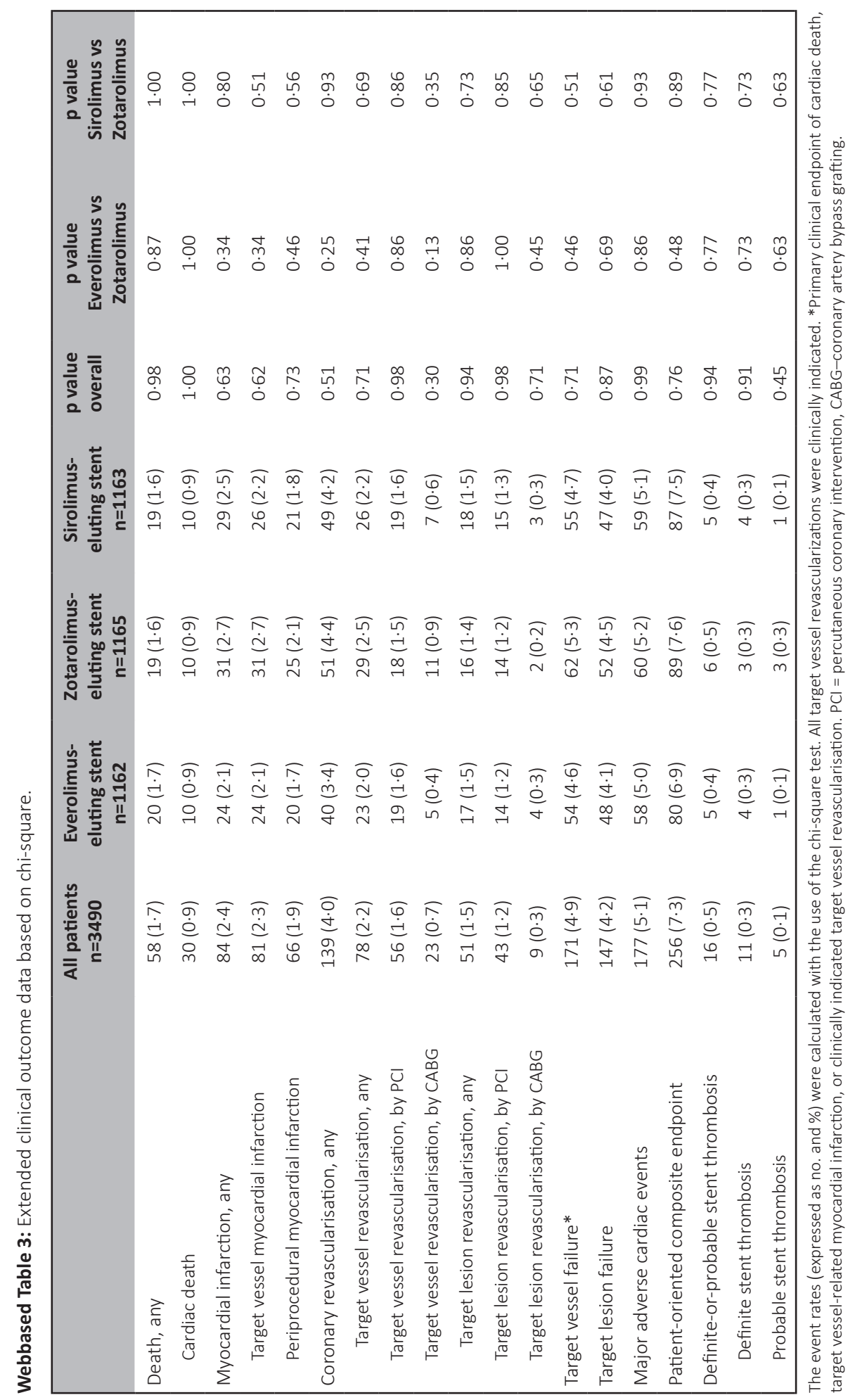




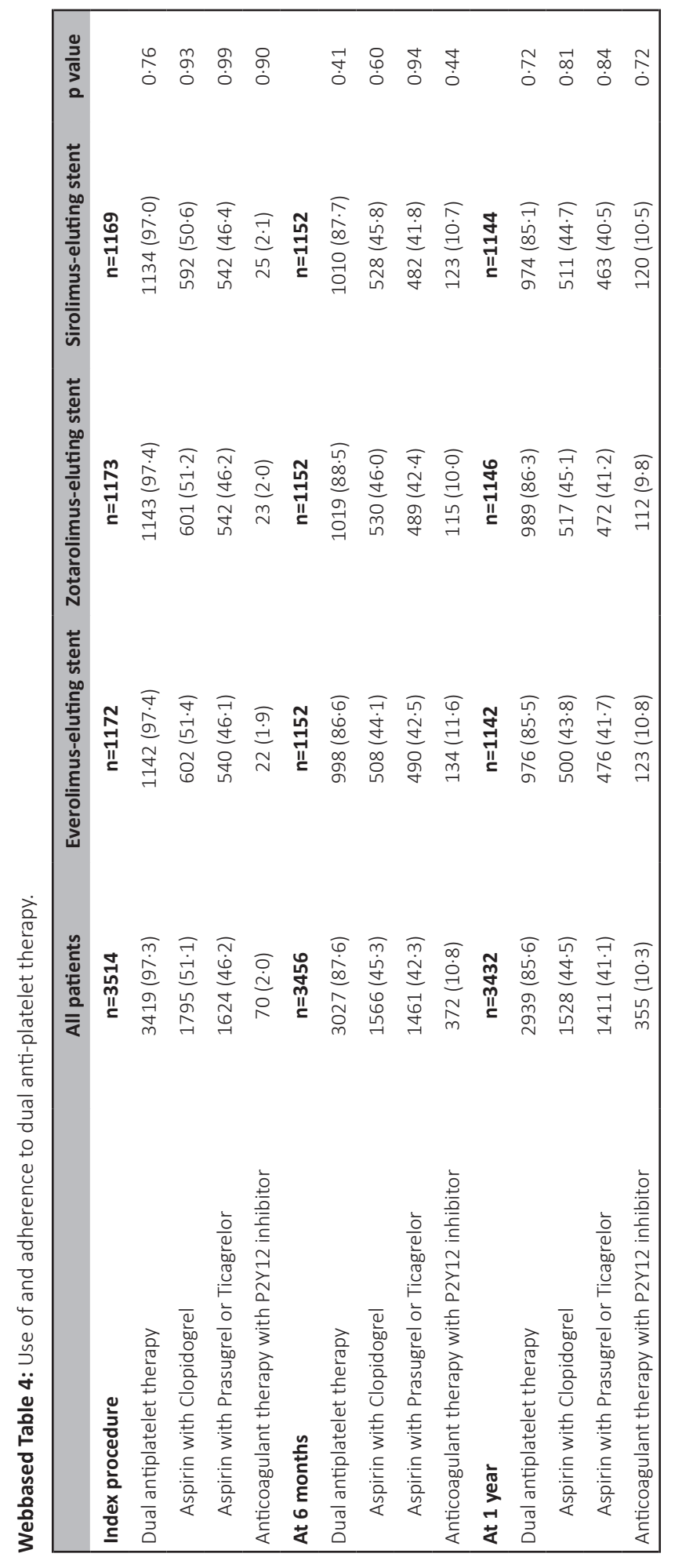




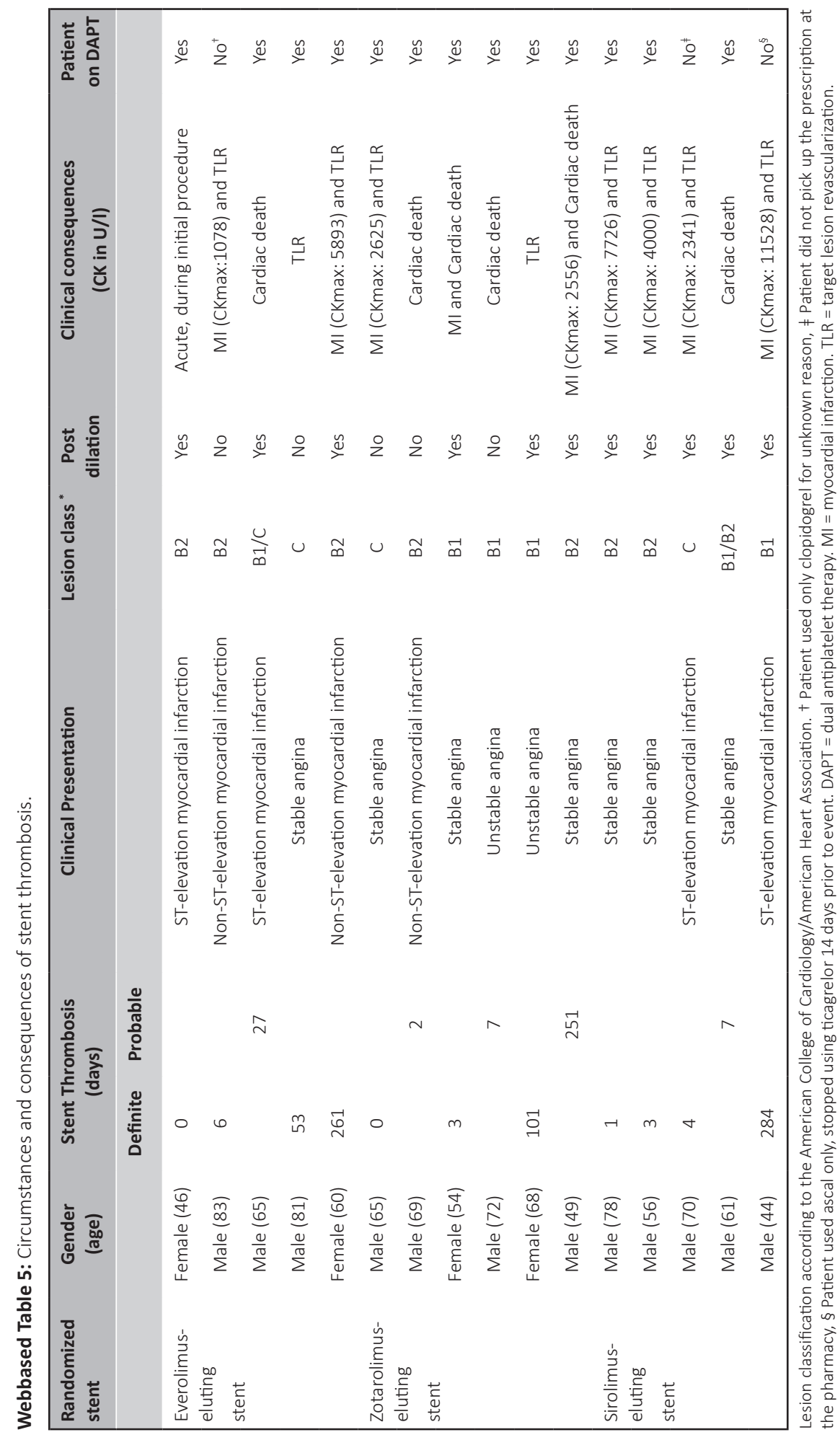




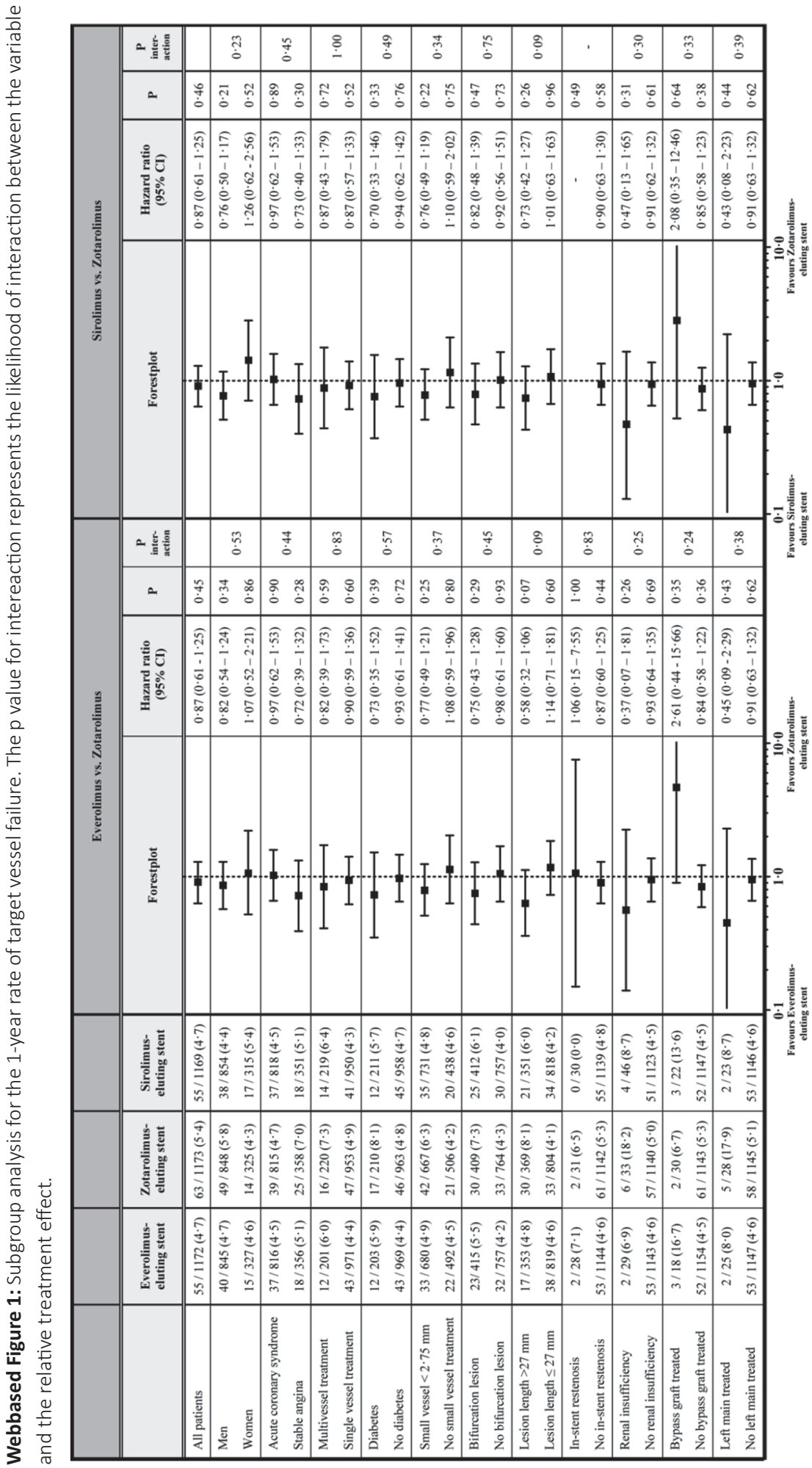





\section{Conflicts of interest statement}

Dr. von Birgelen has received moderate lecture fees from AstraZeneca and Biotronik, and his institution has received significant research grants from AstraZeneca, Biotronik, Boston Scientific, and Medtronic. The other authors reported no conflict of interest.

\section{Copyright}

Reprinted with permission from

International Journal of Cardiology 2016 Oct 15;221:1087-94

(c) Elsevier 


\section{CHAPTER 7}

\section{Safety and Efficacy of Everolimus-Eluting Bioresorbable Vascular Scaffolds Versus Durable Polymer Everolimus-Eluting Metallic Stents Assessed at 1-Year Follow-Up}

A Systematic Review and Meta-Analysis of Studies

Bertrand N. Mukete*, Liefke C. van der Heijden*, Kenneth Tandjung, Hassan Baydoun, Kapil Yadav, Qusai A. Saleh, Carine J.M. Doggen, Nidal Abi Rafeh, Thierry H. Le Jemtel, Clemens von Birgelen

\footnotetext{
* Both authors contributed equally to this manuscript.
}

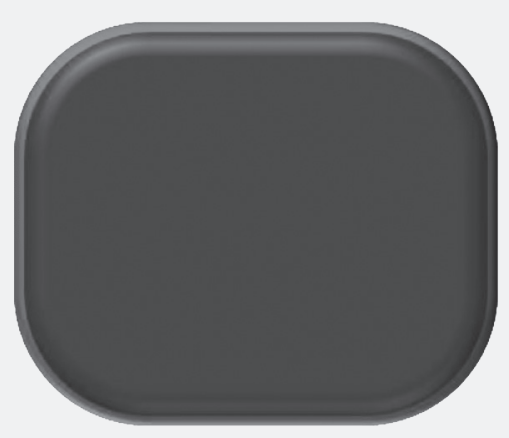




\section{Abstract}

Background: The Absorb bioresorbable vascular scaffold (BVS) was developed to address long-term safety issues of metallic drug-eluting stents. However, it may be associated with an increased event risk during the first year.

Methods: A systematic literature search was performed (in MEDLINE/PubMed, Cochrane CENTRAL, EMBASE, and scientific meeting abstracts) to identify studies that compared BVS and cobalt-chromium durable polymer everolimus-eluting stents (EES). For randomized clinical trials and non-randomized propensity score matched studies that reported 1-year outcome data, fixed/random-effects models were used to generate pooled estimates of outcomes, presented as odds ratios (OR) with 95\%-confidence intervals $(\mathrm{Cl})$.

Results: The 1-year follow-up data of 6 trials with 5,588 patients were analyzed. A device-oriented composite endpoint (DOCE-cardiac death, target vessel myocardial infarction (MI), or target lesion revascularization (TLR)) was reached by 308 BVS or EES patients $(195 / 3,253$ vs 113/2,315). Metaanalysis showed that patients who received BVS had an increased risk of MI ( $4.3 \%$ vs $2.3 \%$, OR $1.63,95 \%$ $\mathrm{Cl}: 1.18-2.25, \mathrm{p}<0.01)$ and definite-or-probable scaffold thrombosis (1.3\% vs $0.6 \%, \mathrm{OR} 2.10,95 \% \mathrm{Cl}$ : $1.13-3.87, p=0.02)$. However, there was no significant between-group difference in risk of DOCE $(6.0 \%$ vs $4.9 \%$, OR 1.19, 95\% Cl: 0.94-1.52, $\mathrm{p}=0.16$ ), cardiac death (0.8\% vs $0.7 \%$, OR 1.14, 95\% Cl: 0.54-2.39, $\mathrm{p}=0.73$ ), or TLR (2.5\% vs $2.5 \%$, OR 0.98, 95\% Cl: 0.69-1.40, $\mathrm{p}=0.92)$.

Conclusions: During the first year of follow-up, patients treated with BVS had a higher incidence of MI and scaffold thrombosis. The risk of DOCE was not significantly different. As BVS may pay off later, future robust data on long-term clinical outcome will be of paramount importance. 


\section{Introduction}

Advancement in coronary device technology aims at reducing and ultimately eliminating late and very late stent thrombosis (ST), continued neointimal tissue growth with formation of neo-atherosclerosis, and long-term caging of the coronary vessel-issues that are still relevant to the latest generation of durable polymer drug-eluting stents (DES). ${ }^{1}$ The durable polymer coating of DES may account for impaired arterial healing and incomplete endothelial covering of the metallic struts with an increased risk of late ST.

The latest technical development is the bioresorbable vascular scaffold (BVS) that aims at providing a finite period of vascular support after stent implantation. ${ }^{2,3}$ The potential advantages of BVS include the preservation of vessel geometry, adaptive vascular remodeling, and restoration of physiologic vasomotion, which offer the prospect of late luminal expansion. 2,4,5 The Absorb BVS (Abbott Vascular, Santa Clara, CA), a device that elutes the same drug as the widely used cobalt chromium everolimuseluting stent $(E E S)^{6}$, is the first BVS that received the Conformite European (CE) mark of approval for clinical use. In registries, the BVS showed clinical event rates that were overall comparable to durable polymer DES. ${ }^{7-11}$ Nevertheless, safety and efficacy of the BVS has not yet been fully established, and there are concerns for a greater risk of scaffold thrombosis before the human body has resorbed the device. $^{12}$

Recently multiple randomized clinical trials evaluating the Absorb BVS were published; however, by themselves they do not have sufficient power to detect differences in important but relative infrequent safety endpoints, such as cardiac death, myocardial infarction, and stent thrombosis. Therefore, in order to gain more insight into the safety and efficacy of the BVS versus EES during the first year from implantation, we performed a meta-analysis of studies that compared both devices and reported 1-year clinical outcome data.

\section{Methods}

\section{Search strategy and selection criteria}

A systematic search of randomized controlled clinical trials (and non-randomized) comparing Absorb BVS versus new-generation durable polymer cobalt-chromium EES using Medline, the Cochrane Central Register of Controlled Trials (CENTRAL), Embase, scientific sessions abstracts, and relevant websites (www.heart.org, www.cardiosource.com, www.tctmd.com, www.clinicaltrialresults.org, www.escardio. org) was performed according to the PRISMA guidelines. ${ }^{13}$ Independent major search term used included "bioresorbable vascular scaffold" and "Absorb stents". Minor search terms used in combination with the major terms included "everolimus-eluting stents", "clinical trials, and "randomized trials" was performed to retrieve peer reviewed published articles and presentations between January 2006 and October 2015. 


\section{Inclusion and exclusion criteria}

We included studies that had a randomized or a propensity score matching design, and compared the Absorb BVS versus durable polymer cobalt-chromium EES. We included only studies that reported clinical outcome data at 12-month follow-up. Included studies provided numeric data on clinical endpoint of interest: target vessel myocardial infarction (MI); target lesion revascularization (TLR); cardiac death; definite-or-probable scaffold/stent thrombosis (ST). The ST criteria was based on the definition of the Academic Research Consortium (ARC). ${ }^{14}$

We excluded studies if they met any of the following criteria: duplicate publication; outcomes of interest not clearly reported or impossible to extract or calculate from the published results; follow-up duration <12-months; single-arm studies; and studies that compared BVS to a nonpermanent polymer DES (i.e., bioabsorbable or bioresorbable polymer DES). Figure 1 depicts a flow diagram for the selection of trials included in this review, using the PRISMA guidelines. ${ }^{13}$

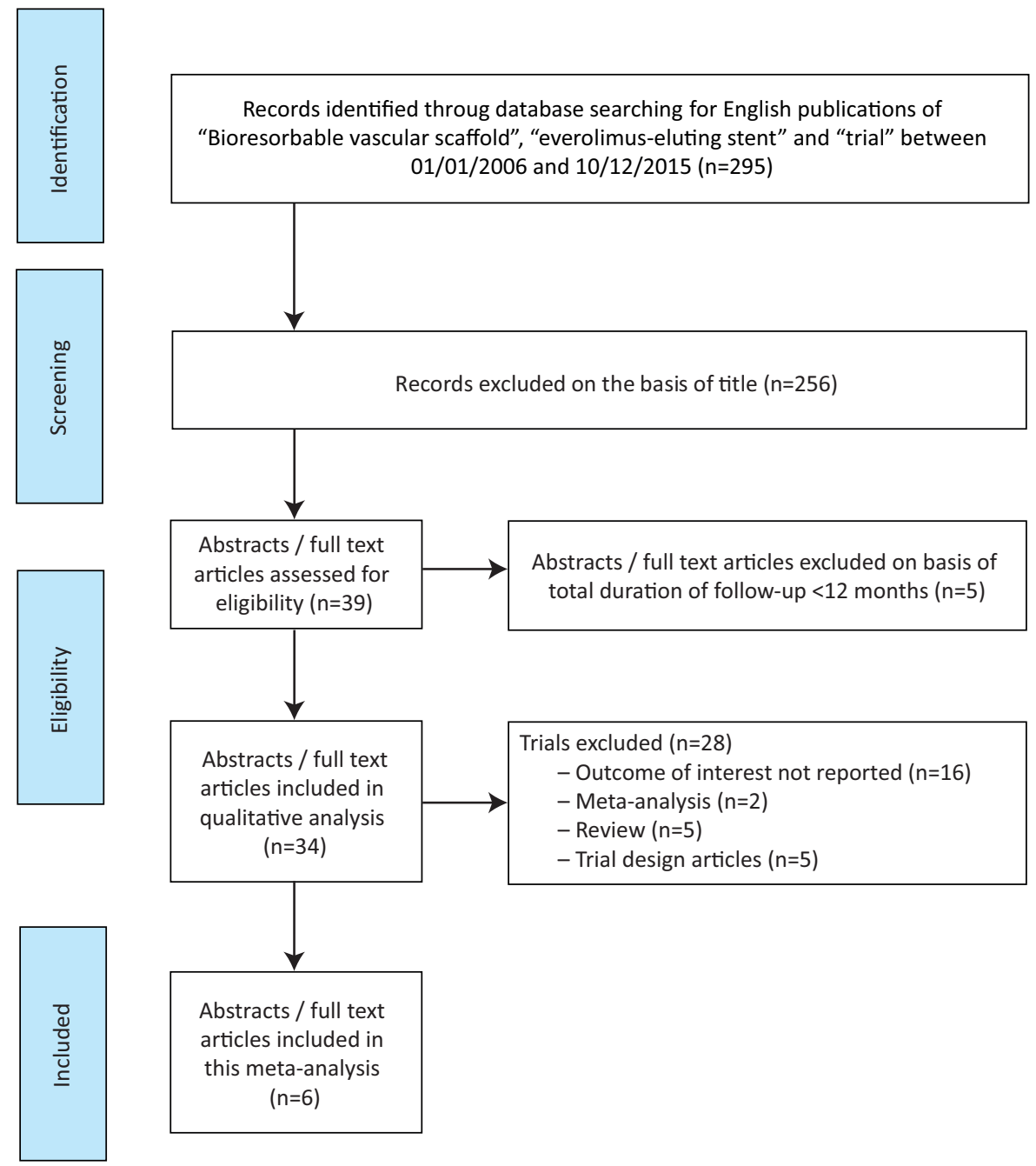

Figure 1: Flow diagram of literature search and study selection. 


\section{Inclusion and exclusion criteria of selected studies}

In general, patients aged 18 years and older, presenting with stable angina, unstable angina or silent ischemia, who underwent percutaneous coronary intervention for one or two de novo native coronary artery lesions in separate epicardial coronary vessels, were eligible for enrollment in the ABSORB II, ABSORB Japan, ABSORB III, ABSORB China, and ABSORB Extend trial. ${ }^{15-19}$ Patients with acute myocardial infarction were excluded in all ABSORB trials. In addition, left ventricular ejection fraction $<30 \%, \mathrm{PCl}$ of the target vessel during the last 12 months, and patients with a high bleeding risk were excluded. Main angiographic exclusion criteria were left main or ostial location of the lesion, excessive vessel tortuosity, heavy calcification proximal to or within the target lesion, and bifurcation lesions with side branch $\geq 2.0 \mathrm{~mm}$ requiring guidewire or dilation. ${ }^{15-19}$ The BVS-EXAMINATION Study is an observational study, in which all consecutive STEMI patients treated with BVS were enrolled. ${ }^{20}$

\section{Data extraction and assessment of bias}

All publications were independently assessed for eligibility at the title or abstract level by 3 independent investigators (BNM, LCVdH, KT). We collected information about the study design, clinical and procedural characteristics, and clinical and safety outcomes. The Cochrane Collaboration's tool was used to assess the risk of bias based on the quality of each eligible trial. ${ }^{21}$ In addition, we visually assessed for publication bias with a funnel plot (Figure 2) while the Begg and Mazumdar test ${ }^{22}$ was used to quantify the amount of publication bias.

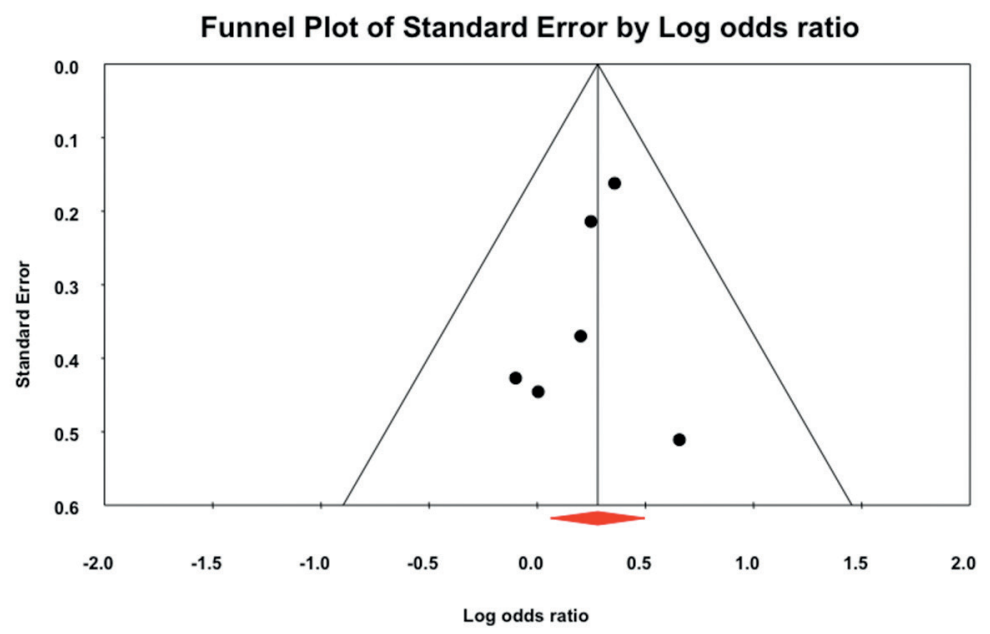

Figure 2: Funnel plot of standard error by log odds ratio. A total of 6 studies was analyzed for effect size. Abbreviations: BVS=bioresorbable vascular scaffold; EES=everolimus-eluting stent.

\section{Study endpoints}

We assessed a device-oriented composite endpoint (DOCE-cardiac death, target vessel myocardial infarction, or target lesion revascularization), reflecting efficacy and safety. Individual endpoints of efficacy were TVR and TLR. Individual safety endpoints were cardiac death, target vessel MI, and definite-or-probable ST, as defined by the ARC. ${ }^{14}$ 


\section{Statistical analysis}

For each clinical and combined clinical endpoint, the independent odds ratio (OR) and weighted mean difference was calculated with the $95 \%$ confidence interval $(\mathrm{CI})$. A summary OR was then derived for the comparison of BVS with EES. The summary effect size was determined using a fixed/random effect model based on the absence/presence of heterogeneity respectively. Heterogeneity was assessed using the $I^{2}$ statistic. ${ }^{23}$ We assumed heterogeneity among the studies when the degree of inconsistency (using $\mathrm{I}^{2}$ statistics) was $>50 \%$ with an associated $p$-value $\leq 0.05$. We used the Mantel-Haenszel ${ }^{24}$ fixedeffect model and DerSimonian and Liard ${ }^{25}$ random-effect model to calculate the summary effect size based on the absence or presence of heterogeneity among studies. A chi-square statistic was used to determine difference between baseline clinical and angiographic characteristics and Unpaired twotailed student T-test was used to calculate difference in mean of the procedural characteristics between the two groups. We used the Comprehensive Meta-Analysis (CMA) version 2.0 program for outcomes statistical analysis. ${ }^{26}$

\section{Results}

A total of 6 studies met the inclusion criteria with a sample size of 5,588 patients $(3,263$ in the BVS arm versus 2,325 in the EES arm). The patients of the EES arm received cobalt-chromium-based stents (Xience V, Xience Prime, or Xience Xpedition, Abbott Vascular, Santa Clara, CA, USA) and those of the BVS group were treated with Absorb BVS. Five (ABSORB $\|^{15}, A B S O R B$ Japan ${ }^{16}, A B S O R B \| I^{17}$, ABSORB China ${ }^{18}$, and $A B S O R B$ Extend $^{19}$ out of 6 studies enrolled patients with stable angina or silent ischemia and one study (BVS-EXAMINATION ${ }^{20}$ ) enrolled only patients with ST-elevation MI. The BVS-EXAMINATION study included a third treatment arm that received a bare metal stent (Multilink Vision, Abbott Vascular) but was excluded from our analysis.

Patients were on average $62.1 \pm 10.7$ vs. $61.5 \pm 10.5$ years old ( $p=0.09)$ and $28 \%$ vs $27 \%(p=0.32$ ) diabetics for BVS vs. EES group. More than $70 \%$ of all patients were treated for ACC/AHH class B2 or C lesions. The follow-up period was 1 year in all studies. Tables 1, 2, and 3 shows the design of the individual studies, baseline clinical and angiographic characteristics, and the procedural characteristics. Between the BVS and EES groups there was no significant difference in the proportion of patients with diabetes, previous $\mathrm{MI}$, previous $\mathrm{PCl}$, presentation with unstable angina, or in the proportion of multivessel treatment. However, there were significantly more active smokers $(p<0.01)$ and type $B / C$ lesions $(p<0.01)$ in the BVS group (Table 2). Between BVS and EES, there was no difference in mean reference vessel diameter $(2.68 \pm 0.41 \mathrm{~mm}$ vs $2.70 \pm 0.40 \mathrm{~mm}, p=0.16)$, mean lesion length $(13.1 \pm 5.3 \mathrm{~mm}$ vs $13.4 \pm 5.3 \mathrm{~mm}, p=0.11)$, mean scaffold/stent length $(21.0 \pm 7.5 \mathrm{~mm}$ vs $21.1 \pm 8.2 \mathrm{~mm}, \mathrm{p}=0.79)$ and mean scaffold/stent diameter $(3.14 \pm 0.40 \mathrm{~mm}$ vs $3.12 \pm 0.41 \mathrm{~mm}, \mathrm{p}=0.12)$. Between groups there was no significant difference in predilatation minimum lumen diameter $(0.96 \pm 0.34 \mathrm{~mm}$ vs $0.95 \pm 0.30 \mathrm{~mm}, \mathrm{p}=0.38)$. However, the final minimum lumen diameter was lower $(2.36 \pm 0.36 \mathrm{~mm}$ vs $2.53 \pm 0.35 \mathrm{~mm}, \mathrm{p}<0.01)$ and the acute gain was higher $(1.41 \pm 0.41 \mathrm{~mm}$ vs $1.58 \pm 0.38 \mathrm{~mm}, \mathrm{p}<0.01)$ in patients with BVS. 


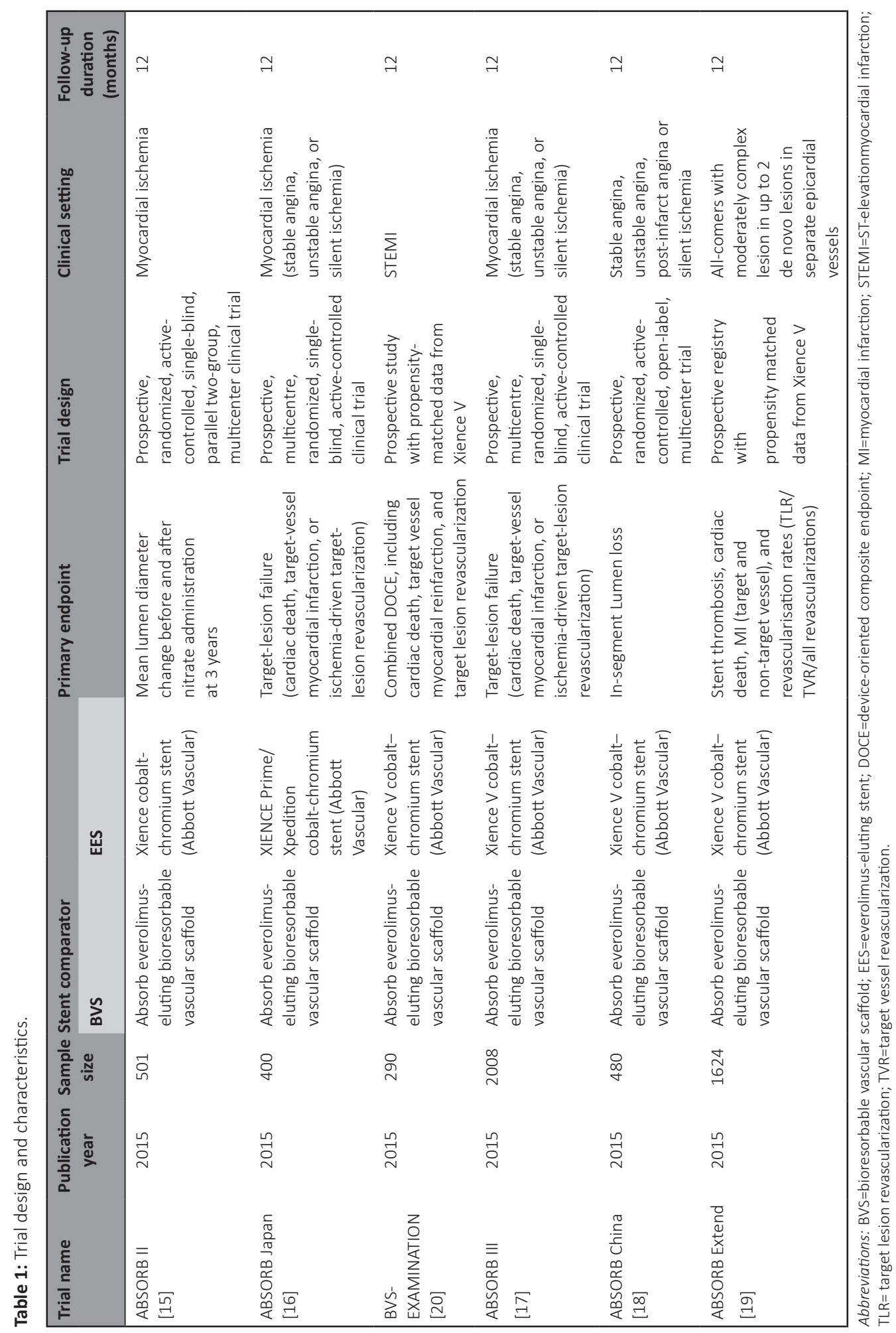


Table 2: Patient and lesion characteristics of included studies.

\begin{tabular}{|c|c|c|c|c|c|c|c|c|}
\hline & \multicolumn{2}{|c|}{ ABSORB II } & \multicolumn{2}{|c|}{ ABSORB Japan } & \multicolumn{2}{|c|}{ BVS EXAMINATION } & \multicolumn{2}{|c|}{ ABSORB III } \\
\hline & BVS & EES & BVS & EES & BVS & EES & BVS & EES \\
\hline Age & $61.5 \pm 10.0$ & $60.9 \pm 10.0$ & $67.1 \pm 9.4$ & $67.3 \pm 9.6$ & $56.01 \pm 12.75$ & $57.57 \pm 12.01$ & $63.5 \pm 10.6$ & $63.6 \pm 10.3$ \\
\hline Diabetes & $80 / 332(24 \%)$ & $40 / 166(24 \%)$ & $96 / 266$ (36\%) & $48 / 134(36 \%)$ & $37 / 290(13 \%)$ & $37 / 290(13 \%)$ & $416 / 1320(32 \%)$ & $224 / 686(33 \%)$ \\
\hline $\begin{array}{l}\text { Active } \\
\text { smoking }\end{array}$ & $79 / 335$ (24\%) & $36 / 166(22 \%)$ & $53 / 266(20 \%)$ & $29 / 134(22 \%)$ & $177 / 290(61 \%)$ & $220 / 290(76 \%)$ & $281 / 1322$ (21\%) & $142 / 686(21 \%)$ \\
\hline $\begin{array}{l}\text { Previous } \\
\mathrm{Ml}\end{array}$ & $93 / 335(28 \%)$ & $48 / 166(29 \%)$ & $42 / 262(16 \%)$ & $32 / 134(24 \%)$ & $10 / 290(4 \%)$ & $10 / 290(4 \%)$ & $282 / 1311(22 \%)$ & $150 / 681(22 \%)$ \\
\hline $\begin{array}{l}\text { Previous } \\
\mathrm{PCl}\end{array}$ & $14 / 120(12 \%)$ & $5 / 56(9 \%)$ & $9 / 266(3 \%)$ & $7 / 134(5 \%)$ & $10 / 290(3 \%)$ & $11 / 290(4 \%)$ & $96 / 1249(8 \%)$ & $38 / 651(6 \%)$ \\
\hline $\begin{array}{l}\text { Unstable } \\
\text { angina }\end{array}$ & $68 / 335(20 \%)$ & $37 / 166(22 \%)$ & $26 / 266(10 \%)$ & $22 / 134(16 \%)$ & NA & NA & $355 / 1321$ (27\%) & $168 / 686(25 \%)$ \\
\hline $\begin{array}{l}\text { Multivessel } \\
\text { disease }\end{array}$ & $57 / 335(17 \%)$ & $25 / 166(15 \%)$ & NR & NR & $24 / 290(8 \%)$ & $28 / 290(10 \%)$ & NR & NR \\
\hline$L A D$ & $163 / 364(45 \%)$ & $84 / 182(46 \%)$ & $127 / 275(46 \%)$ & $58 / 137(42 \%)$ & $145 / 290(50 \%)$ & $117 / 290(40 \%)$ & $617 / 1385$ (45\%) & $301 / 713$ (42\%) \\
\hline $\mathrm{LCX}$ & $106 / 364$ (29\%) & $42 / 182(23 \%)$ & $63 / 275(23 \%)$ & $36 / 137$ (26\%) & $29 / 290(10 \%)$ & $45 / 290(16 \%)$ & $363 / 1385$ (26\%) & $218 / 713$ (31\%) \\
\hline RCA & $95 / 364(26 \%)$ & $56 / 182(31 \%)$ & $85 / 275$ (31\%) & 43/137 (31\%) & $114 / 290$ (39\%) & $126 / 290(43 \%)$ & 404/1385 (29\%) & $194 / 713(27 \%)$ \\
\hline ACC/AHA le & esion class & & & & & & & \\
\hline B1 & $193 / 363(53 \%)$ & $90 / 180(50 \%)$ & $55 / 275(20 \%)$ & $28 / 137$ (20\%) & NR & NR & NR & NR \\
\hline B2 & $159 / 363(44 \%)$ & $87 / 180(48 \%)$ & $154 / 275(56 \%)$ & $68 / 137(50 \%)$ & & & & \\
\hline C & $6 / 363(2 \%)$ & $2 / 180(1 \%)$ & $55 / 275(20 \%)$ & $36 / 137$ (26\%) & & & & \\
\hline $\begin{array}{l}\text { Type B2/C } \\
\text { lesion class }\end{array}$ & $165 / 363(45 \%)$ & $89 / 180(49 \%)$ & $209 / 275(76 \%)$ & 104/137 (76\%) & NR & NR & $949 / 1381$ (69\%) & $513 / 708(73 \%)$ \\
\hline
\end{tabular}

Values are $\mathrm{n}(\%)$ or mean \pm SD. *ABSORB Extend was not included in "total" because no baseline data was available for the EES group. Abbreviations: ACC=American College of Cardiology; AHA=American Heart Association; BVS=bioresorbable vascularscaffold; $E E S=$ everolimus-eluting stent; $L A D=l$ eft anterior descending artery; $L C X=l$ eft circumflex artery; $M I=m y o c a r d i a l$ infarction; $\mathrm{NA}=$ not available; $\mathrm{NR}=$ not reported; $\mathrm{PCl}=$ percutaneous coronary intervention; $\mathrm{RCA}=$ right coronary artery. 


\begin{tabular}{|c|c|c|c|c|c|c|c|}
\hline \multicolumn{2}{|c|}{ ABSORB China } & \multicolumn{2}{|c|}{ ABSORB Extend } & \multicolumn{4}{|c|}{ Total* } \\
\hline BVS & EES & BVS & EES & BVS & EES & $\begin{array}{l}\text { difference } \\
(95 \%-\mathrm{Cl})\end{array}$ & p \\
\hline $57.2 \pm 11.4$ & $57.6 \pm 9.6$ & 61 & NR & $62.1 \pm 10.7$ & $61.5 \pm 10.5$ & 0.60 (-0.09 to 1.29$)$ & 0.09 \\
\hline $60 / 238(25 \%)$ & $55 / 237(23 \%)$ & $219 / 812(27 \%)$ & NR & $689(28 \%)$ & $404(27 \%)$ & $0.01(-0.01$ to 0.04$)$ & 0.32 \\
\hline $78 / 238$ (33\%) & $84 / 237$ (35\%) & $187 / 812(23 \%)$ & NR & $668(27 \%)$ & $511(34 \%)$ & $-0.07(-0.09$ to -0.04$)$ & $<0.01$ \\
\hline 40/238 (17\%) & $38 / 237$ (16\%) & $235 / 812(29 \%)$ & NR & 467 (19\%) & $278(18 \%)$ & $0.01(-0.02$ to 0.03$)$ & 0.57 \\
\hline 23/238 (10\%) & $19 / 237(8 \%)$ & NR & NR & $152(7 \%)$ & $80(6 \%)$ & $0.01(-0.01$ to 0.03$)$ & 0.17 \\
\hline $154 / 238(65 \%)$ & $152 / 237(64 \%)$ & $219 / 812(27 \%)$ & NR & $603(28 \%)$ & $379(31 \%)$ & $-0.03(-0.06$ to 0.001$)$ & 0.06 \\
\hline 42/238 (18\%) & $51 / 237(22 \%)$ & NR & NR & & & & \\
\hline $139 / 251(55 \%)$ & $132 / 252(52 \%)$ & NR (45\%) & NR & $1191(46 \%)$ & $692(44 \%)$ & $0.02(-0.01$ to 0.06$)$ & 0.12 \\
\hline 49/251 (20\%) & $61 / 252(24 \%)$ & NR $(26 \%)$ & NR & $610(24 \%)$ & $402(26 \%)$ & $-0.02(-0.04$ to 0.01$)$ & 0.20 \\
\hline $63 / 251(25 \%)$ & $59 / 252(23 \%)$ & NR $(28 \%)$ & NR & $761(30 \%)$ & $478(30 \%)$ & $-0.01(-0.04$ to 0.02$)$ & 0.63 \\
\hline $53 / 251(21 \%)$ & $56 / 252(22 \%)$ & NR $(53 \%)$ & NR & & & & \\
\hline $120 / 251(48 \%)$ & $126 / 252(50 \%)$ & NR $(42 \%)$ & & & & & \\
\hline $68 / 251(27 \%)$ & $55 / 252(22 \%)$ & NR (3\%) & & & & & \\
\hline $241 / 251$ (96\%) & $236 / 251$ (94\%) & NR $(98 \%)$ & NR & $1564(69 \%)$ & $942(74 \%)$ & $-0.04(-0.08$ to -0.02$)$ & 0.002 \\
\hline
\end{tabular}




\begin{tabular}{|c|c|c|c|c|c|c|c|c|c|}
\hline \multirow{3}{*}{ 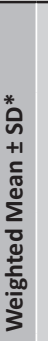 } & \multicolumn{2}{|c|}{$\begin{array}{l}\frac{0}{2} \\
\frac{0}{\pi} \\
\frac{10}{2} \\
\frac{1}{2}\end{array}$} & $\stackrel{\text { Iี }}{0}$ & 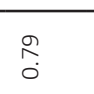 & $\begin{array}{l}0 \\
\stackrel{-1}{0}\end{array}$ & $\begin{array}{l}ت-1 \\
0 \\
0\end{array}$ & $\stackrel{\substack{\infty \\
\hdashline}}{0}$ & $\begin{array}{l}\overrightarrow{0} \\
\dot{0} \\
\dot{v}\end{array}$ & $\begin{array}{l}-\overrightarrow{0} \\
\dot{0}\end{array}$ \\
\hline & 岂 & $\vec{m}$ & 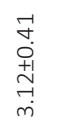 & $\begin{array}{l}\sim \\
\infty \\
+1 \\
+1 \\
\stackrel{-}{N}\end{array}$ & $\begin{array}{l}q \\
+ \\
o \\
+1 \\
\stackrel{1}{2} \\
i\end{array}$ & $\begin{array}{l}m \\
\stackrel{m}{+} \\
\stackrel{+}{+} \\
\ddot{\eta} \\
\stackrel{+}{+}\end{array}$ & 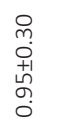 & 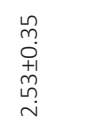 & 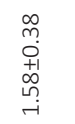 \\
\hline & 气 & 오ำ & $\begin{array}{l}0 \\
+ \\
o \\
\text { +⿱ } \\
\stackrel{+}{-} \\
m\end{array}$ & $\begin{array}{l}\stackrel{n}{2} \\
\text { +1 } \\
0 \\
\dot{i}\end{array}$ & $\begin{array}{l}\vec{f} \\
0 \\
+1 \\
0 \\
0 \\
i \\
i\end{array}$ & 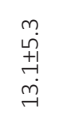 & $\begin{array}{l}\stackrel{0}{m} \\
0 \\
0 \\
+1 \\
0 \\
o \\
0\end{array}$ & $\begin{array}{l}\stackrel{0}{n} \\
0 \\
0 \\
0 \\
\sim \\
i\end{array}$ & $\begin{array}{l}\overrightarrow{7} \\
0 \\
+1 \\
+ \\
+ \\
+\end{array}$ \\
\hline 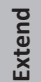 & 嵒 & $\stackrel{\frac{c}{z}}{2}$ & $\frac{\kappa}{z}$ & $\stackrel{\frac{a}{z}}{2}$ & $\frac{\alpha}{z}$ & $\stackrel{\mathscr{c}}{z}$ & $\frac{\mathscr{c}}{z}$ & $\frac{\mathscr{c}}{z}$ & $\frac{\mathscr{c}}{z}$ \\
\hline 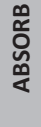 & 藏 & $\underset{\infty}{\stackrel{+}{*}}$ & $\stackrel{\frac{\alpha}{z}}{2}$ & $\frac{\alpha}{z}$ & $\begin{array}{l}0 \\
\tilde{m} \\
0 \\
+1 \\
0 \\
0 \\
i\end{array}$ & $\begin{array}{l}m \\
\stackrel{n}{+} \\
\stackrel{+}{m} \\
\underset{\sim}{\sim}\end{array}$ & 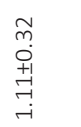 & $\frac{\alpha}{z}$ & $\frac{\alpha}{z}$ \\
\hline 衽 & 总 & $\stackrel{\sim ్ N}{\sim}$ & $\begin{array}{l}\stackrel{+}{o} \\
\stackrel{+}{+} \\
\stackrel{+}{m}\end{array}$ & 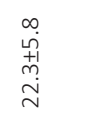 & $\begin{array}{l}m \\
0 \\
i+1 \\
\stackrel{+}{1} \\
\infty \\
i\end{array}$ & $\begin{array}{l}m \\
0 \\
0 \\
+ \\
m \\
m\end{array}$ & $\begin{array}{l}\text { m } \\
0 \\
\text { o } \\
+1 \\
\text { +1 } \\
\text { - }\end{array}$ & $\begin{array}{l}m \\
0 \\
0 \\
+1 \\
\stackrel{1}{N} \\
\text { N }\end{array}$ & 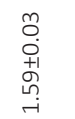 \\
\hline 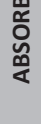 & 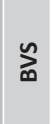 & 亗 & $\begin{array}{l}\stackrel{+}{\circ} \\
\stackrel{+}{+} \\
\stackrel{+}{m}\end{array}$ & 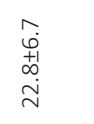 & $\begin{array}{l}m \\
0 \\
0 \\
0 \\
+1 \\
\infty \\
i \\
i\end{array}$ & $\begin{array}{l}m \\
\stackrel{0}{+} \\
\stackrel{+}{+} \\
\dot{+} \\
+\end{array}$ & $\begin{array}{l}m \\
0 \\
0 \\
+1 \\
\infty \\
o \\
o \\
0\end{array}$ & 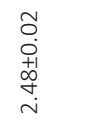 & 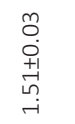 \\
\hline $\begin{array}{l}\equiv \\
\equiv \\
\propto\end{array}$ & 总 & $\stackrel{m}{\wedge}$ & 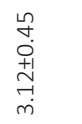 & $\begin{array}{l}0 \\
\stackrel{1}{+} \\
\stackrel{1}{1} \\
\stackrel{i}{0}\end{array}$ & $\begin{array}{l}0 \\
y \\
0 \\
0 \\
11 \\
0 \\
i\end{array}$ & $\begin{array}{l}\infty \\
\stackrel{\infty}{\omega} \\
\stackrel{+}{+} \\
\dot{\sim} \\
\dot{r}\end{array}$ & 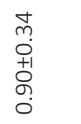 & 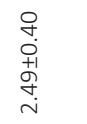 & 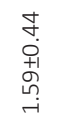 \\
\hline$\frac{\infty}{<}$ & 畣 & 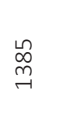 & \begin{tabular}{l}
$m$ \\
\multirow{2}{*}{} \\
0 \\
+1 \\
0 \\
0 \\
$\dot{m}$
\end{tabular} & 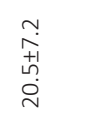 & 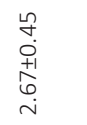 & $\begin{array}{l}\stackrel{+}{+} \\
\stackrel{+}{+1} \\
\stackrel{0}{+} \\
\stackrel{+}{+}\end{array}$ & 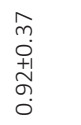 & $\begin{array}{l}\stackrel{o}{+} \\
0 \\
+1 \\
\stackrel{+1}{m} \\
i\end{array}$ & 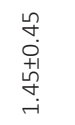 \\
\hline$\frac{z}{\frac{2}{4}}$ & 总 & $\frac{\alpha}{z}$ & 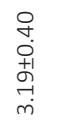 & 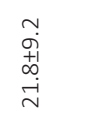 & $\frac{\alpha}{z}$ & $\stackrel{\frac{\alpha}{z}}{ }$ & $\stackrel{\frac{\alpha}{z}}{2}$ & $\frac{\alpha}{z}$ & $\frac{\alpha}{z}$ \\
\hline $\begin{array}{l}\vec{x} \\
\dot{u} \\
\dot{\omega}\end{array}$ & בัฒ & $\stackrel{\frac{c}{z}}{2}$ & 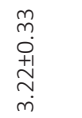 & $\begin{array}{l}\infty \\
\infty \\
+1 \\
\stackrel{1}{1} \\
\underset{\sim}{\sim}\end{array}$ & $\frac{\mathscr{c}}{z}$ & $\stackrel{\frac{\alpha}{z}}{ }$ & $\stackrel{\frac{c}{z}}{2}$ & $\frac{\alpha}{z}$ & $\stackrel{\propto}{z}$ \\
\hline $\begin{array}{l}\frac{c}{\pi} \\
\frac{0}{\pi} \\
\frac{10}{5}\end{array}$ & 岂 & $\hat{m}$ & 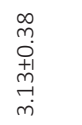 & 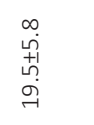 & \begin{tabular}{l}
0 \\
\multirow{1}{*}{} \\
0 \\
+1 \\
$\stackrel{1}{1}$ \\
$i$
\end{tabular} & 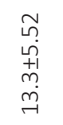 & $\begin{array}{l}0 \\
m \\
0 \\
+1 \\
O \\
0 \\
0\end{array}$ & $\begin{array}{l}o \\
0 \\
0 \\
+1 \\
0 \\
i \\
i\end{array}$ & 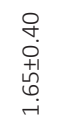 \\
\hline 嘀 & 气ั & 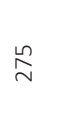 & $\begin{array}{l}\hat{n} \\
0 \\
0 \\
+1 \\
0 \\
\text { ri } \\
\dot{1}\end{array}$ & \begin{tabular}{l}
$\infty$ \\
$\stackrel{\infty}{+}$ \\
\multirow{1}{*}{} \\
$\stackrel{\sim}{0}$
\end{tabular} & \begin{tabular}{l} 
i \\
\multirow{2}{*}{} \\
$\stackrel{+}{+}$ \\
$\stackrel{i}{+}$
\end{tabular} & $\begin{array}{l}\stackrel{\infty}{\sim} \\
\stackrel{\sim}{+1} \\
\stackrel{1}{11} \\
\stackrel{n}{\sim}\end{array}$ & $\begin{array}{l}m \\
m \\
0 \\
0 \\
+1 \\
o \\
0 \\
0\end{array}$ & 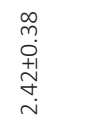 & 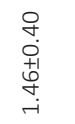 \\
\hline$=$ & 总 & $\underset{\sim}{\sim}$ & 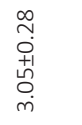 & \begin{tabular}{l}
+ \\
\multirow{1}{*}{} \\
\multirow{2}{+}{} \\
$\dot{\sim}$
\end{tabular} & $\begin{array}{l}0 \\
\stackrel{0}{0} \\
0 \\
+1 \\
0 \\
0 \\
i\end{array}$ & $\begin{array}{l}0 \\
\dot{\omega} \\
+1 \\
\infty \\
\dot{H} \\
\ddot{H}\end{array}$ & $\begin{array}{l}\tilde{y} \\
\text { m. } \\
+1 \\
\text { +1 } \\
0 \\
-i\end{array}$ & 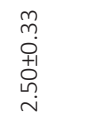 & 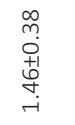 \\
\hline$\stackrel{\infty}{<}$ & 畣 & $\underset{ల}{\mathbb{N}}$ & $\begin{array}{l}\vec{m} \\
0 \\
0 \\
+1 \\
\dot{0} \\
\dot{m}\end{array}$ & $\begin{array}{l}\infty \\
\infty \\
+1 \\
+1 \\
\stackrel{i}{+}\end{array}$ & $\begin{array}{l}\infty \\
\stackrel{\infty}{0} \\
0 \\
+1 \\
\stackrel{1}{0} \\
i\end{array}$ & 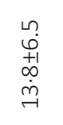 & $\begin{array}{l}\stackrel{n}{n} \\
0 \\
o \\
+1 \\
0 \\
-1\end{array}$ & $\begin{array}{l}\stackrel{m}{0} \\
\stackrel{+}{+} \\
\stackrel{N}{N}\end{array}$ & 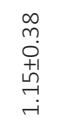 \\
\hline & & 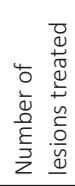 & 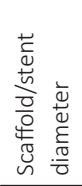 & 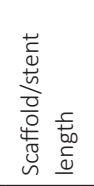 & 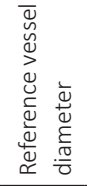 & 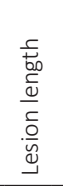 & 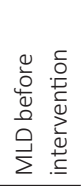 & 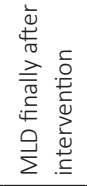 & 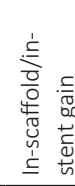 \\
\hline
\end{tabular}




\section{Efficacy outcomes}

Study-level outcomes after 12 months follow-up for DOCE, the individual components of DOCE, TVR, and ST were shown in Table 4 and Figure 3. Follow-up was available in 3,253 patients treated with BVS and in 2,315 patients treated with EES. Slightly more than $80 \%$ of patients were on dual antiplatelet therapy at 12 -month follow-up.

DOCE occurred in $6.0 \%$ of the patients treated with BVS and in $4.9 \%$ of the patients treated with EES (OR 1.19, 95\% Cl: 0.94-1.52, $p=0.16$; heterogeneity: $\left.I^{2}=0, Q=1.49, d f=5, p=0.91\right)$. The rate of cardiac death and TLR were similar for both patients treated with BVS and EES (OR 1.14, 95\% Cl: 0.54-2.39, $p=0.73$ and OR 0.98, 95\% Cl: 0.69-1.40, p=0.92, respectively).

\section{Safety outcomes}

During 1-year follow-up, definite-or-probable stent thrombosis occurred more often in the BVS-group (1.3\% vs $0.6 \%$, OR 2.10, 95\% Cl: $1.13-3.87, p=0.02$; heterogeneity: $\left.I^{2}=0, Q=1.63, d f=5, p=0.90\right)$. In addition, more patients treated with BVS developed target vessel $\mathrm{MI}$ as compared to patients treated with EES (4.3\% vs $2.3 \%$, OR 1.63, 95\% Cl: $1.18-2.25, p<0.01$; heterogeneity: $\left.I^{2}=0, Q=3.16, d f=5, p=0.68\right)$.

\section{Discussion}

The present meta-analysis shows that treatment of in patients with obstructive coronary artery disease with the Absorb BVS was similarly efficacious as implantation of durable polymer cobalt-chromium EES with regard to the risk of repeat revascularization (i.e. both TLR and TVR). In addition, there was no between-group difference in cardiovascular mortality. Patients who received BVS had an increased risk of MI and definite-or-probable scaffold thrombosis. The composite endpoint DOCE showed no significant difference between both device groups.

The present meta-analysis differs from previous meta-analyses in that we (1) included only trials with a minimum follow-up duration of 12-months and (2) compared the BVS only to cobalt-chromiumbased EES. Cassese and colleagues ${ }^{27}$ published a meta-analysis that compared the efficacy and safety outcomes in patients treated with Absorb BVS versus EES but included two studies with 6-month followup duration (TROFI II and EVERBIO II).

Despite the overall relatively small number of definite-or-probable stent thromboses in both groups, treatment with BVS showed a significantly increased risk of $S T(p=0.02)$ as compared to cobalt-chromium EES. The two-fold higher rate of ST with BVS, noted in our study, was similar to previous findings by Cassese et al. and Lipinski et al. ${ }^{27,28}$ Our study also confirmed a significantly higher rate of Ml associated with BVS implantation versus DES. ${ }^{28}$ Similar to Cassese and colleagues ${ }^{27}$, our study confirmed the known favorable safety profile of the EES. In addition, at 1-year follow-up, patients with predominantly ACC/ AHA lesion types B or C treated with BVS showed no significant difference in mortality, revascularization rate, or DOCE. 


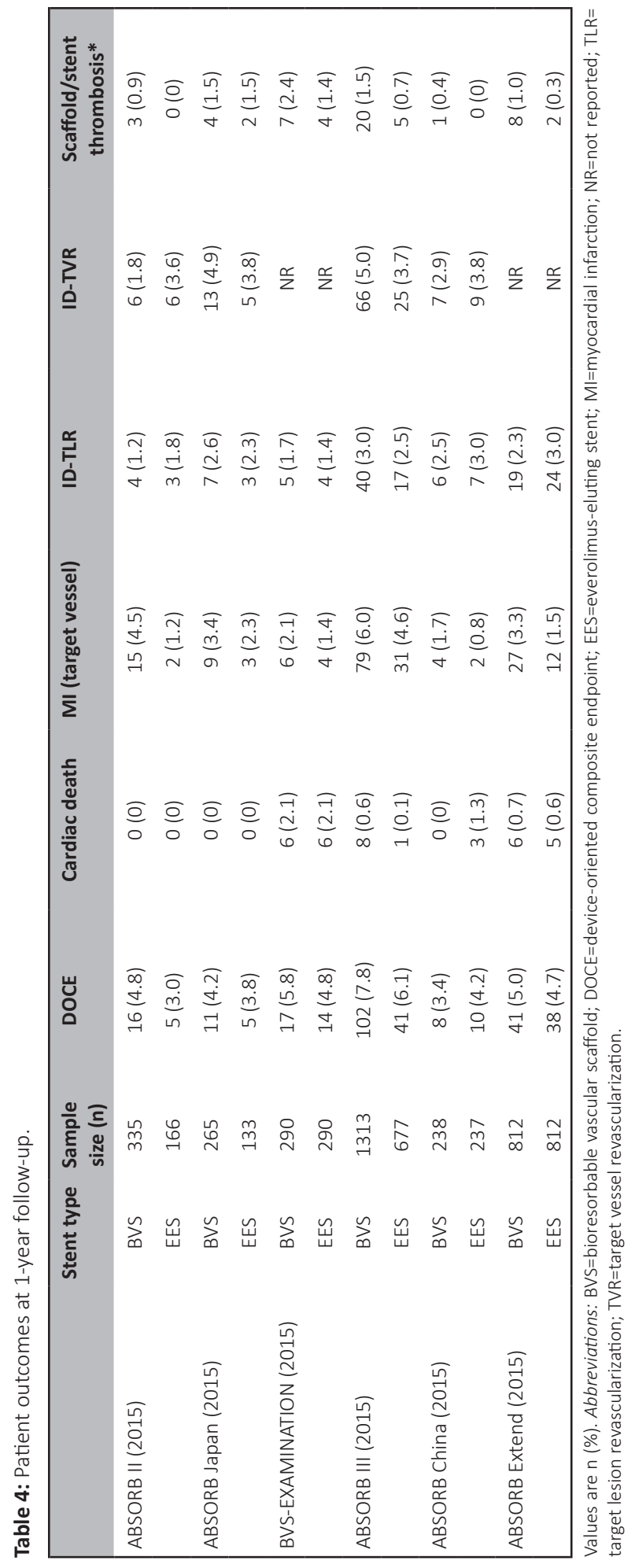


A. Device-oriented composite endpoint

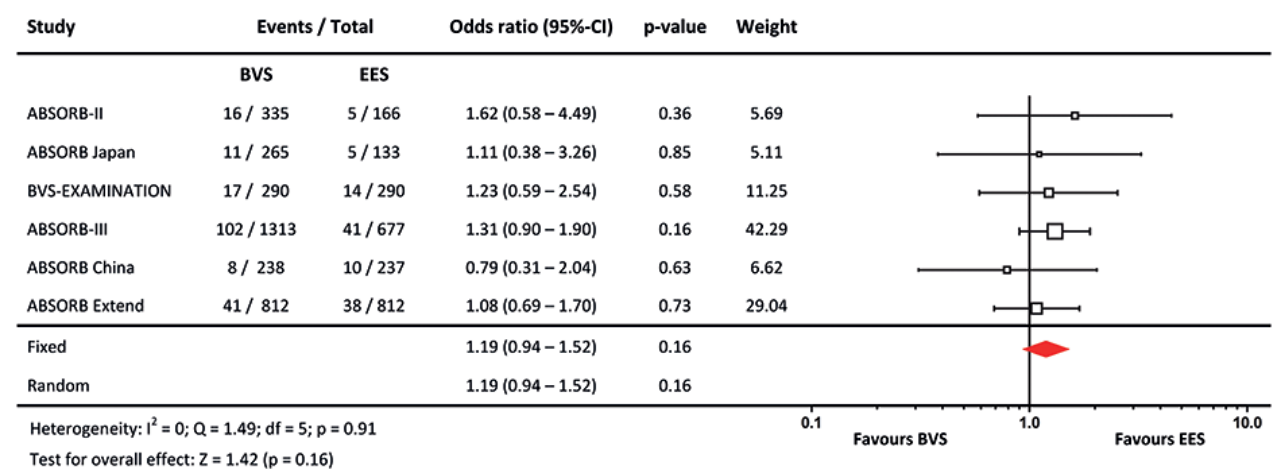

\section{B. Cardiac death}

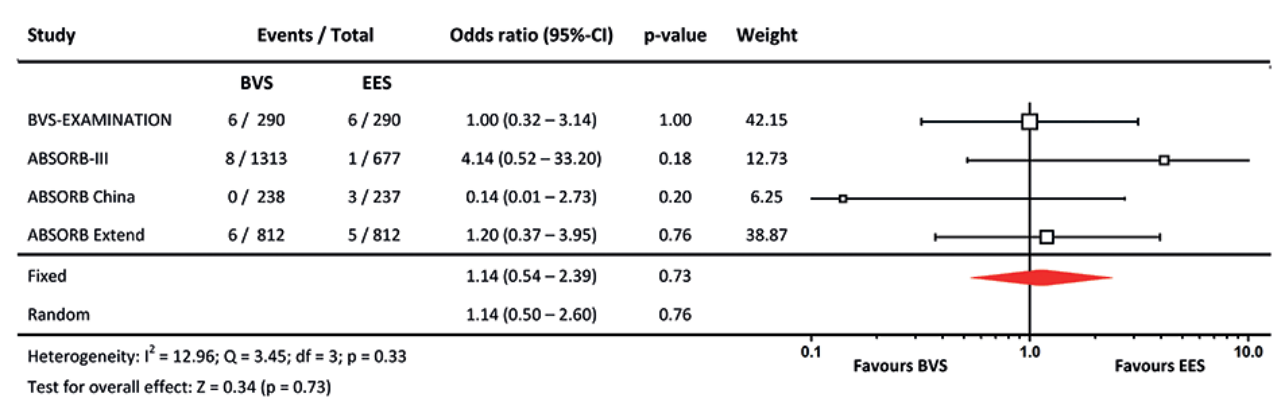

\section{Target vessel myocardial infarction}

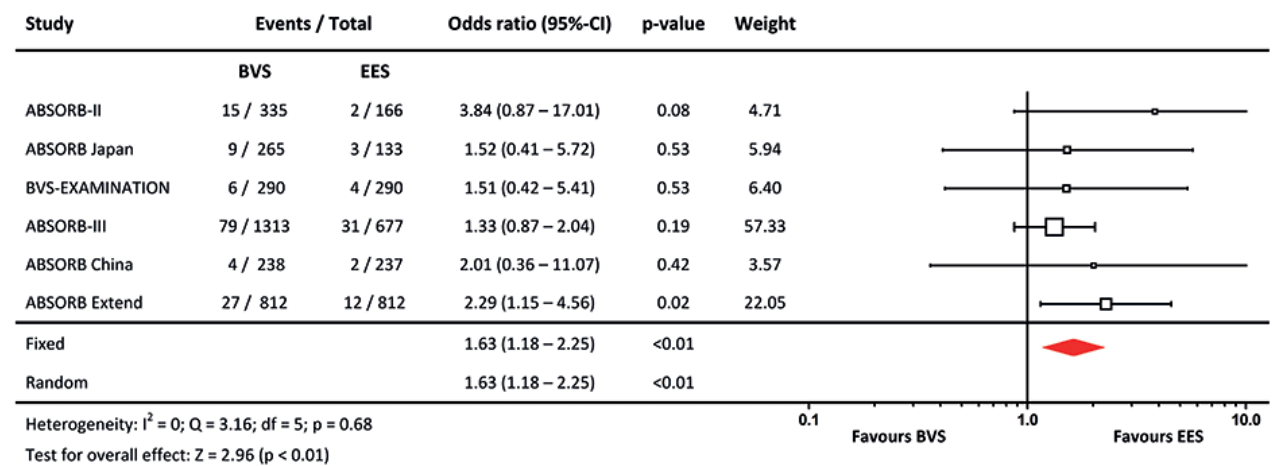

Figure 3: Meta-analysis comparing 1-year clinical outcomes of patients treated with Absorb BVS vs EES panes (A-F) A: device-oriented composite endpoint; B: cardiac death; C: target vessel myocardial infarction; D: target lesion revascularization; E: target vessel revascularization; F: definite-or-probable scaffold/stent thrombosis. Abbreviations: BVS=bioresorbable vascular scaffold; EES=durable-polymer everolimus-eluting stent. 


\section{Target lesion revascularization}

\begin{tabular}{|c|c|c|c|c|c|c|c|}
\hline \multirow[t]{2}{*}{ Study } & \multicolumn{2}{|c|}{ Events / Total } & \multirow[t]{2}{*}{ Odds ratio $(95 \%-\mathrm{Cl})$} & \multirow[t]{2}{*}{ p-value } & \multirow[t]{2}{*}{ Weight } & & \\
\hline & BVS & EES & & & & & \\
\hline ABSORB-II & $4 / 335$ & $3 / 166$ & $0.66(0.15-2.97)$ & 0.59 & 5.44 & 1 & \\
\hline ABSORB Japan & $7 / 265$ & $3 / 133$ & $1.18(0.30-4.62)$ & 0.82 & 6.61 & & \\
\hline BVS-EXAMINATION & $5 / 290$ & $4 / 290$ & $1.25(0.33-4.72)$ & 0.74 & 7.06 & & \\
\hline ABSORB-III & $40 / 1313$ & $17 / 677$ & $1.22(0.69-2.17)$ & 0.50 & 37.45 & & - \\
\hline ABSORB China & $6 / 238$ & $7 / 237$ & $0.85(0.28-2.57)$ & 0.77 & 10.14 & $\longrightarrow$ & \\
\hline ABSORB Extend & $19 / 812$ & $24 / 812$ & $0.79(0.43-1.45)$ & 0.44 & 33.31 & $\longmapsto \square$ & $\longrightarrow$ \\
\hline Fixed & & & $0.98(0.69-1.40)$ & 0.92 & & & \\
\hline Random & & & $0.98(0.69-1.40)$ & 0.92 & & & \\
\hline rogeneity: $1^{2}=c$ & 59 & 90 & & & & Favours BVS & Favours EES 10.0 \\
\hline
\end{tabular}

\section{E. Target vessel revascularization}

\begin{tabular}{|c|c|c|c|c|c|c|c|}
\hline Study & Event & Total & Odds ratio $(95 \%-\mathrm{Cl})$ & p-value & Weight & & \\
\hline & BVS & EES & & & & & \\
\hline ABSORB-II & $6 / 335$ & $6 / 166$ & $0.49(0.15-1.53)$ & 0.22 & 10.58 & $\longrightarrow$ & \\
\hline ABSORB Japan & $13 / 265$ & $5 / 133$ & $1.32(0.46-3.79)$ & 0.61 & 12.56 & & $\rightarrow$ \\
\hline ABSORB-III & $66 / 1313$ & $25 / 677$ & $1.38(0.86-2.21)$ & 0.18 & 63.06 & 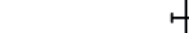 & $-\square$ \\
\hline ABSORB China & $7 / 238$ & $9 / 237$ & $0.77(0.28-2.10)$ & 0.61 & 13.80 & $\longrightarrow$ & \\
\hline Fixed & & & $1.13(0.78-1.65)$ & 0.51 & & & \\
\hline Random & & & $1.09(0.71-1.67)$ & 0.70 & & & \\
\hline Heter & 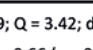 & $=0.33$ & & & 0 & Favours BVS & Favours EES 10.0 \\
\hline
\end{tabular}

\section{F. Definite-or-probable scaffold/stent thrombosis}

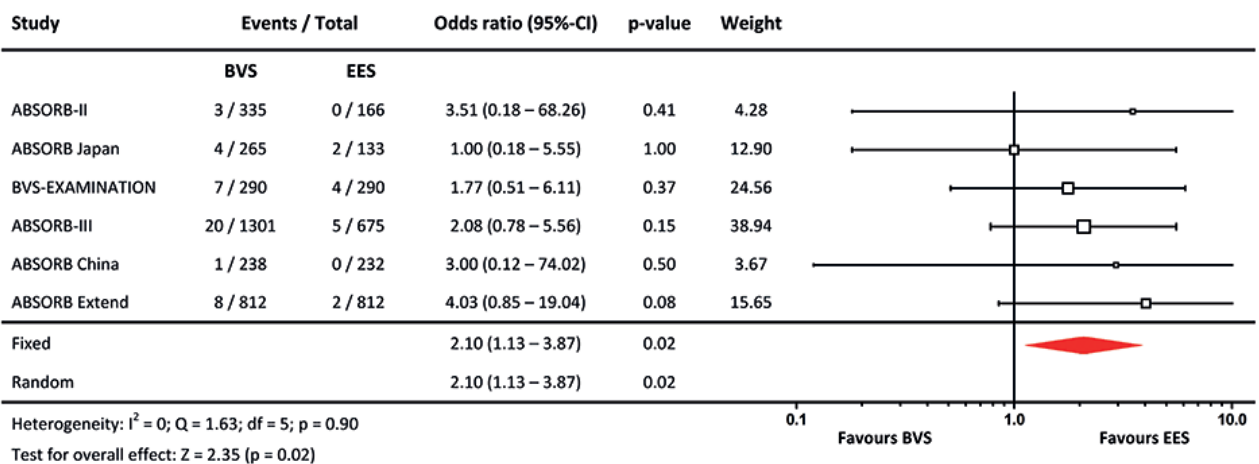

Figure 3: Continued

Theoretically, one would expect a reduction in very late stent thrombosis with BVS after resorption of the polymeric scaffold after 2 to 3 years. Imaging studies have shown persistent presence of the BVS at 12 months and bioresorption at 24 to 26 months. ${ }^{2,3,29}$ One of the anticipated benefits of BVS is minimization of very late stent thrombosis, which occurs after complete bioresorption of the vascular scaffold and return of normal vessel function. ${ }^{3}$ In cohort A of the ABSORB trial, there was no reported stent thrombosis at 3-year follow-up although at 2 and 3 years only a single patient was on dual anti- 
platelet therapy (i.e. aspirin plus thienopyridine). ${ }^{30}$ After 2 years, optical coherence tomography (OCT) assessment of the BVS in the ABSORB cohort A showed that the polymeric struts were no longer recognizable, and on angiography there was very late lumen enlargement. ${ }^{30}$ Similarly, the ABSORB trial cohort B showed at 2-year follow-up no scaffold thrombosis, a return of vasomotion, and very late lumen enlargement. ${ }^{7}$

There are various factors that may have contributed to the higher incidence of device thrombosis up to 12 -months in patients treated with BVS versus EES, as seen in the current meta-analysis: The thicker struts of the BVS may trigger platelet aggregation. ${ }^{31}$ Moreover, the lower final in-device minimum lumen diameter achieved with BVS when compared to EES, may have contributed to the increased thrombotic risk. Suboptimal implantation may result in device malapposition and underexpansion, which are known to affect coronary flow pattern and may subsequently activate the thrombotic cascade. ${ }^{12,32,33}$ Well-controlled post-dilatation and overexpansion of BVS is likely to prevent a substantial proportion of potential coronary thromboses by improving the apposition of the scaffold to the vessel wall; on the other hand, excessive overexpansion can lead to fractures of the polymeric BVS that may induce thrombus formation. ${ }^{34}$ Late scaffold recoil, which may occur more frequently in the absence of adequate lesion preparation, has also been associated with BVS thrombosis. ${ }^{35}$

Further potential mechanisms for very late BVS thrombosis include the presence of uncovered BVS struts after 12 months with discontinuation of dual antiplatelet therapy. ${ }^{32,36}$ In our present analysis, slightly more than $80 \%$ of patients were on dual antiplatelet therapy at 12 -month follow-up. Despite the relatively low rates of BVS thrombosis beyond 12 months, it may be considered to continue dual antiplatelet therapy until the polymeric scaffold is expected to be fully dissolved. ${ }^{37}$ Prolongation of dual antiplatelet therapy might be particularly useful in patients or procedural results with increased thrombotic risk (e.g. diabetics, patients with renal failure, overlapping BVS). Finally, late scaffold discontinuity may cause dislocation of strut remnants into the coronary lumen, which may result in flow disturbance and shear stress to the vessel wall with subsequent platelet recruitment and thrombus formation. ${ }^{36}$

\section{Study Limitations}

In this meta-analysis, we used 4 randomized clinical trials in combination with 2 observational studies to detect differences between Absorb BVS and EES; nevertheless, both observation studies used propensity score matching, and as such meaningful comparisons could be made. While a respectable number of patients $(n=5,588)$ were included in this meta-analysis, it may still be too few to assess true differences in the occurrence of rare adverse events such as stent thrombosis. The BVS technology is still relative new, and as such, from several randomized trials only 12-month outcome data is available. As a consequence, more data on a longer follow-up are required to assess the long-term safety and efficacy of BVS beyond the first year after treatment. 


\section{Conclusion}

During the first year of follow-up, treatment with everolimus-eluting BVS was associated with a higher incidence of target vessel $\mathrm{MI}$ and scaffold thrombosis as compared to metallic EES, but the composite endpoint DOCE showed no significant difference between groups. As BVS may pay off beyond the follow-up period of 1-year, additional robust data on long-term clinical outcome will be of paramount importance. 


\section{References}

1. Wiebe J, Nef HM, Hamm CW. Current status of bioresorbable scaffolds in the treatment of coronary artery disease. J Am Coll Cardiol 2014;64:2541-51.

2. Waksman R. Biodegradable stents: they do their job and disappear. J Invasive Cardiol 2006;18:70-4.

3. Iqbal J, Onuma Y, Ormiston J, Abizaid A, Waksman R, Serruys P. Bioresorbable scaffolds: rationale, current status, challenges, and future. Eur Heart J 2014;35:765-76.

4. Gomez-Lara J, Garcia-Garcia HM, Onuma Y, et al. A comparison of the conformability of everolimus-eluting bioresorbable vascular scaffolds to metal platform coronary stents. J Am Coll Cardiol Intv 2010;3:1190-8.

5. Serruys PW, Garcia-Garcia HM, Onuma Y. From metallic cages to transient bioresorbable scaffolds: change in paradigm of coronary revascularization in the upcoming decade? Eur Heart J 2012;33:16-25b.

6. Tandjung K, Sen H, Lam MK, et al. Clinical outcome following stringent discontinuation of dual antiplatelet therapy after 12 months in real-world patients treated with second-generation zotarolimus-eluting resolute and everolimus-eluting Xience V stents: 2-year follow-up of the randomized TWENTE trial. J Am Coll Cardiol 2013;61:2406-16.

7. Ormiston JA, Serruys PW, Onuma Y, et al. First serial assessment at 6 months and 2 years of the second generation of absorb everolimus-eluting bioresorbable vascular scaffold: a multi-imaging modality study. Circ Cardiovasc Interv 2012;5:620-32.

8. Serruys PW, Onuma $Y$, Dudek D, et al. Evaluation of the second generation of a bioresorbable everolimuseluting vascular scaffold for the treatment of de novo coronary artery stenosis: 12-month clinical and imaging outcomes. J Am Coll Cardiol 2011;58:1578-88.

9. Capodanno D, Gori T, Nef H, et al. Percutaneous coronary intervention with everolimus-eluting bioresorbable vascular scaffolds in routine clinical practice: early and midterm outcomes from the European multicentre GHOST-EU registry. Eurolntervention 2015;10:1144-53.

10. Jaguszewski M, Ghadri JR, Zipponi M, et al. Feasibility of second-generation bioresorbable vascular scaffold implantation in complex anatomical and clinical scenarios. Clin Res Cardiol 2015;104:124-35.

11. Kraak RP, Hassell ME, Grundeken MJ, et al. Initial experience and clinical evaluation of the Absorb bioresorbable vascular scaffold (BVS) in real-world practice: the AMC Single Centre Real World PCI Registry. Eurolntervention 2015;10:1160-8.

12. Raber L, Brugaletta S, Yamaji K, et al. Very Late Scaffold Thrombosis: Intracoronary Imaging and Histopathological and Spectroscopic Findings. J Am Coll Cardiol 2015;66:1901-14.

13. Moher D, Liberati A, Tetzlaff J, Altman DG, Group P. Preferred reporting items for systematic reviews and metaanalyses: the PRISMA statement. Ann Intern Med 2009;151:264-9,W64.

14. Food and Drug Administration. Circulatory System Devices Panel Meeting. 2006.

15. Serruys PW, Chevalier B, Dudek D, et al. A bioresorbable everolimus-eluting scaffold versus a metallic everolimuseluting stent for ischaemic heart disease caused by de-novo native coronary artery lesions (ABSORB II): an interim 1-year analysis of clinical and procedural secondary outcomes from a randomised controlled trial. Lancet 2015;385:43-54.

16. Kimura T, Kozuma K, Tanabe K, et al. A randomized trial evaluating everolimus-eluting Absorb bioresorbable scaffolds vs. everolimus-eluting metallic stents in patients with coronary artery disease: ABSORB Japan. Eur Heart J 2015;36:3332-42.

17. Ellis SG, Kereiakes DJ, Metzger DC, et al. Everolimus-Eluting Bioresorbable Scaffolds for Coronary Artery Disease. N Engl J Med 2015;373:1905-15.

18. Gao R, Yang Y, Han Y, et al. Bioresorbable Vascular Scaffolds Versus Metallic Stents in Patients With Coronary ase: ABSORB China Trial. J Am Coll Cardiol 2015;66:2298-309.

19. Abizaid A. ABSORB EXTEND: One-Year Results in Moderately Complex Lesions (abstr.). Presented at: EuroPCR. Paris, France2015.

20. Brugaletta S, Gori T, Low AF, et al. Absorb bioresorbable vascular scaffold versus everolimus-eluting metallic stent in ST-segment elevation myocardial infarction: 1-year results of a propensity score matching comparison: the BVS-EXAMINATION Study (bioresorbable vascular scaffold-a clinical evaluation of everolimus eluting coronary stents in the treatment of patients with ST-segment elevation myocardial infarction). J Am Coll Cardiol Intv 2015;8:189-97. 
21. Higgins JP, Altman DG, Gotzsche PC, et al. The Cochrane Collaboration's tool for assessing risk of bias in randomised trials. BMJ 2011;343:d5928.

22. Begg CB, Mazumdar M. Operating characteristics of a rank correlation test for publication bias. Biometrics 1994;50:1088-101.

23. Higgins JP, Thompson SG. Quantifying heterogeneity in a meta-analysis. Stat Med 2002;21:1539-58.

24. Mantel N, Haenszel W. Statistical aspects of the analysis of data from retrospective studies of disease. J Natl Cancer Inst 1959;22:719-48.

25. DerSimonian R, Laird N. Meta-analysis in clinical trials. Control Clin Trials 1986;7:177-88.

26. Borenstein M. Introduction to meta-analysis. Chichester: Wiley; 2009.

27. Cassese S, Byrne RA, Ndrepepa G, et al. Everolimus-eluting bioresorbable vascular scaffolds versus everolimuseluting metallic stents: a meta-analysis of randomised controlled trials. Lancet 2015;387:537-44.

28. Lipinski MJ, Escarcega RO, Baker NC, et al. Scaffold Thrombosis After Percutaneous Coronary Intervention With ABSORB Bioresorbable Vascular Scaffold: A Systematic Review and Meta-Analysis. J Am Coll Cardiol Intv 2016;9:12-24.

29. Onuma Y, Serruys PW, Perkins LE, et al. Intracoronary optical coherence tomography and histology at 1 month and 2, 3, and 4 years after implantation of everolimus-eluting bioresorbable vascular scaffolds in a porcine coronary artery model: an attempt to decipher the human optical coherence tomography images in the ABSORB trial. Circulation 2010;122:2288-300.

30. Onuma Y, Serruys PW, Ormiston JA, et al. Three-year results of clinical follow-up after a bioresorbable everolimus-eluting scaffold in patients with de novo coronary artery disease: the ABSORB trial. Eurolntervention 2010;6:447-53.

31. Bourantas CV, Papafaklis MI, Garcia-Garcia HM, et al. Short- and long-term implications of a bioresorbable vascular scaffold implantation on the local endothelial shear stress patterns. J Am Coll Cardiol Intv 2014;7:100-1.

32. Karanasos A, Van Mieghem N, van Ditzhuijzen N, et al. Angiographic and optical coherence tomography insights into bioresorbable scaffold thrombosis: single-center experience. Circ Cardiovasc Interv 2015;8:doi 10.1161/ CIRCINTERVENTIONS.114.002369.

33. Puricel S, Cuculi F, Weissner M, et al. Bioresorbable Coronary Scaffold Thrombosis: Multicenter Comprehensive Analysis of Clinical Presentation, Mechanisms, and Predictors. J Am Coll Cardiol 2016;67:921-31.

34. von Birgelen C, Basalus MWZ. Oversized post-dilatation of current bioresorbable vascular scaffolds: kill or cure? Eurolntervention 2016;11:1330-3.

35. Cortese B, Piraino D, lelasi A, Steffenino G, Orrego PS. Very late bioresorbable vascular scaffold thrombosis due to late device recoil. Int J Cardiol 2015;189:132-3.

36. Timmers L, Stella PR, Agostoni P. Very late bioresorbable vascular scaffold thrombosis following discontinuation of antiplatelet therapy. Eur Heart J 2015;36:393.

37. Karanasos A, van Geuns RJ, Zijlstra F, Regar E. Very late bioresorbable scaffold thrombosis after discontinuation of dual antiplatelet therapy. Eur Heart J 2014;35:1781. 



\section{Conflicts of interest statement}

CVB and LCvdH reported significant institutional research grants to the Research Department of Thoraxcentrum Twente from: AstraZeneca, Biotronik, Boston Scientific, and Medtronic. CvB has been an unpaid consultant to various device-manufacturing companies. BNM reported no conflict of interest.

\section{Copyright}

Reprinted with permission from

International Journal of Cardiology. Article accepted.

(C) Elsevier 


\section{CHAPTER 7.1}

\section{Reply to Letter to the Editor entitled: \\ "Bioresorbable stent thrombosis, lactic acid release and Kounis syndrome"}


We thank Dr. Kounis and colleagues for sharing their interesting thoughts. Although only supported by few data, we cannot exclude that the suggested alternative mechanisms may sometimes apply. An inflammatory environment is a risk factor for bioresorbable vascular scaffold (BVS) thrombosis, but vascular inflammation is often present in acute coronary syndromes, even without scaffolds. ${ }^{1}$

Firstly, we would like to emphasize that finding an increased thrombosis risk in Absorb BVS does not mean that other scaffolds or bioresorbable stents have the same problem. Sirolimus-eluting bioresorbable magnesium stents may even have a particularly low thrombosis risk. ${ }^{2}$ Hence, we can only warn against using the misleading term "bioresorbable stent", when referring to our findings.

Secondly, everolimus-dose and delivery profile of BVS are similar to everolimus-eluting fluoro-polymercoated metal stents which have a very low thrombosis risk. ${ }^{3}$ Therefore, it is highly unlikely that body reactions to everolimus play a major role for BVS thrombosis.

Thirdly, optical coherence tomography supports other mechanisms. Incomplete lesion coverage, BVS under-deployment and mal-apposition, as well as alterations in laminar blood flow and thrombogenicity induced by very thick (especially mal-apposed) struts are important mechanisms of (sub-)acute thromboses. ${ }^{1}$ Late and very late BVS thrombosis have been related to non-embedded and nonabsorbed struts in complex lesions and to late structural discontinuity or device dismantling of malapposed struts, in particular after cessation of dual anti-platelet therapy. ${ }^{1,4}$

Finally, while BVS thromboses have been related to sub-optimal implantation techniques ${ }^{1}$, real-world data of the randomized AIDA trial do not support this notion 4 . Nowadays, contemporary drug-eluting metal stents have excellent clinical outcomes in all-comers, including very low stent thrombosis rates. ${ }^{5}$ Therefore, use of current-generation BVS preferably should be limited to clinical trials. 


\section{References}

1. Y. Sotomi, P. Suwannasom, P.W. Serruys and Y. Onuma. Possible mechanical causes of scaffold thrombosis: insights from case reports with intracoronary imaging, Eurolntervention 12, 2017, 1747-56.

2. R. Waksman, P. Zumstein, M. Pritsch, et al. Second-generation magnesium scaffold Magmaris, device design, and preclinical evaluation in a porcine coronary artery model, Eurolntervention 2017, http://dx.doi.org/ 10.4244/ EIJ-D-16-00915, (in press, online published on March 7, 2017).

3. C. von Birgelen, L.C. van der Heijden, M.W. Basalus, et al. Five-year outcome after implantation of zotarolimusand everolimus-eluting stents in randomized trial participants and nonenrolled eligible patients: a secondary analysis of a randomized clinical trial, JAMA Cardiol. 2, 2017, 268-76.

4. J.J. Wykrzykowska, R.P. Kraak, S.H. Hofma, et al. Bioresorable scaffolds versus metallic stents in routine $\mathrm{PCl}, \mathrm{N}$. Engl. J. Med. 2017,http://dx.doi.org/10.1056/NEJMoa1614954, (in press, online published on March 29, 2017).

5. C. von Birgelen, M.M. Kok, L.C. van der Heijden, et al. Very thin strut biodegradable polymer everolimus-eluting and sirolimus-eluting stents versus durable polymer zotarolimus-eluting stents in allcomers with coronary artery disease (BIO-RESORT): a three-arm, randomised, non-inferiority trial, Lancet 388, 2016,2607-17. 


\section{Conflicts of interest statement}

CvB has been an unpaid consultant to Biotronik, Boston Scientific, and Medtronic. The research department of Thoraxcentrum Twente has received research grants provided by Biotronik, Boston Scientific, and Medtronic. All other authors declared that they have no conflict of interest. 


\title{
CHAPTER 8
}

\section{Bioresorbable Polymer-Coated Orsiro Versus Durable Polymer-Coated Resolute Onyx Stents (BIONYX)}

\author{
Rationale and Design of the \\ Randomized TWENTE IV Multicenter Trial
}

Liefke C. van der Heijden*, Marlies M. Kok*, Paolo Zocca, Gillian A.J. Jessurun, Carl E. Schotborgh, Ariel Roguin, Edouard Benit, Adel Aminian, Peter W. Danse, Marije M. Löwik, Gerard C.M. Linssen, Job van der Palen, Carine J.M. Doggen,

Clemens von Birgelen

*Both authors contributed equally to this manuscript.
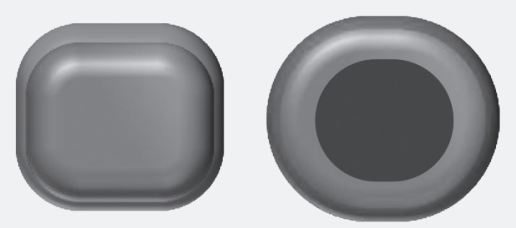


\begin{abstract}
Aim: To compare in a non-inferiority trial the efficacy and safety of 2 contemporary drug-eluting stents (DES): a novel, durable polymer-coated stent versus an established bioabsorbable polymer-coated stent. Methods and Results: The BIONYX trial (ClinicalTrials.gov-no.NCT02508714) is an investigatorinitiated, prospective, randomized, patient and assessor-blinded, international, multicenter study in all-comer patients with all types of clinical syndromes and lesions, who require percutaneous coronary interventions (PCI) with DES. Patients at 7 study sites in the Netherlands, Belgium, and Israel, were randomly assigned (1:1, stratified for gender and diabetes mellitus) to treatment with the novel, zotarolimus-eluting, durable polymer-coated Resolute Onyx stent that has a radiopaque, thin-strut, CoreWire stent platform, versus the sirolimus-eluting, bioresorbable polymer-coated Orsiro stent (reference device) that has a very thin-strut, cobalt-chromium stent backbone. The primary endpoint is the 1-year incidence of the composite clinical endpoint target vessel failure (TVF), consisting of cardiac death, target vessel-related myocardial infarction, or clinically indicated target vessel revascularization. A power calculation, assuming a TVF rate of $6.0 \%$ (non-inferiority margin 2.5\%), revealed that 2,470 study patients would give the study $80 \%$ power (alpha-level $5 \%$ ), allowing for up to $3 \%$ loss to followup. The first patient was enrolled on October 7,2015; on December 23,2016 the last patient entered the study.
\end{abstract}

Conclusions: BIONYX is a large-scale, prospective, randomized, international, multicenter trial, comparing a novel DES with durable coating versus a reference DES with biodegradable coating in allcomers. The study is the first randomized assessment of the Resolute Onyx stent, which is an often-used DES outside the United States. 


\section{Background}

Persistent concerns about the limited safety of the first-generation durable polymer coronary drugeluting stents $(D E S)^{1-3}$ prompted the development of newer-generation DES with more biocompatible durable polymer coatings and better safety profiles. ${ }^{4-6}$ These DES showed favorable outcomes following percutaneous coronary interventions ( $\mathrm{PCl}$ ) in broad patient populations and in all-comers of several clinical trials. ${ }^{7-10}$

Most recently, the Resolute Onyx stent (Medtronic Vascular, Santa Rosa, CA, USA) has been developed, which is a newer-generation durable polymer DES that uses a novel, thin-strut metallic stent backbone with increased radiographic visibility and elutes zotarolimus from its BioLinx polymer coating. In parallel with the progress in durable polymer DES, bioresorbable polymer-coated DES have been developed, of which recently introduced devices have very thin struts and flexible stent designs. ${ }^{4,10,11}$ The Orsiro stent (Biotronik, Bülach, Switzerland), which is such a DES, elutes sirolimus from a circumferential coating that is located mainly on the abluminal side of the very thin-strut, cobalt-chromium stent platform. ${ }^{11,12}$ The efficacy and safety of the Orsiro stent has previously been demonstrated in several randomized trials. ${ }^{10,13,14}$ Resolute Onyx and Orsiro represent the respective summit of the engineering efforts of two major device-manufacturing companies in order to optimize the safety and efficacy of coronary DES. Both devices are clinically used in all types of patients and lesion anatomies.

While data from a head-to-head comparison of both stents would be of interest, such data are not available yet. The aim of the present BIONYX (Bioresorbable Polymer Orsiro Versus Durable Polymer Resolute Onyx Stents) trial is to compare the safety and efficacy of the Resolute Onyx and Orsiro stents in all-comers who truly reflect routine clinical practice, addressing a non-inferiority hypothesis.

\section{Investigational products}

\section{Resolute Onyx stent}

The Resolute Onyx stent is a Conformité Européenne-certified, newer-generation permanent polymercoated DES. The stent is circumferentially covered with the $5.6 \mu \mathrm{m}$-thin BioLinx polymer system that elutes zotarolimus as the anti-proliferative agent during a period of 6 months and consists of a blend of three different polymers: (1) the hydrophobic $\mathrm{C} 10$ polymer, which aids in the control of drug release; (2) the hydrophilic C19 polymer, which supports biocompatibility; and (3) polyvinyl pyro-lidinone, which increases the initial drug burst and enhances the elution rate. The stent platform of Resolute Onyx is made from a single strand, swaged shape corewire that is manufactured into a sinusoidal waveform and automatically welded at predefined connection sites. The corewire consists of a denser core made from a platinum-iridium alloy, surrounded by a cobalt-chromium alloy; the inner core increases the stent's radiopacity. The struts of Resolute Onyx (uncoated $81 \mu \mathrm{m}$ in $3.00 \mathrm{~mm}$ stents) are thinner than the struts of its predecessors-Resolute and Resolute Integrity (Medtronic) (uncoated $91 \mu \mathrm{m}$ ). The increased radiopacity and the modified stent design of Resolute Onyx aim to improve visibility and deliverability of the stent while maintaining its longitudinal and radial strength. Stents with nominal diameters ranging from $2.00 \mathrm{~mm}$ to $5.00 \mathrm{~mm}$ were available. Long-term safety and efficacy of other DES that employed the 
same coating as Resolute Onyx has previously been demonstrated. ${ }^{15-17}$ Nevertheless, the BIONYX trial is the first randomized clinical study to assess the novel Resolute Onyx stent in a broad and unrestricted patient population.

\section{Orsiro stent}

The Orsiro stent is a Conformité Européenne-certified DES with a circumferential, asymmetrical, bioresorbable coating that is thicker on the abluminal stent side (7.4 $\mu \mathrm{m}$ vs $3.5 \mu \mathrm{m})$; this configuration of the coating results in a higher drug dose on the abluminal side of the stent. ${ }^{12}$ The PLLA (poly[L-lactide] acid) coating elutes the drug sirolimus within slightly more than 3 months and is gently resorbed within 1 to 2 years, thereby attempting to minimize inflammation. ${ }^{12,18}$ The active coating covers a thin passive coating of amorphous silicon carbide that encapsulates the metal stent to prevent ion leakage (hybrid coating). ${ }^{12,19}$ Orsiro is based on the PRO-Kinetic double-helix stent platform, which is made from a thinstrut cobalt-chromium alloy and has $60 \mu \mathrm{m}$ struts (in stents with a nominal diameter $<3.50 \mathrm{~mm}$ ) or 80 $\mu \mathrm{m}$ struts (in stents with nominal diameters $\geq 3.50 \mathrm{~mm}$ ). During study enrollment, stents with a nominal diameter ranging from $2.25 \mathrm{~mm}$ to $4.00 \mathrm{~mm}$ were available. The efficacy of Orsiro in preventing excessive neointimal proliferation has been assessed in the angiographic endpoint-study BIO-FLOW II, ${ }^{20}$ in which Orsiro demonstrated non-inferiority versus the fluoropolymer-coated, everolimus-eluting Xience Prime stent (Abbott, Santa Clara, CA) with a low in-stent late lumen loss (0.10 $\pm 0.32 \mathrm{~mm}$ vs $0.11 \pm 0.29 \mathrm{~mm})$ and similar binary restenosis rates (4.0\% vs 4.7\%, respectively). The Orsiro stent was previously tested against 3 other DES in large-sized, randomized, non-inferiority studies, which ascertained the safety and efficacy of Orsiro in greatly unrestricted patient populations. ${ }^{10,13,14}$

\section{Methods}

\section{Main study hypothesis and study design}

The BIONYX trial (ClinicalTrials.gov no. NCT02508714) is an international, multicenter, assessor and patient-blinded, randomized clinical trial in an all-comer population. The main objective is to compare the 1-year safety and efficacy of the novel Resolute Onyx durable-polymer stent with that of the established Orsiro bioresorbable-polymer stent (the reference device) in all-comer patients who undergo $\mathrm{PCl}$ with DES implantation. The study assesses whether the safety and efficacy of the novel Resolute Onyx stent is non-inferior to that of Orsiro. The randomization for DES type was performed in a 1:1 ratio after stratification for gender and presence of diabetes mellitus. The investigator-initiated trial was planned by cardiologists of Thoraxcentrum Twente and is performed in close cooperation with cardiologists of all study sites. Biotronik and Medtronic provided equal financial support.

\section{Study population and study sites}

The study examines at least 2,470 all-comer patients. Patients with a minimum age of 18 years, who required $\mathrm{PCl}$ with DES implantation, were eligible for enrollment. Patients with all clinical syndromes were allowed, irrespective of number, type, location, vessel size, or length of lesions to be treated, as long as operators considered them suitable for treatment with both stents. In order to assess patients 
and lesions that reflect routine clinical practice, only few exclusion criteria were applied (Table 1). The study complies with the Declaration of Helsinki and was approved by the Ethical Review Board Twente and by the study centers' institutional review boards. All patients provided written informed consent. Patient enrollment was performed in the Netherlands, in Belgium, and in Israel at 7 study sites (in the Netherlands: Thoraxcentrum Twente, Medisch Spectrum Twente, Enschede; Haga Hospital, The Hague; Treat Zorggroep, Emmen; Rijnstate Hospital, Arnhem; in Belgium: Virga Jessa Hospital, Hasselt; University Hospital Charleroi, Charleroi; in Israel: Rambam Hospital, Haifa). The first patient was enrolled in BIONYX on October 7, 2015; study enrollment was finished on December 23, 2016.

At least 2,470 all-comer patients with coronary artery disease requiring $\mathrm{PCl}$ with DES implantation

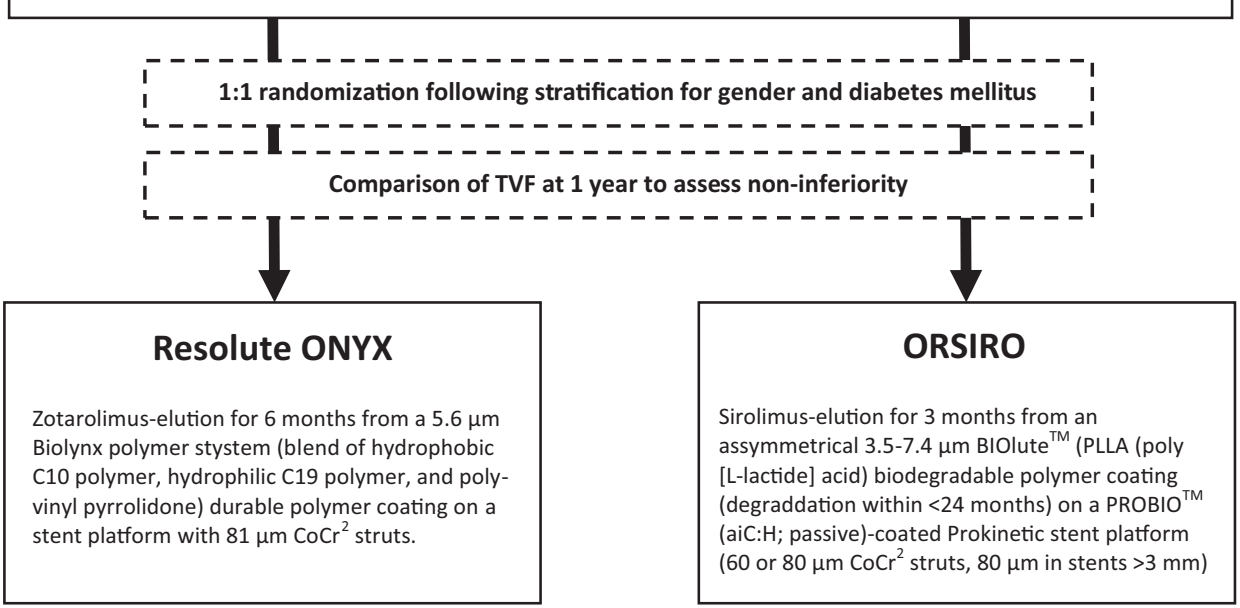

Clinical follow-up $\quad 30$ days, 1 year, 2 years (a follow-up beyond 2 years is intended)

\begin{tabular}{|ll} 
Primairy endpoint & $\begin{array}{l}\text { Incidence of target vessel failure (TVF) at 1-year, a composite of cardiac death, target } \\
\text { vessel-related myocardial infarction, or clinically driven target vessel } \\
\text { revascularization. }\end{array}$ \\
Major secondary endpoint & $\begin{array}{l}\text { Incidence of TVF at 2-year follow-up. } \\
\text { Secondary endpoints }\end{array}$ \\
$\begin{array}{l}\text { Death, myocardial infarction, revascularization, stent thrombosis, target lesion } \\
\text { failure, MACE, patient-oriented composite endpoint }\end{array}$
\end{tabular}

Figure 1: Study Flow Chart.

\section{Study protocol, patient demographics, and medical data}

At baseline, patient demographics and clinical data were collected online in an electronic database of the clinical research organization Diagram (Zwolle, the Netherlands). Laboratory tests were performed in the local laboratories of the participating centers as part of the routine clinical practice. Cardiac biomarkers measurements were scheduled prior to $\mathrm{PCl}$, and 4-18 hours after $\mathrm{PCl}$ or before discharge, with subsequent serial measurements in case of relevant biomarker elevation or complaints until the peak elevation has been measured. Analysts, blinded for the stent type used, will perform angiographic 
analyses and offline quantitative coronary angiographic measurements according to present standards (QangioXA, version 7.3).

Table 1: BIONYX Inclusion and exclusion criteria.

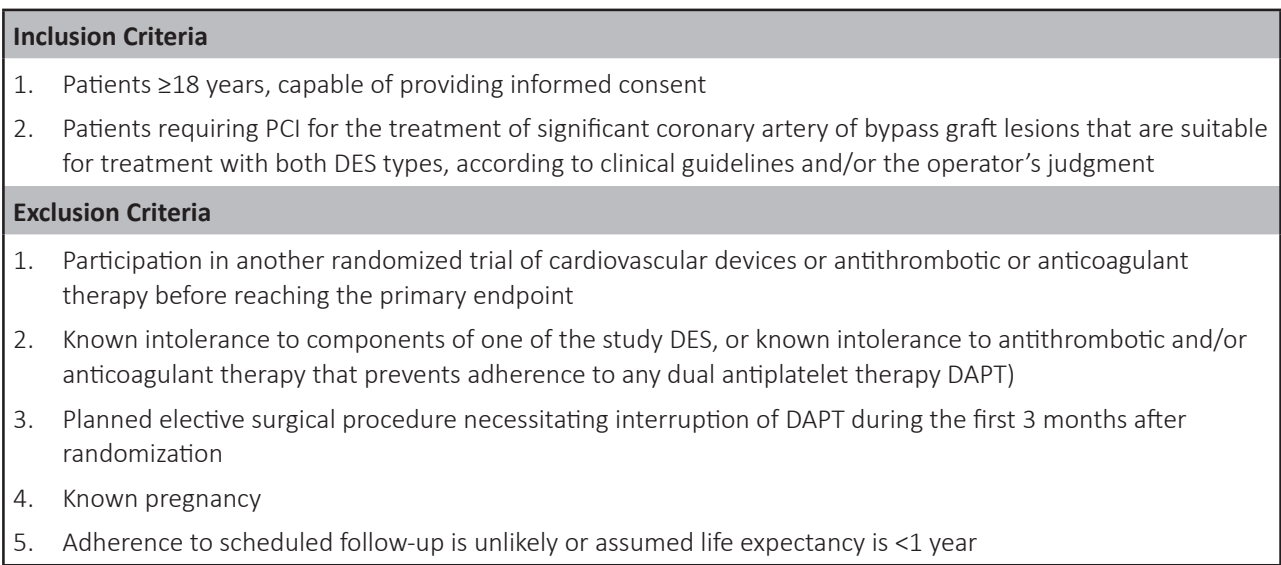

Abbreviations: DAPT=dual antiplatelet therapy; DES=drug-eluting stent; $\mathrm{PCl}=$ percutaneous coronary intervention

$\mathrm{PCl}$ was performed according to routine medical practice and current guidelines. Fractional Flow Reserveassessment of angiographically intermediate stenosis was recommended in accordance with current guidelines. If clinically indicated, use of intravascular ultrasound or optical coherence tomography was permitted for guidance of the $\mathrm{PCl}$ procedure at the operator's discretion. If the operator was unable to insert the randomized study stent, crossover to a stent of choice was allowed. Mixture of stents was generally avoided but was permitted if the operator was unable to insert the randomized stent. Outside the setting of primary $\mathrm{PCl}$, we generally encouraged the treatment of all target lesions in one session, if this was reasonable and safe. However, staged PCI procedures (defined as procedures planned at the time of the index procedure or shortly thereafter and performed within 6 weeks with the same type of study stent) were permitted. During follow-up, in patients with potential restenosis and visually determined lumen narrowing $\leq 80 \%$, the use of Fractional Flow Reserve is encouraged to evaluate its hemodynamic significance and the potential indication for reintervention. In case of additional unplanned revascularization procedures, the use of the allocated DES type was recommended, except for the treatment of an in-stent restenosis of one of the study stents.

Medical therapy during the $\mathrm{PCl}$ procedure was performed according to local routine medical treatment. The use of glycoprotein IIb/IIla inhibitors was left at the operators' discretion. Dual antiplatelet therapy was recommended for 6 to 12 months, according to current medical guidelines. In patients on oral anticoagulation, triple therapy was recommended for at least 1 month, after which oral anticoagulation in combination with a P2Y12 receptor-antagonist, generally clopidogrel, was prescribed for 6 to 12 months. Moreover, in patients on oral anticoagulation, gastric protection with a proton pump inhibitor was generally suggested. 


\section{Follow-up data collection}

After 1 month, $12( \pm 1)$ months, and $24( \pm 1)$ months, follow-up data will be collected during routine visits to the outpatient clinic, or if not feasible, by a medical questionnaire and/or a telephone follow-up, carried out by staff that is blinded to the allocated treatment. Patients will be interviewed regarding repeat hospitalization, revascularization procedures, and myocardial infarction during followup. Survival is checked from the municipal population register. In case of death, information will be obtained from the patients' medical chart, general practitioner, or the referring cardiologist. Pending approval of additional financial support, the investigators are willing to extend the open-label follow-up beyond 24 months until 5 years.

\section{Clinical endpoints and definitions}

The primary composite endpoint is the incidence of target vessel failure (TVF) at 1-year follow-up, which evaluates both, device efficacy and patient safety. Components of TVF are in hierarchical order: cardiac death, target vessel myocardial infarction (MI), or clinically indicated target vessel revascularization. Cardiac death is defined as any death caused by proximate cardiac cause, unwitnessed death, death of unknown cause, and all procedure-related deaths, including those related to concomitant therapy. As in our previous trials, ${ }^{8-10,21}$ target vessel $\mathrm{Ml}$ is defined as any creatine kinase concentration of more than double the upper limit of normal with elevated values of a confirmatory cardio biomarker, and can be related to a target vessel or cannot be related to another vessel. Clinically indicated target vessel revascularization includes revascularization procedures by $\mathrm{PCl}$ and coronary artery bypass grafting. TVF at 2-year follow-up is a major secondary endpoint. Other secondary endpoints include device and patient-oriented efficacy and safety parameters such as target lesion failure, major adverse cardiac events, patient-oriented composite endpoint, and stent thrombosis according to the definitions of the Academic Research Consortium. ${ }^{22}$

\section{Sample size calculation}

The main outcome parameter is the difference in TVF between the two treatment arms after 12 months. At least 2,470 patients had to be enrolled based on a power calculation that assumed TVF rates of $6.0 \%$ at 1-year follow-up, based on outcome data of the DUTCH PEERS (Durable Polymer-Based Stent Challenge of Promus Element Versus Resolute Integrity) trial, ${ }^{9}$ with a $2.5 \%$ non-inferiority margin, giving the study a power of $80 \%$ with an alpha level of 0.05 and allowing for at least $3 \%$ loss to followup. If the upper limit of the 1-sided $95 \%$ confidence interval for the between-DES difference is less than $2.5 \%$, non-inferiority will be declared. Sample size calculation was performed with PASS software (NCSS, Kaysville, Utah, USA).

\section{Randomization}

Randomization was performed by a computer program, and the randomization was done in blocks of 8 and 4 in random order. Moreover, patients were stratified according to the presence of diabetes mellitus and gender before randomization. 


\section{Statistical considerations}

The primary endpoint TVF at 1-year will be analyzed by the log-rank test by comparing the time to the primary endpoint using Kaplan-Meier method. Non-inferiority will be achieved, if the upper limit of the 1-sided $97.5 \%$ confidence interval of the absolute risk difference is less than the non-inferiority margin. The primary analyses will be performed based on intention-to-treat. Pre-specified subgroup analyses will be performed in analogy to previous clinical trials of our group..$^{8-10}$ Subgroup analyses will be performed to assess consistency of treatment effect across different subsets. $P<0.05$ will be considered statistically significant, except for the primary analyses, as outlined.

\section{Trial organization}

Trial organization and data management will be performed by Cardio Research Enschede, Enschede, the Netherlands. Study monitoring will be carried out by an independent external contract research organization (Diagram, Zwolle, the Netherlands). An independent external clinical event committee will adjudicate all potential adverse clinical events. Moreover, an independent Data Safety Monitoring Board will evaluate safety interim analyses of all-cause mortality in both stent arms, performed after the inclusion of $33 \%$ and $66 \%$ of the patient population. The authors are solely responsible for the design and conduct of this study, all study analyses, the drafting and editing of the manuscript, and its final contents. Sponsors will have no access to the study database and are not involved in the interpretation of data or manuscript preparation.

\section{Discussion}

The prospective, international, multicenter BIONYX trial performs in all-comer patients who undergo $\mathrm{PCl}$ a randomized head-to-head comparison of the safety and efficacy of implanting the novel, zotarolimuseluting Resolute Onyx stent with a durable polymer coating versus the established, sirolimus-eluting Orsiro stent with a bioresorbable polymer coating (reference device). The study will evaluate clinical outcome in a non-inferiority setting by comparing the composite primary endpoint TVF at 1-year follow-up. In addition, the study will assess established device- and patient-oriented composite clinical endpoints (e.g. target-lesion failure, major adverse cardiac events, and patient oriented composite endpoint), acute angiographic results as well as the rate of stent thrombosis. All-comer studies are particularly valuable, as their results best reflect the device performance in routine clinical practice and the study findings may be generalized to a great extent. ${ }^{6-10,13-17,23-25}$ Based on the findings of previous clinical studies that examined BioLinx-coated zotarolimus-eluting stents other than Resolute Onyx $x^{10,15,16,17}$ and on the results of randomized trials that compared durable polymer DES with early or newer bioresorbable polymer DES $10,13,14,23-26$, non-inferiority of Resolute Onyx versus Orsiro may be expected. Nevertheless, so far this hypothesis has never been tested, and Resolute Onyx and Orsiro stents have never been compared in clinical practice.

The novel stent platform that is employed in the Resolute Onyx stent uses cobalt-chromium struts with a platinum-iridium core. Because of the more dense strut core, radiographic visibility of the novel stent is increased. This might decrease the risk of geometrical miss, for instance when treating ostial 
disease and short or severely calcified target lesions. Moreover, it may facilitate the identification of stents with suboptimal expansion that may benefit from vigorous postdilation. Similar to Resolute Onyx, platinum-chromium-based everolimus-eluting stents have a superior angiographic visibility. ${ }^{9,10,27}$ In the DUTCH PEERS trial, lesions that had been treated with the more radiopaque Promus Element stent (Boston Scientific, Natick, MA) were significantly more often postdilated than lesions in which an angiographically less visible cobalt-chromium stent had been implanted (79\% vs $74 \%, p=0.002){ }^{.}$It has been argued that the excellent radiographic visibility of the platinum-chromium stents might have contributed to that higher frequency of stent postdilation. ${ }^{9}$ On the other hand, if stents have a high radiographic visibility, longitudinal stent deformation, which sporadically occurs in most contemporary $D E S,{ }^{28,29}$ can be more easily identified and corrected. The latter may explain to some extent why in the DUTCH PEERS trial longitudinal deformation of the Promus Element stent turned out to be rather benign - even after cessation of dual anti-platelet therapy. ${ }^{9,30}$

Adding platinum to stent strut material is known to provide solid solution strengthening that permits a significant reduction in strut thickness. ${ }^{31}$ In the Resolute Onyx stent, the novel corewire strut material and subtle changes in stent design allowed to reduce the thickness of the (uncoated) strut from $91 \mu \mathrm{m}$ in the stent's predecessor (Resolute Integrity) ${ }^{9}$ to now $81 \mu \mathrm{m}$. Previously, thin struts were shown to reduce strut malapposition, flow disturbance, thrombogenicity, and restenosis. ${ }^{32-34}$ In the Orsiro stents with a nominal diameter of $3.5 \mathrm{~mm}$ or larger, the thickness of the uncoated stent strut is $80 \mu \mathrm{m}$ and thus similar to Resolute Onyx, while Orsiro stents $<3.5 \mathrm{~mm}$ have even thinner struts. ${ }^{10}$

Most DES only use 2 basic stent designs to cover their entire range of stent diameters provided..$^{35}$ This is not the case for Resolute Onyx that has 4 basic stent designs to cover its range of stent diameters $(2.0 \mathrm{~mm}$ to $5.0 \mathrm{~mm}$ ), which currently is wider than for any other DES type, including the predecessor Resolute Integrity. ${ }^{35,36}$ In large sized vessels, such as the left main stem and some saphenous vein graft, the availability of an extra large stent may avoid incomplete stent apposition, which is an important risk factor of stent thrombosis in DES. ${ }^{37}$ In very small target vessels - typically side branches or very distal vessels-stents with a nominal diameter of $2.0 \mathrm{~mm}$ may be useful to avoid incomplete stent expansion which is related to a higher risk of restenosis and stent thrombosis. ${ }^{38}$ Moreover, in Resolute Onyx the risk of thrombus formation may be expected to be low, as in previous clinical studies of other BioLinxcoated (Resolute-type) zotarolimus-eluting stents the risk of definite stent thrombosis was found to be low. ${ }^{16,39}$

In the BIONYX trial, the role of the Orsiro stent as reference device is more than justified. As a matter of fact, three randomized all-comer studies demonstrated noninferiority of Orsiro versus the respective comparator DES and showed excellent clinical outcomes for Orsiro. ${ }^{10,13,14}$ In the BIOSCIENCE trial, Orsiro was compared with the everolimus-eluting Xience stent (Abbott Vascular); both DES showed at 12 -month follow-up similar rates of the primary composite endpoint target lesion failure (6.5\% vs $6.6 \%, p=0.95) .{ }^{13}$ The BIO-RESORT trial, which so far is the only randomized trial that compared Orsiro with the Resolute Integrity stent-the predecessor of Resolute Onyx, revealed after 12 months similar rates of the primary endpoint TVF for both stents ( $4.7 \%$ vs $5.4 \%, p=0.46) .{ }^{10}$ While BIO-RESORT has three treatment arms, it was formally not designed to compare the Orsiro stent with the Synergy stent (Boston Scientific ${ }^{27}$ which is another modern, very thin-strut, bioresorbable polymer DES. Nevertheless, it is undeniably of interest that the TVF rates were low and similar for these two DES $(4.7 \%$, for Orsiro as 
well as Synergy). ${ }^{10}$ Moreover, in the SORT OUT VII trial Orsiro was noninferior to the early-generation, bioresorbable polymer, biolimus-eluting Nobori stent (Terumo, Japan) for target lesion failure at 12 months ( $3.8 \%$ vs $4.6 \%, p=0.34$ ); however, the definite stent thrombosis rate was lower in the Orsiro stent group ( $0.4 \%$ vs $1.2 \%, p=0.03) .{ }^{14}$ The results of all three aforementioned trials represent strong evidence for the safety and clinical efficacy of the Orsiro stent and support the choice of Orsiro as the reference device in the BIONYX trial.

Thus, BIONYX is a large-scale, prospective, randomized (1:1), controlled, international, multicenter trial with 2 arms, comparing in all-comer patients two dissimilar contemporary DES. The study will provide new insights into the clinical outcome of $\mathrm{PCl}$ with modern bioresorbable polymer versus permanent polymer DES in patients who reflect routine clinical practice. In addition, the study is the first randomized assessment of the Resolute Onyx stent, which is an often-used DES outside the United States. 


\section{References}

1. Camenzind E, Steg PG, Wijns W. Stent thrombosis late after implantation of first generation drug-eluting stents: a cause for concern. Circulation 2007;115:1440-55.

2. Joner M, Finn AV, Farb A, et al. Pathology of drug-eluting stents in humans: delayed healing and late thrombotic risk. J Am Coll Cardiol 2006;48:193-202.

3. Basalus MW, Joner M, von Birgelen C, Byrne RA. Polymer coatings on drug-eluting stents: Samson's hair and Achilles' heel? Eurolntervention 2013;9:302-5.

4. Stefanini GG, Holmes DR Jr. Drug-eluting coronary-artery stents. N Engl J Med 2013;368:254-65.

5. Stone GW, Rizvi A, Newman W, et al.; SPIRIT IV Investigators. Everolimus-eluting versus paclitaxel-eluting stents in coronary artery disease. N Engl J Med. 2010;362:1663-74.

6. Kedhi E, Joesoef KS, McFadden E, et al. Second-generation everolimus-eluting and paclitaxel-eluting stents in real-life practice (COMPARE): a randomised trial. Lancet 2010;375:201-9.

7. Serruys PW, Silber S, Garg S, et al. Comparison of zotarolimus-eluting and everolimus-eluting coronary stents. N Engl J Med 2010;363:136-46.

8. von Birgelen C, Basalus MW, Tandjung K, et al. A randomized controlled trial in second-generation zotarolimuseluting Resolute stents versus everolimus-eluting Xience $V$ stents in real-world patients: the TWENTE trial. J Am Coll Cardiol 2012;59:1350-61.

9. von Birgelen C, Sen H, Lam MK, et al. Third-generation zotarolimus-eluting and everolimus-eluting stents in all-comer patients requiring a percutaneous coronary intervention (DUTCH PEERS): a randomised, single-blind, multicentre, non-inferiority trial. Lancet 2014;383:413-23.

10. von Birgelen C, Kok MM, van der Heijden LC, et al. Very thin strut biodegradable polymer everolimus-eluting and sirolimus-eluting stents versus durable polymer zotarolimus-eluting stents in allcomers with coronary artery disease (BIO-RESORT): a three-arm, randomised, non-inferiority trial. Lancet 2016;388:2607-17.

11. Stefanini GG, Taniwaki M, Windecker S. Coronary stents: novel developments. Heart 2014;100:1051-61.

12. Tittelbach M, Diener T. Orsiro: the first hybrid drug-eluting stent, opening up a new class of drug-eluting stents for superior patient outcomes. Interventional Cardiology 2011;6:142-4.

13. Pilgrim T, Heg D, Roffi M, et al. Ultrathin strut biodegradable polymer sirolimus-eluting stent versus durable polymer everolimus-eluting stent for percutaneous coronary revascularisation (BIOSCIENCE): a randomised, single-blind, non-inferiority trial. Lancet 2014;384:2111-22.

14. Jensen LO, Thayssen P, Maeng M, et al. Randomized comparison of a biodegradable polymer ultrathin strut sirolimus-eluting stent with a biodegradable polymer biolimus-eluting stent in patients treated with percutaneous coronary intervention: the SORT OUT VII trial. Circ Cardiovasc Interv 2016;9:e003610.

15. Iqbal J, Serruys PW, Silber S, et al. Comparison of zotarolimus- and everolimus-eluting coronary stents: final 5-year report of the RESOLUTE All-Comers trial. Circ Cardiovasc Interv 2015;8:e002230.

16. von Birgelen C, van der Heijden LC, Basalus MWZ, et al. Five-year outcome after implantation of zotarolimusand everolimus-eluting stents in randomized trial participants and nonenrolled eligible patients: a secondary analysis of a randomized clinical trial. JAMA Cardiol 2017; in press, e-published ahead of print: January 18, 2017 (doi:10.1001/jamacardio.2016.5190).

17. van der Heijden LC, Kok MM, Löwik MM, et al. Three-year safety and efficacy of treating all-comers with newergeneration Resolute Integrity or Promus Element stents in the randomized DUTCH PEERS TWENTE II) trial. Eurolntervention 2017; in press (doi:10.4244/EIJ-D-16-00571).

18. Hamon M, Niculescu R, Deleanu D, Dorobantu M, Weissman NJ. Waksman R. Clinical and angiographic experience with a third-generation drug-eluting Orsiro stent in the treatment of single de novo coronary artery lesions (BIOFLOW-I): a prospective, first-in-man study. Eurolntervention 2013;8:1006-11.

19. Heublein B, Pethig K, Özbek C, Elsayed M, Bolz A, Schaldach M. Silicon carbide coating-a new hybrid design of coronary stents. Prog Biomed Res 1998;1:33-9.

20. Windecker S, Haude M, Neumann FJ, et al. Comparison of a novel biodegradable polymer sirolimus-eluting stent with a durable polymer everolimus-eluting stent: results of the randomized BIOFLOW-II trial. Circ Cardiovasc Interv 2015;8:e001441. 
21. Tandjung K, Basalus MW, Sen H, et al. DUrable polymer-based sTent CHallenge of Promus ElemEnt versus ReSolute integrity (DUTCH PEERS): rationale and study design of a randomized multicenter trial in a Dutch allcomers population. Am Heart J 2012;163:557-62.

22. Vranckx P, Cutlip DE, Mehran R, et al. Myocardial infarction adjudication in contemporary all-comer stent trials: balancing sensitivity and specificity. Eurolntervention 2010;5:871-4.

23. Windecker S, Serruys PW, Wandel S, et al. Biolimus-eluting stent with biodegradable polymer versus sirolimuseluting stent with durable polymer for coronary revascularisation (LEADERS): a randomised non-inferiority trial. Lancet 2008;372:1163-73.

24. Christiansen EH, Jensen LO, Thayssen $\mathrm{P}$, et al. Bioloimus-eluting biodegradable polymer-coated stent versus durable polymer-coated sirolimus-eluting stent in unselected patients receiving percutaneous coronary intervention (SORT OUT V): a randomised non-inferiority trial. Lancet 2013;381:661-69.

25. Smits PC, Hofma S, Togni M, et al. Abluminal biodegradable polymer biolimus-eluting stent versus durable polymer everolimus-eluting stent (COMPARE II): a randomised, controlled, non-inferiority trial. Lancet 2013;381:651-60.

26. Natsuaki M, Kozuma K, Morimoto T, et al. Final 3-year outcome of a randomized trial comparing secondgeneration drug-eluting stents using either biodegradable polymer or durable polymer Nobori biolimus-eluting versus Xience/Promus everolimus-eluting stent trial. Circ Cardiovasc Interv 2015;8:e002817.

27. Kereiakes DJ, Meredith IT, Windecker S, et al. Efficacy and safety of a novel bioabsorbable polymer-coated, everolimus-eluting coronary stent: the EVOLVE II randomized trial. Circ Cardiovasc Interv 2015;8:e002372.

28. Ormiston JA, Webber B, Ubod B, White J, Webster MW. Stent longitudinal strength assessed using point compression: insights from a second-generation, clinically related bench test. Circ Cardiovasc Interv 2014;7:629.

29. Ota $\mathrm{H}$, Kitabata $\mathrm{H}$, Magalhaes MA, et al. Comparison of frequency and severity of longitudinal stent deformation among various drug-eluting stents: an intravascular ultrasound study. Int J Cardiol 2014;175:261-7.

30. Sen H, Lam MK, Löwik MM, et al. Clinical events and patient-reported chest pain in all-comers treated with Resolute Integrity and Promus Element stents: 2-year follow-up of the DUTCH PEERS randomized trial (TWENTE II). J Am Coll Cardiol Interv 2015;8:889-99.

31. O'Brien B, Carroll W. The evolution of cardiovascular stent materials and surfaces in response to clinical drivers: a review. Acta Biomaterialia 2009;5:945-58.

32. Kolandaivelu K, Swaminathan R, Gibson WJ, et al. Stent thrombogenicity early in high-risk interventional settings is driven by stent design and deployment and protected by polymer-drug coatings. Circulation 2011;123:1400-9.

33. Kastrati A, Mehilli J, Dirschinger J, et al. Intracoronary stenting and angiographic results: strut thickness effect on restenosis outcome (ISAR-STEREO) trial. Circulation 2001;103:2816-21.

34. Briguori C, Sarais C, Pagnotta P, et al. In-stent restenosis in small coronary arteries: impact of strut thickness. J Am Coll Cardiol 2002;40:403-9.

35. Ng J, Foin N, Ang HY, et al. Over-expansion capacity and stent design model: an update with contemporary DES platforms. Int J Cardiology 2016;221:171-179.

36. Foin $\mathrm{N}$, Alegria $\mathrm{E}$, Sen $\mathrm{S}$, et al. Importance of knowing stent design threshold diameters and post-dilatation capacities to optimise stent selection and prevent stent overexpansion/incomplete apposition during PCl. Int J Cardiol 2013;166:755-8.

37. Cook S, Wenaweser $\mathrm{P}$, Togni $\mathrm{M}$, et al. Incomplete stent apposition and very late stent thrombosis after drugeluting stent implantation. Circulation 2007;115:2426-34.

38. Fujii K, Carlier SG, Mintz GS, et al. Stent underexpansion and residual reference segment stenosis are related to stent thrombosis after sirolimus-eluting stent implantation: an intravascular ultrasound study. J Am Coll Cardiol 2005;45:995-8

39. Silber S, Kirtane AJ, Belardi JA, Liu M, Brar S, Rothman M, Windecker S. Lack of association between dual antiplatelet therapy use and stent thrombosis between 1 and 12 months following Resolute zotarolimus-eluting stent implantation. Eur Heart J 2014;35:1949-56. 




\section{Part III}

Contemporary drug-eluting stents for complex lesions 


\section{Conflicts of interest statement}

CVB has been unpaid consultant to various device-manufacturing companies. Thoraxcentrum Twente has received research grants provided by Abbott Vascular, AstraZeneca, Biotronik, Boston Scientific, and Medtronic. All other authors declare that they have no conflict of interest. 


\section{CHAPTER 9}

Long-Term Outcome of Consecutive Patients With Previous Coronary Bypass Surgery, Treated With Newer-Generation Drug-Eluting Stents

Liefke C. van der Heijden, Marlies M. Kok, Paolo Zocca, Hanim Sen, Marije M. Löwik, Silvia Mariani, Frits H.A.F. de Man, Marc Hartmann, Martin G. Stoel, K. Gert van Houwelingen, J.(Hans) W. Louwerenburg, Gerard C.M. Linssen, Carine J.M. Doggen, Jan G. Grandjean, Clemens von Birgelen
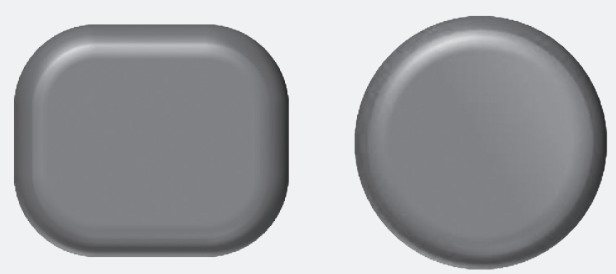


\begin{abstract}
Background: Percutaneous coronary intervention (PCI) in patients with previous coronary artery bypass grafting $(C A B G)$ is associated with adverse clinical events. While the use of newer-generation drugeluting stents (DES) showed favorable short-term safety profiles, there is a lack of long-term outcome data. We evaluated the impact of previous CABG on 5-year clinical outcome of patients treated with $\mathrm{PCl}$ using newer-generation DES.
\end{abstract}

Methods and Results: In this patient-level pooled analysis of the prospective TWENTE trial and NonEnrolled TWENTE registry, we assessed a consecutive series of patients who underwent $\mathrm{PCI}$ with newergeneration DES for non-ST-elevation acute coronary syndromes or stable angina.

Of all 1,709 patients, 202 (11.8\%) had a history of CABG. Patients with previous CABG had significantly higher 5 -year rates of cardiac death $(10.4 \%$ vs $4.3 \%$; $p<0.001)$ and target vessel revascularization $(25.0 \%$ vs $8.1 \% ; \mathrm{p}<0.001)$. These differences remained statistically significant after adjustment for differences in baseline characteristics. Landmark analysis revealed that from 1 to 5 -year follow-up, the rates of cardiac death $(8.1 \%$ vs $3.2 \% ; p<0.001)$ and target vessel revascularization $(17.1 \%$ vs $5.9 \% ; p<0.001)$ were significantly higher in patients with previous CABG. Among patients with a history of CABG, PCI of an obstructed vein graft was associated with a higher rate of 5 -year target vessel revascularization $(\mathrm{p}=0.003)$.

Conclusions: Until 5-year follow-up after PCI with newer-generation DES, the risk of cardiac death and target vessel revascularization was significantly higher in patients with previous CABG. The target vessel revascularization rate was highest in patients who underwent $\mathrm{PCl}$ of obstructed vein grafts. 


\section{Introduction}

Patients with a history of coronary artery bypass grafting (CABG) are often older, have more comorbidities, and require treatment of more complex target lesions. ${ }^{1-3}$ Gradual failure of a bypass graft, lesion recurrence, and disease progression in native coronary vessels are the main causes of repeat revascularization in patients with a history of $\mathrm{CABG}^{4-7}$ Current guidelines recommend percutaneous coronary intervention ( $\mathrm{PCl}$ ) as the first choice for treating late (>1 month) graft failure, because of an increased mortality risk associated with redo-CABG. 8,9

$\mathrm{PCl}$ in patients with previous bypass surgery are often complex and more often associated with adverse clinical outcome..$^{10-12}$ Several studies have shown significantly higher rates of repeat target vessel revascularization ${ }^{7,13}$ - in particular if $\mathrm{PCl}$ was performed in degenerated saphenous vein grafts (SVG), which also bear an increased risk for embolizing friable debris and atherothrombotic material., ${ }^{2,14-18}$ The introduction of the early drug-eluting stents (DES) improved clinical outcomes of $\mathrm{PCl}$ in bypass grafts as compared to bare metal stents. ${ }^{19-21}$ Newer-generation DES showed even more favorable short-term safety profiles ${ }^{14}$, but there is a lack of long-term outcome data after $\mathrm{PCl}$ with newer-generation DES, comparing patients with versus without previous CABG. ${ }^{15,16}$

Therefore, in the present study we investigated the 5-year clinical outcome of a consecutive series of patients treated with $\mathrm{PCl}$ with newer-generation DES for stable angina or non-ST-elevation acute coronary syndromes ${ }^{22}$, and evaluated the impact of previous CABG on the long-term outcome. In addition, among patients with a history of $C A B G$, we compared the long-term clinical results following $\mathrm{PCl}$ in native coronary arteries versus bypasses.

\section{Methods}

\section{Study design and patient population}

This analysis was performed using the patient-level pooled data $(n=1,709)$ from the prospective TWENTE trial and the Non-Enrolled TWENTE registry, ${ }^{22}$ details of these studies have previously been reported. ${ }^{23,24}$ In brief, the TWENTE trial (Clinicaltrials.gov NCT01066650) is an investigator-initiated, patient-blinded, randomized, comparative DES trial with limited exclusion criteria. ${ }^{23} \mathrm{~A}$ total of 1,391 patients were enrolled between June 18, 2008 and August 26, 2010 at Thoraxcentrum Twente, Enschede, the Netherlands, and were 1:1 randomized to treatment with Resolute zotarolimus-eluting stents (Medtronic Vascular, Santa Rosa, CA) or Xience V everolimus-eluting stents (Abbott Vascular, Santa Clara, CA) ${ }^{23}$ During the course of the randomized trial, 318 eligible but non-enrolled patients were treated at the operator's discretion with one of the DES that were examined in the randomized trial, using the same routine clinical and procedural strategies. ${ }^{24}$ Both studies complied with the Declaration of Helsinki for investigation in human beings and were approved by the Medical Ethical Committee Twente and the institutional review board. All participants in the randomized trial provided written informed consent. For the registry, patients were not required to change behavior or take action other than following their regular treatment; therefore, according to Dutch law, and approved by the Medical Ethical Committee Twente, written informed consent from patients in this registry was not required. 
Pooling data from both studies permitted the assessment of a consecutive series of 1,709 patients who were treated at a high-volume tertiary center for cardiac intervention (Thoraxcentrum Twente, Enschede, the Netherlands) by PCI with second-generation DES for non-ST-elevation acute coronary syndromes or stable angina. The current study evaluated the impact of previous CABG on 5-year outcome. Baseline characteristics and 1-year clinical outcome of patients with versus without previous CABG have been reported. ${ }^{14}$

\section{Clinical procedures}

Patients from the TWENTE trial and the Non-enrolled TWENTE registry were treated by the same operators. Interventional procedures were performed according to standard techniques, routine clinical procedures, and current medical guidelines. Details of the intervention, medical treatment, ECG assessment and laboratory testing have been described ${ }^{23,24}$ and did not differ between studies.

\section{Definitions of clinical endpoints}

Endpoint definitions, which did not differ between the randomized trial and registry ${ }^{23,24}$, were based on suggestions from the Academic Research Consortium (ARC) ${ }^{25}$, including the addendum on the definition of myocardial infarction (MI). ${ }^{26}$ In brief, death was considered cardiac, unless an evident non-cardiac cause could be established. MI was defined by any creatine kinase concentration of more than double the upper limit of normal with elevated values of confirmatory cardiac biomarkers. Target vessel MI was related to the target vessel or could not be related to another vessel. Target vessel revascularization was defined as any repeat coronary revascularization of the target vessel by re-PCl or surgery.

\section{Acquisition and analysis of clinical follow-up data}

Clinical follow-up data were obtained at visits to outpatient clinics or, if not feasible, by telephone or medical questionnaire. Monitoring was performed by an independent, external clinical research organization (Diagram, Zwolle, the Netherlands). Independent contract research organizations (Cardialysis, Rotterdam, the Netherlands, and (Diagram, Zwolle, the Netherlands) performed the adjudication of adverse clinical events for both randomized trial participants and non-enrolled eligible patients.

\section{Statistical analysis}

Data were reported as frequencies and percentages for dichotomous and categorical variables and as mean \pm standard deviation for continuous normally distributed variables. Chi-square test and Fisher's exact test were used as appropriate. Differences between groups in continuous variables were assessed with the Student's $t$ test. The time to clinical endpoints was assessed according to Kaplan-Meier methods and the Logrank-test was applied for between-group comparisons. Hazard ratios were computed using Cox proportional hazards regression analysis. Parameters were considered as potential confounders if associations were found with a $p$-value $\leq 0.15$ in univariate analyses. Propensity score analysis was used for adjustment of potential confounders. The propensity score was estimated using multiple logistic regression analysis. A multivariate Cox regression model, including the propensity score as an independent variable, was then used to adjust for the propensity score. A two-sided $p$-value $<0.05$ was considered significant. Data analysis was performed with SPSS (version 22.0, SPSS Inc., Chicago, IL, USA). 


\section{Results}

\section{Baseline characteristics}

Of all 1,709 patients, 202 (11.8\%) had a history of CABG, which had been performed $11.2 \pm 8.5$ years prior to their index $\mathrm{PCl}$. Sole use of arterial grafts only was present in $18 \%$ of the patients, while $63 \%$ were treated with a combination of arterial graft(s) and SVG. Obstructed bypass grafts were present in 141 (69.8\%) patients with previous CABG (arterial graft: 22.7\%, SVG: 77.3\%). In 111 of these patients, the obstructed graft was the culprit lesion (i.e. lesion causing complaints), but only 65 (58.6\%) patients underwent graft $\mathrm{PCl}$, while the other $46(41.4 \%)$ patients were treated in native coronary vessels. Differences in baseline characteristics are shown in Table 1.

Table 1: Baseline characteristics.

\begin{tabular}{|c|c|c|c|}
\hline & \multicolumn{2}{|c|}{$\begin{array}{c}\text { All patients } \\
n=1709\end{array}$} & \multirow[b]{2}{*}{ p-value } \\
\hline & $\begin{array}{c}\text { Previous CABG } \\
n=202\end{array}$ & $\begin{array}{c}\text { No previous CABG } \\
n=1507\end{array}$ & \\
\hline Age (yrs) & $68.5 \pm 9.4$ & $64.1 \pm 10.7$ & $<0.001$ \\
\hline Women & $41(20.3)$ & 435 (28.9) & 0.011 \\
\hline Diabetes mellitus & $58(28.7)$ & 315 (20.9) & 0.012 \\
\hline Hypertension & $113(55.9)$ & $845(56.1)$ & 0.972 \\
\hline Hypercholesterolemia & 143/199 (71.9) & $853 / 1476(57.8)$ & $<0.001$ \\
\hline Current smoker & $22(10.9)$ & $388(25.7)$ & $<0.001$ \\
\hline Family history of coronary artery disease & 108/181( 59.7) & $734 / 1403$ (52.3) & 0.062 \\
\hline Previous myocardial infarction & $82(40.6)$ & $505(33.5)$ & 0.046 \\
\hline Previous percutaneous coronary intervention & $81(40.1)$ & $299(19.8)$ & $<0.001$ \\
\hline Clinical syndrome at presentation & & & 0.023 \\
\hline Non-ST-elevation myocardial infarction & $40(19.8)$ & 435 (28.9) & \\
\hline Unstable angina & $51(25.2)$ & $358(23.8)$ & \\
\hline Stable angina & $111(55.0)$ & $714(47.4)$ & \\
\hline Chronic renal failure & $13(6.4)$ & $46(3.1)$ & 0.013 \\
\hline Left ventricular ejection fraction $<30 \%$ & 10/144 (6.9) & $35 / 1106(3.2)$ & 0.022 \\
\hline Peripheral arterial disease & $26(14.0)$ & $122(9.0)$ & 0.032 \\
\hline Multivessel treatment & $52(25.7)$ & $345(22.9)$ & 0.368 \\
\hline Total number of lesions treated per patient & & & 0.381 \\
\hline One & $133(65.8)$ & $927(61.5)$ & \\
\hline Two & $49(24.3)$ & $436(28.9)$ & \\
\hline Three or more & $20(9.9)$ & $144(9.6)$ & \\
\hline At least one chronic total occlusion treated & $12(5.9)$ & $111(7.4)$ & 0.462 \\
\hline
\end{tabular}

Values are $\mathrm{n}(\%)$ unless otherwise stated. Abbreviation: $\mathrm{CABG}=$ coronary artery bypass grafting. 


\section{Clinical event rates at 5-year follow-up}

Patients with previous CABG had a significantly higher 5-year rate of target vessel revascularization than patients without previous CABG $(25.0 \%$ vs $8.1 \%$; $p<0.001$ ) (Table 2), while the rate of definite stent thrombosis was low and similar for both groups (0.6\% vs. $0.8 \%)$. Cardiac death occurred more often in patients with previous CABG (10.4\% vs $4.3 \%$; $p<0.001)$. There was no between-group difference in the incidence of non-target vessel-related revascularization (7.9\% vs $7.5 \%$ ). The time-to-event curves of several outcome parameters are displayed in Figure 1.

Multivariate analysis with propensity score adjustment demonstrated, after adjustment for all available known potential confounders, that the 5-year rates of target vessel revascularization (adjusted HR 3.10, $95 \% \mathrm{Cl}$ 2.09-4.61) and cardiac death (adjusted HR 1.87, 95\% Cl 1.03-3.38) were significantly higher in patients with previous CABG (Table 2).

Table 2: 5 -year clinical event rates in patients with previous CABG versus patients without a history of bypass surgery.

\begin{tabular}{|c|c|c|c|c|c|c|}
\hline & & & $\begin{array}{l}\text { All patien } \\
n=1,70\end{array}$ & & & \\
\hline & $\begin{array}{c}\text { Previous } \\
\text { CABG } \\
n=202\end{array}$ & $\begin{array}{c}\text { No previous } \\
\text { CABG } \\
n=1,507\end{array}$ & $\begin{array}{l}\text { Unadjusted hazard } \\
\text { ratio }(95 \% \mathrm{Cl})\end{array}$ & p-value & $\begin{array}{l}\text { Adjusted hazard } \\
\text { ratio }(95 \% \mathrm{Cl})\end{array}$ & p-value \\
\hline Any death & $35(17.4)$ & $150(10.1)$ & $1.81(1.25-2.61)$ & 0.001 & $1.37(0.89-2.11)$ & 0.15 \\
\hline Cardiac death & $20(10.4)$ & $63(4.3)$ & $2.46(1.49-4.06)$ & $<0.001$ & $1.87(1.03-3.38)$ & 0.04 \\
\hline Any myocardial infarction & $22(11.5)$ & $107(7.3)$ & $1.58(1.00-2.50)$ & 0.05 & $1.63(0.97-2.75)$ & 0.07 \\
\hline $\begin{array}{l}\text { Target vessel myocardial } \\
\text { infarction }\end{array}$ & 19 (9.9) & $94(6.4)$ & $1.55(0.94-2.53)$ & 0.08 & $1.71(0.98-2.98)$ & 0.06 \\
\hline $\begin{array}{l}\text { Target vessel } \\
\text { revascularization }\end{array}$ & $47(25.0)$ & $116(8.1)$ & $3.41(2.43-4.79)$ & $<0.001$ & $3.10(2.09-4.61)$ & $<0.001$ \\
\hline $\begin{array}{l}\text { Target lesion } \\
\text { revascularization }\end{array}$ & $38(20.3)$ & $85(5.9)$ & $3.71(2.53-5.44)$ & $<0.001$ & $3.49(2.23-5.45)$ & $<0.001$ \\
\hline $\begin{array}{l}\text { Non-target vessel } \\
\text { revascularization }\end{array}$ & 15 (7.9) & $107(7.5)$ & $1.08(0.63-1.86)$ & 0.78 & $0.86(0.47-1.59)$ & 0.63 \\
\hline Target vessel failure & $73(37.6)$ & $231(15.7)$ & $2.66(2.04-3.46)$ & $<0.001$ & 2.69 (1.99-3.65) & $<0.001$ \\
\hline Definite stent thrombosis & $1(0.6)$ & $11(0.8)$ & $0.69(0.09-5.36)$ & 0.72 & $0.69(0.07-6.43)$ & 0.74 \\
\hline
\end{tabular}

Values are $\mathrm{n}(\%)$. Data were analyzed using the Kaplan-Meir method, which implies that patients who could not be followed up for the entire 5 years because of death, consent withdrawal, or loss to follow-up were censored at the exact moment of dropout. Therefore, the percentages provided in the Table may slightly differ from the results of straightforward calculations of nominator divided by denominator. Abbreviation: $\mathrm{CABG}=$ coronary artery bypass grafting.

Landmark analysis between 1- and 5-year follow-up showed a significantly higher cardiac mortality in patients with previous CABG (8.1\% vs $3.2 \%$; $p<0.001$ ) (Figure 2$)$, while there was no significant betweengroup difference in cardiac death during the first 12 months (2.5\% vs $1.1 \% ; p=0.11$ ). In addition, target vessel revascularization occurred more often in patients with previous CABG during both the first 12 months and from 1 - to 5 -year follow-up (0-1 year: $9.5 \%$ vs $2.4 \%$, p<0.001; $1-5$ years: $17.1 \%$ vs. $5.9 \%$, $\mathrm{p}<0.001$ ) (Figure 2). 

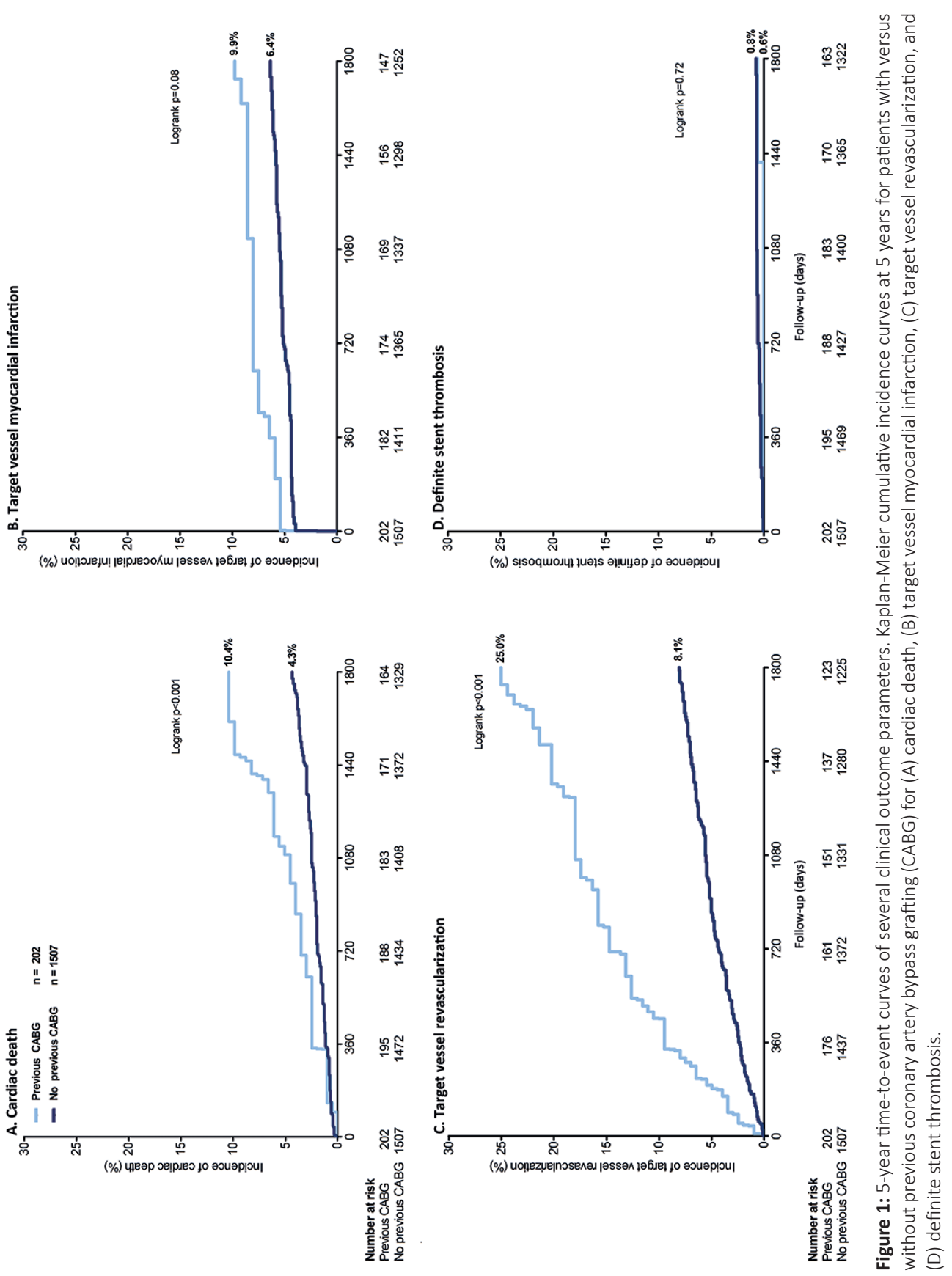


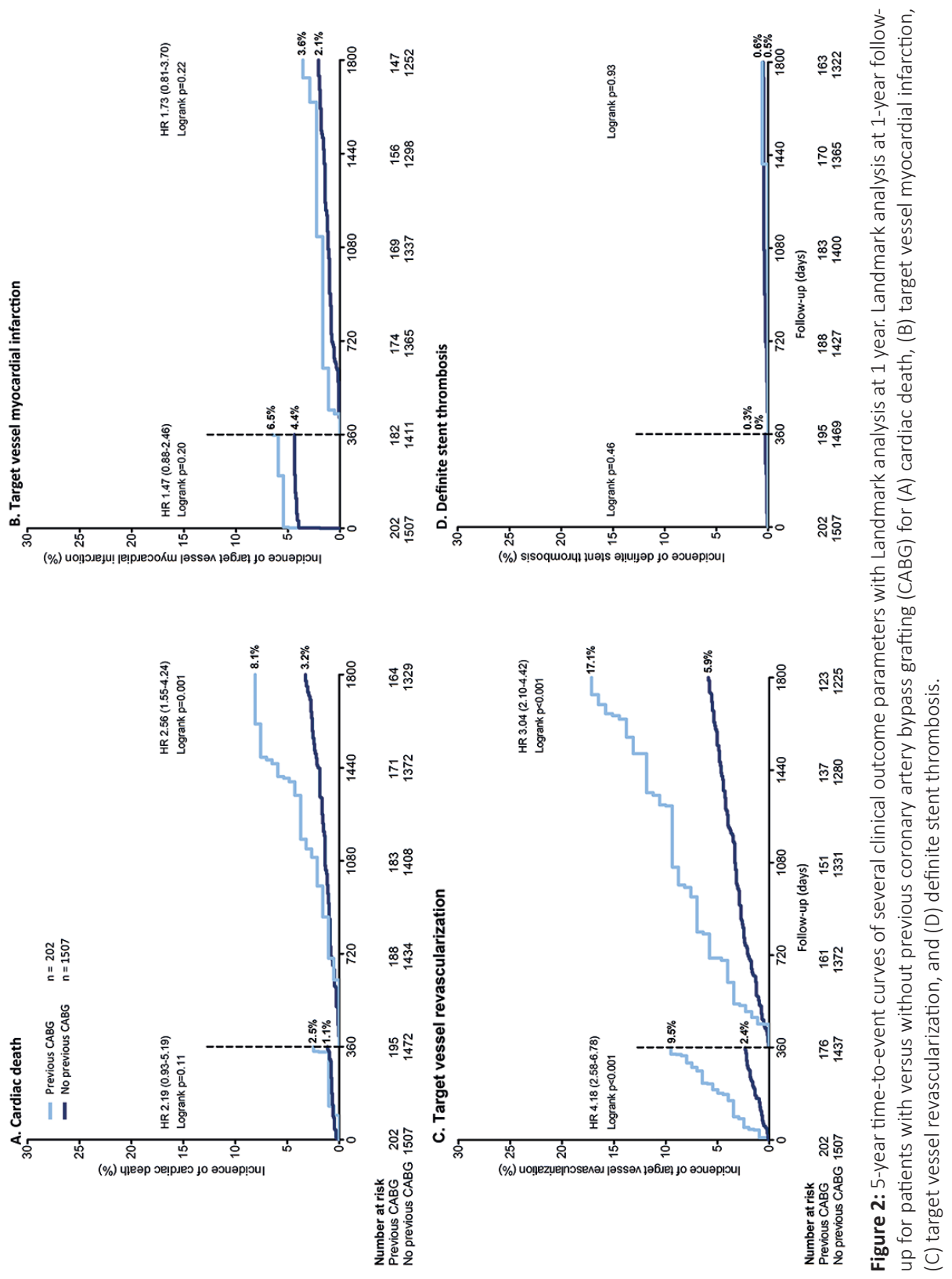




\section{Subgroup analysis among patients with previous CABG}

The outcome of patients with a history of CABG was assessed according to the actually treated vessel. $\mathrm{PCl}$ of a diseased bypass graft was associated with a worse 5-year clinical outcome (Table 3). Target vessel revascularization rates were higher in patients with graft lesions who underwent $\mathrm{PCl}$ of the bypass graft than in patients (with or without graft lesions) who were treated exclusively in native coronary arteries (39.6\% vs $18.5 \%$ and $18.1 \%$; $=0.003$ ) (Figure 3 ).

Table 3: Subgroup analysis in patients with previous CABG, based on culprit and target vessels: 5-year outcome.

\begin{tabular}{|lcccc|}
\hline & \multicolumn{4}{c|}{$\begin{array}{c}\text { Patients with previous CABG } \\
\mathbf{n = 2 0 2}\end{array}$} \\
& $\begin{array}{c}\text { Culprit vessel is } \\
\text { native vessel that } \\
\text { was stented } \\
\mathbf{n = 9 1}\end{array}$ & $\begin{array}{c}\text { Culprit vessel is bypass } \\
\text { graft, but native vessel } \\
\text { was stented } \\
\mathbf{n = 4 6}\end{array}$ & $\begin{array}{c}\text { Culprit vessel is } \\
\text { bypass graft that } \\
\text { was stented } \\
\mathbf{n = 6 5}\end{array}$ & p-value \\
Cardiac death & $9(10.6)$ & $7(15.3)$ & $4(6.5)$ & 0.33 \\
Target vessel myocardial infarction & $6(6.9)$ & $4(9.1)$ & $9(14.7)$ & 0.30 \\
Target vessel revascularization & $15(18.1)$ & $8(18.5)$ & $24(39.6)$ & 0.003 \\
\hline
\end{tabular}

Values are $\mathrm{n}(\%)$. Data were analyzed using the Kaplan-Meir method, which implies that patients who could not be followed up for the entire 5 years because of death, consent withdrawal, or loss to follow-up were censored at the exact moment of dropout. Therefore, the percentages provided in the Table may slightly differ from the results of straightforward calculations of nominator divided by denominator. Abbreviation: $\mathrm{CABG}=$ coronary artery bypass grafting.

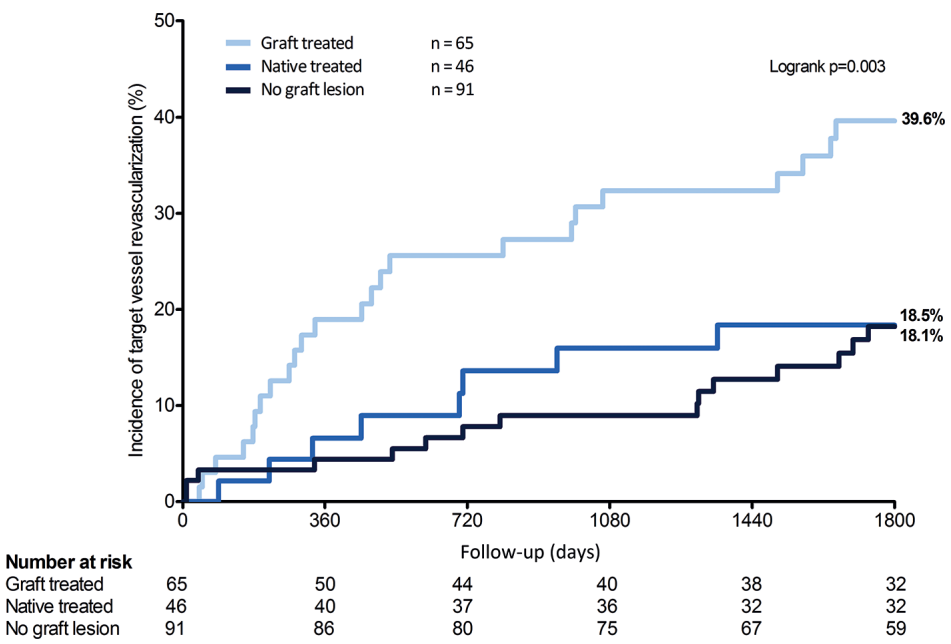

Figure 3: 5-year time-to-event curves of target vessel revascularization in subgroups of patients with previous CABG. Kaplan-Meier cumulative incidence curves for target vessel revascularization for subgroups of patients with a history of CABG 


\section{Discussion}

\section{Main findings}

We assessed the 5-year outcome of a consecutive series of 1,709 patients, treated with $\mathrm{PCl}$ with secondgeneration DES for non-ST-elevation acute coronary syndromes or stable angina, and then compared the long-term outcome of 202 patients with previous CABG versus 1,507 patients who had no history of bypass surgery. This is the first study to assess the impact of previous CABG on 5-year outcome after PCI with newer-generation DES. Patients with previous CABG had significantly higher 5 -year rates of target vessel revascularization ( $25.0 \%$ vs $8.1 \%)$ and cardiac death $(10.4 \%$ vs $4.3 \%)$ than patients who had no history of CABG. These differences remained statistically significant after adjustment for between-group differences in baseline characteristics. When the analysis was confined to events that occurred within the second to fifth year of follow-up, patients with versus without previous CABG still showed significant differences in the aforementioned clinical endpoints. Among patients with a history of CABG, $\mathrm{PCl}$ of an obstructed bypass graft was associated with a higher rate of 5 -year target vessel revascularization.

\section{Previous CABG and cardiovascular event risk}

Previous studies reported conflicting mortality data following $\mathrm{PCl}$ in patients with versus without a history of CABG. 7,10-13,15,17 In patients presenting with ST-elevation MI, there was no baseline-adjusted difference in 1-year outcome between patients with versus without previous $C_{A B G^{7,13}}$, but in other studies, among patients treated for various types of acute coronary syndromes, 1-year mortality was significantly higher in patients with a history of CABG. ${ }^{10,12}$ In addition, among patients with various types of acute coronary syndromes, the long-term baseline-adjusted risk of all-cause and cardiac mortality following treatment with or without PCl were not significantly increased in patients who had a history of CABG. ${ }^{11,15,17}$

In the present study, following adjustment for potential confounders, 5-year cardiac mortality risk was significantly higher in all-comer patients who previously had undergone CABG (adjusted HR 1.87). This difference was mainly based on a significantly higher rate of cardiac death during the second to fifth year of follow-up ( $8.1 \%$ vs $3.2 \%$ ). This difference in cardiac mortality from the aforementioned studies may be largely explained by differences in study population and treatment. In the current study, we assessed a consecutive series of patients who were treated with newer-generation DES for all clinical syndromes, except for ST-elevation MI, 14,22 while other studies with long-term follow-up exclusively examined patients with ST-elevation MI or patients with acute coronary syndromes. ${ }^{10,11,17}$ Furthermore, these patients had been treated with or without PCl-prior to the introduction of the newer-generation DES. ${ }^{11,17}$

In our study, patients with a history of CABG, treated with PCI with newer-generation DES, had a 3.1-times higher baseline-adjusted risk of repeat target vessel revascularization during the 5-year follow-up as compared to patients who had no history of CABG. In the era of early-generation DES, the risk of repeat target vessel revascularization was also higher in patients with previous CABG. ${ }^{10,11,15,17}$ 


\section{Venous and arterial bypass grafts}

We observed that the risk of repeat revascularization was highest in patients who had undergone a stent implantation in a diseased SVG, which corroborates previous studies. ${ }^{15-17,27,28}$ In addition, we found that patients with a stenosis in a bypass graft who were treated in the native vessel had a target vessel revascularization rate that was similar to patients with non-obstructed (i.e. patent) bypass grafts who were treated for native vessel lesions (18.5\% vs $18.1 \%)$. Both subtroups of patients had relatively high rates of target vessel revascularization as compared to patients without a history of CABG, which may be due to high lesion complexity, comorbidities, and aggressive atherosclerosis and disease progression in native coronary vessels in patients with a history of CABG.

A known disadvantage of using saphenous veins as bypass material is the accelerated progression of atherosclerosis in SVG, which leads to friable plaques with a high risk of embolization and thrombosis and ultimately high rates of graft stenosis and bypass occlusion. ${ }^{1-3}$ Ten years after CABG, approximately $75 \%$ of SVG are severely diseased or occluded. ${ }^{29,30}$ Attrition in SVG and the development of new native vessel stenoses (distal to the bypass anastomosis) due to progression of coronary atherosclerosis are typical causes of late graft failure. ${ }^{2}$ Therefore, PCI in SVG is associated with a higher risk of atheroma embolization, resulting in no-reflow phenomenon, graft perforation, and restenosis as compared to $\mathrm{PCl}$ in native coronary vessels. ${ }^{18}$

In contrast to SVG, arterial bypass grafts are significantly less susceptible to attrition and accelerated atherosclerosis; therefore, they are more likely to remain patent than SVG. ${ }^{4}$ The most frequent causes of arterial graft dysfunction are neo-intimal hyperplasia secondary to a vascular trauma during surgical preparation of the graft or the anastomosis and general disease progression in the native coronary vasculature.$^{31} \mathrm{~A}$ greater patency of arterial grafts was also observed in the present study population. Patients with previous $C A B G$ required less often treatment of the left descending artery than patients without previous CABG (17.3\% vs 55.4\%), due to (generally) proper functioning of the left internal mammary artery to left descending artery-bypass grafts.

\section{Long-term outcome of $\mathrm{PCl}$ with newer-generation DES after previous CABG}

Most CABG-related studies examined patients treated with bare metal stents or first-generation DES. ${ }^{12,13,15,16}$ The use of first generation drug-eluting stents (DES) in graft vessel lesions was associated with improved long-term clinical outcomes as compared to bare metal stents. ${ }^{19-21}$ Little is known about the use of second-generation DES in patients with previous CABG. ${ }^{14,32,33}$ Long-term follow-up data are scarce, which are of particular interest as a previous study suggested the presence of a late catch-up in target vessel revascularization after PCl with first-generation DES in SVG. ${ }^{34}$

A relatively small, retrospective, observational study that included patients during a period of 9 years (with inevitable changes in PCl-technique, devices, and medication) found significantly lower 1- and 2 -year rates of a composite endpoint of safety and efficacy in patients treated with newer- versus firstgeneration DES..$^{32}$ Another retrospective analysis used a historical control group to show, at 1-year followup, no significant differences in target vessel revascularization between early-generation versus newergeneration DES (10.2\% vs $10.7 \%) .{ }^{33}$ Our group previously reported a target vessel revascularization rate of $9.4 \%$ in patients with a history of CABG at 1-year follow-up. ${ }^{14}$ The difference in event rate from the abovementioned study may be best explained by dissimilarities in study population. 


\section{Implications of the study}

This first analysis of 5-year follow-up after $\mathrm{PCl}$ with newer-generation DES in patients with versus without previous $C A B G$ has demonstrated a persistently low risk of $\mathrm{MI}$ and stent thrombosis despite a history of CABG. PCI with newer-generation DES in patients with previous CABG is safe, but the high long-term rate of target vessel revascularization shows that, after treatment with newer-generation DES, the risk of repeat revascularizations remains high. In times of increasingly low adverse event rates of randomized all-comer trials $s^{35}$, it will be of paramount importance to keep enrolling patients with a history of CABG in order to ensure adequate statistical power. In clinical practice, knowledge of the safety but increased risk of repeat target vessel revascularization following $\mathrm{PCl}$ in patients with previous coronary bypass surgery - in particular if a diseased SVG requires treatment- will be relevant to cardiologists and other physicians involved in heart team discussions and informed consent.

\section{Limitations}

The sample size of the CABG group $(n=202)$ limits the power of the study to show statistically significant differences in outcomes, especially in subgroup analyses. The results of this post-hoc analysis are hypothesis generating, and the findings should not be transferred to the setting of primary PCl, as patients with acute ST-elevation MI were not assessed. The choice of treating native vessels or bypass grafts were left to the operator's discretion; as a native vessel $\mathrm{PCl}$ is generally the preferred approach ${ }^{9}$, patients with the most complex native vessel disease may have undergone bypass treatment because a native vessel PCl was not an option anymore. However, in the present study the high TVR rate in patients treated in SVG supports the "native vessel first" strategy.

\section{Conclusions}

Until 5-year follow-up after $\mathrm{PCI}$ with newer-generation DES, the risk of cardiac death and target vessel revascularization was significantly higher in patients with previous CABG. The target vessel revascularization rate was highest in patients who underwent $\mathrm{PCl}$ of obstructed vein grafts.

\section{Sources of Funding}

Abbott Vascular and Medtronic equally supported the randomized TWENTE trial. The present study was performed without external funding. 


\section{References}

1. Tejada JG, Velazquez M, Hernandez F, et al. Percutaneous revascularization in patients with previous coronary artery bypass graft surgery. Immediate and 1-year clinical outcomes. Int J Cardiol 2009;134:201-6.

2. Scarsini R, Zivelonghi C, Pesarini G, Vassasnelli C, Ribichini FL. Repeat revascularization: percutaneous coronary intervention after coronary bypass graft surgery. Cardiovasc Revasc Med 2016;17:272-8.

3. Ribichini F, Pugno F, Ferrero V, Wijns W, Vacca G, Vassanelli C, Virmani R. Long-term histological and immunohistochemical findings in human venous aorto-coronary bypass grafts. Clin Sci (Lond) 2008;114:211-20.

4. Sabik JF, Blackstone EH, Gillinov AM, Smedira NG, Lytle BW. Occurrence and risk factors for reintervention after coronary artery bypass grafting. Circulation 2006;114[Suppl. I]:I-454-60.

5. Tatoulis J, Buxton BF, Fuller JA. Patency of 2127 arterial to coronary conduits over 15 years. Ann Thorac Surg 2004; 77:93-101.

6. Escaned J. Secondary revascularization after CABG surgery. J Nat Rev Cardiol 2012;9:540-9.

7. Garg P, Kamaruddin H, Iqbal J, Wheeldon N. Outcomes of primary percutaneous coronary intervention for patients with previous coronary artery bypass grafting presenting with ST-segment elevation myocardial infarction. Open Cardiovasc Med J 2015;9:99-104.

8. Morrison DA, Sethi G, Sacks J, Henderson WG, Grover F, Sedlis S, Esposito R. Percutaneous coronary intervention versus repeat bypass surgery for patients with medically refractory myocardial ischemia: AWESOME randomized trial and registry experience with post-CABG patients. J Am Coll Cardiol 2002;40:1951-4.

9. Windecker S, Kohl P, Alfonso F, et al. 2014 ESC/EACTS Guidelines on myocardial revascularization: the Task Force on myocardial revascularization of the European Society of Cardiology (ESC) and the European Association for Cardio-Thoracic Surgery (EACTS) developed with the special contribution of the European Association of Percutaneous Cardiovascular Interventions (EAPCI). Eur Heart J 2014;35:2541-19.

10. Nikolsky E, McLaurin BT, Cox DA, Manoukian SV, Xu K, Mehran R, Stone GW. Outcomes of patients with prior coronary artery bypass grafting and acute coronary syndromes: analysis from the ACUITY (acute catheterization and urgent intervention triage strategy) trial. J Am Coll Cardiol Intv 2012;5:919-26.

11. Berry C, Pieper KS, White HD, et al. Patients with prior coronary artery bypass grafting have a poor outcome after myocardial infarction: an analysis of the valsartan in acute myocardial infarction trial (VALIANT). Eur Heart J 2009;30:1450-6.

12. Teixeira R, Vieira MJ, Ribeiro MA, Goncalves L, Gersh BJ. Prognosis following acute coronary syndromes according to prior coronary artery bypass grafting: meta-analysis. Eur Heart J Acute Cardiovasc Care 2015;4:518-27.

13. Iqbal J, Kwok CS, Kontopantelis E, et al. Outcomes following primary percutaneous coronary intervention in patients with previous coronary artery bypass surgery. Circ Cardiovasc Interv 2016;9:e003151.

14. Sen H, Lam MK, Tandjung K, et al. Impact of previous coronary artery bypass surgery on clinical outcome after percutaneous interventions with second generation drug-eluting stents in TWENTE trial and Non-Enrolled TWENTE registry. Int J Cardiol 2014;176:885-90.

15. Yamaji K, Kimura T, Morimoto T, Nakagawa Y, Inoue K, Nobuyoshi M, Mitsudo K. Percutaneous coronary intervention in patients with previous coronary artery bypass grafting (from the J-Cypher Registry). Am J Cardiol 2013;112:1110-9.

16. Xanthopoulou I, Davlouros P, Tsigkas G, Panagiotou A, Hahalis G, Alexopoulos D. Long-term clinical outcome after percutaneous coronary intervention in graft vs native vessels in patients with previous coronary artery bypass grafting. Can J Cardiol 2011;27:716-24.

17. Nikolsky E, Mehran R, Yu J, et al. Comparison of outcomes of patients with ST-segment elevation myocardial infarction with versus without previous coronary artery bypass grafting (from the harmonizing outcomes with revascularization and stents in acute myocardial infarction [HORIZONS-AMI] trial). Am J Cardiol 2013;111:137786.

18. Brilakis ES, Saeed B, Banerjee S. Drug-eluting stents in saphenous vein grafts interventions: a systematic review. Eurolntervention 2010;5:722-30.

19. Mehilli J, Pache J, Abdel-Wahab M, et al. Drug-eluting versus bare-metal stents in saphenous vein graft lesions (ISAR-CABG): a randomised controlled superiority trial. Lancet 2011;378:1071-8.

20. Brilakis ES, Lichtenwalter C, Abdel-Karim AR, et al. Continued benefit from paclitaxel-eluting compared with bare-metal stent implantation in saphenous vein graft lesions during long-term follow-up of the SOS (stenting of saphenous vein grafts) trial. J Am Coll Cardiol Intv 2011;4:176-82. 
21. Agostoni P, Vermeersch P, Semeraro O, et al. Intravascular ultrasound comparison of sirolimus-eluting stent versus bare metal stent implantation in diseased saphenous vein grafts (from the RRISC [reduction of restenosis in saphenous vein grafts with Cypher sirolimus-eluting stent] trial). Am J Cardiol 2007;100:52-8.

22. von Birgelen C, van der Heijden LC, Basalus MW, et al. Five-year outcome after implantation of zotarolimus- and everolimus-eluting stents in randomized participants and nonenrolled eligible patients: a secondary analysis of a randomized trial. JAMA Cardiol 2017;2:268-76.

23. von Birgelen C, Basalus MWZ, Tandjung K, et al. A randomized controlled trial in second-generation zotarolimuseluting Resolute stents versus everolimus-eluting Xience $V$ stents in real-world patients: the TWENTE trial. J Am Coll Cardiol 2012;59:1350-61.

24. Sen H, Tandjung K, Basalus MWZ, et al. Comparison of eligible non-enrolled patients and the randomized TWENTE trial population treated with Resolute and XIENCE V drug-eluting stents. Eurolntervention 2012;8:66471.

25. Cutlip DE, Windecker S, Mehran R, et al. Clinical endpoints in coronary stent trials: a case for standardized definitions. Circulation 2007;115:2344-51.

26. Vranckx P, Cutlip DE, Mehran R, Kint PP, Silber S, Winecker S, Serruys PW. Myocardial infarction adjudication in contemporary all-comer stent trials: balancing sensitivity and specificity. Addendum to the historical MI definitions used in stent studies. Eurolntervention 2010;5:871-4.

27. Lopes RD, Mehta RH, Hafley GE, et al. Relationship between vein graft failure and subsequent clinical outcomes after coronary artery bypass surgery. Circulation 2012;125:746-56.

28. Brilakis ES, Rao SV, Banerjee S, et al. Percutaneous coronary intervention in native arteries versus bypass grafts in prior coronary artery bypass grafting patients: a report from the National Cardiovascular Data Registry. J Am Coll Cardiol Intv 2011;4:844-50.

29. FitzGibbon GM, Leach AJ, Kafka HP, Keon WJ. Coronary bypass graft fate: long-term angiographic study. J Am Coll Cardiol 1991;17:1075-80.

30. Campeu L, Enjalbert M, Lesperance J, Vaisilic C, Grondin CM, Bourassa MG. Atherosclerosis and late closure of aortocoronary saphenous vein grafts: sequential angiographic studies at 2 weeks, 1 year, 5 to 7 years, and 10 to 12 years after surgery. Circulation 1983;68:II-1-7.

31. Hwang MH, Meadows WR, Palac RT, Piao ZE, Pfiarre R, Loeb HS, Gunnar RM. Progression of native coronary artery disease at 10 years: insights from a randomized study of medical versus surgical therapy for angina. J Am Coll Cardiol 1990;16:1066-70.

32. Kitabata H, Loh JP, Pendyala LK, et al. Two-year follow-up of outcomes of second-generation everolimus-eluting stents versus first-generation drug-eluting stents for stenosis of saphenous vein grafts used as aortocoronary conduits. Am J Cardiol 2013;112:61-7.

33. Costopoulos C, Latib A, Naganuma T, et al. Comparison of first- and second-generation drug-eluting stents in saphenous vein grafts used as aorto-coronary conduits. Am J Cardiol 2013;112:318-22.

34. Yang TH, Kim DI, Jin HY, et al. Angiographic late catch-up phenomenon after sirolimus-eluting stent implantation. Int J Cardiol 2012;160:48-52.

35. von Birgelen C, Kok MM, van der Heijden LC, et al. Very thin strut biodegradable polymer everolimus-eluting and sirolimus-eluting stents versus durable polymer zotarolimus-eluting stents in allcomers with coronary artery disease (BIO-RESORT): a three-arm, randomised, non-inferiority trial. Lancet 2016;388:2607-17. 



\section{Conflicts of interest statement}

CVB has been consultant to and has received lecture fees or travel expenses from Abbott Vascular, Boston Scientific, and Medtronic; he received lecture fees from MSD and AstraZeneca. The institution has received research grants, provided by Abbott Vascular, Biotronik, Boston Scientific, and Medtronic. MJIJ is consultant to PANAXEA b.v.; he has received payments for lectures from Roche, Pfizer, and Sanofi Aventis. All other authors declare that they have no conflict of interest.

\section{Acknowledgments}

The authors thank Dr. M. Brusse-Keizer from Medisch Spectrum Twente in Enschede for statistical advice.

\section{Copyright}

Reprinted with permission of

Clinical Research in Cardiology 2016 Mar;105(3): 206-15.

(C) Springer 


\title{
CHAPTER 10
}

\author{
Bifurcation Treatment With Novel, \\ Highly Flexible Drug-Eluting Coronary Stents \\ in All-Comers
}

2-Year Outcome in Patients of the DUTCH PEERS Trial

Liefke C. van der Heijden, Marlies M. Kok, Ming Kai Lam, Peter W. Danse, Alexander R. Schramm, Gillian A.J. Jessurun, R. Melvyn Tjon Joe Gin,

K. Gert van Houwelingen, Raymond W.M. Hautvast, Gerard C.M. Linssen, Hanim Sen, Marije M. Löwik, Maarten J. IJzerman, Carine J.M. Doggen, Clemens von Birgelen
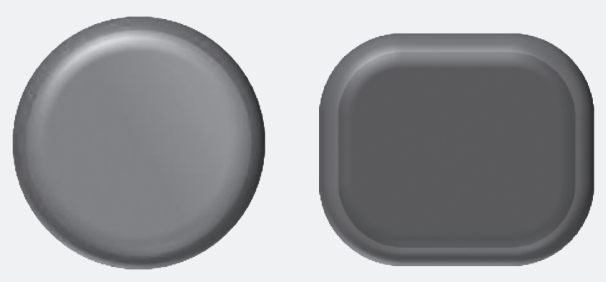

Clin Res Cardiol. 2016;105:206-15. 


\section{Abstract}

Background: Percutaneous coronary intervention (PCI) in bifurcated lesions with second-generation drug-eluting stents (DES) was associated with increased myocardial infarction (MI) rates. Flexible stent designs that accommodate well to vessel tapering may be of benefit in challenging anatomies such as bifurcated target lesions, but so far data are scarce.

Methods: We analyzed the 2-year follow-up data of the DUTCH PEERS (TWENTE II) trial, which randomized 1,811 all-comer patients to $\mathrm{PCl}$ with newer generation Resolute Integrity zotarolimuseluting (Medtronic) or Promus Element everolimus-eluting stents (Boston Scientific). In bifurcated lesions, provisional stenting was generally performed. Target vessel failure is a composite endpoint, consisting of cardiac death, target vessel MI, or target vessel revascularization.

Results: Patients with at least one bifurcated lesion ( $n=465,25.7 \%$ ) versus patients with non-bifurcated target lesions only ( $n=1,346,74.3 \%$ ) showed similar rates of clinical endpoints including target vessel failure $(9.2 \%$ vs $7.9 \%, p=0.36)$ and definite stent thrombosis $(0.4 \%$ vs $1.0 \%, p=0.38)$. Target vessel $\mathrm{Ml}$ was more common in patients with bifurcated lesions ( $3.4 \%$ vs $1.6 \%, p=0.02$ ); but after multivariate analysis with propensity score adjustment, bifurcation treatment was found not to be an independent predictor of target vessel MI (HR 1.40, 95\% Cl: 0.71-2.76, p=0.34). Among patients with bifurcated lesions, DES type and side-branch size did not affect outcome, but periprocedural $\mathrm{Ml}$ occurred more often after two-stent approaches ( $9.0 \%$ vs $2.1 \%, p=0.002$ ).

Conclusion: All-comer patients treated for bifurcated and non-bifurcated target lesions showed similar and low rates of clinical endpoints, suggesting that the DES used are efficacious and safe for treating bifurcated target lesions. 


\section{Introduction}

Stenting of bifurcated target lesions is among the most challenging procedures in the field of percutaneous coronary intervention ( $\mathrm{PCl})^{1,{ }^{1,2}}$ In bifurcated lesions, the introduction of the first generation of drug-eluting stents (DES) reduced the need for repeat revascularization as compared to the previously used bare metal stents. ${ }^{2-8}$ DES of the second generation, which employed coatings with an improved biocompatibility and thinner struts than the first generation, have shown favorable clinical results in both broad patient populations ${ }^{9-18}$ and bifurcated lesions ${ }^{19-21}$. Despite generally encouraging clinical outcomes, the rate of myocardial infarction (MI), in particular of periprocedural MI (PMI), was still higher following stenting of bifurcated lesions as compared to non-bifurcated lesions. ${ }^{20,21}$

Recently, novel DES have been developed with thinner struts and/or more flexible stent designs that accommodate well to vessel tapering, which may be of benefit in challenging anatomies such as bifurcated lesions..$^{22,23}$ The DUTCH PEERS randomized trial compares two such DES in an all-comer patient population and has shown similar and favorable results for both devices up to 2-year follow-up in the overall study population..$^{24,25}$

While the use of highly flexible DES has resulted in an overall low $\mathrm{MI}$ rate ${ }^{24}$, it is unknown whether the implantation of such modern stents may still be associated with an increased risk of MI in bifurcated target lesions. In the present study, we assessed the hypothesis that there may be no difference in safety and efficacy of these flexible DES in treating patients with bifurcated versus non-bifurcated target lesions. In addition, among patients with bifurcated lesions, we evaluated the potential impact of stent type, side-branch size, kissing-balloon inflation, and technical complexity of the procedure on 2-year clinical outcome.

\section{Methods}

\section{Patient population and study design}

The present study was performed using the 2-year follow-up data of the randomized, patient-blinded, multicenter DUTCH PEERS trial. ${ }^{25}$ Details of the DUTCH PEERS (TWENTE II) trial ClinicalTrials.gov NCT01331707) have previously been reported. ${ }^{24}$ In brief, the trial compares (1:1 randomization) the Resolute Integrity zotarolimus-eluting cobalt-chromium stent (Medtronic Vascular, Santa Rosa, CA) and the Promus Element everolimus-eluting platinum-chromium stent (Boston Scientific, Natick, MA) in 1,811 all-comer patients. Patients were enrolled between November 25, 2010 and May 24, 2012. The trial complies with the Declaration of Helsinki and was approved by the Medical Ethics Committee Twente and the institutional review boards of all participating centers. All patients provided written informed consent. Interventional procedures and application of concomitant medication were performed in accordance to medical guidelines, clinical standards, and the physician's judgment. The generally recommended approach of bifurcation lesion treatment was provisional stenting, but the technique of stenting, medical treatment strategy, and use of final kissing-balloon inflation were left at the operator's discretion. ${ }^{24}$ 


\section{Clinical follow-up, monitoring, adjudication, and angiographic analysis}

A detailed description of the 2-year follow-up data has previously been reported. ${ }^{25}$ Data monitoring was performed by the independent contract research organization (CRO) Diagram (Zwolle, the Netherlands). The independent CRO Cardialysis (Rotterdam, the Netherlands) performed the processing of clinical outcome data and clinical event adjudication. Experienced angiographic analysts from Thoraxcentrum Twente, blinded for the stent-type and clinical outcome, performed offline quantitative coronary angiographic analyses according to current standards for all patients from the four study centers (Qangio XA 7.2, Medis, Leiden, the Netherlands).

\section{Data analysis}

For the purpose of the present analysis, patients were categorized into patients with at least one bifurcated target lesion versus patients with non-bifurcated lesions. A relevant side-branch was defined, according to the definition in the SYNTAX score, as a junction of a main vessel and a side-branch with minimum lumen diameter $\geq 1.5 \mathrm{~mm}$ (after intracoronary administration of nitrates and before $\mathrm{PCl}$ ), as measured by quantitative coronary angiography. ${ }^{26}$ Further analyses among patients with bifurcated lesions involved comparisons between [1] the two allocated stents; [2] bifurcated lesions with sidebranch $\geq 2.0 \mathrm{~mm}$ versus side-branch $<2.0 \mathrm{~mm}$, as measured by quantitative coronary angiography; [3] the use of final kissing-balloon inflation versus no final kissing; and [4] single versus two-stent approach.

\section{Clinical endpoints}

Clinical endpoints were defined according to the Academic Research Consortium (ARC), including the addendum on myocardial infarction. ${ }^{27,28}$ Death was considered cardiac, unless an evident noncardiac cause could be established. Myocardial infarction (MI) was defined by any creatine kinase concentration of more than double the upper limit of normal with elevated values of a confirmatory cardiac biomarker. PMI was defined as target vessel MI within 48 hours after PCI. Stent thrombosis was classified according to the ARC definitions. The composite endpoint target vessel failure was defined as cardiac death, target vessel MI, or clinically driven target vessel revascularization. Target lesion failure was defined as a composite of cardiac death, target vessel $\mathrm{MI}$, and clinically indicated target lesion revascularization. A patient-oriented composite endpoint consisted of all-cause death, any MI, and any repeat revascularization. Major adverse cardiac events were classified as a composite of allcause death, any Ml, emergent coronary artery bypass grafting, and clinically indicated target lesion revascularization.

\section{Statistical analysis}

Data were reported as frequencies and percentages for dichotomous and categorical variables, as mean \pm standard deviation for continuous normally distributed variables and as median and interquartile range for not normally distributed variables. Chi-square test and Fisher's exact test were used as appropriate. Differences between groups in continuous variables were assessed with the Student's $t$ test or the Wilcoxon rank-sum test. The Kaplan-Meier method was used to calculate the time to clinical endpoint and the Log-rank test was applied to compare groups. Parameters were considered as potential confounders if associations were found with a $p$-value $\leq 0.15$ in univariate analyses. For 
adjustment of potential confounders, propensity score analysis was used. The propensity score was estimated using multiple logistic regression analysis. Gender, clinical syndrome, RCA treatment, LAD treatment, multivessel disease, small vessel treatment, total stent length, number of stents per patient, postdilation, and lesion length longer than $27 \mathrm{~mm}$ were used to calculate the propensity score for having a bifurcated target lesion. A multivariate Cox regression model, including the propensity score as independent variable, was then used to adjust for the propensity score. All p-values and confidence intervals were two-sided and $p$-values $<0.05$ were considered significant. Data analysis was performed with SPSS (version 22.0, SPSS Inc., Chicago, IL, USA).

\section{Results}

\section{Baseline, lesion, and procedural characteristics}

Of all 1,811 randomized trial participants, 465 patients (25.7\%) were treated for at least one bifurcated lesion. Patients with bifurcated lesions were predominantly men, presented more often with stable angina, and underwent more often treatment of multiple vessels and lesions in left anterior descending arteries (Tables 1 and 2). Most patients (83.2\%) were treated with single stents. If a two-stent technique was applied, T-stenting (73.1\%) was generally preferred above (mini-)crush (17.9\%), culotte (2.6\%), and other two-stent approaches (6.4\%) (Table 2). Final kissing-balloon inflation was performed in 139 (29.9\%) patients with bifurcated target lesions.

Among all patients with bifurcated lesions, 244 (52.5\%) were treated with Resolute Integrity and 221 (47.5\%) with Promus Element stents. The characteristics of patients, lesions, and procedures did not differ between the two stent-groups (data not shown) except for a higher rate of kissing-balloon inflation in Resolute Integrity stents ( $36.1 \%$ vs $23.1 \%, p=0.002$ ).

\section{Clinical event rates and multivariate analysis at 2-year follow-up}

Two-year follow-up data were available for 1,810 (99.9\%) patients. Time-to-event analysis of patients with bifurcated lesions and patients with non-bifurcated lesions showed no significant differences in the rates of target vessel failure $(9.2 \%$ vs $7.9 \%, p$-Log-rank $=0.33)$, cardiac death $(1.7 \%$ vs $2.3 \%, p$-Logrank $=0.45$ ), and target vessel revascularization ( $4.5 \%$ vs $4.8 \%$, p-Log-rank $=0.77$ ) (Figure 1 ). Target vessel MI was higher in patients with bifurcation lesions (3.4\% vs 1.6\%, p-Log-rank=0.02) (Figure 1, Table 3), but after multivariate analysis with propensity score adjustment, bifurcation treatment was found not to be an independent predictor of target vessel MI (HR 1.40, 95\% Cl: $0.71-2.76, p=0.34)$. The rates of definite stent thrombosis after 2 years were low and comparable for both patients with bifurcated and non-bifurcated lesions ( $0.4 \%$ vs $1.0 \%, p=0.38)$.

\section{Clinical outcome among patients with bifurcated lesions}

Among patients with bifurcated target lesions, the rates of various clinical endpoints were similar for patients treated with Resolute Integrity versus Promus Element stents (Table 4). There was also no significant difference in any clinical endpoint between bifurcated lesions with side-branch $\geq 2.0 \mathrm{~mm}$ versus $<2.0 \mathrm{~mm}$, and between the use of final kissing-balloon inflation versus no use of final kissing 
balloons (Table 4, Figure 2). The use of a two-stent approach resulted in significantly higher PMI rates than the use of a single stent ( $9.0 \%$ vs $2.1 \%, p=0.002$ ).

Table 1: Patient characteristics of all study patients comparing patients with bifurcated versus non-bifurcated target lesions.

\begin{tabular}{|c|c|c|c|}
\hline & \multicolumn{3}{|c|}{$\begin{array}{c}\text { All patients } \\
n=1811\end{array}$} \\
\hline & $\begin{array}{c}\text { BL } \\
n=465\end{array}$ & $\begin{array}{l}\text { non-BL } \\
n=1346\end{array}$ & $\mathbf{p}$ \\
\hline Age (yrs) & $63.8 \pm 11.3$ & $64.0 \pm 10.7$ & 0.74 \\
\hline Female & $100(21.5)$ & $389(28.9)$ & 0.002 \\
\hline $\mathrm{BMI}\left(\mathrm{kg} / \mathrm{m}^{2}\right)$ & $27.9 \pm 4.5^{*}$ & $28.0 \pm 4.8^{+}$ & 0.71 \\
\hline Diabetes mellitus & $83(17.8)$ & $241(17.9)$ & 0.98 \\
\hline Previous MI & $112(24.1)$ & $285(21.2)$ & 0.19 \\
\hline Previous $\mathrm{PCl}$ & $85(18.3)$ & $264(19.6)$ & 0.53 \\
\hline Previous CABG & $42(9.0)$ & $131(9.7)$ & 0.66 \\
\hline Clinical syndrome at index $\mathrm{PCl}$ procedure & & & 0.005 \\
\hline Stable angina pectoris & $218(46.9)$ & $531(39.5)$ & \\
\hline Unstable angina pectoris & $57(12.3)$ & $188(14.0)$ & \\
\hline Non-ST-elevation myocardial infarction & $118(25.4)$ & $329(24.4)$ & \\
\hline ST-elevation myocardial infarction & $72(15.5)$ & $298(22.1)$ & \\
\hline
\end{tabular}

Values are mean \pm SD or $n(\%)$. BL bifurcated target lesion, CABG coronary artery bypass grafting, MI myocardial infarction, nonBL non-bifurcated target lesion, $\mathrm{PCl}$ percutaneous coronary intervention. * $\mathrm{n}=375,+\mathrm{n}=1049$. 

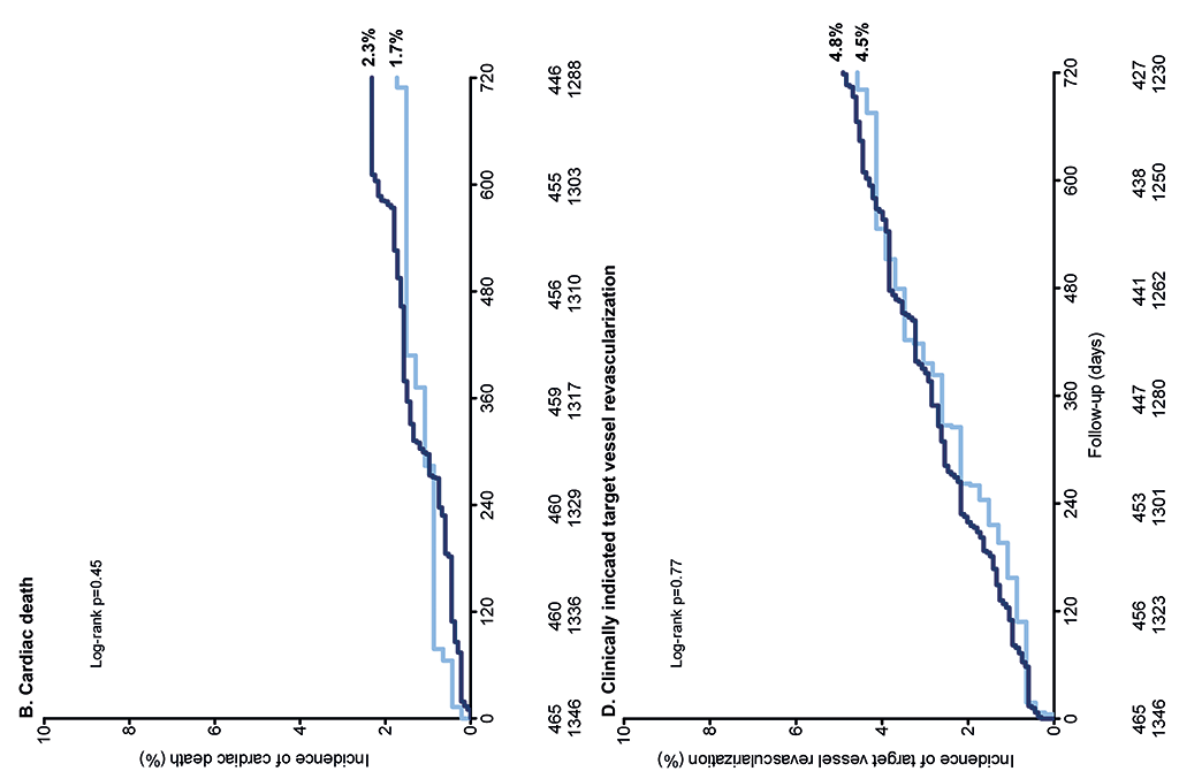

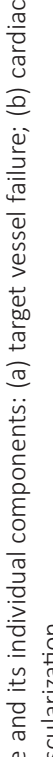

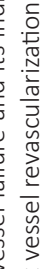

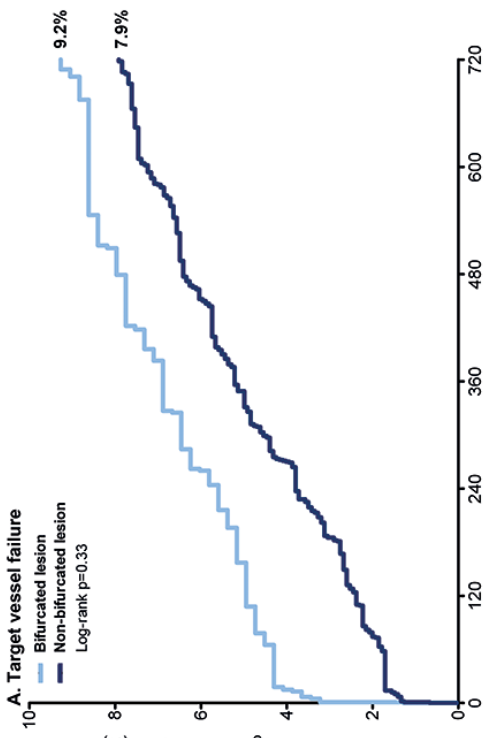

啇
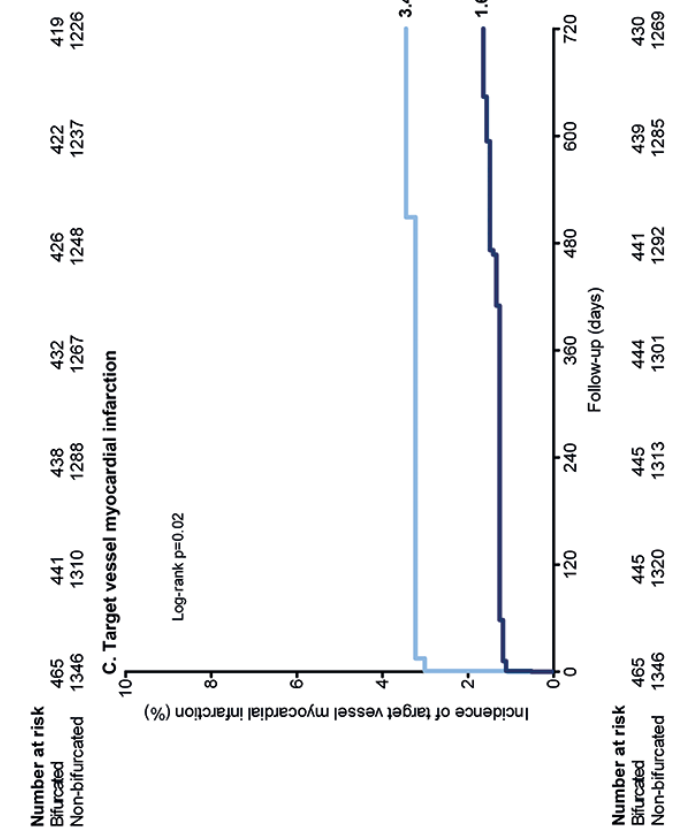

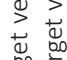

这势

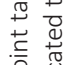

응 믐

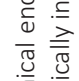

凉

$\stackrel{\oplus}{\frac{\pi}{n}}$ 으

हैं 
Table 2: Lesion and procedural characteristics of all study patients comparing patients with bifurcated versus non-bifurcated target lesions.

\begin{tabular}{|c|c|c|c|}
\hline \multirow[b]{2}{*}{ Lesion/procedural characteristics } & \multicolumn{2}{|c|}{$\begin{array}{c}\text { All patients } \\
n=1811\end{array}$} & \multirow[b]{2}{*}{$\mathbf{p}$} \\
\hline & $\underset{n=465}{B L}$ & $\begin{array}{l}\text { non-BL } \\
n=1346\end{array}$ & \\
\hline Multivessel treatment & $123(26.5)$ & $173(12.9)$ & $<0.001$ \\
\hline \multicolumn{4}{|l|}{ Treated coronary vessels } \\
\hline Right coronary artery & $91(19.6)$ & $578(42.9)$ & $<0.001$ \\
\hline Left anterior descending artery & $336(72.3)$ & $518(38.5)$ & $<0.001$ \\
\hline Circumflex artery & $148(31.8)$ & 375 (27.9) & 0.10 \\
\hline De novo lesion & $423(91.0)$ & $1204(89.5)$ & 0.35 \\
\hline Severe calcification & $106(22.8)$ & $301(22.4)$ & 0.85 \\
\hline At least one chronic total occlusion & $19(4.1)$ & $57(4.2)$ & 0.89 \\
\hline At least one in-stent restenosis & $17(3.7)$ & $38(2.8)$ & 0.37 \\
\hline At least one small-vessel & $298(64.1)$ & $770(57.2)$ & 0.01 \\
\hline At least one lesion length $>27 \mathrm{~mm}$ & $95(20.4)$ & $223(16.6)$ & 0.06 \\
\hline \multicolumn{4}{|l|}{ Medina classification of bifurcation lesions } \\
\hline 0.0 .1 & $37(8.0)$ & & \\
\hline 0.1 .0 & $67(14.4)$ & & \\
\hline 0.1 .1 & $21(4.5)$ & & \\
\hline 1.0.0 & $55(11.8)$ & & \\
\hline 1.0.1 & $39(8.4)$ & & \\
\hline 1.1 .0 & $148(31.8)$ & & \\
\hline 1.1 .1 & $98(21.1)$ & & \\
\hline Total stent length & $36.0(22.0-56.0)$ & $28.0(18.0-48.0)$ & $<0.001$ \\
\hline Number of stents per patient & $2.1(1.3)$ & $1.7(1.0)$ & $<0.001$ \\
\hline Longest lesion length (mm) & $19.4(12.0)$ & $18.3(12.1)$ & 0.09 \\
\hline Degree of stenosis (pre-PCl)* & $70.2(16.9)$ & $71.1(18.2)$ & 0.36 \\
\hline Residual in-stent stenosis (post-PCI)* & $17.9(8.7)$ & $17.3(8.1)$ & 0.22 \\
\hline Postdilation & $397(85.4)$ & $1006(74.7)$ & $<0.001$ \\
\hline \multicolumn{4}{|l|}{ Stenting approach } \\
\hline 1-stent & $387(83.2)$ & & \\
\hline 2-stent & $78(16.8)$ & & \\
\hline T-stenting & $57(73.1)$ & & \\
\hline (Mini)crush & 14 (17.9) & & \\
\hline Culotte & $2(2.6)$ & & \\
\hline Other & $5(6.4)$ & & \\
\hline Final kissing-balloon inflation & $139(29.9)$ & & \\
\hline
\end{tabular}

Values are mean $\pm S D, n(\%)$, or median (IQR). BL bifurcated target lesion, non-BL non-bifurcated target lesion, $\mathrm{PCl}$ percutaneous coronary intervention. *A reference vessel diameter of $>2.75 \mathrm{~mm}$ is defined as a small vessel. + In the case of multiple target lesions, the most severe diameter stenosis is presented. 
Table 3: Two-year clinical outcome in patients with bifurcated lesions versus non-bifurcated lesions.

\begin{tabular}{|c|c|c|c|}
\hline & \multicolumn{3}{|c|}{$\begin{array}{l}\text { All patients } \\
n=1810\end{array}$} \\
\hline & $\begin{array}{c}B L \\
n=465\end{array}$ & $\begin{array}{l}\text { non-BL } \\
n=1345\end{array}$ & $p$ \\
\hline Cardiac death & $8(1.7)$ & $31(2.3)$ & 0.45 \\
\hline Target vessel myocardial infarction & $16(3.4)$ & $22(1.6)$ & 0.02 \\
\hline Periprocedural myocardial infarction* & $15(3.2)$ & $15(1.1)$ & 0.002 \\
\hline Target vessel revascularization $^{+}$ & $21(4.5)$ & $65(4.8)$ & 0.78 \\
\hline 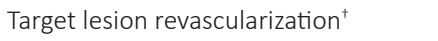 & $16(3.4)$ & $50(3.7)$ & 0.78 \\
\hline Definite stent thrombosis & $2(0.4)$ & $13(1.0)$ & 0.38 \\
\hline Definite or probable stent thrombosis & $4(0.9)$ & $16(1.2)$ & 0.56 \\
\hline Target vessel failure & $43(9.2)$ & 106 (7.9) & 0.36 \\
\hline Target lesion failure & $38(8.2)$ & $93(6.9)$ & 0.37 \\
\hline Major adverse cardiac events & $43(9.2)$ & $112(8.3)$ & 0.54 \\
\hline Patient-oriented composite endpoint & $60(12.9)$ & $168(12.5)$ & 0.82 \\
\hline
\end{tabular}

Values are $\mathrm{n}(\%)$. Two-year follow-up was available for 1810 of 1811 patients (99.9\%). BL bifurcated target lesion, Non-BL nonbifurcated target lesion. * Periprocedural myocardial infarction is a sub-classification of (any) target vessel myocardial infarction; + clinically indicated.

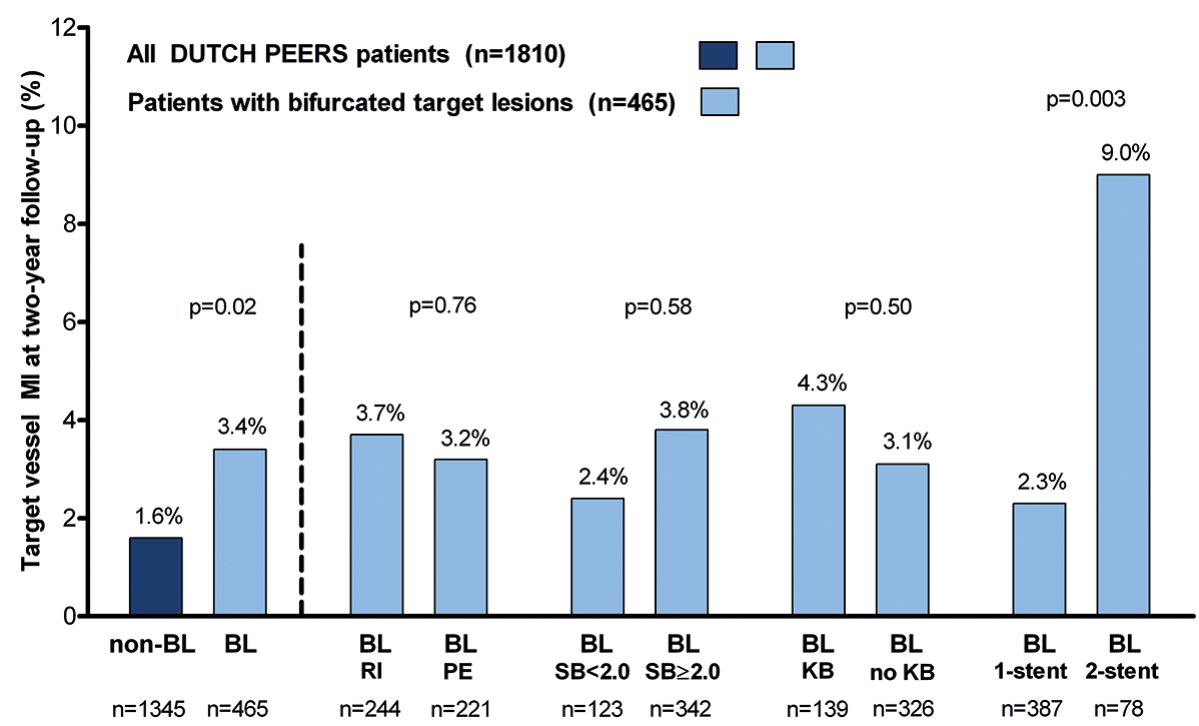

Figure 2: Target vessel $\mathrm{MI}$ rate at 2-year follow-up. Abbreviations: $\mathrm{BL}=$ bifurcated target lesion; $\mathrm{KB}=$ final kissingballoon inflation; $\mathrm{MI}=$ myocardial infarction, $\mathrm{PE}=$ promus element; $\mathrm{RI}=$ resolute integrity; $\mathrm{SB}=$ side-branch. Two-year follow-up was available for 1810 of 1811 patients (99.9\%). 


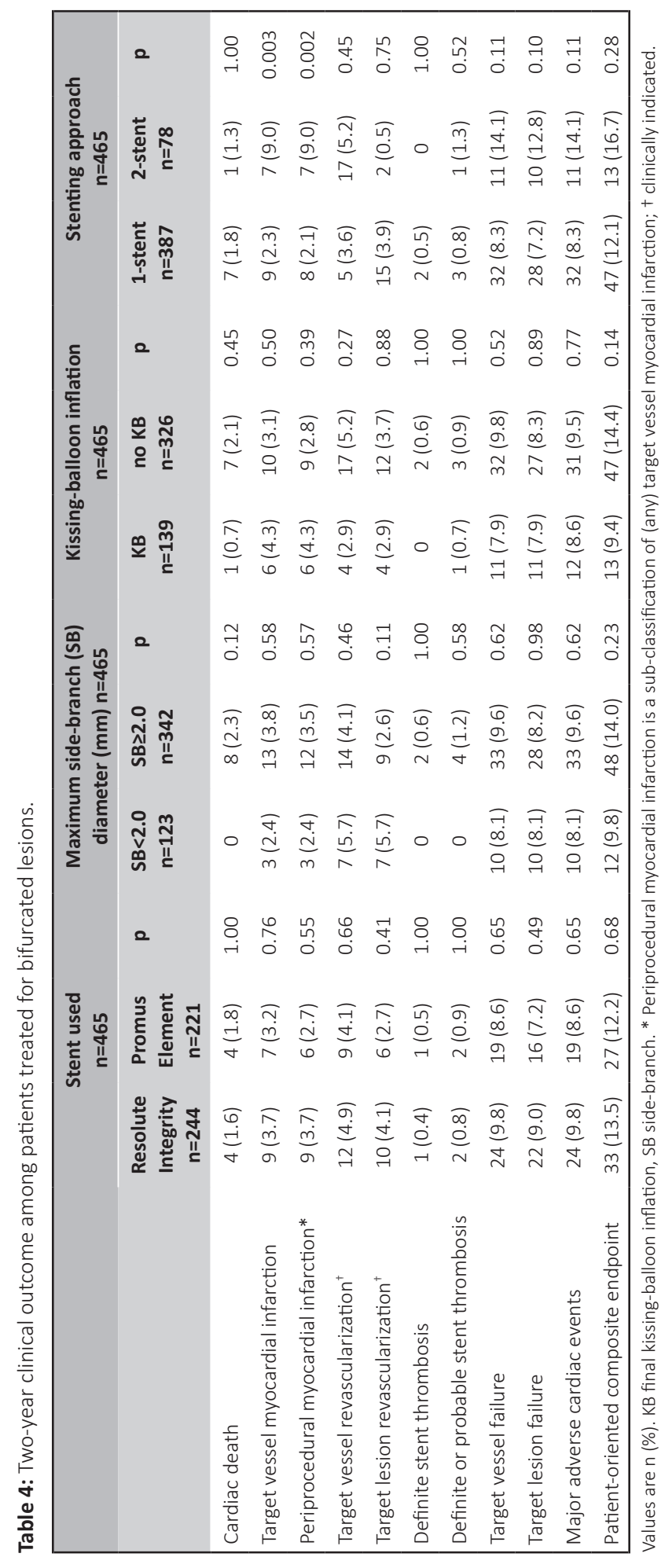




\section{Discussion}

\section{Major findings}

In the present subanalysis of the DUTCH PEERS randomized trial, all-comer patients treated for at least one bifurcated lesion versus patients with non-bifurcated target lesions showed similar 2-year rates of various clinical endpoints. Target vessel MI was more common in patients with bifurcation lesions $13.4 \%$ vs $1.6 \%$ ), but after multivariate analysis, bifurcation treatment was found not to be an independent predictor of target vessel MI. Among patients with bifurcated lesions, we found no impact of DES type, side-branch size, or final kissing-balloon inflation on various clinical endpoints. PMI was more common among patients with bifurcation lesions treated with a two-stent technique. Our findings suggest that the novel, flexible DES used are efficacious and safe for treating bifurcated target lesions.

\section{Stent design and outcome of $\mathrm{PCl}$ in bifurcated lesions}

Previous studies with second-generation DES examined devices with the same drug-eluting coatings, but rather different designs of the stent platforms, as used in the devices of the present study. ${ }^{20,21}$ In a sub-analysis of the TWENTE trial, patients treated for bifurcation lesions with second-generation Resolute (Medtronic) or Xience V stents (Abbott Vascular Devices, Santa Clara, CA) showed favorable 3-year outcomes that were similar to the outcomes in patients with non-bifurcated lesions (e.g. target vessel failure rate $13.1 \%$ vs $12.6 \%$ ), but in patients with bifurcated lesions the PMI rate was more than twice as high $(6.9 \%$ vs $3.1 \%, p<0.01) .{ }^{20}$ These data corroborated the results of a sub-study of the RESOLUTE All Comers trial, which had also reported a higher PMI rate in 392 patients with bifurcated lesions (6.5\% vs $3.4 \%$, unadjusted $p=0.009) .{ }^{21}$ In a pooled analysis of the RESOLUTE All Comer trial and the RESOLUTE International Registry, the incidence of target lesion failure and the individual components thereof was higher during the first 30 days after $\mathrm{PCl}$ of patients who were treated for bifurcation lesions as compared to patients treated for non-bifurcated lesions. However, during the remainder of the 3-year follow-up, clinical event rates were similar for both patient groups. ${ }^{29} \mathrm{PMI}$ in treatment of bifurcated lesions may result from (stent-induced) closure of side-branches, flow-limiting dissections, distal (micro)embolization of atherothrombotic debris, and the occurrence of slow flow or no-reflow. ${ }^{20,30}$

The development of newer-generation DES and the progression into devices with highly flexible stent platforms have reduced the need for repeat revascularization and the rate of target vessel MI following PCl of bifurcated lesions..$^{3-9,11,19-22,31}$ Burzotta et al. used virtual bench tests to assess the impact of technical characteristics of DES platforms on stenting in bifurcated lesions, showing that technical features of DES platforms lead to differences in response to similar procedural steps of provisional stenting, such as final kissing-balloon inflation. ${ }^{23}$ Therefore, technical characteristics of stents should be taken into account in the selection process of the most appropriate DES for treatment of bifurcated lesions. ${ }^{23}$

Both Resolute Integrity and Promus Element stents have demonstrated favorable results in the allcomer patient population of the DUTCH PEERS randomized trial. ${ }^{24}$ The present study of patients with bifurcated lesions has shown similar rates of various clinical endpoints for both stent groups. The baseline characteristics of patients with bifurcated lesions in both DES arms were comparable, but in 
Promus Element stents final kissing-balloon inflation was less often performed. As the actual motives of the operators were not documented in this context, we can only speculate that knowledge about the somewhat increased risk of longitudinal deformation of the Promus Element stent ${ }^{22}$ might have held some operators back from performing final simultaneous kissing-balloon inflations.

\section{Final kissing-balloon inflation}

The potential impact of a final kissing-balloon inflation on clinical outcome following stenting of bifurcated lesions is still unclear. ${ }^{20,32-34}$ Niemelä et al. investigated the use of routine final kissing-balloon inflation after successful stenting of the main branch with a single stent. Despite a reduced rate of angiographic side-branch (re)stenosis following kissing-balloon inflation, clinical outcome (PMI was not included) was similar for patients treated with versus without final kissing-balloon inflation. ${ }^{32}$ Threeyear outcome data of the TWENTE trial have also shown similar target vessel failure rates in patients with bifurcated lesions who were treated with or without final kissing-balloon inflation ${ }^{20}$, while final kissing-balloon inflation was reported to be beneficial following treatment of true bifurcation lesions with single, predominantly first-generation DES in patients with acute coronary syndromes. ${ }^{34} \mathrm{It}$ is likely that the inconsistent results of final kissing-balloon inflation with different stent types are caused by differences in the specific technical stent characteristics, leading to different stent strut distributions after final kissing-balloon inflation. ${ }^{23}$

\section{Side-branch size}

Previous studies of stenting in bifurcations used different criteria to define relevant side-branches and studied dissimilar patient populations, which renders comparison of their event rates difficult.,3,8,19,21,32,35 In contrast to several other trials that considered side-branches $\geq 1.75 \mathrm{~mm}^{4}, \geq 2.0 \mathrm{~mm}^{5,19,33,34,36-38}$, $\geq 2.25 \mathrm{~mm}^{31,32,35}$, or $\geq 2.5 \mathrm{~mm}^{3}$ as relevant, the present study defined side-branches to be relevant if they had a minimum lumen diameter $\geq 1.5 \mathrm{~mm}$ by quantitative coronary angiography, as suggested by the investigators of the SYNTAX trial. ${ }^{26}$ Nevertheless, when comparing clinical outcome of patients with bifurcated lesions and side-branches $<2.0 \mathrm{~mm}$ versus side-branches $\geq 2.0 \mathrm{~mm}$, we found no relation between the side-branch size and the risk of various clinical endpoints including target vessel MI.

\section{Single versus two-stent approach}

Previous studies that compared the outcome of bifurcation treatment with two-stent strategies versus the use of a single stent suggested more often, similar to the results of the present substudy, a higher risk of PMI following two-stent procedures.,21,29,35,39 It has been speculated that during the more complex two-stent procedures the longer duration of vessel instrumentation, the more frequent balloon and stent passages through vessel segments proximal to the bifurcation, and the generally higher frequency of stent postdilation may contribute to the higher PMI risk. ${ }^{35} \mathrm{~A}$ slight disadvantage of the single-stent approach may be the somewhat higher risk of side-branch occlusion after stenting the main branch. ${ }^{37,40}$ Predictors of side-branch occlusion are: a high pre-procedural degree of side-branch stenosis; a calcified side-branch lesion; a long obstructed side-branch segment; proximal disease in the main branch; and treatment for an acute coronary syndrome. ${ }^{40}$ In such bifurcation lesions with an 
increased risk of jeopardizing the side-branch, the straightforward use of a two-stent technique will often increase the likelihood of keeping the side-branch patent. ${ }^{41}$

\section{Limitations}

Because of the post hoc nature of the present analysis, the results must be considered hypothesis generating. Nevertheless, in the absence of published data on $\mathrm{PCl}$ in bifurcated lesions with these novel, flexible DES, the findings may be of interest. Similar to previous studies ${ }^{20,21}$, the sample size of subgroups among patients with bifurcated lesions was limited. Therefore, the results of subgroup analyses should be interpreted with caution.

\section{Conclusion}

All-comer patients treated for bifurcated and non-bifurcated target lesions showed similar and low rates of clinical endpoints, suggesting that the DES used are efficacious and safe for treating bifurcated target lesions. 


\section{References}

1. Lassen JF, Holm NR, Stankovic G, et al. Percutaneous coronary intervention for coronary bifurcation disease: consensus from the first 10 years of the European Bifurcation Club meetings. Eurolntervention 2014;10:545-60.

2. von Birgelen C, van Houwelingen KG, Lam MK. Coronary bifurcations: still the touchstone of drug-eluting stents and bioresorbable vascular scaffolds? Rev Esp Cardiol 2014;67:797-803.

3. Colombo A, Moses JW, Morice MC, et al. Randomized study to evaluate sirolimus-eluting stents implanted at coronary bifurcation lesions. Circulation 2004;109:1244-9.

4. Thuesen L, Kelbaek H, Klovgaard L, et al. Comparison of sirolimus-eluting and bare metal stents in coronary bifurcation lesions: subgroup analysis of the Stenting Coronary Arteries in Non-Stress/Benestent Disease Trial (SCANDSTENT). Am Heart J 2006;152:1140-5.

5. Colombo F, Biondi-Zoccai G, Infantino V, et al. A long-term comparison of drug-eluting versus bare metal stents for the percutaneous treatment of coronary bifurcation lesions. Acta Cardiol 2009;64:583-8.

6. Romagnoli E, De Servi S, Tamburino C, et al. Real-world outcome of coronary bifurcation lesions in the drugeluting stent era: results from the 4,314-patient Italian Society of Invasive Cardiology (SICI-GISE) Italian Multicenter Registry on Bifurcations (I-BIGIS). Am Heart J 2010;160:535-42.

7. Tommasino A, Burzotta F, Sciahbasi A, et al. Procedural and clinical evaluation of the novel zotarolimus-eluting resolute stent in patients with unselected bifurcated coronary stenosis treated by provisional approach: a multicenter registry. J Invasive Cardiol 2011;23:50-4.

8. Costopoulos C, Latib A, Ferrarello S, et al. First versus second-generation drug-eluting stents for the treatment of coronary bifurcations. Cardiovasc Revasc Med 2013;14:311-5.

9. von Birgelen C, Basalus MW, Tandjung K, et al. A randomized controlled trial in second-generation zotarolimuseluting Resolute stents versus everolimus-eluting Xience $V$ stents in real-world patients: the TWENTE trial. J Am Coll Cardiol 2012;59:1350-61.

10. Kedhi E, Joesoef KS, McFadden E, et al. Second-generation everolimus-eluting and paclitaxel-eluting stents in real-life practice (COMPARE): a randomised trial. Lancet 2010;375:201-9.

11. Serruys PW, Silber S, Garg S, et al. Comparison of zotarolimus-eluting and everolimus-eluting coronary stents. N Engl J Med 2010;363:136-46.

12. Akin I, Hochadel M, Abdel-Wahab M, et al. Clincial outcomes of different first- and second-generation drugeluting stents in routine clinical practice: results from the prospective multicenter German DES.DE registry. Clin Res Cardiol 2013;102:371-381.

13. Wöhrle J, Rottbauer W, Imhof A. Everolimus-eluting stents for treatment of chronic total coronary occlusions. Clin Res Cardiol 2012;101:23-8.

14. Mehilli J, Richardt G, Valgimigli M, et al. Zotarolimus- versus everolimus-eluting stents for unprotected left main coronary artery disease. J Am Coll Cardiol 2013;62:2075-82.

15. Ndrepepa G, Tada T, Fusaro M, et al. Association of coronary atherosclerotic burden with clinical pressentation and prognosis in patients with stable and unstable coronary artery disease. Clin Res Cardiol 2012;101:1003-11.

16. Akin I, Naber C, Sabin G, et al. Outcome of percutaneous coronary intervention with drug-eluting stents in unprotected left main versus non-left main native coronary artery disease: results from the prospective multicenter German DES.DE registry. Clin Res Cardiol 2013;102:679-86.

17. Sen H, Lam MK, Tandjung K, et al. Impact of previous coronary artery bypass surgery on clinical outcome after percutaneous interventions with second generation drug-eluting stents in TWENTE trial and Non-Enrolled TWENTE registry. Int J Cardiol 2014;176:885-90.

18. Akin I, Nienaber CA, Richardt G, et al. Risk factors for clinical events at 1-year follow-up after drug-eluting stent implantation: results from the perspective multicenter German DES.DE registry. Clin Res Cardiol 2014;103:36372.

19. Pan M, Burzotta F, Trani C, et al. Three-year follow-up of patients with bifurcation lesions treated with sirolimusor everolimus-eluting stents: SEAside and CORpal cooperative study. Rev Esp Cardiol 2014;67:797-803.

20. Lam MK, Sen H, van Houwelingen KG, et al. Three-year clinical outcome of patients with bifurcation treatment with second-generation Resolute and Xience V stents in the randomized TWENTE trial. Am Heart J 2015;169:6977. 
21. Diletti R, Garcia-Garcia HM, Bourantas CV, et al. Clinical outcomes after zotarolimus and everolimus drug eluting stent implantation in coronary artery bifurcation lesions: insights from the RESOLUTE All Comers Trial. Heart 2013;99:1267-74.

22. Ormiston JA, Webber B, Webster MW. Stent longitudinal integrity bench insights into a clinical problem. J Am Coll Cardiol Intv 2011;4:1310-7.

23. Burzotta F, Mortier P, Trani C. Characteristics of drug-eluting stent platforms potentially influencing bifurcated lesion provisional stenting procedure. Eurolntervention 2014;10:124-32.

24. von Birgelen C, Sen H, Lam MK, et al. Third-generation zotarolimus-eluting and everolimus-eluting stents in all-comer patients requiring a percutaneous coronary intervention (DUTCH PEERS): a randomised, single-blind, multicentre, non-inferiority trial. Lancet 2014;383:413-23.

25. Sen H, Lam MK, Löwik MM, et al. Clinical events and patient-reported chest pain in all-comers treated with Resolute Integrity and Promus Element stents: two-year follow-up of the randomized DUTCH PEERS (TWENTE II) trial. J Am Coll Cardiol Intv 2015;8:889-99.

26. Sianos S, Morel MA, Kappetein AP, et al. The SYNTAX Score: an angiographic tool grading the complexity of coronary artery disease. Eurolntervention 2005;1:219-27.

27. Cutlip DE, Windecker S, Mehran R, et al. Clinical end points in coronary stent trials: a case for standardized definitions. Circulation 2007;115:2344-51.

28. Vranckx P, Cutlip DE, Mehran R, et al. Myocardial infarction adjudication in contemporary all-comer stent trials: balancing sensitivity and specificity. Addendum to the historical MI definitions used in stent studies. Eurolntervention 2010;5:871-4.

29. Ferenc M, Kornowski R, Belardi J, et al. Three-year outcomes of percutaneous coronary intervention with nextgeneration zotarolimus-eluting stents for de novo coronary bifurcation lesions. J Invasive Cardiol 2014;26:630-8.

30. Park DW, Kim YH, Yun SC, et al. Impact of the angiographic mechanisms underlying periprocedural myocardial infarction after drug-eluting stent implantation. Am J Cardiol 2004;113:1105-10.

31. Roversi S, Biondi-Zoccai G, Romagnoli E, et al. Early and long-term outlook of percutaneous coronary intervention for bifurcation lesions in young patients. Int J Cardiol 2013;167:2995-9.

32. Niemelä M, Kervinen K, Erglis A, et al. Randomized comparison of final kissing balloon dilatation versus no final kissing balloon dilatation in patients with coronary bifurcation lesions treated with main vessel stenting: the Nordic-Baltic Bifurcation Study III. Circulation 2011;123:79-86.

33. Hariki $\mathrm{H}$, Shinke $\mathrm{T}$, Otake $\mathrm{H}$, et al. Potential benefit of final kissing balloon inflation after single stenting for the treatment of bifurcation lesions-insights from optical coherence tomography observations. Circ J 2013;77:1193-201.

34. Kim TH, Lee HJ, Jang $\mathrm{H}$, et al. Impact of final kissing balloon inflation after simple stent implantation for the treatment of non-left main true coronary bifurcation lesions in patients with acute coronary syndrome. Int J Cardiol 2014;177:907-11.

35. Hildick-Smith D, de Belder AJ, Cooter N, et al. Randomized trial of simple versus complex drug-eluting stenting for bifurcation lesions: the British Bifurcation Coronary Study: old, new and evolving strategies. Circulation 2010;121:1235-43

36. Schulz A, Hauschild T, Kleber FX, et al. Treatment of coronary de novo bifurcation lesions with DCB only strategy. Clin Res Cardiol 2014;103:451-456.

37. Kherada NI, Sartori S, Tomey MI, et al. Dedicated two-stent technique in complex bifurcation percutaneous coronary intervention with use of everolimus-eluting stents: the EES-bifurcation study. Int J Cardiol 2014;174:137.

38. Song PS, Song YB, Yang JH, et al. Periprocedural myocardial infarction is not associated with an increased risk of long-term cardiac mortality after coronary bifurcation stenting. Int J Cardiol 2013;167:1251-6.

39. Niccoli G, Ferrante G, Porto I, et al. Coronary bifurcation lesions: to stent one branch or both? A meta-analysis of patients treated with drug eluting stents. Int J Cardiol 2015;139:80-91

40. Hahn JY, Chun WJ, Kim JH, et al. Predictors and outcomes of side branch occlusion after main vessel stenting in coronary bifurcation lesions: results from the COBIS II Registry (COronary BIfurcation Stenting). J Am Coll Cardiol 2013;62:1654-9.

41. Freixa $\mathrm{X}$, Almasood $\mathrm{AA}$, Asif $\mathrm{N}$, et al. Long-term outcomes using a two-stent technique for the treatment of coronary bifurcations. Int J Cardiol 2013;168:446-51. 


\section{Conflicts of interest statement}

CVB has been consultant to Abbott Vascular, Boston Scientific, and Medtronic, and received lecture fees from MSD and AstraZeneca. The institution has received research grants from Abbott Vascular, Biotronik, Boston Scientific, and Medtronic. MIJJ is consultant to PANAXEA b.v., and he has received lecture fees from Roche, Pfizer, and Sanofi Aventis. All other authors declare that they have no conflict of interest. The investigatorinitiated, randomized TWENTE trial was equally funded by Abbott Vascular and Medtronic until the 2-year follow-up. The 3-year follow-up was supported by an unrestricted research grant from Medtronic. The present sub-study was performed without specific funding.

\section{Copyright}

Reprinted with permission from

Heart and Vessels 2016 Nov;31(11):1731-1739

(C) Springer 


\section{CHAPTER 11}

\section{Long-Term Outcome and Chest Pain in Patients With True Versus Non-True Bifurcation Lesions Treated With Second-Generation Drug-Eluting Stents in the TWENTE Trial}

K. Gert van Houwelingen, Liefke C. van der Heijden, Ming Kai Lam, Marlies M. Kok, Marije M. Löwik, J.(Hans) W. Louwerenburg, Gerard C.M. Linssen, Maarten J. IJzerman, Carine J.M. Doggen, Clemens von Birgelen
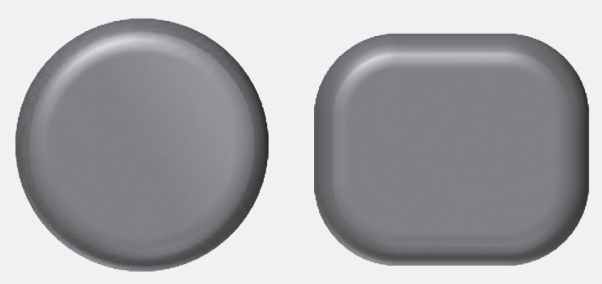


\begin{abstract}
Objectives: To assess 3-year clinical outcome of patients with true bifurcation lesions (TBL) versus non-true bifurcation lesions (non-TBL) following treatment with second-generation drug-eluting stents (DES).

Background: TBL are characterized by the obstruction of both main vessel and side-branch. Limited data are available on long-term clinical outcome following TBL treatment with newer generation DES.

Methods: We performed an explorative sub-study of the randomized TWENTE trial among 287 patients who had bifurcated target lesions with side-branches $\geq 2.0 \mathrm{~mm}$. Patients were categorized into TBL (Medina classes: $1,1,1 ; 1,0,1 ; 0,1,1$ ) versus non-TBL to compare long-term clinical outcome.

Results: A total of 116 (40.4\%) patients had TBL while 171 (59.6\%) had non-TBL only. Target lesion revascularization rates were similar (3.5\% vs $3.5 \%, \mathrm{p}=1.0)$ and definite-or-probable stent thrombosis rates were low (both $<1.0 \%$ ). The target-vessel myocardial infarction (MI) rate was $11.3 \%$ vs $5.3 \%$ $(p=0.06)$, mostly driven by (periprocedural) $\mathrm{MI} \leq 48$ hours from $\mathrm{PCl}$. All-cause mortality and cardiac death rates were $8.7 \%$ vs $3.5 \%(p=0.06)$, and $3.5 \%$ vs $1.2 \%(p=0.22)$, respectively. The 3 -year major adverse cardiac event (MACE) rate for patients with TBL vs. non-TBL was $20.0 \%$ vs $11.7 \%(p=0.05)$. At 1 , 2, and 3 -year follow-up, $6.5 \%, 13.0 \%$, and $11.0 \%$ of patients reported chest-pain at $\leq$ moderate physical effort, without any between-group difference.
\end{abstract}

Conclusions: Patients treated with second-generation DES for TBL had somewhat higher adverse event rates than patients with non-TBL, but dissimilarities did not reach statistical significance. Up to 3-year follow-up, the vast majority of patients of both groups remained free from chest pain. 


\section{Introduction}

True bifurcation lesions (TBL) are characterized by an advanced atherosclerotic disease burden that obstructs at bifurcation level both the main vessel and the side-branch. Percutaneous coronary interventions ( $\mathrm{PCl}$ ) of TBL are often technically more challenging, require more often two-stent techniques ${ }^{1,2}$, and have previously been associated with somewhat lower technical success rate and a higher restenosis risk ${ }^{3-5}$. Meanwhile, second-generation drug-eluting stents (DES) were developed, which have shown favorable outcomes in broad patient populations. ${ }^{6,7}$ In bifurcation lesions, the use of these contemporary DES reduced the incidence of restenosis as compared to early DES ${ }^{8-11}$, which might partly be related to an improved side-branch access. ${ }^{12}$ Nevertheless, the incidence of periprocedural myocardial infarction (MI) is still increased in patients with bifurcated target lesions. ${ }^{13-15}$ This might be related to the increased procedural complexity of bifurcation stenting or the atherosclerotic disease itself, which both are generally higher in patients with TBL. Only few large randomized clinical trials have reported data on the long-term performance of second-generation DES in bifurcated target lesions. ${ }^{13,14,16}$ However, these studies comprised target lesions with a variety of bifurcation types, and clinical outcome was generally reported at the group-level without specifying outcome for patients with TBL versus non-TBL. ${ }^{13,14}$ As a consequence, long-term outcome data of patients who were treated with second-generation DES in TBL are of interest but scarce. ${ }^{17}$

For that reason, we performed an explorative sub-study of the TWENTE trial,18 in patients with bifurcated target lesions and a side-branch size of at least $2 \mathrm{~mm}$, comparing the long-term clinical outcome of patients with TBL versus patients, who were treated for non-TBL only. In addition, we analyzed the patient-reported chest pain to detect potential differences between patients with TBL versus non-TBL, and to assess the relation between chest pain after bifurcation stenting and hard clinical endpoints.

\section{Methods}

\section{Study Population}

The randomized TWENTE trial (ClinicalTrials.gov NCT01066650) enrolled 1,391 patients between June 2008 and August 2010 without any limit for target lesion length, reference size, and number of lesions or diseased vessels to be treated. The few inclusion and exclusion criteria (no STEMI within 48 hours), and details of the study have previously been reported. ${ }^{7,19}$ In brief, a broad and heterogeneous population of $\mathrm{PCl}$ patients were randomized for treatment with the zotarolimus-eluting Resolute (Medtronic Inc., Santa Rosa, CA) or everolimus-eluting Xience V stent (Abbott Vascular, Santa Clara, CA). The TWENTE trial was approved by the accredited Medical Ethics Committee Twente and complied with the Declaration of Helsinki, and study participants provided a written informed consent. The three-year clinical outcome of the TWENTE trial population has recently been reported. ${ }^{18}$

The present sub-study assessed patients who had bifurcated target lesions with a side-branch reference lumen diameter $\geq 2.0 \mathrm{~mm}$, as measured by quantitative coronary angiography (QCA). Based on the lesion classification provided by the angiographic core lab, we categorized the study population into patients with at least one TBL that involved both main vessel and side-branch (i.e. Medina classes: 1,1,1; $1,0,1 ; 0,1,1$ ) versus patients with non-TBL (i.e. Medina classes: $1,1,0 ; 1,0,0 ; 0,1,0 ; 0,0,1$ ) only. ${ }^{20}$ 


\section{Coronary Intervention}

The interventional procedure was performed according to standard techniques, and the choice of the concomitant medication was based on routine institutional protocols and current guidelines. In bifurcated target lesions, provisional T-stenting of the side-branch was generally preferred. ${ }^{21}$ The treatment strategy, the technique of stenting, and the decision to perform final kissing balloon inflations were left at the discretion of the operator. In general, dual anti-platelet therapy was prescribed for 1 year.

\section{Coronary Angiographic Analysis}

Analysts of the angiographic core lab at Thoraxcentrum Twente, blinded to the stent type used, classified the lesion types and performed off-line quantitative coronary angiography of all cases according to current standards with the use of dedicated edge-detection software (QAngio XA version 7.1; Medis, the Netherlands). ${ }^{7}$ Bifurcated target lesions, according to the definition of the present sub-study, were defined as lesions at junctions of a main vessel and a side-branch that had (after administration of intracoronary nitrates and before $\mathrm{PCl}$ ) a diameter $\geq 2.0 \mathrm{~mm}$ by QCA.

\section{Assessment of Clinical Follow-Up}

The follow-up procedures of the TWENTE trial have previously been reported., ${ }^{7,19}$ In brief, systematic laboratory and electrocardiographic testing were performed to identify peri-procedural myocardial infarction (MI). Research nurses and analysts, blinded to the treatment arm, obtained information on clinical endpoints and chest pain by use of a medical records and a medical questionnaire or, in the absence of a response, a telephone follow-up that was based on the same questions.

Patient-reported chest pain, the principal symptom of angina pectoris and a surrogate for myocardial ischemia, was classified into scores: patients with chest pain score 0-1 were symptom-free or experienced chest pain only at the very maximum level of physical exertion but were not limited in daily activities; patients with score 2 had chest pain at moderate physical effort (during moderate/normal daily activities); and patients with score 3 had chest pain at mild physical effort or even at rest. ${ }^{22}$

\section{Definition of Clinical Endpoints}

Clinical endpoints were defined according to the Academic Research Consortium (ARC). ${ }^{23,24}$ Cardiac death was defined as any death due to proximate cardiac cause (e.g. MI, low-output failure, fatal arrhythmia). Myocardial Infarction (MI) was defined by any creatine kinase concentration of more than double the upper limit of normal with elevated values of a confirmatory cardiac biomarker (creatine kinase MB fraction or troponin), based on the updated ARC definition of MI. Periprocedural MI (PMI) was defined as target-vessel-related MI within 48 hours after PCl. ${ }^{23,24}$ The cardiac markers were systematically assessed with subsequent serial measurements in case of relevant elevation or complaints. Stent thrombosis was defined according to the ARC as definite or probable. Target-Lesion Failure (TLF) was defined as composite of cardiac death, target-vessel-related MI, or clinically indicated Target-Lesion Revascularization (TLR), and Major Adverse Cardiac Events (MACE) was defined as a composite of all-cause mortality, any $\mathrm{MI}$, emergent coronary bypass surgery, or TLR. ${ }^{7}$ 
Clinical event adjudication was performed by independent, external clinical event committees, organized by independent clinical research organizations (Cardialysis, Rotterdam, the Netherlands; and Diagram, Zwolle, the Netherlands). The TWENTE trial is an investigator-initiated study, supported by equal unrestricted research grants from Abbott Vascular and Medtronic. The authors are solely responsible for the study design, conduct of the study, statistical analysis, and reporting of outcomes.

\section{Statistical Analysis}

Continuous variables were expressed as mean \pm standard deviation (SD), and categorical data were presented as numbers and percentages. Baseline characteristics were compared using chi-square test or Fisher's exact test for categorical variables and Student's $t$ test for continuous variables. The time to clinical endpoint was assessed according to the Kaplan Meier method, and the log-rank test was applied to compare the incidence of MACE in patients with TBL versus non-TBL. Confidence intervals and p-values were two-sided. Analyses were performed using (SPSS 15.0 SPSS Inc., Chicago, IL, USA). P-values $<0.05$ were considered significant.

\section{Results}

\section{Demographics and Cardiovascular Risk Profile of Patients}

A total of $287(20.6 \%)$ patients of all 1,391 TWENTE trial participants had bifurcated target lesions with side-branches $\geq 2.0 \mathrm{~mm}$. Based on the Medina classification of the bifurcation lesion, patients were categorized into the TBL ( $n=116,40.4 \%$ ) versus the non-TBL groups ( $n=171,59.6 \%)$. Patients of the two groups did not differ in demographics and cardiovascular risk profile (Table 1).

\section{Lesion Characteristics and Interventional Procedure}

The lesion characteristics (other than the Medina class) were similar for both groups, with the only exception being a slightly smaller side-branch lumen diameter in the TBL group $(2.3 \pm 0.3 \mathrm{~mm}$ vs $2.4 \pm 0.4 \mathrm{~mm}, \mathrm{p}=0.01$ ) (Table 2). The rate of stent postdilatation was high and simi lar in both groups (95.2\% vs $94.2 \%, p=0.57)$. However, as may be expected, in patients with TBL a two-stent bifurcation approach was more often performed $(41.4 \%$ vs $11.1 \%, p<0.01)$, and the total number of stents implanted and the rate of final kissing balloon inflation were higher in this group (Table 2). If two-stent technique was applied, T-stenting (61.2\%) was generally preferred above (mini-)crush (20.9\%), culotte (10.3\%), and other two-stent approaches (7.5\%). Final kissing balloon inflation was performed in $36.4 \%$ of patients treated with the single-stent approach and in $77.6 \%$ of patients treated with two-stent techniques. 
Table 1: Characteristics of patients with true versus non-true bifurcation lesions with side-branches $\geq 2 \mathrm{~mm}$.

\begin{tabular}{|c|c|c|c|}
\hline & & $\begin{array}{c}\text { All patients } \\
n=287\end{array}$ & \\
\hline Patient characteristics & $\begin{array}{l}\text { True bifurcation (TBL) } \\
\text { group } \\
n=116\end{array}$ & $\begin{array}{c}\text { Non-true bifurcation } \\
\text { (non-TBL) group } \\
n=171\end{array}$ & p \\
\hline Age (years) & $65.4 \pm 10.6$ & $64.0 \pm 10.4$ & 0.26 \\
\hline Female gender & $30(25.9)$ & $44(25.7)$ & 0.98 \\
\hline Diabetes mellitus & $24(20.7)$ & $32(18.7)$ & 0.68 \\
\hline Arterial hypertension & $64(55.2)$ & $90(52.6)$ & 0.67 \\
\hline Hypercholesterolemia & $60(54.1)$ & $87(51.5)$ & 0.67 \\
\hline Current smoker & $33(28.4)$ & $42(24.6)$ & 0.46 \\
\hline Family history of CAD & $53(45.7)$ & $90(52.6)$ & 0.25 \\
\hline Previous MI & $41(35.3)$ & $48(28.1)$ & 0.19 \\
\hline Previous $\mathrm{PCl}$ & $22(19.0)$ & $29(17.0)$ & 0.66 \\
\hline Previous CABG & $12(10.3)$ & $11(6.4)$ & 0.23 \\
\hline Clinical syndrome & & & 0.97 \\
\hline Stable angina pectoris & $55(47.4)$ & $81(47.4)$ & \\
\hline Unstable angina & $31(26.7)$ & $44(25.7)$ & \\
\hline Non-ST-elevation MI & $30(25.9)$ & 46 (27.9) & \\
\hline
\end{tabular}

Values are $n(\%)$ or mean $( \pm S D)$. Patients of the TBL group were treated for at least one TBL. CABG coronary artery bypass grafting, CAD coronary artery disease, $\mathrm{MI}$ myocardial infarction, $\mathrm{PCl}$ percutaneous coronary intervention, $\mathrm{TBL}$ true bifurcation lesion.

\section{Long-Term Clinical Outcome}

Three-year follow-up was available in 286 (99.7\%) patients; 1 patient withdrew consent during follow-up. The TLR rate was low in both groups (3.5\% vs 3.5\%, p=1.00) (Table 3). The rates of definite-or-probable stent thrombosis were very low; a single definite stent thrombosis occurred after 17 months in a patient with TBL. The target-vessel MI rate was $11.3 \%$ vs $5.3 \%(p=0.06)$, mostly driven by (periprocedural) MI $\leq 48$ hours from $\mathrm{PCl}(9.6 \%$ vs $4.7 \%, \mathrm{p}=0.10)$. All-cause mortality and cardiac death rates were $8.7 \%$ vs $3.5 \%(p=0.06)$, and $3.5 \%$ vs $1.2 \%(p=0.22)$, respectively.

The 3-year major adverse cardiac event (MACE) rate for patients with TBL vs non-TBL was $20.0 \%$ vs $11.7 \%(p=0.05)$. A Kaplan-Meier analysis for MACE in Figure 1 shows the time-to-event curves, which reflect a numerically dissimilar incidence of periprocedural events and, during the second year of follow-up, a somewhat further diverging course. Landmark analysis revealed that during the first 48 hours from stenting and from 48 hours until 3-year follow-up, MACE was not significantly different between patients treated for TBL versus non-TBL (9.6\% vs 4.7\%, p-Logrank=0.12, and $11.4 \%$ vs $7.4 \%$, $p$-Logrank $=0.26$, respectively) (Figure 2). All but 2 MACE (related to additional non-bifurcated target lesions in patients with multivessel treatment) were related to the bifurcated target lesions. 
Table 2: Lesion and procedural characteristics of patients with true versus non-true bifurcation lesions.

\begin{tabular}{|c|c|c|c|}
\hline \multirow[b]{2}{*}{ Lesion characteristics } & \multicolumn{2}{|c|}{$\begin{array}{c}\text { All patients } \\
\mathrm{n}=\mathbf{2 8 7}\end{array}$} & \multirow[b]{2}{*}{ 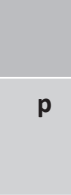 } \\
\hline & $\begin{array}{l}\text { True bifurcation (TBL) } \\
\text { group } \\
n=116\end{array}$ & $\begin{array}{c}\text { Non-true bifurcation } \\
\text { (non-TBL) group } \\
n=171\end{array}$ & \\
\hline De novo lesions & $99(85.3)$ & $154(90.1)$ & 0.23 \\
\hline Severe calcification & $21(18.1)$ & $35(20.5)$ & 0.62 \\
\hline At least one aorto-ostial lesion & $8(6.9)$ & $15(8.8)$ & 0.57 \\
\hline \multicolumn{4}{|l|}{ Treated coronary vessels } \\
\hline Left main & $11(9.5)$ & $19(11.1)$ & 0.66 \\
\hline Right coronary artery & $17(14.7)$ & $36(21.1)$ & 0.17 \\
\hline Left anterior descending artery & $91(78.4)$ & $124(72.5)$ & 0.26 \\
\hline Circumflex artery & $41(35.3)$ & $62(36.3)$ & 0.87 \\
\hline Medina classification & & & $<0.01$ \\
\hline 0.1 .1 & $39(33.6)$ & 0 & \\
\hline 1.0 .1 & $18(15.5)$ & 0 & \\
\hline 1.1 .1 & $59(50.9)$ & 0 & \\
\hline 0.0 .1 & 0 & $25(14.6)$ & \\
\hline 0.1 .0 & 0 & $51(29.8)$ & \\
\hline 1.0.0 & 0 & $44(25.7)$ & \\
\hline 1.1 .0 & 0 & $51(29.8)$ & \\
\hline Bifurcation angle $\left({ }^{\circ}\right)$ & $55.7 \pm 22.1$ & $62.1 \pm 41.0$ & 0.14 \\
\hline Longest lesion length (mm) & $20.1 \pm 1.1$ & $20.1 \pm 12.3$ & 0.78 \\
\hline Degree of stenosis before $\mathrm{PCl}(\%)$ & $67.5 \pm 13.3$ & $67.1 \pm 13.3$ & 0.76 \\
\hline Residual in-stent stenosis post $\mathrm{PCl}(\%)$ & $15.2 \pm 6.2$ & $14.3 \pm 6.2$ & 0.25 \\
\hline \multicolumn{4}{|l|}{ Side-branch characteristics } \\
\hline Lumen diameter SB before $\mathrm{PCI}(\mathrm{mm})$ & $2.3 \pm 0.3$ & $2.4 \pm 0.4$ & 0.01 \\
\hline Degree of SB stenosis before $\mathrm{PCl}(\%)$ & $62.8 \pm 13.0$ & $65.4 \pm 18.8$ & 0.40 \\
\hline Longest SB lesion length (mm) & $10.0 \pm 6.3$ & $10.8 \pm 8.1$ & 0.58 \\
\hline \multicolumn{4}{|l|}{ Procedural characteristics } \\
\hline Number of stents per patient & $2.6 \pm 1.4$ & $2.2 \pm 1.2$ & 0.01 \\
\hline Total stent length per patient (mm) & $50.0 \pm 29.8$ & $43.4 \pm 27.4$ & 0.06 \\
\hline Predilatation & $90(77.6)$ & $115(67.3)$ & 0.06 \\
\hline Stent postdilation & $111(95.2)$ & $161(94.2)$ & 0.57 \\
\hline Final kissing balloon inflation & $65(56.0)$ & $67(39.2)$ & $<0.01$ \\
\hline Single vs. two-stent approach & & & $<0.01$ \\
\hline Single stent approach & $68(58.6)$ & $152(88.9)$ & \\
\hline Two-stent approach & $48(41.4)$ & $19(11.1)$ & \\
\hline
\end{tabular}

Values are $\mathrm{n}(\%)$ or mean ( \pm SD) unless otherwise stated. In case of multiple target lesions with side-branches $\geq 2 \mathrm{~mm}$, quantitative coronary angiographic data of the lesion with the most severe lumen diameter obstruction are presented. In case of multiple bifurcated target lesions, a two-stent approach was scored if applied in at least one target lesion. Abbreviations: $\mathrm{PCl}=$ percutaneous coronary intervention; $\mathrm{SB}=$ side-branch; $\mathrm{TBL}=$ true bifurcation lesion. 
Table 3: Three-year clinical outcome of patients with true versus non-true bifurcation lesions.

\begin{tabular}{|c|c|c|c|}
\hline & \multicolumn{3}{|c|}{$\begin{array}{l}\text { All patients } \\
n=286\end{array}$} \\
\hline & $\begin{array}{l}\text { True bifurcation (TBL) } \\
\text { group } \\
n=115^{a}\end{array}$ & $\begin{array}{l}\text { Non-true bifurcation } \\
\text { (non-TBL) group } \\
n=171\end{array}$ & $\mathbf{p}$ \\
\hline \multicolumn{4}{|l|}{ Adverse events } \\
\hline All-cause mortality & $10(8.7)$ & $6(3.5)$ & 0.06 \\
\hline Cardiac death & $4(3.5)$ & $2(1.2)$ & 0.22 \\
\hline Any myocardial infarction & $13(11.3)$ & $9(5.3)$ & 0.06 \\
\hline Target-vessel related myocardial infarction & $13(11.3)$ & $9(5.3)$ & 0.06 \\
\hline Periprocedural myocardial infarction & $11(9.6)$ & $8(4.7)$ & 0.10 \\
\hline Myocardial infarction $>48 \mathrm{hr}$ post $\mathrm{PCl}$ & $2(1.7)$ & $1(0.6)$ & 0.57 \\
\hline Target-lesion revascularization (TLR) & $4(3.5)$ & $6(3.5)$ & 1.00 \\
\hline Emergent coronary bypass surgery & 0 & 0 & \\
\hline Definite or probable stent thrombosis & $1(0.9)$ & 0 & 0.40 \\
\hline \multicolumn{4}{|l|}{ Composite clinical endpoints } \\
\hline Target-lesion failure (TLF) & $19(16.5)$ & $16(9.4)$ & 0.07 \\
\hline Major adverse cardiac events (MACE) & $23(20.0)$ & $20(11.7)$ & 0.05 \\
\hline
\end{tabular}

Values are $\mathrm{n}(\%)$. $\mathrm{PCl}$ percutaneous coronary intervention. ${ }^{\text {a }}$ Due to one withdrawal of consent in the true bifurcation lesion (TBL) group, the number of patients with 3-year follow-up is one lower as compared to baseline. Target-lesion failure (TLF) is a composite of cardiac death, target-vessel-related myocardial infarction, or clinically indicated target-lesion revascularization (TLR); major adverse cardiac event (MACE) is a composite endpoint of all-cause mortality, any myocardial infarction, emergent coronary bypass surgery, or TLR; periprocedural myocardial infarctions occurred during the first $48 \mathrm{~h}$ after an index procedure.

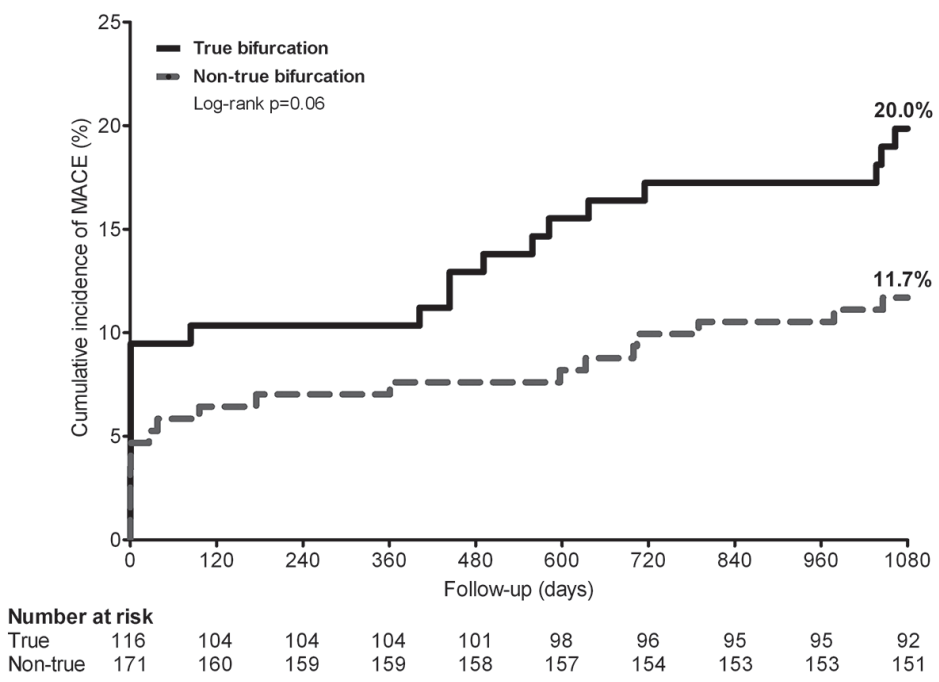

Figure 1: Cumulative incidence of MACE following $\mathrm{PCl}$ with secondgeneration DES in patients of the true versus non-true bifurcation lesion groups. All patients had been treated for at least one bifurcated target lesion with a side-branch $\geq 2 \mathrm{~mm}$. DES drug-eluting stents, MACE major adverse cardiac event, a composite endpoint of allcause mortality, any myocardial infarction, emergent coronary bypass surgery, and target-lesion revascularization, $\mathrm{PCl}$ percutaneous coronary intervention. 


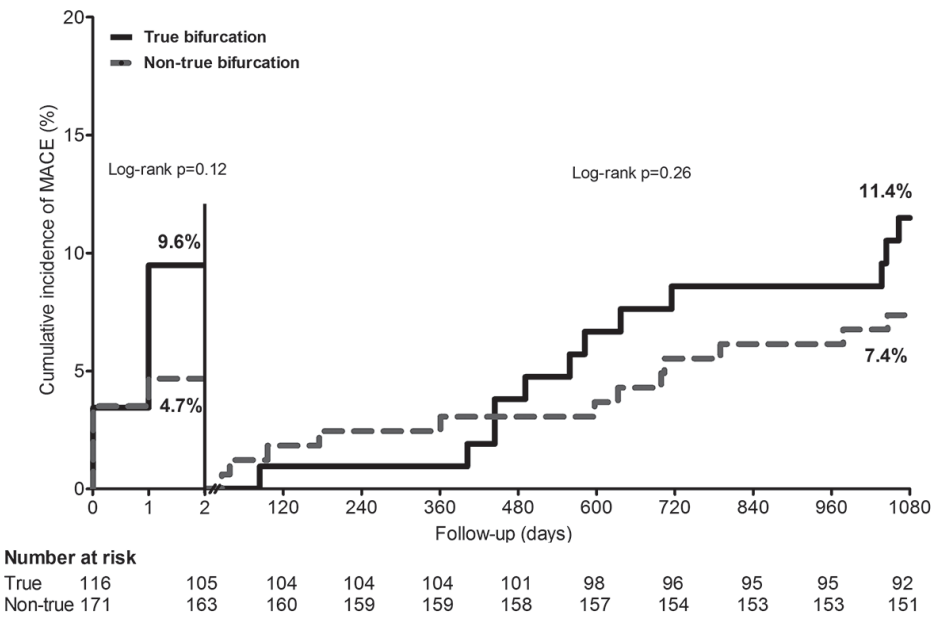

Figure 2: Landmark analysis of MACE at 2 days. MACE major adverse cardiac events, a composite endpoint of all-cause mortality, any myocardial infarction, emergent coronary artery bypass surgery, and target-lesion revascularization.

\section{Chest Pain at Follow-Up, and Adverse Events During Consecutive Time Intervals}

At 30-day, 1, 2, and 3-year follow-up, overall 3.0\%, 6.5\%, 13.0\%, and $11.0 \%$ of patients reported chest-pain at $\leq$ moderate physical effort. The percentages of patients with clinically relevant chest pain at 30-day, 1-year, 2-year, and 3-year follow-up are presented in Figure 3. There was no significant between-group difference in chest pain, and the vast majority of these patients was free from chest pain or had pain only occasionally at the very maximum level of physical exertion. MACE and coronary revascularization during subsequent time intervals were rare in both, patients with and without chest pain.

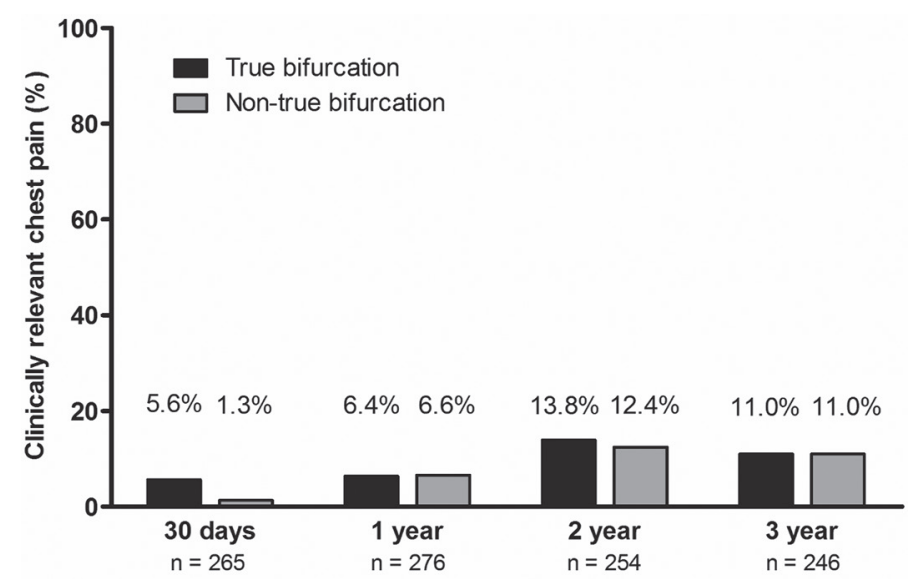

Figure 3: Clinically relevant chest pain at four time points of followup. Clinically relevant chest pain was defined as chest pain at moderate physical effort (during moderate/normal daily activities), at mild physical effort, or even at rest. 


\section{Discussion}

In the present study, we assessed the long-term outcome of 287 patients with bifurcated target lesions and side-branches $\geq 2 \mathrm{~mm}$ from the TWENTE trial, who were treated with second-generation DES. The 3-year TLR rate did not differ between 115 patients with TBL versus 171 patients with non-TBL only (3.5\% both); and in both patient groups the risk of definite-or-probable stent thrombosis was very low (both $<1.0 \%$ ). During the 3 -year follow-up of the present study, similar in both groups, the vast majority of patients was free from chest pain. The MACE rates of patients with TBL and non-TBL were $20 \%$ and $11.7 \%$. This numerical but non-significant dissimilarity in MACE $(p=0.05)$ was related to the incidence of all-cause mortality as well as Ml; the latter occurred mostly within 48 hours from stenting (i.e. periprocedural MI).

\section{Bifurcation Treatment With Second-Generation DES}

Second-generation DES have only been used in a few prospective studies that investigated clinical outcome following $\mathrm{PCl}$ for bifurcation lesions. ${ }^{9,25-28}$ In the Z-SEAside study, patients with bifurcation lesions who were treated with the Resolute stent showed a lower rate of a procedure-related composite endpoint than patients who were treated with the first-generation sirolimus-eluting stent $(n=75$, each $)^{25}$; and a multicenter registry of 180 patients who were treated with Resolute in bifurcated lesions also showed a low 9-month MACE rate. ${ }^{28}$ Long-term outcome data from dedicated bifurcation studies with second-generation DES are scarce. ${ }^{17} \mathrm{~A}$ recent pooled analysis of the 3-year clinical outcome of the randomized SEAside and CORpal studies ${ }^{9,10}$ showed a favorable MACE rate beyond 1 year in patients treated with Xience $V$ stent as compared to the first-generation sirolimus-eluting stent. ${ }^{16}$ Moreover, a retrospective study in 237 patients found acceptable clinical outcomes up to 2 years after the implantation of Xience $V$ and Resolute in bifurcation lesions. ${ }^{29}$ Recent sub-studies of the RESOLUTE All Comers, TWENTE, and DUTCH PEERS trials revealed similar and favorable 2 and 3-year clinical outcomes for patients who were treated with newer-generation DES for bifurcated versus non-bifurcated target lesions. ${ }^{13-15}$

\section{True Bifurcation Stenting}

Percutaneous interventions of TBL, which are characterized by an advanced atherosclerotic disease burden that obstructs the main vessel and the side-branch, are often technically more demanding, require more frequently complex techniques that involve the implantation of 2 stents, and were previously associated with a higher restenosis risk. ${ }^{1,3-5}$ Besides the increased procedural complexity of stenting, a more diffuse distribution of atherosclerotic plaque and a more advanced disease stage may account for an increased risk of adverse events in patients treated for TBL.

So far only few studies addresses TBL treatment with second-generation DES. A small randomized study in 69 patients revealed similar angiographic and clinical 9-month results after treatment of (predominantly true) bifurcation lesions with Xience $V$ stents, using a simple versus a complex strategy. ${ }^{26}$ These findings were confirmed by a retrospective study in 319 patients, who were treated with Xience for TBL, showing favorable angiographic and 1-year clinical outcomes in patients treated with a 2-stent technique. ${ }^{30}$ Despite the overall favorable outcome of bifurcation treatment with second-generation DES, there has been a higher incidence of periprocedural MI. 13,14,27 
Our present study suggests that the risk of periprocedural MI might be higher in TBL. This could be related to the often-greater atherosclerotic burden in TBL, which may lead to more plaque displacement (with an occlusion of small side-branches) and/or distal embolization of atherothrombotic material during stenting. ${ }^{31}$ While there is still an ongoing debate on the clinical impact of periprocedural $\mathrm{MI}^{32,33}$, it has recently been shown that periprocedural MI after treatment of TBL with a wide variety of DES types was associated with a significant increase in 1-year mortality. ${ }^{34}$

In the present study, both 3 -year mortality $(8.7 \%$ vs $3.5 \%, p=0.06)$ and MACE rate $(20.0 \%$ vs $11.7 \%$, $\mathrm{p}=0.05$ ) were, albeit statistically non-significant, numerically higher in patients with TBL versus nonTBL. While a play of chance cannot be excluded, this numeric difference might also be related to a more diffuse distribution of atherosclerosis that cannot be explained from patient demographics and cardiovascular risk factors, which did not differ between both, patients with TBL and non-TBL of the present study.

\section{Chest Pain During Follow-Up of Patients With Bifurcation Stenting}

Chest pain following successful PCI with DES is clinically and economically relevant, as it often initiates the consultation of a general practitioner or cardiologist with further cardiac assessment. ${ }^{35}$ While there is growing interest in this issue ${ }^{36}$, most recent studies on bifurcation treatment with DES have focused on device-oriented clinical endpoints. ${ }^{5}$ Overall, data on chest pain in patients treated with secondgeneration DES are scarce. ${ }^{5,36}$ The randomized DUTCH PEERS trial, found no difference in chest pain between two DES at 1 and 2-year follow-up.22

In the present study, we documented, similar to the DUTCH PEERS trial, patient-reported chest pain in relation to the patients individual range of physical activities. The majority of patients treated for TBL or non-TBL was free from clinically relevant chest pain. This symptom will generally determine whether a patient seeks further medical assessment. The absence of chest pain in the majority of our patients is supported by previous studies in bifurcation lesions, which showed that provisional stenting of the main branch generally does not result in a significant reduction in fractional flow reserve of the jailed side-branch, which means that there is usually no ischemia in the myocardium subtended by the jailed branch. ${ }^{37-39}$

\section{Study Limitations}

Due to the explorative nature and the sample size of the present study, findings should be considered hypothesis generating. The comparison of findings from different bifurcation stent studies is hampered by the fact that there is no uniformity in the minimum size of (relevant) side-branches and no general consent on whether to determine side-branch size visually or per QCA. ${ }^{4,10,13,14,25,40}$ Bifurcation lesions with side-branches $\geq 2 \mathrm{~mm}$, as addressed in our present study, were also examined in the Z-SEAside and the SEAside studies. ${ }^{9,25}$ Recently, advanced, user-friendly three-dimensional reconstruction and analysis software for bifurcation lesions has become available ${ }^{41}$, but such software was not available at the time of angiographic analysis of the TWENTE trial. 


\section{Conclusion}

Patients treated with second-generation DES for TBL had somewhat higher adverse event rates than patients with non-TBL, but dissimilarities did not reach statistical significance. Up to 3-year follow-up, the vast majority of patients of both groups remained free from chest pain. 


\section{References}

1. Jim MH, Wu EB, Fung RC, et al. Angiographic result of T-stenting with small protrusion using drug-eluting stents in the management of ischemic side branch: the ARTEMIS study. Heart Vessels 2015;30:427-431.

2. Fan L, Chen L, Luo Y, et al. DK mini-culotte stenting in the treatment of true coronary bifurcation lesions: a propensity score matching comparison with T-provisional stenting. Heart Vessels 2016;31:308-21.

3. Al Suwaidi J, Berger PB, Rihal CS, et al. Immediate and longterm outcome of intracoronary stent implantation for true bifurcation lesions. J Am Coll Cardiol 2000;35:929-936.

4. Niemelä M, Kervinen K, Erglis A, et al. Randomized comparison of final kissing balloon dilatation versus no final kissing balloon dilatation in patients with coronary bifurcation lesions treated with main vessel stenting: the Nordic-Baltic Bifurcation Study III. Circulation 2011;123:79-86.

5. Louvard Y, Lefevre T, Morice MC. Bifurcation lesions. In: Eekhout E, Serruys PW, Wijns W, Vahanian A, van Sambeek M, and de Palma R, editors. Percutaneous interventional cardiovascular medicine: the PCR-EAPCI textbook. 2012; Toulouse, France: Europa Edition, pp 283-320.

6. Serruys PW, Silber S, Garg S, et al. Comparison of zotarolimus-eluting and everolimus-eluting coronary stents. N Engl J Med 2010;363:136-146.

7. von Birgelen C, Basalus MW, Tandjung K, et al. A randomized controlled trial in second-generation zotarolimuseluting Resolute stents versus everolimus-eluting Xience $V$ stents in real-world patients: the TWENTE trial. J Am Coll Cardiol 2012;59:1350-1361.

8. Burzotta F, Trani C, Todaro D, et al. Prospective randomized comparison of sirolimus- or everolimus-eluting stent to treat bifurcated lesions by provisional approach. J Am Coll Cardiol Intv 2011;4:327-335.

9. Pan M, Medina A, Suarez de Lezo J, et al. Randomized study comparing everolimus- and sirolimus-eluting stents in patients with bifurcation lesions treated by provisional side-branch stenting. Catheter Cardiovasc Interv 2012;80:1165-1170.

10. Costopoulos C, Latib A, Ferrarello S, et al. First- versus second-generation drug-eluting stents for the treatment of coronary bifurcations. Cardiovasc Revasc Med 2013;14:311-315.

11. Herrador JA, Fernandez JC, Guzman M, Aragon V. Comparison of zotarolimus- versus everolimus-eluting stents in the treatment of coronary bifurcation lesions. Catheter Cardiovasc Interv 2011;78:1086-1092.

12. Burzotta F, Mortier P, Trani C. Characteristics of drug-eluting stent platforms potentially influencing bifurcated lesion provisional stenting procedure. Eurolntervention 2014;10:124-132.

13. Diletti R, Garcia-Garcia HM, Bourantas CV, et al. Clinical outcomes after zotarolimus and everolimus drug eluting stent implantation in coronary artery bifurcation lesions: insights from the RESOLUTE All Comers Trial. Heart 2013;99:1267-1274.

14. Lam MK, Sen H, van Houwelingen KG, et al. Three-year clinical outcome of patients with bifurcation treatment with second-generation Resolute and Xience V stents in the randomized TWENTE trial. Am Heart J 2015;169:6977.

15. van der Heijden LC, Kok MM, Lam MK, et al. Bifurcation treatment with novel, highly flexible drug-eluting coronary stents in all-comers: 2-year outcome in patients of the DUTCH PEERS trial. Clin Res Cardiol 2016; 105:206-15.

16. Pan M, Burzotta F, Trani C, et al. Three-year follow-up of patients with bifurcation lesions treated with sirolimusor everolimus-eluting stents: SEAside and CORpal Cooperative Study. Rev Esp Cardiol 2014;67:797-803.

17. von Birgelen C, van Houwelingen KG, Lam MK. Coronary bifurcations: still the touchstone of drug-eluting stents and bioresorbable vascular scaffolds? Rev Esp Cardiol (Engl Ed) 2014;67:787-789.

18. Löwik MM, Lam MK, Sen H, et al. Safety of second-generation drug-eluting stents three years after randomized use in the TWENTE trial. Eurolntervention 2015;10:1276-1279.

19. Tandjung K, Sen H, Lam MK, et al. Clinical outcome following stringent discontinuation of dual antiplatelet therapy after 12 months in real-world patients treated with second-generation zotarolimus-eluting resolute and everolimus-eluting Xience V stents: 2-year follow-up of the randomized TWENTE trial. J Am Coll Cardiol 2013;61:2406-2416.

20. Medina A, Suarez de Lezo J, Pan M. A new classification of coronary bifurcation lesions. Rev Esp Cardiol (Engl Ed) $2006 ; 59: 183$ 
21. Lassen JF, Holm NR, Stankovic G, et al. Percutaneous coronary intervention for coronary bifurcation disease: consensus from the first 10 years of the European Bifurcation Club meetings. Eurolntervention 2014;10:545560.

22. Sen H, Lam MK, Löwik MM, et al. Clinical events and patient-reported chest pain in all-comers treated with Resolute Integrity and Promus Element stents: two-year follow-up of the randomized DUTCH PEERS (TWENTE II) trial. J Am Coll Cardiol Intv 2015;8:889-899.

23. Cutlip DE, Windecker S, Mehran R, et al. Clinical end points in coronary stent trials: a case for standardized definitions. Circulation 2007;115:2344-2351.

24. Vranckx P, Cutlip DE, Mehran R, et al. Myocardial infarction adjudication in contemporary all-comer stent trials: balancing sensitivity and specificity. Addendum to the historical MI definitions used in stent studies. Eurolntervention 2010;5:871-874.

25. Burzotta F, Trani C, Talarico GP, et al. Resolute zotarolimus-eluting stent to treat bifurcated lesions according to the provisional technique: a procedural performance comparison with sirolimus- and everolimus-eluting stents. Cardiovasc Revasc Med 2013;14:122-127.

26. Ruiz-Salmerón RJ, Valenzuela LF, Pérez I, et al. Approach to coronary bifurcation lesions using the everolimuseluting stent: comparison between a simple strategy and a complex strategy with T-stenting. Rev Esp Cardiol 2013;66:636-643.

27. Džavík V, Kaul U, Guagliumi G, et al. Two-year outcomes after deployment of XIENCE V everolimus-eluting stents in patients undergoing percutaneous coronary intervention of bifurcation lesions: a report from SPIRIT V single arm study. Catheter Cardiovasc Interv 2013;82:E163-E172.

28. Tommasino A, Burzotta F, Sciahbasi A, et al. Procedural and clinical evaluation of the novel zotarolimus-eluting resolute stent in patients with unselected bifurcated coronary stenosis treated by provisional approach: a multicenter registry. J Invasive Cardiol 2011;23:50-54

29. Ferrarello S, Costopoulos C, Latib A, et al. The role of everolimus-eluting and resolute zotarolimus-eluting stents in the treatment of coronary bifurcations. J Invasive Cardiol 2013;25:436-440.

30. Kherada NI, Sartori S, Tomey MI, et al. Dedicated two-stent technique in complex bifurcation percutaneous coronary intervention with use of everolimus-eluting stents: the EES-bifurcation study. Int J Cardiol 2014;174:1317.

31. Medina A, Martín P, Suárez de Lezo J, et al. Ultrasound study of the prevalence of plaque at the carina in lesions that affect the coronary bifurcation. Implications for treatment with provisional stent. Rev Esp Cardiol 2011;64:43-50.

32. Prasad A, Herrmann J. Myocardial infarction due to percutaneous coronary intervention. N Engl J Med 2011;364:453-464.

33. Arai $T$, Yuasa $S$, Miyata $H$, et al. Incidence of periprocedural myocardial infarction and cardiac biomarker testing after percutaneous coronary intervention in Japan: results from a multicenter registry. Heart Vessels 2013;28:714-9.

34. Chen SL, Zhang JJ, Ye F, et al. Periprocedural myocardial infarction is associated with increased mortality in patients with coronary artery bifurcation lesions after implantation of a drug-eluting stent. Catheter Cardiovasc Interv 2015;85:696-705

35. Jaworski R, Jankowska EA, Ponikowski P, Banasiak W. Costs of management of patients with coronary artery disease in poland: The multicenter RECENT study. Pol Arch Med Wewn 2012;122:599-607.

36. Stone, GW. Demonstration of Clinical Superiority with a Bioresorbable Vascular Scaffold: The ABSORB III + IV Clinical Trial Program. Presented at EuroPCR 2014. May 20, Paris.

37. Ahn JM, Lee JY, Kang SJ, et al. Functional assessment of jailed side branches in coronary bifurcation lesions using fractional flow reserve. J Am Coll Cardiol Interv 2012;5:155-161.

38. Kumsars I, Narbute I, Thuesen L, et al. Side branch fractional flow reserve measurements after main vessel stenting: a Nordic-Baltic Bifurcation Study III substudy. Eurolntervention 2012;7:1155-1161.

39. Koo BK, Park KW, Kang HJ, et al. Physiological evaluation of the provisional side-branch intervention strategy for bifurcation lesions using fractional flow reserve. Eur Heart J 2008;29:726-732.

40. Girasis C, Onuma Y, Schuurbiers JC, et al. Validity and variability in visual assessment of stenosis severity in phantom bifurcation lesions: a survey in experts during the fifth meeting of the European Bifurcation Club. Catheter Cardiovasc Interv 2012;79:361-368. 
41. Tu S, Echavarria-Pinto M, von Birgelen C, et al. Fractional flow reserve and coronary bifurcation anatomy: a novel quantitative model to assess and report the stenosis severity of bifurcation lesions. J Am Coll Cardiol Intv 2015;8:564-574. 


\section{Conflicts of interest statement}

CVB was in the past consultant to several device-manufacturing companies; the institution has received research grants, provided by AstraZeneca, Abbott Vascular, Biotronik, Boston Scientific, and Medtronic. The other authors declared to have no potential conflict. 


\title{
CHAPTER 12
}

\section{Clinical Outcome of All-Comer Patients With Severely Calcified Bifurcation Lesions Treated With New-Generation Coronary Drug-Eluting Stents}

\author{
A Patient-Level Pooled Analysis From TWENTE and \\ DUTCH PEERS (TWENTE II)
}

Jennifer Huisman, Liefke C. van der Heijden, Marlies M. Kok, Peter W. Danse,

Rutger L. Anthonio, K. Gert van Houwelingen, Marije M. Löwik, Paolo Zocca, Gerard C.M. Linssen, Carine J.M. Doggen, Clemens von Birgelen
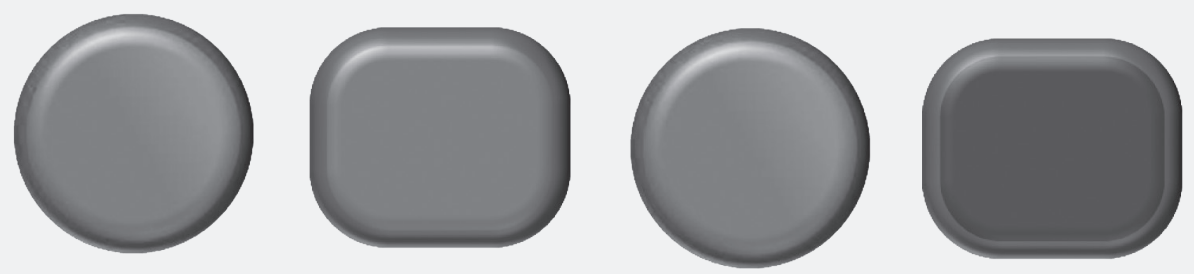


\begin{abstract}
Background: Percutaneous coronary interventions $(\mathrm{PCl})$ of both bifurcated lesions and severely calcified lesions still have an increased event risk.

Aim: We evaluated the hypothesis that, in patients undergoing $\mathrm{PCl}$ of bifurcated lesions with newergeneration drug-eluting stents (DES), clinical event risk may be higher if these lesions are severely calcified.

Methods: In a patient-level pooled analysis of data from the TWENTE and DUTCH PEERS randomized clinical trials (ClinicalTrials.gov NCT01066650 and NCT01331707), we assessed the impact of severe target lesion calcification on 2-year clinical outcomes of patients who underwent $\mathrm{PCl}$ with newergeneration DES for bifurcated lesions.

Results: Of 827 patients with bifurcated target lesions, 168 (20.3\%) had at least one severe target lesion calcification. These patients were older $(p<0.001)$, more often diabetics ( $p=0.002)$, and less often active smokers $(p=0.02$ ) than patients who had no severely calcified lesion. They underwent more often lesion pretreatment ( $86.3 \%$ vs $75.6 \% ; p=0.003$ ) and treatment of long lesions ( $31.0 \%$ vs $17.5 \% ; p<0.001$ ), but single-stent treatment ( $80.4 \%$ vs $80.7 \% ; p=0.91$ ) and kissing-balloon inflation ( $36.9 \%$ vs $36.4 \% ; p=0.91$ ) rates were similar. There was no significant between-group difference in the composite clinical endpoint target vessel failure (11.9\% vs $10.2 \%$; $p$-Logrank $=0.51$ ) or its components (cardiac death, target vessel $\mathrm{MI}$, or target vessel revascularization). Definite-or-probable stent thrombosis rates were low and similar (0.6\% vs $0.9 \%$; $p$-Logrank=1.0). Multivariate analyses with propensity score adjustment showed that severe calcification was not an independent predictor of events.
\end{abstract}

Conclusion: Among all-comers treated with newer-generation DES in bifurcation lesions, the presence of a severe calcification was not associated with significant increase in adverse event risk. 


\section{Introduction}

In the treatment of coronary artery disease with percutaneous coronary intervention ( $\mathrm{PCl}$ ), bifurcation lesions account for $15-20 \%$ of all lesions treated. ${ }^{1}$ Despite improvement in stent design and $\mathrm{PCl}$ technique over the last decades, bifurcated target lesions and severely calcified target lesions remain challenges to $\mathrm{PCl}$ and show an increased risk of suboptimal results and adverse outcomes. ${ }^{1-7}$ The introduction of first-generation drug-eluting stents (DES) reduced the risk of in-stent restenosis as compared to $\mathrm{PCl}$ with bare metal stents. ${ }^{8-11}$ Meanwhile, newer-generation permanent polymer DES with improved biocompatibility, thinner stent struts, and more flexible designs have demonstrated great efficacy and favorable safety in broad and almost unrestricted study populations. ${ }^{12-21}$

The TWENTE and DUTCH PEERS randomized trials assessed such newer-generation DES in broad study populations that reflect patients treated in routine clinical practice, including many patients treated for bifurcated and/or severely calcified lesions. In both trials, the 2-year clinical outcome of the overall study population was similar for the DES compared. ${ }^{12,13}$ Recent sub-studies based on TWENTE and DUTCH PEERS data showed higher event rates following PCl in bifurcated coronary lesions ${ }^{6,7}$ as well as after $\mathrm{PCl}$ in severely calcified lesions. ${ }^{22}$ The hypothesis is quite conceivable that patients undergoing $\mathrm{PCl}$ in severely calcified bifurcation lesions may have an even higher adverse event risk than patients who are treated either in bifurcated lesions or in calcified lesions. Yet there is a lack of data about the outcome of patients who underwent $\mathrm{PCl}$ in bifurcated lesions that were severely calcified. ${ }^{23}$ Therefore, in the present patient-level pooled analysis of data from the TWENTE and DUTCH PEERS randomized trials, we assessed the impact of severe target lesion calcification on the 2-year outcome of patients who were treated with newer-generation permanent polymer-coated DES in bifurcated coronary lesions.

\section{Methods}

\section{Patient population, procedures, and study design}

The present post hoc analysis of the TWENTE (The Real-World Resolute Versus Xience V Drug-Eluting Stent Study in Twente; ClinicalTrials.gov NCT01066650) and DUTCH PEERS (TWENTE II) (Durable PolymerBased Stent Challenge of Promus Element vs. Resolute Integrity; ClinicalTrials.gov NCT01331707) trial $^{12,13}$ assessed the impact of severe target lesion calcification on the 2-year clinical outcome in 827 patients treated in at least one bifurcated target lesion. Both trials complied with the Declaration of Helsinki and were approved by the Medical Ethics Committee Twente and the institutional review boards of all participating centers. All patients provided written informed consent.

The study design, definitions of clinical endpoints, characteristics of patients, lesions, procedures, and the 1 and 2-year clinical outcomes of both trials have previously been reported. ${ }^{12,13,24,25}$ TWENTE and DUTCH PEERS are investigator-initiated, patient-blinded, randomized trials in which respectively 1,391 and 1,811 patients with stable or acute coronary syndromes were enrolled (an acute ST-elevation MI was an exclusion criterion in TWENTE, but not in DUTCH PEERS). ${ }^{12,13,24,25}$ The TWENTE trial compared, after 1:1 randomization, the Resolute zotarolimus-eluting stent (Medtronic Vascular, Santa Rosa, CA) 
and the Xience V everolimus-eluting stent (Abbott Vascular, Santa Clara, CA). All-comer patients in the DUTCH PEERS trial were 1:1 randomized to treatment with the Resolute Integrity zotarolimus-eluting stent (Medtronic Vascular, Santa Rosa, CA) or the Promus Element everolimus-eluting stent (Boston Scientific, Natick, MA). Interventional procedures and application of concomitant medication were performed in accordance with contemporary medical guidelines, clinical standards, and the physician's judgment. The stenting technique, medical treatment strategy, and the use of final kissing-balloon inflation were left to the operator's discretion. The generally recommended approach for bifurcation treatment was provisional stenting. 6,7 Processing of clinical outcome data and clinical event adjudication were performed by independent external clinical research organizations (Cardialysis, Rotterdam, and Diagram, Zwolle, the Netherlands). Experienced angiographic analysts from Thoraxcentrum Twente, blinded to stent-type and clinical outcome, performed offline quantitative coronary angiographic analyses according to current standards for all patients (Qangio XA 7.1 and 7.2, Medis, Leiden, the Netherlands).

For the purpose of the present analysis, all trial participants treated for at least one bifurcated lesion were categorized into patients with versus without severe calcification of at least one bifurcated target lesion. A subgroup analysis was performed in patients with only a single target lesion. A relevant bifurcation was defined, according to the definition in the SYNTAX score and analogues to previous studie, as a junction of a main vessel and a side-branch with a minimum lumen diameter $\geq 1.5 \mathrm{~mm}$ (after intracoronary administration of nitrates and before $\mathrm{PCl}$ ), as measured by quantitative coronary angiography. ${ }^{6,7,26}$ Target lesion calcification was, analogous to previous studies, defined as readily apparent densities or x-ray absorbing masses, noted within the apparent vascular wall at the site of the target lesion prior to any contrast injection; severe target lesion calcification was noted without cardiac motion before contrast injection and generally compromised both sides of the arterial wall. ${ }^{22,26-28}$

\section{Clinical endpoints}

Clinical endpoints were defined according to the Academic Research Consortium (ARC), including the addendum on myocardial infarction ${ }^{27,29}$, analogues to previous studies. $6,7,22,28$ Death was considered cardiac, unless an evident non-cardiac cause could be determined. Myocardial infarction (MI) was defined by any creatine kinase concentration of more than double the upper limit of normal with elevated values of a confirmatory cardiac biomarker. Periprocedural MI was defined as target vessel $\mathrm{MI}$ within 48 hours after $\mathrm{PCl}$. Stent thrombosis was classified according to the ARC definitions. The composite endpoint target vessel failure (TVF) was defined as cardiac death, target vessel $\mathrm{MI}$, or clinically driven target vessel revascularization. Major adverse cardiac events (MACE) consisted of allcause death, any MI, emergent coronary bypass surgery, or clinically indicated TLR.

\section{Statistical analysis}

Data were reported as frequencies and percentages for dichotomous and categorical variables, and as mean \pm standard deviation or median (interquartile range) for continuous variables. Chi-square tests and Fisher's exact tests were used for dichotomous and categorical variables, and continuous variables were assessed using Student's t tests or the Wilcoxon rank-sum tests, as appropriate. The Kaplan-Meier method was used to determine the time to clinical endpoint and the Log-rank test was 
applied to compare between-group differences. Parameters were considered as potential confounders if in univariate analyses associations were found with a $p$-value $<0.15$. For adjustment of potential confounders, propensity score analysis was used. The propensity score was estimated using multiple logistic regression analysis. A multivariate Cox regression model, including the propensity score as independent variable, was then used to adjust for the propensity score. All p-values and confidence intervals were two-sided and $p$-values $<0.05$ were considered significant. Data analysis was performed with SPSS (version 22.0, SPSS Inc., Chicago, IL, USA).

\section{Results}

\section{Baseline patient, lesion, and procedural characteristics}

A total of 827 patients in the randomized TWENTE and DUTCH PEERS trial were treated for a bifurcated lesion. Of these patients, 168 (20.3\%) were treated for at least one severely calcified bifurcation lesion. Patients with severely calcified bifurcated target lesions were older, had more often diabetes mellitus and hypercholesterolemia, and were less often active smokers (Table 1) than the 659 (79.7\%) patients with bifurcated target lesions that were not severely calcified. Patients with severely calcified bifurcated target lesions underwent more often multi-vessel treatment (37.5\% vs $28.4 \% ; p=0.02$ ) and treatment of long (>27 mm) lesions (31.0\% vs 17.5\%; $p<0.001$ ) (Table 2). In addition, lesion pretreatment was more often performed in patients with severely calcified lesions ( $86.3 \%$ vs $75.6 \%$; $p=0.003$ ) (Table 2 ). In most patients ( $80.4 \%$ vs $80.7 \% ; p=0.91$ ), the bifurcation lesions were treated with a single-stent approach. The rate of final kissing-balloon inflation did not differ between patients with versus without severe target lesion calcification ( $36.9 \%$ vs $36.4 \% ; p=0.91$ ).

\section{Clinical event rates at 2-year follow-up}

Two-year follow-up data were available in all patients (100\%). Patients with versus without severe calcification of the bifurcated target lesions showed no statistically significant difference in the incidence of the composite clinical endpoint target vessel failure (11.9\% vs $10.2 \%$; $p$-Logrank $=0.51)$ and its components: cardiac death (2.4\% vs $1.5 \%$; p-Logrank=0.50), target vessel-related $\mathrm{MI}$ ( $4.8 \%$ vs $5.5 \%$; p-Logrank $=0.72$ ), and target vessel revascularization ( $5.4 \%$ vs $4.1 \%$; $p$-Logrank $=0.48$ ) (Table 3, Figure 1). In addition, the rates of definite or probable stent thrombosis after 2 years were low and similar for both groups (0.6\% vs $0.9 \%$; plogrank $=1.0)$.

\section{Multivariate analysis, and subgroup analysis of patients with a single target lesion}

Multivariate analyses with the use of propensity score adjustment revealed that the presence of severe lesion calcification in patients with bifurcated lesions was not an independent predictor of various adverse clinical events (Table 3, adjusted p-values). In addition, among patients treated with $\mathrm{PCl}$ for single bifurcated lesions, the rates of the various clinical endpoints (Table 4) were similar for patients with versus without severe target lesion calcification. 
Table 1: Baseline characteristics of patients with at least one bifurcated target lesion, comparing patients with versus without severe calcification of bifurcated target lesions.

\begin{tabular}{|c|c|c|c|}
\hline \multirow[b]{2}{*}{ Patient characteristics } & \multicolumn{3}{|c|}{$\begin{array}{l}\text { Patients with at least one bifurcated target lesion } \\
\qquad n=827\end{array}$} \\
\hline & $\begin{array}{l}\text { Severe calcification } \\
\qquad n=168\end{array}$ & $\begin{array}{l}\text { No severe calcification } \\
\qquad n=659\end{array}$ & p \\
\hline Age (yrs) & $67.5 \pm 9.9$ & $63.1 \pm 11.0$ & $<0.001$ \\
\hline Men & $126(75.0)$ & $512(77.7)$ & 0.46 \\
\hline $\mathrm{BMI}\left(\mathrm{kg} / \mathrm{m}^{2}\right)$ & $27.3 \pm 3.9$ & $27.8 \pm 4.3$ & 0.17 \\
\hline Diabetes mellitus & $46(27.4)$ & $112(17.0)$ & 0.002 \\
\hline Hypertension & $91(54.2)$ & $348(52.8)$ & 0.75 \\
\hline Hypercholesterolemia & $95(57.2)$ & $321(49.3)$ & 0.07 \\
\hline Active smoking & $28(16.7)$ & $167(25.3)$ & 0.02 \\
\hline Family history of CAD & $89(53.0)$ & 329 (49.9) & 0.48 \\
\hline Chronic renal failure* & $5(3.0)$ & $15(2.3)$ & 0.60 \\
\hline Peripheral artery disease & $12(7.2)$ & $45(6.9)$ & 0.91 \\
\hline Previous MI & $54(32.1)$ & $172(26.1)$ & 0.12 \\
\hline Previous $\mathrm{PCl}$ & $29(17.3)$ & $122(18.5)$ & 0.71 \\
\hline Previous CABG & $28(16.7)$ & 39 (5.9) & $<0.001$ \\
\hline Clinical syndrome & & & 0.89 \\
\hline ST-elevation myocardial infarction & $13(7.9)$ & $59(9.0)$ & \\
\hline Non-ST-elevation myocardial infarction & $47(28.0)$ & 171 (25.9) & \\
\hline Unstable angina & $28(16.7)$ & $120(18.2)$ & \\
\hline Stable angina & $80(47.6)$ & 309 (46.9) & \\
\hline
\end{tabular}

Values are mean $\pm \mathrm{SD}$, or $\mathrm{n}(\%) .{ }^{*}$ Chronic renal failure was defined as a serum creatinine level $>130 \mu \mathrm{mol} / \mathrm{L}$. Abbreviations: $\mathrm{BMI}=$ body mass index; $\mathrm{CABG}=$ coronary artery bypass grafting; $\mathrm{CAD}=$ coronary artery disease; $\mathrm{Ml}=$ myocardial infarction; $\mathrm{PCl}=$ percutaneous coronary intervention. 
Table 2: Lesion and procedural characteristics of patients with at least one bifurcated target lesion, comparing patients with versus without severe calcification of bifurcated target lesions.

\begin{tabular}{|c|c|c|c|}
\hline \multirow[b]{2}{*}{ Lesion / procedural characteristics } & \multicolumn{3}{|c|}{$\begin{array}{l}\text { Patients with at least one bifurcated target lesion } \\
\qquad n=827\end{array}$} \\
\hline & $\begin{array}{l}\text { Severe calcification } \\
n=168\end{array}$ & $\begin{array}{l}\text { No severe calcification } \\
\qquad n=659\end{array}$ & $\mathbf{p}$ \\
\hline Multi-vessel treatment & $63(37.5)$ & $187(28.4)$ & 0.02 \\
\hline \multicolumn{4}{|l|}{ Treated coronary vessels } \\
\hline Right coronary artery & $35(20.8)$ & $126(19.1)$ & 0.62 \\
\hline Left anterior descending artery & $125(74.4)$ & $480(72.8)$ & 0.68 \\
\hline Circumflex artery & $59(35.1)$ & $224(34.0)$ & 0.78 \\
\hline De novo lesions & $150(89.3)$ & $593(90.0)$ & 0.79 \\
\hline At least one chronic total occlusion & $12(7.1)$ & $33(5.0)$ & 0.28 \\
\hline At least one lesion length $>27 \mathrm{~mm}$ & $52(31.0)$ & $115(17.5)$ & $<0.001$ \\
\hline At least one in-stent restenosis & $6(3.6)$ & $25(3.8)$ & 0.89 \\
\hline At least one ostial lesion & $23(13.7)$ & $35(5.3)$ & $<0.001$ \\
\hline Medina classification & & & 0.09 \\
\hline 0.0 .1 & $14(8.3)$ & $53(8.0)$ & \\
\hline 0.1 .0 & $19(11.3)$ & $111(16.8)$ & \\
\hline 0.1 .1 & $11(6.5)$ & $53(8.0)$ & \\
\hline 1.0 .0 & $22(13.1)$ & $92(14.0)$ & \\
\hline 1.0.1 & $9(5.4)$ & $51(7.7)$ & \\
\hline 1.1 .0 & $42(25.0)$ & $168(25.5)$ & \\
\hline 1.1 .1 & $51(30.4)$ & $131(19.9)$ & \\
\hline True bifurcation (Medina 0.1.1; 1.0.1; 1.1.1) & $71(42.3)$ & $235(35.7)$ & 0.11 \\
\hline Lesion predilation & $145(86.3)$ & $498(75.6)$ & 0.003 \\
\hline Rotational atherectomy & $9(5.4)$ & $1(0.2)$ & $<0.001$ \\
\hline Cutting balloon & $20(11.9)$ & $11(1.7)$ & $<0.001$ \\
\hline Stenting approach & & & 0.91 \\
\hline 1-stent technique & $135(80.4)$ & $532(80.7)$ & \\
\hline 2-stent technique & $33(19.6)$ & $127(19.3)$ & \\
\hline Maximum diameter stenosis (\%) pre $\mathrm{PCI}$ * & $70.0(61.0-77.6)$ & $66.5(57.9-76.0)$ & 0.01 \\
\hline Maximum diameter stenosis (\%) post $\mathrm{PCl} *$ & $14.5(10.8-17.9)$ & $15.6(11.3-20.4)$ & 0.003 \\
\hline Maximum implantation pressure stent (atm) & $15.8 \pm 2.6$ & $15.2 \pm 2.6$ & 0.006 \\
\hline Postdilation & $157(93.5)$ & $583(88.5)$ & 0.06 \\
\hline Maximale pressure postdilation* (atm) & $23.4 \pm 4.6$ & $22.1 \pm 5.5$ & 0.008 \\
\hline Final kissing balloon inflation & $62(36.9)$ & $240(36.4)$ & 0.91 \\
\hline Minimum lumen diameter post $\mathrm{PCl}(\mathrm{mm})$ & $2.04 \pm 0.60$ & $2.04 \pm 0.54$ & 0.94 \\
\hline Total stent length & $45.0(28.0-67.5)$ & $36.0(22.0-56.0)$ & $<0.001$ \\
\hline
\end{tabular}

Values are mean $\pm \mathrm{SD}$, or $\mathrm{n}(\%)$. ${ }^{*} \mathrm{In}$ the case of multiple target lesions, the most severe is presented. $\mathrm{Abbreviations:} \mathrm{PCl}=$ percutaneous coronary intervention 


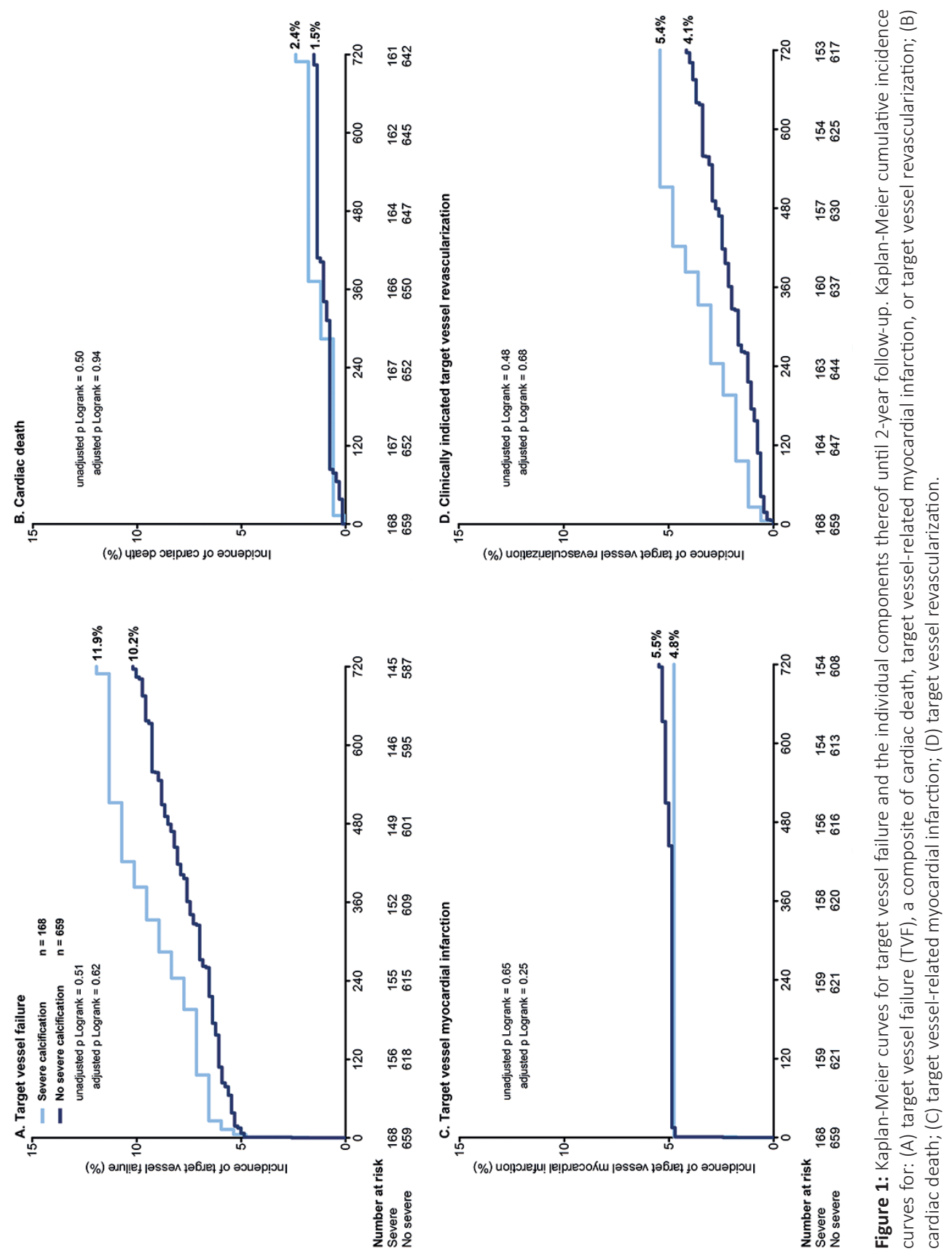


Table 3: Clinical outcomes at 2 years of patients with at least one bifurcated target lesion, comparing patients with versus without severe calcification of bifurcated target lesions.

\begin{tabular}{|c|c|c|c|c|}
\hline & \multicolumn{4}{|c|}{$\begin{array}{l}\text { Patients with at least one bifurcated target lesion } \\
\qquad \mathrm{n}=827\end{array}$} \\
\hline & $\begin{array}{l}\text { Severe calcification } \\
n=168\end{array}$ & $\begin{array}{l}\text { No severe calcification } \\
\qquad \mathrm{n}=659\end{array}$ & $\begin{array}{l}\text { Unadjusted } \\
\mathbf{p}\end{array}$ & $\begin{array}{c}\text { Adjustec } \\
\mathbf{p}\end{array}$ \\
\hline Cardiac death & $4(2.4)$ & $10(1.5)$ & 0.50 & 0.94 \\
\hline $\begin{array}{l}\text { Target vessel myocardial } \\
\text { infarction }\end{array}$ & $8(4.8)$ & $36(5.5)$ & 0.72 & 0.25 \\
\hline Target vessel revascularization* & $9(5.4)$ & $27(4.1)$ & 0.48 & 0.68 \\
\hline Target lesion revascularization* & $6(3.6)$ & $20(3.0)$ & 0.72 & 0.82 \\
\hline Target vessel failure & 20 (11.9) & $67(10.2)$ & 0.51 & 0.62 \\
\hline Major adverse cardiac events & $22(13.1)$ & $69(10.5)$ & 0.33 & 0.73 \\
\hline $\begin{array}{l}\text { Definite or probable stent } \\
\text { thrombosis }\end{array}$ & $1(0.6)$ & $6(0.9)$ & 1.00 & 0.58 \\
\hline
\end{tabular}

Values are $\mathrm{n}(\%) .{ }^{*}$ Clinically indicated. Adjusted P values were derived from propensity score-adjusted analyses.

Table 4: Clinical outcomes at 2 years of patients with single target lesions, comparing patients with versus without severe calcification of bifurcated target lesion.

\begin{tabular}{|lccc|}
\hline & \multicolumn{2}{c|}{$\begin{array}{c}\text { Patients with a single target lesion, bifurcated lesions only } \\
\mathbf{n = 4 6 4}\end{array}$} & $\begin{array}{c}\text { No severe calcification } \\
\mathbf{n = 3 7 6}\end{array}$ \\
\cline { 2 - 2 } & $\begin{array}{c}\text { Severe calcification } \\
\mathbf{n = 8 8}\end{array}$ & $6(1.6)$ & 0.65 \\
Cardiac death & $2(2.3)$ & $11(2.9)$ & 1.00 \\
Target vessel myocardial infarction & $2(2.3)$ & $15(4.0)$ & 0.77 \\
Target vessel revascularization* & $4(4.5)$ & $12(3.2)$ & 1.00 \\
Target lesion revascularization* & $2(2.3)$ & $29(7.7)$ & 0.67 \\
Major adverse cardiac events & $8(9.1)$ & $33(8.8)$ & 0.67 \\
Definite or probable stent thrombosis & $9(10.2)$ & $1(0.3)$ & 1.00 \\
\hline
\end{tabular}

Values are $\mathrm{n}(\%) .{ }^{*}$ Clinically indicated.

\section{Discussion}

The technical evolution from bare metal stents to first-generation and, most recently, to newergeneration DES has reduced the risk of lesion recurrence and other adverse events, as demonstrated by several clinical trials that examined broad study populations. 8,15,30-33 Nevertheless, bifurcated and/ or calcified lesions remain a challenge in terms of procedural success and clinical outcome. ${ }^{1,2,34}$ In this patient-level pooled analysis of data from the TWENTE and DUTCH PEERS (TWENTE II) trials, the rates 
of the composite clinical endpoint TVF at 2-year follow-up (11.9\% vs $10.2 \%$ ) did not differ significantly between patients who underwent stenting in severely calcified bifurcated lesions versus patients with bifurcated target lesions that did not show a severe calcification. Moreover, the 2-year stent thrombosis rates $(0.6 \%$ vs $0.9 \%)$ were low and similar. Thus, treatment of bifurcated lesions with newer-generation DES in the presence of severe calcification was not associated with a significant increase in clinical event risk.

\section{Treatment of bifurcated coronary lesions}

Stenting of bifurcation lesions bears an increased (periprocedural) MI risk. ${ }^{6,720,21}$ Recent analyses of data from the TWENTE and DUTCH PEERS trials demonstrated low and similar rates of several clinical endpoints following coronary stenting in bifurcated and non-bifurcated target lesions, but periprocedural $\mathrm{MI}$ rates were still higher in patients with bifurcated target lesion. ${ }^{6,7}$ Such events may result from distal (micro)embolization of athero-thrombotic debris, closure of side-branches, flow-limiting dissections, or the occurrence of slow flow or no-reflow after stenting. ${ }^{6,7,35}$ The impact of routine final kissing-balloon inflation on clinical outcome is still a matter of discussion. $., 7,36-38$ The Nordic-Baltic Bifurcation Study III explored the effect of routine final kissing-balloon inflation after successful provisional stenting of the main branch and found that the incidence of angiographic side-branch (re)stenosis was lowered without significant improvement in clinical outcome; however, periprocedural MI was not included in the measure of clinical outcome. ${ }^{36}$ Previous analyses of data from the TWENTE and DUTCH PEERS trials demonstrated similar rates of the composite clinical endpoint target vessel failure in patients with bifurcated target lesions who underwent final kissing balloon inflation and those who did not. ${ }^{6,7}$

\section{Challenges of calcified target lesions}

Severe target lesion calcification remains an independent predictor of all-cause mortality despite recent improvements in DES technology, as demonstrated by a patient-level pooled analysis of seven stent trials. ${ }^{39}$ In addition, in patients with calcified target lesions, the incidence of the combined endpoint of all-cause mortality, MI, or any revascularization was significantly higher. ${ }^{39}$ In general, it is unattractive to directly stent calcified target lesions, as this increases the risk of stent under-expansion, which may promote restenosis and even stent thrombosis. ${ }^{40}$ Furthermore, the polymer coatings of DES may be damaged in highly calcified vessels, which can reduce the delivered drug dosage and might affect the prevention of restenosis. ${ }^{34}$ Certain properties of DES (e.g. strut thickness, device flexibility, and biocompatibility) have been improved in newer-generation durable polymer devices. ${ }^{33}$ However, recent analyses demonstrated that at 2-year follow-up severe target lesion calcification was still an independent predictor of target vessel failure in both patients with stable angina and patients with acute coronary syndromes. ${ }^{22,28}$

\section{Stenting bifurcated coronary lesions with severe calcification}

In most studies, both bifurcated lesion and target lesion calcification are associated with an increased adverse event risk. ${ }^{6,720-22,28,39}$ This easily leads to the assumption that the combination of these challenging lesion characteristics might further increase risk. However, the findings of our present analysis suggest that severe target lesion calcification does not represent an independent additional risk to bifurcation 
treatment. Nevertheless, patients undergoing $\mathrm{PCl}$ of severely calcified bifurcation lesions required more often predilation and aggressive pretreatment of their target lesions, and stents were implanted and postdilated at higher pressures. More aggressive lesion pretreatment comprised a higher use of rotational atherectomy and cutting balloons in order to favorably modify calcified target lesions, which facilitates stent delivery and improves stent expansion with the ultimate goal of optimizing the final procedural result. ${ }^{40,42}$ In the present study, rotational atherectomy and cutting balloon inflation were more frequently used in patients with severely calcified bifurcated target lesions (5.4\% and $11.9 \%$, respectively) than in patients treated in bifurcated lesions without severe calcification $(0.2 \%$ and $1.7 \%$, respectively). Nevertheless, both patient groups were similar in their high adherence to provisional stenting ( $80.4 \%$ vs $80.7 \%)$; and kissing balloon inflations were only performed in slightly more than one third of the cases (36.9\% vs $36.4 \%$ ). The various factors discussed above may have contributed to the quite favorable clinical outcomes of severely calcified bifurcated lesions. The low rates of both target vessel-related $\mathrm{MI}$ and stent thrombosis represent a safety signal for the newer-generation durable polymer metallic DES used in TWENTE and DUTCH PEERS. However, operators may not take these favorable results for granted, for instance if severely calcified bifurcated lesions are treated without appropriate measures to optimize delivery and expansion of stents.

\section{Limitations}

The patients with severely calcified bifurcation lesions were identified from 3,202 participants in largescale DES trials with liberal patient enrolment. Nevertheless, the sample size does not have sufficient power to reliably assess very rare adverse events, such as stent thrombosis. Due to the study's posthoc nature, our findings should be considered hypothesis generating only. Future prospective studies will still be of great value. We defined the presence of target lesion calcification on X-ray appearance; superior intracoronary imaging techniques (e.g., intravascular ultrasound or optical coherence tomography) were only infrequently used. Nevertheless, this X-ray-based approach of classifying lesion calcification has previously been used by other researchers. ${ }^{22,39}$

\section{Conclusion}

Among all-comers in the TWENTE and DUTCH PEERS trials treated with newer-generation DES in bifurcation lesions, the presence of a severe calcification of the bifurcated target lesion was not associated with a significant increase in adverse event risk. 


\section{References}

1. Lassen JF, Holm NR, Stankovic G, et al. Percutaneous coronary intervention for coronary bifurcation disease: consensus from the first 10 years of the European Bifurcation Club meetings. Eurolntervention. 2014;10:545-60.

2. von Birgelen C, van Houwelingen KG, Lam MK. Coronary bifurcations: still the touchstone of drug-eluting stents and bioresorbable vascular scaffolds? Rev Esp Cardiol. 2014;67:797-803.

3. Madhavan MV, Tarigopula M, Mintz GS, Maehara A, Stone GW, Généreux P. Coronary artery calcification: pathogenesis and prognostic implications. J Am Coll Cardiol. 2014;63:1703-14.

4. Reimers B, von Birgelen C, van der Giessen WJ, Serruys PW. A word of caution on optimizing stent deployment in calcified lesions: acute coronary rupture with cardiac tamponade. Am Heart J. 1996;131:192-4.

5. Bourantas CV, Zhang YJ, Garg S, et al. Prognostic implications of coronary calcification in patients with obstructive coronary artery disease treated by percutaneous coronary intervention: a patient-level pooled analysis of 7 contemporary stent trials. Heart. 2014;100:1158-64.

6. Lam MK, Sen H, van Houwelingen KG, et al. Three-year clinical outcome of patients with bifurcation treatment with second-generation Resolute and Xience V stents in the randomized TWENTE trial. Am Heart J. 2015;169:6977.

7. van der Heijden LC, Kok MM, Lam MK, et al. Bifurcation treatment with novel, highly flexible drug-eluting coronary stents in all-comers: 2-year outcome in patients of the DUTCH PEERS trial. Clin Res Cardiol. 2016;105:206-15.

8. Colombo F, Biondi-Zoccai G, Infantino V, et al. A long-term comparison of drug-eluting versus bare metal stents for the percutaneous treatment of coronary bifurcation lesions. Acta Cardiol. 2009;64:583-8.

9. Thuesen L, Kelbaek H, Kløvgaard L, et al. Comparison of sirolimus-eluting and bare metal stents in coronary bifurcation lesions: subgroup analysis of the Stenting Coronary Arteries in Non-Stress/Benestent Disease Trial (SCANDSTENT). Am Heart J. 2006;152:1140-5.

10. Romagnoli E, De Servi S, Tamburino C, et al. Real-world outcome of coronary bifurcation lesions in the drugeluting stent era: results from the 4,314-patient Italian Society of Invasive Cardiology (SICI-GISE) Italian Multicenter Registry on Bifurcations (I-BIGIS). Am Heart J. 2010;160:535-42.

11. Colombo A, Moses JW, Morice MC, et al. Randomized study to evaluate sirolimus-eluting stents implanted at coronary bifurcation lesions. Circulation. 2004;109:1244-9.

12. von Birgelen C, Basalus MWZ, Tandjung K, et al. A randomized controlled trial in second-generation zotarolimuseluting Resolute stents versus everolimus-eluting Xience V stents in real-world patients: the TWENTE trial. J Am Coll Cardiol. 2012;59:1350-1361.

13. von Birgelen C, Sen H, Lam MK, et al. Third-generation zotarolimus-eluting and everolimus-eluting stents in all-comer patients requiring a percutaneous coronary intervention (DUTCH PEERS): a randomised, single-blind, multicentre, non-inferiority trial. Lancet. 2014;383:413-23.

14. Burzotta F, Trani C, Todaro D, et al. Prospective randomized comparison of sirolimus- or everolimus-eluting stent to treat bifurcated lesions by provisional approach. JACC Cardiovasc Interv. 2011;4:327-35.

15. Costopoulos C, Latib A, Ferrarello S, et al. First versus second-generation drug-eluting stents for the treatment of coronary bifurcations. Cardiovasc Revasc Med. 2013;14:311-5.

16. Ferrarello S, Costopoulos C, Latib A, et al. The role of everolimus-eluting and resolute zotarolimus-eluting stents in the treatment of coronary bifurcations. J Invasive Cardiol. 2013;25:436-440.

17. Tommasino A, Burzotta F, Sciahbasi A, et al. Procedural and clinical evaluation of the novel zotarolimus-eluting resolute stent in patients with unselected bifurcated coronary stenosis treated by provisional approach: a multicenter registry. J Invasive Cardiol. 2011;23:50-4.

18. Kedhi E, Joesoef KS, McFadden E, et al. Second-generation everolimus-eluting and paclitaxel-eluting stents in real-life practice (COMPARE): a randomised trial. Lancet. 2010;375:201-9.

19. Serruys PW, Silber S, Garg S, et al. Comparison of zotarolimus-eluting and everolimus-eluting coronary stents. N Engl J Med. 2010;363:136-46.

20. Akin I, Hochadel M, Abdel-Wahab M, et al. Clincial outcomes of different first- and second-generation drugeluting stents in routine clinical practice: results from the prospective multicenter German DES.DE registry. Clin Res Cardiol. 2013;102:371-381.

21. Diletti R, Garcia-Garcia HM, Bourantas CV, et al. Clinical outcomes after zotarolimus and everolimus drug eluting stent implantation in coronary artery bifurcation lesions: insights from the RESOLUTE All Comers Trial. Heart. 2013;99:1267-74. 
22. Huisman J, van der Heijden LC, Kok MM, et al. Impact of severe lesion calcification on clinical outcome of patients with stable angina, treated with newer generation permanent polymer-coated drug-eluting stents: A patient-level pooled analysis from TWENTE and DUTCH PEERS (TWENTE II). Am Heart J. 2016;175:121-129.

23. Butman SM. Warning: this report does not address heavily calcified coronary arteries. Catheter Cardiovasc Interv. 2010;76:643.

24. Tandjung K, Sen H, Lam MK, et al. Clinical outcome following stringent discontinuation of dual antiplatelet therapy after 12 months in real-world patients treated with second-generation zotarolimus-eluting resolute and everolimus-eluting Xience V stents: 2-year follow-up of the randomized TWENTE trial. J Am Coll Cardiol. 2013;18;61:2406-16.

25. Sen H, Lam MK, Löwik MM, et al. Clinical events and patient-reported chest pain in all-comers treated with Resolute Integrity and Promus Element stents: two-year follow-up of the randomized DUTCH PEERS (TWENTE II) trial. J Am Coll Cardiol Intv. 2015;8:889-99.

26. Sianos G, Morel MA, Kappetein AP, et al. The SYNTAX Score: an angiographic tool grading the complexity of coronary artery disease. Eurolntervention. 2005;1:219-27.

27. Vranckx P, Cutlip DE, Mehran R, et al. Myocardial infarction adjudication in contemporary all-comer stent trials: balancing sensitivity and specificity. Addendum to the historical MI definitions used in stent studies. Eurolntervention. 2010;5:871-4.

28. Huisman J, van der Heijden LC, Kok MM, et al. Two-year outcome after treatment of severely calcified lesions with newer-generation drug-eluting stents in acute coronary syndromes: A patient-level pooled analysis from TWENTE and DUTCH PEERS. J Cardiol. 2017;69:660-5.

29. Cutlip DE, Windecker S, Mehran R, et al. Clinical end points in coronary stent trials: a case for standardized definitions. Circulation. 2007;115:2344-51.

30. Bangalore S, Vlachos HA, Selzer F, et al. Percutaneous coronary intervention of moderate to severe calcified coronary lesions: insights from the National Heart, Lung, and Blood Institute Dynamic Registry. Catheter Cardiovasc Interv. 2011;77:22-8.

31. Palmerini T, Biondi-Zoccai G, Della Riva D, et al. Stent thrombosis with drug-eluting and bare-metal stents: evidence from a comprehensive network meta-analysis. Lancet. 2012;379:1393-402.

32. Navarese EP, Tandjung K, Claessen B, et al. Safety and efficacy outcomes of first and second generation durable polymer drug eluting stents and biodegradable polymer biolimus eluting stents in clinical practice: comprehensive network meta-analysis. BMJ. 2013;347:f6530.

33. Stefanini GG, Taniwaki M, Windecker S. Coronary stents: novel developments. Heart. 2014;100:1051-61.

34. Wiemer M, Butz T, Schmidt W, Schmitz KP, Horstkotte D, Langer C. Scanning electron microscopic analysis of different drug eluting stents after failed implantation: from nearly undamaged to major damaged polymers. Catheter Cardiovasc Interv. 2010;75:905-11.

35. Park DW, Kim YH, Yun SC, et al. Impact of the angiographic mechanisms underlying periprocedural myocardial infarction after drug-eluting stent implantation. Am J Cardiol. 2004;113:1105-10.

36. Niemelä M, Kervinen K, Erglis A, et al. Randomized comparison of final kissing balloon dilatation versus no final kissing balloon dilatation in patients with coronary bifurcation lesions treated with main vessel stenting: the Nordic-Baltic Bifurcation Study III. Circulation. 2011;123:79-86.

37. Hariki $H$, Shinke $T$, Otake $H$, et al. Potential benefit of final kissing balloon inflation after single stenting for the treatment of bifurcation lesions-insights from optical coherence tomography observations. Circ J. 2013;77:1193-201.

38. Kim TH, Lee HJ, Jang $\mathrm{H}$, et al. Impact of final kissing balloon inflation after simple stent implantation for the treatment of non-left main true coronary bifurcation lesions in patients with acute coronary syndrome. Int J Cardiol. 2014;177:907-11.

39. Généreux P, Madhavan MV, Mintz GS, et al. Ischemic outcomes after coronary intervention of calcified vessels in acute coronary syndromes. Pooled analysis from the HORIZONS-AMI (Harmonizing Outcomes With Revascularization and Stents in Acute Myocardial Infarction) and ACUITY (Acute Catheterization and Urgent Intervention Triage Strategy) trials. J Am Coll Cardiol. 2014;63:1845-54.

40. Abdel-Wahab M, Baev R, Dieker P, et al. Long-term clinical outcome of rotational atherectomy followed by drugeluting stent implantation in complex calcified coronary lesions. Catheter Cardiovasc Interv. 2013;81:285-91

41. Abdel-Wahab M, Richardt G, Joachim Büttner H, et al. High-speed rotational atherectomy before paclitaxeleluting stent implantation in complex calcified coronary lesions: the randomized ROTAXUS (Rotational Ahterectomy prior to taxus stent treatment for complex native coronary artery disease) trial. J Am Coll Cardiol Intv. 2013;6:10-9. 


\section{Conflicts of interest statement}

CVB has been consultant to and has received lecture fees or travel expenses from Abbott Vascular, Boston Scientific, and Medtronic; he received lecture fees from MSD and AstraZeneca. The institution has received research grants, provided by Abbott Vascular, Biotronik, Boston Scientific, and Medtronic. All other authors declare that they have no conflict of interest.

\section{Copyright}

Reprinted with permission from

American Heart Journal 2016 Jun;176:28-35.

(c) Elsevier 


\section{CHAPTER 13}

\section{Small Vessel Treatment With Contemporary Newer-Generation Drug-Eluting Coronary Stents in All-Comers}

Insights From 2-Year DUTCH PEERS (TWENTE II)

Randomized Trial

Liefke C. van der Heijden, Marlies M. Kok, Peter W. Danse, Alexander R. Schramm, Marc Hartmann, Marije M. Löwik, Gerard C.M. Linssen, Martin G. Stoel, Carine J.M. Doggen, Clemens von Birgelen
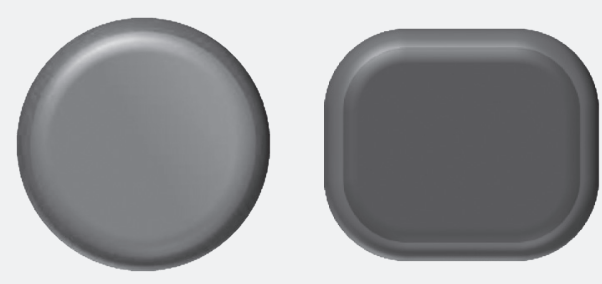


\begin{abstract}
Background: Treatment of lesions in small vessels was associated with worse clinical outcome, and various definitions of 'small vessels' have been used. Data with novel drug-eluting stents (DES) are scarce.

Methods: To compare the outcome of patients with versus without small vessel treatment, we assessed 2-year follow-up data of the DUTCH PEERS randomized trial (clinicaltrial.gov:NCT01331707), in which 1,811 all-comers were treated with contemporary zotarolimus-eluting (Resolute Integrity) or everolimus-eluting (Promus Element) stents. Primary endpoint was target lesion failure (TLF), a composite of cardiac death, target vessel myocardial infarction (MI), and target lesion revascularization (TLR).

Results: The rates of TLF (9.5\% vs 5.4\%, p-Logrank=0.001) and two individual components thereof target vessel MI (3.1\% vs 1.3\%, p-Logrank $=0.006)$ and TLR ( $4.8 \%$ vs $2.8 \%$, $p$-Logrank $=0.02)$ - were higher among $798(44.1 \%)$ patients treated in at least one small vessel $(<2.50 \mathrm{~mm}$ by quantitative coronary angiography). Multivariate analysis with propensity score adjustment demonstrated that treatment of small vessel-lesions independently predicted TLF at 2-year follow-up (HR 1.60, 95\% Cl: 1.09-2.34). Patients with the smallest target vessel being $<2.25 \mathrm{~mm}$ had TLF rates similar to patients with smallest target vessels of 2.25 to $<2.50 \mathrm{~mm}$; however, patients treated in vessels no smaller than 2.50 to $<3.00$ $\mathrm{mm}$ and patients treated in vessels $\geq 3.00 \mathrm{~mm}$ had lower TLF rates $(9.3 \%, 9.8 \%, 5.0 \%, 5.8 \%$, respectively, p-Logrank=0.009).
\end{abstract}

Conclusion: Patients treated with novel DES in small vessel lesions had higher adverse event rates than patients who had no small vessel treatment. Our data suggest that with current stents a vessel diameter $<2.50 \mathrm{~mm}$ is a suitable threshold to identify small target vessels. 


\section{Introduction}

Treatment of lesions in small coronary vessels is a challenge for percutaneous coronary intervention (PCI) and has been associated with an increased risk of adverse clinical events. ${ }^{1-6}$ Several studies have shown an impact of the stent type used on clinical outcome following PCl in small vessels, especially on the occurrence of restenosis. ${ }^{2,7-12}$ In contrast to bare metal stents ${ }^{2-6}$, the use of first-generation DES in small vessels reduced the need for repeat revascularization due to less in-stent neointimal proliferation. ${ }^{8,13-16}$ Nevertheless, early DES still showed a certain neointima-induced late lumen loss during follow-up, which may be related to more unfavorable consequences and adverse clinical events in small target vessels compared to large vessels. ${ }^{17}$ Strut thickness, lesion length, and the minimum stent lumen diameter were previously identified as independent predictors of restenosis in DES. 4,6,18,19 Treatment of small vessel lesions with second-generation durable polymer DES may result in somewhat more favorable results, but data from randomized clinical all-comer trials are scarce.-11,20,21 As a consequence, it is of interest to investigate the clinical outcome of patients who were treated with novel DES in small target vessels. ${ }^{22}$

The randomized DUTCH PEERS trial compares in all-comer patients two recent DES that combine the coating and drug of second-generation DES with modified metallic stent platforms that aim at an increase in flexibility and deliverability. ${ }^{23}$ In this study, the zotarolimus-eluting Resolute Integrity (Medtronic, Santa Rosa, CA) and the everolimus-eluting Promus Element stent (Boston Scientific, Natick, MA) have shown similar and favorable safety and efficacy up to 2 years. ${ }^{24}$

In the present substudy of the DUTCH PEERS trial, we analyzed the 2-year clinical follow-up data of allcomer patients treated for lesions in at least one small coronary vessel $(<2.50 \mathrm{~mm})$ versus patients with target lesions in larger sized vessels ( $\geq 2.50 \mathrm{~mm}$ ). In addition, adverse clinical event rates of patients with various different minimum target vessel sizes (i.e. previous definitions of small vessels) were compared to further evaluate the impact of target vessel size on clinical outcome.

\section{Methods}

\section{Study design and patient population}

The randomized, patient-blinded, multicenter DUTCH PEERS (TWENTE II) trial compares the Resolute Integrity zotarolimus-eluting cobalt-chromium stent (Medtronic Vascular, Santa Rosa, CA) and the Promus Element everolimus-eluting platinum-chromium stent (Boston Scientific, Natick, MA). The study design and procedures of this investigator-initiated trial (ClinicalTrials.gov:NCT01331707) have been described in detail23,25 and 2-year clinical outcome has been reported. ${ }^{24}$ In brief, 1,811 all-comer patients who were treated by $\mathrm{PCl}$ for de-novo or restenotic lesions in coronary arteries or bypass grafts were enrolled. There was no limit for lesion length, reference size, or number of lesions or diseased vessels to be treated. Both Resolute Integrity stents as Promus Element stents were available with nominal sizes ranging from $2.25 \mathrm{~mm}$ to $4.00 \mathrm{~mm}$. The trial complied with the Declaration of Helsinki and was approved by the independent Medical Ethics Committee Twente and the institutional review boards of all participating centers. All patients provided written informed consent. Study enrollment was performed between November 2010 and May 2012. 
In the present sub-study, patients who were treated for at least one small vessel lesion were compared to patients treated for lesions in larger vessels only. A small vessel was defined as a coronary artery with a reference vessel diameter of less than $2.50 \mathrm{~mm}$, as measured by quantitative coronary angiography (QCA).

\section{Clinical follow-up, monitoring, event adjudication, and angiographic analysis}

Interventional procedures and application of concomitant medication were performed in accordance to medical guidelines, clinical standards, and the physician's judgment. Lesion predilation, direct stenting, stent postdilation and use of glycoprotein $\mathrm{Ilb} / \mathrm{Illa}$ receptor antagonists were left at the operator's discretion. In general, dual antiplatelet therapy was prescribed for 12 months. ${ }^{23}$

Research nurses, blinded to the treatment arm, obtained information on clinical events through a medical questionnaire or, in the absence of a response, a telephone interview that was based on the same questions. Data monitoring was performed by the independent contract research organization (CRO) Diagram (Zwolle, the Netherlands). The independent CRO Cardialysis (Rotterdam, the Netherlands) performed the processing of clinical outcome data and clinical event adjudication.

For all patients, offline quantitative coronary angiographic analysis was performed according to current standards by angiographic analysts from Thoraxcentrum Twente, who were blinded for the stent-type and clinical outcome (Qangio XA 7.2, Medis, Leiden, the Netherlands).

\section{Clinical endpoints}

Definitions of all predefined clinical endpoints have previously been described in detail ${ }^{23,25}$ and follow the suggestions from the Academic Research Consortium (ARC), including the addendum on myocardial infarction. ${ }^{26,27}$ Death was considered cardiac, unless an evident non-cardiac cause could be established. Myocardial infarction (MI) was defined by any creatine kinase concentration of more than double the upper limit of normal with elevated values of a confirmatory cardiac biomarker. A target vessel-related $\mathrm{MI}$ was related to the target vessel or could not be related to another vessel. Side branches of bifurcated target lesions were only classified as target vessel when being stented. Periprocedural MI (PMI) was defined as target vessel-related $\mathrm{MI}$ within 48 hours after $\mathrm{PCl}$. Both clinical outcomes as well as enzyme elevation as reported by the extended historical MI definition were used for defining periprocedural $\mathrm{MI}$ in case of elevated CK or (CKMB) from index MI that has not yet reached its maximum level. ${ }^{27}$ Stent thrombosis was classified according to the ARC definitions. ${ }^{26,27}$

The composite endpoint target lesion failure (TLF) was defined as cardiac death, target vessel-related MI or clinically indicated target lesion revascularization (TLR). TLF, which is based on these three individual event components with different mechanisms and time courses, reflects the device and lesion-related part of the entire spectrum of adverse events that may occur during the years of follow-up. TLR is considered clinically indicated if the angiographic diameter stenosis was $\geq 70 \%$, or $\geq 50 \%$ in the presence of ischemic signs or symptoms. The use of FFR was left to the operator's discretion. Major Adverse Cardiac Events (MACE) was defined as a composite of all-cause death, any MI, emergent coronary artery bypass surgery (CABG), or clinically indicated TLR. 


\section{Statistical analysis}

Data were reported as frequencies and percentages for dichotomous and categorical variables, and as mean \pm standard deviation for continuous variables. Categorical variables were assessed with the chi-square test or Fisher's exact test as appropriate, whereas continuous variables were assessed with the Student's $t$ test or Wilcoxon rank-sum test as appropriate. The Kaplan-Meier analysis was used to calculate the time to clinical endpoint and the Log-rank test was applied to compare between-group differences. A two-sided p-value of less than 0.05 was considered significant. Variables were considered as potential confounders if associations were found with a $p$-value $<0.15$ in univariate analysis. For adjustment of potential confounders, propensity score analysis was used. The propensity score was estimated using multiple logistic regression analysis. Diabetes mellitus, current smoker, previous acute $\mathrm{MI}$, clinical syndrome at presentation, multivessel treatment, total number of stents implanted, treatment of at least one CTO treatment, LAD treatment, RCX treatment, bifurcation treatment, percentage diameter stenosis post $\mathrm{PCl}$, Syntax score, and postdilatation were used to calculate the propensity score for treatment of small vessel lesions. Subsequently, a Cox-regression analysis was performed using small vessel treatment and the propensity score as independent variables. Statistical analyses were performed with SPSS (version 22.0, SPSS Inc., Chicago, IL, USA).

\section{Funding}

The investigator-initiated DUTCH PEERS (TWENTE II) study is equally funded by Boston Scientific and Medtronic; there are no other sponsors than indicated. The authors are solely responsible for the design and conduct of this study, all study analyses and drafting and editing of the paper.

\section{Results}

\section{Characteristics of patients, lesions, and $\mathrm{PCl}$ procedures}

A total of $798(44.1 \%)$ of all 1,811 randomized trial participants were treated in at least one small vessel (diameter $<2.50 \mathrm{~mm}$ ). 1,013 (55.9\%) patients were treated for larger vessels only. Patients treated for small vessel lesions more often had a history of diabetes mellitus ( $20.4 \%$ vs $15.9 \% ; p=0.01$ ) and a previous $\mathrm{MI}$, more often presented with stable angina, and more often underwent treatment of multiple vessels (Table 1). Treatment was more frequently performed in bifurcation lesions $(28.2 \%$ vs 23.7\%; $p=0.03$ ) and lesions longer than $27 \mathrm{~mm}$ (20.2\% vs 15.5\%; $p=0.009)$, and thereby, as expected, the total number of stents implanted and the total stent length were higher in patients with small vessel lesions (Table 1). 
Table 1: Baseline characteristics of study population, comparing patients with vs without small-vessel treatment.

\begin{tabular}{|c|c|c|c|}
\hline \multirow[b]{2}{*}{ Patient characteristics } & \multicolumn{2}{|c|}{$\begin{array}{c}\text { All patients } \\
n=1811\end{array}$} & \multirow[b]{2}{*}{ p-value } \\
\hline & $\begin{array}{c}\text { Small vessel } \\
n=798\end{array}$ & $\begin{array}{c}\text { No small vessel } \\
n=1013\end{array}$ & \\
\hline Age (yrs) & $64.2 \pm 10.7$ & $63.8 \pm 10.8$ & 0.40 \\
\hline Female & $228(28.6)$ & $261(25.8)$ & 0.18 \\
\hline Diabetes mellitus & $163(20.4)$ & $161(15.9)$ & 0.01 \\
\hline Current smoker & $167(20.9)$ & $277(27.3)$ & 0.002 \\
\hline Arterial hypertension & $430(53.9)$ & $554(54.7)$ & 0.73 \\
\hline Hypercholesterolaemia & $381(47.7)$ & $467(46.1)$ & 0.49 \\
\hline Family history of coronary artery disease & $404(50.6)$ & $499(49.3)$ & 0.56 \\
\hline Previous MI & $208(26.1)$ & $189(18.7)$ & $<0.001$ \\
\hline Previous $\mathrm{PCl}$ & $159(19.9)$ & $190(18.8)$ & 0.53 \\
\hline Previous CABG & $84(10.5)$ & $89(8.8)$ & 0.21 \\
\hline Stable angina pectoris & $384(48.1)$ & $365(36.0)$ & $<0.001$ \\
\hline \multicolumn{4}{|l|}{ Lesion/procedural characteristics } \\
\hline Stent type used & & & 0.14 \\
\hline Zotarolimus-eluting stent & $415(52.0)$ & $491(48.5)$ & \\
\hline Everolimus-eluting stent & $383(48.0)$ & $522(51.5)$ & \\
\hline Multivessel treatment & $196(24.6)$ & $100(9.9)$ & $<0.001$ \\
\hline \multicolumn{4}{|l|}{ Treated coronary vessels } \\
\hline Right coronary artery & $240(30.1)$ & $429(42.3)$ & $<0.001$ \\
\hline Left anterior artery & $423(53.0)$ & $431(42.5)$ & $<0.001$ \\
\hline Circumflex artery & $315(39.5)$ & $208(20.5)$ & $<0.001$ \\
\hline De novo lesions & $706(88.5)$ & $921(90.9)$ & 0.09 \\
\hline At least one chronic total occlusion & $54(6.8)$ & $22(2.2)$ & $<0.001$ \\
\hline At least one in-stent restenosis & $17(2.1)$ & $38(3.8)$ & 0.05 \\
\hline At least one severe calcification & $175(21.9)$ & $232(22.9)$ & 0.62 \\
\hline At least one bifurcation & $225(28.2)$ & $240(23.7)$ & 0.03 \\
\hline At least one lesion length $>27 \mathrm{~mm}$ & $161(20.2)$ & $157(15.5)$ & 0.009 \\
\hline Total stent length & $44.9 \pm 30.2$ & $32.5 \pm 21.4$ & $<0.001$ \\
\hline Number of stents per patient & $2.1 \pm 1.2$ & $1.5 \pm 0.8$ & $<0.001$ \\
\hline Postdilation & $602(75.4)$ & $801(79.1)$ & 0.07 \\
\hline Degree of stenosis pre $\mathrm{PCl}$ & $71.4 \pm 18.4$ & $70.4 \pm 17.5$ & 0.24 \\
\hline Degree of stenosis post $\mathrm{PCl}$ & $18.5 \pm 8.7$ & $16.6 \pm 7.8$ & $<0.001$ \\
\hline
\end{tabular}

Values are $\mathrm{n}(\%)$ or mean \pm SD. A small vessel was defined by a reference vessel diameter $<2.5 \mathrm{~mm}$ by quantitative coronary angiography. Abbreviation: $\mathrm{CABG}$, Coronary artery bypass grafting. 
Table 2: Two-year clinical outcome, comparing patients with vs without small-vessel treatment.

\begin{tabular}{|c|c|c|c|c|c|}
\hline & & & $\begin{array}{l}\text { All patients } \\
n=1810\end{array}$ & & \\
\hline & $\begin{array}{c}\text { Small vessel } \\
n=798\end{array}$ & $\begin{array}{l}\text { No small vessel } \\
n=1012\end{array}$ & $\begin{array}{l}\text { Unadjusted } \\
\text { p-Logrank }\end{array}$ & $\begin{array}{l}\text { Hazard ratio } \\
\qquad(95 \% \mathrm{CI})\end{array}$ & $\begin{array}{l}\text { Adjusted } \\
p=\text { Logrank }\end{array}$ \\
\hline Death & $28(3.5)$ & $29(2.9)$ & 0.44 & $1.33(0.75-2.36)$ & 0.33 \\
\hline Cardiac death & $20(2.5)$ & 19 (1.9) & 0.36 & $1.37(0.69-2.74)$ & 0.37 \\
\hline Target vessel MI & $25(3.1)$ & $13(1.3)$ & 0.006 & $1.40(0.67-2.93)$ & 0.37 \\
\hline Periprocedural MI & $19(2.4)$ & $11(1.1)$ & 0.03 & $1.05(0.46-2.38)$ & 0.90 \\
\hline $\begin{array}{l}\text { Clinically indicated target lesion } \\
\text { revascularization }\end{array}$ & $38(4.8)$ & $28(2.8)$ & 0.03 & $1.83(1.07-3.13)$ & 0.03 \\
\hline Target lesion failure & $76(9.5)$ & $55(5.4)$ & 0.001 & $1.60(1.05-2.10)$ & 0.02 \\
\hline Major adverse cardiac events & $86(10.8)$ & $69(6.8)$ & 0.003 & $1.48(1.05-2.10)$ & 0.03 \\
\hline $\begin{array}{l}\text { Definite or probable stent } \\
\text { thrombosis }\end{array}$ & $12(1.5)$ & $8(0.8)$ & 0.15 & $1.54(0.57-4.13)$ & 0.39 \\
\hline
\end{tabular}

Values are $\mathrm{n}(\%)$. A small vessel was defined by a reference vessel diameter $<2.5 \mathrm{~mm}$ by quantitative coronary angiography. Twoyear follow-up was available for 1,810 of all 1,811 patients (99.9\%).

\section{Clinical outcome and multivariate analysis}

Two-year follow-up data was available in 1,810 (99.9\%) patients (Table 2). ${ }^{25}$ A Kaplan-Meier analysis for TLF is presented in Figure 1 (9.5\% vs 5.4\%; p-Logrank=0.003, unadjusted HR 1.79; $95 \% \mathrm{Cl}$ : 1.27-2.54). These time-to-event curves reflect a higher incidence of target vessel MI (3.1\% vs 1.3\%; $\mathrm{p}$-Logrank=0.006) and clinically indicated TLR (4.8\% vs $2.8 \%$; $\mathrm{p}$-Logrank=0.02) in patients treated for small vessel lesions. Landmark analysis revealed that during the first 48 hours from stenting the rate of target vessel MI was significantly higher among patients treated for small vessel lesions versus patients treated for lesions in vessels $\geq 2.50 \mathrm{~mm}$ ( $2.4 \%$ vs $1.1 \%$; $p$-Logrank=0.03). From 48 hours until 2 years of follow-up the rate of target vessel MI was numerically, but not significantly higher in patients treated for small vessel lesions $(0.8 \%$ vs $0.2 \%$; $p$-Logrank $=0.07)$. In patients with multivessel $\mathrm{PCl}$ and small vessel lesions, all but three target vessel $\mathrm{MI}$ were related to a small vessel lesion. Multivariate analysis with propensity score adjustment demonstrated that, after adjustment for all potential confounders, treatment of small vessel lesions was an independent predictor of TLF at 2-year follow-up (HR 1.60, 95\% Cl: 1.09-2.34). Patients treated with Resolute Integrity versus Promus Element stents had similar rates of the composite main clinical endpoint TLF (9.9\% vs 9.1\%; $p=0.72$ ). In patients with single vessel treatment, the TLF rate was higher in patients the smallest target vessel being $<2.50 \mathrm{~mm}$ vs $\geq 2.50 \mathrm{~mm}$ ( $8.6 \%$ vs $5.4 \%, p=0.01)$. The rates of target vessel MI were $2.7 \%$ vs $1.2 \%(p=0.04)$.

\section{Vessel sizes}

A total of $493(61.8 \%)$ patients were treated for at least one lesion in a very small vessel $(<2.25 \mathrm{~mm})$; in these patients, the rates of the composite endpoint TLF and various other clinical endpoints were comparable to those of 305 (38.2\%) patients with the smallest target vessel diameter being 2.25 to $<2.50 \mathrm{~mm}$ (Table 3, Figure 2). Both, patients treated in vessels no smaller than 2.50 to $<3.00 \mathrm{~mm}$ and patients treated in vessels $\geq 3.00 \mathrm{~mm}$ had lower TLF rates ( $p$-Logrank=0.009, Figure 2). 

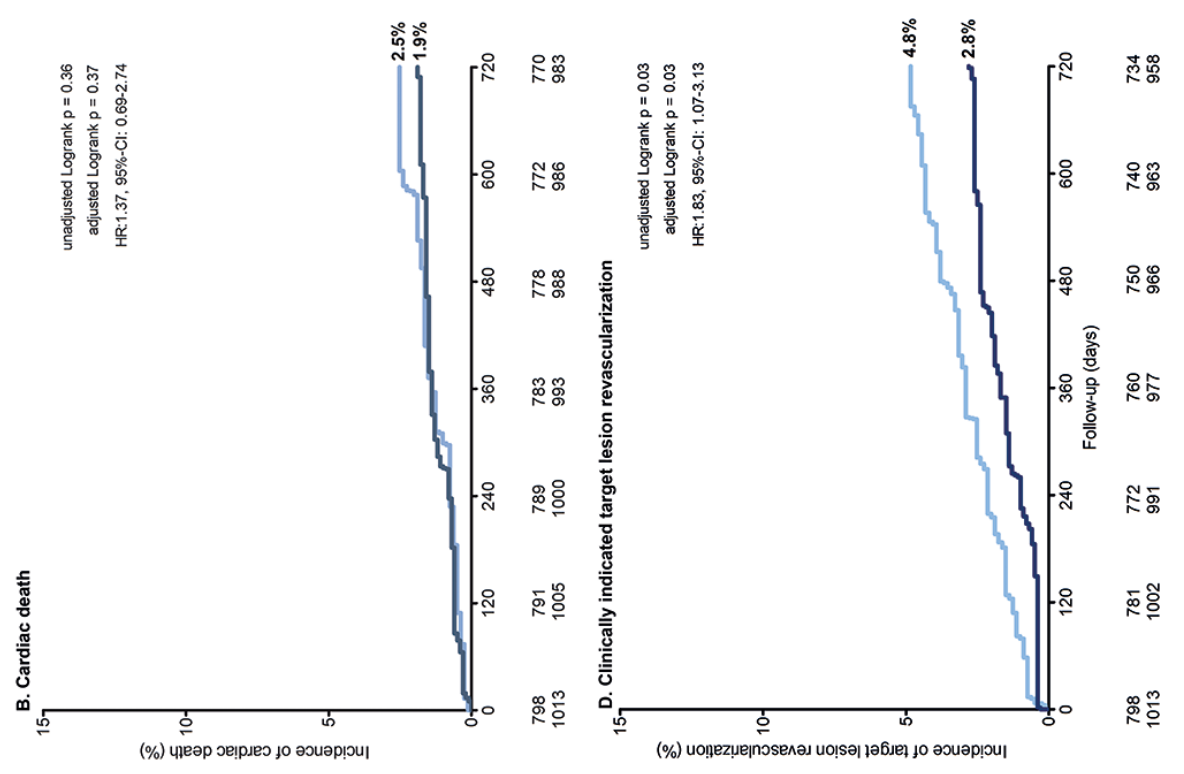

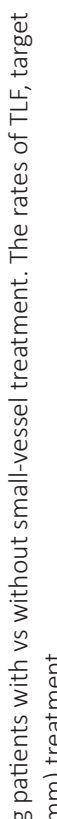
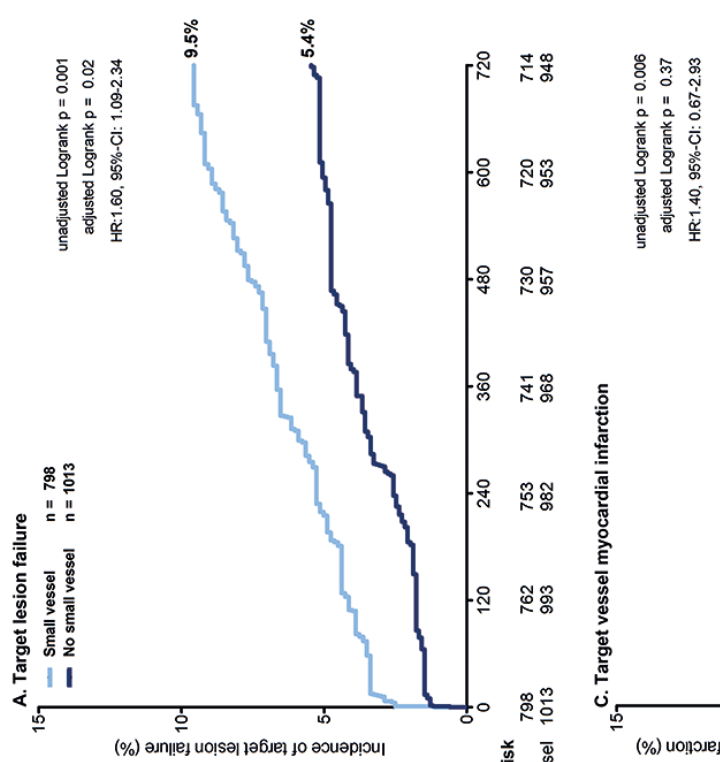

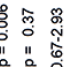

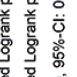

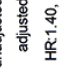

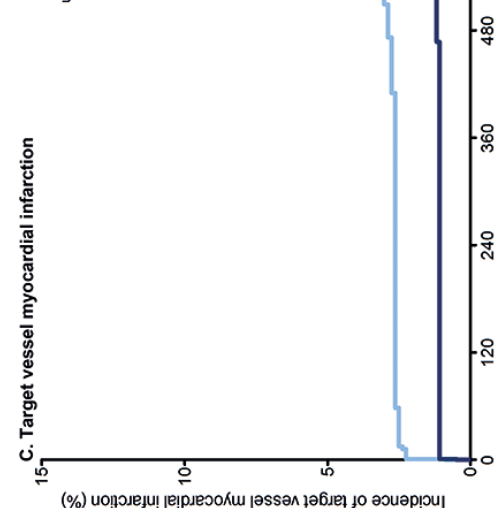

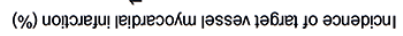




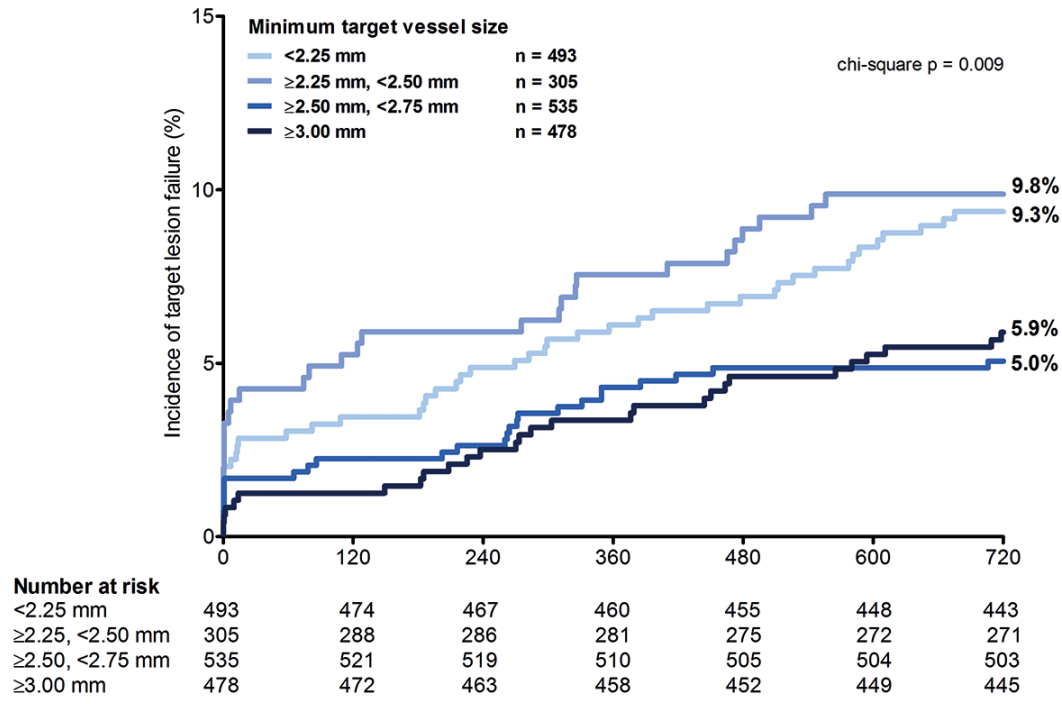

Figure 2: Target lesion failure in subgroups of patients with increasingly smaller minimum target vessel size. Patients with the smallest target vessel being $<2.25 \mathrm{~mm}$ had TLF rates similar to patients with a smallest target vessels being 2.25 to $<2.50 \mathrm{~mm}$, whereas patients treated in vessels no smaller than 2.50 to $<3.00 \mathrm{~mm}$ as well as patients treated in vessels $\geq 3.00 \mathrm{~mm}$ only had lower TLF rates.

Table 3: Two-year clinical outcome of all patients treated in small target vessels, comparing patients treated in very small-vessel lesions $(<2.25 \mathrm{~mm}$ ) vs patients treated in target vessels being 2.25 to $<2.50 \mathrm{~mm}$.

\begin{tabular}{|c|c|c|c|c|c|}
\hline & \multicolumn{5}{|c|}{$\begin{array}{l}\text { Small vessel } \\
\qquad n=798\end{array}$} \\
\hline & $\begin{array}{c}<2.25 \mathrm{~mm} \\
\mathrm{n}=493\end{array}$ & $\begin{array}{c}\geq 2.25 \mathrm{~mm} \\
\mathrm{n}=305\end{array}$ & $\begin{array}{l}\text { Unadjusted } \\
\text { p-Logrank }\end{array}$ & $\begin{array}{l}\text { Hazard ratio } \\
(95 \% \mathrm{CI})\end{array}$ & $\begin{array}{l}\text { Adjusted } \\
\text { p-Logrank }\end{array}$ \\
\hline Death & $17(3.4)$ & $11(3.6)$ & 0.91 & $1.03(0.47-2.24)$ & 0.94 \\
\hline Cardiac death & $13(2.6)$ & $7(2.3)$ & 0.76 & $0.88(0.34-2.25)$ & 0.79 \\
\hline Target vessel MI & $13(2.6)$ & $12(3.9)$ & 0.31 & $1.90(0.84-4.28)$ & 0.12 \\
\hline Periprocedural MI & $9(1.8)$ & $10(3.3)$ & 0.19 & $2.50(0.98-6.38)$ & 0.06 \\
\hline $\begin{array}{l}\text { Clinically indicated target lesion } \\
\text { revascularization }\end{array}$ & $24(4.9)$ & $14(4.6)$ & 0.76 & $1.02(0.52-2.01)$ & 0.95 \\
\hline Target lesion failure & $46(9.3)$ & $30(9.8)$ & 0.81 & $1.20(0.75-1.93)$ & 0.45 \\
\hline Major adverse cardiac events & $51(10.3)$ & $35(11.5)$ & 0.62 & $1.24(0.80-1.93)$ & 0.34 \\
\hline Definite or probable stent thrombosis & $8(1.6)$ & $4(1.3)$ & 1.00 & $1.02(0.30-3.53)$ & 0.97 \\
\hline
\end{tabular}

Values are $\mathrm{n}(\%)$. A small vessel was defined by a reference vessel diameter $<2.50 \mathrm{~mm}$ by quantitative coronary angiography. 


\section{Discussion}

\section{Major findings}

All-comer patients treated for at least one small vessel lesion had a significantly higher incidence of target lesion failure at 2-year follow-up, after adjustment for any potential confounders (HR 1.60, 95\% Cl: 1.09-2.34). This difference resulted mostly from a higher rate of target vessel $\mathrm{MI}$ and clinically indicated TLR. Within the subgroup of patients treated for lesions in small vessels, there was no difference in clinical endpoints between patients treated for very small vessel $(<2.25 \mathrm{~mm})$ lesions versus patients who underwent stenting of vessels with a minimum diameter of 2.25 to less than $2.50 \mathrm{~mm}$. Patients with target vessels no smaller than 2.50 to $<3.00 \mathrm{~mm}$ as well as patients treated for lesions in vessels $\geq 3.00 \mathrm{~mm}$ only had lower TLF rates than patients with small vessel lesions.

\section{Outcome of $\mathrm{PCl}$ in small vessel lesions in previous trials with DES}

The treatment of lesions in small vessels has always been associated with a higher incidence of restenosis as compared to the treatment of larger vessel lesions. ${ }^{2,6,28}$ In a study in which 2,058 patients were treated with either sirolimus-eluting or a paclitaxel-eluting early-generation DES, higher restenosis rates at angiographic follow-up were seen in patients treated in vessels with a diameter smaller than $2.41 \mathrm{~mm}$, as compared to patients treated in larger vessels $(>2.41 \mathrm{~mm})$. The restenosis rates in the subgroups of patients treated in larger vessels (vessel diameter of $2.41-2.84 \mathrm{~mm}$ and $>2.84 \mathrm{~mm}$ ) were similar $(8.4 \%$ vs $8.0 \%){ }^{28}$

A recent sub-study of the LEADERS trial, in which 1,707 patients were treated with either a biolimuseluting or sirolimus-eluting stent, revealed comparable findings. A significantly higher rate of TLR $(9.6 \%$ vs $2.6 \%$ ) and MACE ( $12.1 \%$ vs $7.1 \%)$ was seen in patients treated in small vessels $(<2.75 \mathrm{~mm}) .{ }^{29}$

It has been hypothesized that the difference in restenosis rates is mainly caused by late lumen loss. ${ }^{3,4,12}$ In small vessels, a relatively higher loss of lumen diameter will occur, resulting in a higher risk of restenosis when vessel sizes get smaller., ${ }^{2,17}$ Apart from the higher risk caused by late lumen loss, patients with lesions in smaller vessels differ significantly on baseline characteristics from patients with lesions in larger vessels. Female gender ${ }^{30}$ and diabetes ${ }^{8,31}$ are often associated with smaller vessel diameters. Furthermore, patients with smaller vessels were more often treated for longer lesions ${ }^{32}$, multiple vessels ${ }^{1,2,7}$, and had more often a history of $\mathrm{PCl}^{1,2}$.

Drug-coated balloons have been suggested as an alternative to DES in order to avoid luminal obstruction by the stent struts, which is most important in small target vessels. While studies with early drugcoated balloons showed inconsistent results in small vessels ${ }^{33}$, a recent, prospective registry revealed encouraging results with a paclitaxel-coated balloon in de-novo lesions in small vessels ${ }^{34}$.

\section{Differences in definitions and cut-off points}

In previous trials, the reference vessel diameter has been assessed in various ways, ranging from visual assessment to QCA analysis. ${ }^{4,21}$ Consequently, this has led to different definitions of 'small vessels', being either based on reference vessel diameter or stent diameter ${ }^{20,21}$, which renders a comparison of studies difficult. With bare metal stents and early DES, a reference vessel diameter of less than $3.00 \mathrm{~mm}$ was most often used to define a small vessel., ${ }^{5,13,16,19}$ However, with the development of smaller stent 
diameters, the cut-off value changed to $2.50 \mathrm{~mm} \cdot{ }^{20,21}$ With the ongoing trend toward the development of DES with an increasingly smaller minimum stent size, even smaller vessels will become part of the treatable range of coronary vessel dimensions.

\section{Implications}

The evolution of the definition of 'small vessels' has paralleled the development of devices with increasingly smaller minimum sizes. So what is currently the most appropriate definition of a 'small vessel' with the use of contemporary newer-generation DES? The results of the present study suggest that the risk of TLF (1) is larger in patients treated in vessels smaller than $2.50 \mathrm{~mm}$; (2) remains similar even for smaller minimum target vessels $(<2.25 \mathrm{~mm})$; and (3) is lower in patients with minimum target vessel sizes of more than $2.50 \mathrm{~mm}$ (with no difference between medium and larger sized vessels). Thus, a cut-off value of $2.50 \mathrm{~mm}$ is a legitimate threshold to distinguish between small and larger target vessels in the setting of PCI with contemporary DES as used in the DUTCH PEERS trial and should be considered as a cut-off value in future studies on small vessel treatment. In addition, in clinical practice, knowledge of the fact that adverse events may be higher following treatment of coronary vessels $<2.50$ $\mathrm{mm}$ may help taking therapeutic decisions in patients with coronary disease that involves small vessels. Considering the recent enlargement of the interventional armamentarium by ultra-small DES $(2.00 \mathrm{~mm})$, the findings of the present study may be of particular interest and of clinical relevance. Our data suggest that clinical follow-up of interventions with such devices in very small coronary arteries deserves attention, as the event risk after such procedures might be increased.

\section{Limitations}

Because of the post-hoc nature of the present analysis, the results should be considered hypothesis generating. Based on the data presented, we cannot rule out that a threshold of $2.75 \mathrm{~mm}$ might also have been suitable to define small vessels. In future studies, it may be of interest to evaluate the optimal cut-off value, based on smaller increments in vessel size and to validate the derived threshold in a separate patient cohort. The use of the cut-off value of $2.25 \mathrm{~mm}$ in the absence of smaller stents might be considered a limitation. Since patients did not receive a routine angiographic follow-up, there is no data on potential differences in angiographic restenosis rate. We did not apply angiographic exclusion criteria; therefore, quantitative coronary angiographic analysis was challenging in some cases. Angiography is limited in its capacity to distinguish between true small vessel size and apparently small vessels in patients with diffuse coronary disease. Intravascular ultrasound is superior to angiography in assessing true vessel size and coronary remodeling. ${ }^{35,36}$ In diabetic patients, the lack of angiographic follow-up, may be associated with some underestimation of events. Nevertheless, we obtained relevant data on clinically indicated target lesion revascularization rates without amplification by routine angiographic follow-up, which most hospitals do not consider a routine procedure. In addition, due to systematic assessment of post-PCl cardiac markers and ECG changes, rigorous monitoring, and the availability of follow-up data in as much as $99.9 \%$ of the patients, potential underreporting of TLR or other major adverse events is very unlikely, while due to the lack of angiographic follow-up, some cases with clinically "silent" obstruction or occlusion of the target vessel may not have been observed. Because of the various cut-off values used to define 'small vessels' in previous trials, it is difficult to 
compare studies. Nevertheless, the current analysis of the different thresholds shows that we used a legitimate cut-off value to examine the effect of small vessel treatment.

\section{Conclusion}

Patients treated with novel DES in small vessel lesions had higher adverse event rates than patients who had no small vessel treatment. Our data suggest that with current stents a diameter of $2.50 \mathrm{~mm}$ is a suitable threshold value to identify small target vessels. 


\section{References}

1. Schunkert H, Harrell L, Palacios IF. Implications of small reference vessel diameter in patients undergoing percutaneous coronary revascularization. J Am Coll Cardiol 1999;34:40-8.

2. Elezi S, Kastratie A, Neumann FJ, Hadamitzky M, Dirschinger J, Schömig A. Vessel size and long-term outcome after coronary stent placement. Circulation 1998;98:1875-80.

3. Biondi-Zoccai G, Moretti C, Abbate A, Sheiban I. Percutaneous coronary intervention for small vessel coronary artery disease. Cardiovasc Revasc Med 2010;11:189-98.

4. Akiyama T, Moussa I, Reimers B, et al. Angiographic and clinical outcome following coronary stenting of small vessels: a comparison with coronary stenting of large vessels. J Am Coll Cardiol 1998;32:1610-8.

5. Agostoni P, Biondi-Zoccai GG, Gasparini GL, et al. Is bare-metal stenting superior to balloon angioplasty for small vessel coronary artery disease? Evidence from a meta-analysis of randomized trials. Eur Heart J 2005;26:881-9.

6. Kasaoka S, Tobis JM, Akiyama T, et al. Angiographic and intravascular ultrasound predictors of in-stent restenosis. J Am Coll Cardiol 1998;32:1630-5.

7. Tanimoto S, Daemen J, Tsuchida K, et al. Two-year clinical outcome after coronary stenting of small vessels using 2.25-mm sirolimus- and paclitaxel-eluting stents: insight into the RESEARCH end T-SEARCH registries. Catheter Cardiovasc Interv 2007;69:94-103.

8. Claessen BE, Smits PC, Kereiakes DJ, et al. Impact of lesion length and vessel size on clinical outcomes after percutaneous coronary intervention with everolimus- versus paclitaxel-eluting stents: pooled analysis from the SPIRIT (clinical evaluation of the XIENCE V everolimus eluting coronary stent system) and COMPARE (secondgeneration everolimus-eluting and paclitaxel-eluting stents in real-life practice) randomized trials. J Am Coll Cardiol Intv 2011:11;1209-15.

9. Teirstein PS, Meredith IT, Feldman RL, et al. Two-year safety and effectiveness of the platinum chromium everolimus-eluting stent for the treatment of small vessels and longer lesions. Catheter Cardiovasc Interv 2015;85:207-15.

10. Bartorelli AL, Serruys PW, Miquel-Hébert K, Yu S, Pierson W, Stone GW. An everolimus-eluting stent versus a paclitaxel-eluting stent in small vessel coronary artery disease: a pooled analysis from the SPIRIT II and SPIRIT III trials. Catheter Cardiovasc Interv 2010;76:60-6.

11. Cho SC, Jeong MH, Kim W, et al. Clinical outcomes of everolimus- and zotarolimus eluting stents in patients with acute myocardial infarction for small coronary artery disease. J Cardiol 2014;63:409-17.

12. Togni M, Eber S, Widmer J, et al. Impact of vessel size on outcome after implantation of sirolimus-eluting and paclitaxel-eluting stents. J Am Coll Cardiol 2007;50:1123-31.

13. Schampaert $E$, Cohen EA, Schlüter $M$, et al. The Canadian study of sirolimus-eluting stent in the treatment of patients with long de novo lesions in small native coronary arteries (C-SIRIUS). J Am Coll Cardiol 2004;43:1110-5.

14. Puyrimat E, Mangiacapra F, Peace A, et al. Long-term clinical outcome in patients with small vessel disease treated with drug-eluting versus bare-metal stenting. Am Heart J 2011;162:907-13.

15. Ardissino D, Cavallini C, Bramucci E, et al. Sirolimus-eluting vs uncoated stents for prevention of restenosis in small coronary arteries: a randomized trial. JAMA 2004;292:2727-34.

16. Parikh SV, Luna M, Selzer F, et al. Outcomes of small coronary artery stenting with bare-metal stents versus drugeluting stents: results from the NHLBI Dynamic Registry. Catheter Cardiovasc Interv 2014:83:192-200.

17. Mauri L, Orav EJ, Kuntz RE. Late loss in lumen diameter and binary restenosis for drug-eluting stent comparison. Circulation 2005;111:3435-42.

18. Kastrati A, Mehilli J, Dirschinger J, et al. Intracoronary stenting and angiographic results: strut thickness effect on restenosis outcome (ISAR-STEREO) trial. Circulation 2001;103:2816-21.

19. Briguori C, Sarais C, Pagnotta P, et al. In-stent restenosis in small coronary arteries: impact of strut thickness. J Am Coll Cardiol 2002;40:403-9.

20. Hermiller JB, Rutledge DR, Mao VW, et al. Clinical outcomes in real-world patients with small vessel disease treated with Xience $V$ everolimus-eluting stents: one year results from the Xience $V$ USA condition of approval post-market study. Catheter Cardiovasc Interv 2014;84:7-16.

21. Caputo R, Leon M, Serruys $P$, et al. Performance of the Resolute Zotarolimus-eluting stent in small vessels. Catheter Cardiovasc Interv 2014;84:17-23. 
22. Srour JF, Abbott JD. Small vessel and long lesions: changing label indications for drug eluting stents. Catheter Cardiovasc Interv 2015;85:216-7.

23. von Birgelen C, Sen H, Lam MK, et al. Third-generation zotarolimus-eluting and everolimus-eluting stents in all-comer patients requiring a percutaneous coronary intervention (DUTCH PEERS): a randomised, single-blind, multicentre, non-inferiority trial. Lancet 2014;383:413-23.

24. Sen H, Lam MK, Löwik MM, et al. Clinical events and patient-reported chest pain in all-comers treated with Resolute Integrity and Promus Element stents: two-year follow-up of the randomized DUTCH PEERS (TWENTE II) trial. J Am Coll Cardiol Intv 2015;8:889-99.

25. Tandjung K, Basalus MW, Sen H, et al. Durable polymer-based sTent Challenge of promus ElemEnt versus ReSolute integrity (DUTCH PEERS): Rationale and study design of a randomized multicenter trial in a Dutch allcomers population. Am Heart J 2012;163:557-62.

26. Cutlip DE, Windecker $S$, Mehran R, et al. Clinical end points in coronary stent trials: a case for standardized definitions. Circulation 2007;115:2344-51.

27. Vranckx P, Cutlip DE, Mehran R, et al. Myocardial infarction adjudication in contemporary all-comer stent trials: balancing sensitivity and specificity. Addendum to the historical $\mathrm{MI}$ definitions used in stent studies. Eurolntervention 2010;5:871-4.

28. Elezi S, Dibra A, Mehilli J, et al. Vessel size and outcome after coronary drug-eluting stent placement: results from a large cohort of patients treated with sirolimus- or pacliaxel-eluting stents. J Am Coll Cardiol 2006;48:1304-9.

29. Wykrzykowska JJ, Serruys PW, Onuma Y, et al. Impact of vessel size on angiographic and clinical outcomes of revascularization with biolimus-eluting stent with biodegradable polymer and sirolimus-eluting stent with durable polymer: the LEADERS trial substudy. J Am Coll Cardiol Intv 2009;2:861-70.

30. Dodge JT, Brown BG, Bolson EL, Dodge HT. Lumen diameter of normal human coronary arteries: influence of age, sex, anatomic variation, and left ventricular hypertrophy or dilation. Circulation 1992;86:232-46.

31. Nicholls SJ, Murat Tuzcu E, Kalidindi S, et al. Effect of diabetes on progression of coronary atherosclerosis and arterial remodeling: a pooled analysis of 5 intravascular ultrasound trials. J Am Coll Cardiol 2008;52:255-62.

32. Hsieh $\mathrm{I}-\mathrm{C}$, Chien $\mathrm{C}-\mathrm{C}$, Chang $\mathrm{H}-\mathrm{J}$, et al, Acute and long-term outcomes of stenting in coronary vessel $>3.0 \mathrm{~mm}$, 3.0-2.5 mm, and <2.5 mm. Catheter Cardiovasc Interv 2001;53:341-22.

33. Loh JP, Waksman R. Paclitaxel drug-coated balloons: a review of current status and emerging applications in native coronary artery de novo lesions. J Am Coll Cardiol Intv 2012;5:1001-12.

34. Zeymer $U$, Waliszewski M, Spiecker M, et al. Prospective 'real world' registry for the use of the 'PCB only' strategy in small vessel de novo lesions. Heart 2014;100:311-6.

35. von Birgelen C, Mintz GS, de Vrey EA, et al. Atherosclerotic coronary lesions with inadequate compensatory enlargement have smaller plaque and vessel volumes: observations with three dimensional intravascular ultrasound in vivo. Heart 2998;79:137-42.

36. von Birgelen C, Kutryk MJB, Gil R, et al. Quantification of the minimal luminal cross-sectional area after coronary stenting by two- and three-dimensional intravascular ultrasound versus edge detection and vidodensitometry. Am J Cardiol 1996;78:520-5. 



\section{Conflicts of interest statement}

CvB has been consultant to Boston Scientific and Medtronic and has received lecture fees from AstraZeneca. The institution has received research grants, provided by Abbott Vascular, Biotronik, Boston Scientific, and Medtronic. MJIJ is consultant to PANAXEA b.v., and he has received lecture fees from Roche, Pfizer, and Sanofi Aventis. All other authors declare that they have no conflict of interest.

\section{Copyright}

Reprinted with permission from

American Heart Journal 2016 May;175:121-9.

(c) Elsevier 


\title{
CHAPTER 14
}

Impact of Severe Lesion Calcification on Clinical Outcome of Patients with Stable Angina, Treated with Newer Generation Permanent Polymer-Coated Drug-Eluting Stents

\author{
A Patient-Level Pooled Analysis From TWENTE and \\ DUTCH PEERS (TWENTE II)
}

Jennifer Huisman, Liefke C. van der Heijden, Marlies M. Kok, Peter W. Danse,

Gillian A.J. Jessurun, Martin G. Stoel, K. Gert van Houwelingen, Marije M. Löwik,

Raymond W.M. Hautvast, Maarten J. IJzerman, Carine J. Doggen, Clemens von Birgelen
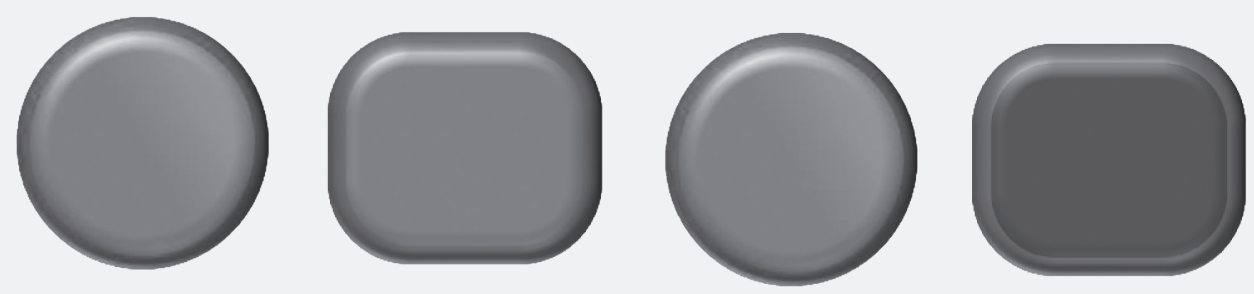


\section{Abstract}

Background: The outcome of percutaneous coronary intervention (PCI) with newer generation permanent polymer-coated drug-eluting stents (DES) in patients with severely calcified lesions is greatly unknown. We assessed the impact of severe lesion calcification on clinical outcome in patients with stable angina who underwent PCI with newer generation DES.

Methods: TWENTE and DUTCH PEERS randomized trials enrolled 1,423 patients with stable angina, who were categorized into patients with versus without severe target lesion calcification. A patient-level pooled analysis assessed clinical outcome, including target vessel failure (TVF), a composite of cardiac death, target vessel-related $\mathrm{MI}$, or target vessel revascularization (TVR).

Results: Patients with severe calcification ( $n=342)$ were older (66.6 \pm 9.1 vs $64.2 \pm 9.8$ years, $p<0.001$ ) and had more diabetes $(25.7 \%$ vs $20.4 \%, p=0.04$ ) than other patients $n=1,081)$. Patients with calcified lesions had higher rates of TVF ( $16.4 \%$ vs $9.8 \%, p$-Logrank $=0.001)$, cardiac death ( $4.4 \%$ vs $1.5 \%, p=0.03)$, target vessel $\mathrm{MI}(7.6 \%$ vs $3.4 \%, p=0.001)$, and definite stent thrombosis ( $1.8 \%$ vs $0.4 \%, p=0.02)$. Multivariate analysis demonstrated that severe calcification was an independent risk factor of 2-year TVF (HR 1.42, 95\% Cl: 1.02-1.99, p-Logrank=0.04); landmark analysis showed that this was based on a difference during the first year (periprocedural: $5.8 \%$ vs $3.1 \%$, p-Logrank $=0.02$; first year: $7.5 \%$ vs $3.8 \%$, p-Logrank=0.007; second year: $4.1 \%$ vs $3.3 \%$, p-Logrank $=0.54$ ).

Conclusion: In patients with stable angina, severe target lesion calcification is associated with an increased risk of adverse cardiovascular events following treatment with newer generation permanent polymer-coated DES. This increase in risk is restricted to the first year of follow-up, which is an encouraging finding. 


\section{Introduction}

Patients with severely calcified target lesions had an increased risk of adverse clinical events following percutaneous coronary interventions (PCI). ${ }^{1}$ While first generation drug-eluting stents (DES) reduced the incidence of restenosis and the need for repeat revascularization as compared to bare metal stents, ${ }^{2,3}$ target lesion calcification remained a strong predictor of adverse outcome, as recently shown in a pooled analysis of patients treated for acute coronary syndromes with mostly first generation DES. ${ }^{4}$ Due to an increased risk of very late stent thrombosis in early DES, ${ }^{5-8}$ newer generation permanent polymer-coated DES with an improved biocompatibility were developed. ${ }^{9}$

Newer generation DES have demonstrated a great efficacy and favorable safety in clinical studies of patients with a mild-to-moderate cardiovascular event risk ${ }^{10-13}$ as well as in broad and unrestricted patient populations. ${ }^{14-20}$ The TWENTE and DUTCH PEERS (TWENTE II) trials are two prospective randomized clinical studies that assess newer generation permanent polymer-coated zotarolimuseluting and everolimus-eluting stents in patients that reflect routine clinical practice. ${ }^{17,18,21}$

Data of patients with severely calcified target lesions, treated with newer generation permanent polymer-coated DES, are scarce..$^{10,22}$ In this context, the outcome of clinically stable patients is of particular interest, as [1] these patients have the highest prevalence of severely calcified target lesions, [2] insight into the performance of newer generation DES in calcified lesions is more likely to affect future therapeutic decisions (that are typically based on heart team discussions that consider all therapeutic options), and [3] a periprocedural cardiac marker release can unequivocally be related to the interventional procedure itself.1,23-25

In the present patient-level analysis of pooled data from the TWENTE and DUTCH PEERS trials, we therefore evaluated the impact of severe target lesion calcification on the clinical outcome of patients with stable angina who underwent $\mathrm{PCI}$ with newer generation permanent polymer-coated DES.

\section{Methods}

\section{Study population, procedures, and design}

This study was performed in all patients with stable coronary syndromes in the TWENTE (The RealWorld Resolute Versus Xience V Drug-Eluting Stent Study in Twente; ClinicalTrials.gov NCT01066650) and DUTCH PEERS (TWENTE II) (Durable Polymer-Based Stent Challenge of Promus Element versus Resolute Integrity; ClinicalTrials.gov NCT01331707) trials. ${ }^{17,18}$ Details of the TWENTE and DUTCH PEERS (TWENTE II) trials ${ }^{17,18}$ and the 2-year clinical follow-up of both trials have previously been reported. ${ }^{26,27}$ In brief, the TWENTE and DUTCH PEERS trials are investigator-initiated, patient-blinded, randomized studies, in which 3,202 patients with stable or acute coronary syndromes were enrolled and treated with newer generation permanent polymer-coated DES. After 1:1 randomization, patients in the TWENTE trial $(n=1,391)$ were treated with the Resolute zotarolimus-eluting stent (Medtronic Vascular, Santa Rosa, CA) or the Xience V everolimus-eluting stent (Abbott Vascular, Santa Clara, CA). Patients in the DUTCH PEERS trial $(n=1,811)$ were randomized to treatment with the Resolute Integrity zotarolimuseluting stent (Medtronic Vascular, Santa Rosa, CA) or the Promus Element everolimus-eluting stent 
(Boston Scientific, Natick, MA). Each of the two randomized non-inferiority trials reported similar clinical outcomes for the respective zotarolimus-eluting and everolimus-eluting stents. ${ }^{26,27}$ In addition, the primary composite clinical outcome parameter did not differ between the patient populations with stable angina of both trials at 2-year follow-up. As a consequence, the present analysis of pooled data is warranted. Both trials were approved by the accredited Medical Ethics Committee Twente and the institutional review boards of all participating centers, and complied with the Declaration of Helsinki. All patients provided written informed consent.

The aim of this patient-level pooled analysis was to assess the impact of severe target lesion calcification on clinical outcome in patients with stable angina. For the purpose of the present analysis, all TWENTE and DUTCH PEERS trial participants with stable coronary syndrome were categorized into patients with versus without angiographically determined severe target lesion calcification.

\section{Definition of target lesion calcification and angiographic analysis}

Analysts from the angiographic core lab of Thoraxcentrum Twente, blinded to randomization and patient outcome, performed the qualitative and quantitative coronary angiographic analyses according to current standards, using the software Qangio XA (Version 7.1 and 7.2, Medis, Leiden, the Netherlands). The angiographic analysts prospectively classified the target lesion calcification in analogy with previous studies.,11 Target lesion calcification was defined as readily apparent densities or $x$-ray absorbing masses, noted within the apparent vascular wall at the site of the target lesion prior to any contrast injection; in addition, severe target lesion calcification was noted without cardiac motion before contrast injection and generally compromised both sides of the arterial wall.3,4,11

\section{Interventional procedures, medical treatment, and event adjudication}

Interventional procedures were performed according to standard techniques, routine clinical protocols, and current medical guidelines, which did not differ between trials. Details of the intervention, such as lesion predilation or stent postdilation, and the application of concomitant medication, were left at the operator's discretion. Importantly, medical treatment did not differ between trials: unfractionated heparin was usually administered as an anticoagulant during $\mathrm{PCl}$, and dual anti-platelet therapy, which commonly consisted of aspirin and clopidogrel, was generally prescribed for 12 months. ${ }^{17,18}$ Electrocardiograms and laboratory tests were systematically performed.

Monitoring was performed by an independent, external clinical research organization (Diagram, Zwolle, the Netherlands). Clinical follow-up data were obtained at visits to outpatient clinics or, if not feasible, by telephone and/or medical questionnaire. In both trials, independent clinical research organizations processed the clinical outcome data, and independent external clinical events committees, blinded to the assigned treatment, adjudicated the adverse clinical events (Cardialysis, Rotterdam, the Netherlands).

\section{Definition of clinical endpoints}

The two trials used the same clinical endpoint definitions which have previously been reported. ${ }^{17,26}$ The definitions were in accordance with the Academic Research Consortium (ARC), including the addendum on myocardial infarction. ${ }^{28,29}$ Death was considered cardiac, unless an evident non-cardiac cause could 
be established. Myocardial infarction (MI) was defined by any creatine kinase concentration of more than double the upper limit of normal with elevated values of a confirmatory cardiac biomarker. A target vessel-related MI was related to the target vessel or could not be related to another vessel. Periprocedural MI (PMI) was defined as target vessel-related MI within 48 hours after PCl. Stent thrombosis was classified according to the ARC definitions.

The composite endpoint target vessel failure (TVF), which at 1-year was the primary endpoint of both the TWENTE and DUTCH PEERS trials, was defined as a composite of cardiac death, target vessel-related $\mathrm{MI}$, or clinically driven target vessel revascularization (TVR). TVR and target lesion revascularization (TLR) were considered clinically indicated if the angiographic diameter stenosis was $\geq 70 \%$, or $\geq 50 \%$ in the presence of ischemic signs or symptoms.

Target lesion failure (TLF) was defined as a composite of cardiac death, target vessel-related MI, or clinically indicated TLR; Major Adverse Cardiac Events (MACE) as a composite of all-cause death, any $\mathrm{MI}$, emergent coronary bypass surgery, or clinically indicated TLR; and a patient-oriented composite endpoint (POCE) as a composite of all-cause mortality, any MI, or any repeat (target and non-target vessel) revascularization.

\section{Statistical analysis}

Data were reported as frequencies and percentages for dichotomous and categorical variables, and as mean \pm standard deviation for continuous variables. Dichotomous and categorical variables were assessed using Chi-square tests and Fisher's exact tests, and continuous variables were assessed using Student's t tests or the Wilcoxon rank-sum tests, as appropriate. The Kaplan-Meier method was used to calculate the time to clinical endpoint and the Logrank test was applied to compare betweengroup differences. For TVF, a landmark analysis at 1-year follow-up was performed. All p-values and confidence intervals were two-sided and $p$-values $<0.05$ were considered significant. Parameters were considered as potential confounders if in univariate analyses associations were found with a $p$-value $<0.15$. A multivariate Cox regression model was then used to adjust for potential confounders. Data analysis was performed with SPSS (version 17.0, SPSS Inc., Chicago, IL, USA).

\section{Funding}

The TWENTE and DUTCH PEERS (TWENTE II) randomized trials were supported by equal unrestricted grants from Abbott Vascular and Medtronic, and from Boston Scientific and Medtronic, respectively. The authors are solely responsible for the design and conduct of this study, all study analyses and drafting, and editing of the paper.

\section{Results}

\section{Baseline, lesion, and procedural characteristics}

Of all 3,202 trial participants, 1,423 patients were treated for stable angina pectoris. A total of 342 (24.0\%) patients were treated for at least one severely calcified target lesion. They were older and had diabetes mellitus more often than patients without severe target lesion calcification ( $n=1,081)$ (Table 
1). The rate of renal failure showed no significant difference between the two patient groups ( $4.7 \%$ vs $3.3 \%, p=0.25)$. Patients with severely calcified lesions more often underwent treatment of multiple vessels $(28.4 \%$ vs $18.7 \%, p<0.001)$, were more often treated for long lesions ( $34.5 \%$ vs $17.0 \%, p<0.001)$, and underwent more lesion pretreatment $(86.0 \%$ vs $71.9 \%, p<0.001)$ (Table 2$)$.

Table 1: Baseline characteristics of all study patients comparing patients with versus without severe target lesion calcification.

\begin{tabular}{|c|c|c|c|}
\hline \multirow[b]{2}{*}{ Patient characteristics } & \multicolumn{3}{|c|}{$\begin{array}{l}\text { All patients } \\
n=1423\end{array}$} \\
\hline & $\begin{array}{l}\text { Severe calcification } \\
\qquad \mathrm{n}=\mathbf{3 4 2}\end{array}$ & $\begin{array}{l}\text { No severe calcification } \\
\qquad n=1081\end{array}$ & p-value \\
\hline Age (yrs) & $66.6 \pm 9.1$ & $64.2 \pm 9.8$ & $<0.001$ \\
\hline Women & $84(24.6)$ & $276(25.5)$ & 0.72 \\
\hline $\mathrm{BMI}\left(\mathrm{kg} / \mathrm{m}^{2}\right)$ & $28.1 \pm 4.9$ & $28.0 \pm 4.3$ & 0.60 \\
\hline Diabetes mellitus & $88(25.7)$ & $220(20.4)$ & 0.04 \\
\hline Hypertension & $210(61.4)$ & $661(61.1)$ & 0.93 \\
\hline Hypercholesterolemia & $223(65.6)$ & $645(60.2)$ & 0.08 \\
\hline Current smoker & $50(14.6)$ & 194 (17.9) & 0.16 \\
\hline Family history of CAD & $188(55.0)$ & $612(56.6)$ & 0.59 \\
\hline Chronic renal failure* & $16(4.7)$ & $36(3.3)$ & 0.25 \\
\hline Hemodialysis & $1(0.3)$ & $2(0.2)$ & 0.56 \\
\hline Previous MI & $77(22.5)$ & $251(23.2)$ & 0.79 \\
\hline Previous $\mathrm{PCl}$ & $82(24.0)$ & $253(23.4)$ & 0.83 \\
\hline Previous CABG & $56(16.4)$ & $127(11.7)$ & 0.03 \\
\hline
\end{tabular}

Values are mean $( \pm S D)$ or $n(\%) .{ }^{*}$ Chronic renal failure was defined as a serum creatinine level $\geq 130 \mu \mathrm{mol} / \mathrm{l}$. Abbreviations: $\mathrm{BMI}=$ body mass index; $\mathrm{CABG}=$ coronary artery bypass grafting; $\mathrm{CAD}=$ coronary artery disease.

\section{Clinical event rates at 2-year follow-up}

Two patients withdrew consent during the first year, but 2-year follow-up data were available for all remaining 1,421 patients (99.9\%). A time-to-event analysis of TVF revealed a significantly higher event rate in patients with severely calcified target lesions ( $16.4 \%$ vs $9.8 \%, \mathrm{p}$-Logrank $=0.001$; Figure 1). Of the individual components of TVF (Table 3, Figure 1), both cardiac death and target vesselrelated $\mathrm{MI}$ showed significantly higher rates in patients with severely calcified lesions (4.4\% vs $1.5 \%$, $p=0.03$, and $7.6 \%$ vs $3.4 \%, p=0.001$, respectively), while a difference in clinically indicated TVR did not reach statistical significance ( $7.6 \%$ vs $5.8 \%, p=0.24)$. During the two years of follow-up, definite stent thrombosis occurred more often in patients with severely calcified lesions ( $1.8 \%$ vs $0.4 \%, p=0.02$; Figure 2). When patients with stent thrombosis and at the same time) target vessel myocardial infarction were excluded from the analysis, patients with severe target lesion calcification still showed a higher TVF rate ( $14.4 \%$ vs. $9.1 \%, p=0.006)$. 
Table 2: Characteristics of lesions and interventional procedures.

\begin{tabular}{|c|c|c|c|}
\hline & \multicolumn{3}{|c|}{$\begin{array}{c}\text { All patients } \\
n=1423\end{array}$} \\
\hline & $\begin{array}{l}\text { Severe calcification } \\
\qquad n=342\end{array}$ & $\begin{array}{l}\text { No severe calcification } \\
\qquad n=1081\end{array}$ & p-value \\
\hline Multivessel treatment & $97(28.4)$ & $202(18.7)$ & $<0.001$ \\
\hline Two or more lesions treated per patient & $135(39.5)$ & $326(30.2)$ & 0.001 \\
\hline \multicolumn{4}{|l|}{ Treated coronary vessels } \\
\hline Right coronary artery & $152(44.4)$ & $359(33.2)$ & $<0.001$ \\
\hline Left anterior descending artery & $173(50.6)$ & $536(49.6)$ & 0.75 \\
\hline Circumflex artery & $96(28.1)$ & $341(31.5)$ & 0.23 \\
\hline De novo lesions & $302(88.3)$ & $909(84.1)$ & 0.06 \\
\hline At least one chronic total occlusion & $25(7.3)$ & $87(8.0)$ & 0.66 \\
\hline At least one in-stent restenosis & $11(3.2)$ & $49(4.5)$ & 0.29 \\
\hline At least one ostial lesion & $52(15.2)$ & $86(8.0)$ & $<0.001$ \\
\hline At least one small vessel* & $225(65.8)$ & $713(66.0)$ & 0.95 \\
\hline At least one lesion length $>27 \mathrm{~mm}$ & $118(34.5)$ & $184(17.0)$ & $<0.001$ \\
\hline Predilatation & $294(86.0)$ & $777(71.9)$ & $<0.001$ \\
\hline Total stent length (mm) & $44.0(28.0-74.0)$ & $30.0(18.0-51.0)$ & $<0.001$ \\
\hline Number of stents per patient & $2.5 \pm 1.5$ & $1.9 \pm 1.1$ & $<0.001$ \\
\hline Rotablation & $32(9.4)$ & $2(0.2)$ & $<0.001$ \\
\hline Cutting balloon & $46(13.5)$ & $25(2.3)$ & $<0.001$ \\
\hline Maximum \% diameter stenosis pre $\mathrm{PCl}$ & $67.5(58.9-77.5)$ & $65.2(55.9-73.8)$ & 0.005 \\
\hline Maximum \% diameter stenosis post $\mathrm{PCl}$ & $14.0(10.7-17.9)$ & $14.5(10.5-19.5)$ & 0.14 \\
\hline Minimum lumen diameter post $\mathrm{PCl}$ & $2.17 \pm 0.58$ & $2.11 \pm 0.55$ & 0.08 \\
\hline Maximum implantation pressure stent & $16.3 \pm 2.74$ & $15.4 \pm 2.6$ & $<0.001$ \\
\hline Postdilation & $307(89.8)$ & $891(82.4)$ & 0.001 \\
\hline Maximum pressure at postdilation (atm) & $24.9 \pm 4.7$ & $23.4 \pm 5.0$ & $<0.001$ \\
\hline
\end{tabular}

Values are mean $( \pm S D)$ or $n(\%)$ unless otherwise stated. ${ }^{*} A$ small vessel was defined by a reference vessel diameter $<2.75 \mathrm{~mm}$.

\section{Multivariate analysis and landmark analysis of target vessel failure}

Cox regression analysis suggested that the treatment of severely calcified lesions is associated with an increased risk of TVF (unadjusted HR 1.74, 95\% Cl: 1.23-2.40, p=0.001). Following adjustment for all potential confounders, multivariate analysis for TVF demonstrated the presence of severely calcified target lesions to be an independent predictor of TVF at 2-year follow-up (adjusted HR 1.42, 95\% Cl: 1.02-1.99, $\mathrm{p}=0.04)$.

A landmark analysis (Figure 3 ) revealed that, during the first 48 hours and from 48 hours until one year of follow-up, TVF was significantly higher in patients with severely calcified target lesions (5.8\% vs. 3.1\%, $\mathrm{p}$-Logrank $=0.02$, and $7.5 \%$ vs $3.8 \%$, $p$-Logrank $=0.007$, respectively). During the second year of follow-up there was no between-group difference ( $4.1 \%$ vs $3.3 \%, p$-Logrank=0.54). There was no difference in periprocedural myocardial infarction rate between patients with severely calcified target lesions treated with versus without use of rotational atherectomy and/or cutting balloon dilatation. 
Table 3: Two-year clinical outcome of patients with versus without severe target lesion calcification.

\begin{tabular}{|c|c|c|c|}
\hline & \multicolumn{3}{|c|}{$\begin{array}{c}\text { All patients } \\
n=1421\end{array}$} \\
\hline & $\begin{array}{l}\text { Severe calcification } \\
\mathrm{n}=\mathbf{3 4 2}\end{array}$ & $\begin{array}{l}\text { No severe calcification } \\
\qquad n=1079\end{array}$ & p-value \\
\hline Death (any) & $20(5.8)$ & $32(3.0)$ & 0.01 \\
\hline Cardiac death & $15(4.4)$ & $16(1.5)$ & 0.001 \\
\hline Target vessel-related MI (any) & $26(7.6)$ & $37(3.4)$ & 0.001 \\
\hline Periprocedural MI & $20(5.8)$ & $33(3.1)$ & 0.02 \\
\hline Target vessel revascularization* & $26(7.6)$ & $63(5.8)$ & 0.24 \\
\hline Target lesion revascularization* & $20(5.8)$ & $45(4.2)$ & 0.20 \\
\hline Target vessel failure & $56(16.4)$ & $106(9.8)$ & 0.001 \\
\hline Target lesion failure & $51(14.9)$ & $92(8.5)$ & 0.001 \\
\hline Major adverse cardiac events & $57(16.7)$ & $110(10.2)$ & 0.001 \\
\hline Patient oriented composite endpoint & $76(22.2)$ & $155(14.4)$ & 0.001 \\
\hline Definite stent thrombosis & $6(1.8)$ & $4(0.4)$ & 0.02 \\
\hline Definite or probable stent thrombosis & $8(2.3)$ & $10(0.9)$ & 0.04 \\
\hline Acute (0-1 days) & $2(0.6)$ & $3(0.3)$ & 0.60 \\
\hline Subacute (2-30 days) & $2(0.6)$ & $1(0.1)$ & 0.15 \\
\hline Late (31-360 days) & $4(1.2)$ & $3(0.3)$ & 0.06 \\
\hline Very late (361-720 days) & 0 & $3(0.3)$ & 1.00 \\
\hline
\end{tabular}

Values are n (\%). 2-year follow-up was available for 1421 of all 1423 patients (99.9\%). * Clinically indicated. 

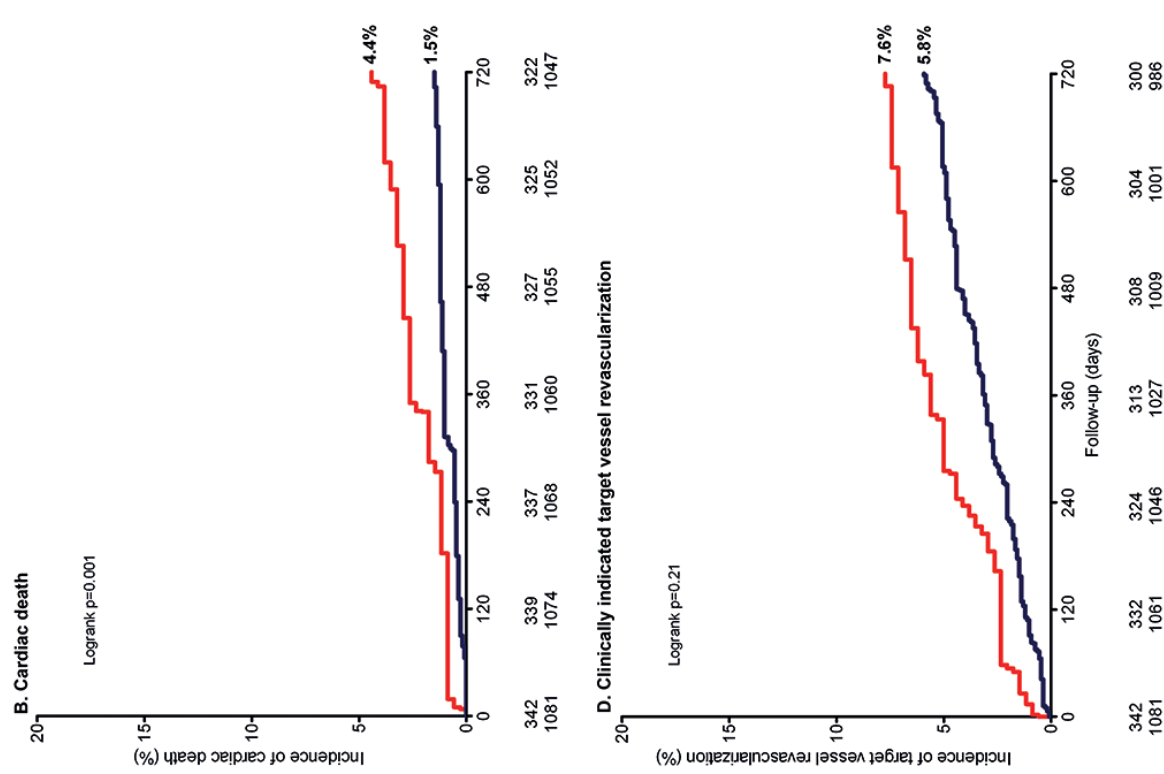

둗ำ

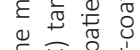
可高嵒 这势 워월

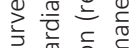
ป ]

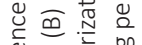
要

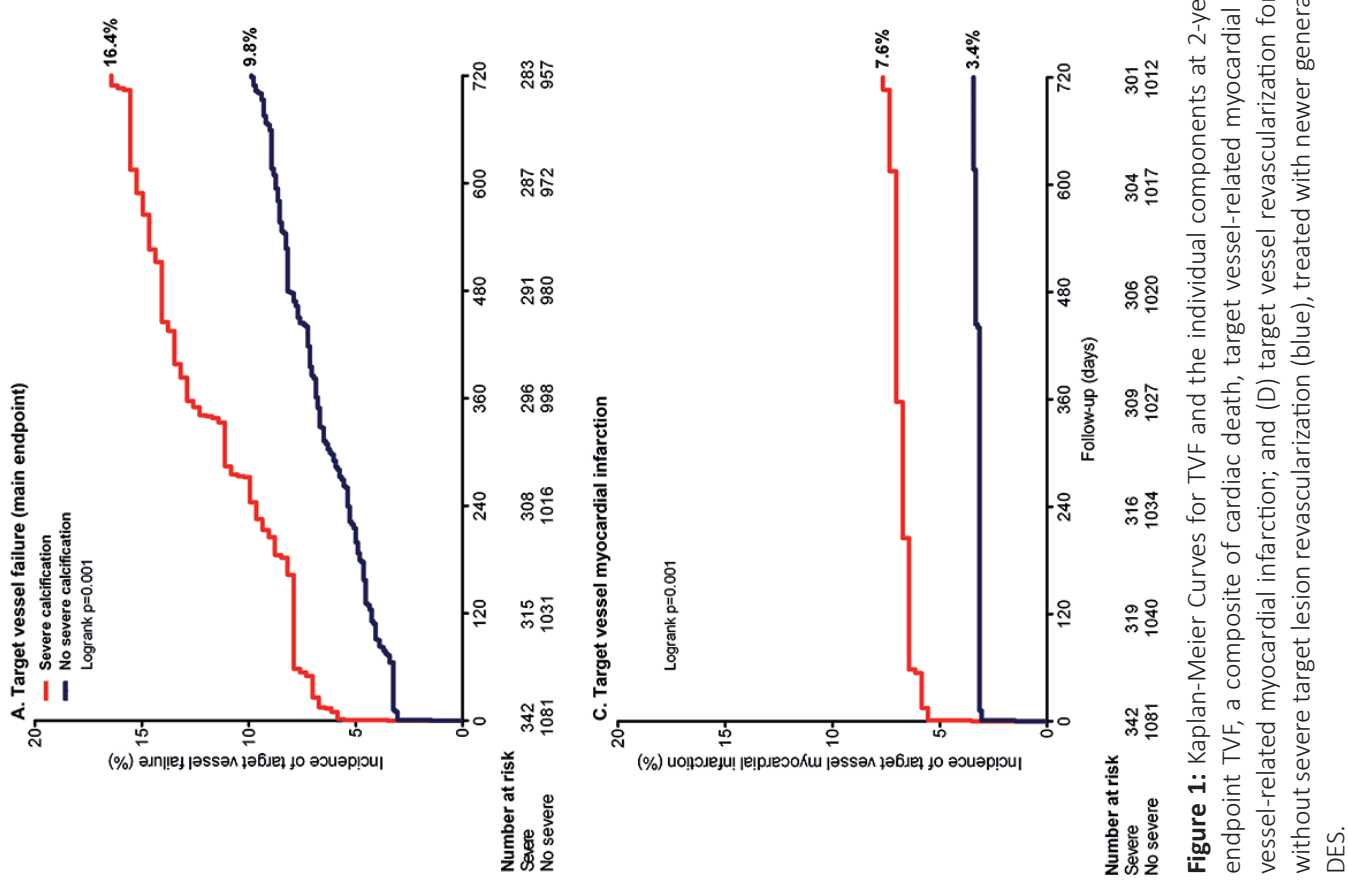




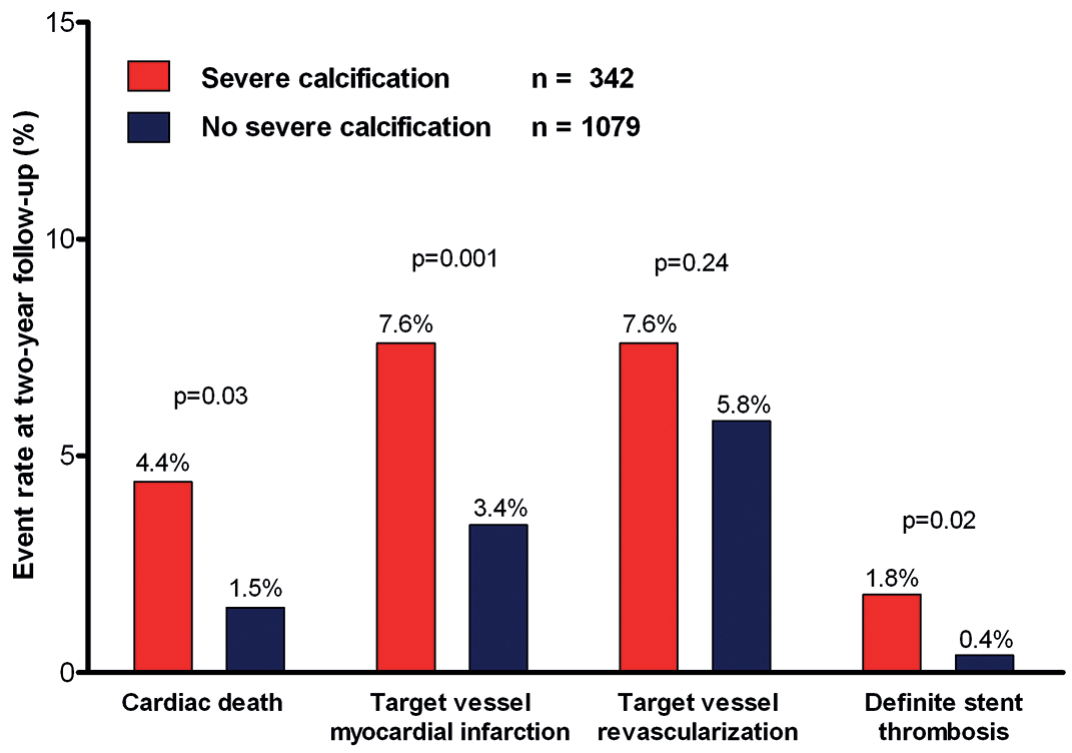

Figure 2: Adverse cardiovascular events at 2-year follow-up. Patients with (red) versus without severe target lesion revascularization (blue), treated with newer generation permanent polymer-coated DES, are compared. Two-year follow-up data were available for 1421 of all 1423 patients (99.9\%).

\section{Discussion}

The present patient-level analysis of pooled clinically stable patients in the TWENTE and DUTCH PEERS trials showed a significantly higher 2-year rate of the composite endpoint TVF in patients treated for severely calcified coronary lesions (16.4\% vs $9.8 \%$ ). In addition, patients with severe lesion calcification had higher rates of cardiac death, target vessel-related $\mathrm{Ml}$, and definite stent thrombosis than the other patients. Multivariate analyses demonstrated severe target lesion calcification to be an independent predictor of TVF (HR 1.42) after the implantation of newer generation permanent polymer-coated DES. According to a landmark analysis, this increased TVF risk is restricted to the first year of follow-up.

\section{Previous studies of DES in calcified lesions}

Since the introduction of $\mathrm{PCl}$ for the treatment of obstructive coronary disease, target lesion calcification has been a predictor of worse clinical outcome. ${ }^{30-32}$ One year after first generation sirolimus-eluting stent implantation, 152 patients with moderately or severely calcified lesions showed significantly more TLR (7.3\% vs $2.8 \%$ ) and MACE (13.8\% vs 6.1\%) than 228 patients with no or mild lesion calcification; in addition, the patients with calcified lesions showed a trend towards a higher incidence of definite stent thrombosis (1.5\% vs $0.3 \%) .{ }^{31}$ Data from a Japanese post-marketing surveillance registry of first generation sirolimus-eluting stent implantation showed in 98 dialysis patients a particularly high 3-year incidence of TLR in the presence of moderate-to-severe lesion calcification, which was higher than the TLR rate in patients with no-or-mild lesion calcification ( $29.8 \%$ vs $9.8 \%) .32$ Of our present study population, only three patients required dialysis. 


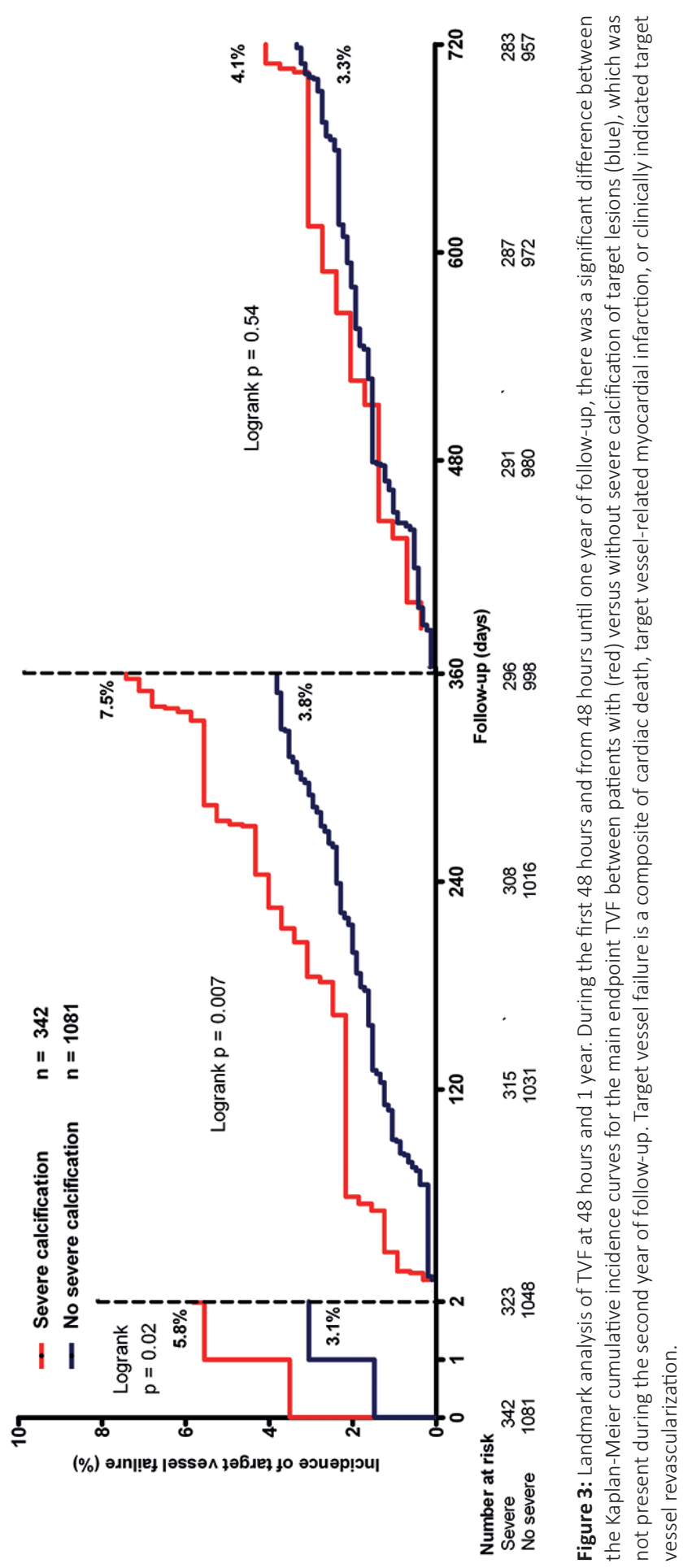


In a patient-level pooled analysis of seven different stent trials, of which six examined first generation DES, severe target lesion calcification (that was present in $20 \%$ of patients) was found to be an independent predictor of all-cause mortality (HR 1.33,95\% Cl: 1.00-1.77) and a combined endpoint of all-cause mortality, myocardial infarction, or any revascularization ( $\mathrm{HR} 1.18,95 \% \mathrm{Cl}$ : 1.01-1.39). ${ }^{30}$ Definite stent thrombosis occurred more often among patients with severely calcified target lesions (3.0\% vs $1.8 \%$ ), but was not independently predicted by the presence of severe lesion calcification. Landmark analyses showed for all-cause mortality and the combined endpoint (i.e. all-cause mortality, myocardial infarction, or any revascularization) a higher incidence in severely calcified lesions during both the first and the second-to-third year of follow-up. ${ }^{30}$ While this meta-analysis comprised patients from a large trial of second generation permanent polymer DES, most patients were treated with other generation DES. Among these patients, many were treated with first generation DES that are known to have higher event rates than newer generation permanent polymer DES beyond 1 year from stenting. We can only speculate that the relatively high proportion of patients with first generation DES in this meta-analysis may have resulted in the persistently increased event risk beyond 1 year, which we did not find in our landmark analysis of TVF in patients treated with newer generation permanent polymer DES only.

In the present study of clinically stable patients treated with newer generation permanent polymercoated DES, we found at 2-year follow-up a rate of definite stent thrombosis that was relatively low in patients treated for severely calcified lesions but significant higher than in patients with less calcified lesions (1.5\% vs $0.4 \%$ ). Both rates are relatively low, but the systematic assessment of post- $\mathrm{PCl}$ cardiac markers and ECG changes, the rigorous monitoring, and the availability of follow-up data in as much as $99.9 \%$ of patients in the TWENTE and the DUTCH PEERS trials make potential underreporting of stent thrombosis, (periprocedural) myocardial infarction, or other important clinical events unlikely. The higher rate of stent thrombosis among patients with severely calcified target lesions is in concordance with results of the aforementioned meta-analysis, ${ }^{30}$ as well as a pooled analysis for calcified lesions in patients who were (mainly) treated with first generation DES for acute coronary syndromes. ${ }^{4}$ Higher rates of stent thrombosis may be due to difficulties with stent delivery, stent underexpansion, or stent damage, which are more common in severely calcified lesions. ${ }^{4,33,34}$

\section{Risk factors and lesion characteristics associated with severe lesion calcification}

Severe lesion calcification was previously found to be associated with advanced age, hypertension, diabetes, and chronic renal failure. ${ }^{1,4}$ Both diabetes and advanced age were previously shown to be independent predictors for cardiac death. ${ }^{4} \mathrm{~A}$ longer lesion length and multi-vessel treatment, both markers of extensive atherosclerotic disease and a worse prognosis, are independent predictors of $\mathrm{MI}$ until 1-year follow-up. ${ }^{4}$ In the present analysis, the rate of patients with chronic renal failure was similar between patients with versus without severely calcified lesions. After adjustment for any potential confounder in our present analysis, severe target lesion calcification was still an independent predictor for TVF (HR 1.42). 


\section{Challenges of coronary DES implantation in severely calcified lesions}

In severely calcified coronary lesions, sufficient lesion preparation is particularly important, as direct stenting may be impossible or associated with inadequate stent expansion. In addition, severely calcified lesions can result in suboptimal stent deployment which may lead to in-stent restenosis or incomplete or delayed endothelization with increased risk of stent thrombosis. ${ }^{34}$ Uneven distribution of stent struts, which is more likely to occur in calcified lesions, may lead to adverse clinical events due to a local imbalance in scaffolding properties and drug release. Rotational atherectomy can favorably modify the morphology of calcified lesions but is associated with an increased trauma to the vessel wall. As a consequence, it was associated with a high risk of in-stent restenosis in bare metal stents, but the use of DES made the approach much more effective with relatively low rates of adverse events. ${ }^{35}$ In a randomized controlled trial, in which routine use of rotational atherectomy followed by paclitaxel eluting stent implantation was compared to standard therapy and paclitaxel stent implantation, no difference in clinical endpoints was observed between both patient groups. ${ }^{36}$ Cutting balloon inflation, rotational atherectomy, and/or predilatation with non-compliant balloons at high pressures, can also be highly effective to prepare calcified lesions. In the present study, $86.0 \%$ of the patients with severely calcified lesions were pretreated versus $71.9 \%$ of the patients without severe calcified lesions $(p<0.001)$. While DES significantly improved outcome, local drug delivery into the coronary vessel wall may sometimes be impaired in highly calcified target lesions. In addition, manipulation with DES in severely calcified vessels can result in damage to the polymer coating, which might locally reduce the effectiveness of a DES in suppressing the proliferation of neointima. ${ }^{37,38}$ Nevertheless, the 2-year outcome of the present study suggests that $\mathrm{PCl}$ with newer generation permanent polymer-coated DES in severely calcified target lesions is not associated with a disproportionately increased target vessel revascularization rate, as compared to patients without severe lesion calcification (7.6\% vs. $5.8 \%$ ).

\section{Limitations}

Event rates of comparative, randomized DES trials may be somewhat lower than those obtained in routine daily practice. Nevertheless, patient and lesion characteristics and previously reported enrollment rates suggest that both trials assessed patients with complex and diffuse coronary artery disease, which reflects routine clinical practice. We did not use intravascular ultrasound or optical coherence tomography to classify lesion calcification further. Nevertheless, previous studies have demonstrated that prognostic information on target lesion calcification can be obtained from the analysis of coronary angiography. ${ }^{30}$ No late angiographic follow-up was obtained. However, due to the systematic assessment of post-PCI cardiac markers and ECG changes, rigorous monitoring, and availability of follow-up data in as much as $99.9 \%$ of patients in TWENTE and the DUTCH PEERS trials make potential underreporting of important clinical events unlikely. 


\section{Conclusions}

In patients with stable angina who undergo $\mathrm{PCl}$ with newer generation permanent polymer-coated DES severe target lesion calcification is associated with an increased risk of adverse cardiovascular events. The increase in risk is restricted to the first year of follow-up, which is encouraging. 


\section{References}

1. Madhavan MV, Tarigopula M, Mintz GS, et al. Coronary artery calcification: pathogenesis and prognostic implications. J Am Coll Cardiol 2014;63:1703-14.

2. Bangalore $\mathrm{S}$, Vlachos HA, Selzer F, et al. Percutaneous coronary intervention of moderate to severe calcified coronary lesions: insights from the National Heart, Lung, and Blood Institute Dynamic Registry. Catheter Cardiovasc Interv 2011;77:22-8.

3. Moussa I, Ellis SG, Jones M, et al. Impact of coronary culprit lesion calcium in patients undergoing paclitaxeleluting stent implantation (a TAXUS-IV sub study). Am J Cardiol 2005;96:1242-7.

4. Généreux P, Madhavan MV, Mintz GS, et al. Ischemic outcomes after coronary intervention of calcified vessels in acute coronary syndromes. Pooled analysis from the HORIZONS-AMI (Harmonizing Outcomes With Revascularization and Stents in Acute Myocardial Infarction) and ACUITY (Acute Catheterization and Urgent Intervention Triage Strategy) trials. J Am Coll Cardiol 2014;63:1845-54.

5. Daemen J, Wenaweser P, Tsuchida K, et al. Early and late coronary stent thrombosis of sirolimus-eluting and paclitaxel-eluting stents in routine clinical practice: data from a large two-institutional cohort study. Lancet 2007;369:667-78.

6. Palmerini T, Biondi-Zoccai G, Della Riva D, et al. Stent thrombosis with drug-eluting and bare-metal stents: evidence from a comprehensive network meta-analysis. Lancet 2012;379:1393-402.

7. Navarese EP, Tandjung K, Claessen B, et al. Safety and efficacy outcomes of first and second generation durable polymer drug eluting stents and biodegradable polymer biolimus eluting stents in clinical practice: comprehensive network meta-analysis. BMJ 2013;347:f6530.

8. Bangalore S, Toklu B, Amoroso N, et al. Bare metal stents, durable polymer drug eluting stents, and biodegradable polymer drug eluting stents for coronary artery disease: mixed treatment comparison meta-analysis. BMJ 2013;347:f6625.

9. Stefanini GG, Taniwaki M, Windecker S. Coronary stents: novel developments. Heart 2014;100:1051-61.

10. Stone GW, Rizvi A, Newman W, et al. Everolimus-eluting versus paclitaxel-eluting stents in coronary artery disease. N Engl J Med 2010;362:1663-74.

11. Onuma Y, Tanimoto S, Ruygrok P, et al. Efficacy of everolimus eluting stent implantation in patients with calcified coronary culprit lesions: two-year angiographic and three-year clinical results from the SPIRIT II study. Catheter Cardiovasc Interv 2010;76:634-42.

12. Stone GW, Teirstein PS, Meredith IT, et al. A prospective, randomized evaluation of a novel everolimus-eluting coronary stent: the PLATINUM trial. J Am Coll Cardiol 2011;57:1700-8.

13. Meredith IT, Teirstein PS, Bouchard A, et al. Three-year results comparing platinum-chromium PROMUS Element and cobalt-chromium XIENCE $V$ everolimus-eluting stents in de novo coronary artery narrowing (from the PLATINUM Trial). Am J Cardiol 2014;113:1117-23.

14. Kedhi E, Joesoef KS, McFadden E, et al. Second-generation everolimus-eluting and paclitaxel-eluting stents in real-life practice (COMPARE): a randomised trial. Lancet 2010;375:201-9.

15. Jensen LO, Thayssen P, Maeng M, et al. Three-year outcomes after revascularization with everolimus- and sirolimus-eluting stents from the SORT OUT IV trial. J Am Coll Cardiol Intv 2014;7:840-8.

16. Serruys PW, Silber S, Garg S, et al. Comparison of zotarolimus-eluting and everolimus-eluting coronary stents. N Engl J Med 2010;363:136-46.

17. von Birgelen C, Basalus MW, Tandjung K, et al. A randomized controlled trial in second-generation zotarolimuseluting Resolute stents versus everolimus-eluting Xience $V$ stents in real-world patients: the TWENTE trial. J Am Coll Cardiol 2012;59:1350-61.

18. von Birgelen $\mathrm{C}$, Sen H, Lam MK, et al. Third-generation zotarolimus-eluting and everolimus-eluting stents in all-comer patients requiring a percutaneous coronary intervention (DUTCH PEERS): a randomised, single-blind, multicentre, non-inferiority trial. Lancet 2014;383:413-23

19. Park KW, Kang SH, Kang HJ, et al. A randomized comparison of platinum chromium-based everolimus-eluting stents versus cobalt chromium-based zotarolimus-eluting stents in all-comers receiving percutaneous coronary intervention: HOST-ASSURE, a randomized, controlled, noninferiority trial. J Am Coll Cardiol 2014;63:2805-16. 
20. Raungaard B, Jensen LO, Tilsted HHet al. Zotarolimus-eluting durable-polymer-coated stent versus a biolimuseluting biodegradable-polymer-coated stent in unselected patients undergoing percutaneous coronary intervention (SORT OUT VI): a randomised non-inferiority trial. Lancet 2015;385:1527-35.

21. Sen $H$, Tandjung K, Basalus M, et al. Comparison of eligible non-enrolled patients and the randomised TWENTE trial population treated with Resolute and XIENCE V drug-eluting stents. Eurolntervention 2012;8:664-71.

22. Butman SM. Warning: this report does not address heavily calcified coronary arteries. Catheter Cardiovasc Interv 2010;76:643.

23. Waters DD, Azar RR. The curse of target lesion calcification: still active after all these years. J Am Coll Cardiol 2014;63:1855-6.

24. Räber L, Zanchin T, Baumgartner S, et al. Differential healing response attributed to culprit lesions of patients with acute coronary syndromes and stable coronary artery after implantation of drug-eluting stents: an optical coherence tomography study. Int J Cardiol 2014;173:259-67.

25. Windecker S, Kolh P, Alfonso F, et al. 2014 ESC/EACTS Guidelines on myocardial revascularization. The Task Force on Myocardial Revascularization of the European Society of Cardiology (ESC) and the European Association for Cardio-Thoracic Surgery (EACTS). Eur Heart J 2014;35:2541-619.

26. Tandjung K, Sen H, Lam MK, et al. Clinical outcome following stringent discontinuation of dual antiplatelet therapy after 12 months in real-world patients treated with second-generation zotarolimus-eluting resolute and everolimus-eluting xience V stents: 2-year follow-up of the randomized TWENTE trial. J Am Coll Cardiol 2013;61:2406-16.

27. Sen H, Lam MK, Löwik MM, et al. Clinical events and patient-reported chest pain in all-comers treated with Resolute Integrity and Promus Element stents: two-year follow-up of the randomized DUTCH PEERS TWENTE II) trial. J Am Coll Cardiol Intv 2015,8:889-99.

28. Cutlip DE, Windecker S, Mehran R, et al. Clinical end points in coronary stent trials: a case for standardized definitions. Circulation 2007;115:2344-51.

29. Vranckx P, Cutlip DE, Mehran R, et al. Myocardial infarction adjudication in contemporary all-comer stent trials: balancing sensitivity and specificity. Addendum to the historical $\mathrm{MI}$ definitions used in stent studies. Eurolntervention 2010;5:871-4.

30. Bourantas CV, Zhang YJ, Garg S, et al. Prognostic implications of coronary calcification in patients with obstructive coronary artery disease treated by percutaneous coronary intervention: a patient-level pooled analysis of 7 contemporary stent trials. Heart 2014;100:1158-64.

31. Kawaguchi $R$, Tsurugaya $H$, Hoshizaki $H$, et al. Impact of lesion calcification on clinical and angiographic outcome after sirolimus-eluting stent implantation in real-world patients. Cardiovasc Revasc Med 2008;9:2-8.

32. Fujimoto $\mathrm{H}$, Nakamura $\mathrm{M}$, Yokoi $\mathrm{H}$. Impact of calcification on the long-term outcomes of sirolimus-eluting stent implantation: subanalysis of the Cypher Post-Marketing Surveillance Registry. Circ J 2012;76:57-64.

33. Liu X, Doi H, Maehara A, Mintz GS, et al. A volumetric intravascular ultrasound comparison of early drug-eluting stent thrombosis versus restenosis. J Am Coll Cardiol Intv 2009;2:428-34.

34. Fujii K, Carlier SG, Mintz GS, et al. Stent underexpansion and residual reference segment stenosis are related to stent thrombosis after sirolimus-eluting stent implantation: an intravascular ultrasound study. J Am Coll Cardiol 2005;45:995-8.

35. Abdel-Wahab M, Baev R, Dieker P, et al. Long-term clinical outcome of rotational atherectomy followed by drugeluting stent implantation in complex calcified coronary lesions. Catheter Cardiovasc Interv 2013;81:285-91.

36. Abdel-Wahab M, Richardt G, Bütnner HJ, et al. High-speed rotational atherectomy before paclitaxel-eluting stent implantation in complex calcified coronary lesions: the randomized ROTAXUS (Rotational Ahterectomy prior to taxus stent treatment for complex native coronary artery disease) trial. J Am Coll Cardiol Intv 2013;6:10-9.

37. Wiemer M, Butz T, Schmidt W, et al. Scanning electron microscopic analysis of different drug eluting stents after failed implantation: from nearly undamaged to major damaged polymers. Catheter Cardiovasc Interv 2010;75:905-11.

38. Basalus MW, Joner M, von Birgelen C, et al. Polymer coatings on drug-eluting stents: Samson's hair and Achilles' heel? Eurolntervention 2013;9:302-5. 



\section{Conflicts of interest statement}

CVB has been consultant to Boston Scientific and Medtronic and has received lecture fees from AstraZeneca. The institution has received research grants, provided by Abbott Vascular, Biotronik, Boston Scientific, Medtronic, and AstraZeneca. MIJ is consultant to PANAXEA b.v., and he has received lecture fees from Roche, Pfizer, and Sanofi Aventis.All other authors declare that they have no conflict of interest.

\section{Copyright}

Reprinted with permission of

Journal of Cardiology 2017 Apr;69(4):660-665.

(c) Elsevier 


\title{
CHAPTER 15
}

\section{Two-Year Outcome After Treatment of Severely Calcified Lesions With Newer-Generation Drug-Eluting Stents in Acute Coronary Syndromes}

\author{
A Patient-Level Pooled Analysis From TWENTE \\ and DUTCH PEERS
}

Jennifer Huisman, Liefke C. van der Heijden, Marlies M. Kok,

(J.)Hans W. Louwerenburg, Peter W. Danse, Gillian A.J. Jessurun,

Frits H.A.F. de Man, Marije M. Löwik, Gerard C.M. Linssen,

Maarten J. IJzerman, Carine J. Doggen, Clemens von Birgelen
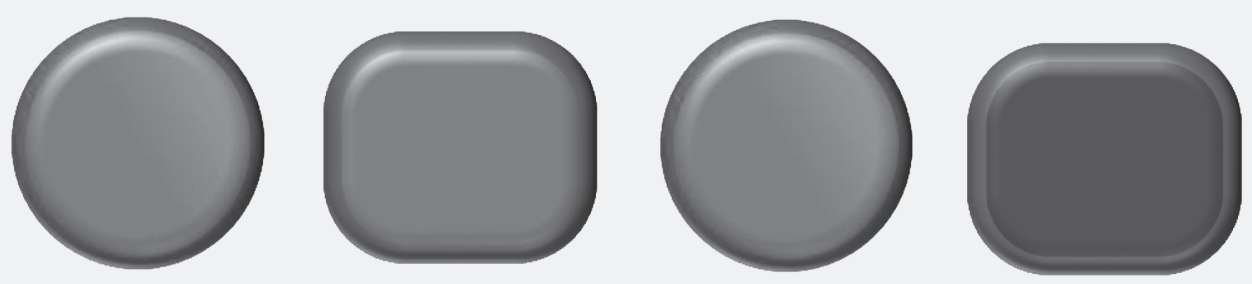


\section{Abstract}

Background: Data on medium-term outcome of patients with ACS, treated with newer-generation durable polymer DES in severely calcified coronary lesions, are scarce. We aimed to assess the impact of severe coronary lesion calcification on clinical outcome of patients with acute coronary syndrome (ACS), treated with newer-generation drug-eluting stents (DES).

Methods: The TWENTE and DUTCH PEERS randomized trials comprise 1,779 ACS patients, who were categorized into patients with versus without severe target lesion calcification. We performed a patientlevel pooled analysis to assess 2-year outcome, including target vessel failure (TVF), a composite of cardiac death, target vessel-related myocardial infarction (MI), or target vessel revascularization (TVR).

Results: Patients with severe target lesion calcification ( $n=340,19.1 \%)$ were older (66.8 \pm 10.6 vs $62.8 \pm 11.5$ years, $p<0.001)$ and had more often diabetes $(22.1 \%$ vs $16.8 \%, p=0.02)$ and hypercholesterolemia ( $51.5 \%$ vs $42.9 \%, p=0.005$ ) than other patients ( $n=1,439,79.9 \%)$. In addition they showed a higher TVF rate ( $12.4 \%$ vs $7.0 \%, p=0.001)$, mainly related to a difference in TVR $(6.8 \%$ vs $3.3 \%$, $\mathrm{p}=0.003)$. There was a borderline significant between-group difference in cardiac death $(3.6 \%$ vs $1.8 \%$, $\mathrm{p}=0.05)$, but not in target vessel $\mathrm{MI}(3.8 \%$ vs $2.6 \%, \mathrm{p}=0.23)$ and definite stent thrombosis $(0.9 \%$ vs $0.6 \%$, $\mathrm{p}=0.71$ ). Multivariate analysis demonstrated that severe lesion calcification was an independent risk factor of TVF (HR 1.51, 95\% Cl: 1.04-2.19, $p=0.03$ ).

Conclusions: In patients with ACS, treatment of severely calcified lesions with newer-generation DES was associated with an overall higher clinical event risk-related in particular to a higher TVR rate, while the risk of MI was low. 


\section{Introduction}

Percutaneous coronary interventions (PCl) in patients with severely calcified lesions are associated with an increased risk of suboptimal procedural results and adverse clinical events. ${ }^{1}$ In the setting of acute coronary syndromes (ACS), which are known to be associated with an increased thrombogenicity, lesion calcification is frequently present and may have a particularly negative impact on outcome. ${ }^{2}$ As is shown in a large pooled analysis of patients with ACS and calcified target lesions, treatment with (mostly) firstgeneration drug-eluting stents (DES) significantly reduced the need for repeat revascularization, ${ }^{3}$ as compared to bare metal stents. ${ }^{2,4}$ Newer-generation permanent polymer-coated DES were developed to increase biocompatibility. ${ }^{5-7}$ These devices demonstrated a favorable safety profile and high efficacy in study populations with mild-to-moderate cardiovascular event risks ${ }^{8-11}$ as well as in broad, greatly unrestricted patient populations. ${ }^{12-18}$ Nevertheless, severely calcified target lesions may still impair delivery and expansion of newer-generation DES and may represent a serious challenge to polymer coatings. ${ }^{19}$ While the treatment of severely calcified coronary lesions with newer-generation DES may still be associated with an increased risk of adverse events-in particular in the setting of ACS - only limited data are available. The TWENTE and DUTCH PEERS trials are two prospective randomized clinical studies that assessed newer-generation zotarolimus-eluting and everolimus-eluting stents in broad patient populations, which reflect routine clinical practice and comprise many high-risk patients with ACS and severe target lesion calcification. ${ }^{15,16}$ In the present patient-level pooled analysis of these two trials, we evaluated the impact of severe target lesion calcification on 2-year outcome of PCl with newer-generation permanent polymer-coated DES in the setting of ACS.

\section{Methods}

Among all 1,779 patients with an acute coronary syndrome in the TWENTE (The Real-World Resolute Versus Xience V Drug-Eluting Stent Study in Twente; NCT01066650) and DUTCH PEERS TWENTE II) (Durable Polymer-Based Stent Challenge of Promus Element vs. Resolute Integrity; NCT01331707) trials, ${ }^{15,16}$ we assessed the impact of severe target lesion calcification on 2-year clinical outcome. Both trials were approved by the accredited Medical Ethics Committee Twente and the institutional review boards of all participating centers and complied with the Declaration of Helsinki. All patients provided written informed consent.

Details of the TWENTE and DUTCH PEERS (TWENTE II) trials ${ }^{15,16}$ and the 2-year clinical follow-up of both trials have been reported. ${ }^{20,21}$ In brief, the two studies are investigator-initiated, patient-blinded, randomized trials in which respectively 1,391 and 1,811 patients with stable or acute coronary syndromes were enrolled. After 1:1 randomization, patients in the TWENTE trial were treated with the Resolute zotarolimus-eluting stent (Medtronic Vascular, Santa Rosa, CA) or the Xience V everolimuseluting stent (Abbott Vascular, Santa Clara, CA). Patients in the DUTCH PEERS trial were randomized to treatment with the Resolute Integrity zotarolimus-eluting stent (Medtronic) or the Promus Element everolimus-eluting stent (Boston Scientific, Natick, MA). For the purpose of the present analysis, 
patients presenting with an ACS were categorized into patients with versus without severe target lesion calcification.

Angiographic analysts of Thoraxcentrum Twente, blinded to randomization and patient outcome, performed the qualitative and quantitative coronary angiographic analyses of all cases according to current standards, using the software Qangio XA (Version 7.1 and 7.2, Medis, Leiden, the Netherlands). The angiographic analysts of the core lab prospectively classified target lesion calcification in analogy with previous studies. ${ }^{4,9}$ The presence of target lesion calcification was defined as readily apparent densities or x-ray absorbing masses, noted within the apparent vascular wall at the site of the target lesion prior to any contrast injection; in addition, severe target lesion calcification was noted without cardiac motion before contrast injection and generally compromised both sides of the arterial wall. Interventional procedures were performed according to standard techniques, routine clinical protocols, and current medical guidelines. Lesion preparation (e.g., use of rotational atherectomy or cutting balloon inflation), stent postdilatation, and the application of concomitant medication were left to the operator's discretion. Medical treatment did not differ between the two trials. Unfractionated heparin was usually administered as anticoagulant during PCl, and dual anti-platelet therapy, which consisted of aspirin and clopidogrel or ticagrelor, was generally prescribed for 12 months. ${ }^{15,16}$ Electrocardiograms and laboratory tests were systematically performed.

An external clinical research organization (Diagram, Zwolle, the Netherlands), performed the monitoring independently. Clinical follow-up data were obtained at visits to outpatient clinics or, if not feasible, by telephone and/or medical questionnaire. In both trials, processing of clinical outcome data were performed by independent clinical research organizations (Cardialysis, Rotterdam, the Netherlands). Independent clinical events committees, blinded to the assigned treatment, adjudicated all major adverse clinical events.

The clinical endpoints were defined according to the Academic Research Consortium (ARC), including the addendum on myocardial infarction, ${ }^{22,23}$ and have previously been described in detail. ${ }^{15,16}$ In brief, death was considered cardiac, unless an evident non-cardiac cause could be established, and myocardial infarction (MI) was defined by any creatine kinase concentration of more than double the upper limit of normal with elevated values of a confirmatory cardiac biomarker. A target vessel-related MI was related to the target vessel or could not be related to another vessel, and a periprocedural MI was defined as target vessel-related $\mathrm{MI}$ within 48 hours after $\mathrm{PCl}$. Stent thrombosis was classified according to the ARC definitions.

The composite clinical endpoint target vessel failure (TVF), which at 1-year was the primary endpoint of both the TWENTE and DUTCH PEERS trials, is defined as a composite of cardiac death, target vessel $\mathrm{MI}$, or clinically driven target vessel revascularization (TVR). TVR and target lesion revascularization (TLR) were considered clinically indicated if the angiographic diameter stenosis was $\geq 70 \%$, or $\geq 50 \%$ in the presence of ischemic signs or symptoms. The composite endpoint target lesion failure is defined as a composite of cardiac death, target vessel-related MI, and clinically indicated TLR; major adverse cardiac events is a composite of all-cause death, any $\mathrm{MI}$, emergent coronary bypass surgery, or clinically indicated TLR; a patient-oriented composite endpoint is a composite of all-cause mortality, any MI, and any repeat (target- and non-target vessel) revascularization. 
Data were reported as frequencies and percentages for dichotomous and categorical variables, and as mean \pm standard deviation (SD) or median with interquartile range (IQR) for continuous variables. Dichotomous and categorical variables were assessed using Chi-square tests and Fisher's exact tests, and continuous variables were assessed using Student's $t$ tests, the Wilcoxon rank-sum tests or MannWhitney-U test, as appropriate. The Kaplan-Meier method was used to calculate the time to clinical endpoint and the Logrank test was applied to compare between-group differences. All p-values and confidence intervals were two-sided and $p$-values $<0.05$ were considered significant. Parameters were considered as potential confounders if in univariate analyses associations were found with a $p$-value $<0.15$. A multivariate Cox regression model was then used to adjust for potential confounders. Data analysis was performed with SPSS (version 17.0, SPSS Inc., Chicago, IL, USA).

\section{Results}

A total of 1,779 patients with ACS were assessed, of whom 340 (19.1\%) patients were treated for at least one severely calcified target lesion. These patients were significantly older, and had more often diabetes (with more antidiabetic treatment) and a history of myocardial infarction (Table 1). The 4 different stent types were equally distributed between patients with versus without severe target lesion calcification. The rate of renal failure was similar in both groups ( $3.2 \%$ vs $2.6 \%, p=0.55)$. Patients with severely calcified target lesions more often underwent treatment of multiple vessels ( $24.7 \%$ vs $17.3 \%$, $\mathrm{p}=0.002$ ) and aorto-ostial lesions ( $12.4 \%$ vs $6.4 \%, \mathrm{p}<0.001)$, and more lesion preparation was required. In addition, patients with severely calcified lesions were significantly more often treated for long lesions (25.3\% vs $15.5 \%, p<0.001$; Table 2 ).

During the first year of follow-up 3 patients withdrew consent. Of all other 1,776 patients (99.8\%), 2-year follow-up data were available. A time-to-event analysis of TVF revealed a significantly higher event rate in patients with severely calcified target lesions ( $12.4 \%$ vs $7.0 \%, p=0.001$; Figure 1 ). Of the individual components of TVF, only TVR and cardiac death showed significantly higher rates in patients with severely calcified lesions (6.8\% vs $3.3 \%, p=0.003$, and $3.6 \%$ vs $1.8 \%, p=0.05$; Table 3, Figure 2). However, there was no significant between-group difference in target vessel $\mathrm{MI}(3.8 \%$ vs $2.6 \%, p=0.23)$ or definite stent thrombosis ( $0.9 \%$ vs $0.6 \%, p=0.71$; Table 3 ).

Multivariate analysis for TVF demonstrated that, after adjustment for potential confounders (i.e. diabetes mellitus, insulin treatment, previous myocardial infarction, lesion length more than $27 \mathrm{~mm}$, and use of cutting balloon), the presence of severely calcified target lesions was an independent predictor of TVF at 2-year follow-up (adjusted HR 1.58, 95\% Cl: 1.23-2.03, p<0.001). 

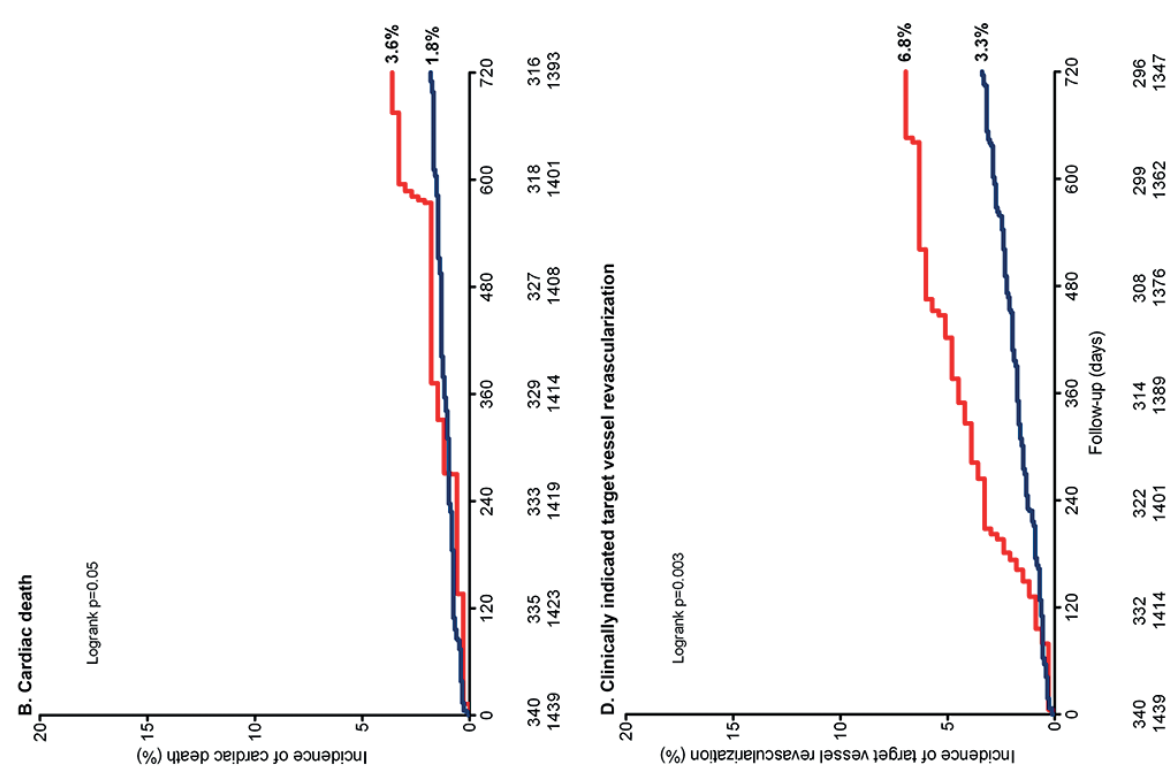

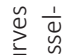

בั 莽

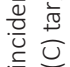
产 证 ह ป 는 $\sum_{1}^{\infty} \cong$ 들

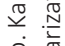
국 空 은 $\stackrel{\pi}{\Perp}$ $\underset{7}{2}+\frac{0}{2}$ 范 茛市 ํํํ
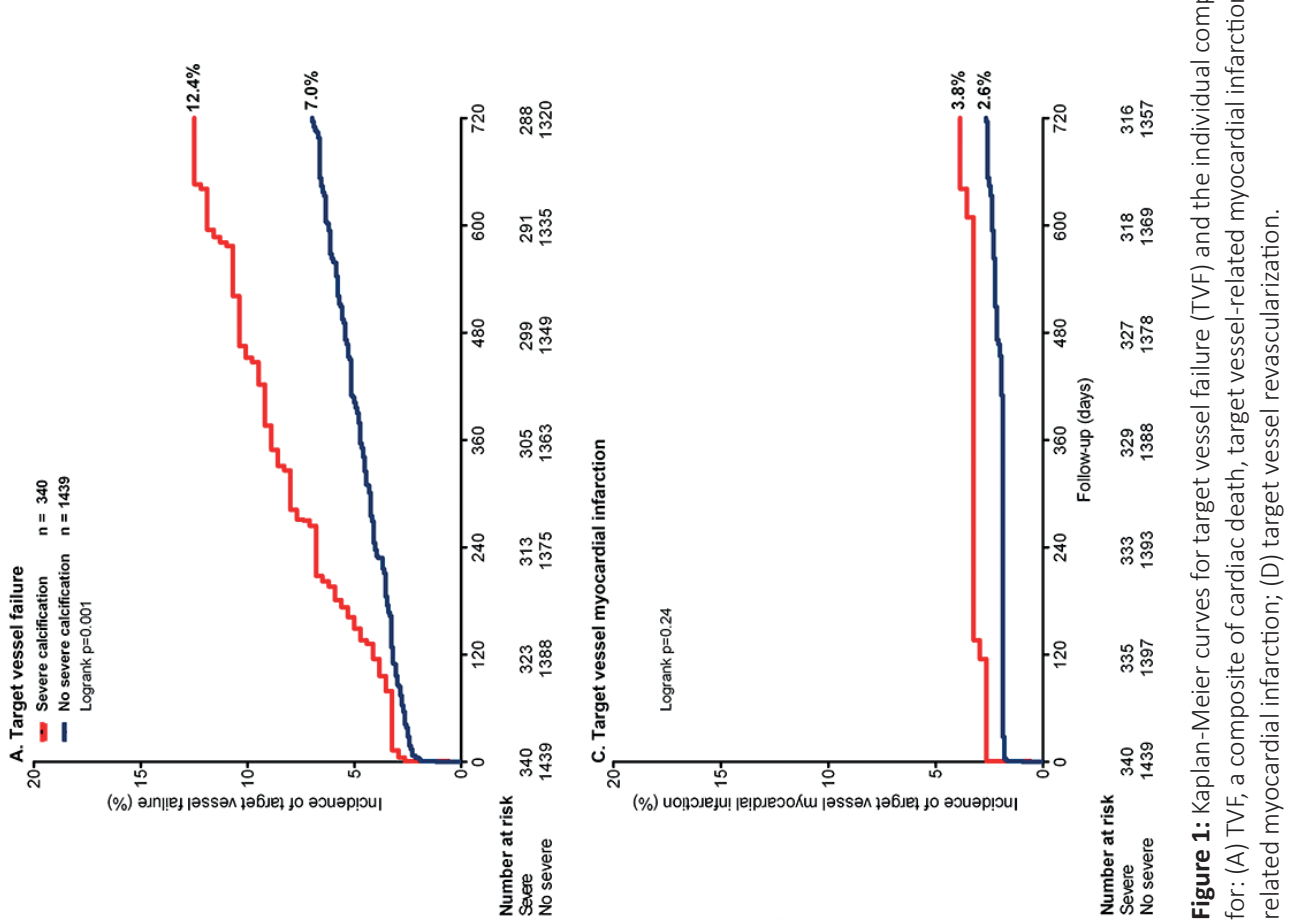
Table 1: Baseline characteristics of all study patients comparing patients with versus without severe target lesion calcification.

\begin{tabular}{|c|c|c|c|}
\hline \multirow[b]{2}{*}{ Patient characteristics } & \multicolumn{2}{|c|}{$\begin{array}{l}\text { All patients } \\
n=1779\end{array}$} & \multirow[b]{2}{*}{$\mathbf{p}$} \\
\hline & $\begin{array}{l}\text { Severe calcification } \\
\qquad n=340\end{array}$ & $\begin{array}{l}\text { No severe calcification } \\
\qquad n=1439\end{array}$ & \\
\hline Age (yrs) & $66.8 \pm 10.6$ & $62.8 \pm 11.5$ & $<0.001$ \\
\hline Women & $106(31.2)$ & $405(28.1)$ & 0.27 \\
\hline $\mathrm{BMI}\left(\mathrm{kg} / \mathrm{m}^{2}\right)$ & $27.8 \pm 4.4$ & $27.7 \pm 4.4$ & 0.76 \\
\hline Diabetes mellitus & $75(22.1)$ & $242(16.8)$ & 0.02 \\
\hline Hypertension & $181(53.2)$ & 705 (49.0) & 0.16 \\
\hline Hypercholesterolemia & $172(51.5)$ & 611 (42.9) & 0.005 \\
\hline Current smoker & $78(22.9)$ & $462(32.1)$ & 0.001 \\
\hline Family history of coronary artery disease & $168(49.4)$ & 675 (46.9) & 0.41 \\
\hline Chronic renal failure* & $11(3.2)$ & $38(2.6)$ & 0.55 \\
\hline Peripheral arterial disease & 40 (11.9) & $111(7.8)$ & 0.02 \\
\hline Previous myocardial infarction & $126(37.1)$ & $393(27.3)$ & $<0.001$ \\
\hline Previous $\mathrm{PCl}$ & $64(18.8)$ & $238(16.5)$ & 0.31 \\
\hline Previous CABG & $35(10.3)$ & $103(7.2)$ & 0.05 \\
\hline Clinical syndrome & & & 0.33 \\
\hline ST-elevation MI & $69(20.3)$ & 301 (20.9) & \\
\hline Non-ST elevation MI & $172(50.6)$ & $667(46.4)$ & \\
\hline Unstable angina pectoris & $99(29.1)$ & $471(32.7)$ & \\
\hline Oral antidiabetics & $62(18.2)$ & $190(13.2)$ & 0.02 \\
\hline Insulin & $34(10.0)$ & $78(5.4)$ & 0.002 \\
\hline Statins & $310(91.2)$ & $1341(93.2)$ & 0.20 \\
\hline ACE inhibitors & $147(43.2)$ & $604(42.0)$ & 0.67 \\
\hline AT1-receptor antagonists & $60(17.6)$ & $228(15.8)$ & 0.42 \\
\hline$\beta$-Blockers & $288(84.7)$ & $1227(85.3)$ & 0.79 \\
\hline Calcium channel blockers & $80(23.5)$ & $178(19.3)$ & 0.08 \\
\hline LDL-cholesterol, mmol/l ( $n=255 / 1102)$ & $2.87 \pm 1.09$ & $3.07 \pm 1.06$ & 0.006 \\
\hline HDL-cholesterol, mmol/l (n=259/1151) & $1.26 \pm 0.38$ & $1.17 \pm 0.34$ & $<0.001$ \\
\hline Triglycerides, mmol/l (n=256/1130) & $1.74 \pm 1.32$ & $1.84 \pm 1.27$ & 0.23 \\
\hline Hemoglobin, mmol/l ( $\mathrm{n=315/1336)}$ & $8.45 \pm 1.06$ & $8.69 \pm 0.94$ & $<0.001$ \\
\hline Creatinine, $\mu \mathrm{mol} / \mathrm{l}(\mathrm{n}=316 / 1337)$ & $83.4 \pm 20.2$ & $84.1 \pm 29.8$ & 0.69 \\
\hline
\end{tabular}

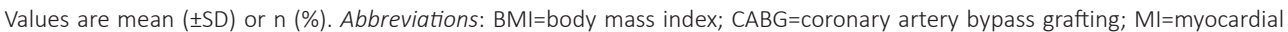
infarction; $\mathrm{PCl}=$ percutaneous coronary interven-tion; $\mathrm{ACE}=$ angiotensin-converting enzyme; $\mathrm{AT} 1=$ angiotensin receptor $1 ; \mathrm{LDL}=$ low-density lipoprotein; HDL=high-density lipoprotein. ${ }^{~}$ Chronic renal failure was defined as a serum creatinine level $\geq 130 \mathrm{mmol} / \mathrm{l}$. 
Table 2: Lesion and procedural characteristics of patients with an acute coronary syndrome, comparing patients with severe calcified lesions versus patients without severe calcified lesions.

\begin{tabular}{|c|c|c|c|}
\hline \multirow{2}{*}{ Lesion and procedural characteristics } & \multicolumn{3}{|c|}{$\begin{array}{c}\text { All patients } \\
n=1779\end{array}$} \\
\hline & $\begin{array}{l}\text { Severe calcification } \\
n=340\end{array}$ & $\begin{array}{l}\text { No severe calcification } \\
\qquad n=1439\end{array}$ & p-value \\
\hline Multivessel treatment & $84(24.7)$ & $249(17.3)$ & 0.002 \\
\hline \multicolumn{4}{|l|}{ Treated coronary vessels } \\
\hline Right coronary artery & $150(44.1)$ & $513(35.6)$ & 0.004 \\
\hline Left anterior artery & $170(50.0)$ & $699(48.6)$ & 0.64 \\
\hline Circumflex artery & $97(28.5)$ & $428(29.7)$ & 0.66 \\
\hline De novo lesions & $307(90.3)$ & $1303(90.5)$ & 0.89 \\
\hline Bifurcation & $98(28.8)$ & $340(23.6)$ & 0.05 \\
\hline At least one chronic total occlusion & $15(4.4)$ & $44(3.1)$ & 0.21 \\
\hline At least one in-stent restenosis & $14(4.1)$ & $50(3.5)$ & 0.57 \\
\hline At least one aorto-ostial lesion & $42(12.4)$ & $92(6.4)$ & $<0.001$ \\
\hline At least one small-vessel* & $198(58.2)$ & $806(56.0)$ & 0.46 \\
\hline At least one lesion length $>27 \mathrm{~mm}$ & $86(25.3)$ & $223(15.5)$ & $<0.001$ \\
\hline Total stent length per patient & $40.0(24.0-60.0)$ & $28.0(18.0-45.0)$ & $<0.001$ \\
\hline Number of stents per patient & $2.1(1.3)$ & $1.7(1.0)$ & $<0.001$ \\
\hline Rotablation & $10(2.9)$ & $4(0.3)$ & $<0.001$ \\
\hline Cutting balloon & $26(7.6)$ & $14(1.0)$ & $<0.001$ \\
\hline Maximal pressure postdilatation & $23.7 \pm 4.9$ & $22.5 \pm 5.2$ & $<0.001$ \\
\hline Maximum ref vessel diameter & $2.9 \pm 0.6$ & $2.8 \pm 0.6$ & 0.14 \\
\hline Max implantation pressure stent & $15.9 \pm 2.75$ & $15.3 \pm 2.55$ & $<0.001$ \\
\hline Postdilatation & $302(88.8)$ & $1125(78.2)$ & $<0.001$ \\
\hline Maximum \% diameter stenosis pre $\mathrm{PCl}$ & $71.6(53.8-79.1)$ & $68.8(59.4-79.2)$ & 0.02 \\
\hline Maximum \% diameter stenosis post $\mathrm{PCl}$ & $13.2(9.5-17.1)$ & $14.7(11.0-20.0)$ & $<0.001$ \\
\hline Minimum lumen diameter post $\mathrm{PCl}$ & $2.22 \pm 0.61$ & $2.18 \pm 0.56$ & 0.30 \\
\hline
\end{tabular}

Values are mean $( \pm S D)$, median $(I Q R)$, or $n(\%)$. $P C l$, percutaneous coronary intervention. a $A$ small-vessel was defined as a reference vessel diameter $<2.75 \mathrm{~mm}$. 
Table 3: 2-year clinical outcome of patients with versus without severe target lesion calcification.

\begin{tabular}{|lccc|}
\hline & & $\begin{array}{c}\text { All patients } \\
\mathbf{n = 1 7 7 6}\end{array}$ & p-value \\
& $\begin{array}{c}\text { Severe calcification } \\
\mathbf{n = 3 3 8}\end{array}$ & $\begin{array}{c}\text { No severe calcification } \\
\mathbf{n = 1 4 3 8}\end{array}$ & 0.003 \\
Death (any) & $22(6.5)$ & $45(3.1)$ & 0.05 \\
$\quad$ Cardiac death & $12(3.6)$ & $26(1.8)$ & 0.23 \\
Target vessel-related MI (any) & $13(3.8)$ & $38(2.6)$ & 0.27 \\
$\quad$ Periprocedural MI & $9(2.7)$ & $25(1.7)$ & 0.003 \\
Target vessel revascularization & $23(6.8)$ & $48(3.3)$ & 0.002 \\
Target lesion revascularization & $19(5.6)$ & $34(2.4)$ & 0.001 \\
Target vessel failure & $42(12.4)$ & $100(7.0)$ & 0.001 \\
Target lesion failure & $39(11.5)$ & $90(6.3)$ & $<0.001$ \\
Major adverse cardiac events & $47(13.9)$ & $113(7.9)$ & $<0.001$ \\
Patient oriented composite endpoint & $63(18.6)$ & $166(11.5)$ & 0.71 \\
Definite stent thrombosis & $3(0.9)$ & $9(0.6)$ & 0.49 \\
Definite or probable stent thrombosis & $5(1.5)$ & $15(1.0)$ & . \\
\hline
\end{tabular}

Values are $\mathrm{n}(\%)$. 2-year follow-up was available for 1776 of all 1779 patients (99.8\%). MI, myocardial infarction.

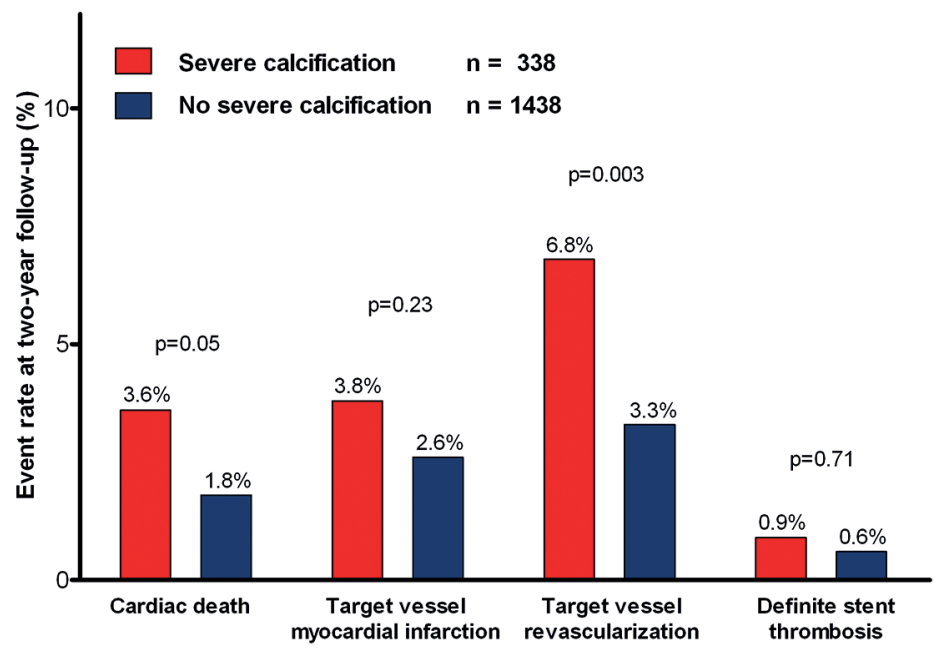

Figure 2: Adverse cardiovascular events at 2-year follow-up. 2-year follow-up data were available for 1776 of all 1779 patients $(99.8 \%)$.

\section{Discussion}

In this patient-level pooled data analysis from TWENTE and DUTCH PEERS (TWENTE II), patients with ACS, who were treated with newer-generation zotarolimus-eluting and everolimus-eluting stents for severely 
calcified coronary lesions, showed significantly higher rates of the composite clinical endpoint TVF at 2-year follow-up (12.4\% vs 7.0\%). A multivariate analysis confirmed severe target lesion calcification to be an independent predictor of TVF (adjusted HR 1.51). Insulin treatment and cholesterol levels (LDL-C and HDL-C) were no independent predictors of TVF. The overall higher risk of clinical events in ACS patients with severely calcified target lesions was primarily related to a higher incidence of TVR $(6.8 \%$ vs 3.3\%), which is first and foremost a parameter of treatment efficacy. The fact that the MI and stent thrombosis rates were low may be interpreted as a safety signal for the treatment of ACS patients with severe target lesions calcification using newer-generation DES. The early (periprocedural) increase in $\mathrm{MI}$ in both patients with and without severe target lesion calcification was often not caused by a stent thrombosis, but it may have been the result of procedure-related embolization of atherothrombotic material or the occlusion of (very) small side branches from predilatation, stenting, and/or postdilatation of stents.

In a histopathological study, microcalcifications within the thin fibrous cap of atheromatous plaques - in particular when $>5 \mu \mathrm{m}$ - were related to plaque rupture. ${ }^{24}$ In addition, coronary calcification is known to increase with a plaque burden; however, there is still an ongoing debate on whether coronary calcification is just (or mainly) a marker of plaque burden or also indicates unstable lesions with an increased risk of rupture. ${ }^{24,25}$ Genereux et al. recently showed that at least one moderate-to-severe target lesion calcification is present in $27 \%$ and $38 \%$ of patients with non-ST elevation ACS and STEMI, respectively. ${ }^{2}$ In the present study, at least one severe target lesion calcification was present in $19 \%$ of patients with ACS, which fits into this scope.

Direct stenting of severely calcified coronary lesions is often impossible and in many cases undesirable, as this approach bears a higher risk of stent under-expansion and delayed or incomplete stent endothelialization, which may lead to stent thrombosis and/or in-stent restenosis. ${ }^{26}$ In addition, manipulations with a DES in a severely calcified coronary vessel and/or lesion might cause damage to the polymer coating, which may locally impair both drug delivery from the coating and its capacity to prevent restenosis. ${ }^{19}$ Coronary macro-calcification, a marker of extensive atherosclerotic disease, was previously shown to predict an increased risk of TLR after stenting. 2,27 Rotational atherectomy may be used to favorably modify severely calcified lesions, facilitate stent delivery, and improve lesion expansion and the procedural result. ${ }^{26}$ As an alternative, severely calcified lesions can also be pre-treated with cutting-balloons. In the present study, rotational atherectomy or cutting balloon inflations were used in no more than $11 \%$ of patients with severe target lesion calcification. As the crossing-profiles of modern DES have become increasingly small and flexible, stenting can be performed in many cases following a pre-dilatation with a (non-compliant) balloon catheter. In addition, stent postdilatation at higher balloon pressures, which was frequently performed in our patients with severe lesion calcification (89\%), is most helpful to achieve a good procedural result with adequate stent expansion and apposition.

Despite recent improvements in DES, the presence of a severely calcified coronary target lesion still is a predictor of worse outcome. ${ }^{2,27-29}$ A recent retrospective pooled analysis of 7 stent trials that used various first and second-generation DES for the treatment of different patient populations demonstrated that the presence of a severely calcified lesion is an independent predictor of all-cause death, myocardial infarction, and any revascularization ( $\mathrm{HR} 1.18,95 \% \mathrm{Cl}: 1.01-1.39, \mathrm{p}=0.04$ ), but not of stent thrombosis. ${ }^{27}$ In a pooled analysis of patients treated for ACS with (mostly) first-generation DES, 
a higher TVR rate was observed in patients with moderate-to-severe target lesion calcification $(9.4 \%$ vs $7.5 \%, p=0.02$ ), and a multivariate analysis showed an independent association between moderate-tosevere target lesion calcification and 1-year TLR (HR 1.44, 95\% Cl: 1.17-1.78, p<0.001). ${ }^{2}$ In addition, in that study, patients with severely calcified lesions had a higher rate of stent thrombosis. While in the setting of ACS the risk of stent thrombosis is generally increased, ${ }^{6}$ in our present study the rate of stent thrombosis was low. This may partly be due to the more biocompatible nature of the polymer coatings of the newer-generation DES used, while, due to the systematic assessment of post-PCI cardiac markers and ECG changes and the availability of follow-up in as much as $99.8 \%$ of patients, underreporting of stent thrombosis or other vital clinical events is unlikely.

The present study is limited by its post-hoc nature, and therefore its findings should be considered hypothesis generating. Nevertheless, as data on medium-term outcome of patients with ACS treated in severely calcified coronary lesions with newer-generation DES are scarce, the findings may be of interest. In our current study that made no routine use of intracoronary imaging techniques, we evaluated in a relatively large population of all-comer patients the presence of target lesion calcification based on x-ray appearance. Previous studies have shown that prognostic information on target lesion calcification can be obtained from angiographic assessment. ${ }^{27-29}$ However, intravascular ultrasound and optical coherence tomography allow a more detailed assessment of coronary atherosclerosis and plaque composition. ${ }^{30-35}$ This includes a better identification and classification of target lesion calcification, as these techniques are more sensitive in detecting calcium and also reveal information on the exact location, magnitude, and distribution of calcium (e.g. superficial versus deep location). ${ }^{35-39}$

\section{Conclusion}

In conclusion, in patients who presented with ACS, treatment of severely calcified lesions with newergeneration permanent polymer-coated DES was associated with an overall increased risk of adverse cardiovascular events at 2-year follow-up-related in particular to a higher TVR rate - while the risk of $\mathrm{Ml}$ remained low. 


\section{References}

1. Madhavan MV, Tarigopula M, Mintz GS, et al. Coronary artery calcification: pathogenesis and prognostic implications. J Am Coll Cardiol 2014;63:1703-14.

2. Généreux P, Madhavan MV, Mintz GS, et al. Ischemic outcomes after coronary intervention of calcified vessels in acute coronary syndromes. Pooled analysis from the HORIZONS-AMI (Harmonizing Outcomes With Revascularization and Stents in Acute Myocardial Infarction) and ACUITY (Acute Catheterization and Urgent Intervention Triage Strategy) TRIALS. J Am Coll Cardiol 2014;63:1845-54.

3. Bangalore S, Vlachos HA, Selzer F, et al. Percutaneous coronary intervention of moderate to severe calcified coronary lesions: insights from the National Heart, Lung, and Blood Institute Dynamic Registry. Catheter Cardiovasc Interv 2011;77:22-8.

4. Moussa I, Ellis SG, Jones M, et al. Impact of coronary culprit lesion calcium in patients undergoing paclitaxeleluting stent implantation (a TAXUS-IV sub study). Am J Cardiol 2005;96:1242-7.

5. Stefanini GG, Taniwaki M, Windecker S. Coronary stents: novel developments. Heart. 2014;100:1051-1061.

6. Palmerini T, Biondi-Zoccai G, Riva DD, et al. Stent thrombosis with drug-eluting and bare-metal stents: evidence from a comprehensive network meta-analysis. Lancet 2012;379:1393-402.

7. Navarese EP, Tandjung K, Claessen B, et al. Safety and efficacy outcomes of first and second generation durable polymer drug eluting stents and biodegradable polymer biolimus eluting stents in clinical practice: comprehensive network meta-analysis. BMJ 2013;347:f6530.

8. Stone GW, Rizvi A, Newman W, et al. Everolimus-eluting versus paclitaxel-eluting stents in coronary artery disease. N Engl J Med 2010;362:1663-74.

9. Onuma $Y$, Tanimoto S, Ruygrok P, et al. Efficacy of everolimus eluting stent implantation in patients with calcified coronary culprit lesions: two-year angiographic and three-year clinical results from the SPIRIT II study. Catheter Cardiovasc Interv 2010;76:634-42.

10. Stone GW, Teirstein PS, Meredith IT, et al. A prospective, randomized evaluation of a novel everolimus-eluting coronary stent: the PLATINUM trial. J Am Coll Cardiol 2011;57:1700-8.

11. Meredith IT, Teirstein PS, Bouchard A, et al. Three-year results comparing platinum-chromium PROMUS Element and cobalt-chromium XIENCE $\vee$ everolimus-eluting stents in de novo coronary artery narrowing (from the PLATINUM Trial). Am J Cardiol 2014;113:1117-23.

12. Kedhi E, Joesoef KS, McFadden E, et al. Second-generation everolimus-eluting and paclitaxel-eluting stents in real-life practice (COMPARE): a randomised trial. Lancet 2010;375:201-9.

13. Jensen LO, Thayssen P, Maeng M, et al. Three-year outcomes after revascularization with everolimus- and sirolimus-eluting stents from the SORT OUT IV trial. J Am Coll Cardiol Intv 2014;7:840-8.

14. Serruys PW, Silber S, Garg S, et al. Comparison of zotarolimus-eluting and everolimus-eluting coronary stents. N Engl J Med 2010;363:136-46.

15. von Birgelen C, Basalus MWZ, Tandjung K, et al. A randomized controlled trial in second-generation zotarolimuseluting Resolute stents versus everolimus-eluting Xience V stents in real-world patients: the TWENTE trial. J Am Coll Cardiol 2012;59:1350-1361.

16. von Birgelen C, Sen H, Lam MK, et al. Third-generation zotarolimus-eluting and everolimus-eluting stents in all-comer patients requiring a percutaneous coronary intervention (DUTCH PEERS): a randomised, single-blind, multicentre, non-inferiority trial. Lancet 2014;383:413-23.

17. Park KW, Kang SH, Kang HJ, et al. A randomized comparison of platinum chromium-based everolimus-eluting stents versus cobalt chromium-based zotarolimus-eluting stents in all-comers receiving percutaneous coronary intervention: HOST-ASSURE, a randomized, controlled, noninferiority trial. J Am Coll Cardiol 2014;63:2805-16.

18. Raungaard B, Jensen LO, Tilsted $\mathrm{HH}$, et al. Zotarolimus-eluting durable-polymer-coated stent versus a biolimuseluting biodegradable-polymer-coated stent in unselected patients undergoing percutaneous coronary intervention (SORT OUT VI): a randomised non-inferiority trial. Lancet 2015; 385:1527-5.

19. Wiemer M, Butz T, Schmidt W, et al. Scanning electron microscopic analysis of different drug eluting stents after failed implantation: from nearly undamaged to major damaged polymers. Catheter Cardiovasc Interv 2010;75:905-11. 
20. Tandjung K, Sen H, Lam MK, et al. Clinical outcome following stringent discontinuation of dual antiplatelet therapy after 12 months in real-world patients treated with second-generation zotarolimus-eluting resolute and everolimus-eluting xience V stents: 2-year follow-up of the randomized TWENTE trial. J Am Coll Cardiol 2013;61:2406-16.

21. Sen H, Lam MK, Löwik MM, et al. Clinical events and patient-reported chest pain in all-comers treated with Resolute Integrity and Promus Element stents: two-year follow-up of the randomized DUTCH PEERS (TWENTE II) trial. J Am Coll Cardiol Intv 2015;8:889-99.

22. Cutlip DE, Windecker S, Mehran R, et al. Clinical end points in coronary stent trials: a case for standardized definitions. Circulation 2007;115:2344-51.

23. Vranckx P, Cutlip DE, Mehran R, et al. Myocardial infarction adjudication in contemporary all-comer stent trials: balancing sensitivity and specificity. Addendum to the historical MI definitions used in stent studies. Eurolntervention 2010;5:871-4.

24. Otsuka F, Sakakura K, Yahagi K, et al. Has our understanding of calcification in human coronary atherosclerosis progressed? Arterioscler Thromb Vasc Biol 2014;34:724-36.

25. Virmani R, Burke AP, Farb A, Kolodgie FD. Pathology of the vulnerable plaque. J Am Coll Cardiol 2006;47:C13-8.

26. Abdel-Wahab M, Baev R, Dieker P, et al. Long-term clinical outcome of rotational atherectomy followed by drugeluting stent implantation in complex calcified coronary lesions. Catheter Cardiovasc Interv 2013;81:285-91.

27. Bourantas CV, Zhang YJ, Garg S, et al. Prognostic implications of coronary calcification in patients with obstructive coronary artery disease treated by percutaneous coronary intervention: a patient-level pooled analysis of 7 contemporary stent trials. Heart 2014;100:1158-64.

28. Kawaguchi R, Tsurugaya $\mathrm{H}$, Hoshizaki H, et al. Impact of lesion calcification on clinical and angiographic outcome after sirolimus-eluting stent implantation in real-world patients. Cardiovasc Revasc Med 2008;9:2-8.

29. Fujimoto H, Nakamura M, Yokoi H. Impact of calcification on the long-term outcomes of sirolimus-eluting stent implantation: subanalysis of the Cypher Post-Marketing Surveillance Registry. Circ J 2012;76:57-64.

30. Sato H, Kawasak Mi, Morita N, et al. Distribution of tissue characteristics of coronary plaques evaluated by integrated backscatter intravascular ultrasound: Differences between the inner and outer vessel curvature. J Cardiol 2015;66:489-495.

31. Erbel R, Ge J, Görge G, et al. Intravascular ultrasound classification of atherosclerotic lesions according to American Heart Association recommendation. Coron Artery Dis 1999;10:489-99.

32. Okura H. Intravascular ultrasound-derived tissue characterization of the in-stent neointima: Are they "true colors" shining through? J Cardiol 2014;64:421-422.

33. von Birgelen C, Klinkhart W, Mintz GS, et al. Plaque distribution and vascular remodeling of ruptured and nonruptured coronary plaques in the same vessel: An intravascular ultrasound study in vivo. J Am Coll Cardiol 2001;37:1864-70.

34. Lee SY, Shin DH, Shehata I, et al. Association between fractional flow reserve and coronary plaque characteristics assessed by optical coherence tomography. J Cardiol. In press (electronically published ahead of print on 18 November 2015 (doi: 10.1016/j.jjcc.2015.10.012).

35. Huisman J, Hartmann M, von Birgelen C. Ultrasound and light: friend or foe? On the role of intravascular ultrasound in the era of optical coherence tomography. Int J Cardiovasc Imaging 2011;27:209-14.

36. Hibi K, Kimura K, Umemura S. Clinical utility and significance of intravascular ultrasound and optical coherence tomography in guiding percutaneous coronary interventions. Circ J 2015;79:24-33.

37. von Birgelen C, Mintz GS, Sieling C, et al. Relation between plaque composition and vascular remodeling in coronary lesions with different degrees of lumen narrowing as assessed with three-dimensional intravascular ultrasound in patients with stable angina pectoris. Am J Cardiol 2003;91:1103-7.

38. Mintz GS. Intravascular imaging of coronary calcification and its clinical implications. JACC Cardiovasc Imaging 2015;8:461-71.

39. von Birgelen C, Mintz GS, Böse D, et al. Impact of moderate lesion calcium on mechanisms of coronary stenting as assessed with three-dimensional intravascular ultrasound in vivo. Am J Cardiol 2003;92:5-10. 


\section{Conflicts of interest statement}

CVB was consultant to various stent-manufacturing companies, among them being Abbott Vascular and Boston Scientific. Thoraxcentrum Twente has received research grants, provided by Abbott Vascular, AstraZeneca, Biotronik, Boston Scientific, and Medtronic. All other authors declare that they have no conflict of interest. 


\section{CHAPTER 16}

\section{Three-Year Outcome of Patients With Severely Calcified Coronary Lesions Treated With Different Everolimus-Eluting Stents}

A Propensity Score-Adjusted Analysis of TWENTE and DUTCH PEERS Trial Participants, Treated by the Same Operators

Liefke C. van der Heijden, Jennifer Huisman, Marlies M. Kok, Marije M. Löwik, Marc Hartmann, K. Gert van Houwelingen, Martin G. Stoel, Frits H.A.F. de Man, J.(Hans) W. Louwerenburg, Gerard C.M. Linssen, Carine J.M. Doggen,

Clemens von Birgelen
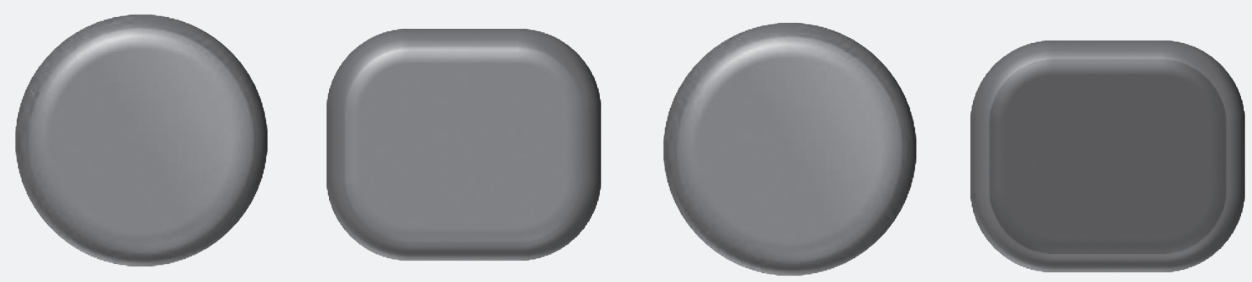


\begin{abstract}
Objectives: To analyze the 3-year clinical outcome of 282 participants in the TWENTE and DUTCH PEERS trials, who had severely calcified lesions that were treated with one of two fluoro-polymer-coated everolimus-eluting stents: the novel platinum-chromium Promus Element stent or its predecessor, the cobalt-chromium Xience $V$ stent.
\end{abstract}

Background: Severe target lesion calcification can lead to impaired expansion, incomplete apposition, and decreased visibility of coronary drug-eluting stents (DES). Therefore, patients with calcified lesions may benefit most from refinements in stent material and design.

Methods: Patients were treated by the same operators at Thoraxcentrum Twente for stable angina or non-ST-elevation acute coronary syndromes and received the same anti-platelet therapy. The independently adjudicated main clinical endpoint was target vessel failure (TVF), a composite of cardiac death, target vessel myocardial infarction (MI), or target vessel revascularization.

Results: In patients treated with Promus Element $(n=133)$ versus Xience $V(n=149)$ the incidence of TVF was $14.8 \%$ vs $18.9 \%$ ( $p$-Logrank=0.33). There was no significant between-DES difference in cardiac death and target vessel revascularization rates. Target vessel $\mathrm{MI}$ occurred less often in the Promus Element group (2.0\% vs 9.1\%; p-Logrank=0.02); after propensity score adjustment, Promus Element use was still associated with a lower risk of target vessel MI (adjusted HR: 0.23, 95\% Cl:0.06-0.85, adjusted $\mathrm{p}$-Logrank=0.03). Definite-or-probable stent thrombosis rates were similar for both DES groups $(2.0 \%$ vs $2.3 \%$; $p$-Logrank=0.89).

Conclusions: In patients with severely calcified target lesions, the 3-year risk of various cardiovascular events did not differ significantly between DES groups. The target vessel MI rate was lower in the Promus Element group. 


\section{Introduction}

Severe calcification of coronary lesions has been associated with an increased risk of unfavourable outcome following stenting, including higher rates of repeat revascularization, myocardial infarction, and death. ${ }^{1}$ The increased event risk has been related to impaired stent expansion and incomplete stent apposition. ${ }^{2-4}$ In addition, as calcium is radiopaque, it interferes with the X-ray-based visualization of stents. ${ }^{5-6}$ Therefore, refinements in strut material and stent design may be particularly important for the treatment of severely calcified lesions. ${ }^{5}$

The early-generation drug-eluting stents (DES) reduced the restenosis risk as compared to bare metal stents $^{7}$, but severe target lesion calcification remained an independent predictor of adverse events ${ }^{8}$. Newer-generation DES had more biocompatible coatings and showed favourable results in all-comers, which included many patients with complex and calcified target lesions. ${ }^{9-13}$ Nevertheless, in all-comers treated with newer-generation DES, severe calcification was still associated with a higher incidence of various clinical events. ${ }^{14,15}$

Everolimus-eluting stents (EES) with durable fluoro-polymer coating have been studied in various clinical trials that demonstrated their efficacy and favourable safety in patients with mild-to-moderate cardiovascular risk ${ }^{16-19}$ and in broad and unrestricted study populations ${ }^{10,11,20-23}$. The Xience V EES (Abbott Vascular, Santa Rosa, CA) has a cobalt-chromium backbone, while the more recently introduced Promus Element EES (Boston Scientific, Nattick, MA) uses the same coating and drug as the Xience V, but its stent backbone has a novel, flexible design and is made from a platinum-chromium alloy that increases radial force and angiographic visibility., ${ }^{54-27}$ Considering these characteristics of Promus Element, the device theoretically may show advantages in the treatment of patients with severely calcified lesions. Therefore, we compared in the present patient-level pooled, propensity-adjusted analysis of the TWENTE and DUTCH PEERS trials the impact of EES type (Promus Element vs. Xience V) on 3-year clinical outcome of patients with severely calcified target lesions, who were treated by the same operators and received the same dual anti-platelet therapy.

\section{Methods}

\section{Study design and patient population}

This study was performed in patients of the TWENTE (The Real-World Resolute Versus Xience $V$ Drug-Eluting Stent Study in Twente; ClinicalTrials.gov NCT01066650) and DUTCH PEERS (TWENTE II) (Durable Polymer-Based Stent Challenge of Promus Element versus Resolute Integrity; ClinicalTrials.gov NCT01331707) trials, who were treated at Thoraxcentrum Twente. Details of the TWENTE and DUTCH PEERS (TWENTE II) trials have previously been reported. ${ }^{11,22}$

In brief, the TWENTE and DUTCH PEERS trials are investigator-initiated, patient-blinded, randomized studies, in which 3,202 patients with stable angina or acute coronary syndromes were treated with newer-generation durable polymer DES. After 1:1 randomization, patients in the TWENTE single-centre trial were treated with Resolute zotarolimus-eluting stents (Medtronic Vascular, Santa Rosa, CA) or Xience V everolimus-eluting stents (Abbott Vascular, Santa Clara, CA). Patients in the DUTCH PEERS 
multicentre trial were randomized to treatment with Resolute Integrity zotarolimus-eluting stents (Medtronic Vascular, Santa Rosa, CA) or Promus Element everolimus-eluting stents (Boston Scientific, Natick, MA). Both trials were approved by the Medical Ethics Committee Twente and the institutional review board, and complied with the Declaration of Helsinki. All patients provided written informed consent.

In this analysis, we evaluated the impact of use of Promus Element versus Xience V EES on clinical outcome in patients with severely calcified lesions. In order to obtain equivalent patient populations for both EES groups and to minimize potential confounders, we assessed patients who suffered from stable angina or non-ST-elevation acute coronary syndromes (ST-elevation myocardial infarction (MI) within the last 48 hours was an exclusion criterion in TWENTE) and were treated by the same operators at Thoraxcentrum Twente.

\section{Stent characteristics}

The Xience $V$ stent consist of a thin-strut $(81 \mu \mathrm{m})$ Vision-Multilink cobalt-chromium platform and elutes everolimus from a thin $(7.8 \mu \mathrm{m})$ layer of durable fluoro-polymer coating. The Promus Element stent also elutes everolimus from a thin $(7 \mu \mathrm{m})$ layer of fluoro-polymer coating, however, its thin struts (81 $\mu \mathrm{m})$ are made from platinum-chromium alloy. In contrast to Xience $V$ stent, the laser-cut stent platform consists of serpentine rings, each joined to the next by two connectors, and has offset segment peaks. ${ }^{5}$

\section{Procedures and angiographic analysis}

The same operators treated the patients of both trials. Interventional procedures were performed according to standard techniques, routine clinical protocols, and current medical guidelines, which did not differ between trials. Details of the intervention, such as lesion predilation or stent postdilation, and the application of concomitant medication, were left at the operator's discretion. Medical treatment did not differ between trials: unfractionated heparin was usually administered as an anticoagulant during $\mathrm{PCl}$, and dual anti-platelet therapy, which commonly consisted of aspirin and clopidogrel, was generally prescribed for 12 months. ${ }^{11,22}$ Electrocardiograms and laboratory tests were systematically performed. Analysts of the angiographic core lab of Thoraxcentrum Twente, blinded to randomization and patient outcome, performed the qualitative and quantitative coronary angiographic analysis according to current standards, using the software Qangio XA (version 7.1 and 7.2, Medis, Leiden, the Netherlands). The angiographic analysts prospectively classified the target lesion calcification in analogy with previous studies. ${ }^{17}$ Target lesion calcification was defined as readily apparent densities or $\mathrm{x}$-ray absorbing masses, noted within the apparent vascular wall at the site of the target lesion, prior to any contrast injection. Severe target lesion calcification was noted without cardiac motion before contrast injection and generally compromised both sides of the arterial wall.., 17

\section{Follow-up, monitoring and adjudication}

Clinical follow-up data were obtained at visits to outpatient clinics or, if not feasible, by telephone or medical questionnaire. Monitoring was performed by an independent, external clinical research organization (Diagram, Zwolle, the Netherlands). In both trials, independent clinical research organizations processed the clinical outcome data; independent external clinical events committees, 
blinded to the assigned treatment, adjudicated the adverse clinical events (Cardialysis, Rotterdam, the Netherlands, and Diagram, Zwolle, the Netherlands).

\section{Clinical endpoints}

Both trials used the same definitions of the predefined clinical endpoints, which have previously been described in detail ${ }^{11,22}$ and follow the suggestions of the Academic Research Consortium (ARC). ${ }^{27,28}$ In brief, death was considered cardiac, unless an evident non-cardiac cause could be established; MI was defined by any creatine kinase concentration of more than double the upper limit of normal with elevated values of a confirmatory cardiac biomarker; a target vessel-related MI was related to the target vessel or could not be related to another vessel. Periprocedural MI was defined as a target vesselrelated $\mathrm{MI}$ during the first 48 hours after stenting.

The composite endpoint target vessel failure (TVF) was defined as cardiac death, target vessel-related $\mathrm{MI}$, or clinically indicated target vessel revascularization (TVR). TVR is considered clinically indicated if the angiographic diameter stenosis was $\geq 70 \%$, or $\geq 50 \%$ in the presence of ischemic signs or symptoms. The composite endpoint target lesion failure was defined as cardiac death, target vessel-related MI or clinically indicated target lesion revascularization.

\section{Statistical analysis}

Data were reported as frequencies and percentages for dichotomous and categorical variables, as mean \pm standard deviation for continuous normally distributed variables. Chi-square test and Fisher's exact test were used as appropriate. Differences between groups in continuous variables were assessed with the Student's $t$ test. The Kaplan-Meier method was used to calculate the time to clinical endpoints and the Logrank-test was applied to compare groups. A two-sided p-value of less than 0.05 was considered significant. Variables were considered as potential confounders if associations were found with a $p<0.15$ in univariate analysis. For adjustment of all potential confounders, propensity score analysis was used. The propensity score was estimated using multiple logistic regression analysis. Previous MI, clinical syndrome, left anterior descending artery treatment, lesion length $<27 \mathrm{~mm}$, rotablation, maximum implantation pressure of the stent, maximum pressure of postdilation, maximum final reference vessel diameter, and postdilation were used to calculate the propensity score for being treated with Promus Element versus Xience $V$ stents. A multivariate Cox-regression analysis was then performed using treatment with Xience $V$ versus Promus Element stents and the propensity score as independent variables. Data analysis was performed with SPSS (version 22.0, SPSS Inc., Chicago, IL, USA).

\section{Results}

\section{Baseline, lesion, and procedural characteristics}

A total of 282 patients were treated for at least one severely calcified lesion. Of them, 149 (52.8\%) patients were treated with Promus Element and 133 (47.2\%) with Xience V. Patients treated with Xience $\mathrm{V}$ had more often a history of $\mathrm{MI}(26 . \%$ vs $38.3 \%, \mathrm{p}=0.03)$ and non-ST-elevation acute coronary syndromes at baseline (38.3\% vs $53.4 \%, p=0.001$ ) (Table 1 ). The rate of renal failure did not differ 
significantly ( $4.7 \%$ vs $2.3 \%, p=0.34)$. Rotational atherectomy was more often performed in patients treated with Promus Element (8.1\% vs 1.5\%, p=0.01) (Table 2). The rates of lesion pretreatment were similar for both EES, but the maximum balloon pressure for stent implantation was higher in Xience $\mathrm{V}$ $(15.8 \pm 2.5 \mathrm{~atm}$ vs $16.7 \pm 3.0 \mathrm{~atm}, \mathrm{p}=0.01)$. The rate of postdilatation was similar and very high in both EES-groups. Postdilation was performed at higher pressures in Xience $V(23.9 \pm 4.6 \mathrm{~atm}$ vs $25.3 \pm 4.6 \mathrm{~atm}$, $\mathrm{p}=0.02)$.

Table 1: Demographics and patient characteristics.

\begin{tabular}{|lccc|}
\hline & $\begin{array}{c}\text { Promus Element } \\
\mathbf{n = 1 4 9}\end{array}$ & $\begin{array}{c}\text { Xience } \mathbf{~} \\
\mathbf{n = 1 3 3}\end{array}$ & $\mathbf{p}$ \\
Age $(\mathrm{yrs})$ & $68.0 \pm 9.6$ & $67.6 \pm 10.1$ & 0.70 \\
Women & $47(31.5)$ & $37(27.8)$ & 0.50 \\
$\mathrm{BMI}\left(\mathrm{kg} / \mathrm{m}^{2}\right)$ & $28.1 \pm 5.2$ & $27.4 \pm 4.0$ & 0.26 \\
Diabetes mellitus & $36(24.2)$ & $26(19.5)$ & 0.35 \\
Chronic renal failure & $7(4.7)$ & $3(2.3)$ & 0.34 \\
Previous MI & $39(26.2)$ & $51(38.3)$ & 0.03 \\
Previous PCl & $29(19.5)$ & $24(18.0)$ & 0.76 \\
Previous CABG & $22(14.8)$ & $23(17.3)$ & 0.56 \\
Clinical syndrome & & & 0.001 \\
\multicolumn{1}{|c|}{ Stable angina pectoris } & $92(61.7)$ & $62(46.6)$ & $32(24.1)$ \\
$\quad$ Unstable angina pectoris & $12(8.1)$ & $39(29.3)$ & \\
\hline
\end{tabular}

Values are $\mathrm{n}(\%)$ or mean $\pm \mathrm{SD}$. Abbreviations: $\mathrm{BMI}=$ body mass index; $\mathrm{Ml}=$ myocardial infarction; $\mathrm{PCl}=$ percutaneous coronary intervention; $\mathrm{CABG}=$ coronary artery bypass grafting.

\section{Clinical event rates at 3-year follow-up}

The rates of cardiac death and target vessel revascularization were similar for both stent groups $(5.4 \%$ vs $6.1 \%, \mathrm{P}_{\text {Log-rank }}=0.77$, and $8.1 \%$ vs $7.6 \%, p$-Logrank $=0.89$, respectively) (Table 3, Figure 1 ). Time-toevent analysis revealed a significantly lower rate of target vessel $\mathrm{Ml}$ for patients treated with Promus Element versus Xience $\mathrm{V}(2.0 \%$ vs $9.1 \%$, $\mathrm{p}$-Logrank=0.02), mainly due to a higher rate of periprocedural MI (1.4\% vs $6.1 \%$, p-Logrank=0.03). A numeric difference in TVF did not reach statistical significance (14.8\% vs $18.9 \%$, p-Logrank=0.33) (Table 3 ). The rate of definite-or-probable stent thrombosis did not differ between both EES ( $2.0 \%$ vs $2.3 \%$, p-Logrank=0.89).

Multivariate analysis with use of propensity score adjustment demonstrated that, after adjustment for all potential confounders, Promus Element was an independent predictor of a lower risk of target vessel MI (adjusted HR: 0.23, 95\% Cl:0.06-0.85, adjusted p-Logrank=0.03) (Table 3). 
Table 2: Lesion and procedural characteristics.

\begin{tabular}{|c|c|c|c|}
\hline & $\begin{array}{l}\text { Promus Element } \\
\qquad n=149\end{array}$ & $\begin{array}{c}\text { Xience V } \\
n=133\end{array}$ & $\mathbf{p}$ \\
\hline Multivessel treatment & $40(26.8)$ & $41(30.8)$ & 0.46 \\
\hline \multicolumn{4}{|l|}{ Treated coronary vessels } \\
\hline Right coronary artery & $65(43.6)$ & $54(40.6)$ & 0.61 \\
\hline Left anterior descending artery & $75(50.3)$ & $82(61.7)$ & 0.06 \\
\hline Circumflex artery & $37(24.8)$ & $36(27.1)$ & 0.67 \\
\hline De novo lesions & $129(86.6)$ & $115(86.5)$ & 0.98 \\
\hline At least one bifurcation & $42(28.2)$ & $40(30.1)$ & 0.73 \\
\hline At least one chronic total occlusion & $8(5.4)$ & $13(9.8)$ & 0.16 \\
\hline At least one in-stent restenosis & $7(4.7)$ & $4(3.0)$ & 0.46 \\
\hline At least one small-vessel & $91(61.1)$ & $80(60.2)$ & 0.87 \\
\hline At least one lesion length $>27 \mathrm{~mm}$ & 55 (36.9) & $34(25.6)$ & 0.04 \\
\hline Rotablation & $12(8.1)$ & $2(1.5)$ & 0.01 \\
\hline Cutting balloon & $19(12.8)$ & $16(12.0)$ & 0.85 \\
\hline Predilation & $132(88.6)$ & $112(84.2)$ & 0.28 \\
\hline Maximum implantation pressure (atm) & $15.8 \pm 2.5$ & $16.7 \pm 3.0$ & 0.01 \\
\hline Postdilation & $129(86.6)$ & $124(93.2)$ & 0.07 \\
\hline Postdilation pressure, maximum (atm) & $23.9 \pm 4.6$ & $25.3 \pm 4.6$ & 0.02 \\
\hline Final reference diameter, maximum (mm) & $2.8 \pm 0.6$ & $2.9 \pm 0.6$ & 0.44 \\
\hline Final lumen diameter, minimum (mm) & $2.2 \pm 0.6$ & $2.2 \pm 0.7$ & 0.98 \\
\hline
\end{tabular}

Values are $\mathrm{n}(\%)$ or mean \pm SD.

Table 3: Clinical endpoints at 3-year follow-up.

\begin{tabular}{|lccccc|}
\hline Clinical Endpoints & $\begin{array}{c}\text { Promus } \\
\text { Element } \\
\mathbf{n = 1 4 9}\end{array}$ & Xience V & $\begin{array}{c}\text { Unadjusted } \\
\mathbf{p} \text {-Logrank }\end{array}$ & $\begin{array}{c}\text { Adjusted Hazard } \\
\text { ratio (95\% CI) }\end{array}$ & $\begin{array}{c}\text { Adjusted } \\
\mathbf{p} \text {-Logrank }\end{array}$ \\
$\begin{array}{l}\text { Cardiac death } \\
\begin{array}{l}\text { Target vessel myocardial } \\
\text { infarction }\end{array}\end{array}$ & $3(2.0)$ & $12(9.1)$ & 0.02 & $0.23(0.06-0.85)$ & 0.03 \\
$\quad \begin{array}{l}\text { Periprocedural myocardial } \\
\text { infarction }\end{array}$ & $2(1.3)$ & $8(6.1)$ & 0.06 & $0.23(0.05-1.17)$ & 0.08 \\
$\begin{array}{l}\text { Target vessel revascularization } \\
\quad \begin{array}{l}\text { Target lesion } \\
\text { revascularization }\end{array}\end{array}$ & $12(8.1)$ & $10(7.6)$ & 0.89 & $0.91(0.37-2.22)$ & 0.83 \\
TVF & $10(6.7)$ & $6(4.5)$ & 0.44 & $1.32(0.45-3.86)$ & 0.62 \\
$\begin{array}{l}\text { TLF } \\
\text { Definite or probable stent }\end{array}$ & $22(14.8)$ & $25(18.9)$ & 0.33 & $0.60(0.33-1.11)$ & 0.10 \\
thrombosis & $20(13.4)$ & $23(17.4)$ & 0.34 & $0.58(0.31-1.10)$ & 0.10 \\
\hline
\end{tabular}

Values are $\mathrm{n}$ (\%). 2 patients withdrew consent during follow-up. Event rates were calculated with Kaplan-Meier method. Abbreviations: TVF=target vessel failure; $T L F=$ target lesion failure. 

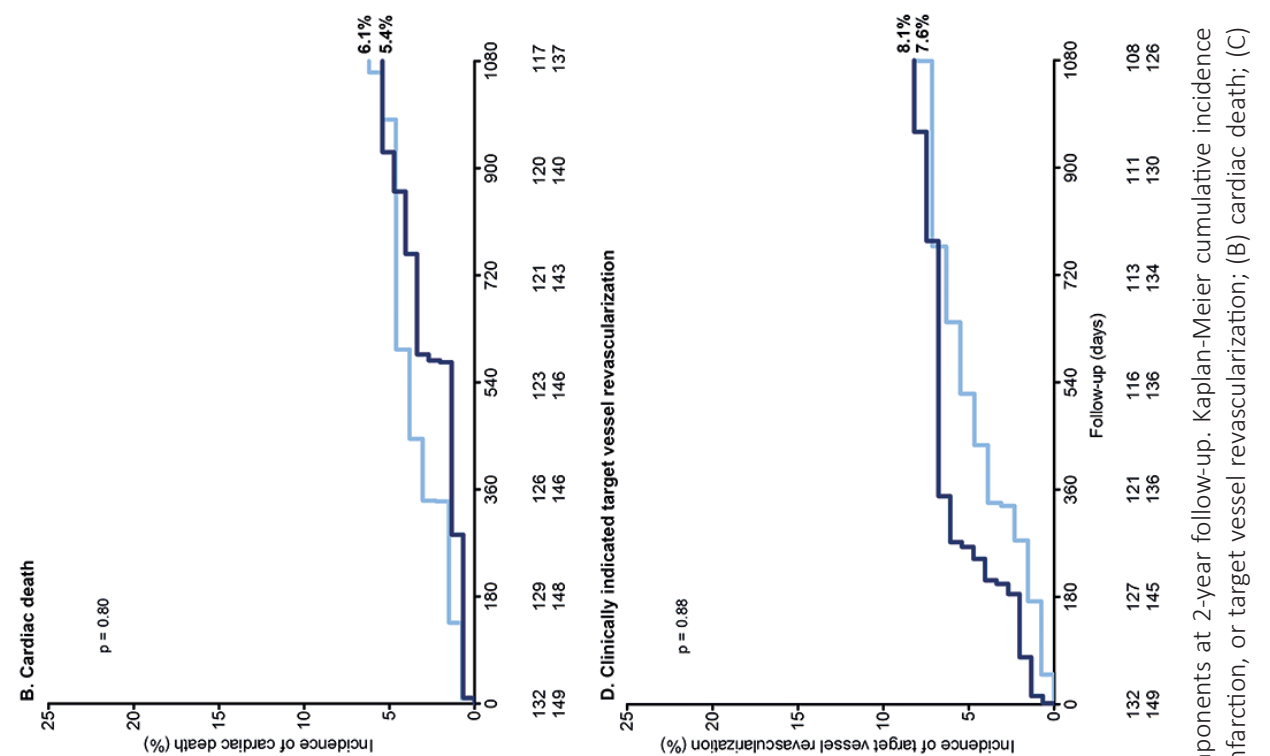

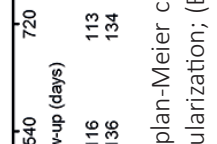

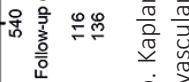

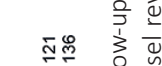

o ल

กย

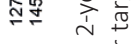

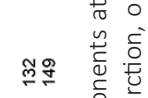

है
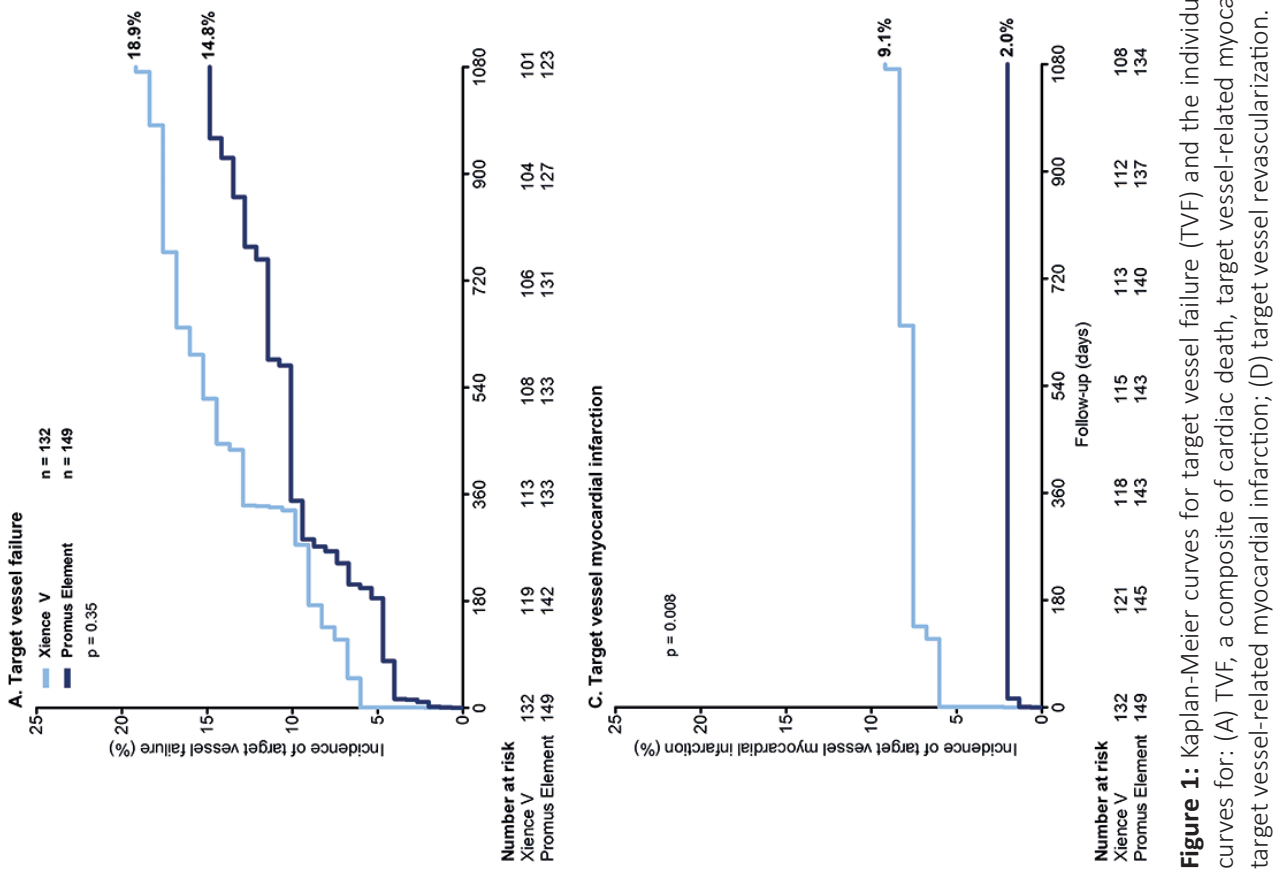


\section{Discussion}

In the present patient-level pooled, propensity score-adjusted analysis of participants in the TWENTE and DUTCH PEERS trials ${ }^{11,22}$, we compared clinical outcome after PCl with Promus Element versus Xience $V$ stents in patients with severely calcified lesions. To further minimize potentially confounding factors, we assessed only patients who were treated by the same operators at Thoraxcentrum Twente. At 3-year follow-up, event rates were relatively low for both EES. There was no significant difference between the Promus Element and Xience $V$ stent groups in TVF, cardiac death, or target vessel revascularization rates. Nevertheless, patients treated with Promus Element showed a significantly lower risk of target vessel MI (adjusted HR 0.23).

\section{Xience $\mathbf{V}$ cobalt-chromium fluoro-polymer EES}

The newer-generation cobalt-chromium Xience $V$ EES has been evaluated in various clinical trials that demonstrated significantly better clinical outcomes versus bare metal stents. ${ }^{29,30}$ Other clinical trials, such as the SPIRIT IV and COMPARE trials, demonstrated advantages of Xience $V$ over early-generation DES. ${ }^{20,31,32}$ In addition, in the RESOLUTE AC and TWENTE trials Xience $V$ showed clinical results similar to a newer-generation, durable polymer, zotarolimus-eluting stent. ${ }^{10,11}$

Despite all progress in the development of $\mathrm{DES}^{9}$, the treatment of severely calcified lesions remains a challenge ${ }^{8,14,15}$. Based on medium-term follow-up data of the SPIRIT II trial, Onuma et al. reported that $\mathrm{PCl}$ with Xience $\mathrm{V}$ cobalt-chromium EES was associated with a higher ischemia-driven target lesion revascularization rate in 68 patients with calcified target lesions versus 144 patients with non-calcified lesions (6.9\% vs $0.8 \%, p=0.03) .{ }^{17}$ The presence of severe target lesion calcification represented, among others, an angiographic exclusion criterion in SPIRIT $\|^{17}$, but not in TWENTE and DUTCH PEERS ${ }^{11,22}$. Considering evident differences in study population ${ }^{11,17,22}$, it is very difficult to compare the SPIRIT II data with the findings of our present study, which showed a numerically lower target lesion revascularization rate (4.5\%) after treating severely calcified lesions with Xience $V$ stents.

\section{Promus Element platinum-chromium fluoro-polymer EES}

Clinical studies with Promus Element EES showed favourable 1-year outcomes in the rather selected study population of the PLATINUM tria| ${ }^{18}$ and in all-comer populations. ${ }^{22,23}$ In addition, the long-term safety and efficacy of Promus Element has been reported. ${ }^{33,34} \mathrm{~A}$ recent meta-analysis confirmed the favourable outcomes of Promus Element. ${ }^{35}$ For the Promus Element stent, an increased risk of longitudinal stent deformation was found as a trade-off of the novel design. 22,24,36 However, as demonstrated in the DUTCH PEERS trial, angiographically overt longitudinal stent deformation is rare and generally unrelated to future clinical events. ${ }^{22}$ This lack of clinical sequelae may be related to the high visibility of platinumchromium alloy, which facilitates the detection and, if required, correction of longitudinally compressed DES (for instance by a careful postdilation or the implantation of an additional stent). On the hand, other thin-strut DES may also be susceptible to longitudinal stent compression ${ }^{36,37}$, which in less radiopaque stents is more difficult to recognize and may pose a higher clinical risk while being undetected. 


\section{Promus Element versus Xience $\mathbf{V}$}

Promus Element and Xience $V$ are both EES with fluoro-polymer coatings, which metal backbones with thin stent struts accomplish device flexibility. ${ }^{24}$ The platinum-chromium backbone of the Promus Element EES differs in design from the cobalt-chromium backbone of Xience $\mathrm{V}^{5,18,19,22,24-26}$ The Promus Element stent was developed to compensate for the somewhat lower radial force and angiographic visibility of thin struts by use of a platinum-chromium backbone and a modified design to improve conformability. ${ }^{5,24-26}$ An angiographic study confirmed the higher radial force of Promus Element, which showed significantly less recoil than Xience $V^{25}$ No significant differences were observed in the extent of acute stent recoil between the Xience Prime and Promus Premier stent in a registry of 100 all-comer patients. ${ }^{38}$ The higher radial force may be particularly us eful when treating heavily calcified lesions while the improved conformability of the stent may avoid extreme deformations to the vessel geometry.

A meta-analysis recently suggested that clinical outcome of platinum-chromium Promus Element EES is favourable and similar to various newer-generation DES, including the cobalt-chromium Xience EES. ${ }^{36}$ These findings do not exclude potential between-stent differences in specific sub-sets of target lesions or sub-groups of patients. ${ }^{36}$ Patients with severe target lesion calcification may represent a particularly interesting sub-group of patients that deserves further attention.

In the PLATINUM QCA study, which excluded patients with moderate or severe lesion calcification (besides other exclusion criteria), Promus Element showed an in-stent late lumen loss that was similar to Xience V. ${ }^{38}$ Incomplete apposition of Promus Element (5.7\%) was infrequently seen with intravascular ultrasound, which was a significantly lower rate than previously seen in Xience $\mathrm{V}$ (34.4\% in historical data of SPIRIT III). ${ }^{38}$ Differences in material and design of Promus Element and Xience $\mathrm{V}$ might have played a role, but the exceptionally high stent postdilation rate $(91.0 \%)$ and the high postdilation pressure $(18.0 \pm 3.6 \mathrm{~atm})$ in Promus Element stents of the PLATINUM QCA study may be an important explanation (maximum balloon pressure was $14.8 \pm 2.9 \mathrm{~atm}$ in Spirit III). ${ }^{39}$

In patients with severely calcified lesions, assessed in our present study, the postdilation rates of Promus Element and Xience $V$ stents (86.6\% versus 93.2\%) were similar and very high, as may be expected. Postdilation, which was performed based on clinical judgment of the operator, was done at higher pressures in Xience $V(23.9 \pm 4.6 \mathrm{~atm}$ versus $25.3 \pm 4.6 \mathrm{~atm})$. Furthermore, when the stents were deployed, Xience $V$ required a higher balloon pressure for stent implantation $(15.8 \pm 2.5$ atm versus $16.7 \pm 3.0 \mathrm{~atm}$ ). While reasons for the higher rate of target vessel MI (mostly periprocedural) in the Xience V-group can only be assumed, one may speculate that the higher balloon pressures might have played a role. In addition, dissimilarities in flexibility and conformability of the two devices might have contributed to the difference observed.

\section{Implications of the Study}

All-comer studies represented for more than one decade the pinnacle of clinical DES research. Premises are changing, as many contemporary stent studies showed (very) low event rates, rendering future assessment of novel stents difficult. ${ }^{20,22,23,28}$ While certain stents may be safe and efficacious in a broad patient population with various types of target lesions, it may reveal its higher adverse event risk only in certain high-risk subsets. Moreover, there is growing interest in customizing DES use (e.g. for calcified lesions, small vessels, bifurcations, and patients with high bleeding risk). As suggested by this study in 
patients with severely calcified target lesions, stents with higher radial force, improved conformability, and better angiographic visibility may help reduce the risk of target vessel MI. However, a prudent interpretation of this finding is required, as it did not show an impact on cardiac mortality.

\section{Limitations}

The results of this post-hoc study are hypothesis generating and should not be transferred to patients undergoing primary $\mathrm{PCl}$, as such patients were not analyzed. The study is limited by the fact that the two EES groups are from clinical trials that were consecutively performed. Nevertheless, all patients were treated within a reasonably short period of time by the same operators of a tertiary $\mathrm{PCl}$ center, who applied uniform clinical protocols and prescribed the same dual anti-platelet therapy. In addition, both trials used systematic assessment of post-PCI cardiac markers and ECG changes, the same clinical endpoint definitions, and independent external adjudication of clinical events. ${ }^{11,22}$

\section{Conclusions}

In patients with severely calcified target lesions, the 3-year risk of various cardiovascular events did not differ significantly between the Promus Element and Xience $V$ stent groups. Only the target vessel MI rate was lower in the Promus Element group. 


\section{References}

1. Madhavan MV, Tarigopula M, Mintz GS, Maehara A, Stone GW, Généreux P. Coronary artery calcification: pathogenesis and prognostic implications. J Am Coll Cardiol 2014;63:1703-14.

2. Gudmundsdottir I, Adamson P, Gray C, et al. Optical coherence tomography versus intravascular ultrasound to evaluate stent implantation in patients with calcific coronary artery disease. Open Heart 2015;2:e000225.

3. Reimers B, von Birgelen C, van der Giessen WJ, Serruys PW. A word of caution on optimizing stent deployment in calcified lesions: acute coronary rupture with cardiac tamponade. Am Heart J 1996;131:192-4.

4. von Birgelen C, Mintz GS, Böse D, et al. Impact of moderate lesion calcium on mechanisms of coronary stenting as assessed with three-dimensional intravascular ultrasound in vivo. Am J Cardiol 2003;92:5-10.

5. Menown IBA, Noad R, Garcia EJ, Meredith I. The platinum chromium element stent platform: from alloy, to design, to clinical practice. Adv Ther 2010;27:129-41.

6. Maehara A, Fitzgerald PJ. Coronary calcification: assessment by intravascular ultrasound imaging. Clin Res Cardiol 2000;89:supp2:S112-6.

7. Bangalore S, Vlachos HA, Selzer F, Wilensky RL, Kip KE, Williams DO, Faxon DP. Percutaneous coronary intervention of moderate to severe calcified coronary lesions: insights from the National Heart, Lung, and Blood Institute Dynamic Registry. Catheter Cardiovasc Interv 2011;77:22-8.

8. Généreux P, Madhavan MV, Mintz GS, et al. Ischemic outcomes after coronary intervention of calcified vessels in acute coronary syndromes. Pooled analysis from the HORIZONS-AMI (Harmonizing Outcomes With Revascularization and Stents in Acute Myocardial Infarction) and ACUITY (Acute Catheterization and Urgent Intervention Triage Strategy) trials. J Am Coll Cardiol 2014;63:1845-54.

9. Stefanini GG, Taniwaki M, Windecker S. Coronary stents: novel developments. Heart 2014;100:1051-61.

10. Serruys PW, Silber S, Garg S, et al. Comparison of zotarolimus-eluting and everolimus-eluting coronary stents. N Engl J Med 2010;363:136-46.

11. von Birgelen C, Basalus MW, Tandjung K, et al. A randomized controlled trial in second-generation zotarolimuseluting Resolute stents versus everolimus-eluting Xience $V$ stents in real-world patients: the TWENTE trial. J Am Coll Cardiol 2012;59:1350-61.

12. Fajadet J, Neumann FJ, Hildick-Smith D, et al. Twelve-month results of a prospective, multicentre trial to assess the everolimus-eluting coronary stent system (PROMUS Element): the PLATINUM PLUS all-comers randomised trial. Eurointervention 2017;12:1595-604.

13. Teirstein PS, Meredith IT, Feldman RL, et al. Two-year safety and effectiveness of the platinum chromium everolimus-eluting stent for the treatment of small vessels and longer lesions. Catheter Cardiovasc Interv 2015;85:207-15.

14. Huisman J, van der Heijden LC, Kok MM, et al. Impact of severe lesion calcification on clinical outcome of patients with stable angina, treated with newer generation permanent polymer-coated drug-eluting stents: a patient-level pooled analysis from TWENTE and DUTCH PEERS (TWENTE II). Am Heart J 2016;175:121-9.

15. Huisman J, van der Heijden LC, Kok MM, et al. Two-year outcome after treatment of severely calcified lesions with newer-generation drug-eluting stents in acute coronary syndromes: a patient-level pooled analysis from TWENTE and DUTCH PEERS. J Cardiol 2017;69:660-5.

16. Stone GW, Rizvi A, Newman W, et al. Everolimus-eluting versus paclitaxel-eluting stents in coronary artery disease. N Engl J Med 2010;362:1663-74.

17. Onuma $Y$, Tanimoto S, Ruygrok $P$, et al. Efficacy of everolimus eluting stent implantation in patients with calcified coronary culprit lesions: two-year angiographic and three-year clinical results from the SPIRIT II study. Catheter Cardiovasc Interv 2010;76:634-42.

18. Stone GW, Teirstein PS, Meredith IT, et al. A prospective, randomized evaluation of a novel everolimus-eluting coronary stent: the PLATINUM trial. J Am Coll Cardiol 2011;57:1700-8.

19. Meredith IT, Teirstein PS, Bouchard A, Carrié D, Möllmann H, Oldroyd KG, Hall J, Allocco DJ, Dawkins KD, Stone GW. Three-year results comparing platinum-chromium PROMUS Element and cobalt-chromium XIENCE V everolimus-eluting stents in de novo coronary artery narrowing (from the PLATINUM Trial). Am J Cardiol 2014;113:1117-23.

20. Kedhi E, Joesoef KS, McFadden E, Wassing J, van Mieghem C, Goedhart D, Smits PC. Second-generation everolimus-eluting and paclitaxel-eluting stents in real-life practice (COMPARE): a randomised trial. Lancet 2010;375:201-9. 
21. Jensen LO, Thayssen P, Maeng M, et al. Three-year outcomes after revascularization with everolimus- and sirolimus-eluting stents from the SORT OUT IV trial. J Am Coll Cardiol Intv 2014;7:840-8.

22. von Birgelen C, Sen H, Lam MK, et al. Third-generation zotarolimus-eluting and everolimus-eluting stents in all-comer patients requiring a percutaneous coronary intervention (DUTCH PEERS): a randomised, single-blind, multicentre, non-inferiority trial. Lancet 2014;383:413-23

23. Park KW, Kang SH, Kang HJ, et al. A randomized comparison of platinum chromium-based everolimus-eluting stents versus cobalt chromium-based zotarolimus-eluting stents in all-comers receiving percutaneous coronary intervention: HOST-ASSURE, a randomized, controlled, noninferiority trial. J Am Coll Cardiol 2014;63:2805-16.

24. O'Brien BJ, Stinson JS, Larsen SR, Eppihimer MJ, Carroll WM. A platinum-chromium steel for cardiovascular stents. Biomaterials 2010;31:3755-61.

25. Ota T, Ishii H, Sumi T, et al. Impact of coronary stent designs on acute stent recoil. J Cardiol 2014;64:347-52.

26. Bennett J, Dubois C. A novel platinum chromium everolimus-eluting stent for the treatment of coronary artery disease. Biologics 2013;7:149-59.

27. Cutlip DE, Windecker S, Mehran R, et al. Clinical end points in coronary stent trials: a case for standardized definitions. Circulation 2007;115:2344-51.

28. Vranckx P, Cutlip DE, Mehran R, Kint PP, Silber SS, Windecker S, Serruys PW. Myocardial infarction adjudication in contemporary all-comer stent trials: balancing sensitivity and specificity. Addendum to the historical MI definitions used in stent studies. Eurolntervention 2010;5:871-4.

29. Sabaté M, Burgaletta $S$, Cequier A, et al. The EXAMINATION trial (everolimus-eluting stents versus bare-metal stents in ST-segment elevation myocardial infarction): 2-year results from a multicenter randomized controlled trial. J Am Coll Cardiol 2014;7:64-71.

30. Pedersen SH, Pfisterer M, Kaiser C, et al. Drug-eluting stents and bare metal stents in patients with NSTE-ACS: 2-year outcome from the randomised BASKET-PROVE trial. Eurolntervention 2014;10:58-64.

31. Stone GW, Rizvi A, Sudhir K, et al. Randomized comparison of everolimus- and paclitaxel-eluting stents: 2-year follow-up from the SPIRIT (clinical evaluation of the XIENCE V everolimus eluting coronary stent system) IV trial. J Am Coll Cardiol 2011;58:19-25

32. Toyota T, Shiomi H, Morimoto T, Kimura T. Meta-analysis of long-term clinical outcomes of everolimus-eluting stents. Am J Cardiol 2015;116:187-94.

33. Meredith IT, Teirstein PS, Bouchard A, et al. Three-year results comparing platinum-chromium PROMUS element and coablt-chromium Xience $V$ everolimus-eluting stents in de novo coronary artery narrowing (from the PLATINUM trial). Am J Cardiol 2014;113:1117-23.

34. van der Heijden LC, Kok MM, Löwik MM, et al. Three-year safety and efficacy of treating all-comers with newergeneration Resolute Integrity or Promus Element stents in the randomised DUTCH PEERS (TWENTE II) trial. Eurolntervention 2017;12:2128-31.

35. Cassese S, Ndrepepa G, Byrne RA, et al. Outcomes of patients treated with durable-polymer platinumchromium everolimus-eluting stents: a meta-analysis of randomized trials. Eurolntervention 2017; doi 10.4244/ EIJ-D-16-00871 [Epub ahead of print].

36. Abdel-Wahab M, Sulimov DS, Kassner G, Geist V, Toelg R, Richardt G. Longitudinal deformation of contemporary coronary stents: an integrated analysis of clinical experience ans observations from the bench. J Interv Cardiol 2012;25:576-85.

37. Dvir D, Kitabata $H$, Barbash IM, et al. In vivo evaluation of axial integrity of coronary stents using intravascular ultrasound: insights on longitudinal stent deformation. Catheter Cardiovasc Interv. 2014;84:397-405.

38. Van Bommel RJ, Lemmert ME, van Mieghem NM, van Geuns RJ, van Domburg RT, Daemen J. Occurrence and predictors of acute stent recoil-A comparison between the Xience Prime cobalt chromium stent and the Promus Premier platinum-chromium stent. Catheter Cardiovasc Interv 2017. Doi 10.1002 [Epub ahead of print].

39. Meredith I, Whitbourn R, Scott D, et al. Platinum QCA: a prospective, multicentre study assessing clinical, angiographic, and intravascular ultrasound outcomes with the novel platinum chromium thin-strut Promus Element everolimus-eluting stent in de novo coronary stenoses. Eurolntervention 2011;7:84-90. 



\section{Part IV}

General discussion and future perspectives 


\section{CHAPTER 17}

General Discussion and Future Perspectives

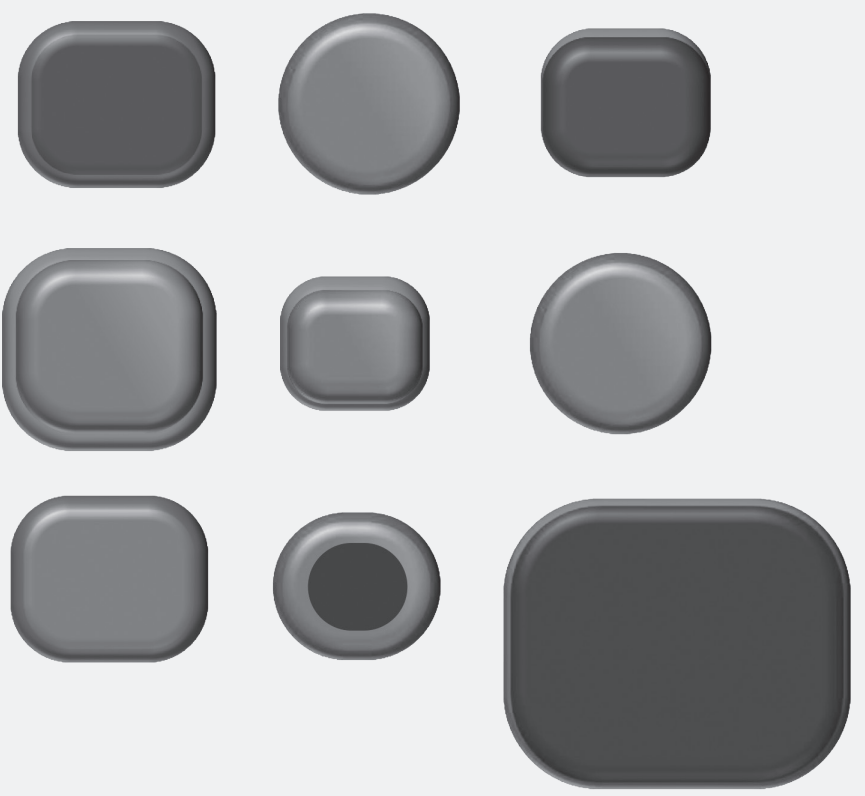




\section{General Discussion and Future Perspectives}

Drug-eluting stents (DES) are still subject to continued refinements in order to optimize clinical outcome. Second-generation DES were improved as compared to first-generation DES in terms of biocompatibility, which reduced the potential of the stents to induce an inflammatory response to the vessel wall (Chapter 1). Five-year clinical outcome of the TWENTE trial showed that the secondgeneration Resolute and Xience $V$ DES have favorable and similar long-term safety and efficacy in a broad patient population (Chapter 2). In newer-generation DES, metallic stent platforms became more flexible to increase deliverability and stent apposition to the vessel wall in order to tackle the most challenging lesions and vessel anatomies. The DUTCH PEERS randomized trial showed similar safety and efficacy of the newer-generation Resolute Integrity and Promus Element stents in treating all-comer patients up to 3-year follow-up (Chapter 3). In addition, the Resolute Integrity and Promus Element stents were similarly safe and efficacious in treating high-risk patients, who had significantly higher adverse event rates than patients with a lower risk (Chapter 4).

With the improvements of DES and their overall relatively low rates of adverse events, it becomes increasingly difficult to demonstrate a benefit of DES refinements. ${ }^{1-4}$ Therefore, in all-comer trials, it will be important to also enroll patients with increased adverse event risk (e.g. patients with a history of coronary artery bypass grafting (CABG)) and patients with particularly challenging target lesions (Chapters 2, 4, and $\mathbf{9}$ ). However, minor selection cannot be excluded in patients with end-stage coronary heart disease or excessive co-morbidities. In addition, while certain stents may be safe and efficacious in a broad patient population with various types of target lesions, they may reveal a higher adverse event risk only in certain high-risk patient subsets. Moreover, there is growing interest in customizing the use of DES, for instance for bifurcations, small vessels, calcified lesions, and patients with high bleeding risk. In bifurcated target lesions, highly flexible stent platforms have reduced the rates of repeat revascularization and target vessel myocardial infarction after percutaneous coronary inter-vention. ${ }^{5-12}$ True bifurcation lesions, which are characterized by an advanced atherosclerotic disease burden that obstructs the main vessel and the side-branch, are often technically more demanding and require more frequently complex techniques with implantation of two stents than bifurcation lesions with atherosclerotic involvement of the main vessel or side branch only. ${ }^{13-16}$ Virtual bench tests assessed the impact of technical characteristics of DES platforms on stenting in bifurcated lesions and showed that dissimilar technical features of DES platforms lead to differences in response to similar procedural steps in provisional stenting, such as the final kissing-balloon inflation. ${ }^{17}$ Therefore, technical characteristics of stents should be taken into account in the selection process of the most appropriate DES for treating bifurcated lesions. ${ }^{17}$ Up to 3-year follow-up, patients treated with second-generation DES for true bifurcation lesions had somewhat higher adverse event rates than patients with less complex bifurcation lesions (Chapter 11). As shown in Chapter 10, patients treated for bifurcated versus nonbifurcated lesions showed similar and low rates of clinical endpoints at 2-year follow-up, suggesting that the newer-generation DES used are efficacious and safe for treating bifurcated target lesions. In this context, the thin struts and flexible stent designs that accommodate well to vessel tapering may have been beneficial. 
Stenting small coronary vessels has also been associated with an increased risk of adverse events..$^{18-22}$ Strut thickness was previously identified as an independent predictor of restenosis in DES. ${ }^{21-24}$ Restenosis rates are related to neointimal ingrowth, which results in a reduction of lumen size and is addressed as angiographic late lumen loss in studies with quantitative coronary angiographic assessment. ${ }^{20,21,25}$ As the thickness of neointimal ingrowth is similar in stents with struts of various sizes, in small vessels a relatively greater loss of lumen cross-sectional area will occur that results in a higher restenosis risk. ${ }^{19,26}$ In small vessels, thinner stent struts are thus particularly valuable. As shown in Chapter 13, all-comer patients treated with newer-generation DES in small vessel lesions had higher adverse event rates than patients treated with DES in larger sized vessels. Our data suggest that, with current stents, a diameter of $2.5 \mathrm{~mm}$ is a suitable threshold value to identify small target vessels. The minimum stent strut thickness required to ensure sufficient radial force to prevent elastic recoil of the dilated vessel depends on both stent material and design. Platinum-chromium and cobalt chromium alloys, as used in the newer-generation Promus Element and Resolute Integrity stents, respectively, permit the construction of stents with thin struts and flexible designs. ${ }^{27}$ Such designs reduce stent thrombogenicity by reducing the incidence of strut malapposition and lowering coronary flow disturbance. ${ }^{28}$ However, thin stent struts might have the disadvantage of providing insufficient radial force. In addition, the lower radiographical visibility of thin stent struts might theoretically increase the risk of geometrical miss that could result in higher event rates.

Local drug delivery into the coronary vessel wall might be impaired in highly calcified target lesions. Furthermore, manipulation with DES in severely calcified vessels can result in damage to the polymer coating, which might locally reduce the effectiveness of DES in suppressing the proliferation of neointima. ${ }^{29,30}$ In addition, the treatment of calcified lesions may sometimes result in a suboptimal stent deployment, which may lead to in-stent restenosis or to incomplete or delayed strut endothelization with an increased risk of stent thrombosis. ${ }^{31}$ Uneven distribution of stent struts, which is more likely to occur in calcified lesions, may lead to adverse clinical events due to a local imbalance in scaffolding properties and drug delivery. Stents with higher radial force, improved conformability, and better angiographic visibility may help to reduce the risk of adverse events. In a patient-level pooled analysis of TWENTE and DUTCH PEERS, we found that severe target lesion calcification was associated with an increased risk of adverse clinical events in patients presented with stable angina and acute coronary syndromes, both treated with second- or newer-generation DES (Chapter 14, Chapter 15). In addition, the presence of severe lesion calcification among all-comers treated with newer-generation DES in bifurcated lesions was not associated with a higher adverse event risk (Chapter 12). As shown in Chapter 16, patients with severely calcified target lesions showed lower 3-year rates of target vessel myocardial infarction when being treated with newer-generation Promus Element versus second-generation Xience $V$ stents. However, the composite clinical endpoint target vessel failure and its individual components other than target vessel myocardial infarction showed no significant between-stent difference (Chapter 16). While these findings were obtained from a propensity-score adjusted analysis rather than a randomized controlled trial, our data suggest that assessing the potential benefits of stent refinements in selected high risk patient populations is of interest.

Alternative DES technologies (Chapter 5) and stents with biodegradable coatings were developed in parallel with the refinement of durable polymer-coated DES. The lifelong presence of a durable polymer 
in a coronary artery might induce vessel wall inflammation, delay arterial healing, and occasionally cause serious complications such as stent thrombosis and myocardial infarction. ${ }^{32}$ Growing awareness of this risk motivated the development of stents with biodegradable coatings that leave only a bare metal stent after polymer resorption. ${ }^{27,33-41}$ While early biodegradable polymer stents had thick stainless steel struts and did not show non-inferiority as compared to early-generation durable polymer stents ${ }^{36}$, currently novel biodegradable polymer-coated stents are available that have very thin struts. The 1-year outcome of data from the BIO-RESORT trial shows non-inferiority of two biodegradable polymer-coated DES as compared to the newer-generation Resolute Integrity stent in treating a complex all-comer patient population (Chapter 6). While the potential benefits (or disadvantages) of biodegradable polymer DES might only be noted later than after 1 year ${ }^{36,37,42}$, the absence of a loss of 1-year safety and efficacy with these devices is a prerequisite before assessing their potential longer-term effects. In the ongoing BIONYX trial, which was introduced and discussed in Chapter 8, the very thin strut Orsiro biodegradable polymer-coated stent, which is one of the two biodegradable polymer-coated stents assessed in the BIO-RESORT trial, will be compared with the novel durable polymer-coated Resolute Onyx DES that uses a new thin-strut metallic stent backbone with increased radiographic visibility. It is of interest to assess the safety and efficacy of next-generation metallic DES in sub-populations of patients who may benefit the most from the refinements made. The following device innovations and potential applications might be considered. Thin stent struts may be of benefit for the treatment of small vessel lesions, while thick stent struts might be useful in ostial locations. In addition, an open cell design may be particularly valuable in major bifurcations; and stents with a high radial force are likely to facilitate the treatment of severely calcified lesions. Finally, in patients with diabetes mellitus, DES with specific drug doses and a prolonged delivery of the drug may be tested.

Bioresorbable vascular scaffolds that aim at providing a finite period of vascular support after device implantation represent the most recent technical development. ${ }^{43,44}$ The potential benefits of bioresorbable vascular scaffolds include the preservation of vessel geometry, adaptive vascular remodeling, restoration of physiologic vasomotion, and finally the prospect of late luminal expansion. ${ }^{43,45,46}$ Our meta-analysis (Chapter 7) showed that during the first year of follow-up, patients treated with polymer-based Absorb bioresorbable vascular scaffolds had a significantly higher incidence of myocardial infarction and scaffold thrombosis than patients treated with durable polymer DES. The mechanisms of scaffold thrombosis are still a matter of further investigation. There are various factors that may have contributed to the higher incidence of device thrombosis. The thicker struts of the bioresorbable vascular scaffold may disturb coronary blood flow and trigger platelet aggregation. ${ }^{47}$ Moreover, the lower final in-device minimum lumen diameter achieved with bioresorbable vascular scaffolds (as compared to DES) may have contributed to the increased thrombotic risk. Incomplete lesion coverage, bioresorbable scaffold under-deployment and malapposition, as well as alterations in laminar blood flow and thrombogenicity, induced by very thick (especially malapposed) struts are important mechanisms of (sub-)acute scaffold thrombosis. ${ }^{48-51}$ Late and very late bioresorbable vascular scaffold thrombosis have been related to non-embedded and non-absorbed struts in complex lesions and to late structural discontinuity or device dismantling of malapposed struts, in particular after the cessation of dual anti-platelet therapy. ${ }^{51,52}$ Well-controlled post-dilatation and overexpansion of bioresorbable vascular scaffolds is likely to prevent a substantial proportion of potential coronary 
thromboses by improving scaffold apposition to the vessel wall. ${ }^{51,53}$ While bioresorbable scaffold thrombosis has been related to sub-optimal implantation techniques, real-world data from the lesion analysis in the randomized AIDA trial do not support this. ${ }^{51,52}$

Contemporary DES have shown excellent clinical outcomes, including very low stent thrombosis rates, in all-comers and high-risk patient populations. Therefore, it is reasonable to suggest that the use of current-generation bioresorbable vascular scaffolds should preferably be limited to clinical trials. Ongoing and future controlled clinical studies that carefully assess the safety and efficacy of nextgeneration devices will determine whether there is a future role for polymer-based bioresorbable vascular scaffolds in clinical practice. 


\section{References}

1. von Birgelen C, Basalus MW, Tandjung K, et al. A randomized controlled trial in second-generation zotarolimuseluting Resolute stents versus everolimus-eluting Xience $V$ stents in real-world patients: the TWENTE trial. J Am Coll Cardiol 2012;59:1350-61.

2. Jensen LO, Thayssen P, Hansen HS, et al. Randomized comparison of everolimus-eluting and sirolimus-eluting stents in patients treated with percutaneous coronary intervention: the Scandinavian Organization for Randomized Trials with Clinical Outcome IV (SORT OUT IV). Circulation 2012;125:1246-55.

3. Serruys PW, Silber S, Garg S, et al. Comparison of zotarolimus-eluting and everolimus-eluting coronary stents. N Engl J Med 2010;363:136-46.

4. von Birgelen $\mathrm{C}$, Sen H, Lam MK, et al. Third-generation zotarolimus-eluting and everolimus-eluting stents in all-comer patients requiring a percutaneous coronary intervention (DUTCH PEERS): a randomized, single-blind, multicenter, non-inferiority trial. Lancet 2014;383:413-23.

5. Colombo A, Moses JW, Morice MC, et al. Randomized study to evaluate sirolimus-eluting stents implanted at coronary bifurcation lesions. Circulation 2004;109:1244-9.

6. Thuesen L, Kelbaek H, Klovgaard L, et al. Comparison of sirolimus-eluting and bare metal stents in coronary bifurcation lesions: subgroup analysis of the Stenting Coronary Arteries in Non-Stress/Benestent Disease Trial (SCANDSTENT). Am Heart J 2006;152:1140-5.

7. Colombo F, Biondi-Zoccai G, Infantino V, et al. A long-term comparison of drug-eluting versus bare metal stents for the percutaneous treatment of coronary bifurcation lesions. Acta Cardiol 2009;64:583-8.

8. Romagnoli E, De Servi S, Tamburino C, et al. Real-world outcome of coronary bifurcation lesions in the drugeluting stent era: results from the 4,314-patient Italian Society of Invasive Cardiology (SICl-GISE) Italian Multicenter Registry on Bifurcations (I-BIGIS). Am Heart J 2010;160:535-42. Pan M, Burzotta F, Trani C, et al. Three-year follow-up of patients with bifurcation lesions treated with sirolimus- or everolimus-eluting stents: SEAside and CORpal cooperative study. Rev Esp Cardiol 2014;67:797-803.

9. Lam MK, Sen H, van Houwelingen KG, et al. Three-year clinical outcome of patients with bifurcation treatment with second-generation Resolute and Xience V stents in the randomized TWENTE trial. Am Heart J 2015;169:6977 .

10. Diletti R, Garcia-Garcia HM, Bourantas CV, et al. Clinical outcomes after zotarolimus and everolimus drug eluting stent implantation in coronary artery bifurcation lesions: insights from the RESOLUTE All Comers Trial. Heart 2013;99:1267-74.

11. Ormiston JA, Webber B, Webster MW. Stent longitudinal integrity bench insights into a clinical problem. J Am Coll Cardiol Intv 2011;4:1310-7.

12. Roversi S, Biondi-Zoccai G, Romagnoli E, et al. Early and long-term outlook of percutaneous coronary intervention for bifurcation lesions in young patients. Int J Cardiol 2013;167:2995-9.

13. $\operatorname{Jim} M H$, Wu EB, Fung RC, et al. Angiographic result of T-stenting with small protrusion using drug-eluting stents in the management of ischemic side branch: the ARTEMIS study. Heart Vessels 2015;30:427-431.

14. Al Suwaidi J, Berger PB, Rihal CS, et al. Immediate and longterm outcome of intracoronary stent implantation for true bifurcation lesions. J Am Coll Cardiol 2000;35:929-936.

15. Niemelä M, Kervinen K, Erglis A, et al. Randomized comparison of final kissing balloon dilatation versus no final kissing balloon dilatation in patients with coronary bifurcation lesions treated with main vessel stenting: the Nordic-Baltic Bifurcation Study III. Circulation 2011;123:79-86.

16. Louvard Y, Lefevre T, Morice MC. Bifurcation lesions. In: Eekhout E, Serruys PW, Wijns W, Vahanian A, van Sambeek M, and de Palma R, editors. Percutaneous interventional cardiovascular medicine: the PCR-EAPCI textbook. 2012; Toulouse, France: Europa Edition, pp 283-320.

17. Burzotta F, Mortier P, Trani C. Characteristics of drug-eluting stent platforms potentially influencing bifurcated lesion provisional stenting procedure. Eurolntervention 2014;10:124-32.

18. Schunkert H, Harrell L, Palacios IF. Implications of small reference vessel diameter in patients undergoing percutaneous coronary revascularization. J Am Coll Cardiol 1999;34:40-8.

19. Elezi S, Kastratie A, Neumann FJ, Hadamitzky M, Dirschinger J, Schömig A. Vessel size and long-term outcome after coronary stent placement. Circulation 1998;98:1875-80.

20. Biondi-Zoccai G, Moretti C, Abbate A, Sheiban I. Percutaneous coronary intervention for small vessel coronary artery disease. Cardiovasc Revasc Med 2010;11:189-98. 
21. Akiyama T, Moussa I, Reimers B, et al. Angiographic and clinical outcome following coronary stenting of small vessels: a comparison with coronary stenting of large vessels. J Am Coll Cardiol 1998;32:1610-8.

22. Kasaoka S, Tobis JM, Akiyama T, et al. Angiographic and intravascular ultrasound predictors of in-stent restenosis. J Am Coll Cardiol 1998;32:1630-5.

23. Kastrati A, Mehilli J, Dirschinger J, et al. Intracoronary stenting and angiographic results: strut thickness effect on restenosis outcome (ISAR-STEREO) trial. Circulation 2001;103:2816-21.

24. Briguori C, Sarais C, Pagnotta P, et al. In-stent restenosis in small coronary arteries: impact of strut thickness. J Am Coll Cardiol 2002;40:403-9.

25. Togni M, Eber S, Widmer J, et al. Impact of vessel size on outcome after implantation of sirolimus-eluting and paclitaxel-eluting stents. J Am Coll Cardiol 2007;50:1123-31.

26. Mauri L, Orav EJ, Kuntz RE. Late loss in lumen diameter and binary restenosis for drug-eluting stent comparison. Circulation 2005;111:3435-42.

27. Stefanini GG, Taniwaki M, Windecker S. Coronary stents: novel developments. Heart 2014; 100: 1051-61.

28. Kolandaivelu K, Swaminathan R, Gibson WJ, et al. Stent thrombogenicity early in high-risk interventional settings is driven by stent design and deployment and protected by polymer-drug coatings. Circulation 2011; 123: 140009.

29. Wiemer M, Butz T, Schmidt W, et al. Scanning electron microscopic analysis of different drug eluting stents after failed implantation: from nearly undamaged to major damaged polymers. Catheter Cardiovasc Interv 2010;75:905-11.

30. Basalus MW, Joner M, von Birgelen C, et al. Polymer coatings on drug-eluting stents: Samson's hair and Achilles' heel? Eurolntervention 2013;9:302-5.

31. Fujii K, Carlier SG, Mintz GS, et al. Stent underexpansion and residual reference segment stenosis are related to stent thrombosis after sirolimus-eluting stent implantation: an intravascular ultrasound study. J Am Coll Cardiol 2005;45:995-8.

32. Joner M, Finn AV, Farb A, et al. Pathology of drug-eluting stents in humans: delayed healing and late thrombotic risk. J Am Coll Cardiol 2006; 48: 193-202.

33. Räber L, Kelbæk H, Ostojic M, et al. Effect of biolimus-eluting stents with biodegradable polymer vs bare-metal stents on cardiovascular events among patients with acute myocardial infarction: the COMFORTABLE AMI randomized trial. JAMA 2012; 308: 777-87.

34. Windecker S, Serruys PW, Wandel S, et al. Biolimus-eluting stent with biodegradable polymer versus sirolimuseluting stent with durable polymer for coronary revascularisation (LEADERS): a randomised non-inferiority trial. Lancet 2008; 372: 1163-73.

35. Serruys PW, Farooq V, Kalesan B, et al. Improved safety and reduction in stent thrombosis associated with biodegradable polymer-based biolimus-eluting stents versus durable polymer-based sirolimus-eluting stents in patients with coronary artery disease: final 5-year report of the LEADERS (limus eluted from a durable versus erodable stent coating) randomized, noninferiority trial. JACC Cardiovasc Interv 2013; 6: 777-89.

36. Christiansen EH, Jensen LO, Thayssen P, et al. Biolimus-eluting biodegradable polymer-coated stent versus durable polymer-coated sirolimus-eluting stent in unselected patients receiving percutaneous coronary intervention (SORT OUT V): a randomised non-inferiority trial. Lancet 2013; 381: 661-69.

37. Byrne RA, Kastrati A, Massberg S, et al; ISAR-TEST 4 Investigators. Biodegradable polymer versus permanent polymer drug-eluting stents and everolimus- versus sirolimus-eluting stents in patients with coronary artery disease: 3-year outcomes from a randomized clinical trial. J Am Coll Cardiol 2011; 58: 1325-31.

38. Smits PC, Hofma S, Togni M, et al. Abluminal biodegradable polymer biolimus-eluting stent versus durable polymer everolimus-eluting stent (COMPARE II): a randomised, controlled, non-inferiority trial. Lancet 2013; 381: 651-60.

39. Raungaard B, Jensen LO, Tilsted $\mathrm{HH}$, et al. Zotarolimus-eluting durable-polymer-coated stent versus a biolimuseluting biodegradable-polymer-coated stent in unselected patients undergoing percutaneous coronary intervention (SORT OUT VI): a randomised non-inferiority trial. Lancet 2015; 385: 1527-35.

40. Palmerini T, Biondi-Zoccai G, Della Riva D, et al. Clinical outcomes with bioabsorbable polymer- versus durable polymer-based drug-eluting and bare-metal stents: evidence from a comprehensive network meta-analysis. J Am Coll Cardiol 2014; 63: 299-307.

41. Kaiser C, Galatius S, Jeger R, et al. Long-term efficacy and safety of biodegradable-polymer biolimus-eluting stents: main results of the Basel Stent Kosten-Effektivitäts Trial-PROspective Validation Examination II (BASKETPROVE II), a randomized, controlled noninferiority 2-year outcome trial. Circulation 2015; 131: 74-81. 
42. Stefanini GG, Byrne RA, Serruys PW, et al. Biodegradable polymer drug-eluting stents reduce the risk of stent thrombosis at 4 years in patients undergoing percutaneous coronary intervention: a pooled analysis of individual patient data from the ISAR-TEST 3, ISAR-TEST 4, and LEADERS randomized trials. Eur Heart J 2012; 33: 1214-22.

43. Waksman R. Biodegradable stents: they do their job and disappear. J Invasive Cardiol 2006;18:70-4.

44. Iqbal J, Onuma Y, Ormiston J, Abizaid A, Waksman R, Serruys P. Bioresorbable scaffolds: rationale, current status, challenges, and future. Eur Heart J 2014;35:765-76.

45. Gomez-Lara J, Garcia-Garcia HM, Onuma Y, et al. A comparison of the conformability of everolimus-eluting bioresorbable vascular scaffolds to metal platform coronary stents. J Am Coll Cardiol Intv 2010;3:1190-8.

46. Serruys PW, Garcia-Garcia HM, Onuma Y. From metallic cages to transient bioresorbable scaffolds: change in paradigm of coronary revascularization in the upcoming decade? Eur Heart J 2012;33:16-25b.

47. Bourantas CV, Papafaklis MI, Garcia-Garcia HM, et al. Short- and long-term implications of a bioresorbable vascular scaffold implantation on the local endothelial shear stress patterns. J Am Coll Cardiol Intv 2014;7:100-1.

48. Raber L, Brugaletta S, Yamaji K, et al. Very Late Scaffold Thrombosis: Intracoronary Imaging and Histopathological and Spectroscopic Findings. J Am Coll Cardiol 2015;66:1901-14.

49. Karanasos A, Van Mieghem N, van Ditzhuijzen N, et al. Angiographic and optical coherence tomography insights into bioresorbable scaffold thrombosis: single-center experience. Circ Cardiovasc Interv 2015;8:doi 10.1161/ CIRCINTERVENTIONS.114.002369.

50. Puricel S, Cuculi F, Weissner M, et al. Bioresorbable Coronary Scaffold Thrombosis: Multicenter Comprehensive Analysis of Clinical Presentation, Mechanisms, and Predictors. J Am Coll Cardiol 2016;67:921-31.

51. Sotomi Y, Suwannasom P, Serruys PW, Onuma Y. Possible mechanical causes of scaffold thrombosis: insights from case reports with intracoronary imaging. Eurolntervention 2017;12:1747-56.

52. Wykrzykowska JJ, Kraak RP, Hofma SH, et al. Bioresorbable scaffolds versus metallic stents in routine PCl, N Engl J Med 2017; In press, online published on March 29, 2017 (DOI: 10.1056/NEJMoa1614954).

53. von Birgelen C, Basalus MWZ. Oversized post-dilatation of current bioresorbable vascular scaffolds: kill or cure? Eurolntervention 2016;11:1330-3. 



\title{
CHAPTER 18
}

\author{
Summary and Conclusions
}

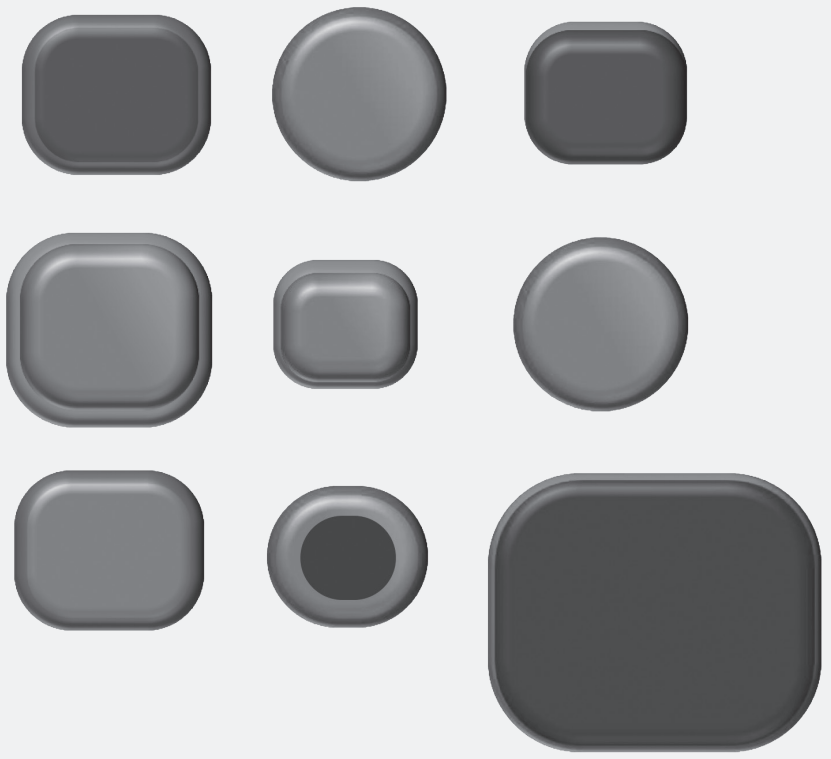




\section{Summary}

Drug-eluting stents (DES) have become indispensable in the treatment of coronary artery disease. These coronary implants are constantly being improved to optimize safety and efficacy of treatment. Secondgeneration DES were improved, as compared to first-generation DES, in terms of biocompatibility, which reduced their potential of inducing an inflammatory response to the vessel wall. In newer-generation DES, metallic stent platforms became more flexible to increase deliverability and apposition of the stent to the vessel wall in order to tackle the most challenging lesions and anatomies.

While short-term follow-up of second-generation and newer-generation DES are well examined in randomized clinical trials in all-comer patients, there is a lack of data about the long-term safety and efficacy of these stents. In addition, little is known about the performance of newer-generation DES in patients with challenging target lesions.

This thesis evaluated the impact of various stent types on clinical outcome in broad patient populations and in patients with complex lesions. In addition, novel developments as biodegradable polymercoated stents, the bioresorbable vascular scaffold, and the COMBO stent with dual-therapy technology were discussed.

Chapter 1 provided a brief summary of the impact of the development of drug-eluting stents (DES) and the advantages of second- and newer-generation DES on clinical outcome. In addition, it is shown that it is of interest to assess the impact of the implanted DES on clinical outcome in patients of randomized all-comers trials with complex lesions.

\section{Part II: Assessment of broad patient populations}

Chapter $\mathbf{2}$ assessed the 5-year safety and efficacy of two newer-generation DES in randomized TWENTE trial participants. In addition, long-term outcomes of non-enrolled but eligible patients treated with the same DES were compared. Of all 1,391 trial participants, the incidence of the primary endpoint target vessel failure-a composite of cardiac death, target vessel-related myocardial infarction, or clinically indicated target vessel revascularization- was comparable between patients treated with zotarolimuseluting Resolute Integrity versus everolimus-eluting Promus Element stents ( $16.1 \%$ vs $18.1 \%$ ). In addition, the rates of definite stent thrombosis were low in both stent-groups. The 318 non-enrolled eligible patients had significantly higher rates of target vessel failure, while in all eligible patients (i.e. trial participants plus non-enrolled, eligible patients) the rate of target vessel failure was merely slightly higher than in the trial participants only (18.3\% vs $17.1 \%)$. The long-term outcome data from nonenrolled eligible patients support the validity of the randomized trial and represent, together with the randomized trial, a strong case of long-term safety and efficacy for the second-generation drug-eluting stents used.

Chapter 3 provided data about 3-year safety and efficacy of percutaneous coronary interventions (PCI) with newer-generation zotarolimus-eluting Resolute Integrity and everolimus-eluting Promus Element stents in participants in the DUTCH PEERS randomized trial. Of all 1,811 all-comer patients included in the DUTCH PEERS trial, 3-year follow-up data were available in 1,807 (99.8\%) patients. At three-year follow-up, the incidence of target vessel failure was low and similar for both DES (10.7\% vs $10.3 \%$ ). 
In addition, the rate of the individual components of target vessel failure and the rate of definite-orprobable stent thrombosis were low and comparable in both groups. This chapter demonstrated that the safety and efficacy of treating all-comers with newer-generation Resolute Integrity and Promus Element stents were extended up to 3 years.

Chapter 4 evaluated the 3-year clinical outcome in patients with a high risk for adverse clinical events in the DUTCH PEERS trial. Patients were categorized as "high-risk" if they fulfilled at least one of these criteria: (1) diabetes mellitus, (2) previous myocardial infarction, (3) previous coronary revascularization, (4) chronic renal failure, (5) left ventricular ejection fraction $\leq 30 \%$, or (6) age $\geq 75$ years. Of all 1,811 patients who were randomized in the DUTCH PEERS trial, 957 (52.8\%) were categorized as high-risk. The 3-year incidence of the composite endpoint target vessel failure was higher in high-risk patients versus low-to-intermediate risk patients (13.2\% vs 7.5\%). The newer-generation Resolute Integrity and Promus Element stents showed similar results in terms of safety and efficacy for treating high-risk patients (target vessel failure $13.3 \%$ vs $13.1 \%$ ).

Chapter 5 described a propensity score matched analysis of the COMBO stent versus the newergeneration Resolute Integrity and Promus Element stents. The COMBO stent combines an abluminal sirolimus-eluting layer with a luminal endothelial progenitor cell-capturing layer, which aims to promote true vessel healing. After propensity score matching, 771 patients treated with COMBO stents were compared to 771 patients treated with Resolute Integrity or Promus Element stents. At 2-year followup, the rate of target lesion failure was not significantly different between both groups (7.9\% vs $6.4 \%$ ). The rate of definite stent thrombosis was also similar ( $0.8 \%$ vs $0.7 \%$ ). This chapter demonstrated that, in this propensity score matched cohort, no significant differences were observed between patients treated with the COMBO stent versus Resolute Integrity or Promus Element stents.

Chapter 6 presented the 1-year follow-up data of the BIO-RESORT trial. The BIO-RESORT trial is an investigator-initiated, multicenter, randomized trial comparing the safety and efficacy of treating allcomer patients with two novel biodegradable polymer-coated DES (sirolimus-eluting Orsiro stent and everolimus-eluting Synergy stent) versus the established durable polymer newer-generation zotarolimus-eluting Resolute Integrity stent. At 1-year follow-up, both very thin strut DES with dissimilar biodegradable polymer coatings were non-inferior to the durable polymer Resolute Integrity stent. The absence of loss of 1-year safety and efficacy with the use of these two biodegradable polymer-coated stents is a prerequisite before assessing their potential longer-term benefits.

Chapter $\mathbf{7}$ evaluated the safety and efficacy of the Absorb bioresorbable vascular scaffold at 1-year follow-up. This scaffold was developed to address long-term safety issues of metallic DES. However, this meta-analysis shows that during the first year of follow-up, patients treated with bioresorbable vascular scaffolds had a higher incidence of myocardial infarction and scaffold thrombosis as compared to patients treated with durable polymer everolimus-eluting stents. As bioresorbable scaffolds may still prove beneficial, future robust data on long-term clinical outcome will be of paramount importance.

In Chapter 7.1, we discussed potential mechanisms of bioresorbable vascular scaffold thrombosis, in response to a letter to the editor. We concluded that, while the mechanisms of scaffold thrombosis are not yet well known and contemporary drug-eluting metal stents have excellent clinical outcomes in allcomers, the use of bioresorbable scaffolds preferably should be limited to clinical trials. 
Chapter 8 introduced the rationale and study design of the BIONYX trial. The BIONYX trial is an investigator-initiated, international, multicenter, randomized clinical trial. Patients at seven study sites in the Netherlands, Belgium, and Israel were randomly (1:1, stratified for gender and diabetes mellitus) assigned to treatment with the novel newer-generation zotarolimus-eluting durable polymer-coated Resolute Onyx stent versus the sirolimus-eluting bioresorbable polymer-coated Orsiro stent. While both devices are clinically used in all types of patients and lesion anatomies, data from a head-to-head comparison of both stents are not available yet. The aim of the BIONYX trial is therefore to assess the (1-year) safety and efficacy of treating all-comer patients with the durable-polymer coated Resolute Onyx stent versus the biodegradable polymer-coated Orsiro stent (as the reference).

\section{Part III: Contemporary drug-eluting stents for complex lesions}

Chapter 9 assessed a consecutive series of patients who underwent PCl with newer-generation DES for non-ST-segment elevation acute coronary syndromes or stable angina in order to demonstrate the impact of previous coronary artery bypass grafting (CABG) on 5-year clinical outcome. Of all 1,709 patients in the TWENTE trial and non-enrolled TWENTE registry, 202 (11.8\%) had a history of CABG. Patients with previous CABG had significantly higher 5-year rates of cardiac death (10.4\% vs $4.3 \%)$ and target vessel revascularization (25.0\% vs $8.1 \%$ ). These differences remained statistically significant after adjustment for differences in baseline characteristics. The target vessel revascularization rate was highest in patients who underwent $\mathrm{PCl}$ of obstructed vein grafts. Knowledge of the safety but increased risk of repeat revascularization following $\mathrm{PCl}$ in patients with previous CABG will be relevant to cardiologists and other physicians involved in heart team discussions and informed consent.

Chapter 10 provided data about the 2-year clinical outcome following treatment of bifurcated target lesions in all-comer patients treated with newer-generation DES in the DUTCH PEERS trial. Of the 1,811 trial participants, 465 (25.7\%) were treated for at least one bifurcated lesion. The incidence of target vessel failure was not statistically significantly different between both groups. Target vessel myocardial infarction was more common in patients with bifurcation lesions (3.5\% vs 1.6\%), but after multivariate analysis, bifurcation treatment was found not to be an independent predictor of target vessel myocardial infarction. Among patients with bifurcation lesions, we found no impact of DES-type, side-branch size, or final kissing-balloon inflation on various clinical endpoints. Periprocedural myocardial infarction was more common among patients with bifurcation lesion treated with a two-stent technique. Our findings suggest that the flexible newer-generation DES used are efficacious and safe for treating bifurcated target lesions.

Chapter 11 demonstrated the impact of treatment of true bifurcation lesions on 3-year clinical outcomes. True bifurcation lesions are characterized by the obstruction of both the main vessel and side-branch. Patients treated with second-generation Resolute or Xience $V$ stents for true bifurcation lesions ( $n=115$ ) had somewhat higher adverse events rates than patients with non-true bifurcation lesions ( $n=171)$, but dissimilarities did not reach statistical significance (20.0\% vs 11.7\%). Up to 3-year follow-up, the vast majority of patients in both groups remained free from chest pain. 
Chapter 12 evaluated the hypothesis that, in patients undergoing PCl of bifurcated lesions with newergeneration DES, clinical event risk may be higher if these lesions are severely calcified. We performed a patient-level pooled analysis of TWENTE and DUTCH PEERS randomized trials. Of 827 patients with bifurcation lesions, 168 (20.3\%) had at least one severe target lesion calcification. These patients were older, more often diabetics and more often underwent lesion pretreatment. No significant betweengroup difference in the composite endpoint target vessel failure was observed at 2-year follow-up (11.9\% vs $10.2 \%)$. The rates of definite-or-probable stent thrombosis were low and similar $(0.6 \%$ vs $0.9 \%)$. In conclusion, this chapter demonstrated that the presence of severe target lesion calcification among all-comers treated with newer-generation DES in bifurcation lesions was not associated with significant increase in adverse event risk.

Chapter 13 provided insight in the impact of small vessel treatment on 2-year clinical outcome in patients treated with newer-generation DES. Of 1,811 DUTCH PEERS trial participants, 798 (44.1\%) were treated in at least one small vessel (diameter $<2.50 \mathrm{~mm}$ ). The rate of target lesion failure was significantly higher in patients treated in small vessels. Multivariate analysis with propensity score adjustment demonstrated that treatment of small vessel-lesions independently predicted target lesion failure at 2-year follow-up (hazard ratio 1.60). Patients treated with Resolute Integrity and Promus Element stents had similar rates of the composite endpoint target lesion failure ( $9.9 \%$ vs $9.1 \%$ ). Patients whose smallest target vessel was $<2.25 \mathrm{~mm}$ had similar target lesion failure rates as patients whose smallest target vessel was 2.25 to $<2.50 \mathrm{~mm}$. Patients treated in vessels no smaller than 2.50 to $<3.00$ $\mathrm{mm}$ and patients treated in vessels $\geq 3.00 \mathrm{~mm}$ had lower target lesion failure rates than patients treated in smaller sized vessels $(2.50 \mathrm{~mm})$. Our data suggest that, with newer-generation DES, a vessel diameter $<2.50 \mathrm{~mm}$ is a suitable threshold to identify small target vessels.

Chapter 14 assessed the impact of severe lesion calcification on clinical outcome in patients with stable angina who underwent PCI with newer-generation DES. Of all patients in the TWENTE and DUTCH PEERS randomized trials, 1,423 were treated for stable angina. A total of 342 (24.0\%) patients were treated for at least one severely calcified target lesion. At 2-year follow-up, the rate of target vessel failure was significantly higher among patients with at least one severely calcified lesion (25.7\% vs $20.4 \%$ ). Multivariate analysis demonstrated that severe calcification was an independent predictor of reaching the primary composite endpoint target vessel failure at 2-year follow-up. This difference was restricted to the first year of follow-up. For therapeutic decision-making, information on the performance of newer-generation DES in patients with severe lesion calcification should be taken into account.

Chapter 15 described the influence of severe lesion calcification in patients who presented with an acute coronary syndrome and were treated with newer-generation DES in the TWENTE and DUTCH PEERS trials. Of 1,779 patients presenting with an acute coronary syndrome, 340 (19.1\%) were treated in at least one severely calcified lesion. The rate of target vessel failure was at 2-year followup significantly higher in patients with severely calcified target lesions ( $12.4 \%$ vs $7.0 \%$ ). Multivariate analysis demonstrated that severe lesion calcification was an independent risk factor of target vessel failure. This chapter demonstrated that, despite the improvements in DES, the presence of a severely calcified coronary target lesion is still a predictor of worse outcome.

Chapter 16 evaluated, in a patient-level pooled, propensity score adjusted analysis of TWENTE and DUTCH PEERS, the impact of stent type (Xience $V$ versus Promus Element) on 3-year clinical outcome 
in patients with severely calcified target lesions. The Promus Element everolimus-eluting stent uses the same coating and drug as the Xience $\mathrm{V}$, but its stent backbone has a novel, flexible design and is made from a platinum-chromium alloy that increases radial force and angiographic visibility. At 3-year follow-up, the rate of target vessel myocardial infarction was significantly higher in patients treated with Xience $V$ versus Promus Element stents (9.1\% vs $2.0 \%$ ). After propensity score adjustment, Promus Element use was still associated with a lower risk of target vessel myocardial infarction. Cardiac death and revascularizations were not significantly different between both groups. This chapter demonstrated that, while certain stents may be safe and efficacious in a broad patient population with various types of target lesions, they may reveal a potential higher adverse event risk only in certain high-risk subsets.

Chapter 17 conducted a general discussion of the findings of this thesis. In addition, we outlined future perspectives. 


\section{Conclusions}

Drug-eluting stents are widely used in daily clinical practice. They are still subject to refinement in order to further optimize clinical outcome. Second-generation DES and newer-generation DES have shown excellent outcomes in terms of safety and efficacy both at short-term and long-term follow-up. With the improvements of stents and the overall relatively low rates of adverse events, it is becoming increasingly difficult to demonstrate a benefit of stent refinement. Therefore, it may be of interest to focus the assessment on high-risk patients or patients with complex lesions. High-risk patients can be defined according to previous studies and were shown, in the randomized DUTCH PEERS trial, to have increased rates of target vessel failure at 3-year follow-up, but there was no between-stent difference in clinical outcome. No difference in clinical outcome was observed following treatment of bifurcated versus non-bifurcated target lesions; however, patients with a history of coronary bypass surgery, patients treated for at least one lesion in a small vessel $(<2.50 \mathrm{~mm})$, and patients with at least one severely calcified target lesion had significant higher adverse event rates than patients without these complex lesion characteristics. A sub-analysis of the patient-level pooled data of TWENTE and DUTCH PEERS showed that, in patients with severely calcified target lesions, the 3-year risk of target vessel myocardial infarction was lower after implantation of newer-generation Promus Element stents versus second-generation Xience $V$ stents, while other outcome parameters showed no significant betweenstent difference. While this was a propensity-adjusted analysis and not a randomized controlled trial, and the results should be considered hypothesis generating only, the analysis suggests that it may be valuable to investigate the potential benefits of stent refinements in subgroups of selected high-risk patients.

In parallel with the refinement of durable-polymer coated DES, stents with biodegradable coatings were developed. The 1-year outcome data of the BIO-RESORT trial show non-inferiority of two biodegradable polymer-coated DES as compared to the newer-generation Resolute Integrity stent in treating a complex all-comers population. The absence of loss of 1-year safety and efficacy with the use of biodegradable polymer-coated DES is a prerequisite before assessing their potential longer-term benefits.

The latest technical development is a bioresorbable vascular scaffold that aims at providing only a finite period of vascular support after device implantation. Our meta-analysis showed that during the first year of follow-up, patients treated with bioresorbable vascular scaffolds had a significantly higher incidence of myocardial infarction and scaffold thrombosis as compared to patients treated with durable polymercoated metallic DES. The mechanisms of scaffold thrombosis are still a matter of further investigation. Contemporary DES have shown excellent clinical outcomes, including very low stent thrombosis rates, in all-comer patients and high-risk patient populations. Therefore, it is reasonable to suggest that the use of current generation bioresorbable scaffolds should preferably be limited to clinical studies. 


\section{CHAPTER 19}

Samenvatting en Conclusies

Dankwoord

Portfolio
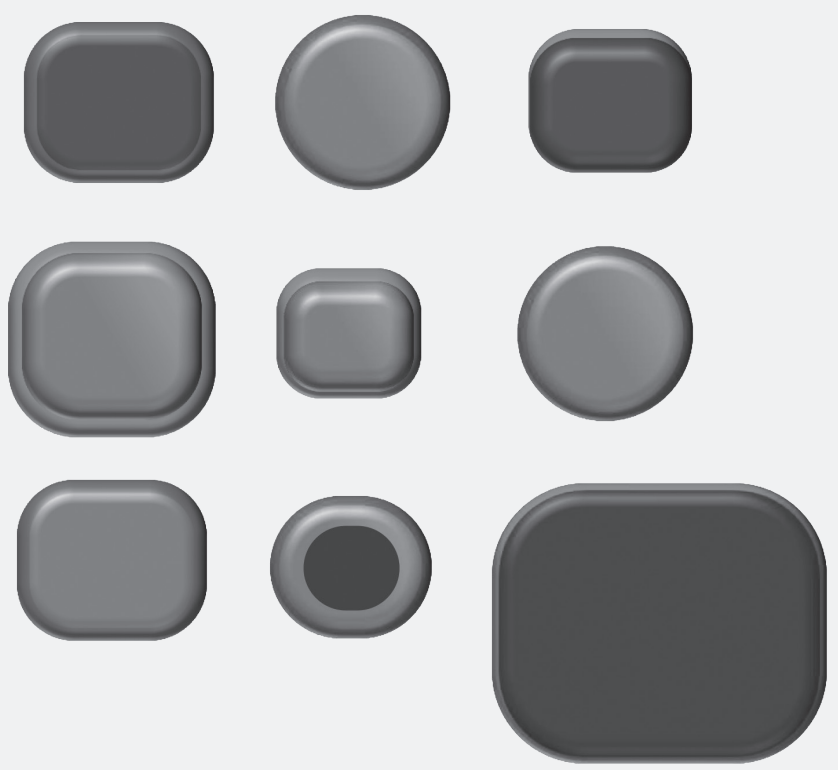


\section{Samenvatting}

Medicijn-afgevende stents (drug-eluting stents; DES) zijn onmisbaar geworden voor de behandeling van coronair lijden. Er vinden continu ontwikkelingen plaats om de veiligheid en effectiviteit van deze stents te optimaliseren. De tweede generatie DES zijn ten opzichte van de eerste generatie DES verbeterd op het gebied van biocompatibiliteit, waardoor zij minder invloed hebben op de inductie van een inflammatoire respons in de vaatwand. Bij de nieuwe-generatie stents is de flexibiliteit van de stent platforms verbeterd, waardoor de plaatsbaarheid en de hechting van de stents aan de vaatwand aanzienlijk is verbeterd. Dit kan voornamelijk van voordeel zijn bij de behandeling van coronair lijden waarbij er sprake is van een uitdagende anatomie en/of complexe laesies.

Terwijl de korte-termijn follow-up van tweede- en nieuwe generatie stents goed zijn onderzocht in gerandomiseerde studies in een brede groep van patiënten, is er nog onvoldoende informatie beschikbaar over de lange termijn veiligheid en effectiviteit van deze stents. Daarnaast is er nog weinig bekend over de prestaties van nieuwe-generatie DES bij patiënten met complexe laesies.

Dit proefschrift evalueert de impact van verschillende stents op de klinische uitkomst in zowel een algemene patiënten populatie als in patiënten die complexe laesies hebben. Daarnaast zullen nieuwe types stents, zoals stents met biologisch afbreekbare polymeren, geheel oplosbare scaffolds en de COMBO stent die van een nieuwe soort technologie gebruik maakt bediscussieerd worden.

Hoofdstuk 1 geeft een korte samenvatting over de ontwikkelingen van DES en de voordelen die tweedeen nieuwe-generatie DES op klinische uitkomst kunnen hebben. Daarnaast wordt laten zien dat het interessant is om de impact van de geïmplanteerde stent op de klinische uitkomst te onderzoeken in hoog-risico patiënten en patiënten met complexe laesies.

\section{Deel II: Onderzoek in brede patiënten populaties}

Hoofdstuk 2 laat de 5-jaar follow-up zien van de gerandomiseerde TWENTE trial, waarbij de veiligheid en effectiviteit van twee nieuwe-generatie DES zijn vergeleken. Daarnaast zijn de lange termijn uitkomsten van patiënten die geschikt waren voor deelname aan de TWENTE trial, maar die niet geïncludeerd zijn onderzocht. In totaal zijn 1,391 patiënten geïncludeerd in de TWENTE trial. De incidentie van het primaire eindpunt "target vessel failure", een gecombineerd eindpunt bestaande uit cardiale dood, myocard infarct en target vessel-gerelateerde revascularisatie, was gelijk onder patiënten die met de zotarolimus-afgevende stent versus de everolimus-afgevende stent behandeld werden ( $16.1 \% \mathrm{vs}$. 18.1\%). Definitieve stent trombose kwam bij beide stents weinig voor. Target vessel failure kwam vaker voor onder de 318 patiënten die niet geïncludeerd, maar wel geschikt waren voor inclusie. Als je de populatie van deelnemers aan de TWENTE trial en de niet-geïncludeerden bij elkaar optelt, dan was het totale percentage target vessel failure slechts licht verhoogd vergeleken met het percentage target vessel failure bij alleen de deelnemers van de TWENTE trial optrad (18.3\% vs. 17.1\%). De lange termijn uitkomsten van de niet-geïncludeerde patiënten bevestigt de validiteit van de gerandomiseerde trial en representeert samen met de studie resultaten een sterk argument voor lange termijn veiligheid van de tweede-generatie DES die gebruikt zijn. 
Hoofdstuk 3 presenteert data betreffende de driejaars veiligheid en effectiviteit van stent plaatsing met nieuwe generatie zotarolimus-afgevende Resolute Integrity en everolimus-afgevende Promus Element stents in de gerandomiseerde DUTCH PEERS trial. Van alle 1,811 patiënten die geïncludeerd zijn in de DUTCH PEERS trial is driejaars follow-up beschikbaar in 1,807 (99.8\%) patiënten. Na drie jaar follow-up is het optreden van target vessel failure gelijk voor beide stentgroepen (10.7\% vs. 10.3\%). Bij de verschillende componenten van het gecombineerde eindpunt target vessel failure wordt ook geen verschil gezien, evenals dat er geen verschil wordt gezien in het percentage definitieve-ofwaarschijnlijke stent trombose. Dit hoofdstuk laat zien dat nieuwe generatie Resolute Integrity en Promus Element stents veilig en effectief zijn voor het behandelen van obstructief coronair lijden in een all-comer patiënten populatie.

Hoofdstuk 4 evalueert de klinische uitkomsten na drie jaar bij patiënten van de DUTCH PEERS trial die een verhoogd risico hebben op nadelige klinische uitkomsten. Patiënten vallen onder de hoogrisico categorie als ze aan tenminste één van de volgende voorwaarden voldoen: (1) diabetes mellitus, (2) myocard infarct in de voorgeschiedenis, (3) coronaire revascularisatie in de voorgeschiedenis, (4) chronische nierinsufficiëntie, (5) linker ventriculaire ejectie fractie $\leq 30 \%$, of (6) leeftijd $\geq 75$ jaar. Van alle 1,811 gerandomiseerde patiënten in de DUTCH PEERS trial kunnen 957 (52.8\%) als hoog-risico patiënten geclassificeerd worden. De 3-jaars incidentie van het gecombineerde eindpunt target vessel failure is hoger in hoog-risico patiënten dan in patiënten met een laag-tot-intermediair risico (13.2\% vs. 7.5\%). De veiligheid en effectiviteit van de nieuwe generatie Resolute Integrity en Promus Element stents in hoog risico patiënten is vergelijkbaar (target vessel failure 13.3\% vs. 13.1\%).

Hoofdstuk 5 beschrijft een propensity score gematchte analyse waarin de COMBO stent met een gecombineerde populatie van patiënten vergeleken is die behandeld waren met de nieuwe generatie Resolute Integrity en Promus Element stents. De COMBO stent combineert een abluminale, sirolimusafgevende laag met een luminale endotheel progenitorcel-bevattende laag, wat als doel heeft om herstel van de vaatwand na stentplaatsing te bevorderen. Na propensity score matching is een populatie van 771 patiënten die behandeld zijn met de COMBO stent en 771 patiënten die behandeld zijn met de Resolute Integrity of Promus Element stent met elkaar vergeleken. Twee jaar follow-up toont geen significant verschil in target laesie failure tussen beide groepen (7.9\% vs. 6.4\%). Definitieve stent trombose is ook vergelijkbaar tussen beide groepen (0.8\% vs. $0.7 \%$ ). Dit hoofdstuk laat zien dat, in dit gematchte cohort, geen significant verschil in klinische uitkomst is geobserveerd tussen patiënten behandeld met de COMBO stent versus patiënten behandeld met nieuwe generatie Resolute Integrity of Promus Element DES.

Hoofdstuk 6 toont de 1 jaar follow-up van de BIO-RESORT trial. De BIO-RESORT trial is een onderzoeker geïnitieerde, multicenter, gerandomiseerde trial met als doel de veiligheid en effectiviteit van twee nieuwe DES met biologisch afbreekbare polymeer coatings (sirolimus-afgevende Orsiro stent en everolimus-afgevende Synergy stent) te vergelijken met de nieuwe-generatie Resolute Integrity stent met permanente polymere coating. Na 1-jaar follow-up zijn beide biologisch afbreekbare polymeer coating stents niet-inferieur aan de Resolute Integrity stent. Dat er na 1 jaar geen verslechterde veiligheid en effectiviteit gezien wordt is een belangrijk feit voordat de mogelijke lange termijn voordelen van deze stents onderzocht worden. 
Hoofdstuk 7 evalueert de veiligheid en effectiviteit van de Absorb biologisch afbreekbare vasculaire scaffold na één jaar follow-up. Deze scaffold is ontwikkeld met de gedachte de lange-termijn veiligheidsaspecten van metalen DES te verbeteren. Echter, deze meta-analyse laat zien dat gedurende het eerste jaar van follow-up de patiënten die behandeld zijn met biologisch afbreekbare scaffolds een hogere incidentie hebben van myocard infarcten en scaffold trombose in vergelijking tot patiënten die met everolimus-afgevende stents met permanente polymeren behandeld zijn. Aangezien het voordelige effect van de scaffold mogelijk pas op de langere termijn zichtbaar is, is toekomstige langetermijn follow-up van deze scaffold erg belangrijk.

In hoofdstuk 7.1 discussiëren we over de mogelijke mechanismen van trombose van biologisch afbreekbare vasculaire scaffolds. Wij concluderen dat, gezien het feit dat het mechanisme van scaffold trombose nog niet volledig begrepen wordt en moderne metalen DES excellente uitkomsten laten zien in all-comers, biologische afbreekbare scaffolds bij voorkeur alleen gebruikt zouden moeten worden in klinische trials.

Hoofdstuk 8 introduceert de rationale en studie design van de BIONYX trial. De BIONYX trial is een onderzoek geïnitieerde, internationale, multicenter, gerandomiseerde klinische studie. Patiënten van 7 studiecentra uit Nederland, België en Israël zullen worden gerandomiseerd (1:1, gestratificeerd voor geslacht en diabetes mellitus) naar behandeling met de nieuwste-generatie zotarolimus-afgevende permanente polymeer-coating Resolute Onyx stent of naar behandeling met de sirolimus-afgevende biologisch afbreekbare polymeer-coating Orsiro stent. Terwijl beide stents al gebruikt worden voor de behandeling van diverse patiënten met verschillende soorten laesies, is er nog nooit een vergelijking uitgevoerd tussen deze beide stents. De doelstelling van de BIONYX trial is dan ook te onderzoeken of de veiligheid en effectiviteit na 1 jaar vergelijkbaar is in een all-comer patiënten populatie die behandeld wordt met de permanent polymeer-coating Resolute Onyx stent versus de biologisch afbreekbare polymeer-coating Orsiro stent (als referentie).

\section{Deel III: Medicijn-afgevende stents voor de behandeling van complexe laesies}

Hoofdstuk 9 onderzoekt een opeenvolgende serie aan patiënten die vanwege stabiele angina pectoris of een niet-ST-elevatie myocard infarct een $\mathrm{PCl}$ hebben ondergaan met plaatsing van een tweede-generatie DES. Het doel is om de impact van een coronaire bypass operatie (CABG) in de voorgeschiedenis te bepalen op de 5-jaar uitkomst na PCI. Van alle 1,709 patiënten die hebben meegedaan aan de TWENTE trial en die niet geïncludeerd zijn maar gevolgd zijn in de non-enrolled TWENTE registratie, hebben 202 (11.8\%) patiënten een CABG in de voorgeschiedenis. Cardiale dood (10.4\% vs. 4.3\%) en target vessel revascularisaties (25.0\% vs. $8.1 \%$ ) treden na 5 -jaar vaker op bij patiënten met een bypass operatie in de voorgeschiedenis. Deze verschillen blijven bestaan nadat er voor verschillen in patiënt- en laesie karakteristieken is gecorrigeerd. Target vessel revascularisatie kwam het vaakst voor in patiënten die een $\mathrm{PCl}$ ondergingen van een venegraft. Wetenschap over de veiligheid, maar het verhoogde risico op herhaalde revascularisaties bij patiënten met een bypass operatie in de voorgeschiedenis die een PCl ondergaan is belangrijk voor cardiologen en andere medici betrokken bij het hartteam en is belangrijk voor de informed consent procedure. 
Hoofdstuk 10 geeft data weer van de klinische uitkomsten twee jaar na behandeling van bifurcatielaesies in een all-comer patiënten populatie van de DUTCH PEERS trial, allen behandeld met nieuwe-generatie DES. Van alle 1,811 patiënten die geïncludeerd waren in de trial hebben 465 (25.7\%) ten minste één bifurcatielaesie waarvoor zij behandeld zijn. De incidentie van target vessel failure was gelijk voor patiënten met - en patiënten zonder bifurcatielaesies. Target vessel myocard infarcten kwamen vaker voor in de bifurcatie-groep (3.5\% vs. 1.6\%). Na multivariate analyse bleek echter dat behandeling van een bifurcatie laesie geen onafhankelijk voorspeller voor het optreden van target vessel myocard infarcten was. Als alleen naar de groep patiënten gekeken werd die voor ten minste één bifurcatielaesie behandeld werden, dan was er geen impact van type DES, het formaat van de zijtak, of het wel of niet gebruiken van kissing-balloon inflatie op diverse klinische eindpunten. Periprocedureel myocard infarct kwam vaker voor bij patiënten die met twee stents behandeld werden ten opzichte van patiënten die met één stent behandeld werden. Onze bevindingen suggereren dat de flexibele nieuwe-generatie DES veilig en effectief zijn voor de behandeling van bifurcatielaesies.

Hoofdstuk 11 laat de impact zien van een echte bifurcatie laesie op 3-jaar klinische uitkomst. Echte bifurcatielaesies zijn laesies waarbij zowel het hoofdvat als het zijvat aangedaan zijn. Patiënten die met tweede-generatie stents behandeld werden voor echte bifurcatie-laesies $(n=115)$ hadden een wat hoger percentage events dan patiënten die behandeld werden voor niet-echte bifurcatielaesies $(n=171)$. Dit verschil was echter niet statistisch significant (20.0\% vs. $11.7 \%)$. Tot aan 3-jaar follow-up was het merendeel van de patiënten in beide groepen vrij van klachten van pijn op de borst.

Hoofdstuk 12 evalueert de hypothese dat patiënten die een bifurcatielaesie hebben en behandeld worden met een nieuwe-generatie DES, een hoger percentage events hebben als er ook nog sprake is van een ernstige calcificatie in die bifurcatielaesie. Om deze hypothese te toetsen hebben we alle patiënten van de TWENTE en DUTCH PEERS trials die voor tenminste één bifurcatie laesie behandeld werden onderzocht. Van de 827 patiënten met een bifurcatie laesie hadden 168 (20.3\%) van de patiënten tevens ernstige calcificaties in de bifurcatie laesie. Patiënten met ernstige calcificaties waren ouder, hadden vaker diabetes mellitus en de laesies werden vaker voorbehandeld tijdens de $\mathrm{PCl}$ procedure. Het samengestelde eindpunt target vessel failure was niet significant verschillend na 2-jaar tussen beide groepen (11.9\% vs. 10.2\%). Het percentage definitieve-of-waarschijnlijke stent trombose was ook laag en gelijk (0.6\% vs. 0.9\%). Dit hoofdstuk laat zien dat de aanwezigheid van ernstige calcificatie van de bifurcatie laesie geen significante verhoging gaf van het risico op nadelige events bij patiënten die met nieuwe-generatie DES behandeld zijn.

Hoofdstuk 13 geeft inzicht in de impact van het behandelen van kleine vaten op resultaten na 2-jaar bij patiënten die behandeld zijn met nieuwe-generatie DES. Van de 1,811 deelnemers aan de DUTCH PEERS trial zijn 798( 44.1\%) van de patiënten behandeld in tenminste één klein vat (diameter $<2.50$ mm). Het gecombineerde eindpunt target laesie falen was significant hoger bij patiënten die in tenminste één klein vat behandeld werden. Multivariate analyse met propensity score correctie laat zien dat het behandelen van een klein vat een onafhankelijk voorspeller is voor het optreden van target laesie falen na 2-jaar follow-up (hazard ratio 1.60). Patiënten behandeld met Resolute Integrity stents en patiënten behandeld met Promus Element stents ervaren even vaak target laesie falen (9.9\% vs. 9.1\%). Patiënten die het kleinst behandelde vat $<2.25 \mathrm{~mm}$ hebben, hebben een vergelijkbaar aantal events als patiënten met het kleinst behandelde vat tussen de 2.25 en $2.50 \mathrm{~mm}$. Patiënten die in vaten behandeld worden 
die niet kleiner zijn dan 2.50 tot $<3.00 \mathrm{~mm}$ en patiënten die enkel behandeld worden in vaten $\geq 3.00 \mathrm{~mm}$ hebben minder vaak target lesion failure. Deze data suggereren dat bij gebruik van nieuwe-generatie DES, een vaatdiameter van $<2.50 \mathrm{~mm}$ een goed afkappunt zou kunnen zijn om een klein vat te definiëren. Hoofdstuk 14 onderzoekt de impact van ernstige laesie calcificaties op de klinische uitkomst bij patiënten die zich presenteren met stabiele angina pectoris en een $\mathrm{PCl}$ ondergaan met plaatsing van een nieuwe-generatie DES. Van alle patiënten van de TWENTE en DUTCH PEERS trial zijn in totaal 1,423 patiënten behandeld voor stabiele angina pectoris. Van deze patiënten hebben 342 (24.0\%) ernstige calcificaties in tenminste één laesie. Na 2-jaar follow-up, is de incidentie target vessel falen significant hoger in de groep die tenminste één ernstige laesie calcificatie had ( $25.7 \%$ vs. $20.4 \%$ ). Multivariate analyse laat zien dat ernstige laesie calcificatie een onafhankelijke voorspeller is van het optreden van het primaire eindpunt target vessel falen na 2-jaar follow-up. Het verschil komt voort uit een verschil in het eerste jaar. De informatie over de resultaten van $\mathrm{PCI}$ met nieuwe-generatie DES bij patiënten met ernstige laesie calcificaties kan meegenomen worden bij het maken van therapeutische beslissingen.

Hoofdstuk 15 beschrijft de invloed van ernstige calcificaties bij patiënten van de TWENTE en DUTCH PEERS trial die zich presenteren met een acuut coronair syndroom waarvoor ze behandeld worden met nieuwe-generatie DES. Van de 1,779 patiënten met een acuut coronair syndroom hebben 340 (19.1\%) een behandeling ondergaan in tenminste één ernstig gecalcificeerde laesie. Target vessel falen kwam vaker voor bij patiënten met ernstige laesie calcificaties (12.4\% vs. 7.0\%). Multivariate analyse laat zien dat ernstige laesie calcificatie een onafhankelijke voorspeller is voor het optreden van target vessel falen. Dit hoofdstuk laat zien dat, ondanks de verbeteringen in DES, de aanwezigheid van ernstige calcificaties nog steeds een voorspeller is van een slechtere uitkomst.

Hoofdstuk 16 evalueert in een gepoolde database van deelnemers aan de TWENTE en DUTCH PEERS trials de impact van het type stent dat gebruikt is (Xience $V$ versus Promus Element) op 3-jaar klinische uitkomst bij patiënten met ernstige calcificaties. De Promus Element stent maakt gebruik van dezelfde coating als de Xience $V$ stent, maar heeft een nieuw flexibel stent design gemaakt van platinumchromium, waardoor de radiale kracht en de angiografische zichtbaarheid van deze stent moet zijn verbeterd. Na 3-jaar follow-up wordt gezien dat er na behandeling met de Promus Element stent minder vaak target vessel myocard infarcten op getreden zijn dan na behandeling met Xience $V$ stents $(2.0 \% \mathrm{vs}$. 9.1\%). Na propensity score correctie is gebruik van de Promus Element nog steeds geassocieerd met een lager risico op target vessel gerelateerde myocard infarcten. Wat betreft cardiale dood en aantal revascularisaties wordt er geen verschil gezien tussen beiden. Dit hoofdstuk laat zien dat bepaalde stents mogelijk een hoger risico op nadelige uitkomsten kan laten zien in bepaalde hoog risico groepen, terwijl de stents wel veilig en effectief gevonden zijn in een brede patiëntenpopulatie met verschilende type laesies.

Hoofdstuk 17 bediscussiëren we kort de bevindingen van dit proefschrift en geven we de toekomstperspectieven weer. 


\section{Conclusies}

Medicijn-afgevende stents worden veel gebruikt in de dagelijkse klinische praktijk. Er vinden continu ontwikkelingen plaats van deze stents om de klinische uitkomst te verbeteren. De tweede-generatie stents en de nieuwe-generatie stents hebben in termen van veiligheid en effectiviteit beide uitstekende uitkomsten laten zien op zowel de korte- als de lange-termijn follow-up. Door lage hoeveelheden events wordt het moeilijk om voordeel aan te tonen van nieuwe ontwikkelingen van stents boven de al bestaande stents. Om toch voldoende statistische power te bereiken kan het daarom interessant zijn om de focus van het onderzoek te leggen op hoog-risico patiënten of patiënten met complexe laesies. Het is mogelijk om hoog-risico patiënten te definiëren op basis van eerdere studies. In de gerandomiseerde DUTCH PEERS trial wordt laten zien dat volgens die definitie hoog-risico patiënten inderdaad vaker target vessel failure hebben na 3-jaar follow-up, zonder dat er verschil was tussen de twee nieuwe-generatie stents. De klinische uitkomst bleek niet verschillend te zijn tussen patiënten die wel of niet voor bifurcatielaesies behandeld werden. Wel bleek dat patiënten met een bypass operatie in de voorgeschiedenis, patiënten die behandeld zijn in tenminste één klein vat $(<2.50 \mathrm{~mm}$ ) en patiënten die in tenminste één van de behandelde laesies ernstige calcificaties had zitten een hoger risico op adverse event hebben dan patiënten die dit niet hadden. Een sub-analyse van de op patiënten niveau gepoolde data van de TWENTE en DUTCH PEERS trial laat zien dat bij patiënten die behandeld zijn met de nieuwe generatie Promus Element stent na 3-jaar follow-up minder vaak een target vessel myocard infarct geobserveerd werd dan bij patiënten die behandeld zijn met de tweede-generatie Xience $V$ stent. Andere uitkomsten zijn niet verschillend tussen beide groepen. Omdat dit een propensityscore gecorrigeerde analyse is en niet een gerandomiseerde studie, moeten de resultaten met enige voorzichtigheid geïnterpreteerd worden en kan de conclusie alleen als hypothese genererend gezien worden. Echter suggereren deze wel dat het waardevol zou kunnen zijn om potentiële voordelen van nieuwe stent-ontwikkelingen te onderzoeken in een subgroep van patiënten met een hoog risico.

Naast de ontwikkeling van de permanente polymere coatings DES zijn er nu ook stents met biologisch afbreekbare polymere coatings ontwikkeld. In de BIO-RESORT wordt gezien dat na één jaar behandeling met twee nieuwe biologisch afbreekbaar polymeer-coating stents non-inferieur is gebleken aan behandeling met de nieuwe generatie Resolute Integrity stent in een all-comer patiënten populatie. Voordat mogelijke lange termijn voordelen van deze stents onderzocht worden is het belangrijk de veiligheid en effectiviteit na 1 -jaar te beschrijven.

De laatste ontwikkeling die plaatsgevonden heeft is de ontwikkeling van biologisch afbreekbare vasculaire scaffolds. Doelstelling van deze scaffolds is om te voorkomen dat er levenslang een device in het coronaire vat aanwezig blijft. Onze meta-analyse laat zien dat gedurende het eerste jaar follow-up er een significant hoger percentage myocard infarcten en scaffold tromboses optreedt bij patiënten die met een biologisch afbreekbare scaffold behandeld worden ten opzichte van patiënten die met een permanente metalen DES behandeld worden. De mechanismen achter scaffold trombose moeten nog verder onderzocht worden. Moderne DES hebben uitstekende resultaten laten zien, waaronder een laag percentage stent trombose, bij zowel all-comer patiënten populaties als in hoog-risico patiënten groepen. Het is daarom redelijk om te suggereren dat het gebruik van de huidige generatie biologisch oplosbare scaffolds bij voorkeur beperkt zou moeten worden tot klinische studies. 


\section{Dankwoord}

En ja hoor, het is zo ver... mijn proefschrift is klaar. Ik ben er heel erg blij mee!

$\mathrm{Nu}$ was dit proefschrift nooit tot stand gekomen zonder hulp. ledereen die in welke mate dan ook betrokken geweest is bij het tot stand komen van dit proefschrift wil ik dan ook hartelijk bedanken. Een aantal personen wil ik hierbij in het bijzonder noemen.

Allereerst Clemens von Birgelen, mijn promotor. Waar ik in het begin nooit had gedacht dat ik een geschikte PhD-kandidaat zou zijn, heb je mij vanaf het begin af aan het volle vertrouwen gegeven dat het goed zou komen. Dankjewel voor alle tijd, energie, motivatie en vertrouwen die ik de afgelopen jaren heb gekregen! Als ik vrijdagavond wat opstuurde, wist ik dat er maandag vaak al weer een antwoord in mijn mailbox zat waar ik mee vooruit kon. En als het even niet lukte, waren er altijd goede adviezen om weer verder te gaan. Mede daardoor is het gelukt om in de afgelopen periode dit proefschrift tot stand te brengen. Dankjewel!

Dan mijn co-promotor, Carine Doggen. Eens in de twee weken, soms wat minder vaak, stond er een afspraak gepland om de achtergrond en de statistiek van de nieuwe manuscripten door te spreken. Deze momenten heb ik altijd als heel leerzaam ervaren. Het was elke keer zeer verhelderend om de uitgevoerde analyses en de gevonden resultaten ook vanuit een ander oogpunt te bekijken. De discussies die we hebben gevoerd en de gedachtewisselingen over het hoe en waarom van het uitvoeren van bepaalde analyses en de interpretatie hiervan hebben gezorgd dat ik nu ook met een hele andere achtergrond naar wetenschappelijk onderzoek kan kijken. Dankjewel hiervoor!

Vervolgens wil ik alle collega's van het CRE (CardioResearch Enschede) bedanken. Harald, Marlies, Paolo, Marleen, Tim, Linnea, Martina, Renate, Ilona, Jacqueline, Marije, Tamara, Jan, Wendy, Petra, Gera, Henny, Jolande, Magda, Judith en Ben, dank jullie wel voor alle hulp en vooral ook voor alle gezelligheid! Hoewel we eerst verspreid over twee locaties zaten, heb ik het als heel waardevol ervaren dat we later in het nieuwe ziekenhuis in één hok terecht zijn gekomen. Dit is de samenwerking zeker ten goede gekomen. Dank jullie wel voor jullie eindeloze inzet waardoor het mogelijk is om de TWENTE studies uit te voeren.

Marlies, nu hoor jij natuurlijk bij de CRE collega's, maar een aparte vermelding voor jou is wel het minste wat ik kan doen. Wat ben ik blij met jou als maatje bij het onderzoek! Als ik het even niet zag zitten, dan wist je me te motiveren. Als het allemaal onmogelijk leek om af te krijgen, konden we samen de schouders eronder zetten. En als er iets te vieren was, dan grepen wij deze kans altijd aan. Dankjewel voor alle gezelligheid (bij de congressen, maar ook op het CRE), de goede adviezen, de momentjes klagen als het gewoon even niet ging, de motivatie om door te gaan als het een uitzichtloze stapel werk leek, de ontspannen momenten tussendoor, het meedenken over de manuscripten, eigenlijk gewoon alles! 
Job van der Palen en Marjolein Brusse, dank jullie wel voor al jullie statistiek uitleg. Op het moment dat we jullie nodig hadden, konden we altijd snel terecht. Dat heb ik erg op prijs gesteld.

Behalve het CRE in het MST hebben ook heel veel andere medewerkers uit het MST meegewerkt aan de studies. Ik wil dan ook alle (interventie)cardiologen, hartcatherisatie laboranten, artsassistenten, verpleegkundig specialisten, verpleegkundigen, secretaresses en iedereen die verder heeft meegewerkt aan het onderzoek bedanken voor de enorme inzet en hulp. Zonder jullie allemaal kunnen de TWENTE studies niet lopen.

Behalve in het MST hebben ook veel medewerkers van externe centra meegewerkt aan onze studies. Ik wil dan ook graag alle medewerkers bedanken van de cardiologie in het Treant Zorggroep locatie Emmen, Rijnstate ziekenhuis te Arnhem, Haga ziekenhuis in Den Haag, Medisch Centrum Alkmaar in Alkmaar, Albert Schweitzer ziekenhuis te Dordrecht, Virga Jessa Hospital in Hasselt, België, University Hospital Charleroi te Charleroi, België en het Rambam Hospital in Haifa, Israël.

Alle co-auteurs op de manuscripten, dank jullie wel voor de bijdrage die jullie hebben geleverd aan de manuscripten. De waardevolle aanvullingen en feedback en natuurlijk ook de aanmoedigingen op de congressen als we iets mochten presenteren heb ik als zeer prettig ervaren.

Behalve al deze mensen die direct vanuit het onderzoek betrokken waren bij mijn promotietraject, zijn er ook een heleboel mensen om me heen die me de hele onderzoeksperiode hebben gesteund.

Allereerst wil ik mijn paranimfen, Laila van der Heijden en Marianne van der Veen-van Engeland (beter bekend als Ed (;) bedanken. Laila, als grote zus van me ben je altijd een goed voorbeeld voor me geweest. De manier waarop je dingen aanpakt, hoe je zo veel dingen tegelijk weet te combineren en dan ook nog eens zusjestijd tussendoor hebt en daarnaast een promotietraject weet te combineren met je specialisatie... wonderbaarlijk! Dankjewel voor al je motiverende woorden en alle tips die me verder geholpen hebben. In ben echt heel blij dat jij mijn zus bent en dat je mijn paranimf wil zijn.

Ed, het is inmiddels al meer dan 10 jaar geleden dat we elkaar hebben leren kennen. Al vanaf het eerste jaar van de studie zijn we goed bevriend met elkaar. Tijdens het vijfde en zesde studiejaar zagen we elkaar een stuk minder omdat we wat verder uit elkaar woonden. Op het moment dat je belde met de vraag hoe nu verder wist ik het wel.... wat zou het leuk zijn als we uiteindelijk in dezelfde stad aan het werk zouden zijn! En ja hoor, ook jij kwam naar Enschede toe. Ik vind het super dat we elkaar nu regelmatig tegenkomen en gewoon weer dicht bij elkaar in de buurt wonen. Fijn om zo'n maatje te hebben waar je altijd bij terecht kan. Dankjewel!

Pappie en mammie, dankjewel voor de steun die ik van jullie heb gekregen. Jullie staan altijd voor me klaar, of het nu gaat om een telefoontje, even langskomen, verhuizen, of wat dan ook... dat stel ik zo enorm op prijs! Joost, heerlijk om een broertje te hebben waarmee je gewoon op een hele andere manier over onderzoek kan praten. Anneke en Joanne, bedankt voor alle heerlijke kopjes koffie met 
grote melkschuimlaag erop. Ik heb er van genoten om elke keer als er een artikel geaccepteerd was een gebakje met elkaar te eten. In het vervolg vinden we vast een andere goede reden om zo nu en dan een gebakje te eten. Jan Robert, dankjewel voor alle adviezen, steun, motivatie, luisterende oren, hulp en liefde die ik van je gekregen heb. Ik vind je heel erg lief! 


\section{Portfolio}

\section{Curriculum Vitae}

Liefke Clementine van der Heijden werd geboren op 30 juli 1989 te Apeldoorn. In 2007 behaalde zij haar VWO diploma aan de Heemgaard te Apeldoorn, waarna zij datzelfde jaar startte met de studie Geneeskunde aan de Rijksuniversiteit Groningen. In het laatste jaar van de studie volgde zij haar semiarts stage Cardiologie in het Medisch Spectrum Twente te Enschede en verrichte zij daar ook haar wetenschapsstage. Na haar afstuderen in december 2013 is zij begin 2014 gestart als arts-assistent Cardiologie in het Medisch Spectrum Twente te Enschede. Na zes maanden verruilde zij het klinische werk voor een promotietraject bij de interventie-cardiologie onder begeleiding van C. von Birgelen, wat geleid heeft tot dit proefschrift. Zij startte 1 april 2017 met de opleiding Cardiologie onder leiding van de opleiders dr. P.M.J. Verhorst en dr. M.F. Scholten en de eerste twee jaren vooropleiding Interne Geneeskunde onder leiding van dr. W.M. Smit.

\section{Publications}

1. Kalkman DN*, Kok MM*, van der Heijden LC*, Woudstra P, Beijk MA, Tijssen JG, von Birgelen C, de Winter RJ. Clinical outcomes after percutaneous coronary intervention with the COMBO stent versus Resolute Integrity and Promus Element stetns: a propensity-matched analysis. Eurointervention 2017 [Epub ahead of print]. *First, second, and third author contributed equally to this work.

2. Zocca P, van der Heijden LC, Kok MM, Löwik MM, Hartman M, Stoel MG, Louwerenburg JW, de Man FHAH, Linssen GCM, Knottnerus I, Doggen CJM, van Houwelingen KG, von Birgelen C. Clopidogrel or ticagrelor in acute coronary syndrome patients treated with newer-generation drug-eluting stents: CHANGE DAPT. Eurointervention. Accepted.

3. von Birgelen C, van der Heijden LC, Mukete BN. Reply to Letter to the Editor entitled: Bioresorbable stent thrombosis, lactic acid release and Kounis syndrome". Int J Cardiol 2017;247:16.

4. van der Heijden LC, Kok MM, Löwik MM, Danse PW, Jessurun GAJ, Hartmann M, Stoel MG, van Houwelingen KG, Hautvast RWM, Linssen GCM, Doggen CJM, von Birgelen C. Three-year outcome of patients with coronary disease and increased event risk treated with newer-generation drugeluting stents: from the randomized DUTCH PEERS trial. Cardiology 2017;137:207-17.

5. Kok MM, Weernink MGM, von Birgelen C, Fens A, van der Heijden LC, van Til JA. Patient preference for radial versus femoral vascular access for elective coronary procedures: the PREVAS study. Catheter Cardiovasc Interv 2017 [Epub ahead of print].

6. Huisman J, van der Heijden LC, Kok MM, Louwerenburg JW, Danse PW, Jessurun GAJ, de Man FHAH, Löwik MM, Linssen GCM, IJzerman MJ, Doggen CJM, von Birgelen C. Two-year outcome after treatment of severely calcified lesions with newer-generation drug-eluting stents in acute coronary syndromes: a patient-level pooled analysis from TWENTE and DUTCH PEERS. J Cardiol 2017;69:6605.

7. von Birgelen $C^{*}$, van der Heijden LC*, Basalus MW, Kok MM, Sen H, Louwerenburg HW, van Houwelingen KG, Stoel MG, de Man FHAH, Linssen GCM, Tandjung K, Doggen CJM, van der Palen J, Löwik MM. Five-year outcome after implantation of zotarolimus- and everolimus-eluting 
stents in randomized trial participants and nonenrolled eligible patients: a secondary analysis of a randomized clinical trial. JAMA Cardiol 2017;2:268-76. *First and second author contributed equally to this work.

8. van der Heijden LC, Kok MM, Löwik MM, Danse PW, Jessurun GAJ, Hautvast RWM, van Houwelingen KG, Stoel MG, Hartmann M, Linssen GCM, Dogen CJM, von Birgelen C. Three-year safety and efficacy of treating all-comers with newer-generation Resolute Integrity or Promus Element stents in the randomized DUTCH PEERS (TWENTE II) trial. Eurointervention 2017;12:2128-31.

9. von Birgelen C*, Kok MM*, van der Heijden LC*, Danse PW, Schotborgh C, Scholte M, Tjon Joe Gin RM, Somi S, van Houwelingen KG, Stoel MG, de Man FHAH, Louwerenburg JW, Hartmann M, Zocca P, Linssen GCM, van der Palen J, Doggen CJM, Löwik MM. Very thin-strut biodegradable polymer everolimus-eluting and sirolimus-eluting stents versus durable polymer zotarolimus-eluting stents in all-comers with coronary artery disease (BIO-RESORT): a three-arm, randomised, non-inferiority trial. Lancet 2016;388:2607-17 * First, second, and third author contributed equally to this work.

10. van Houwelingen KG, van der Heijden LC, Lam MK, Löwik MM, Louwerenburg JW, Linssen GCM, IJzerman MJ, Doggen CJM, von Birgelen C. Long-term outcome and chest pain in patients with true versus non-true bifrucation lesions treated with second-generation drug-eluting stents in the TWENTE trial. Heart and Vessels 2016;31:1731-39.

11. Huisman J, van der Heijden LC, Kok MM, Danse PW, Jessurun GAJ, Stoel MG, van Houwelingen KG, Löwik MM, Hautvast RWM, IJzerman M, Doggen CJM, von Birgelen C. Impact of severe lesion calcification on clinical outcome of patients with stable angina, treated with newer generation permanent polymer-coated drug-eluting stents: a patient-level pooled analysis from TWENTE and DUTCH PEERS (TWENTE II). Am Heart J 2016;175:121-9.

12. van der Heijden LC, Kok MM, Danse PW, Schramm AR, Hartmann M, Löwik MM, Linssen GCM, Stoel MG, Doggen CJM, von Birgelen C. Small-vessel treatment with contemporary newer-generation drug-eluting stents in all-comers: insights from 2-year DUTCH PEERS (TWENTE II) randomized trial. Am Heart J 2016;176:28-35.

13. Tu S, Westra J, Yang J, von Birgelen C, Ferrara A, Pellicano M, Nef H, Tebaldi M, Murasato Y, Lansky A, Barbato E, van der Heijden LC, Reiber JH, Holm NR, Wijns W. Diagnostic accuracy of fast computational approaches to derive fractional flow reserve from diagnostic coronary $\mathrm{X}$-ray angiography in the international multicenter FAVOR (Functional Assessment by Various FlOw Reconstructions) pilot study. J Am Coll Cardiol Intv 2016;9:2024-35.

14. Mukete BN*, van der Heijden LC*, Tandjung K, Baydoun H, Yadav K, Saleh QA, Doggen CJM, Abi Rafeh N, Le Jemtel TH, von Birgelen C. Safety and efficacy of everolimus-eluting bioresorbable vascular scaffolds versus durable polymer everolimus-eluting metallic stents assessed at 1-year follow-up: a systematic review and meta-analysis of studies. Int J Cardiol 2016;221:1087-94. *First and second author contributed equally to this work.

15. Kok MM, van der Heijden LC, Sen H, Danse PW, Löwik MM, Anthonio RL, Louwerenburg JW, de Man FHAH, Linssen GCM, IJzerman M, Doggen CJM, Maas AH, Mehran R, von Birgelen C. Gender difference in chest pain after implantation of newer generation coronary drug-eluting stents: a patient-level pooled analysis from TWENTE and DUTCH PEERS. J Am Coll Cardiol Intv 2016;9:553-61. 
16. van der Heijden LC, Kok MM, Lam MK, Danse PW, Schramm AR, Jessurun GAJ, Tjon Joe Gin RM, van Houwelingen KG, Hautvast RWM, Linssen GCM, Sen H, Löwik MM, IJzerman MJ, Doggen CJM, von Birgelen C. Bifurcation treatment with novel, highly flexible drug-eluting coronary stents in allcomers: two-year outcome in patients of the DUTCH PEERS trial. Clin Res Cardiol 2016;105:206-15.

17. Lam MK, Sen H, van Houwelingen KG, Löwik MM, van der Heijden LC, Kok MM, de Man FHAH, Linssen GCM, Tandjung K, Doggen CJM, von Birgelen C. Three-year clinical outcome of patients with bifurcation treatment with second-generation Resolute and Xience $V$ stents in the randomized TWENTE trial. Am Heart J 2015;169:69-77.

18. von Birgelen C, van der Heijden LC, Sen H, Löwik MM. Response to a letter to the editor by Tavella et al. Entitled: Post-PCl angina- a new performance measure? J Am Coll Cardiol 2015;8:1640-41.

19. Sen H, Lam MK, Löwik MM, Danse PW, Jessurun GAJ, van Houwelingen KG, Anthonio RL, Tjon Joe Gin RM, Hautvast RWM, Louwerenburg JW, de Man FHAH, Stoel MG, van der Heijden LC, Linssen GCM, IJzerman MJ, Tandjung K, Doggen CJM, von BIrgelen C. Clinical events and patient-reported chest pain in all-comers treated with Resolute Integrity and Promus Element stents: 2-year followup of the DUTCH PEERS (Durable polymer-based stent challenge of Promus Element versus Resolute Integrity) Randomized Trial (TWENTE II). J Am Coll Cardiol Intv 2015;8:889-99.

\section{Oral Presentations}

- Myocardial infarction rates in bifurcation lesions: still elevated after use of contemporary, highly deliverable drug-eluting stents with novel, flexible designs? EuroPCR 2015, Paris.

- Two-year outcome and chest pain in 1,811 all-comer patients, treated for bifurcated versus nonbifurcated lesions with highly deliverable drug-eluting coronary stents. TCT 2015, San Francisco.

- Safety and efficacy of treating all-comers with novel zotarolimus-eluting and everolimus-eluting coronary stents: 3-year outcome of the randomized DUTCH PEERS trial. EuroPCR 2016, Paris.

- Two-year clinical outcome in 1,811 all-comers treated with novel drug-eluting coronary stents in at least one small vessel versus larger sized vessels only. EuroPCR 2016, Paris.

- Small vessel treatment with novel newer-generation drug-eluting stents in all-comers: which threshold is most appropriate for defining 'small coronary vessels'? EuroPCR 2016, Paris.

- Small vessel treatment with contemporary drug-eluting stents: a propensity score adjusted analysis in 1,811 all-comer patients. ESC 2016, Rome.

\section{Poster Presentations}

- Long-term outcome and patient self-reported chest pain following treatment of true bifurcation lesions with second-generation stents in the TWENTE trial. EuroPCR 2015, Paris.

- 3-year clinical outcome of the DUTCH PEERS (TWENTE II) randomized trial: cobalt-chromium zotarolimus-eluting Resolute Integrity versus platinum-chromium everolimus-eluting Promus Element stents in all-comer patients. TCT 2015, San Francisco.

- Clinical relevant chest pain and 3-year clinical outcome in high-risk patients after percutaneous coronary intervention with novel drug-eluting stents: from the randomized DUTCH PEERS trial. ESC 2016, Rome. 
- Platinum-chromium versus cobalt-chromium durable polymer everolimus-eluting stents in calcified lesions: a patient-level pooled, propensity score-adjusted analysis in participants of clinical trials. EuroPCR 2017, Paris. 